\title{
ASSESSMENT OF THE GROUNDWATER PROTECTION PROGRAM Y-12 NATIONAL SECURITY COMPLEX, OAK RIDGE, TENNESSEE
}

\author{
December 2005
}

\author{
Prepared by
}

Groundwater Services, Inc.

Under Subcontract No.4300046269

for the

\section{Environmental Compliance Department Environment, Safety, and Health Division Y-12 National Security Complex Oak Ridge, Tennessee 37831}

\section{Managed by}

BWXT Y-12, L.L.C.

For the U.S. Department of Energy Under Contract No. DE-AC05-00OR22800 


\section{DISCLAIMER}

This report was prepared as an account of work sponsored by an agency of the United States Government. Neither the United States Government nor any agency thereof, nor any of their employees, makes any warranty, express or implied, or assumes any legal liability or responsibility for the accuracy, completeness, or usefulness of any information, apparatus, product, or process disclosed, or represents that its use would not infringe privately owned rights. Reference herein to any specific commercial product, process, or service by trade name, trademark, manufacturer, or otherwise, does not necessarily constitute or imply its endorsement, recommendation, or favoring of the United States Government or any agency thereof. The views and opinions of authors expressed herein do not necessarily state or reflect those of the United States Government or any agency thereof. 


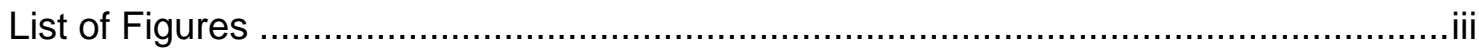

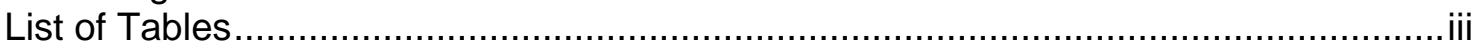

List of Acronyms and Abbreviations .........................................................................

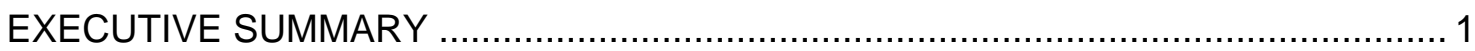

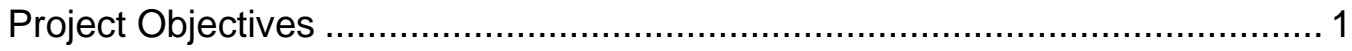

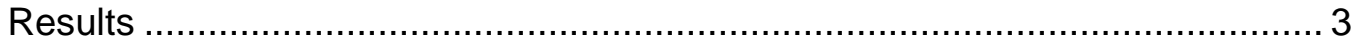

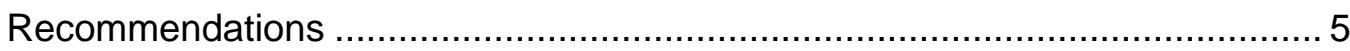

SECTION I QUALITATIVE ASSESSMENT

1.0 INTRODUCTION

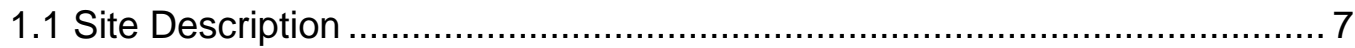

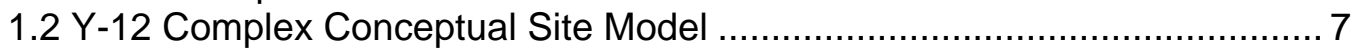

2.0 REGULATORY FRAMEWORK AND MONITORING OBJECTIVES ................... 15

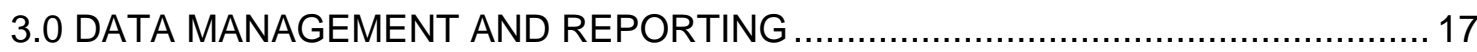

3.1 Monitoring Program Overview............................................................ 17

3.2 Data Management for the GWPP........................................................ 19

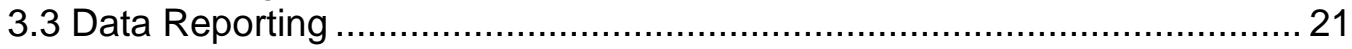

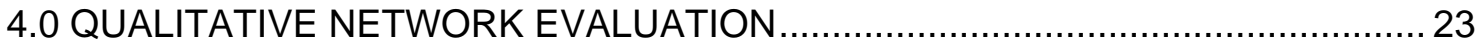

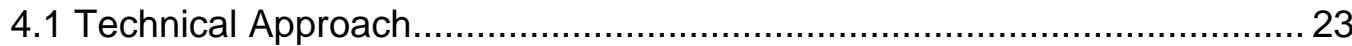

4.2 Decision Logic and Location Scoring …………………...................... 24

SECTION II QUANTITATIVE ASSESSMENT

1.0 INTRODUCTION

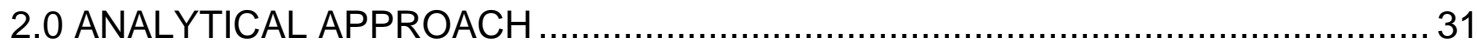

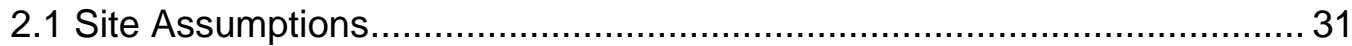

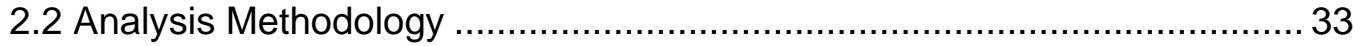

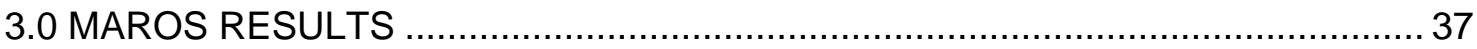

3.1 Bear Creek Hydrogeologic Regime ...................................................... 37

3.2 East Fork Poplar Creek Hydrogeologic Regime ......................................5 51

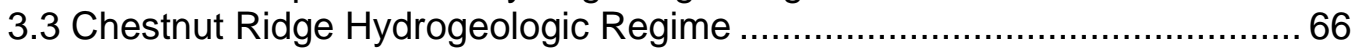

4.0 COMBINED QUALITATIVE AND QUANTITATIVE ANALYSIS ….......................75

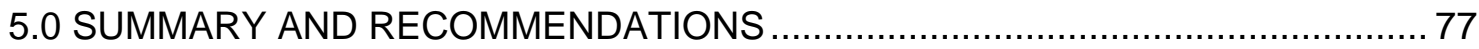

5.1 Monitoring Program Evaluation

5.2 Quantitative Network Evaluation

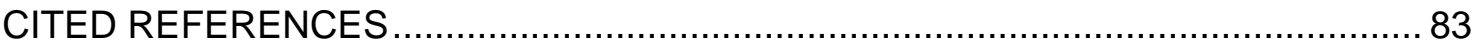




\section{APPENDICES:}

\section{A FIGURES}

B TABLES

C MAROS 2.1 METHODOLOGY

D QUALITATIVE REVIEW AND MAROS REPORTS BY ANALYSIS GROUP Appendix D.1 Bear Creek Regime West S-3

Appendix D.2 Bear Creek Regime Oil Landfarm WMA

Appendix D.3 Bear Creek Regime Bear Creek Burial Grounds

Appendix D.4 East Fork Regime East S-3 Area

Appendix D.5 East Fork Regime Central Y-12 Area

Appendix D.6 East Fork Regime Fuel Station Area

Appendix D.7 East Fork Regime East Y-12

Appendix D.8 Chestnut Ridge Regime West Chestnut Ridge

Appendix D.9 Chestnut Ridge Regime Security Pits Area

Appendix D.10 Chestnut Ridge Regime Landfills V and VII Area

Appendix D.11 Chestnut Ridge Regime East Kerr Hollow Area 


\section{List of Figures}
A.1 MAROS Analysis Sample Locations
A.2 Bear Creek Regime Exceedance of Screening Levels for Priority Constituents
A.3 East Fork Regime Exceedance of Screening Levels for Priority Constituents
A.4 Chestnut Ridge Regime Exceedance of Screening Levels for Priority Constituents
A.5 MAROS Results Bear Creek Regime OLFA and West S-3
A.6 MAROS Results Bear Creek Burial Grounds
A.7 MAROS Results East Fork Regime East S-3 and Central Y-12
A.8 MAROS Results East Fork Regime East Y-12
A.9 MAROS Results Chestnut Ridge Regime
A.10 Well Decision Logic

\section{List of Tables}

B.1 Major Constituents and Screening Levels

B.2 Location Analysis Groups for MAROS Evaluation

B.3 MAROS Results West S3 Area

B.4 MAROS Results Oil Landfarm WMA

B.5 MAROS Results Bear Creek Burial Grounds

B.6 MAROS Results East S-3 Area

B.7 MAROS Results Central Y-12 Area

B.8 MAROS Results Fuel Station Area

B.9 MAROS Results East Y-12 Area

B.10 MAROS Results West Chestnut Ridge

B.11 MAROS Results Chestnut Ridge Security Pits

B.12 MAROS Results Landfills $V$ and VII Areas

B.13 MAROS Results East Chestnut Ridge

B.14 Summary Sampling Recommendations Bear Creek Regime

B.15 Summary Sampling Recommendations East Fork Regime

B.16 Summary Sampling Recommendations Chestnut Ridge Regime 


\section{List of Acronyms and Abbreviations}

\begin{tabular}{|c|c|}
\hline ACO & Analytical Chemistry Organization \\
\hline ADMS & Analytical Data Management System \\
\hline AFCEE & Air Force Center for Environmental Excellence \\
\hline AOI & Area of Interest \\
\hline bgs & Below Ground Surface \\
\hline $\mathrm{BJC}$ & Bechtel Jacobs Company, L.L.C. \\
\hline BWXT & BWXT Y-12, L.L.C. \\
\hline CES & Cost Effective Sampling \\
\hline $\mathrm{cm} / \mathrm{s}$ & Centimeters per Second \\
\hline CY & Calendar Year \\
\hline $11 \mathrm{DCA}$ & 1,1-Dichloroethane \\
\hline 11DCE & 1,1-Dichoroethene \\
\hline 12DCE & 1,2-Dichloroethene \\
\hline CDCE & cis-1,2-Dichloroethene \\
\hline tDCE & trans-1,2-Dichloroethene \\
\hline CERCLA & Comprehensive Environmental Response, Compensation and Liability Act \\
\hline CRSP & Chestnut Ridge Security Pits \\
\hline COC & Constituent of Concern \\
\hline cov & Coefficient of Variation \\
\hline CTET & Carbon Tetrachloride \\
\hline DOE & United States Department of Energy \\
\hline DNAPL & Dense Non-Aqueous Phase Liquid \\
\hline EDDs & Electronic Data Deliverables \\
\hline EM & Environmental Management \\
\hline
\end{tabular}




\begin{tabular}{|c|c|}
\hline EMWMF & Environmental Management Waste Management Facility \\
\hline EQCAS & Environmental Quality Control and Analysis System \\
\hline $\mathrm{ft} / \mathrm{yr}$ & Feet per Year \\
\hline GA & Gross Alpha Activity \\
\hline GB & Gross Beta Activity \\
\hline GIMS & Groundwater Information Management System \\
\hline GIS & Geographic Information System \\
\hline GSI & Groundwater Services, Inc. \\
\hline GWPP & Groundwater Protection Program \\
\hline HSCB & Hypothetical Statistical Compliance Boundary \\
\hline LIMS & Laboratory Information Management System \\
\hline LTM & Long-Term Monitoring \\
\hline LTMO & Long-Term Monitoring Optimization \\
\hline MAROS & Monitoring and Remediation Optimization Software \\
\hline MCES & Modified Cost Effective Sampling \\
\hline$M C L$ & Maximum Contaminant Level \\
\hline MNA & Monitored Natural Attenuation \\
\hline MSL & Mean Sea Level \\
\hline MTBE & tert-Butyl Methyl Ether \\
\hline NAPL & Non-Aqueous Phase Liquid \\
\hline NNSA & National Nuclear Security Administration \\
\hline NPDES & National Pollution Discharge Elimination System \\
\hline PCE & Tetrachloroethene \\
\hline PLSF & Preliminary Location Sampling Frequency \\
\hline
\end{tabular}




\begin{tabular}{|c|c|}
\hline POE & Point of Exposure \\
\hline PRG & Preliminary Remediation Goal \\
\hline QA/QC & Quality Assurance/Quality Control \\
\hline RCRA & Resource Conservation and Recovery Act \\
\hline RI & Remedial Investigation \\
\hline ROD & Record of Decision \\
\hline SAIC & Science Applications International Corporation \\
\hline SF & Slope Factor \\
\hline SMDP & Scientific Management Decision Points \\
\hline SVOCs & Semi-volatile Organic Compounds \\
\hline SWDF & Solid Waste Disposal Facility \\
\hline Tc-99 & Technetium 99 \\
\hline $1,1,1-\mathrm{TCA}$ & 1,1,1-Trichloroethane \\
\hline TCE & Trichloroethene \\
\hline TDEC & Tennessee Department of Environment and Conservation \\
\hline UEFPC & Upper East Fork Poplar Creek \\
\hline USACOE & United States Army Corps of Engineers \\
\hline USEPA & United States Environmental Protection Agency \\
\hline VC & Vinyl chloride \\
\hline VOC & Volatile Organic Compound \\
\hline WMA & Waste Management Area \\
\hline WRRP & Water Resources Restoration Program \\
\hline
\end{tabular}




\section{EXECUTIVE SUMMARY}

The following report contains an assessment of the Y-12 Groundwater Protection Program (GWPP) for the Y-12 National Security Complex at the Oak Ridge Reservation, Tennessee. The GWPP is administered by BWXT Y-12, L.L.C. for the purpose of groundwater surveillance monitoring. After over 20 years of extensive site characterization and delineation efforts, groundwater in the three hydrogeologic areas that comprise the Y-12 Complex requires a long-term monitoring network strategy that will efficiently satisfy surveillance monitoring objectives set forth in DOE Order 450.1. The GWPP assessment consisted of two phases, a qualitative review of the program and a quantitative evaluation of the groundwater monitoring network using the Monitoring and Remediation Optimization System (MAROS) software methodology.

The specific objective of the qualitative section of the review of the GWPP was to evaluate the methods of data collection, management, and reporting and the function of the monitoring network for the $\mathrm{Y}-12$ facility using guidance from regulatory and academic sources. The results of the qualitative review are used to suggest modifications to the overall program that would be consistent with achieving objectives for long-term groundwater monitoring. While cost minimization is a consideration in the development of the monitoring program, the primary goal is to provide a comprehensive strategy to provide quality data to support site decision making during facility operations, long-term resource restoration, and property redevelopment.

The MAROS software is designed to recommend an improved groundwater monitoring network by applying statistical techniques to existing historic and current site analytical data. The MAROS methodology also considers hydrogeologic factors, regulatory framework, and the location of potential receptors. The software identifies trends and suggests components for an improved monitoring plan by analyzing individual monitoring wells in the current monitoring system as well as identifying area-wide trends.

The MAROS analysis was conducted as part of the review of the GWPP. The analysis was conducted to develop the future monitoring strategy for chlorinated solvents, radioactive constituents, metals, and nitrate affected groundwater. Because of the complicated geologic and hydrologic conditions, the MAROS method has been supplemented with results from the qualitative network assessment and decision logic methods to determine an effective monitoring strategy consistent with the unique objectives of the current GWPP.

\section{Project Objectives}

The overall objectives of the assessment of the GWPP included

- Determine if the monitoring network is sufficient to accurately represent plume behavior;

- Determine if any parts of the long-term monitoring program (LTM) are redundant (i.e., do not provide new information);

- Determine if there are any ways to improve the accuracy and reliability of the LTM program; 
- Determine if there any ways to reduce the life-cycle costs of the LTM program without sacrificing accuracy and protectiveness

The qualitative assessment of the GWPP included a review of sample collection, handling, analysis and data management and reporting. Additionally the monitoring network was analyzed by applying qualitative decision logic described in a variety of long-term monitoring guidance documents such as the joint USEPA/USACOE Roadmap to Long-Term Monitoring Optimization (USEPA 2005) and in American Society of Civil Engineers Long-Term Groundwater Monitoring document (ASCE, 2003). Implementation of qualitative methods was guided by site-wide monitoring objectives and historic and current site data. The key objectives of the qualitative analysis included:

- Determining if site characterization and historic data collection efforts support the design of a long-term monitoring optimization (LTMO) process.

- Determine if data management and reporting activities comply with regulatory requirements and efficiently support site management decision making.

- Determine if analytical and well sampling techniques are appropriate and consistent with current regulatory guidance.

- Performing a qualitative evaluation (to support the quantitative evaluation) of how each sample location in the network contributes to each of the following objectives:

o Regulatory compliance;

o Horizontal delineation of the plume;

o Vertical delineation of the plume;

o Monitoring exit pathways;

o Early detection of off-site migration or plume expansion;

o Determination of background water quality;

o Monitoring source behavior.

As part of the quantitative evaluation of the GWPP, the groundwater monitoring network in the vicinity of the Y-12 facility was analyzed by applying the MAROS 2.1 statistical and decision support methodology to historic and current site data. The key objectives of the analysis included:

- Determine overall stability of individual plumes through trend analysis and moment analysis;

- Evaluate individual well constituent concentration trends over time;

- Address adequate and effective sampling through reduction of redundant wells or reduction of sampling effort without information loss;

- Identify areas of higher uncertainty which should be addressed by future sampling events;

- Assess sampling frequency needed to provide sufficient plume stability information; and,

- Compare the current (2004) monitoring strategy with the improved plan suggested by the MAROS methodology combined with qualitative evaluation of the monitoring network. 


\section{Results}

\section{Qualitative Assessment}

- Overall, the GWPP appears to be effectively and efficiently achieving monitoring goals set forth in Department of Energy (DOE) Order 450.1.

- Site Characterization efforts at the Y-12 Complex have an extensive history, and over 1900 sample locations are identified in the analytical database. Based on a qualitative analysis of source areas, geology and transport pathways, the monitoring network currently available is adequate to describe site-wide and offsite affected groundwater and potential transport pathways. There is sufficient data to support development of a LTMO plan.

- Groundwater sampling techniques used by the GWPP are appropriate to the size and scale of the program. The transition to low-flow techniques is supported by USEPA guidance (Puls and Barcelona, 1996) and the use of dedicated pumps and tubing at wells provides confidence in the quality of the data collected from these locations.

- Laboratory methods currently provide data that satisfy data quality objectives, meeting standards for quantization limits, quality assurance/quality control standards and documentation. High quality, reliable analytical data are generally available from 1996 to the present. The historic data set (prior to 1996), may not be entirely comparable to recent data due to changes in groundwater sampling techniques, sample handling and laboratory methods.

- The analytical data management system (ADMS) at the Y-12 Complex is efficient, well designed, and highly documented. The BWXT Groundwater Information Management System (GIMS) provides a high quality data system to support site decision making. The automated data review feature ensures possible errors or changes in plume status are identified early. The GIMS analytical database is thorough and easy to use.

- Site monitoring reports, while well written, may benefit from better quality maps and data visualization instruments. More effort should be directed toward improving the geographic information system (GIS) that links with the excellent analytical database.

\section{Quantitative Assessment}

The MAROS 2.1 sampling optimization software/methodology has been applied to the Y-12 groundwater monitoring well network for data collected during the period January 1996 through December 2004. Because the facility has three main hydrogeologic areas, several constituent classes, and multiple potential source zones, the facility was divided into 11 analysis groups, each analyzed separately. The site-wide results for 443 sample locations have been combined and are summarized below, as recommendations for the entire network. 
- COC Choice: Priority constituents were identified in the MAROS constituents of concern (COC) Choice module. For most site areas, the volatile organic compound (VOC) constituents of greatest interest are tetrachloroethene/perchloroethylene (PCE) and trichloroethene (TCE). Priority inorganic constituents include nitrate and Gross Alpha (GA) and Gross Beta (GB) activity. Metals are a secondary but persistent concern in many areas of the Complex.

- Moment Analysis. The MAROS Moment Analysis module estimates the total dissolved mass (Zeroth Moment), distance of the center of mass from the source (First Moment) and distribution of mass within the plume (Second Moments). Moment analyses indicated that most plumes are classified as having "Stable" or "Decreasing" trends. Stable or decreasing plumes are good candidates for a reduced monitoring effort. Some areas have high variance in the data, indicating that more samples from a consistent set of wells would improve the statistical analysis.

- Well Redundancy: Due to the fractured geology and variety of depths of the wells, the Well Redundancy tool in MAROS could not be used to determine redundant locations (the redundancy tool is based on a two-dimensional analysis for diffuse flow plumes). Wells were recommended for removal from the monitoring program based on the qualitative evaluation and lines of evidence from the statistical trend evaluation and Moment analyses.

- Well Sufficiency: The Well Sufficiency tool was used to identify areas within the plume that may require additional monitoring due to inter-well concentration uncertainty. No new well locations are recommended. An increased monitoring effort (i.e. more frequent sampling) is suggested for some areas along the perimeter of the plume in the area of New Hope Pond and on the northern edge of the East Fork plumes.

- Sampling Frequency: The MAROS well sampling frequency tool uses a Modified Cost Effective Sampling (MCES) method to recommend an optimized sampling schedule for each well in the network. The results of the MCES method recommend moving from quarterly and semi-annual monitoring of all wells to an annual and biennial sampling program. Specific sampling frequency recommendations were developed using both quantitative and qualitative methods and are presented in Tables B.14-16 for each of the Regimes discussed in this document.

- Comparison of the current monitoring program (BWXT, 2004) with the MAROS recommended sampling plan indicates that MAROS suggests including more well locations, but at reduced frequency. The final recommendation includes a list of monitoring locations to review and re-evaluate after two to four years of data collection. The final number and identity of wells in the monitoring program will depend on the number of review wells to be sampled each year. 
Based on the analysis of the current monitoring plan, the recommended long-term monitoring strategy results in a redirection in sampling effort from frequent sampling of a limited number of wells to less frequent sampling of a larger number of wells. This strategy examines more locations, which is consistent with the monitoring objectives articulated in DOE Order 450.1. The improved plan allows site personnel to focus attention on potential problem areas and decrease effort in areas where concentrations are shrinking or not detected. Regions of the plumes with higher rates of concentration change have been assigned higher monitoring frequencies, whereas areas of stability or concentration reduction have a reduced number of sampling points or frequency. The total number of wells can be reduced after a sufficient data set (i.e. four to six samples in a 10 year period) has been accumulated to examine the recent concentration trends.

\section{Recommendations}

\section{Qualitative Assessment}

- Data management, storage, review and reporting activities of the Y-12 GWPP are, excellent. Electronic data management is notable in its efficacy and efficiency. Reports would benefit from stronger data visualization tools. Consider developing stronger links between the analytical database and sophisticated mapping and visualization software.

- Based on examination of the GWPP analytical database, laboratory practices and analyses, particularly since the late 1990's, provide high quality analytical data to support site management decisions. Prior to the 1990's, some apparent outliers were detected in the analytical database, particularly for certain radioactive constituents. Consider reviewing and flagging data that may not be comparable to current sample events.

- The Y-12 Complex has been well characterized with many 'active' well locations, some suitable for constituent delineation and others for hydrologic investigation. Active well locations used to collect chemical analytical data should be clearly distinguished from those used for hydrogeologic data collection in the analytical database.

- The constituent 1,4-dioxane should be included in the analytical program. 1,4Dioxane was used as a stabilizer in commercial 1,1,1-trichloroethane (111TCA) preparations. This constituent (1,4-dioxane) should be analyzed for in areas where 1,1-dichloroethene (11DCE) or 1,1-dichloroethane (11DCA) are detected.

- Sample a consistent suite of constituents at all locations. Because plumes are commingled and many constituents were used or disposed of plant-wide, analysis of all COCs provides information on the distribution of affected groundwater. Target constituents for the entire site include VOCs, metals, nitrate, Gross Alpha (GA) and Gross Beta (GB) activity, and uranium.

- Low-flow sampling techniques are recommended for groundwater sample locations across the site. 


\section{Quantitative Assessment}

- The results of the analysis of the Y-12 GWPP well network indicate that the sampling frequency of most wells can be reduced from quarterly and semiannual monitoring to annual, biennial or even greater time intervals between samples.

- By reducing sample intervals, more locations in the network can be sampled without increasing cost or effort. Sampling of more locations is important at Y-12 due to the diversity of sources and constituent classes and the complex hydrology. Increasing the diversity of sample locations while reducing the frequency of sampling will better fulfill the monitoring objectives set forth in DOE Order 450.1.

- While the current monitoring network had sufficient data to evaluate the efficacy of the network for long-term monitoring, the statistical tools used for the trend analysis could be more definitive with a larger, more consistent data set for many locations that currently have limited data. Before wells can be excluded from the sample network, sufficient data must be available to statistically support elimination of the well. The short-term recommendation is to sample wells with limited recent data for four sampling events and statistically reevaluate the contribution of each well after two years of sampling data has been obtained.

- For all analytical locations, sampling intervals should provide a minimum of four to six sample events in a 10-year time frame.

- Sample a consistent suite of constituents at all locations. Because many plumes are commingled and many constituents are distributed plant-wide, analysis of all COCs provides information on the distribution of affected groundwater. Target constituents for the entire site include VOCs, metals, nitrate, Gross Alpha (GA) and Gross Beta (GB) activity, and uranium.

- No new wells are recommended at this time.

- Evaluation of 443 sample locations resulted in a recommendation for removing 130 locations from the analytical program. Sample frequencies for 113 locations are specified under one of the regulatory orders. A well function review and possible supplemental sampling is recommended for 89 locations. No locations are recommended for quarterly sampling, while 20 locations are suggested for semi-annual sampling, 54 for annual sampling and 36 for biennial sampling. 


\section{SECTION I--QUALITATIVE ASSESSMENT}

\subsection{INTRODUCTION}

\section{$1.1 \quad$ Site Description}

The U.S. Department of Energy (DOE) Y-12 National Security Complex is part of the larger Oak Ridge Reservation located in eastern Tennessee. The Complex is operated by BWXT Y-12, L.L.C. (BWXT) for the National Nuclear Security Administration (NNSA), under the broader supervision of the DOE. The facility has been engaged in research and manufacturing activities in support of security and defense missions for more than 60 years. Construction on the complex began in 1943 as part of the Manhattan Project, and $\mathrm{Y}-12$ was actively involved in developing the first generation of nuclear weapons used during the Second World War. In the intervening years the primary missions of the site have evolved. Security related research and manufacturing activities have continued, and programs in basic scientific research and environmental management have been instituted at the facility.

Historic manufacturing and research operations at the facility have resulted in widespread areas of affected soil, surface water and groundwater in the Y-12 Complex. Environmental investigation and characterization activities have been conducted at the facility for over 30 years. Over 1900 surface and groundwater environmental monitoring locations are listed in the BWXT Analytical Database for locations sampled historically and currently by BWXT and Bechtel Jacobs Company, L.L.C. (BJC), the DOE environmental management (EM) contractor in Oak Ridge, TN. The Y-12 Complex within the larger Oak Ridge Reservation is regulated under the Comprehensive Environmental Response, Compensation and Liability Act (CERCLA). Areas within the Complex are covered by Resource Conservation and Recovery Act (RCRA) programs as well as broader supervision by the Tennessee Department of Environment and Conservation (TDEC). Broader environmental monitoring and restoration supervision is also mandated under DOE orders for the Y-12 Complex.

\section{$1.2 \quad$ Y-12 Conceptual Site Model}

The groundwater and surface water monitoring program at $\mathrm{Y}-12$ is guided by a detailed conceptual site model that encompasses site topography, surface water drainage, groundwater flow systems, contaminant source areas, and contaminant migration pathways. Background information regarding this conceptual site model is outlined in Section 2.0 of the Calendar Year 2003 Groundwater Monitoring Report, Y-12 National Security Complex (BWXT Y-12, 2004a).

The conceptual model for the Y-12 site includes three hydrogeologic regimes. The Bear Creek Regime and the Upper East Fork Poplar Creek Regime (East Fork Regime) are in Bear Creek Valley, which is bounded to the north by Pine Ridge and to the south by Chestnut Ridge. The Bear Creek Regime includes several miles of Bear Creek Valley between the west end of Bear Creek Valley and a topographic and hydrologic divide near the west end of the Y-12 industrial complex. The East Fork Regime, which includes the $\mathrm{Y}-12$ industrial complex, is located east of the topographic/hydrologic divide and west of Scarboro Road. 
The Chestnut Ridge Regime is located directly south of the Y-12 industrial complex and is bounded by Bear Creek Valley to the north, Scarboro Road to the east, Bethel Valley Road to the south, and Dunaway Branch to the west. The following sections describe the hydrogeology, source areas, and migration pathways for the three hydrogeologic regimes.

\subsubsection{Hydrogeology}

Bedrock Geology: Bedrock geology in the area consists of thrust-faulted southeastdipping sequences of shale, siltstone, limestone, and dolostone strata of Cambrian and Ordovician age. In Bear Creek Valley the outcropping geologic units are in the Conasauga Group, which includes the Maynardville Limestone, Nolichucky Shale, Maryville Limestone, Rogersville Shale, Rutledge Limestone, and Pumpkin Valley Shale. Bedrock is overlain by up to 40 feet of unconsolidated materials in Bear Creek Valley, and extensive areas of fill materials typically ranging from 5 to 25 feet in thickness are present beneath the $\mathrm{Y}-12$ industrial complex.

On Chestnut Ridge the formations that outcrop are carbonates of the Knox Group and overlying interbedded limestones and shales of the Chickmauga Group. Unweathered bedrock is overlain by up to 100 feet of unconsolidated residuum on Chestnut Ridge.

Surface Water Drainage: Surface water in the Bear Creek Regime flows along Bear Creek and its tributaries. Bear Creek acts as a major conduit of the shallow karst network in the Maynardville Limestone, and discharge from springs is an important component of flow in the main channel of Bear Creek. Bear Creek includes gaining reaches (i.e., groundwater discharge areas) and losing reaches. Major sections of upper and middle Bear Creek are seasonally dry.

In the East Fork Regime, surface water is drained by Upper East Fork Poplar Creek. The main channel and all the northern tributaries in the western and central portions of the East Fork Regime were filled and replaced by an extensive network of underground stormwater drain lines that were constructed during development of the Y-12 complex. A lined surface impoundment (Lake Reality) is located on the eastern side of the East Fork Regime and regulates the quantity and quality of surface water exiting Upper East Fork Poplar Creek. Lake Reality was built to replace New Hope Pond, an unlined surface impoundment that was closed and capped in 1988.

Aquifer and Aquitard Hydrogeologic Units: For the conceptual site model, geologic strata have been divided into two hydrogeologic units. The aquifer includes the Maynardville Limestone and the overlying dolomite formations of the Knox Group. The aquitard includes several formations that underlie the Maynardville Limestone, including the Nolichucky Shale, Maryville Limestone, Rogersville Shale, Rutledge Limestone, Pumpkin Valley Shale, and Rome Formation.

Groundwater Flow in Maynardville Limestone: The Maynardville Limestone outcrops along the axis of Bear Creek Valley. Most groundwater flow in the Maynardville Limestone is reported to occur at depths less than 100 feet below ground surface in a shallow karst network of interconnected solution conduits and cavities. In the deeper 
groundwater flow system within the Maynardville Limestone, fractures are the primary flow pathways.

Potentiometric surface elevations in Bear Creek Valley show a hydrologic divide near the western end of the Y-12 industrial complex. In the Bear Creek Regime, groundwater flow occurs parallel to geologic strike (i.e., along Bear Creek Valley) toward the westsouthwest. In the Upper East Fork Regime, groundwater flow occurs parallel to geologic strike toward the east-southeast.

Groundwater Flow in Knox Group Formations: Groundwater flow underlying Chestnut Ridge occurs in a very shallow "stormflow zone" in the unconsolidated residuum and in fractures within unweathered bedrock of the Knox Group formations. The bedrock has enlarged fractures and other solution features characteristic of karst aquifers. Most groundwater flux occurs near the transition between the residuum and unweathered bedrock, where the water table is located.

Potentiometric surface elevations at the Chestnut Ridge Regime generally mirror surface topography. Along the crest of the ridge groundwater generally flows from west to east, parallel to geologic strike, with radial flow components to the north and south. In the central portion of the Chestnut Ridge Regime, groundwater flow is radial from local flow divides along hilltops. Groundwater flow directions in the southern part of the regime are generally toward the south.

Groundwater Flow in the Aquitard Formations: Most groundwater flow in the aquitard occurs within a permeable water table interval near the interface between unconsolidated materials and bedrock. Some groundwater flow also occurs in fractures within the bedrock. Only a small percentage of groundwater flow in the aquitard recharges deeper bedrock. In the Bear Creek Regime, it is estimated that about $94 \%$ of available water in the aquitard discharges to tributaries of Bear Creek. In the East Fork Regime, surface water infiltration and recharge to the water table interval are significantly influenced by impervious surface cover, extensive areas of fill, and subsurface networks of stormwater drains, sewer lines, and process lines. The presence of these underground utilities and operation of basement dewatering sumps strongly influences local groundwater flow patterns at some locations in the East Fork Regime.

\subsubsection{Source Areas}

Groundwater quality monitoring data show that the most widespread groundwater contaminants at Y-12 are nitrate, volatile organic compounds (VOCs), uranium isotopes and technetium-99 (Tc-99). Gross alpha (GA) and gross beta (GB) activity are evaluated routinely to monitor the overall uranium isotope and Tc-99 distribution across the site. GA and GB analyses are supplemented with confirmatory isotopic analyses to better define the specific constituents in groundwater. The major constituents found in groundwater at the $\mathrm{Y}-12$ Complex along with the applicable screening levels are listed in Table B.1. 
In the Bear Creek Regime, the primary sources of groundwater contamination include the S-3 Site located to the east, a group of sites known as the Oil Landfarm Waste Management Area (WMA), and a group of sites known as the Bear Creek Burial Grounds Waste Management Area. The principal constituents of concern (COCs) at the source areas in the Bear Creek Regime are summarized in the table below, which was developed based on descriptions in the Calendar Year 2003 Groundwater Monitoring Report, Y-12 National Security Complex (BWXT Y-12, 2004a).

\begin{tabular}{|c|c|c|}
\hline \multicolumn{3}{|c|}{ Source Areas and COCs at the Bear Creek Regime } \\
\hline & Major COCs & Other COCs \\
\hline \multicolumn{3}{|l|}{ S-3 Site } \\
\hline Former S-3 Ponds & $\begin{array}{l}\text { Nitrate, Tc-99, uranium isotopes; } \\
\text { VOCs, (with possible DNAPL); } \\
\text { trace metals }\end{array}$ & -- \\
\hline \multicolumn{3}{|c|}{ Oil Landfarm Waste Management Area } \\
\hline Boneyard/Burnyard & Uranium isotopes & -- \\
\hline $\begin{array}{l}\text { Hazardous } \\
\text { Chemical Disposal } \\
\text { Area }\end{array}$ & VOCs & -- \\
\hline Oil Landfarm & $\begin{array}{l}\text { 111TCA; 11DCA; 11DCE; PCE; } \\
\text { TCE; tDCE; } \\
\text { CDCE }\end{array}$ & -- \\
\hline Sanitary Landfill I & $\begin{array}{l}\text { 11DCA; tDCE; } \\
\text { cDCE; boron }\end{array}$ & -- \\
\hline \multicolumn{3}{|c|}{ Bear Creek Waste Management Area } \\
\hline $\begin{array}{l}\text { Burial Ground A } \\
\text { (North) }\end{array}$ & $\begin{array}{l}\text { PCE; TCE; cDCE } \\
\text { (possible DNAPL) }\end{array}$ & $\begin{array}{l}\text { Other VOCs, (including } \\
111 T C A ; 1,1-D C A ; \text { and } \\
1,2-D C A \text { ) }\end{array}$ \\
\hline $\begin{array}{l}\text { Burial Ground A } \\
\text { (South) }\end{array}$ & $\begin{array}{l}\text { PCE; TCE; cDCE } \\
\text { (confirmed DNAPL) }\end{array}$ & $\begin{array}{l}\text { Other VOCs (including } \\
\text { 111TCA; 11DCA; and } \\
\text { 12DCA); } \\
\text { boron; uranium isotopes }\end{array}$ \\
\hline $\begin{array}{l}\text { Burial Ground C } \\
\text { (East) }\end{array}$ & cDCE; vinyl chloride & Boron; uranium isotopes \\
\hline $\begin{array}{l}\text { Burial Ground C } \\
\text { (West) }\end{array}$ & cDCE; vinyl chloride & Boron \\
\hline Walk-In Pits & $\begin{array}{l}\text { PCE } \\
\text { (possible DNAPL) }\end{array}$ & Other VOCs \\
\hline
\end{tabular}


The S-3 Site is located at the eastern end of the Bear Creek Regime in the aquitard unit (Nolichucky Shale) above and north of the transmissive aquifer unit. The former S-3 ponds area is characterized by high nitrate concentrations and uranium isotopes affecting groundwater flowing in the aquitard and into the Maynardville Limestone at the base of the valley. Westward flow is occurring in the aquitard along strike (as evidenced by nitrate concentrations in GW-829, GW-537, and GW-085, see discussion Section II 3.3.1 West S-3). The source areas for the Oil Landfarm WMA and Bear Creek Burial Grounds are mostly underlain by the geologic formations that comprise the aquitard. VOC constituents from the Oil Landfarm WMA and Burial Grounds flow through fractures in the aquitard as well as in surface streams into affected groundwater in the Maynardville aquifer formation. The central and western areas of the Bear Creek Regime are characterized by highly commingled plumes containing diverse VOCs from the western waste management areas and nitrate and radioactive constituents from the eastern S-3 area.

In the East Fork Poplar Creek Hydrogeologic Regime (East Fork Regime), contaminant sources include waste treatment, storage, and disposal sites; bulk product transfer, storage, or use areas; industrial process buildings; waste and product spill areas; process pipelines, drains, and utilities associated with the industrial complex; the former S-3 ponds; petroleum storage tank sites. As in the Bear Creek Regime, intermingling of contaminants from the source areas has produced an extensive groundwater contamination plume beneath the Y-12 industrial complex and extending into Union Valley to the east of Scarboro Road, beyond the boundary of the Oak Ridge Reservation.

Groundwater containing nitrate and radioactive constituents from the S-3 and S-2 areas commingle with tetrachloroethene (PCE), tetrachloroethene (TCE) and carbon tetrachloride (CTET) sources in the industrial areas of the East Fork Regime. Trace metal constituents are found in some areas, but the extent of groundwater affected by metals is not as large as that of VOC or nitrate/radioactive constituents. Commingling of constituents from diverse source locations occurs along the length of the East Fork Regime. The principal constituents of concern (COCs) at the source areas in the East Fork Regime are summarized in the table below, which was developed based on descriptions in the Calendar Year 2003 Groundwater Monitoring Report, Y-12 National Security Complex (BWXT Y-12, 2004a). 


\begin{tabular}{|c|c|c|}
\hline \multicolumn{3}{|c|}{ Source Areas and COCs at the East Fork Regime } \\
\hline & Major COCs & Other COCs \\
\hline \multicolumn{3}{|l|}{ Western Y-12 Area } \\
\hline Former S-3 Ponds & Nitrate, Tc-99, uranium isotopes & VOCs, Trace metals \\
\hline S-2 Site, & Gross alpha and beta activity & Nitrates and trace metals \\
\hline $\begin{array}{l}\text { Waste Coolant } \\
\text { Processing Area } \\
\text { Salvage Yard, Other } \\
\text { Processing and } \\
\text { Manufacturing Areas }\end{array}$ & $\begin{array}{l}\text { PCE; TCE; CDCE; } \\
\text { 11DCE; vinyl chloride, metals }\end{array}$ & Other VOCs \\
\hline \multicolumn{3}{|l|}{ Central Y-12 Area } \\
\hline $\begin{array}{l}\text { Sources in Western } \\
\text { Y-12 Area }\end{array}$ & $\begin{array}{l}\text { Gross alpha and } \\
\text { beta activity, Nitrate }\end{array}$ & Trace metals \\
\hline $\begin{array}{l}\text { Building } 9212 \text { and } \\
\text { Other Industrial } \\
\text { Facilities }\end{array}$ & $\begin{array}{l}\text { PCE; TCE; cDCE; } \\
\text { 11DCE; vinyl chloride; } \\
\text { 111TCA; 11DCA }\end{array}$ & Other VOCs \\
\hline \multicolumn{3}{|l|}{ Eastern Y-12 Area } \\
\hline $\begin{array}{l}\text { Upgradient Industrial } \\
\text { Complex }\end{array}$ & PCE, TCE, CTET & $\begin{array}{l}\text { Gross alpha and } \\
\text { beta activity, Nitrate }\end{array}$ \\
\hline $\begin{array}{l}\text { Building } 9720-6 \text { and } \\
\text { Other Industrial } \\
\text { Facilities }\end{array}$ & $\begin{array}{l}\text { CTET; chloroform; } \\
\text { dichloromethane; BTEX }\end{array}$ & $\begin{array}{l}\text { Other VOCs, Trace } \\
\text { metals }\end{array}$ \\
\hline
\end{tabular}

Groundwater contamination is less extensive at the Chestnut Ridge Hydrogeologic Regime, as no intensive industrial activity occurred in this area of interest (AOI). Chestnut Ridge has several legacy and current solid waste management units. VOCs are the principal groundwater constituents of concern (COCs) in this area. A distinct plume of VOCs has been identified near the Chestnut Ridge Security Pits (CRSP), and Industrial Landfill IV is a suspected source of VOC impacts. The major COCs associated with these two potential source areas are listed in the table below.

\begin{tabular}{|l|l|}
\hline \multicolumn{2}{|c|}{ Source Areas and COCs at the Chestnut Ridge Regime } \\
\hline & COCs \\
\hline $\begin{array}{l}\text { Chestnut Ridge } \\
\text { Security Pits }\end{array}$ & 111TCA; 11DCA; 11DCE; PCE; TCE; CDCE \\
\hline Industrial Landfill IV & 111TCA; 11DCA; 11DCE; boron \\
\hline
\end{tabular}

\subsubsection{Contaminant Migration Pathways}

In the Bear Creek Regime, the principal contaminant migration pathways include the following:

- Migration of COCs into the karst network of the Maynardville Limestone due to direct recharge from overlying source areas, recharge from losing reaches of Bear Creek, and inflow of shallow groundwater from the aquitard. 
- Migration of COCs in deeper intervals of the Maynardville Limestone along fracture flowpaths.

- Discharge of COCs from the karst network and from deeper intervals of the Maynardville Limestone into springs and into the channel of Bear Creek in gaining reaches of the creek.

- Migration of COCs along the water table interval of the aquitard into tributaries or other surface water discharge areas that flow into Bear Creek.

- Limited migration of COCs to deeper intervals of the aquitard bedrock formations along fracture flowpaths.

In the East Fork Regime, the principal contaminant migration pathways are generally similar to those in the Bear Creek Regime, except that there is significant interaction between shallow groundwater and stormdrains, other utilities, building sumps, and buried tributaries. Another important difference is that dry weather flow in Upper East Fork Poplar Creek comes from a variety of sources, including non-contact cooling water, condensate, cooling tower blowdown, groundwater discharge, and a significant flow of water taken from the Clinch River. Water from the Clinch River is discharged into East Fork Poplar Creek for flow management in order to comply with the NPDES permit.

Five primary tributary drainage basins are located on south Chestnut Ridge. The tributaries are largely intermittent and receive flow via surface runoff, stormflow and groundwater baseflow. The tributaries run south toward Bethel Valley. Chestnut Ridge is underlain by Knox Group formations. Three hydrogeologic subsystems occur in this region: stormflow zone, vadose zone and deeper groundwater zone. Groundwater can occur intermittently above the water table in this area, forming a stormflow zone at up to eight feet below ground surface (ft bgs). Stormflow is intermittent after precipitation events. The vadose zone is defined below roughly $8 \mathrm{ft}$ bgs and extends near the bedrock/residuum interface. Recharge, is episodic and occurs along saturated permeable fractures.

Deeper groundwater in the Chestnut Ridge regime occurs in permeable, planar fractures and conduits in a poorly permeable matrix. Hydraulic conductivity can vary over multiple orders of magnitude. Groundwater flow along the crest of the ridge, which functions as a recharge zone, is generally from west to east, parallel to geologic strike, with some radial flow. Groundwater flow south of the crest is generally to the south. 


\subsection{REGULATORY FRAMEWORK AND MONITORING OBJECTIVES}

The current Y-12 Groundwater Protection Program (GWPP) is charged with fulfilling surveillance monitoring objectives as articulated in the United States Department of Energy (DOE) Order 450.1 (DOE, 2004). The Order requires that each DOE site implement an environmental management system that "provides for systematic planning, integrated execution, and evaluation of programs that ensure public health and environmental protection, pollution prevention, and compliance with DOE Directives and applicable laws and statutes" (USDOE, 2004). Sites covered under DOE Order 450.1 are charged with developing a site-wide environmental monitoring program providing data to meet regulatory requirements in the short term and to form the foundation of responsible stewardship in the long-term.

The overall groundwater monitoring program at the $\mathrm{Y}-12$ National Security Complex is divided between two entities with different objectives. The Water Resources Restoration Program (WRRP), managed by the $B J C$, is charged with meeting the short-term regulatory requirements of RCRA, CERCLA and the State of Tennessee, while the longterm surveillance mandate is served by the Y-12 GWPP. The GWPP at Y-12, therefore, has a somewhat unique status among groundwater monitoring programs in that the primary objective of data collection is not to satisfy any one specific external regulatory requirement, but rather to provide proactive monitoring in anticipation of new threats to groundwater resources and to support responsible property management. The Y-12 GWPP serves as a watch-dog to provide management decision support for future regulatory and public health priorities, as both the site use and regulatory environments evolve.

Because of the somewhat unique status of the GWPP, many of the assumptions normally encountered in long-term groundwater monitoring evaluations must be reframed. Design criteria for the monitoring program are not entirely compliance driven and cannot be completely routine. The surveillance mission of the monitoring network means that unique, innovative or emerging issues must be addressed, which can be difficult to incorporate into a routine, fixed monitoring plan. The overall strategy of the surveillance monitoring network design still has the mandate to avoid redundancy, maintain consistent quality control and provide efficient and cost effective data. The apparently competing goals of the surveillance monitoring program provide a significant challenge in the development of an optimized monitoring network.

General objectives of Surveillance monitoring applicable to the Y-12 GWPP are provided in Ground Water Surveillance Monitoring Guide for Use with DOE O 450.1 Environmental Protection Program (USDOE, 2004). The surveillance monitoring network should be able to detect at the earliest possible time impacts on groundwater from facility operations using observation points located in prioritized areas of the site. Surveillance monitoring should track existing contamination in order to quantify the dimension and magnitude of affected groundwater and provide early warning of changes in concentration or extent of affected groundwater. If active remediation is underway, surveillance monitoring should provide data to support evaluation of the efficacy of the remedial action. Data from the surveillance monitoring program may be used to support diverse functions such as model development, remedial decision support or human and ecological risk evaluations. 
Specific requirements of Order 450.1 require that groundwater that is or potentially could be affected by facility operations be monitored with the following objectives.

- Determine baseline groundwater quality and quantity;

- Demonstrate compliance with applicable regulations;

- Ensure early detection of affected groundwater;

- Identify and monitor sources of groundwater contamination;

- Evaluate potential for off-site migration of site constituents;

- Support site management decisions.

In essence, the specific monitoring objectives above provide the scientific questions that the data collected in the monitoring program must answer. The answers to the monitoring questions are then used to support site decisions, or in USEPA terminology, Scientific Management Decision Points (SMDPs) (USEPA, 2004a). SMDPs generally outline what actions will be taken if the weight of evidence indicates that site conditions have changed significantly and implementation of a contingency plan is warranted. In the event constituents are moving toward receptors or exceeding certain risk-based levels, contingent response actions may be implemented. Conversely, as constituent concentrations fall below screening levels, the decision may be made to change remediation strategies (i.e. reduce or turn off pumping wells) or recommend reduced frequency or removal of wells from the sampling plan.

In order to assess the current GWPP, an evaluation of how the current sample locations fulfill each of the objectives listed above has been developed. Results are tabulated in the Qualitative Evaluation section of this report and are carried through to the Quantitative Evaluation section of the report. Evaluation of the contribution of each well to the overall network forms the basis for determining the spatial and temporal sampling frequency.

The majority of regulatory compliance is tasked to the WRRP (BJC), so the qualitative (and quantitative) network analysis will center on a sampling program that satisfies the other goals set forth by the DOE. It is assumed that wells monitored by BJC to satisfy RCRA and CERCLA requirements will be sampled at the same locations and sampling frequency. However, optimized recommendations for these locations are included as part of the assessment in the event supplemental data on these wells is deemed necessary. Often, a limited suite of analytes is sampled for compliance wells, which is problematic when data are used to support modeling efforts or programs with broader goals.

This report will not specifically evaluate the SMDPs or Contingency Plans; however, these elements are essential parts of the overall monitoring program and are specifically mentioned in the DOE guidance document (USDOE, 2004). 


\subsection{SAMPLING, ANALYSIS AND DATA MANAGEMENT PROGRAM}

The design of an efficient long-term groundwater monitoring program includes optimizing methods by which data are collected and organized as much as choosing the locations and sampling frequencies in the physical network. For any long-term monitoring program, significant efficiencies in cost and effort may be achieved by improving sample collection and analysis. For most programs significant enhancements can be achieved by improving data management and reporting. For these reasons, the sampling, analytical and data management activities of the GWPP were assessed as part of the overall evaluation.

\subsection{Monitoring Program Overview}

\subsubsection{Well Sampling and Inspection Techniques}

Due to the size and complexity of the Y-12 Complex, the well sampling program has developed several strategies to improve efficiency. Groundwater wells are grouped by location and sampling events are largely semi-annual, scheduled for alternating quarters. However, because of the number of locations, wells are sampled over many days of each quarter. By careful scheduling, the majority of data are collected by a limited number of trained personnel, providing a high degree of repeatability in data collection methods. The current program includes dedicated pumps and tubing at most locations, which is beneficial in eliminating artifacts from improperly cleaned equipment.

Responsibility for collection, transportation and chain-of-custody control for most surface and groundwater samples is assigned to the $\mathrm{Y}-12$ Analytical Chemistry Organization (ACO). Preparation of bottle lists, container type, preservatives and laboratory test identification is conducted by ACO personnel. Based on the description of the sampling program in the Y-12 GWPP Groundwater and Surface Water Sampling and Analysis Plan for Calendar Year 2005 (BWXT, 2004b), and the results in the GWPP database, no recommendations are made to improve efficiency in the aforementioned aspects of the well sampling and inspection program.

The USEPA currently recommends low-flow sampling techniques to monitor groundwater wells (Puls and Barcelona, 1996), and since 1997, low-flow techniques have been used over much of the site. Low-flow techniques reduce the time and waste generated per sample location and can dramatically reduce cost and effort associated with groundwater sampling. The $\mathrm{Y}-12$ facility historically used a conventional three-well volume purge method to sample groundwater. In order to test comparability in the data sets between the two well sampling techniques, both techniques have been used at a small number of wells to collect samples. In some cases the two techniques give different results that are not necessarily predictable from well to well.

While the dual sampling provides an interesting data set, the recommendation is to commit to one technique per well, with low-flow sampling preferred. At locations where the purge technique appears to provide more representative data, the purge method should be used. Because wells where both methods have been used now have enough data to determine a statistical trend in the recent data and contrast methods with 
previous data, a decision should be made to collect samples using only the best method. By choosing one method, redundancy in sampling will be reduced.

Unfiltered groundwater samples are collected from all monitoring locations, which is consistent with most guidance documents. The GWPP also includes the collection of filtered groundwater samples from limited locations where three-well-volume purging sampling techniques are used. Samples are filtered in the field using a $0.45 \mu \mathrm{m}$ filter. Filtering is indicated for locations with high turbidity in order to quantify concentrations of dissolved metals.

Formal well inspection techniques described in site reports (BWXT, 2003b) provide a good basis for evaluating and correcting any physical problems with the well locations. Well locations should also have an informal inspection during every sample event. The condition of the well, pump and tubing can be noted on the daily work log or physical water quality parameter sheet. In this way, well conditions can be monitored more frequently than is suggested in the report.

\subsubsection{Laboratory Analysis}

Site characterization activities have identified a large number of constituents of concern (COCs) for the Y-12 Complex (see Section 1.0 and Table B.1). Several constituent classes and geochemical parameters have been analyzed at many locations using a variety of analytical techniques. Overall, the approach has been very thorough and recent (1996 to present) laboratory analyses have produced a large, very high quality groundwater data set.

Based on the evaluation of data provided in the GWPP database and procedures outlined in the Quality Assurance Plan for the Analytical Chemistry Organization (BWXT, 2003c) as referenced in the Y-12 Groundwater Protection Program Groundwater and Surface Water Sampling and Analysis Plan for Calendar Year 2005 (BWXT, 2004b), detection limits are appropriate relative to the specified screening levels. Appropriate numbers and types of duplicate samples, trip and field blanks and laboratory quality assurance/quality control (QA/QC) samples are analyzed. Laboratory and sampling methods and dates are documented and stored within the GWPP database. Data qualifiers and flags are included in the data set. As discussed above, GA and GB activity analyses are used to monitor radioactive species routinely. Confirmatory samples are analyzed periodically to identify specific isotopes and constituents in samples with GA and GB activity. The methods used to evaluate error and significance in GA and GB activity are appropriate to these types of analyses. The current laboratory analysis program and methods are supplying excellent data to support site monitoring objectives and decision making.

An examination of the analytical data indicates that the suite of constituents sampled at each location changes periodically. For example, well GW-276 in eastern Bear Creek Valley near the S-3 pits (which is regulated under an RCRA permit) has been sampled 36 times since 1986. In the GWPP database, GW-276 has 36 results for VOC's such as TCE and PCE; 32 results for Total Uranium, 29 results for Uranium, and 24 results for Nitrate as Nitrogen, 9 results for Nitrate/Nitrite, 3 results for Nitrate/Nitrite as Nitrogen and 5 results for the suite of semi-volatile organic compounds (SVOCs). Sampling 
location GW-526 has been sampled 37 times since 1986, with 33 results for vinyl chloride and only 10 results for nitrate as nitrogen. The varying list of constituents can make comparisons between constituent trends problematic. The diverse types of nitrate and uranium analyses, in particular, create problems in comparing nitrate results across time. The monitoring recommendation is to sample a consistent suite of basic constituents at all locations. Additional analyses, such as specific uranium isotopes can be added to the program occasionally, but the core group of constituents should be analyzed using the same methods (with the same parameter names) consistently.

A consistent set of analytes is particularly important across the $\mathrm{Y}-12$ Complex because many plumes are commingled and many constituents were used or disposed of plantwide. Analysis of all COCs provides information on the distribution and transport of affected groundwater through the complex geological formations underlying the Y-12 Complex. Target constituents for the entire site should include VOCs, metals, nitrate, Gross Alpha (GA) and Gross Beta (GB) activity, and total uranium.

One minor constituent of possible concern that should be included in the monitoring program is 1,4-dioxane. 1,4-Dioxane was a common chemical stabilizer in commercial 1,1,1-trichloroethane (111TCA) preparations, and has become a concern among the regulatory community. Federal MCLs and groundwater screening levels have not been widely established for 1,4-dioxane. Regulatory standards for drinking water vary widely by state, from 85 ppb in Michigan to 5 ppb in Florida (Mohr, 2001). California EPA has chosen a Preliminary Remediation Goal (PRG) of near 3 ppb (Spath and Alexeeff 1998) while USEPA Region IX has designated a PRG of 6.1 ppb (USEPA Region IX PRG'S 2004). 1, 4-Dioxane is more mobile than the chlorinated co-constituents and should be analyzed for downgradient of areas where 111TCA, 1,1-dichloroethene (11DCE) or 1,1dichloroethane (11DCA) are detected.

Field parameters (e.g. temperature, dissolved oxygen, conductivity, $\mathrm{pH}$, redox) are important in characterizing background source water and monitoring the passage of water through various formations. Real time monitoring of field parameters during lowflow groundwater sampling is used to determine when the groundwater has stabilized and is representative of formation water. Field parameter data can be included in the site database to indicate general trends, but the accuracy of most field methods is not extremely high. Collection of data on geochemical parameters such as dissolved oxygen, sulfate, and reduced iron using more rigorous techniques can support lines of evidence confirming natural attenuation processes for a monitored natural attenuation (MNA) remedy. However, collection of large quantities of this type of data has limited utility in a routine monitoring program. The recommendation is that geochemical indicators outside of normal field parameters should be collected in support of a specific objective (like confirming MNA) in a supplemental monitoring program.

\subsection{Data Management for the GWPP}

The Y-12 GWPP Data Management Plan (BWXT, 2003a) details the process of data management for the diverse types of data collected to fulfill program objectives. 
Elements of a good data management system include complete, reliable and repeatable data processing, transparent data management, fidelity (data in = data out), data connectivity and a system to question and revise the data. Based on the methods presented in the Data Management Plan and an interview with the GWPP data management team, the GWPP has a high quality data management system.

The laboratory performing the chemical analyses on water samples (Analytical Chemistry Organization) uses an electronic Laboratory Information Management System (LIMS) to document, track and report sample results and data flags. Electronic data deliverables (EDDs) from the laboratory are transmitted to the Analytical Data Management System (ADMS), which is managed by Science Applications International Corporation (SAIC). The ADMS functions as the master analytical data repository for GWPP and other site stakeholders. Field data from the sampling team are entered into the system. Data from both the WRRP (managed by BJC) and the GWPP are included in the ADMS.

The ADMS uses the SAS data management and statistical application to review and store the data in the site analytical database. The GWPP has instituted an automated standardized data-screening tool, the Environmental Quality Control and Analysis System (EQCAS), to identify anomalies in data points as results are delivered in electronic format from the laboratory to the GWPP personnel. In this way, environmental data can be examined immediately and outliers can be questioned and resolved before data are permanently entered into the ADMS. The data review feature is especially important in creating a high quality data set. Data from the ADMS are forwarded to GWPP for inclusion in the Y-12 Groundwater Information Management System (GIMS) managed by BWXT. The GIMS includes sampling information, water levels, well construction and geologic information, location inspection and maintenance information as well as site analytical results.

Based on the review of the analytical data for groundwater from the GIMS, the data reported since the 1996-1998 time period has achieved data quality objectives set forth in most regulatory guidance documents (USEPA, 2004a, USEPA, 1987). Sampling data are reported to GWPP personnel with QA/QC parameters, well and sample information, field parameters and analysis methods included with every record. Sample location information is included in the GIMS database, and contains information on the location, depth, geologic formation, date of installation and other pertinent sample location information. The sample location data is linked to the analytical data, creating easy access to complete sample information.

The GWPP has a model data management system, and no changes are recommended at this time. In terms of data review, historic data points that may be outliers or inaccurate should be flagged so that they can be easily removed from statistical analyses. Additionally, locations designated as 'active' should be separated into two categories, those active for hydrogeologic monitoring only, and those used for routine analytical sampling. Those locations used for hydrogeologic samples such as potentiometric surface measurements should be easy to distinguish from locations where chemical analytical data are being collected. 
With the combination of quality data management and laboratory analyses and cooperation between the WRRP and GWPP, the Y-12 site has an established and powerful tool for supporting site management decisions.

\subsection{Data Reporting}

During review of Calendar Year 2003 Groundwater Monitoring Report, GSI identified three ways that future annual groundwater monitoring reports could be enhanced: i) prepare detailed maps for selected COCs at individual sampling locations for each hydrogeologic regime; ii) highlight exceedances of screening levels on the data tables found in Appendices D, E, and F of the report; and iii) provide more specific conclusions in Section 5.0 of the report.

Prepare Detailed Maps for Selected COCs in Each Hydrogeologic Regime: The calendar year 2003 report includes four figures (Figures. A.7, A.8, A.9, and A.10) that show the generalized extent of nitrate, VOCs, gross alpha activity, and gross beta activity, respectively, in groundwater at and near the Y-12 Complex. However, these maps do not show monitoring locations or COC concentration values at critical locations within the plume. Because of the high spatial heterogeneity of concentrations in Y-12 Complex groundwater, each monitoring location has more spatial significance than in a diffuse flow-type regime. Wells along known conduits or fractures should be highlighted. Wells with low concentrations to non-detect values can be spatially quite close to affected locations. Maps with specific locations identified would assist in communicating a more detailed picture of the extent of affected groundwater.

Future reports could be improved by including more detailed maps for selected COCs for each hydrogeologic regime. These maps would show monitoring locations and the measured COC concentration at each location. Each map would show the outline(s) of the exceedance area(s) for the COC. As an alternative to iso-concentration contour lines, different color dots or dot sizes would be used to indicate the relative magnitude of the measured concentration at each monitoring location compared to the MCL or screening value (i.e., $>1 X,>10 X,>100 X$ ). The magnitude of concentration compared to the screening value is already used in the monitoring optimization scheme (BWXT, $2003 \mathrm{~b}$ ), and the same data can be visualized using mapping software to illustrate concentration distribution and rationale for future monitoring decisions. Examples of the 'dot map' approach to concentration distribution are shown in Figures A.2 through A.4.

The table below shows the COCs for each area that would be most useful for mapping purposes.

\begin{tabular}{|l|l|}
\hline Hydrogeologic Regime & Proposed Key COCs for Mapping \\
\hline Bear Creek & $\begin{array}{l}\text { Nitrate, Uranium, Total VOCs, PCE, TCE, } \\
\text { Gross Alpha Activity, Gross Beta Activity }\end{array}$ \\
\hline $\begin{array}{l}\text { Upper East Fork Poplar } \\
\text { Creek }\end{array}$ & $\begin{array}{l}\text { Nitrate, Uranium, Total VOCs, PCE, TCE, CTET, } \\
\text { Benzene, Gross Alpha Activity, Gross Beta Activity }\end{array}$ \\
\hline Chestnut Ridge & Total VOCs, Metals \\
\hline
\end{tabular}


Our review of the 2003 monitoring report indicates that MCL exceedance areas for the selected VOCs (i.e., PCE, TCE, CTET, or benzene) should in most cases encompass the exceedance areas for all other VOCs not proposed for mapping. Footnotes on the maps can be used to identify exceptions (e.g., elevated concentrations of vinyl chloride or dichloromethane detected at a few monitoring locations where none of the proposed key VOCs exceed their MCLs).

Highlight Exceedances of Screening Levels on Data Tables in Appendices D, E, and F: The tables in Appendices D, E, and F could be enhanced by highlighting screening level exceedances using bold text, cell shading, or some other type of cell formatting. This enhancement would be especially useful for trace metals and VOCs.

Provide Additional Specific Conclusions in Section 5.0 of the Report: The conclusions in Section 5.0 of the 2003 monitoring report are very general and brief. The conclusions section of future reports should mention specific areas where contaminants from Y-12 have migrated or are most likely to migrate beyond the boundaries of the Oak Ridge Reservation. The conclusion should highlight significant trend analysis results to communicate changes in concentrations at critical locations or indicate overall plume stability. Increasing or decreasing trends at individual locations can be discussed as well as plume wide trends (see Section II MAROS analysis for Moments and plume wide evaluation). It would also be very helpful to include a bullet list of key conclusions for each hydrogeologic regime. A summary of how monitoring results can be interpreted to support specific monitoring objectives outlined in DOE Order 450.1 would be useful for future site decision making. 


\subsection{QUALITATIVE NETWORK EVALUTION}

The Y-12 Complex has over 200 known and potential sources of groundwater constituents resulting from the multiple activities including uranium enrichment, fuel and solvent storage, equipment maintenance and research activities. The concept of 'source' area at Y-12 is complicated by multiple primary and secondary sources, commingled groundwater plumes, preferential flow paths and complicated land-use patterns. The hydrogeology of the $\mathrm{Y}-12$ region is further complicated by fracture flow, conduit flow, areas of diffuse flow and connection between surface water and groundwater. Straightforward quantitative techniques for monitoring network optimization do not account for the unique characteristics of the $\mathrm{Y}-12$ site. For this reason, a qualitative evaluation of site wells was performed prior to and along with application of the quantitative MAROS methodology. The results of the merger of qualitative and quantitative approaches are summarized fully in the Section II of this report.

\section{$4.1 \quad$ Technical Approach}

The technical approach for improving the efficiency of the Y-12 GWPP monitoring strategy includes both qualitative and quantitative evaluation strategies. Steps common to most long-term monitoring optimization (LTMO) techniques have been described in a number of guidance documents including the Roadmap to Long-Term Monitoring Optimization (USEPA 2005), the AFCEE Long-Term Monitoring Optimization Guide (AFCEE, 1997) and in Long-Term Groundwater Monitoring: The State of the Art (ASCE, 2003). The technique used to evaluate the GWPP at Y-12 is consistent with the approach presented in these documents. Preliminary steps in the LTMO process include identifying monitoring objectives, reviewing the current monitoring program, and collecting and evaluating historic and current site data, which are detailed above. The qualitative evaluation of the monitoring network was conducted to assess the degree to which the current well network is achieving specific temporal and spatial objectives.

The qualitative hydrogeologic network evaluation involves collecting diverse site data, organizing lines of evidence and forming a decision on spatial and temporal sampling based on a weight of evidence approach. Data included in the qualitative evaluation are discussed below. The results of the qualitative hydrogeologic analysis were combined with the results of the quantitative approach to form a final monitoring recommendation.

The major products of the broader qualitative analysis include a site wide understanding of monitoring objectives, groundwater flow, major constituents, source areas and receptors, which are summarized in the material above. Factors considered for the hydrogeologic analysis for individual wells are presented in Section 4.2, and include information on how each well functions to fulfill the monitoring objectives for the various groundwater flow regimes and constituent classes. Results from the qualitative evaluation were combined with the results of the quantitative analysis in the form of a decision logic flow chart (see Figure A.10) to determine the final temporal and spatial well network.

In order to provide as thorough a review of the monitoring network as possible, all active sample locations were identified in the analytical database and included in the analysis. 
Active sample locations have been designated by BWXT using decision logic detailed in the GWPP Monitoring Optimization Report (BWXT, 2003b). Active locations include those sampled for chemical analytical data and groundwater surface elevations as well as those locations sampled for hydrogeologic information only. The formal groundwater sampling optimization was performed for chemical analytical samples only. Collection of hydrogeologic data should continue at current active locations. Recommendations for locations to be 'removed' from the network indicate that these locations should be sampled for hydrogeologic data rather than both hydrogeologic and analytical data.

Active monitoring locations at the $\mathrm{Y}-12$ Security Complex were divided into eleven different analysis groups. (Surface water sample locations and Westbay wells were not included in the groundwater network analysis.) The analysis groups are listed in Table B.2. The locations were grouped according to the three main hydrogeologic regimes and then further subdivided geographically based on common source areas and similar constituent classes. The analysis groups were formed to simplify data processing and to automate the final recommended temporal and spatial network.

\section{$4.2 \quad$ Decision Logic and Location Scoring}

Sample locations were categorized based on criteria discussed below as part of an overall hydrogeologic qualitative evaluation of the GWPP network. Sample locations were evaluated qualitatively for their utility to the overall program. The qualitative evaluation includes expert judgment of whether the locations provide data for horizontal or vertical delineation, source monitoring, potential exit pathways, etc. The criteria evaluated were identified as critical decision factors controlling the identity and frequency of groundwater sample locations. Many of the criteria are taken directly from monitoring objectives articulated in DOE Order 450.1. Data for criteria scoring were taken from the Y-12 GWPP GIMS (provided by BWXT, 2005) and provided by expert judgment based on the location of the well using GIS data and geological information. Results from the criteria scoring were used along with results from the MAROS analysis in recommending an optimized groundwater sampling network for the Y-12 Complex. Qualitative and quantitative results were combined using the decision logic flow chart outlined in Section II, 4.0 and illustrated in Figure A.10. Results of the qualitative scoring are summarized by sample location analysis group in Appendix D (Tables D.1.1 through D.11.1). An explanation of the decision categories identified for the qualitative evaluation is presented below.

\subsubsection{RCRA/CERCLA Point of Compliance}

Sample locations required under regulatory programs as points of compliance were identified from the Monitoring Optimization Plan and from the Calendar Year 2003 Groundwater Monitoring Report (BWXT, 2004). The location and sampling frequency of this group of wells is fixed by RCRA post-closure permits, CERCLA interim or final Records of Decision (ROD) or other regulatory programs (e.g. EMWMF and SWDF solid waste permits). The majority of wells identified under regulatory programs are sampled as part of the WRRP. Regulated locations were considered very high priority and their identity and sampling frequency were evaluated but not considered for changes. However, in the preliminary MAROS analysis, regulated wells were considered for reduced sampling frequency or elimination, for future reference. Backup and alternate 
sampling locations identified in regulatory documents were also considered high priority. Should an alternate location be included in future regulatory sampling programs, a longterm record of constituent concentrations may be necessary to support sampling frequency or remedial effectiveness decisions. The final network recommendation included primary regulated locations at their current sampling frequency.

\subsubsection{Average Concentration Exceeds Screening Values}

Priority Constituents were chosen for each sampling location from site analytical data (Analytical Database, BWXT, 2005). The full historic analytical record for each well was used to determine an average concentration for the well for each target constituent. The average concentration was determined conservatively using detected results with nondetects assigned a uniform detection limit corresponding to the lowest detection limit in the data set. While this approach may skew the data high, due to historic outlier detections prior to 1996 and assuming the detection limit concentration for non-detects, the approach prevents underestimating concentrations in groundwater. The representative average concentration was divided by the MCL (screening level) for each constituent and a 'normalized' ratio was calculated. For each well, the constituent with the highest historic normalized ratio was chosen as the Priority Constituent. Locations where the average concentration of the representative constituent is above screening levels were identified in the qualitative evaluation. Results of the priority constituent concentrations are displayed graphically for selected locations in Figures A.2 through A.4. (Note: Sample locations where groundwater is unaffected generally had trace metals or GA or GB activity at background levels, triggering these compounds as 'priority constituents'. This artifact was taken into account during the final network analysis.)

\subsubsection{Formation Type}

For the purpose of the qualitative evaluation, each active sample location was assigned a water source formation type. Each location monitors either aquifer water, aquitard water or spring water. Surface water locations apart from springs were not considered in the evaluation; however, data from these surface streams can be important when identifying surface water impact to groundwater. In determining the importance of the monitoring location to the overall network, aquifer locations and springs were given priority. Springs generally discharge from the aquifer formation; however, the springs were considered to have a greater likelihood of becoming a point of exposure (POE) for human or ecological receptors, and were, therefore, considered higher priority locations. Constituents found in the aquifer formation were perceived to have greater possible mobility and access to exit pathways. As no drinking water supply wells are drilled into the aquifer formation near the Y-12 Complex, human exposure via drinking groundwater was not considered.

\subsubsection{Horizontal Delineation}

Wells that provide information for horizontal extent of affected groundwater were given priority in the final analysis. Highest priority was given to wells along the $\mathrm{Y}-12$ property boundary. Horizontal delineation was defined using a combination of data from the 
analytical database and identifying the well location using ARC GIS mapping software. A well was determined to provide horizontal delineation if the well was located on the perimeter of a plume or if the well was in an area with no other wells and COCs were detected routinely at the location.

\subsubsection{Vertical Delineation}

Wells that provide information for vertical delineation were also prioritized. Vertical delineation was somewhat harder to determine from site data. Well construction data were used to identify wells with deep screens or with open holes below the majority of surrounding wells. Locations with high constituent concentrations for deeper locations were prioritized.

\subsubsection{Exit Location}

Exit pathway locations were identified using the Appendix D Tables from the $\mathrm{Y}-12$ GWPP Monitoring Optimization Plan (BWXT, 2003b). Data from these tables were used directly to identify possible exit pathways. All spring locations were identified as exit pathways, even if they were not listed in the Optimization Plan. Exit pathway sample locations were prioritized as they represent more immediate possible exposure pathways for human or ecological receptors. Several exit pathway locations provide data on groundwater emerging as surface water, moving off-site or outside of the immediate Y-12 management area. Data collected from these locations may be used to support remedy choice or effectiveness evaluation or may be useful in supplemental ecological or human health risk evaluations.

\subsubsection{Unique}

The 'unique' category was included in the GWPP Monitoring Optimization Plan, and has been carried forward to this analysis, although the designation was not given high priority. Unique designations were given to locations that had no apparent redundant monitoring locations. Some unique wells provide information on deeper groundwater, potential early detection, or constituent movement, or represent isolated horizontal locations.

\subsubsection{Background Concentration}

Wells with metal and radioactive concentrations below MCLs and no detections of volatile organic constituents, or locations where groundwater samples have always been 'clean' were included in this category. As part of the mission of the GWPP is to determine background water quality, a certain number of statistically clean wells must be included in each hydrogeologic regime.

\subsubsection{Early Detection}

Wells that provide early detection of migration of constituents were identified by their location in transmissive groundwater formations or at spring locations. Data from both the GIMS and ARC GIS maps were used to identify wells where increases in constituent concentrations may signal movement of affected groundwater to more sensitive 
locations downgradient. Wells designated as 'early detection' points are distinguished from 'exit locations', generally, by a location more central to the plume or nearer the source area. Sample locations in less transmissive geology were also considered as early detection rather than exit locations.

\subsubsection{Monitor Source}

Wells that monitor known or potential sources were prioritized. In the final analysis, the number and position of source wells was considered when recommending sample locations and frequency after the quantitative review.

The categories described above were used to identify the function of each well in achieving monitoring objectives and were used along with the results of the MAROS evaluation in a decision logic process to recommend a final sampling network and frequency for wells in the Y-12 Complex. The decision logic flow chart and results of the final analysis are presented in Section II, 4.0. 


\section{SECTION II-QUANTITATIVE ASSESSMENT}

\section{$1.0 \quad$ INTRODUCTION}

Long-term monitoring programs, whether applied for process control, performance measurement, or compliance purposes, require a large scale data collection effort and time commitment, making their cumulative costs very high. With the increasing use of risk-based goals and natural attenuation in recent years as well as the move toward long-term closure upon completion of cleanup activities, the need for better-designed long-term monitoring plans that are cost-effective, efficient, and protective of human and ecological health has greatly increased.

The Air Force Center for Environmental Excellence's (AFCEE's) Monitoring and Remediation Optimization System (MAROS) methodology suggests an improved monitoring network program based on historic monitoring data and location parameters within a complicated groundwater system. The MAROS method does not include a mathematical optimization in the technical sense, but does include a variety of statistical and heuristic evaluations that, when taken together, result in a 'lines of evidence' approach to streamlining a groundwater monitoring network for maximum efficacy. By applying statistical techniques to existing site analytical data, as well as considering hydrogeologic factors and the location of potential receptors, the software suggests an optimal monitoring plan. Section II summarizes the findings of an application of the MAROS 2.1 software methodology to the current Groundwater Protection Program (GWPP) for the Y-12 National Security Complex in Oak Ridge, Tennessee. 


\subsection{ANALYTICAL APPROACH}

The Y-12 Complex has multiple primary and secondary sources of groundwater constituents resulting from site activities including uranium enrichment, manufacturing processes, fuel and solvent storage, equipment maintenance, research and historic solid waste disposal activities that have been conducted at the facility over time. The identification of discrete plumes at the Y-12 Complex is complicated by multiple source locations, preferential flow paths, commingled plumes, surface and groundwater interactions and complex land-use patterns. The MAROS method requires designation of a 'source' area for each analysis and the designation of source area wells. For the purpose of the MAROS analysis, the source area wells were designated as those near known sources, locations farthest upgradient, or with high concentrations of priority constituents.

In part due to the number of diverse sources, complicated hydrogeology, and size of the data set, the active monitoring locations at the $\mathrm{Y}-12$ Security Complex were divided into eleven different analysis groups (see Figure A.1). The wells were grouped according to hydrogeologic regime and then further subdivided based on common source areas and similar constituent classes. The analysis groups are described in Table B.2 and are elaborated in the text below. Detailed descriptions from the qualitative analysis are shown in Appendix D.

Because the hydrogeology of the Y-12 vicinity is distinct from strictly diffuse flow conditions, assumptions about 'normal' plume behavior underlying the MAROS method had to be modified. For example, most areas under consideration do not have a single 'source' and 'tail' zone. Plumes are frequently co-mingled. Due to the karst geology, wells in close proximity on the surface can be unconnected hydraulically, while wells geographically separated on the surface can be connected by preferential flow paths in the subsurface. As opposed to the diffuse flow plume model, wells within the same Y-12 analysis group could have very different major constituents due to the complicated hydrogeology. For this reason, each well was considered to be more independent than in a typical MAROS evaluation. Each individual well was assigned a Priority Constituent, based on historic exceedances, and the concentration trend and recommended sampling frequency were based on that Priority Constituent.

In order to apply the MAROS methodology, several broad assumptions had to be made. These assumptions were applied in order to create general trend and constituent stability results. Many results such as the estimate of total mass in the plume (zeroth moment), should not be interpreted as precise values, but rather as estimates for the purpose of evaluating plume stability and changes over time.

\section{$\underline{2.1 \quad \text { Site Assumptions }}$}

- Each hydrogeologic regime (Bear Creek (BC), East Fork Poplar Creek (EF) and Chestnut Ridge (CR)) was divided into 3-4 analysis groups, roughly based on a common 'source' area and geographic proximity. Analysis groups are listed in Table B.2 and illustrated on Figure A.1 and figures summarizing the MAROS results for each group. 
- Active groundwater sample locations with analytical data in the GWPP GIMS database were included in the analyses. Surface water sample locations and Westbay wells were not included in the quantitative evaluation. Locations where no analytical data were available could not be evaluated. Many groundwater wells currently used for hydrologic monitoring only were included in the quantitative evaluation as potential analytical sample locations in an attempt to provide a thorough analysis of the most effective monitoring network. In the report, some sample locations are recommended for 'removal' from the program. 'Removal' means that chemical analytical samples are not recommended for the purpose of evaluating constituent concentrations, however the well or sample location can be maintained for hydrologic sampling purposes.

- For each analysis group, wells with historically high concentrations of constituents were labeled as 'source' wells. 'Source' wells can be spread widely across an analysis area.

- Target constituents were chosen for the Y-12 facility based on historic exceedance of preliminary screening levels across the site. Target constituents along with preliminary screening levels are shown in Table B.1. The screening levels were based on USEPA MCLs for drinking water, where possible. For constituents without primary or secondary MCLs, USEPA Region IX PRGs were chosen. Historic analytical data for the listed constituents were used to prioritize constituents for each analysis group.

- Priority Constituents were chosen for each sampling location from site analytical data. The full historic analytical record for each well was used to determine an average concentration for the well for each target constituent. Non-detects were assigned a uniform detection limit corresponding to the lowest detection limit. The representative average concentration was divided by the MCL (or screening level) and a 'normalized' ratio was calculated. For each well, the constituent with the highest historic normalized ratio was chosen as the Priority Constituent.

- Groundwater flow directions are generalized for each hydrogeologic regime. The length and width of the plumes for each analysis group were estimated to be roughly the size of the analysis area. MAROS input parameters for each analysis group are shown in Appendix $D$ under each hydrogeologic regime.

- Parameters such as the effective porosity and seepage velocity were estimated from averages of high and low numbers. The porosity was estimated as 0.1 for all locations, while a seepage velocity of $200 \mathrm{ft} / \mathrm{yr}\left(2 \times 10^{-4} \mathrm{~cm} / \mathrm{s}\right)$ was chosen for all hydrogeologic regimes.

- MAROS analyses were conducted with data acquired between January 1996 and December 2004. Historic concentrations (prior to 1996) were used to assess wells that have not been sampled recently.

- Strong connections exist between surface and groundwater in many areas of the Y-12 Complex. Precipitation, surface run-off and stream flow transport 
constituents to sinking locations that affect groundwater; conversely, groundwater exits to surface water bodies in many locations. Determination of seasonal influences on groundwater concentrations is part of site characterization activities, and should be well understood before LTMO occurs. The influence of seasonality in groundwater concentrations may be significant when estimating short-term exposures; however objectives of LTM networks generally involve evaluation of long-term risks and potential for change in status of the plume. For this reason, most LTMO strategies, including MAROS do not address seasonality.

- Ten multi-port Westbay wells have been installed at $\mathrm{Y}-12$, but were not included in the quantitative MAROS analysis. One Westbay location, GW-722, is recommended for annual sampling to characterize groundwater constituents in the New Hope Pond area.

\subsection{Analytical Methodology}

Each monitoring group was evaluated for Constituent Choice and Moments for the entire plume, Statistical Trend and Sampling Frequency for each individual well and Well Redundancy and Well Sufficiency where possible. Details on the calculation and application of each of these methods are provided in Appendix C MAROS 2.1 Methodology. Further details of these methods are included in the MAROS Users Manual (AFCEE, 2003).

\subsubsection{Constituent Choice}

The MAROS method includes a module that evaluates and prioritizes possible constituents of concern based on their prevalence, toxicity and mobility. Concentrations of VOC, radioactive, and metal constituents from each well were entered into the software and evaluated using this tool. The USEPA MCLs as shown in Table B.2 were used as the screening values for the MAROS COC analysis. The COC risk evaluation module was run for data from each analysis group as a preliminary screening tool. Results of the COC selection can be found in Appendix D.

\subsubsection{Moment Analysis}

The role of moment analysis in MAROS is to provide a relative estimate of plume stability in the context of results from other MAROS modules. Stable to decreasing trend results for moments provide evidence that monitoring efforts may be reduced. Results indicating that the plume may be spreading downgradient may indicate that increased monitoring efforts are required.

The moment analysis algorithms in MAROS are simple approximations of complex calculations and are meant to estimate changes in total mass (zeroth moment), center of mass (first moment) and distribution of mass about the center (second moments) for complex well networks. Because of the complex geology at $\mathrm{Y}-12$, uniform aquifer input values were used across the site. For this reason, the estimate of total mass is not as accurate in a diffuse flow type of aquifer, and the actual results are not reported, but rather the trend for the total dissolved mass is indicated in each section. 
A brief explanation of the MAROS moment calculations is given in Appendix $C$ and in the MAROS Users Manual (AFCEE, 2003). MAROS-generated summary reports on the moment analyses are located in Appendix D. Results of the Moment Trend analyses are shown in the text and estimates of the center of mass for the priority constituents are illustrated in figures for each sample group. For the Y-12 GWPP Moment analyses, one or two indicative constituents were chosen for each sample group, and data for these constituents were assumed indicative of the overall stability of the plume in a given area.

\subsubsection{Statistical Trend Analysis}

Within the MAROS software, statistical trend analyses are used to support a conclusion about plume stability (e.g., increasing plume, etc.). The Mann-Kendall and Linear Regression modules are explained in more detail in Appendix $C$ of this document and in the MAROS Users Manual (AFCEE, 2003). As a rule, sample data sufficient to determine a trend with confidence would include at least two years of quarterly data (eight data events) or four years of semi-annual data, with four data points being the minimum necessary to return a statistically meaningful result. The Statistical Trend analysis was performed for the priority constituent for each individual sample location. The final statistical trend is a combination of the results of the Mann-Kendall statistical trend and the Linear Regression analysis to form an Overall Trend. Results of the Trend Analyses are interpreted as follows:

$\begin{array}{llllll}\text { D } & -- & \text { Decreasing Trend } & \text { I } & -- & \text { Increasing Trend } \\ \text { PD } & -- & \text { Probably Decreasing } & \text { PI } & -- & \text { Probably Increasing } \\ \text { S } & -- & \text { Stable Trend } & \text { NT } & -- & \text { No Trend (High Variability } \\ \text { ND } & -- & \text { Non-Detect } & \text { N/A } & -- & \text { Insufficient Data }\end{array}$

\subsubsection{Well Redundancy}

The goal of the well redundancy analysis is to identify wells that are redundant within the existing monitoring network and can be removed from the routine monitoring program. The approach allows elimination of sampling locations that have little impact on the longterm characterization of a contaminant plume. The sampling location module uses the Delaunay triangulation method to determine the significance of the current sampling locations relative to the overall monitoring network. The Delaunay method calculates the network area and average concentration of the plume using data from multiple monitoring wells. A slope factor (SF) is calculated for each well to indicate the significance of the well in the system (i.e. how removing a well changes the average concentration.)

Because wells in the Y-12 GWPP are not necessarily hydraulically connected when they are close to one another on the surface, the Delaunay triangulation was not useful for the site. However, the method was performed for each sample group for the dominant constituents, and no single wells were recommended for removal using this technique. All wells recommended for elimination from the program were designated based on decision logic presented below. Wells recommended for removal were reviewed qualitatively for their larger contribution to the network. Wells with significant sitespecific functions, or high constituent concentrations were maintained in the program. 
Wells suggested for removal from the program should not necessarily be physically removed, but removed from the current regular analytical monitoring program. Groundwater wells may be included in potentiometric surface monitoring, or as plume geometry changes, wells previously eliminated from the monitoring program may be reincorporated into the program.

\subsubsection{Well Sufficiency}

The Well Sufficiency analysis, also based on the Delaunay method, can be used for recommending new sampling locations in areas where additional plume information is needed. It is designed to recommend new wells in areas within the existing monitoring network where there is high uncertainty in groundwater concentrations between established monitoring locations. Specifically, the method evaluates if concentrations at one well can be predicted from concentrations at its nearest neighbors. In many cases, new sampling locations need to be added to the existing network to enhance the spatial plume characterization. The results for determining new sampling locations are derived solely from the two-dimensional spatial configuration of the monitoring network and the spatial pattern of the contaminant plume. Therefore, new well locations needed outside the existing monitoring well network (i.e., a new sentinel well outside the existing plume network) are not assessed.

Because the hydrogeology of the $\mathrm{Y}-12$ facility does not conform to the MAROS conceptual model of a two-dimensional diffuse flow plume, the interpretation of well sufficiency data is complicated. The well sufficiency module was run for each sample group and areas of high uncertainty for dominant constituents are included in the analysis; however, no new wells are suggested. The well sufficiency analysis was used to support quantitative and qualitative evaluations of well removal or sampling frequency. MAROS-generated graphical reports on well sufficiency are provided in Appendix D.

\subsubsection{Sampling Frequency}

The sampling frequency analysis, using the Modified Cost Effective Sampling (MCES) method, was applied to improve sampling frequency efficiency for each sampling location based on the magnitude, direction, and uncertainty of its concentration trend of recent and historical analytical data. The MCES method has both quantitative and qualitative components. Quantitatively, the trend and rate of change are determined by Mann-Kendall and Linear Regression calculations. Qualitative review is then performed to evaluate the position of the well in the network and its relationship to site specific monitoring goals. The MCES method estimates the lowest-frequency sampling schedule for a given groundwater monitoring location while still providing needed information for regulatory and remedial decision-making.

The MCES method requires four to six sample events to evaluate a sampling frequency. Locations with fewer than 4 sample events are automatically assigned a Quarterly sampling frequency until a statistically significant data set can be produced. The MAROS-recommended sampling frequency was adjusted based on the results of the qualitative well evaluation. For example, because the Y-12 GWPP data set has a large number of samples prior to the 1996-2004 analysis period and many historic sample 
locations, all Quarterly monitoring recommendations were reduced to Semi-annual or Annual sample frequencies. For wells with limited data, their utility to the entire network was evaluated and several wells were recommended for removal from the sampling program because they did not uniquely fulfill monitoring objectives detailed in the Qualitative Evaluation Report. Further information on the MCES method is presented in Appendix C and the MAROS Users Manual (AFCEE, 2003).

\subsubsection{Data Sufficiency Analysis}

In the MAROS data sufficiency analysis, statistical techniques are used to assess the sufficiency of monitoring plans for detecting the difference between the mean concentration of a constituent and the cleanup goal. The majority of the analyses are reserved for sites in the later stages of remediation. These analyses require six years of monitoring data for all relevant constituents and are not currently appropriate for wide application at the Y-12 Complex. However, many individual wells may become candidates for this type of analysis within five years. At that time, the calculations could assist site managers in eliminating 'clean' wells from routine monitoring.

\subsubsection{Final Combined Analysis}

Results from both the qualitative and MAROS generated quantitative analyses were combined to recommend final locations and sampling frequencies for the Y-12 Complex network. Details of the final decision logic are provided in Section II, 4.0 below. 


\subsection{MAROS RESULTS}

Results of the MAROS analyses of $\mathrm{Y}-12$ Complex groundwater monitoring locations are discussed below by hydrogeologic regime and by analysis group. Sample locations considered in each regime and analysis group are illustrated on Figures A.1 through A.4. Results from the MAROS analyses are summarized in Tables B.3-B.13 and in Figures A.5-9.

\subsection{Bear Creek Hydrogeologic Regime}

For the purpose of the MAROS analysis, the Bear Creek Hydrogeologic Regime (Bear Creek Regime) was divided into three analysis groups: The Western S-3 and Rust Spoils Area (West S-3), the central Oil Landfarm WMA /EMWMF/Boneyard/Burnyard (OLFA) area and the Bear Creek Burial Grounds (BCBG). Bear Creek has its headwaters in the western portion of the main $\mathrm{Y}-12$ facility and flows west/southwest through Bear Creek Valley until it flows northward into the East Fork Poplar Creek. As in the other hydrogeologic regimes, the subsurface is divided into 'aquifer' group wells in the Maynardville Limestone and Knox Group and the 'aquitard' formations of the Conasauga Group and the Rome Formation, which directly underlie the areas with primary contaminant sources in the Bear Creek Regime. The aquitard areas are hydraulically upgradient of the Maynardville Limestone, and fractures provide the main flow through the unit to the aquifer. Flow from source areas occurs primarily in response to precipitation, resulting in seasonal fluctuations in contaminant transport (BWXT, 2004a).

Results from the three MAROS analyses for the Bear Creek Regime are summarized in tables below and discussed in detail in sections 3.1.1 through 3.1.3. Final network recommendations, based on both qualitative and quantitative approaches are presented in Section II, 4.0 and listed in Table B.14.

\begin{tabular}{|l|c|c|c|l|}
\hline \multirow{2}{*}{ Analysis Group } & \multicolumn{3}{|c|}{ MAROS Results Bear Creek Hydrogeologic Regime } \\
\cline { 2 - 5 } & $\begin{array}{c}\text { Total } \\
\text { Number } \\
\text { Wells }\end{array}$ & $\begin{array}{c}\text { Number wells } \\
\text { Above } \\
\text { Screening } \\
\text { Level }\end{array}$ & Priority COCs & \multicolumn{1}{|c|}{$\begin{array}{c}\text { Moment Analysis } \\
\text { (Plume Stability Analysis) }\end{array}$} \\
\hline West S-3 Area & 44 & $33(75 \%)$ & GB & $\begin{array}{l}\text { Variable total mass, Stable to } \\
\text { Decreasing plume spread }\end{array}$ \\
\hline $\begin{array}{l}|c| \\
\text { Oil Landfarm }\end{array}$ & 59 & $28(47 \%)$ & PCE & $\begin{array}{l}\text { Variability in total mass and } \\
\text { spread results }\end{array}$ \\
\hline BMA & & & $\begin{array}{l}\text { Variability in total mass and } \\
\text { spread results }\end{array}$ \\
\hline BCBG Area & 80 & $36(45 \%)$ & TCE & $\begin{array}{l}\text { Variability in total mass and } \\
\text { spread results }\end{array}$ \\
\hline & & & $\begin{array}{l}\text { Total mass may be Increasing, } \\
\text { spread is Stable to Decreasing }\end{array}$ \\
\hline
\end{tabular}

All analysis areas in the Bear Creek Regime contained sample locations with concentrations above screening levels, with $75 \%$ of locations in the West S-3 area above screening levels. The relative number of locations above screening levels 
decreases across the Regime. Results from the COC Risk evaluation were used to choose representative constituents for plumes in each sample group. Representative COCs were evaluated using the Moment Analysis module in order to determine the relative stability of the plumes. Total mass estimates for plumes in each of the Bear Creek Regime analysis groups indicated variability in the data. Data variability may be the result of limited amount of data (as may be the case in West S-3), varying numbers and identities of wells sampled or actual fluctuations in groundwater concentrations. Plume spread appears to be Stable to Decreasing in the downgradient area of the Bear Creek Regime.

Results for individual sample locations trends in each sample group are tabulated below. Both the number of wells in each category and the percentage of total sample group wells in the category are indicated. Sample locations with NT (No Trend) and N/A (insufficient data) designations may benefit from additional sample collection efforts or removal from the program, if they do not provide important information. Locations with stable to decreasing trends (S, PD, D) or non-detect (ND) results may be candidates for reduced sampling effort. Locations with increasing trends (I or $\mathrm{PI}$ ) should be monitored carefully to determine if the trend indicates an increase in the distribution of the plume.

\begin{tabular}{|l|c|c|c|c|}
\hline \multirow{2}{*}{ Analysis Group } & \multicolumn{4}{|c|}{ Overall MAROS Trend Analysis Bear Creek Regime } \\
\cline { 2 - 5 } & N/A, NT & PD, D, S & I, PI & ND \\
\hline \multicolumn{5}{|c|}{} \\
\hline Western S-3 Area & $38(86 \%)$ & $5(11 \%)$ & $1(2 \%)$ & 0 \\
\hline Oil Landfarm WMA & $41(69 \%)$ & $9(15 \%)$ & $5(8 \%)$ & $4(8 \%)$ \\
\hline BCBG Area & $50(63 \%)$ & $15(19 \%)$ & $9(11 \%)$ & $6(7 \%)$ \\
\hline
\end{tabular}

Note: Insufficient Data (N/A), Decreasing (D), Probably Decreasing (PD), Stable (S), Probably Increasing (PI), and Increasing (I), No Trend (NT); and non-detect for all sample events (ND).

Preliminary sampling frequency recommendations were developed using the MAROS MCES method along with preliminary qualitative evaluation for locations with limited sample data. All wells were included in the analysis, independent of their regulatory status. By considering all wells in the network, a clearer picture of the level of effort required for the site could be developed. Preliminary results for the Bear Creek Regime are presented below, with final results detailed in Section II, 4.0. The preliminary results are compared with the sampling frequency for wells in the Bear Creek Regime from the 2004 calendar year (CY) monitoring program.

Preliminary sampling frequency results comparing the total number of samples for the 2004 program and the recommended program indicate no significant difference in total samples (150 versus 151). However, the number of wells sampled in the optimized program would be much greater than in the 2004 program (156 versus 62 locations). 


\begin{tabular}{|l|c|c|c|}
\hline \multirow{3}{*}{ Monitoring Wells } & \multicolumn{3}{|c|}{ Network Frequency Analysis Bear Creek Regime } \\
\cline { 2 - 4 } & $\begin{array}{c}\text { Sampling } \\
\text { Frequency }\end{array}$ & Number of Wells CY 2004 & $\begin{array}{c}\text { Number of Wells } \\
\text { Preliminary } \\
\text { Recommendation }\end{array}$ \\
\hline \multirow{7}{*}{} & Remove & -- & 27 \\
\cline { 2 - 4 } & Quarterly & 15 & -- \\
\cline { 2 - 4 } & Semi-annual & 43 & 30 \\
\cline { 2 - 4 } & Annual & 4 & 57 \\
\cline { 2 - 4 } & Biennial & 0 & 69 \\
\hline Total Samples (per year) & & $\mathbf{1 5 0}$ & $\mathbf{1 5 1}$ \\
\hline Total Wells & & 62 & 156 \\
\hline
\end{tabular}

* Analysis included wells regardless of regulatory status.

\subsubsection{West S-3 Area (West S-3)}

The eastern region of the Bear Creek Hydrogeologic Regime is dominated by the former S-3 Ponds, but includes the Rust Spoils area to the west and Spoils Area I to the south. The former S-3 Ponds were used to dispose of nitric acid containing Tc-99, uranium isotopes and various trace metals and VOCs. The S-3 site is located in the aquitard unit (Nolichucky Shale) above and north of the transmissive aquifer unit. Groundwater flow across the Regime is generally to the west, but is also characterized by fracture flow and surface water interactions resulting in southerly movement of constituents. The West S3 Area has some of the highest constituent concentrations in the Y-12 Complex, and among the 44 wells evaluated, 33 have average concentrations above screening levels for at least one COC. Sample locations for West S-3 are listed in Table B.3 and illustrated on Figures A.1 and A.2.

\section{Constituent Choice}

The MAROS method COC Risk Evaluation module identified nitrate and Gross Beta activity (GB) as the priority COCs in the S-3 region with Gross Alpha activity (GA) also of concern. Tc-99 and uranium isotopes are likely responsible for GB and GA activity. Among the organic constituents, both PCE and TCE were identified as exceeding screening levels. Printed reports from the MAROS software, including the COC Risk Evaluation are located in Appendix D. Priority constituents were also identified for each individual sample location based on the maximum historic exceedance at that location. Among individual wells, nitrate was identified as the most common COC.

\section{Moment Analysis}

The Moment Analysis was conducted for the both GB activity and PCE in the West S-3 area to assess stability of plumes from the two main constituent source classes. Nitrate affects roughly the same area as GB activity. Results of the First Moment Analyses for GB and PCE are indicated on Figure A.5. MAROS-generated Moment Summary Reports are located in Appendix D.1. Results indicate that the total estimated dissolved mass of GB (Zeroth Moment) in the plume showed No Trend or variability (high COV) 
between 1996 and 2004. GB concentrations show high variability within certain wells with high overall concentrations (e.g. GW-526 ranges between non-detect and 718 $\mathrm{pCi} / \mathrm{L})$, which is reflected in the plume-wide variability in total dissolved mass. First Moment analysis (plume center of mass) indicates a Decreasing trend in the distance of the center of mass from the source, indicating a retreat of the center of mass of the plume upgradient. Estimated First Moment locations over time are illustrated on Figure A.5 (right set of panels) and indicate that the centers of mass are clustered just southwest of the former S-3 Ponds. First moment results provide evidence of stability of the GB plume in this area.

\begin{tabular}{|l|c|l|}
\hline \multirow{2}{*}{$\begin{array}{l}\text { Moment } \\
\text { Type }\end{array}$} & \multicolumn{2}{|c|}{ Gross Beta Activity Moment Trend Analysis West S-3 } \\
\cline { 2 - 3 } Zeroth & No Trend & $\begin{array}{l}\text { Comment } \\
\text { The estimate of total dissolved mass of GB showed high variability (No Trend) } \\
\text { during the sample period 1996-2004. }\end{array}$ \\
\hline First & Decreasing & $\begin{array}{l}\text { The estimated distance from the source to the center of mass showed a } \\
\text { Decreasing trend during the sample period. }\end{array}$ \\
\hline Second & $\begin{array}{l}\text { Stable/ } \\
\text { Stable }\end{array}$ & $\begin{array}{l}\text { The GB plume showed Stable trends in the distribution of constituents in the } \\
\text { direction of groundwater flow and perpendicular to groundwater flow. }\end{array}$ \\
\hline
\end{tabular}

Second Moments for the GB plume indicate that the distribution of mass within the plume is also stable and that mass did not redistribute from the center to the edges of the plume during the sample time frame. Stable second moments are supportive of overall plume stability. Evidence for GB plume stability supports reduced monitoring effort in this area.

Evaluation of plume stability for VOC components is based on data for PCE. Total dissolved mass of PCE in the West S-3 area showed No Trend between 1996 and 2004, indicating concentration variability in either the wells sampled or the concentrations within the wells. Likewise, there was No Trend for PCE First Moments between 1996 and 2004. However, when First Moment values derived from sample events with greater than seven sample locations are plotted (as in Figure A.5) the center of mass appears Stable between 1999 and 2004.

\begin{tabular}{|l|c|l|}
\hline \multirow{2}{*}{$\begin{array}{l}\text { Mype } \\
\text { Type }\end{array}$} & Trend & \multicolumn{1}{c|}{ PCE Moment Trend Analysis West S-3 } \\
\cline { 2 - 3 } Zeroth & No Trend & $\begin{array}{l}\text { The estimate of total dissolved PCE was found to have No Trend during the } \\
\text { sample period 1996-2004. }\end{array}$ \\
\hline First & No Trend & $\begin{array}{l}\text { The estimated distance from the source to the center of mass showed No Trend } \\
\text { during the sample period. }\end{array}$ \\
\hline Second & $\begin{array}{l}\text { No Trend/ } \\
\text { Stable }\end{array}$ & $\begin{array}{l}\text { The PCE plume showed No Trend (high variability) in the distribution of } \\
\text { constituents parallel to groundwater flow, but showed a Stable trend in the } \\
\text { direction perpendicular to groundwater flow. }\end{array}$ \\
\hline
\end{tabular}

Second Moments for the PCE plume show No Trend to Stable results for distribution of mass within the plume. The Moment data for PCE suggest that the VOC plume may not be as stable as the GB plume in this region, but does indicate a need for greater monitoring frequency.

Statistical Trend Results 
Statistical Trend results for sample locations in the West S-3 area are shown in Table B.3 and illustrated on Figure A.5. In order to determine a statistical trend in the data, at least four sample events must be included in the time frame. Of the 44 sample locations in the West S-3 area with historic analytical data in the database, 37 wells did not have sufficient data in the 1996 to 2004 time frame to determine concentration trends. The majority of these wells (27 of 37 ) have historic constituent concentrations significantly above screening levels, particularly for nitrate and radioactive constituents.

Sample locations with Decreasing trends included wells GW-829, GW-311 and GW-276. Location GW-315 had a Probably Decreasing trend while spring SS-1 indicates a Stable trend for nitrate. Taken together, this group of wells monitors the western perimeter of the S-3 Ponds area. Well GW-829 is on the western edge of the analysis group close to the Oil Landfarm WMA (near GW-537), where nitrate concentrations are also Decreasing. Well GW-311 is on the southern perimeter of the plume in the Rust Spoil Area, indicating a Decreasing trend for TCE in this area. Spring SS-1 is located, roughly, between GW-829 and GW-311, in the exit pathway along Bear Creek. SS-1 shows a stable trend for nitrate, supporting Stable to Decreasing nitrate trends in the western area of the plume.

Wells GW-315 and GW-276 are on the eastern edge of the West S-3 area near the divide between Bear Creek and the East Fork Poplar Creek Regimes. GW-276 shows a Decreasing trend for the historic high uranium concentrations in the eastern area. Well GW-315 is located in the Spoils Area I, and indicates that PCE concentrations in the Spoils area are Decreasing.

The only Increasing trend was found at GW-835, located in the center of the West S-3 area. The well (piezometer) has historically high concentrations of uranium, which appear to be Increasing over time. Well GW-835 monitors constituents in the shallow zone in or near the Maynardville Limestone aquifer.

\section{Well Sufficiency}

The well sufficiency analysis, indicating areas within the plume where there is low correlation between constituent concentrations at adjacent wells, has many of the same limitations as the redundancy analysis. However, the data for the West S-3 area were examined using this tool. Well sufficiency results for PCE are indicate a VOC source area between GW-115 and GW-276 and GW-315 in the southern region (high PCE concentrations). Similarly, concentrations between wells GW-243 (high PCE) and GW-615 (low PCE) indicate a source or preferential flow path between these locations. No new wells are recommended for PCE delineation, however, locations GW-276, GW-243 and GW-315 should be monitored routinely to track concentrations in this area.. Well sufficiency data for other constituents do not indicate areas of high uncertainty

Sampling Frequency 
Historic site characterization in the West S-3 area indicates high concentrations of constituents, however recent data collection efforts have been limited and insufficient data are available to determine statistical trends for roughly $80 \%$ of sampling locations. As statistical trend analysis is the basis for the sampling frequency evaluation using the MCES method, qualitative methods and historic data review were used to recommend preliminary sampling frequencies for most locations in the West S-3 area. Preliminary sampling frequencies were used as the basis for determining final sampling frequencies in the decision logic part of the analysis. Final sampling frequency recommendations for the Bear Creek Regime are presented in Table B.14 and are illustrated on Figure A.5.

Nineteen wells were recommended for Biennial sampling, largely as a result of insufficient data. The majority of wells in the Biennial sampling group may be very good candidates for removal from the network; but, the limited recent sample data make determination of trends and exceedances of screening levels difficult to evaluate. The contribution of these wells to the network was further evaluated during the decision logic step, outlined in Section II, 4.0.

Annual sampling was recommended for 14 wells. The wells in this group monitor many of the middle areas of the S-3 plume. Locations GW-122, GW-123, and GW-127 as well as GW-276 were identified in the well sufficiency analysis as requiring monitoring effort to delineate PCE concentrations. Wells GW-345 and GW-829 and spring SS-1 monitor the movement of constituents downgradient toward the Oil Landfarm WMA.

The majority of wells recommended for Semi-annual sampling monitor very high nitrate concentrations in the source area around the former S-3 site. The Increasing uranium trend at GW-835 also triggers a recommendation for Semi-annual monitoring, as does GB activity at GW-243. Monitoring source regions can provide important information supporting long-term site management decisions. While concentrations may not change rapidly in this area, developing a strong data set to support statistical or modeling analyses is important.

\subsubsection{Oil Landfarm WMA (OLFA)}

The Oil Landfarm Waste Management Area (OLFA) is characterized by a number of historic waste management locations. The OLFA is downgradient of the West S-3 area and upgradient of the Bear Creek Burial Grounds (BCBG). The area includes the regions known as the Oil Landfarm, Boneyard/Burnyard, the Hazardous Chemical Disposal Area (HCDA) and Sanitary Landfill I. The disposal areas are located on north of Bear Creek and the transmissive Maynardville Limestone. Constituents migrate via fracture flow and surface water to Maynardville formation. The OLFA area waste management units are suspected sources of VOC constituents, most notably TCE. Constituents such as uranium, Tc-99 and nitrate from the West S-3 area migrate into the OLFA via the aquifer, while the Boneyard/Burnyard is a source of elemental uranium GA activity. Fifty-nine monitoring locations were evaluated in the OLFA analysis group. The sample locations are listed in Table B.4 and illustrated on Figure A.2.

Constituent Choice 
The MAROS method COC Risk Evaluation module identified TCE as the primary VOC constituent for overall plumes in the OLFA with vinyl chloride also of concern. Among the inorganic and radioactive constituents nitrate was identified as exceeding screening levels across the area. Reports from the MAROS software, including the COC Risk Evaluation are located in Appendix D.2. Priority constituents were also identified for each individual sample location based on the maximum historic exceedance at that location. TCE was the main COC at 19 of 59 locations. Elemental uranium (GA activity) was identified as a priority at 11 locations and GB activity was identified as the main constituents at 9 locations, but the majority of these locations had average concentrations below screening levels. Uranium isotopes were detected above screening levels at wells GW-226, GW-227 and GW-229, but the ratio of concentrations to screening levels was still below that of the VOC constituents at these locations.

\section{Moment Analysis}

The Moment Analysis was conducted for the both TCE and nitrate in the OLFA to assess stability of plumes from the two general source regions. MAROS-generated Moment Summary Reports are located in Appendix D.2 and results of First Moment estimates are shown on Figure A.5. Results indicate that the total estimated dissolved mass of TCE (Zeroth Moment) in the plume showed No Trend or variability (high COV) between 1996 and 2004. This may be due to variations in the number and identity of wells sampled during this time. First Moment analysis (plume center of mass) indicates an Increasing trend in the distance of the center of mass from the source, indicating movement of constituents downgradient. However, the estimated First Moments illustrated on Figure A.5 for sample event with $>20$ sample locations indicate that the center of mass has not moved significantly since 2001, when the number of wells sampled in the network stabilized. The center of mass for the TCE plume is localized just southwest of the Boneyard/Burnyard and southeast of Landfill I. The result supports relative stability of the VOC plume in this area since 2001.

\begin{tabular}{|l|c|l|}
\hline \multirow{2}{*}{$\begin{array}{l}\text { Moment } \\
\text { Type }\end{array}$} & \multicolumn{2}{|c|}{ TCE Moment Trend Analysis OLFA } \\
\cline { 2 - 3 } Zeroth & No Trend & $\begin{array}{l}\text { Comment } \\
\text { The estimate of total dissolved mass of TCE showed high variability (No Trend) } \\
\text { during the sample period 1996-2004. }\end{array}$ \\
\hline First & Increasing & $\begin{array}{l}\text { The estimated distance from the source to the center of mass showed an } \\
\text { Increasing trend during the sample period. }\end{array}$ \\
\hline Second & $\begin{array}{c}\text { No Trend/ } \\
\text { Probably } \\
\text { Increasing }\end{array}$ & $\begin{array}{l}\text { The TCE plume showed No Trend in the distribution of constituents in the } \\
\text { direction of groundwater flow and a Probably Increasing trend perpendicular to } \\
\text { groundwater flow (mass distributing to the edges of the plume). }\end{array}$ \\
\hline
\end{tabular}

Second Moments for the TCE plume indicate that the distribution of mass in the plume shows high variability in the direction of groundwater flow. In the direction perpendicular to groundwater flow, mass within the plume is redistributing to the edge, with relative reduction of mass in the center of the well network.

Evaluation of Moments for the nitrate indicates that the estimate of total dissolved mass in the plume shows No Trend, similar to the TCE. High variability in total constituent concentrations may be a result of the position of the OLFA in the center of the Bear 
Creek Regime, where constituents can pass into the area from the West S-3 area and out of the area to the BCBG region. Variability in data may also be due to complex hydrogeology, where surface water runoff in response to rainfall events affects concentrations.

No Trend was found for nitrate First Moments between 1996 and 2004. However, when moments are calculated for sample events with $>10$ sample locations, the distribution appears very stable (Figure A.5). Second Moments for nitrate show increasing distribution of nitrate in the direction of groundwater flow and variability perpendicular to groundwater flow. The plumes in the OLFA area have less stable Moment results, with more variation in the data than those for the West S-3 BCBG areas. Variability in the data indicates that the OLFA area may benefit from consistent data collection efforts.

\begin{tabular}{|l|c|l|}
\hline \multirow{2}{*}{$\begin{array}{l}\text { Moment } \\
\text { Type }\end{array}$} & \multicolumn{2}{|c|}{ Nitrate Moment Trend Analysis OLFA } \\
\cline { 2 - 3 } Zeroth & No Trend & $\begin{array}{l}\text { Comment } \\
\text { The estimate of total dissolved nitrate was found to have No Trend during the } \\
\text { sample period 1996-2004. }\end{array}$ \\
\hline First & No Trend & $\begin{array}{l}\text { The estimated distance from the source to the center of mass showed No Trend } \\
\text { during the sample period. }\end{array}$ \\
\hline Second & $\begin{array}{l}\text { Increasing/ } \\
\text { No Trend }\end{array}$ & $\begin{array}{l}\text { The nitrate plume showed an Increasing trend in spread parallel to GW flow and } \\
\text { No Trend (high variability) in the distribution of constituents perpendicular to GW } \\
\text { flow. }\end{array}$ \\
\hline
\end{tabular}

\section{Statistical Trend Results}

Statistical trend analyses were conducted for constituent concentrations at each sample location between January 1996 and 2004. Trend results are listed on Table B.4 and illustrated on Figure A.5. A total of 59 sample locations at the OLFA area had historic analytical data, of these, 30 wells did not have sufficient data in the recent time frame to determine concentration trends. The function of these wells in the analytical network was reconsidered as part of the comprehensive review of the monitoring network.

Among the 29 wells with sufficient data to determine a trend, four locations had Decreasing or Probably Decreasing trends. All Decreasing locations had average concentrations above the screening level. Well GW-537, which is on-strike with GW-085 and GW-829, shows a Decreasing trend for nitrate GW-829 also has a Decreasing trend for nitrate; however, location GW-085 has a Probably Increasing trend for nitrate. Wells GW-738 and GW-740, which are located south of the waste management areas and represent potential exit pathways, show Decreasing to Probably Decreasing trends for TCE. Well GW-363 with a Probably Decreasing for GB activity does not show concentrations above the screening level and may be unaffected (7 samples since 1996, with the last 3 ND results). Five locations showed Stable trends. Four of five stable locations (GW-006, GW-226, GW-229 and GW-922) are in the Sanitary Landfill 1/Oil Landfarm source area. Stable well GW-724 monitors VOC constituents in the Maynardville formation.

Probably Increasing (PI) trends were found at five locations. The Probably Increasing trends for VOCs were found at locations GW-723 and GW-725 in the Maynardville Limestone exit pathway near Stable well GW-724. These three wells together monitor 
VOC constituents in the transmissive aquifer unit, and all have average concentrations above the screening level. The three locations are recommended for Annual sampling frequency. Other wells with Probably Increasing trends delineate the upgradient northeastern sector of the Boneyard/Burnyard and Oil Landfarm. Wells GW-084 and GW-917 have concentrations well below MCLs, and the trend result is most likely an artifact of variable background concentrations of inorganic constituents and variations in detection limits.

Concentration trends with high variation in the data (NT) were found at 11 locations, but eight of these locations had average concentrations below the screening level, indicating the results may represent noise in low concentration data. Well location GW-225 displayed both high TCE concentrations and high variability in the data indicating that more sampling effort may be required in this area. Wells GW-008 and GW-098 showed lower TCE concentrations with some scatter in the data. These source area wells most likely do not require greater monitoring effort.

\section{Well Sufficiency}

Well sufficiency results for nitrate indicate that the area between well GW-225 and well GW-795 has a great deal of uncertainty. The results for nitrate are displayed graphically in Appendix D.2. Nitrate concentrations in this area drop off rapidly between GW-225 and GW-795. As the wells in the network are screened in different intervals (or represent an open hole in the case of GW-225), the variation in concentrations may represent preferential flow paths, and no new wells are recommended in the area, but continued monitoring of these locations is recommended. Well sufficiency data for other constituents do not indicate areas of high uncertainty

\section{Sampling Frequency}

MAROS-generated recommendations along with a qualitative analysis were used to develop preliminary sampling frequency recommendations that were subsequently used in the final decision logic step to recommend final sampling frequencies. Preliminary recommendations are listed in Table B.4, regardless of regulatory status of the location. Final recommended sampling frequency, accounting for regulatory and qualitative factors is listed in Table B.14 and on displayed on Figure A.5.

Nine locations were identified for removal from the routine monitoring program in the preliminary analysis. No wells were recommended for Quarterly sampling. In the MCES method, wells with less than four sample events in the time frame analyzed are given the default sampling frequency of Quarterly. However, because of the strong historical record of site characterization, reduced sampling frequency is recommended for these locations.

Preliminary recommendations for Semi-annual sampling include wells noted above with Probably Increasing trends, concentrations above screening levels or wells in areas of greater uncertainty. Wells GW-225 and GW-085 have been discussed under trend analysis and well sufficiency. Well GW-226 is adjacent to GW-225, and contributes to constituent monitoring in this area. Well GW-066 monitors the source area south of the Boneyard/Burnyard immediately north of the transmissive exit pathway in the aquifer. 
Wells GW-736 and GW-737 monitor the exit pathway for nitrate, but unlike wells GW-723 and GW-724, these wells do not have sufficient data to determine a trend in the recent time frame.

Sixteen wells have preliminary recommendations for Annual sampling. Many of these wells have constituent concentrations above screening levels, but have sufficient historical sample data to determine that the rate of change of concentrations is not rapid. Many of these wells are currently being sampled at Semi-annual or Quarterly intervals. The majority of wells in the network are recommended for Biennial sampling. Many of these locations have insufficient data to determine concentration trends but have concentrations below screening levels. The recommendation for these wells is to collect four sample points in a 10-year time frame and then re-evaluate the contribution of each well to the entire network. Some wells with high average concentrations and limited sample records are recommended for Biennial sampling. Well GW-601, which has a history of exceedance of TCE screening levels, and has an open hole construction monitoring deeper levels, is recommended for Biennial sampling, which will most likely confirm Decreasing trends at this location.

\subsubsection{Bear Creek Burial Grounds Area Analysis Group}

The Bear Creek Burial Grounds (BCBG) analysis group includes the westernmost region of the $\mathrm{Y}-12$ facility. The DOE owns the property west and downgradient of the BCBG area; consequently, affected groundwater does not, technically, extend off-site beyond the boundaries of the Oak Ridge Reservation. The BCBG area includes two major source regions with distinct constituent profiles. The BCBG are a series of waste management units below the crest of Pine Ridge containing diverse of materials. Groundwater underlying the immediate area of the BCBG is in the aquitard formation, and fracture flow conditions are present. Surface water from the streams that run through the BCBG area interact with groundwater downgradient. The predominant constituents of concern in the waste management areas include VOC compounds, most notably PCE and TCE with 11DCE and VC as secondary compounds. The second major source of constituents to the western Bear Creek Regime is affected groundwater entering from areas to the east (West S-3 and OLFA). Constituents from the BCBG area and the West S-3 area commingle in the aquifer unit.

Active monitoring locations on the western edge of the $\mathrm{Y}-12$ facility were grouped together for the MAROS analysis. In the general area of the BCBG, 90 sample locations were identified as 'active' for both hydrologic and analytical monitoring in the Y-12 database. Of the 90 active locations identified in the database, analytical data were available for 80 locations. These 80 locations were included in the BCBG MAROS analysis group and are listed and described in Table B.5. In calendar year (CY) 2004, 27 of these locations, including groundwater wells and springs were sampled at least once.

\section{Constituent Choice}

Priority analytes were identified for the BCBG area as a whole, using the MAROS method COC Risk Evaluation module. Reports from the MAROS software, including the COC Risk Evaluation are provided in Appendix D.3. Additionally, a priority constituent 
was identified for each sample location based on the maximum historical exceedance at that location. The two priority constituents were chosen for the site analysis, tetrachloroethene (PCE) and Gross Beta (GB) activity. The two constituents represent the two distinct constituent classes. Plumes in the BCBG analysis group are highly commingled, with both VOC and inorganic constituents entering from upgradient sources and the BCBG contributing additional VOC.

\section{Moment Analysis}

The Moment Analysis was conducted for both PCE and GB activity in the BCBG area to determine the relative stability of groundwater concentrations in this area. Results indicate that the total estimated dissolved mass of PCE (Zeroth Moment) in the plume showed a Probably Increasing trend between 1996 and 2004. Changing trends may be due to variations in the well network sampled during this time or Increasing concentrations at specific locations. First Moment results (plume center of mass) are shown on Figure A.6 and indicate a Decreasing trend in the distance of the center of mass from the source. The source was set along the eastern edge of the BCBG for the purpose of the analysis, but precise location of a single source or sources is not possible in this area. The center of mass for the PCE plume is localized just southwest of the $B C B G$ and does not seem to be expanding downgradient. This result supports the overall stability of the VOC plume in this area.

\begin{tabular}{|l|c|l|}
\hline \multirow{2}{*}{$\begin{array}{l}\text { Moment } \\
\text { Type }\end{array}$} & \multicolumn{2}{|c|}{ PCE Moment Trend Analysis BCBG } \\
\cline { 2 - 3 } Zeroth & $\begin{array}{c}\text { Trend } \\
\text { Probably } \\
\text { Increasing }\end{array}$ & $\begin{array}{l}\text { Comment } \\
\text { Increasing during the sample period 1996-2004. }\end{array}$ \\
\hline First & Decreasing & $\begin{array}{l}\text { The estimated distance from the source to the center of mass showed a } \\
\text { Decreasing trend during the sample period. }\end{array}$ \\
\hline Second & $\begin{array}{c}\text { Decreasing/ } \\
\text { Probably } \\
\text { Decreasing }\end{array}$ & $\begin{array}{l}\text { The PCE plume showed Decreasing trends in the distribution of constituents } \\
\text { (more mass in the center of the plume relative to the edges). }\end{array}$ \\
\hline
\end{tabular}

Generally Decreasing Second Moments for the PCE plume indicate that the distribution of mass in the plume is near the center (close to the First Moments) as opposed to expanding toward the edges. Moment results for the PCE plume indicate Stable to Decreasing plume mobility with possible Increasing total dissolved mass.

Evaluation of Moments for the GB activity indicates that the estimate of total dissolved activity in the plume is Stable between 1996 and 2004. Plume stability indicates sampling frequencies can be reduced without loss of information because the plume is not changing rapidly.

The trend of GB First Moments is also Stable between 1996 and 2004, showing some vacillation up and down the Bear Creek Regime, perhaps in response to seasonal influences. Differences in First Moment trends for GB and those for PCE reflect the difference in source areas and transport mechanisms for the two constituent classes in this region of the facility. 
Second Moments for GB show greater variability in the direction of groundwater flow. This may support a seasonal component to constituent distribution in this area. Second Moments perpendicular to groundwater flow are Decreasing, supporting a narrowing of the plume in the north/south direction. Cumulative results of the Moment analyses indicate largely Stable PCE and GB plumes, with some further characterization of PCE locations required to evaluate possible mobilization of mass in this area.

\begin{tabular}{|l|c|l|}
\hline \multirow{2}{*}{$\begin{array}{l}\text { Moment } \\
\text { Type }\end{array}$} & \multicolumn{2}{|c|}{ Gross Beta Activity Moment Trend Analysis BCBG } \\
\cline { 2 - 3 } & Trend & \multicolumn{1}{c|}{ Comment } \\
\hline Zeroth & Stable & $\begin{array}{l}\text { The estimate of total dissolved Gross Beta Activity was found to be Stable during } \\
\text { the sample period 1996-2004. }\end{array}$ \\
\hline First & $\begin{array}{l}\text { The estimated distance from the source to the center of mass showed a Stable } \\
\text { trend during the sample period. }\end{array}$ \\
\hline Second & $\begin{array}{l}\text { No Trend/ } \\
\text { Decreasing }\end{array}$ & $\begin{array}{l}\text { The Gross Beta Activity plume showed No Trend (high variability) in the } \\
\text { distribution of constituents in the direction of groundwater flow and a Decreasing } \\
\text { trend perpendicular to groundwater flow (more mass in the center of the plume } \\
\text { relative to the edges). }\end{array}$ \\
\hline
\end{tabular}

\section{Statistical Trend Results}

Trend analyses were conducted on data collected in the BCBG area between January 1996 and 2004. Concentration trend results for the Bear Creek Regime are presented in Table B.5 and Figure A.6, and are summarized at the beginning of this section. In order to determine a statistical trend for the data, at least four sample events must be available for each location. Of the 80 locations examined, 43 had insufficient analytical data in the time range examined to determine a concentration trend. These locations currently serve the primary function of hydrologic (potentiometric) data collection.

Among wells with a sufficient recent sampling history to determine a trend, six wells had either a Decreasing (D) or Probably Decreasing (PD) concentration trend for the identified priority constituent. Nine other wells had a Stable (S) trend while five wells showed ND (non-detect) for all major constituents. This indicates that 20 out of 37 wells with sufficient data had Decreasing, Stable, or non-detect concentration trends.

Increasing (I) or Probably Increasing (PI) trends were found at 9 wells, three locations each for uranium, PCE and TCE. Increasing trends for VOC constituents were found at wells GW-626, GW-627, GW-289 and GW-653 near the downgradient edge of the source area. Locations GW-082 and GW-069 had increasing trends for vinyl chloride, a degradation product of PCE and TCE. Increasing concentrations of vinyl chloride are frequently encountered in locations of active PCE or TCE degradation, and monitoring data in these locations may be used to support evidence of natural attenuation of these constituents.

Of greater concern, Increasing trends for uranium were found at downgradient and Exit Pathway locations GW-715 and SS-6E and SS-7. The average concentration exceeds the screening level at location SS-6E. The downgradient area of the plume may have been impacted by surface water influence or recent remedial actions in the BCBG area. Continued observation of this region is recommended. 
Concentration trends with high variation in the data (No Trend) were found at 8 locations. Of these locations, 5 had average concentrations above the $\mathrm{MCL}$, including GW-046 and GW-071 in the source area. Concentration trends indicate that many locations have Stable to Decreasing trends, and that Increasing and variable concentration trends are more common close to the BCBG waste management units.

\section{Well Sufficiency}

The well sufficiency analysis, which indicates areas within the plume of greater uncertainty in concentration, indicated an area under Burial Ground A-South as an area of high uncertainty for VOC concentrations. Analysis of landfills or waste management units using the well sufficiency tool frequently results in suggestions to add monitoring locations in the middle of the unit. No new wells are recommended for the BCBG area, but the results of the well sufficiency highlight the need to monitor the source areas carefully.

\section{Sampling Frequency}

The MAROS MCES tool was used to recommend preliminary sampling frequencies for locations in the BCBG. Preliminary sampling frequencies are listed in Table B.5. Preliminary frequencies were used in a decision logic step outlined in Section II, 4.0 to form final network recommendations. The final recommended sampling frequency is discussed in Section II, 4.0 and listed in Table B.14 and illustrated on Figure A.6.

Of the 80 locations analyzed, over half did not have the minimum four sample events in the 10-year time frame to perform the statistical trend analyses. In the case of the BCBG area, recommendations for well removal are based in large part on a qualitative analysis of the function of each well. Designation of wells to be removed is difficult for a commingled plume where different constituents dominate at different locations. The preliminary recommendation is to remove 19 locations from the routine analytical program.

Based on the strength of the historic record and general stability of the plumes, no wells were recommended for Quarterly sampling. Wells recommended for Semi-annual sampling include those with Increasing or Probably Increasing trends for VOC constituents, specifically wells GW-626, GW-627, GW-082, and GW-289. Three locations are recommended for Semi-annual sampling based on uranium concentrations: GW-061, GW-694 and the spring SS-6 (which monitors SS-6E and SS-6W).

The majority of wells in the network are recommended for Annual sampling. Locations with Increasing concentrations but average values below the screening level are recommended for Annual sampling. Eight locations recommended for Annual sampling do not meet minimum data needs to determine a trend. These locations should be sampled until a sufficient data set is established and should be re-evaluated to determine if reduction in sampling frequency is warranted. 
Twenty-five wells are designated for Biennial sampling. Fourteen wells in this group have insufficient data to determine a trend, while other locations have ND trend results and may be approaching 'clean' status. Biennial sampling is an indication that given sufficient data, the well may be deemed redundant or unnecessary in the near future. 


\subsection{Upper East Fork Poplar Creek}

The East Fork Poplar Creek Hydrogeologic Regime extends from the Bear Creek/East Fork divide in the west to the Union Valley area in the east. The general groundwater flow is eastward, distinct from the westward flow in the Bear Creek Regime. The East Fork Regime is characterized by intense industrial development, with the majority of current and historic manufacturing, research and processing activities taking place in the valley formed by the creek. Sources present in the East Fork Regime include the former S-3 Ponds and S-2 Site as well as numerous former underground storage tanks (USTS), the Coal Pile Trench, Scrap Yard, numerous industrial facilities, and the Fire Training Facility. The area is characterized by plumes from a number of sources containing diverse constituents.

Similar to the Bear Creek Regime analysis, the East Fork Regime was divided into four analysis areas divided roughly east to west (see Table B.2, Figure A.1). Analysis groups include the Eastern S-3 (East S-3) area, the Central Y-12 area, the Fuel Station area, and the region around New Hope Pond and Union Valley (East Y-12). As in the other hydrogeologic regimes, the subsurface is divided into 'aquifer' group wells in the Maynardville Limestone and the 'aquitard' Conasauga Group and Rome Formation. The aquitard areas are hydraulically upgradient of the Maynardville Limestone and fractures, and possibly, utility traces provide the main flow paths through the unit to the aquifer.

Results from the four MAROS analyses for the East Fork Regime are summarized in tables below and discussed in detail in sections 3.2.1 through 3.2.3. Final network recommendations, based on both qualitative and quantitative approaches are presented in Section II, 4.0 and listed in Table B.15.

\begin{tabular}{|c|c|c|c|c|}
\hline \multirow[b]{2}{*}{ Analysis Group } & \multicolumn{4}{|c|}{ MAROS Results East Fork Hydrogeologic Regime } \\
\hline & $\begin{array}{c}\text { Total } \\
\text { Number } \\
\text { Wells }\end{array}$ & $\begin{array}{c}\text { Number wells } \\
\text { Above } \\
\text { Screening } \\
\text { Level }\end{array}$ & Priority COCs & $\begin{array}{l}\text { Moment Analysis } \\
\text { (Plume Stability Analysis) }\end{array}$ \\
\hline \multirow[t]{2}{*}{ East S-3 Area } & 49 & $37(75 \%)$ & Nitrate & $\begin{array}{l}\text { Variable total mass, Stable to } \\
\text { Decreasing plume spread }\end{array}$ \\
\hline & & & PCE & $\begin{array}{l}\text { Variability in total mass and } \\
\text { spread results }\end{array}$ \\
\hline \multirow[t]{2}{*}{ Central Y-12 Area } & 44 & $26(59 \%)$ & Uranium & $\begin{array}{l}\text { Variability in total mass and } \\
\text { distribution results, Decreasing } \\
\text { distance to center of mass. }\end{array}$ \\
\hline & & & PCE & $\begin{array}{l}\text { Variability in total mass and } \\
\text { distribution results, Decreasing } \\
\text { distance to center of mass. }\end{array}$ \\
\hline Fuel Station Area & 24 & $5(20 \%)$ & Benzene & $\begin{array}{l}\text { Decreasing total mass, but } \\
\text { increasing distance to center } \\
\text { of mass and distribution of } \\
\text { constituents }\end{array}$ \\
\hline $\begin{array}{l}\text { New Hope Pond } \\
\text { Area }\end{array}$ & 54 & $20(37 \%)$ & CTET & $\begin{array}{l}\text { Probably Increasing dissolved } \\
\text { mass (possibly due to } \\
\text { extraction well), Decreasing } \\
\text { trends in spread of plume }\end{array}$ \\
\hline
\end{tabular}


All analysis areas in the East Fork Regime contained sample locations with concentrations above screening levels, with $75 \%$ of locations in the East S-3 area (as with the West S-3 Area) above screening levels. The relative number of locations above screening levels decreases across the East Fork Regime. The Fuel Station area had the fewest number of exceedances, with many being historic. Results from the COC Risk evaluation were used to choose representative constituents for plumes in each sample group. Representative COCs were evaluated using the Moment Analysis module in order to determine the relative stability of the plumes. Total mass estimates for plumes in each of the East Fork Regime analysis groups indicated variability in the data, with the exception of the Fuel Station Area. Data variability may be the result of limited amount of data (as may be the case in East S-3), variations in the well network (i.e. number and distribution) sampled or actual fluctuations in groundwater concentrations. Plume spread appears to be Decreasing in most areas of the East Fork Regime.

Results for individual sample location trends in each sample group are tabulated below. Both the number of wells in each category and the percentage of total sample group wells in the category are indicated. Sample locations with NT (No Trend) and N/A (insufficient data) designations may benefit from additional sample collection efforts or removal from the program, if they do not provide important information. Locations with stable to decreasing trends (S, PD, D) or non-detect (ND) results may be candidates for reduced sampling effort. Locations with increasing trends (I or $\mathrm{PI}$ ) should be monitored carefully to determine if the trend indicates an increase in the distribution of the plume.

\begin{tabular}{|l|c|c|c|c|}
\hline \multirow{2}{*}{ Analysis Group } & \multicolumn{3}{|c|}{ Overall MAROS Trend Analysis East Fork Regime } \\
\cline { 2 - 5 } & NIA, NT & PD, D, S & I, PI & ND \\
\hline \multicolumn{5}{|c|}{} \\
\hline East S-3 Area & $33(67 \%)$ & $9(18 \%)$ & $1(2 \%)$ & $4(8 \%)$ \\
\hline Central Y-12 Area & $25(57 \%)$ & $16(36 \%)$ & $3(7 \%)$ & 0 \\
\hline Fuel Station Area & $15(63 \%)$ & $1(4 \%)$ & $1(4 \%)$ & $7(29 \%)$ \\
\hline New Hope Pond Area & $24(44 \%)$ & $19(35 \%)$ & $6(11 \%)$ & $5(10 \%)$ \\
\hline
\end{tabular}

Note: Insufficient Data (N/A), Decreasing (D), Probably Decreasing (PD), Stable (S), Probably Increasing (PI), and Increasing (I), No Trend (NT); and non-detect for all sample events (ND).

Preliminary sampling frequency recommendations were developed using the MAROS MCES method along with preliminary qualitative evaluation for locations with limited sample data. All wells were included in the analysis, independent of their regulatory status. By considering all wells in the network, a clearer picture of the level of effort required could be developed. Preliminary results for the East Fork Regime are presented below, with final results detailed in Section II, 4.0 and listed in Table B.15. The preliminary results are compared with the sampling frequency for wells in the sampled during the 2004 calendar year (CY) monitoring program. 


\begin{tabular}{|l|c|c|c|}
\hline \multirow{3}{*}{ Monitoring Wells } & \multicolumn{3}{|c|}{ Network Frequency Analysis East Fork Regime } \\
\cline { 2 - 4 } & $\begin{array}{c}\text { Sampling } \\
\text { Frequency }\end{array}$ & Number of Wells CY 2004 & $\begin{array}{c}\text { Number of Wells } \\
\text { Preliminary } \\
\text { Recommendation }\end{array}$ \\
\hline \multirow{3}{*}{} & Remove & -- & 27 \\
\cline { 2 - 4 } & Quarterly & 1 & -- \\
\cline { 2 - 4 } & Semi-annual & 23 & 21 \\
\cline { 2 - 4 } & Annual & 9 & 63 \\
\cline { 2 - 4 } & Biennial & 0 & 58 \\
\hline Total Samples (per year) & & $\mathbf{5 9}$ & $\mathbf{1 3 4}$ \\
\hline Total Wells & & 34 & 142 \\
\hline
\end{tabular}

Preliminary sampling frequency results comparing the total number of samples for the 2004 program and the recommended program indicate an increase in total samples (59 versus 134) and in total wells sampled (34 versus 142). The preliminary results indicate that more sampling effort may be needed, initially, to better define diverse source areas in the developed part of the $\mathrm{Y}-12$ Complex. Preliminary results were carried forward as the basis for integrating qualitative and quantitative network observations into a final sampling program.

\subsubsection{East S-3 Area}

While the former S-3 Ponds are located in the aquitard area of the Bear Creek Hydrogeologic Regime, constituents from the site have migrated into the East Fork Regime. The S-3 site constitutes one of the major sources of groundwater contaminants in the Y-12 Complex. The East S-3 area analysis group includes data from several source areas including the S-2 Site, Beta-4 Security Pits, Rust Garage Area, Fire Training Facility, Y-12 Salvage Yard, and Waste Coolant Processing Facility (see Figure A.7. A total of 49 sample locations were considered in the evaluation of the Eastern S-3 area. Locations are listed on Table B.6 and are illustrated in Figure A.1 and A.2.

\section{Constituent Choice}

The MAROS method COC Risk Evaluation module identified many constituents as being present in the Eastern S-3 region above screening levels. Metals such as manganese and aluminum may have been mobilized by high nitrate concentrations. While metals are of concern over the site, the primary constituents chosen for site analysis were nitrate for the inorganic compounds and PCE as representative of VOC constituents. Reports from the MAROS software, including the COC Risk Evaluation are located in Appendix D. Priority constituents were also identified for each individual location based on the maximum historic exceedance at that location. Among individual wells, nitrate was identified as the priority constituent. 


\section{Moment Analysis}

The Moment Analysis was conducted for both nitrate and PCE in the East S-3 area to evaluate constituents from the two main constituent classes. MAROS-generated Moment Reports are located in Appendix D.4. Results indicate that the total estimated dissolved mass of nitrate (Zeroth Moment) in the plume showed No Trend or variability between 1996 and 2004. This may be due to variations in the number and identity of wells sampled during this time or the influence of seasonal factors on groundwater flow. First Moment results (plume center of mass) show a Decreasing trend in the distance of the center of mass from the source indicating a retreat of the center of mass of the plume upgradient. The estimated First Moments are illustrated on Figure A.7 (first set of panels) and indicate that the nitrate centers of mass are retreating west. Decreasing First Moment results provide evidence of plume stability, and may indicate some plume shrinkage in this area.

\begin{tabular}{|l|c|l|}
\hline \multirow{2}{*}{$\begin{array}{l}\text { Moment } \\
\text { Type }\end{array}$} & \multicolumn{2}{|c|}{ Nitrate Moment Trend Analysis East S-3 } \\
\cline { 2 - 3 } Zeroth & No Trend & $\begin{array}{l}\text { Comment } \\
\text { The estimate of total dissolved mass of nitrate showed high variability (No Trend) } \\
\text { during the sample period 1996-2004. }\end{array}$ \\
\hline First & Decreasing & $\begin{array}{l}\text { The estimated distance from the source to the center of mass showed a } \\
\text { Decreasing trend during the sample period. }\end{array}$ \\
\hline Second & $\begin{array}{l}\text { Stable/ } \\
\text { No Trend }\end{array}$ & $\begin{array}{l}\text { The nitrate plume showed Stable trends in the distribution of constituents in the } \\
\text { direction of groundwater flow and No Trend perpendicular to groundwater flow. }\end{array}$ \\
\hline
\end{tabular}

Second Moments for the nitrate plume indicate that the distribution of mass within the plume is also stable and that mass did not redistribute from the center to the edges of the plume during the sample time frame. Some variability in mass distribution is seen in the direction perpendicular to groundwater flow. Stable second moments are supportive of overall plume stability.

Stability assessment for the VOC components of the plume is based on data for PCE. Total dissolved mass of PCE in the East S-3 area showed No Trend between 1996 and 2004 , indicating variable concentrations in the wells sampled or the number of wells sampled. Likewise, there was No Trend for PCE First Moments between 1996 and 2004. First Moments appear to be moving back and forth along the axis of groundwater flow, and may be responding to seasonal effects in this location.

\begin{tabular}{|l|c|l|}
\hline \multirow{2}{*}{$\begin{array}{l}\text { Mype } \\
\text { Typent }\end{array}$} & Trend & \multicolumn{1}{c|}{ PCE Moment Trend Analysis East S-3 } \\
\cline { 2 - 3 } Zeroth & No Trend & $\begin{array}{l}\text { The estimate of total dissolved PCE showed No Trend during the sample period } \\
1996-2004 .\end{array}$ \\
\hline First & No Trend & $\begin{array}{l}\text { The estimated distance from the source to the center of mass showed No Trend } \\
\text { during the sample period. }\end{array}$ \\
\hline Second & $\begin{array}{l}\text { No Trend/ } \\
\text { No Trend }\end{array}$ & $\begin{array}{l}\text { The PCE plume showed No Trend (high variability) in the distribution of } \\
\text { constituents }\end{array}$ \\
\hline
\end{tabular}


Second Moments for the PCE plume also show No Trend results for the distribution of mass within the plume. The Moment data for PCE suggest that the VOC plume may not be as stable as the nitrate plume in this region.

\section{Statistical Trend Results}

Trend results for East S-3 Area wells between January 1996 and 2004 are shown in Table B.6 and on Figure A.7. Of the 49 sample locations in the East S-3 area with historic analytical data in the database, 30 wells did not have sufficient data in the 1996 to 2004 time frame to determine concentration trends. The majority of these wells (21 of 30) have historic constituent concentrations significantly above screening levels for one or more of constituents. The limited data from the recent time period limits the quantitative approach to evaluating the monitoring network in this area.

Four sample locations showed Decreasing or Probably Decreasing trends. These included wells GW-274, GW-618, GW-620 and GW-633, which monitor locations in the center of the plume. Five additional wells showed Stable trends, including GW-251, GW-275, GW-619, GW-337 and 55-2C. Combined with the Decreasing trend wells, this group indicates that concentrations are largely Stable to Decreasing along the southern centerline of the plume.

Only two wells had Increasing or Probably Increasing trends. Well GW-253 showed a strongly Increasing trend for the metal cadmium on the southern perimeter of the area. The source of the cadmium in this small zone is nitric acid and plating waste liquids placed in the S-2 site immediately adjacent to well GW-253. The adjacent well GW-251 shows a Stable trend for PCE. The well 55-2B showed a Probably Increasing trend for PCE in the downgradient area of the plume.

No Trend results were found for nitrate in wells GW-108 and GW-109, on the western edge of the area. Concentrations of nitrate at these locations are quite high, and greater monitoring effort may be required in this area. Upgradient well GW-633, also installed in the aquitard unit, showed a Decreasing trend for both nitrate and uranium and much lower average concentrations. GW-633 is completed at shallow depth, where wells GW108 and GW-109 are significantly deeper. (However, GW-633 showed an Increasing trend for benzene and Probably Increasing trends for 11DCE and GB activity. Well GW633 is and example of how different constituents can return different results in locations where multiple sources are present. These results were considered in the final sampling recommendation.)

\section{Well Sufficiency}

Well sufficiency results for PCE and nitrate are illustrated graphically in Appendix D.4. Results show that PCE concentration differences between wells GW-192 in the north (low concentrations) and GW-337 in the south (higher concentrations) and GW-274 and GW-275 to the west create a zone of higher concentration uncertainty. This represents the center of the downgradient plume, and reflects the probability that GW-337 and GW-274 and 275 are hydraulically connected, while GW-192 is out of the main flow path. As the wells in this part of the network are screened in different intervals, the 
variation in concentrations is reasonable, and no new wells are recommended. PCE concentrations in this zone, however, may require continued monitoring effort.

Well sufficiency data for nitrate indicates some uncertainty in the northern and eastern perimeters of the plume. This result stems from higher concentrations at well $55-2 \mathrm{C}$, in the center of the plume and very low concentrations at well GW-192. Very few data points are available between GW-192 and well 55-C2 to describe the change in concentration between these two points. While a new well is not suggested, a review and sampling of well 55-1A would reduce uncertainty in this area.

\section{Sampling Frequency}

The East S-3 area is heavily developed and affected by a number of diverse facility operations. Of the 49 wells evaluated, 37 have average concentrations above screening levels for at least one priority constituent. As with the Western S-3 area, there is limited recent data to evaluate concentration trends in the area. The East and West S-3 areas are source locations with consistent high concentrations located in the center of the property. For these reasons, frequent sampling has been de-prioritized. Because of the limited recent data and the diversity of depths and constituents present, wells were recommended for elimination from the program based on mainly qualitative analysis (see final recommendations in Section II, 4.0).

Preliminary sample frequency and redundancy evaluations are based on the MAROS MCES tool. Results of the preliminary frequency analysis are shown in Table B.6. Preliminary results described here were used along with the decision logic described in Section II, 4.0 of this report to develop the final sampling frequency recommendation. Eight wells were initially recommended for removal. The majority of these wells do not have concentrations above screening levels, provide redundant information with similar wells, and have not been routinely sampled recently. Ten wells are recommended for Biennial sampling, largely as a result of insufficient data or very low concentrations. The majority of wells in the Biennial sampling group may be very good candidates for removal from the network when sufficient data are available to evaluate their contribution to the network

The majority of wells have an Annual monitoring frequency recommendation. Many of these wells delineate the plume both horizontally and vertically. Of the 23 wells in the Annual category, 11 have insufficient data to determine a trend and a subsequent monitoring frequency using the MCES method. Wells recommended for Annual monitoring may be candidates for removal from the network when a sufficient number of samples are collected for statistical analysis. The majority of wells recommended for Semi-annual sampling monitor very high nitrate concentrations, although well GW-253, located in the aquifer formation, has a more frequent monitoring recommendation for cadmium concentrations.

The monitoring network in the East S-3 area functions to monitor the S-3 and S-2 source areas that may provide constituents for transport downgradient, particularly in the more transmissive Maynardville formation. Nitrate concentrations appear stable in the S-3 part of the plume, and source monitoring may require reduced effort. Wells located in the aquitard formation function is to provide information on VOC and metal constituents 
originating from diverse and often poorly defined sources in this area. The general monitoring suggestion for the S-3 area is to collect data at an annual frequency until the wells have sufficient data for statistical evaluation. Final recommendations specific to individual wells are detailed in Table B.15.

\subsubsection{Central Y-12 Area}

The Central Y-12 area is the most highly developed region of the Y-12 Complex. General groundwater flow is to the east. Affected groundwater in the Maynardville aquifer enters the area from the East S-3 region of the East Fork Regime and constituents from other sources commingle with affected groundwater entering the area. The aquifer exits the area to the east and flows into the Fuel Station and New Hope Pond and Union Valley areas. The Central Y-12 area includes the Coal Pile Trench and Uranium Oxide Vault regions. The 45 wells evaluated in this area are listed on Table B.7 and are illustrated in Figures A.1 and A.3.

\section{Constituent Choice}

The MAROS method COC Risk Evaluation module identified many constituents above screening levels in the Central Y-12 region. The VOC constituents PCE and TCE are prioritized based on toxicity, while nitrate and uranium are the inorganic constituents of greatest concern. Reports from the MAROS software, including the COC Risk Evaluation are provided in Appendix D.5. Priority constituents were also identified for each individual location based on the maximum historical exceedance at that location. Among individual wells, PCE (14 locations), GA activity (10 locations), and chromium were identified as the priority constituents.

\section{Moment Analysis}

The Moment Analysis was conducted for both uranium and PCE in the Central Y-12 area to evaluate stability of the VOC and inorganic plumes. MAROS-generated Moment Reports are located in Appendix D.5. Moment results indicate that the total estimated dissolved mass of uranium (Zeroth Moment) in the plume showed No Trend or variability between 1996 and 2004. As stated above, this result may be due to variations in the number and identity of wells sampled during this time or the influence of seasonal factors on groundwater flow. First Moment analysis (plume center of mass) indicates a Decreasing trend in the distance of the center of mass from the source, indicating a retreat of the center of mass between 1996 and 2004. First Moments for uranium are shown on Figure A.7. The estimated First Moments provide evidence of plume stability, and possible shrinkage. 


\begin{tabular}{|l|c|l|}
\hline \multirow{2}{*}{$\begin{array}{l}\text { Moment } \\
\text { Type }\end{array}$} & \multicolumn{2}{|c|}{ Uranium Moment Trend Analysis Central Y-12 } \\
\cline { 2 - 3 } Zeroth & No Trend & \multicolumn{1}{c|}{ Comment } \\
\hline First & $\begin{array}{l}\text { The estimate of total dissolved mass of uranium showed high variability (No } \\
\text { Trend) during the sample period 1996-2004. }\end{array}$ \\
\hline Second & $\begin{array}{c}\text { No Trend/No } \\
\text { Trend }\end{array}$ & $\begin{array}{l}\text { The estimated distance from the source to the center of mass showed a } \\
\text { Decreasing trend during the sample period. }\end{array}$ \\
\hline
\end{tabular}

Second Moments for uranium indicate No Trend in the data. The distribution of mass within the plume is not changing in a definite pattern.

Evaluation of Moments for the VOC components of the plume is based on data for PCE. Total dissolved mass of PCE in the Central Y-12 area showed No Trend between 1996 and 2004, indicating high variability in either the wells sampled or the concentrations within the wells. The First Moment trend for PCE shows Decreasing distance of the center of mass from the defined source area. (The designated 'source' area for both uranium and PCE is in the western portion of the Central Y-12 area, roughly near well 56-2B. While there may be other source areas within the plume, choosing a single location can still indicate relative movement or stability of the center of mass. First Moment movement back upgradient rather than downgradient generally indicates the plume is not expanding.).

\begin{tabular}{|l|c|l|}
\hline \multirow{2}{*}{$\begin{array}{l}\text { Moment } \\
\text { Type }\end{array}$} & \multicolumn{2}{|c|}{ PCE Moment Trend Analysis Central Y-12 } \\
\cline { 2 - 3 } Zeroth & No Trend & $\begin{array}{l}\text { The estimate of total dissolved PCE was found to have No Trend during the } \\
\text { sample period 1996-2004. }\end{array}$ \\
\hline First & Decreasing & $\begin{array}{l}\text { The estimated distance from the source to the center of mass showed No Trend } \\
\text { during the sample period. }\end{array}$ \\
\hline Second & $\begin{array}{c}\text { Probably } \\
\text { Increasing/ } \\
\text { No Trend }\end{array}$ & $\begin{array}{l}\text { The PCE plume showed No Trend (high variability) in the distribution of } \\
\text { constituents. }\end{array}$ \\
\hline
\end{tabular}

Second Moments for PCE indicate that mass is becoming more dilute in the center of the plume, and higher in concentration on the perimeter in the direction of groundwater flow. Second Moments perpendicular to groundwater flow show No Trend. Moment results for PCE and uranium indicate some variation in total dissolved mass estimates and distribution of mass in the plume, but Decreasing trends in the length of the plumes.

\section{Statistical Trend Results}

Trend results for Central Y-12 area wells between January 1996 and 2004 are shown in Table B.7. Of the 44 sample locations in the Central Y-12 area with analytical data in the database, 21 wells did not have sufficient data in the 1996 to 2004 time frame to determine concentration trends. Eight of the wells with insufficient data had historic average constituent concentrations above screening levels. 
Six sample locations showed Decreasing trends while three resulted in Probably Decreasing trends. Wells with Decreasing trends include centrally located aquifer wells GW-193 and GW-219, with GW-700 having a Probably Decreasing trend. Aquitard wells with Decreasing trends included GW-782 and GW-788, while aquitard well 59-1B had a Probably Decreasing trend. Decreasing trend wells GW-605 and GW-606 are in the eastern portion of the plume near the New Hope Pond and are part of the CTET plume in that area. Upgradient aquifer well GW-204 had a Probably Decreasing trend for GA activity.

An additional seven sample locations had Stable concentration trends, including the aquifer wells GW-690 and GW-218. Aquitard wells with Stable trends include 56-2C, GW-656, GW-783, GW-791 and GW-792. Combined together, these sample locations with Stable to Decreasing trends represent 16 of the 24 sample locations with defined trends, providing supporting evidence of overall stability of the plumes.

Three wells had Increasing or Probably Increasing trends. Wells GW-769 and GW-770, located in the center of the plume and screened in the aquitard formation had increasing trends for CTET. Average concentrations of CTET are not particularly high at these locations, but the source of CTET in this region of the plume is not clear. Well GW-781 shows a Probably Increasing trend for PCE in the central portion of the area.

A No Trend result indicates high variability in constituent concentrations. No Trend results were found for TCE (as well as nitrate) at aquifer well GW-698 on the southern perimeter of the area. Other wells with No Trend results include GW-787, GW-789, and GW-820. The latter well has high historic concentrations of PCE and is located on the southern edge of the plume.

\section{Well Sufficiency}

Well sufficiency results for PCE and uranium are illustrated graphically in Appendix D.5. Results show PCE concentration delineation may benefit from greater sampling effort in the region of wells GW-765, well 55-6A, and wells GW-791/792 in the north. The result is due to relatively high concentrations of PCE at the GW-791/792 cluster and low concentrations on the perimeter. While no new wells are recommended in this area, continued sampling effort in this area may be required. An additional area of PCE concentration uncertainty was identified in the region between GW-218, GW-820, and GW-770. Continued monitoring effort should be focused in this area of the plume as well.

Well sufficiency data for uranium indicates some uncertainty in the area around wells GW-788/789 and well GW-193. Uranium concentrations in this area show intermittent detections and the uncertainty may be due to variations in concentration, the presence of secondary sources, or differences in well depth.

\section{Sampling Frequency}

Preliminary sample frequency and redundancy evaluations are based on the MAROS MCES tool. Results of the preliminary frequency analysis are shown in Table B.7. Preliminary results described here were used along with the decision logic described in 
Section II, 4.0 of this report to develop the final sampling frequency recommendation. Final recommendations are listed in Table B.15. Of the 44 locations evaluated, 21 locations have insufficient recent data to confirm a concentration trend, which forms the basis for the sampling frequency recommendation. Twenty-six locations have average concentrations above screening levels for at least one priority constituent.

Eight wells were initially recommended for removal. The majority of these wells do not have concentrations above screening levels, provide redundant information with similar wells, and have not been routinely sampled, recently. Seven wells are recommended for Biennial sampling due to insufficient data or the location of the well. The majority of wells in the Biennial sampling group may be very good candidates for a more reduced sampling frequency (four samples in a 10 year period) when a larger data set is available to evaluate their contribution to the network.

The majority of wells have an Annual monitoring frequency recommendation. Many of these wells delineate the plume both horizontally and vertically. Of the 22 wells in the Annual category, six have insufficient data to determine a trend and a subsequent monitoring frequency using the MCES method. Historic trends were used to recommend frequencies at these locations. Wells recommended for Annual monitoring may be candidates for removal or reduced sampling frequency when a larger data set is available. Wells recommended for Semi-annual sampling monitor very high concentration groundwater with variable trends (GW-820 and GW-698), wells with Increasing trends (GW-769 and GW-770) and wells with insufficient data and average concentrations above the screening level.

The Central Y-12 area has many diverse sources and a large number of priority constituents as well as on-going industrial operations, which can create challenges to the monitoring program. Because inorganic concentrations appear stable to decreasing in many areas of the plume, and most wells monitor water in the aquitard formation, the main function of many of the wells is to provide information on VOC and metal constituents originating from diverse and often poorly defined sources in this area.

\subsubsection{Fuel Station}

The Fuel Station area of the East Fork Regime is a small area near a former fuel station where the predominant COCs are benzene and PCE/TCE. The Fuel Station area is located east of the Central Y-12 area and west of the New Hope Pond area. A group of 24 wells were included in this analysis. The wells are listed on Table A.8.

\section{Constituent Choice}

The MAROS software identified benzene, manganese and TCE/PCE as priority constituents in the Fuel Station area. While manganese is present above the secondary MCLs and lead was identified as the constituent with the highest concentration at several locations, metal contamination appears isolated and the metals do not lead the analysis. For individual wells within the network, benzene, TCE, and PCE were the most common constituents present above screening levels. 


\section{Moment Analysis}

A Moment Analysis for the Fuel Station area was conducted for benzene. MAROSgenerated Moment Reports are located in Appendix D.6. Moment results indicate that the total estimated dissolved mass of benzene (Zeroth Moment) in the plume showed a Decreasing trend between 1996 and 2004. A Decreasing trend for benzene is often seen in plumes with ongoing natural attenuation processes. First Moment analysis (plume center of mass) indicates an Increasing trend in the distance of the center of mass from the source. After 1998, First Moment results were determined from a limited group of monitoring wells, which may have skewed the trend.

An Increasing trend for the center of mass and Decreasing total mass indicates that benzene is degrading at the source while a dilute mass may be moving downgradient. This view of the plume is supported by the Increasing Second Moments which indicate that the plume is becoming more dilute in the center with relatively more mass present at the perimeter. These results indicate a plume shrinking in mass, but moving downgradient as a dilute footprint.

\begin{tabular}{|l|c|l|}
\hline \multirow{2}{*}{$\begin{array}{l}\text { Moment } \\
\text { Type }\end{array}$} & \multicolumn{1}{|c|}{ Benzene Moment Trend Analysis Fuel Station } \\
\cline { 2 - 3 } Zeroth & Decreasing & $\begin{array}{l}\text { Comment } \\
\text { The estimate of total dissolved mass of benzene was found to be Decreasing } \\
\text { during the sample period 1996-2004. }\end{array}$ \\
\hline First & Increasing & $\begin{array}{l}\text { The estimated distance from the source to the center of mass showed an } \\
\text { Increasing trend during the sample period. }\end{array}$ \\
\hline Second & $\begin{array}{l}\text { Increasing/In } \\
\text { creasing }\end{array}$ & $\begin{array}{l}\text { The benzene plume showed Increasing distribution of mass to the edge of the } \\
\text { plume during the sample period. }\end{array}$ \\
\hline
\end{tabular}

\section{Statistical Trend Results}

Trend results for the Fuel Station Area wells between January 1996 and 2004 are shown in Table B.8. In order to determine a statistical trend in the data, at least four sample events must be included in the time frame. Locations that had less than four sample events between 1996 and December 2004 are indicated by an N/A in the Trend category.

Of the 24 sample locations in the Fuel area with analytical data in the database, 10 wells did not have sufficient data in the 1996 to 2004 time frame to determine concentration trends. Three of the wells with insufficient data had historic average concentrations above screening levels.

None of the wells with sufficient data had Decreasing or Probably Decreasing trends for the identified priority constituent. Well GW-776 had a Stable trend for TCE, while well GW-775 in the same cluster had No Trend, for the same compound. GW-775 had low but variable detections of TCE, triggering a No Trend result. In all, five wells showed No Trend results.

A No Trend result can indicate noise in the data, seasonal influence or as in the case of wells GW-753 and GW-754, outliers or spurious, unrepeated single detections. While 
wells GW-753 and GW-754 may be clean, one outlier can skew the result. Well GW-658 also had a No Trend result, but has a history of fairly high benzene concentrations that show some variability.

Seven wells in the network had no detections for the priority constituent during the 1996 to 2004 sample period. These wells are trending toward 'clean' status and are candidates for removal from the monitoring program. The trend analysis indicated that many wells had variable concentrations of priority constituents, and many locations are trending toward clean by displaying intermittent detections.

\section{Well Sufficiency}

The well sufficiency analysis, which indicates areas within the plume where there is low correlation between constituent concentrations at adjacent wells, indicated no areas of high spatial uncertainty for the priority constituents. No new wells are recommended.

\section{Sampling Frequency}

The Fuel Station area is heavily developed but is not affected by as many diverse sources and constituents as other areas of the East Fork Regime. All but one of the wells considered in this area is screened in the aquitard geologic unit, where there is reduced potential for contaminant transport. Of the 24 wells evaluated, 8 have average concentrations above screening levels for at least one priority constituent and 10 locations do not have enough data in the recent time frame to recommend a sampling frequency using the MCES method. The final recommended sampling frequency for individual wells is listed in Table B.15.

Based on the preliminary analysis, nine wells have been recommended for removal. The majority of these wells do not have concentrations above screening levels, provide redundant information with similar wells, and have not been routinely sampled recently. Ten wells are recommended for Biennial sampling. Many Biennial wells had historic exceedances of MCLs but have since dropped near or below detection limits. The majority of wells in the Biennial sampling group may be good candidates for more reduced sampling frequency (four samples over a 10-year period) or elimination from the monitoring program when a larger data set is available to evaluate their contribution to the network.

The benzene plume in the Fuel Station area has a largely Stable to Decreasing mass, with many wells trending toward 'clean' status. TCE is encountered sporadically across the site, with higher concentrations at GW-775 and GW-776, from upgradient source. Groundwater sampling in this region should be directed at collecting sufficient data to confirm natural attenuation of constituents in this area, and to delineate the extent of groundwater affected with chlorinated VOCs.

\subsubsection{East $Y$-12 Area}

The East Y-12 area, including the New Hope Pond and the Union Valley areas, represent the easternmost area of the Y-12 Complex. Affected groundwater in this 
region extends off of the property into the Union Valley/Scarboro Road area, and, therefore, requires greater monitoring effort in order to support management decisions protective of potential off-site receptors. In all, 54 monitoring locations in the East Y-12 area were evaluated using MAROS. The list of wells considered in this monitoring group is shown in Table B.9 and the approximate extent of the analysis area is shown in Figure A.1.

\section{Constituent Choice}

The MAROS Constituent Choice module for the entire area indicates that CTET and PCE are the main VOC constituents of concern (see MAROS COC Reports, Appendix D-7.) Manganese is found above secondary MCL levels, but is not considered a major constituent for the MAROS analysis. For the overall analysis, priority constituents for East Y-12 included CTET, PCE, GA and GB activity. The metals lead, cadmium, and chromium were also included in the analysis, but the majority of exceedances of these compounds occurred prior to 1996. Due to lower mobility, intermittent detections and overall lower concentrations relative to MCLs, metals are not considered as driving the analysis.

The detection of VOCs, metals and radioactive components in the East $\mathrm{Y}-12$ area demonstrates the importance of monitoring a full suite of constituents across the site. A full set of analytes helps to delineate the commingled plumes underlying the easternmost area of the $\mathrm{Y}-12$ Complex.

\section{Moment Analysis}

The Moment Analysis was conducted for the CTET plume in the East Y-12 area. Results indicate that the total estimated dissolved mass of CTET (Zeroth Moment) in the plume Increased between 1996 and 2004. This may be due to mobilization of constituent from the source zones after the installation of the extraction well GW-845 in October 2000. First Moment analysis (plume center of mass) indicates a Decreasing trend in the distance of the center of mass from the estimated source (GW-381). The estimated First Moments are illustrated on Figure A.7. Prior to 1998, the center of dissolved mass for CTET ranged between New Hope Pond and east of Scarboro Road (depending on which wells were sampled). After 2000, the center of mass has stayed relatively stationary between New Hope Pond and GW-845.

The First Moment calculations between 2000 and 2004 were performed for a fairly consistent data set including approximately 15 wells for each sample episode. The consistency of the First Moment data are the result of a very good data set for this area for the time interval evaluated. One of the criteria for a useful long-term monitoring network plan is providing a cohesive data set over a long time interval, which includes the same constituents at the same wells. 


\begin{tabular}{|l|c|l|}
\hline \multirow{2}{*}{$\begin{array}{l}\text { Moment } \\
\text { Type }\end{array}$} & \multicolumn{2}{|c|}{ Carbon Tetrachloride Moment Trend Analysis East Y-12 } \\
\cline { 2 - 3 } & $\begin{array}{c}\text { Trend } \\
\text { Probably } \\
\text { Increasing }\end{array}$ & $\begin{array}{l}\text { Comment } \\
\text { Increastimate of total dissolved mass of CTET was found to be Probably } \\
\text { Increasing during the sample period 1996-2004. }\end{array}$ \\
\hline First & Decreasing & $\begin{array}{l}\text { The estimated distance from the source to the center of mass showed a } \\
\text { Decreasing trend during the sample period. }\end{array}$ \\
\hline Second & $\begin{array}{c}\text { Decreasing/ } \\
\text { Probably } \\
\text { Decreasing }\end{array}$ & $\begin{array}{l}\text { The CTET plume showed Decreasing trends in the distribution of constituents } \\
\text { (more mass in the center of the plume relative to the edges). }\end{array}$ \\
\hline
\end{tabular}

Second Moment results indicate the relative distribution of mass within the plume. Decreasing trends for the Second Moments show the majority of dissolved mass is in the center of the plume rather than trending to the edge of the plume. With the installation of the extraction well, the plume has become stable to retreating in the New Hope Pond area, even though estimates of the total dissolved mass are Increasing.

\section{Statistical Trend Results}

Results of the Statistical Trend analysis are detailed on Table B.9 and summarized in the table above. Increasing concentration trends were found at wells GW-151, and GW220 for CTET and PCE. These trends may be the result of constituent mobilization caused by groundwater pumping at extraction well GW-845 or shallow groundwater influence of the UEFPC Distribution channel underdrain. However, the Increasing trends in this area of the plume require consistent monitoring effort due to the proximity to the property boundary, and the location within the more transmissive Maynardville Limestone. Because of the Increasing trend in this area, monitoring effort should be focused on monitoring locations immediately downgradient of New Hope Pond and along the Y-12 property boundary adjacent to Scarboro Road.

Monitoring wells GW-750 and GW-735 delineate the northern edge of the CTET plume, downgradient from GW-151 and GW-220. The geology south of these wells is complicated, with monitoring location GW-734, drilled into a subterranean cave, providing little useful data. Well GW-722 is a multi-port Westbay well, which was not included in the MAROS analysis, but should be monitored to track concentration trends in this area of the plume. GW-733 is recommended for Annual sampling in this area, and Annual sampling of GW-722 would create a cohesive data set in this location. Monitoring GW-167 showed no detections of CTET in 1991, when it was last sampled for VOCs. Due to the position of GW-161 and the Increasing trends upgradient, this location is recommended for Annual monitoring until sufficient samples are available to determine if VOC constituents are detected at this location.

Three wells had Probably Increasing trends that did not exceed the screening level for the associated constituent. These wells included GW-385 for lead, GW-746 for chromium and GW-747 for GB activity. 


\section{Well Sufficiency}

The well sufficiency analysis, which indicates areas of greater concentration uncertainty within the plume, indicated two areas with larger uncertainty. The results of the well sufficiency analysis for CTET are presented in Appendix D.7. An area of larger uncertainty is located between wells GW-747, GW-750, and GW-170. While no new wells are suggested for this area, continued monitoring of these locations and locations in the general area is recommended. The area of higher uncertainty corresponds with the property boundary between the $\mathrm{Y}-12$ Complex and property in Union Valley.

The second area of higher uncertainty is located in the region of wells GW-154, GW-153, and GW-283 (and their associated nested wells). This location corresponds to the western side of the capped New Hope Pond. As mentioned above, the extraction well at GW-845 may be mobilizing constituents through this area. No new wells are recommended, but the area should be monitored regularly to determine concentration trends at these locations.

\section{Sampling Frequency}

The MAROS MCES method was use to recommend preliminary sampling frequencies for the locations in the East $\mathrm{Y}-12$ area. Preliminary frequencies are listed in Table B.9 with final recommended sampling frequencies listed in Table B.15. Of the 54 locations used in the analysis, 13 have insufficient data to determine a concentration trend, which is the basis for the sampling frequency recommendation. Many wells with Biennial sampling status may be candidates for removal when sufficient data are collected to confirm that they have attained 'clean' status. The MAROS software tool has an option to evaluate if a well is statistically below MCL using an USEPA statistical protocol (see Data Sufficiency in Section 2.2). When applied to the East Y-12 area, several wells were approaching statistical cleanliness for several compounds. However, in order to establish that a well is clean, there must be a sufficiently large data set for all COCs detected at the well. Data sets with a partial suite of analytes or with large gaps in data collection can present challenges to these types of rigorous statistical evaluations. The Data Sufficiency module in MAROS may provide support for removing statistically unaffected locations from the program after approximately two more years of data are collected.

Wells recommended for Semi-annual sampling include those with Increasing trends for CTET, specifically wells GW-151, and GW-220, and wells with high but variable concentrations of PCE (GW-383). Well GW-154 has an Increasing trend for GA activity and is also recommended for Semi-annual sampling.

The preliminary recommended monitoring network in the East $\mathrm{Y}-12$ area reduces the number of samples collected each year, but increases the number of wells surveyed. This recommendation is possible because the extraction well installed at GW-845 appears to have stabilized the plume substantially. By decreasing the frequency of sampling, the overall cost and effort are reduced, and by increasing the number of wells in the program, the sensitive but extensive geographical area around Scarboro Road can be evaluated. 


\subsection{Chestnut Ridge Hydrogeologic Regime}

The Chestnut Ridge Regime is distinct from the Bear Creek and East Fork Poplar Creek Regimes in terms of both geology and source areas. The Chestnut Ridge Regime is underlain by the Knox Group formation and groundwater below the vadose zone occurs in planar fractures within an impermeable matrix. Groundwater flow in the southern area of the regime is generally to the south. Along the ridge, groundwater flow can be to the east, with radial flow in some locations.

The Chestnut Ridge Regime is one of the least developed areas of the Y-12 Complex, with low levels of VOC, metal, and radioactive constituents. The predominant source areas are a series of landfills and solid waste disposal areas, both historic and active, used to dispose of both construction and hazardous wastes. The Chestnut Ridge Regime was divided into four analysis groups based, roughly, on west to east groupings: the West Chestnut Ridge area contains the United Nuclear Corporation site, the Security Pits area along the ridge, Landfill V and VII, and the East Chestnut Ridge/ Kerr Hollow Quarry area.

Results from the four MAROS analyses for the East Fork Regime are summarized in tables below and discussed in detail in sections 3.3.1 through 3.3.3. Final network recommendations, based on both qualitative and quantitative approaches are presented in Section II, 4.0 and detailed in Table B.16.

\begin{tabular}{|c|c|c|c|c|}
\hline \multirow[b]{2}{*}{ Analysis Group } & \multicolumn{4}{|c|}{ MAROS Results Chestnut Ridge Hydrogeologic Regime } \\
\hline & $\begin{array}{l}\text { Total } \\
\text { Number } \\
\text { Wells }\end{array}$ & $\begin{array}{l}\text { Number wells } \\
\text { Above } \\
\text { Screening } \\
\text { Level }\end{array}$ & Priority COCs & $\begin{array}{l}\text { Moment Analysis } \\
\text { (Plume Stability Analysis) }\end{array}$ \\
\hline $\begin{array}{l}\text { West Chestnut } \\
\text { Ridge }\end{array}$ & 24 & $3(12.5 \%)$ & $\begin{array}{l}\text { None identified by } \\
\text { MAROS }\end{array}$ & $\begin{array}{l}\text { Largely unaffected, Stable to } \\
\text { Decreasing trends. GB and Ni } \\
\text { in some locations. }\end{array}$ \\
\hline Security Pits & 29 & $9(31)$ & PCE & $\begin{array}{l}\text { Variability in total mass and } \\
\text { spread results }\end{array}$ \\
\hline Landfill V/VII & 10 & 0 & PCE & $\begin{array}{l}\text { Largely unaffected, Probably } \\
\text { Increasing total mass, Stable } \\
\text { distribution trends }\end{array}$ \\
\hline $\begin{array}{l}\text { East Chestnut } \\
\text { Ridge }\end{array}$ & 26 & $3(15 \%)$ & PCE & $\begin{array}{l}\text { Largely unaffected, PCE } \\
\text { results are for the off-site } \\
\text { locations GW-841-844 }\end{array}$ \\
\hline
\end{tabular}

Analysis areas in the Chestnut Ridge Regime contained relative few sample locations with concentrations above screening levels. The Landfill V and VII area showed no average exceedances. Overall, the Chestnut Ridge Regime is not as heavily impacted as other Y-12 Complex areas. Results from the COC Risk evaluation were used to choose representative constituents for plumes in each sample group. Representative COCs were evaluated using the Moment Analysis module in order to determine the relative stability of the plumes. Due to the low levels of constituents present at these locations, variability in data may be due to concentrations vacillating around the detection limit. 
Results for individual sample location trends in each sample group are tabulated below. Both the number of wells in each category and the percentage of total sample group wells in the category are indicated. Sample locations with NT (No Trend) and N/A (insufficient data) designations may benefit from additional sample collection efforts or removal from the program, if they do not provide important information. Locations with stable to decreasing trends (S, PD, D) or non-detect (ND) results may be candidates for reduced sampling effort. Locations with increasing trends (I or $\mathrm{PI}$ ) should be monitored carefully to determine if the trend indicates an increase in the distribution of the plume.

\begin{tabular}{|l|c|c|c|c|}
\hline \multirow{2}{*}{ Analysis Group } & \multicolumn{4}{|c|}{ Overall MAROS Trend Analysis Chestnut Ridge Regime } \\
\cline { 2 - 5 } & NIA, NT & PD, D, S & I, PI & ND \\
\hline \multicolumn{5}{|c|}{} \\
\hline West Chestnut Ridge & $5(21 \%)$ & $13(54 \%)$ & $3(12.5 \%)$ & $3(12.5 \%)$ \\
\hline CRSP & $23(79 \%)$ & $4(15 \%)$ & $1(3 \%)$ & $1(3 \%)$ \\
\hline Landfills V and VII & 0 & $1(10 \%)$ & $1(10 \%)$ & $8(80 \%)$ \\
\hline East Chestnut Ridge & $15(58 \%)$ & $7(27 \%)$ & 0 & $4(15 \%)$ \\
\hline
\end{tabular}

Note: Insufficient Data (N/A), Decreasing (D), Probably Decreasing (PD), Stable (S), Probably Increasing (PI), and Increasing (I), No Trend (NT); and non-detect for all sample events (ND).

\begin{tabular}{|l|c|c|c|}
\hline \multirow{3}{*}{ Monitoring Wells } & \multicolumn{3}{|c|}{ Network Frequency Analysis Chestnut Ridge Regime } \\
\cline { 2 - 4 } & $\begin{array}{c}\text { Sampling } \\
\text { Frequency }\end{array}$ & Number of Wells CY 2004 & $\begin{array}{c}\text { Number of Wells } \\
\text { Preliminary } \\
\text { Recommendation }\end{array}$ \\
\hline \multirow{7}{*}{} & Remove & - & 13 \\
\cline { 2 - 4 } & Quarterly & 2 & -- \\
\cline { 2 - 4 } & Semi-annual & 56 & 5 \\
\cline { 2 - 4 } & Annual & 2 & 29 \\
\cline { 2 - 4 } & Biennial & 0 & 42 \\
\hline Total Samples (per year) & & $\mathbf{1 2 0}$ & $\mathbf{5 8 . 5}$ \\
\hline Total Wells & & 59 & 76 \\
\hline
\end{tabular}

\subsubsection{West Chestnut Ridge Area}

The West Chestnut Ridge area extends from the crest of Chestnut Ridge in the west, south toward Bethel Valley. The analysis group includes Industrial Landfills II, IV and VI, the United Nuclear Corporation Site, Construction/Demolition Landfill VI and some Oak Ridge Sludge Farm areas. The twenty-four wells included in this analysis group are listed in Table B.10 and the approximate extent of the analysis area is indicated in Figure A.1 and well locations are identified in Figure A.4. 


\section{Constituent Choice}

The MAROS Constituent Choice module for the West Chestnut Ridge area did not indicate any priority constituents above screening levels on an area-wide level. Target constituents were identified for each individual location based on historic detections. Specific locations have historically exceeded screening levels for GA and GB activity, including uranium as well as nickel and lead. Unlike areas in the Bear Creek and East Fork Regimes, no single dominant plume or plumes are located in the West Chestnut Creek area. GA activity is the primary constituent at 12 locations in the area, and was chosen for review using the Moment Analysis tool.

\section{Moment Analysis}

The Moment Analysis was conducted for GA activity in the West Chestnut Ridge area. Estimates of total dissolved activity (Zeroth Moment) Decreased between 1996 and 2004. First Moments (center of mass) for the plume were Stable during this time period. Second Moments, which describe the distribution of mass within the plume showed Stable to No Trend results. The estimate of Moments provides evidence of the overall stability of this plume.

\begin{tabular}{|l|c|l|}
\hline \multirow{2}{*}{$\begin{array}{l}\text { Moment } \\
\text { Type }\end{array}$} & \multicolumn{2}{|c|}{ Gross Alpha Activity Moment Trend Analysis West Chestnut Ridge } \\
\cline { 2 - 3 } Zeroth & Trend & \multicolumn{1}{c|}{ Comment } \\
\hline First & Stable & $\begin{array}{l}\text { The estimate of total dissolved mass of GA was found to be Decreasing during } \\
\text { the sample period 1996-2004. } \\
\text { trend during the sample period. }\end{array}$ \\
\hline Second & $\begin{array}{c}\text { Stable/No } \\
\text { Trend }\end{array}$ & The GA plume showed Stable to No Trend in the distribution of constituents. \\
\hline
\end{tabular}

\section{Statistical Trend Results}

Results of the Statistical Trend analysis are shown in Table B.10 and illustrated on Figure A.9. The statistical trend is a combination of the Mann-Kendall statistical analysis and the Linear Regression method. Of the 24 wells analyzed, only one location had insufficient data to determine a trend. A total of eight locations showed Decreasing trends for the priority constituent, and of these, none had average constituent concentrations above the screening levels. No locations showed Probably Decreasing trends.

Stable to non-detect (ND) trends were found at an additional six sample locations, including two springs analyzed (SCR2.1SP and SCR2.2SP). Fifteen of 24 sample locations showed Stable, Decreasing or non-detect results. These results support the stability of the plume, and indicate that the monitoring effort can be reduced in these locations.

Well locations GW-203 and GW-205 on the southern edge of the area showed Probably Increasing trends for GA and GB activity. Nearby location GW-221 showed No Trend for GA activity. However, these locations did not have concentrations above screening 
levels, and detections may not be significant. Location GW-305 showed an Increasing trend for nickel, the primary constituent, as well as 111TCA. GW-305 is located in Industrial Landfill IV area and surrounding wells did not indicate increasing nickel concentrations.

Well Sufficiency

Well sufficiency analyses were inconclusive for the West Chestnut Ridge group. As concentrations do not exceed reasonable background in many locations, no new wells are recommended and locations chosen for removal from the monitoring program were chosen by qualitative analysis.

\section{Sampling Frequency}

The MAROS recommendations along with a qualitative analysis indicate that sampling frequency can be reduced for most wells from semi-annual to annual or biennial frequency. Because many of these wells are included in regulatory programs, actual reduction in sampling frequency may not be possible. However, for the majority of the area, additional monitoring above that needed for regulatory compliance does not appear to be necessary.

Preliminary sampling frequency results are shown on Table B.10. The final recommended sampling frequency is listed in Section II, 4.0 and on Table B.16 and illustrated on Figure A.9.

The recommended level of monitoring effort in this location is essentially for Biennial sampling (30 wells). Locations recommended for Annual monitoring include those with Probably Increasing trends or with average concentrations close to screening levels.

\subsubsection{Security Pits Area}

The Chestnut Ridge Security Pits (CRSP) are located on the crest of Chestnut Ridge and consist of a series of trenches that received hazardous wastes until the mid-1980's. The Security Pits analysis area extends from the crest of Chestnut Ridge directly south of the Central Y-12 Complex (East Fork Regime) to the Filled Coal Ash Pond, down to Rogers Quarry. The analysis group consisted of 29 sample locations, which are listed on Table B.11 and illustrated on Figures A.1 and A.4.

\section{Constituent Choice}

The MAROS Constituent Choice module identified PCE and 1,1-DCE as priority constituents for the Chestnut Ridge Security Pit analysis group (see MAROS COC Assessment Report in Appendix D.9). However, these constituents were not detected widely (for PCE only 9 out of 29 wells exceeded the screening level) or at high concentrations. 


\section{Moment Analysis}

The Moment Analysis was conducted for PCE in the Security Pit area. Estimates of total dissolved mass of PCE showed No Trend between 1996 and 2004, indicating variability in the data (high coefficient of variation). First Moments (center of mass) for the plume were Stable during this time period. The trend in First Moment locations shows that the center of mass moves around on the ridge, perhaps in response to the number and identity of wells sampled during each event or due to variability in low to non-detect concentrations encountered. Second Moments, which describe the distribution of mass within the plume showed No Trend results. Because concentrations of PCE are relatively low in the plume, small changes in concentrations can result in higher variation in the data. The estimate of Moments provides evidence of the general stability of the plume.

\begin{tabular}{|l|c|l|}
\hline \multirow{2}{*}{$\begin{array}{l}\text { Moment } \\
\text { Type }\end{array}$} & \multicolumn{2}{|c|}{ PCE Moment Trend Analysis CRSP } \\
\cline { 2 - 3 } Zeroth & No Trend & $\begin{array}{l}\text { Comment } \\
\text { The estimate of total dissolved mass of PCE was found to be No Trend during } \\
\text { the sample period 1996-2004. }\end{array}$ \\
\hline First & Stable & $\begin{array}{l}\text { The estimated distance from the source to the center of mass showed a Stable } \\
\text { trend during the sample period. }\end{array}$ \\
\hline Second & $\begin{array}{c}\text { No Trend/No } \\
\text { Trend }\end{array}$ & The GA plume showed Stable to No Trend in the distribution of constituents. \\
\hline
\end{tabular}

\section{Statistical Trend Results}

Results of the Statistical Trend analysis are shown on Table B.11. The statistical trend is a combination of the Mann-Kendall statistical analysis and the Linear Regression method. Of the 29 wells analyzed, 21 locations had insufficient data to determine a trend. Based on historic data, nine locations exceeded the screening level for at least one constituent. Of locations with historic exceedances, six had insufficient recent data to determine a trend. Among the eight locations with sufficient data to determine a trend, two locations had Decreasing concentrations trends, GW-609 and GW-831. Locations GW-175 and GW-608 had Stable trends for PCE. Sample locations GW-609 and GW-175 have average concentrations above screening levels. No Trend was found at spring location SCR3.5SP, with concentrations well below screening levels, and GW612 with concentrations above screening levels. Well GW-177 had non-detect values.

Spring location SCR3.4SP indicated a Probably Increasing trend for GA activity, but this appears to be an artifact of GA activity detection limits and background concentrations. For the data that are available, constituent trends in the Security Pit area appear to be Stable to Decreasing.

\section{Well Sufficiency}

The results of the Well Sufficiency analysis for the Security Pits indicated one area of larger uncertainty in the 11-DCE plume on the crest of Chestnut Ridge in the area of the Security Pit trenches (see MAROS Well Location Report Appendix D.9). No new wells are recommended in this area. 


\section{Sampling Frequency}

The MAROS recommendations along with a qualitative analysis indicate that sampling frequency can be reduced for most locations from semi-annual to annual or biennial frequency. Because several of the wells with sufficient data are included in regulatory programs, actual reduction in sampling frequency may not be possible. However, for the majority of the area, additional monitoring above that for regulatory compliance does not appear to be necessary.

The preliminary recommended sampling frequency is listed in Table B.11. The preliminary frequency does not take regulatory status into account. The recommended changes reduce the frequency for area wells, but the number of wells monitored increases. The majority of wells with Biennial sampling frequency recommendations can be reevaluated after sufficient data have been collected and reduced in frequency or removed from the monitoring program.

\subsubsection{Landfill V and VII}

The Landfill V and VII analysis group encompasses monitoring locations for Industrial Landfill V, construction/Demolition Landfill VII, and South Side of Chestnut Ridge. The analysis group was small, including only 10 sample locations. Well descriptions are provided on Table B.12 with locations shown on Figure A.1.

\section{Constituent Choice}

The MAROS Constituent Choice module for the Landfill $V$ area identified lead as the only constituent with a representative concentration over screening levels (see MAROS COC Assessment Report in Appendix D.10). Individual well constituent choice indicated that PCE has historically been present at several locations. However, in the time frame of 1996 to 2004, eight of 10 wells had non-detect results for PCE, and no detections of metals above reasonable background values. For all wells in the assessment group, none had average concentrations of the priority constituent over the screening level.

\section{Moment Analysis}

While Moment analyses were conducted for the Landfill V and VII area, concentrations are so low that there is very little change in trend to be detected. The estimate of total dissolved mass for PCE showed a Probably Increasing trend, due to increases at well GW-798, the only well with detections of PCE and an Increasing concentration trend.

Trends in estimated First and Second Moments were Stable during the time frame analyzed. This is consistent with the observation that concentrations are increasing at only one well in the network. 


\begin{tabular}{|l|c|l|}
\hline \multirow{2}{*}{$\begin{array}{l}\text { Myment } \\
\text { Type }\end{array}$} & \multicolumn{2}{|c|}{ PCE Moment Trend Analysis Landfill V and VII } \\
\cline { 2 - 3 } Zeroth & $\begin{array}{c}\text { Trend } \\
\text { Probably } \\
\text { Increasing }\end{array}$ & $\begin{array}{l}\text { Comment } \\
\text { The estimate of total dissolved mass of PCE was found to be Probably } \\
\text { Increasing during the sample period 1996-2004. }\end{array}$ \\
\hline First & Stable & $\begin{array}{l}\text { The estimated distance from the source to the center of mass showed a Stable } \\
\text { trend during the sample period. }\end{array}$ \\
\hline Second & $\begin{array}{l}\text { Stable/ } \\
\text { Stable }\end{array}$ & The PCE plume showed Stable trends in the distribution of constituents. \\
\hline
\end{tabular}

\section{Statistical Trend Results}

Results of the Statistical Trend analysis are shown on Table B.12. All wells included in the analysis had sufficient data to analyze trends. Eight of ten wells had no detections of priority constituents and are apparently unaffected. None of the locations had average concentrations above screening levels. Two wells had constituent detections and subsequent trends. Well GW-798, north of Construction/Demolition Landfill VII has an Increasing trend for PCE. The source of PCE in this area is most probably the Security Pits upgradient of the landfill. The second well with a detected trend was GW-797, which showed a Decreasing trend for lead.

\section{Well Sufficiency}

Due to the size of the analysis group, the Well Sufficiency module was not run on the data set.

\section{Sampling Frequency}

The MAROS preliminary recommendations along with a qualitative analysis indicate that sampling frequency can be reduced and many wells can be removed from the routine monitoring program. All sample locations in the Landfill V and VII group are currently covered by regulatory programs; however, locations were considered for modified sample frequency as part of the larger analysis. The final sampling recommendation is to sample as per permit requirements.

\subsubsection{East Chestnut Ridge}

The East Chestnut Ridge area includes monitoring locations in the eastern portion of the Chestnut Ridge down to Bethel Valley. The analysis group consists of 26 sample locations listed in Table B.13 and illustrated on Figure A.1.

\section{Constituent Choice}

The MAROS Constituent Choice identified TCE as the only priority COC in this area (see MAROS COC Assessment Report in Appendix D.10). TCE is found in wells south of the Y-12 Complex in the Scarboro facility wells GW-841 to GW-844. As these wells were included in the database, they were evaluated along with the Y-12 locations. Individual well Constituent Choice indicated that GA activity and lead have historically been 
present at several locations. A review of the data indicates that there have been some historic data outliers for uranium at two wells. Single uranium detections were not repeated in subsequent sample events. For the majority of locations, no COCs were present with average concentrations above screening levels.

\section{Moment Analysis}

Because individual constituents are not distributed widely across the East Chestnut Ridge, the Moment Analysis was not significant to the LTMO in this area. TCE, the priority constituent for East Chestnut Ridge, is found in the offsite wells GW-841 through 844 , so, an analysis of this constituent would not provide the scale of information necessary to support sampling recommendations across the site.

\section{Statistical Trend Results}

Results of the Statistical Trend analysis are shown on Table B.13 and illustrated on Figure A.9. Fifteen of 26 wells included in the analysis had sufficient data to analyze trends. Of these locations, six had Decreasing concentration trends. Well GW-841, with average concentrations of TCE above screening levels, displayed a Decreasing trend. GW-841 is part of a cluster of wells near south of the Chestnut Ridge area that does not appear to be connected to source areas in the Chestnut Ridge area. Other wells in this cluster had non-detect results (GW-844), No Trend results (GW-842) and insufficient data results (GW-843). Because of the diversity of constituents and trends, wells in this area may require more monitoring attention than in other areas of Chestnut Ridge.

Monitoring locations GW-231, GW-142, and GW-143 in the Kerr Hollow Quarry area had Decreasing concentration trends, and did not exceed screening levels. Well GW-145, also in the Kerr Hollow area had a Stable concentration trend. No wells with Increasing or Probably Increasing trends are located in this area. Constituent concentrations are low to non-detect and concentration trends indicate that limited monitoring effort is required.

\section{Well Sufficiency}

Well Sufficiency analyses for the East Chestnut Ridge area did not result in locations identified as exhibiting high uncertainty. No new monitoring locations are recommended.

\section{Sampling Frequency}

The MAROS recommendations along with a qualitative analysis indicate that sampling frequency can be reduced and in the East Chestnut Ridge area. However, the majority of sample locations are included in regulatory programs. All wells that are included in regulatory programs have been retained, but the preliminary sampling frequency recommendations are listed in Table B.13 with final recommendations listed in Table B.16. 


\subsection{Combined Qualitative and Quantitative Analysis}

Results from the qualitative well scoring detailed in Section I were combined with results from the quantitative MAROS analysis in a decision logic format to develop recommendations for sample locations and frequencies for the $\mathrm{Y}-12$ Complex. The decision logic flow chart is illustrated in Figure A.10. Wells identified in RCRA postclosure permits, specified in CERCLA interim or final RODs or related decision documents, or SWDF permits have sampling frequencies that cannot be altered. These regulated wells are recommended for the sampling frequency specified in the decision document; however, a preliminary sampling frequency recommendation can be found for these locations in Tables B.3-B.13. Final recommendations by regime are detailed in Tables B.14-B.16.

Remaining sample locations were evaluated based on whether the average concentration of the priority constituent exceeds the screening level shown in Table B.1. Wells with average concentrations exceeding screening levels were separated into two groups, those with sufficient sample data to determine a concentration trend between 1996 and the present and those with insufficient data. For wells with elevated average concentrations and sufficient data for the MAROS evaluation, the preliminary MAROS sampling frequency was used as the basis for the final recommendation. For locations with less than 4 sample events since 1996, the recommendation is to review the reasons why the locations have not been sampled. Locations that do not provide useful data, either because of well construction or other issues should be removed from the analytical program. For functional sample locations, four data points after 1996 should be collected, and the trend data should be reevaluated. If constituent concentrations are stable to decreasing, or if concentrations are significantly below MCLs, then consider removing the well from the routine analytical program. Redundant source monitoring locations may also be identified and removed from the analytical program, however, fulfilling minimum requirements for trend evaluation is recommended before removal.

Sample locations with concentrations consistently below MCLs required careful consideration. The qualitative data were used to identify wells that function to monitor source areas or provide horizontal or vertical plume delineation. Low concentration wells that did not have an identified function in the network were recommended for removal from the analytical program.

Remaining sample locations with an identified function in the network were carried forward for a more quantitative function score. Locations were scored based on the formation sampled and secondary location characteristics. Springs were assigned 5 points in a scoring system, while aquifer (Maynardville Limestone) locations were assigned 3 points and aquitard wells were assigned 1 point. Exit location wells were assigned an additional 3 points. Wells with unique function and those that provide early detection for constituent mobility or information on background water quality were each assigned 1 additional point.

Sample locations with a score of 5 or more and with sufficient data (>=4 samples 1996 to present) to determine a trend with MAROS were assigned a MAROS based sampling frequency based on the preliminary sampling frequency. Locations with a score of 5 or higher with insufficient data are recommended for further evaluation or removal as 
described above. Sample locations with a score below 5 were considered to provide limited data to support monitoring objectives. Locations with low scores and sufficient data to determine a trend were assigned a reduced monitoring schedule based on the MAROS analysis. Locations with insufficient data and low scores were recommended for removal from the network. Most low scoring locations may be considered for removal in the future.

Results from the decision logic process are summarized below and presented in Tables B.14-B.16. Total samples per year and Total wells include those locations recommended for Quarterly, Semi-annual, Annual or Biennial sampling frequency that are not included in a regulatory program. Sample locations in these categories are recommended for inclusion in the GWPP to meet site-wide monitoring objectives. Wells in the Review category may be included in the GWPP based on their status and as budget considerations allow. Review locations with high scores $(>5)$ and concentrations above screening levels constitute higher priority locations. Detailed recommendations for Review locations are given in Tables B.14-B.16.

Locations identified as Regulated are normally included in the WRRP monitoring program or are the responsibility of the BJC subcontractors. Individual Regulated locations may be sampled by the GWPP if supplemental information on specific groundwater areas is desired. Locations identified under the Remove category can continue as hydrologic monitoring locations and possible alternate sampling locations if plume conditions change.

\begin{tabular}{|c|c|c|c|}
\hline \multirow{2}{*}{ Decision Results } & \multicolumn{3}{|c|}{ Sampling Frequency Results } \\
\hline & Bear Creek & East Fork & Chestnut Ridge \\
\hline Remove & 48 & 58 & 24 \\
\hline Review & 52 & 31 & 6 \\
\hline Regulated & 35 & 33 & 45 \\
\hline Quarterly* & 0 & 0 & 0 \\
\hline Semi-annual & 14 & 6 & 0 \\
\hline Annual & 24 & 25 & 5 \\
\hline Biennial & 10 & 17 & 9 \\
\hline $\begin{array}{l}\text { Total Samples (per } \\
\text { year)* }\end{array}$ & 57 & 45.5 & 9.5 \\
\hline Total Locations* & 48 & 48 & 14 \\
\hline $\begin{array}{l}\text { Total Samples } \\
\text { CY } 2005 \text { Plan }\end{array}$ & 142 & 64 & 10 \\
\hline $\begin{array}{l}\text { Total Locations } \\
\text { CY } 2005 \text { Plan }\end{array}$ & 45 & 32 & 5 \\
\hline
\end{tabular}

* Includes Quarterly, Semi-annual, Annual and Biennial recommendations, wells under Review will increase this number. 


\subsection{SUMMARY AND RECOMMENDATIONS}

The current Y-12 Groundwater Protection Program (GWPP) is charged with fulfilling surveillance monitoring objectives as articulated in DOE Order 450.1 (USDOE, 2004). The Order requires that each DOE site implement an environmental management system that "provides for systematic planning, integrated execution, and evaluation of programs that ensure public health and environmental protection, pollution prevention, and compliance with DOE Directives and applicable laws and statutes" (USDOE, 2004). The current GWPP strives to achieve specific regulatory goals by monitoring a system of groundwater wells and springs on a largely semi-annual basis, maintaining an extensive database on site parameters, and documenting monitoring results in annual and supplemental reports.

\subsection{Monitoring Program Evaluation}

The groundwater monitoring network at the $\mathrm{Y}-12$ Complex is extensive and has achieved a high level of site characterization. Based on the methods of collection, analysis and data management, and the quality and quantity of groundwater data collected, the monitoring network is sufficient to evaluate plume behaviors in the near and long term. While final remedial decisions are pending for some areas in the Y-12 Complex, the current GWPP database has sufficient data to perform a formal long-term monitoring optimization (LTMO) in order to improve data collection in support of the stated monitoring objectives and pending site management decisions. An on-going systematic and coordinated system of qualitative and quantitative analyses is applied to optimize the current monitoring network.

Groundwater sample collection and handling methods are appropriate to the scale of the Y-12 Complex network. Sample collection has been scheduled efficiently to take advantage of labor and laboratory resources. Low-flow sample techniques currently employed are appropriate for the majority of sample locations. Wells where purge techniques provide better quality data should be sampled using this method; however, dual sampling using both methods is redundant and does not directly support monitoring objectives.

The laboratory analysis program has delivered high quality data for the GWPP since approximately 1996. Current laboratory practices as articulated in site documents and as evidenced in the GWPP database meet applicable data quality objectives. While data analyzed before the mid-1990's may have met all of the data quality objectives at the time, detection limits, analytical methods and standards, as well as sampling protocols may have changed since the 1980's, in particular. Data collected before 1996 may be extremely useful, but caution should be exercised in making direct comparisons between historic and recent data sets.

Based on the objectives articulated in DOE 450.1, it is recommended that the GWPP continue to monitor the standardized suite of analytes for each sample taken. Standardizing the parameter list and analytical methods will provide consistency across the data set, and streamline plume analysis. The $\mathrm{Y}-12$ Complex is underlain by numerous commingled plumes, where source and tail areas of one plume merge with elements of another. The benefit of a complete constituent list is that conservative 
constituents (like metals) and constituent ratios can be evaluated to support plume migration analysis, mass flux evaluations, MNA determinations and possible groundwater modeling efforts. Consistent analytical methods will help develop a data set where data from diverse sources are comparable. The benefits derived from reducing uncertainty about constituent delineation outweigh potential cost savings from a limited suite of analytes.

One minor constituent of possible concern that should be included in the monitoring program is 1,4-dioxane. 1,4-Dioxane was a common chemical stabilizer in commercial 111TCA preparations, and has become a concern among the regulatory community (Spath and Alexeeff 1998) with an PRG of near 3 ppb. 1, 4-Dioxane is more mobile than the chlorinated co-constituents and should be analyzed for downgradient of areas where 111TCA, 1,1-dichloroethene (11DCE) or 1,1-dichloroethane are detected.

Based on a review of the Y-12 GWPP Data Management Plan (BWXT, 2003a) and an interview with the data management team, the analytical data management system is of excellent quality. The current Analytical Data Management System (ADMS) and Groundwater Information Management System (GIMS) satisfy data management quality objectives articulated in the documents such as the ASCE Long-Term Groundwater Monitoring document (ASCE, 2003). Early review of data points from the laboratory using an automated system is especially important. Cooperation between the WRRP and GWPP programs in terms of data collection and management should continue.

While site reports are clear and well written, reporting activities would benefit from expanded data visualization techniques. More emphasis should be place on map development. The GWPP would benefit from improving the link between its extensive database and a geographic information system for the purpose of both data analysis and reporting.

The technical approach for improving the efficiency of the Y-12 GWPP monitoring network included both qualitative and quantitative evaluation strategies. The product of the qualitative hydrogeologic analysis for individual wells includes specific information on how each well functions to fulfill the monitoring objectives for the various groundwater flow regimes and constituent classes. Data from the qualitative approach were combined with the results of the quantitative analysis in the form of a decision logic flow chart (see Figure A.10) to determine the final temporal and spatial well network.

\subsection{Quantitative Network Evaluation}

The MAROS 2.1 sampling optimization methodology was applied to the groundwater monitoring network for the Y-12 Security Complex in Oak Ridge, Tennessee. The statistical analytical results of the MAROS evaluation provide the foundation for recommendations to improve the spatial and temporal groundwater monitoring network while supporting the surveillance monitoring objectives articulated in DOE Order 450.1. Results of this qualitative analysis were combined with those of the quantitative analysis to develop an optimized network sampling recommendation.

The optimization of the current monitoring network was achieved through choices in sampling frequency and sampling locations that minimized uncertainty as well as 
redundancy of constituent concentration information. Additionally, identified concentration trends and calculated Moments highlight areas of the plume that require greater or reduced monitoring effort.

Due to the presence of commingled plumes from diverse sources and multiple hydrogeologic regimes, the quantitative analysis was performed by dividing site locations into eleven MAROS analysis groups. Plume level priority constituents were identified by the MAROS COC Risk Evaluation module. Plume level priority COCs were used as representative compounds for evaluating overall plume stability using the Moment Analyses and Well Sufficiency tool. Plume level priority constituents included PCE, TCE, CTET, benzene, GA and GB activity, uranium and nitrate. Priority constituents of concern were identified for each monitoring location based on the magnitude of the exceedance of average constituent concentrations relative to screening levels. Individual well priority COCs were used to evaluate statistical trends and sampling frequencies for each location.

Preliminary recommendations for sample locations and frequencies were developed using the MAROS software. These recommendations were brought into a decision logic flow tool along with a location scoring system based on the qualitative well evaluation. Final location and sampling recommendations resulted from the decision logic step. Results of the quantitative and decision logic analyses for each Regime are presented below.

\subsubsection{Bear Creek Hydrogeologic Regime}

A total of 183 monitoring locations were evaluated in the Bear Creek Regime. The majority of these locations are in the expansive western BCBG area. The Bear Creek Regime contains two main sources of constituents, the former S-3 ponds that are the source of nitrate and radionuclides to the transmissive Maynardville Limestone, and former waste management units in the Nolichucky shale aquitard where chlorinated solvents and some radioactive migrate to the Maynardville formation. Among the 183 possible monitoring locations, 110 locations had limited recent data (fewer than 4 sample events 1996 to 2004) to determine their contribution to the monitoring network. Without a sufficient data set, no quantitative conclusions could be drawn about concentration trends, and recommendations for sample frequency could not be made using MAROS methodology.

Overview statistics (Moment and Statistical Trend analyses) for the Bear Creek regime indicate that the BCBG and West S-3 areas are largely Stable for the two main plumes. Statistical trend analysis indicates that 29 of 73 locations with sufficient data to determine a trend have Stable to Decreasing concentrations. Only one well in the heavily affected West S-3 area had an Increasing trend. Moment analyses do not indicate that plumes are trending downgradient in the recent time frame. The OLFA analysis area, located in the center of the main Bear Creek plume, shows greater variability in concentrations than both the source and tail regions. At the overview level, the plumes have largely stabilized and planning for a reduced long-term monitoring network is appropriate. 
Well Sufficiency analyses for Bear Creek do not reveal any extremely large areas of uncertainty in the plume, indicating that no new wells are necessary. The frequency determination using the MCES method indicates that reduced sample frequency is appropriate for most wells.

Using the combined quantitative and qualitative approach, sampling recommendations were made for 183 locations. Locations covered by regulatory permits or agreements (35) should be sampled according to the regulatory driver (permit, ROD, etc.). However, preliminary sampling recommendations have been made for these locations in the event permits are renegotiated. Forty-eight sample locations are recommended for removal from the analytical network. These wells can be maintained as hydrogeologic monitoring locations.

Fifty-two of the 183 sample locations in the Bear Creek Regime are recommended for review. Sample locations with limited history should be reviewed for function or construction defects. Locations that do not provide quality, representative analytical data should be designated as hydrogeologic monitoring locations, moved to inactive status or plugged and abandoned. For sample locations with limited recent data or for wells that are approaching 'clean' status, the recommendation is to collect samples over the next two years to provide between 4-6 recent data points. All active analytical locations should maintain between 4-6 consistent sample events in a ten-year time frame to evaluate concentrations using statistical tools. Results of the statistical tools can be used to recommend updated sampling frequencies for these locations

No wells are recommended for quarterly sampling. Fourteen locations with Increasing trends or average concentrations above screening levels are recommended for Semiannual sampling. Annual sampling is recommended for 24 locations. The majority of perimeter wells are recommended for Biennial (every 2 years) sampling. In all, 10 locations are recommended for Biennial sampling.

\subsubsection{Upper East Fork Poplar Creek Hydrogeologic Regime}

The Upper East Fork Poplar Creek Regime is the most intensely developed area of the Y-12 Complex. The region is characterized by diverse sources from historic underground storage tanks, influx from the former S-3 Ponds and current industrial activity. The western area of the regime is impacted by constituents originating in the S-3 and S-2 sites, and groundwater concentrations of nitrate and GA and GB activity (and associated isotopes) is quite high in areas. The Central Y-12 analysis area is complicated by multiple small sources of VOCs as well as impacts from the S-3 area. The East Y-12 area, including the New Hope Pond area and Union Valley analysis group represents the only area where affected groundwater migrates off-site. The presence of affected groundwater off-site raises the priority of monitoring in this location.

A network of 171 sample locations was examined quantitatively in the East Fork Regime. Of the wells analyzed, 74 locations had insufficient data to make a quantitative recommendation for sample frequency. Overview statistics for the East Fork regime indicate that the nitrate and uranium/GB activity in the western area of the plume is largely Stable. Individual well trends in the western East Fork are Decreasing to Stable with many wells trending toward non-detect status. VOC constituents in the western 
area of East Fork also appear to have Stable to Decreasing trends supporting the reduction in sample effort over this region. The area around the former Fuel Station also has Stable to Decreasing trends with many locations close to non-detect status.

The East Y-12 area requires careful monitoring due to the presence of affected groundwater off-site. With the installation of an extraction well in the year 2000, the VOC plume in this area has stabilized. The area just south and east of New Hope Pond has some monitoring locations with Increasing trends (GW-220, GW-151 and GW-150), but the First Moment analysis indicates that the center of mass is retreating in this area. The extraction well appears to be controlling migration of constituents downgradient.

The Well Sufficiency analysis indicated some areas of uncertainty between the region of high CTET concentration in the New Hope Pond area and the wells that monitor the property boundary. While no new well locations are recommended in this area, continued monitoring of representative property boundary wells (GW-735, GW-747, and GW-748) is recommended. The overall recommended approach of reducing the frequency of well sampling in favor of monitoring more locations should increase confidence in the delineation of constituents in these locations.

Of the 171 locations evaluated in the East Fork Regime, 33 are covered by regulatory permits. One well (GW-845) is an extraction location, and is monitored as part of the groundwater extraction remedy. Fifty-eight locations are recommended for removal from the analytical monitoring network. Thirty-one locations are recommended for review (as described under the Bear Creek Regime). Routine monitoring is recommended for 48 locations with 6 locations suggested for Semi-annual monitoring, 25 locations for Annual monitoring and 17 locations for Biennial monitoring. The multi-port Westbay well GW-722 is recommended for annual sampling.

\subsubsection{Chestnut Ridge Regime}

The Chestnut Ridge Regime is the least affected area at the $\mathrm{Y}-12$ Complex. The ridge is underlain by the Knox Group formation which consists of groundwater flowing through fractures and solutionally enlarged conduits in an impermeable matrix. A network of 89 sample locations was examined in this area. There are very few constituents detected in this area. VOCs are detected in the Chestnut Ridge Security Pit (CRSP), but the majority of sample locations across the Regime are below screening levels.

In contrast to the other hydrogeologic regimes, wells in the Chestnut Ridge regime are very well sampled with data for as many as 20 sample events since 1996 . Sampling frequency for most wells is determined by regulatory agreement or permit. The well network in Chestnut Ridge functions for detection monitoring rather than compliance monitoring. Because of the strong recent sampling record, statistical trends and MCES sampling frequencies could be determined for most locations. Statistical trend analyses indicate many locations with Decreasing, Probably Decreasing, and Stable trends in addition to those with non-detect results.

Sample frequency results indicate that the majority of locations should be sampled with reduced frequency. Forty-five of the 89 locations in the Chestnut Ridge Regime function as compliance points for regulatory programs and semi-annual sampling is mandated at 
most of these locations. Reduction in sample frequency at these locations may not be possible in the near term. The evaluation indicated that roughly 24 locations could be removed from the program without loss of information. Six locations are recommended for review. Biennial sampling is recommended for nine locations. Locations recommended for Annual sampling include wells in the CRSP area. No wells specific to the surveillance program are recommended for Semi-annual monitoring. The Chestnut Ridge regime appears to require limited monitoring effort relative to the other hydrogeologic regimes.

\section{$\underline{5.3 \quad \text { Conclusion }}$}

The monitoring network at the $\mathrm{Y}-12$ Complex is very extensive and has achieved a high level of site characterization. Based on the quality and quantity of groundwater data collected, the monitoring network is sufficient to evaluate plume behaviors in the near and long term. Many active monitoring locations in the extensive network may provide redundant or irrelevant information, however, an on-going systematic and coordinated system of qualitative and quantitative analysis should be applied to eliminate redundant locations. Part of this systematic approach is to collect at least four samples in a tenyear time frame for wells targeted for future removal from the program.

The current well network appears to provide accurate and reliable information on plume behavior. However, effort should be directed at reducing the sample frequency and collecting information from a wide variety of locations in the future. 


\section{CITED REFERENCES}

AFCEE 2003. Monitoring and Remediation Optimization System (MAROS) 2.1 Software Users Guide. Air Force Center for Environmental Excellence. http://www.gsinet.com/software/MAROS V2 1Manual.pdf

AFCEE (1997). AFCEE Long-Term Monitoring Optimization Guide Version 1.1. Brooks AFB, Brooks City, TX, Air Force Center for Environmental Excellence.

ASCE, Ed. (2003). Long-Term Groundwater Monitoring: The State of the Art. Reston, VA, American Society of Civil Engineers.

Aziz, J. A., C. J. Newell, M. Ling, H. S. Rifai and J. R. Gonzales (2003). "MAROS: A Decision Support System for Optimizing Monitoring Plans." Ground Water 41(3):

BWXT (2004a). Calendar Year 2003 Groundwater Monitoring Report, Y-12 National Security Complex. Elvado Environmental, L.L.C. Y/SUB/04-030332/1. BWXT Y12, LLC.

BWXT (2004b) Y-12 Groundwater Protection Program Groundwater and Surface Water Sampling and Analysis Plan for Calendar Year 2005, Elvado Environmental, L.L.C. September, 2004. Y/SUB/04-030332/2. BWXT, L.L.C.

BWXT, (2003a). Y-12 Groundwater Protection Program Data Management Plan. Y/SUB/03/013288/1. November, 2003. BWXT, LLC.

BWXT, (2003b). Y-12 Groundwater Protection Program Monitoring Optimization Plan for Groundwater Monitoring Wells at the US Department of Energy. Elvado Environmental, L.L.C. September, 2003. Y/SUB/03/013288/1. BWXT, LLC.

BWXT, (2003c). Quality Assurance Plan for the Analytical Chemistry Organization. Analytical Chemistry Organization. Y/P65-9006, Rev. M. BWXT, L.L.C.

Mohr, T.K.G., (2001). Solvent Stabilizers, White Paper. Santa Clara Valley Water District, San Jose, Ca. June 14, 2001.

Puls, R. W. and M. Barcelona (1996). Low-flow (Minimal Drawdown) Groundwater Sampling Procedures. Washington D.C., USEPA: 12.

Spath, D. P. and G. Alexeeff (1998). 1,4-Dioxane Action Level. Sacramento, California, Division of Drinking Water and Environmental Management, Department of Health Services.

USDOE (2005). Ground Water Protection Programs Implementation Guide for Use with DOE O 450.1, Environmental Protection Program. U.S. Department of Energy. DOE G 450.1-9. 
USDOE (2004). Ground Water Surveillance Monitoring Implementation Guide for Use with DOE O 450.1, Environmental Protection Program. U.S. Department of Energy. DOE G 450.1-6.

USDOE (1993). Radiation Protection of the Public and the Environment. DOE Order 5400.5. January 7, 1993 issue date. U.S. Department of Energy Office of Environment, Safety and Health. Washington, D.C.

USEPA (2005). Roadmap to Long-Term Monitoring Optimization. Washington, D.C., United States Environmental Protection Agency; United States Army Corps of Engineers: 42.

USEPA. (2004a). Guidance for Monitoring at Hazardous Waste Sites: Framework for Monitoring Plan Development and Implementation. Washington, D.C. January 2004.

USEPA (1987). Data Quality Objectives for Remedial Response Activities Development Process. Washington, D.C., United States Environmental Protection Agency. 


\section{ASSESSMENT OF THE \\ GROUNDWATER PROTECTION PROGRAM \\ Y-12 NATIONAL SECURITY COMPLEX, OAK RIDGE, TENNESSEE}

Y-12 National Security Complex

Oak Ridge, Tennessee

\section{APPENDIX A: FIGURES}

A.1 MAROS Analysis Sample Locations

A.2 Bear Creek Regime Exceedance of Screening Levels for Priority Constituents

A.3 East Fork Regime Exceedance of Screening Levels for Priority Constituents

A.4 Chestnut Ridge Regime Exceedance of Screening Levels for Priority Constituents

A.5 MAROS Results Bear Creek Regime OLFA/West S-3

A.6 MAROS Results Bear Creek Burial Grounds

A.7 MAROS Results East Fork Regime East S-3 and Central Y-12

A.8 MAROS Results East Fork Regime Fuel Station/East Y-12

A.9 MAROS Results Chestnut Ridge Regime

A.10 Well Decision Logic 


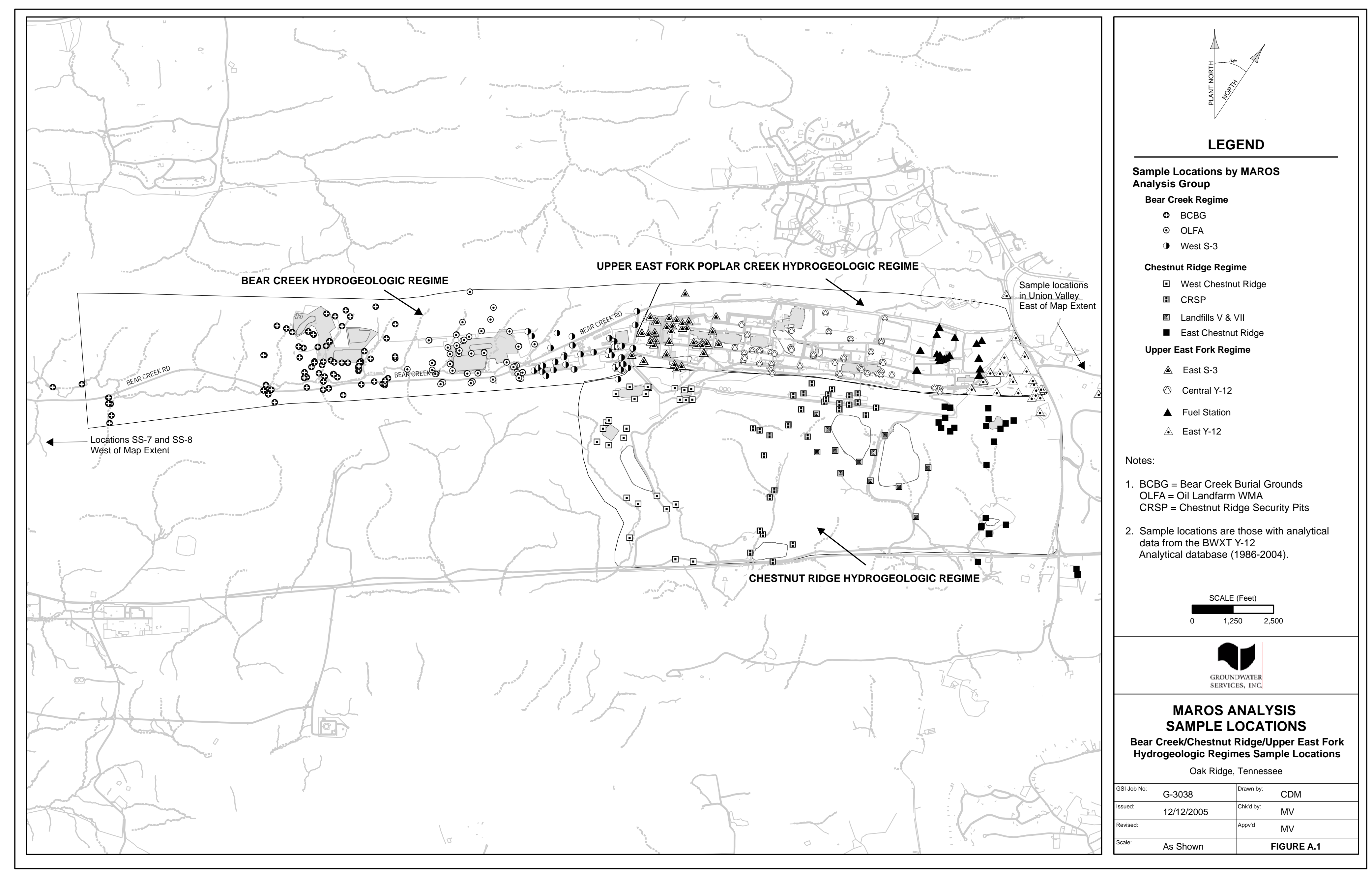




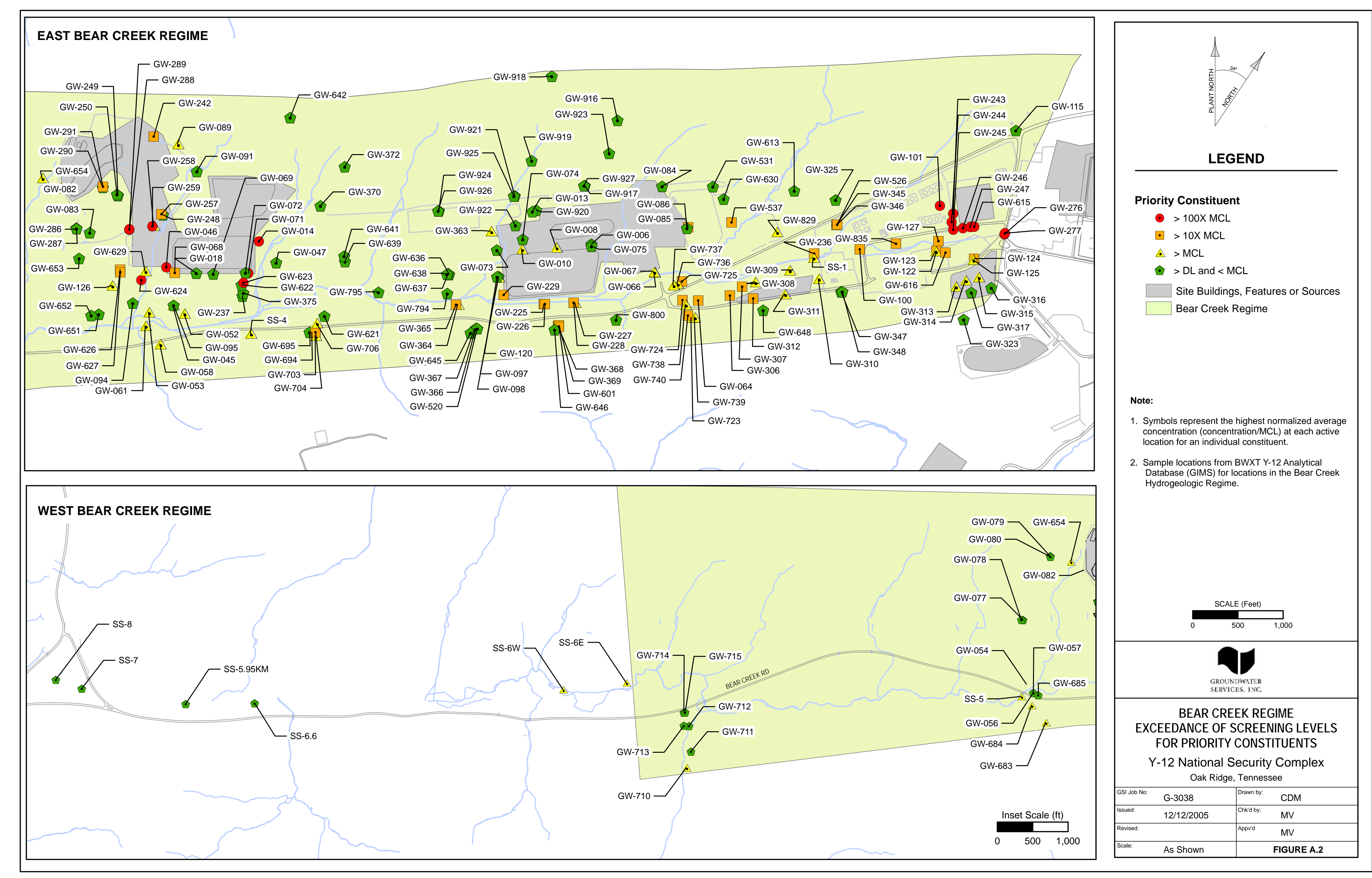



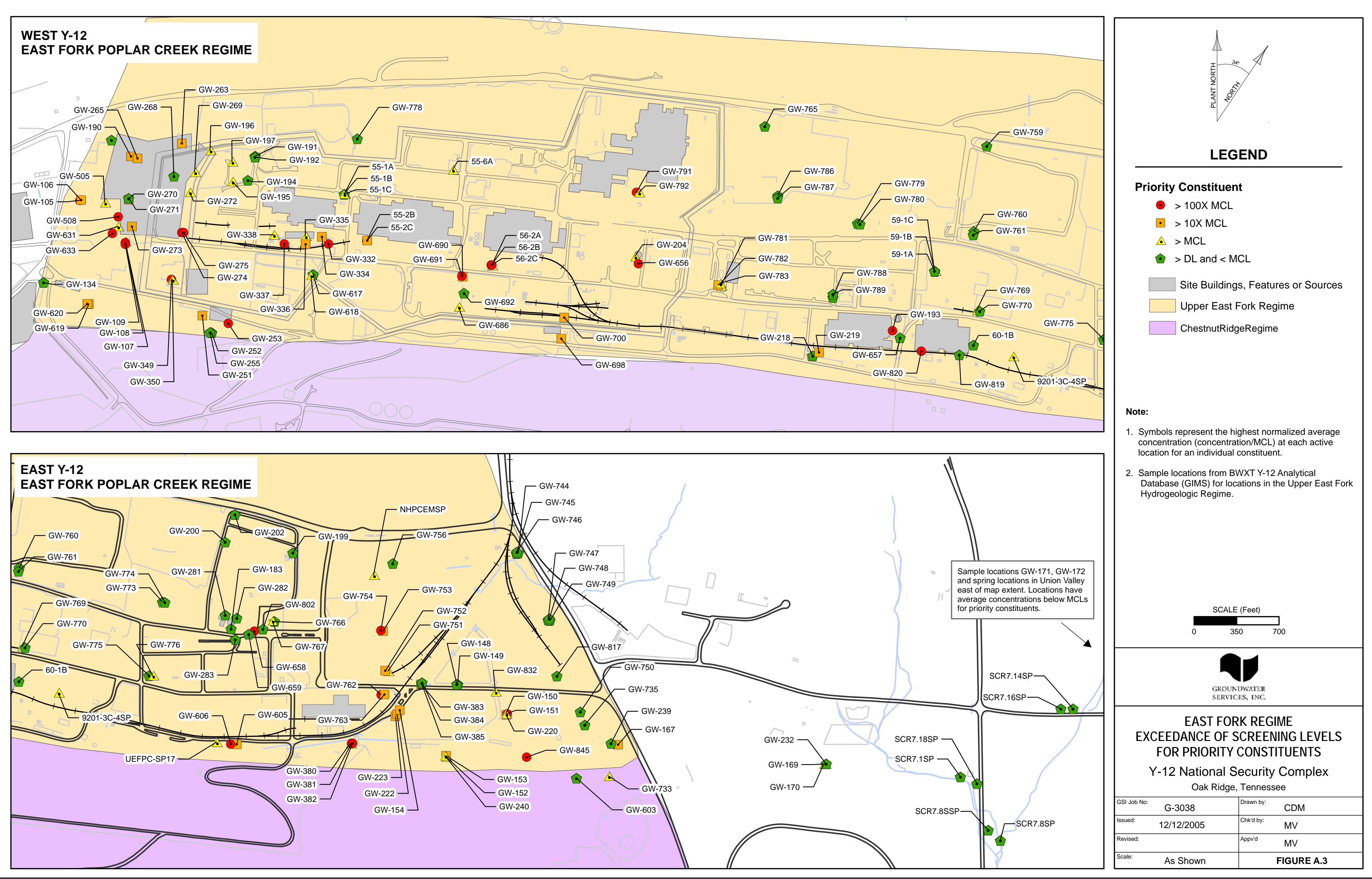


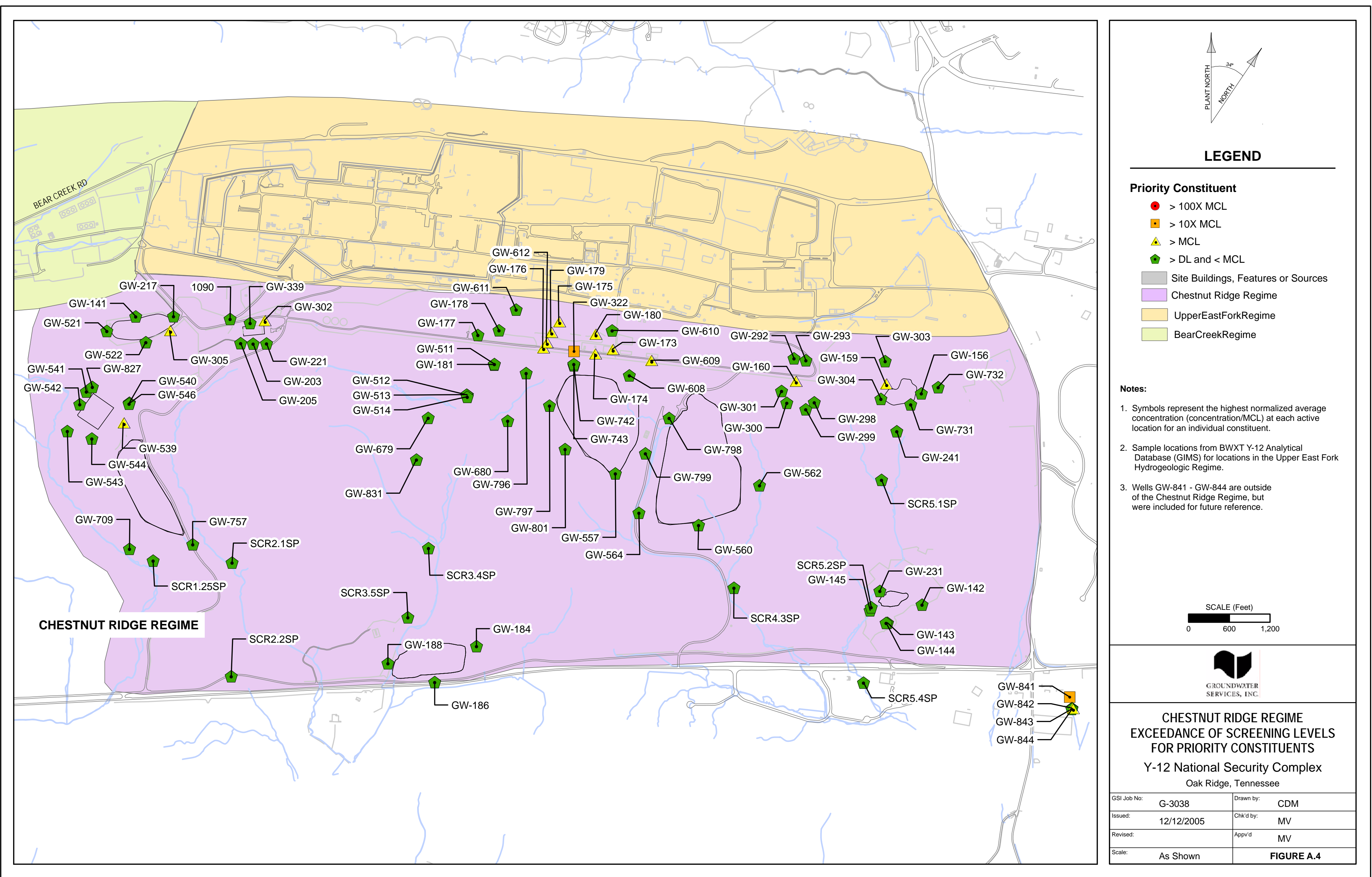



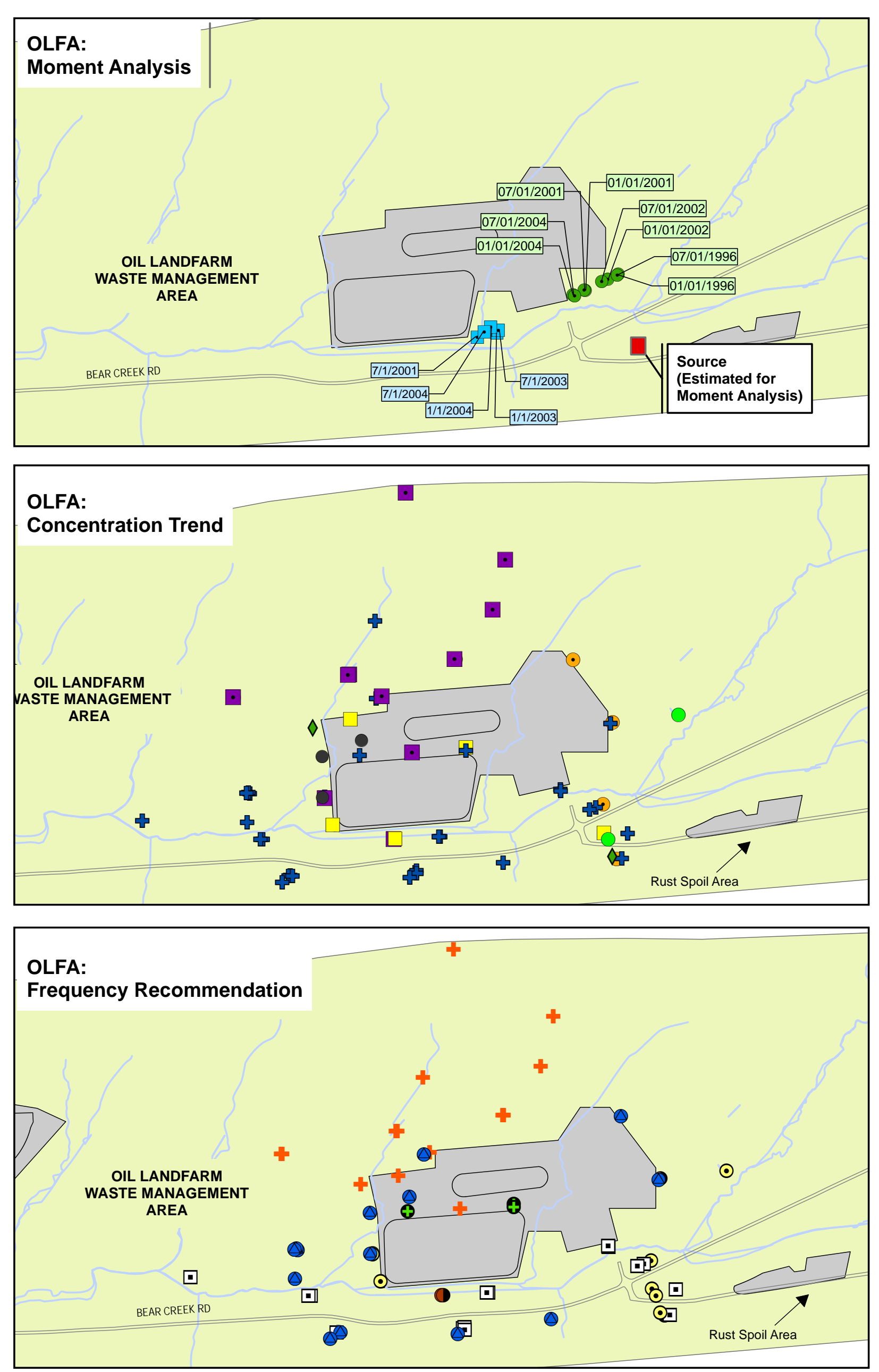

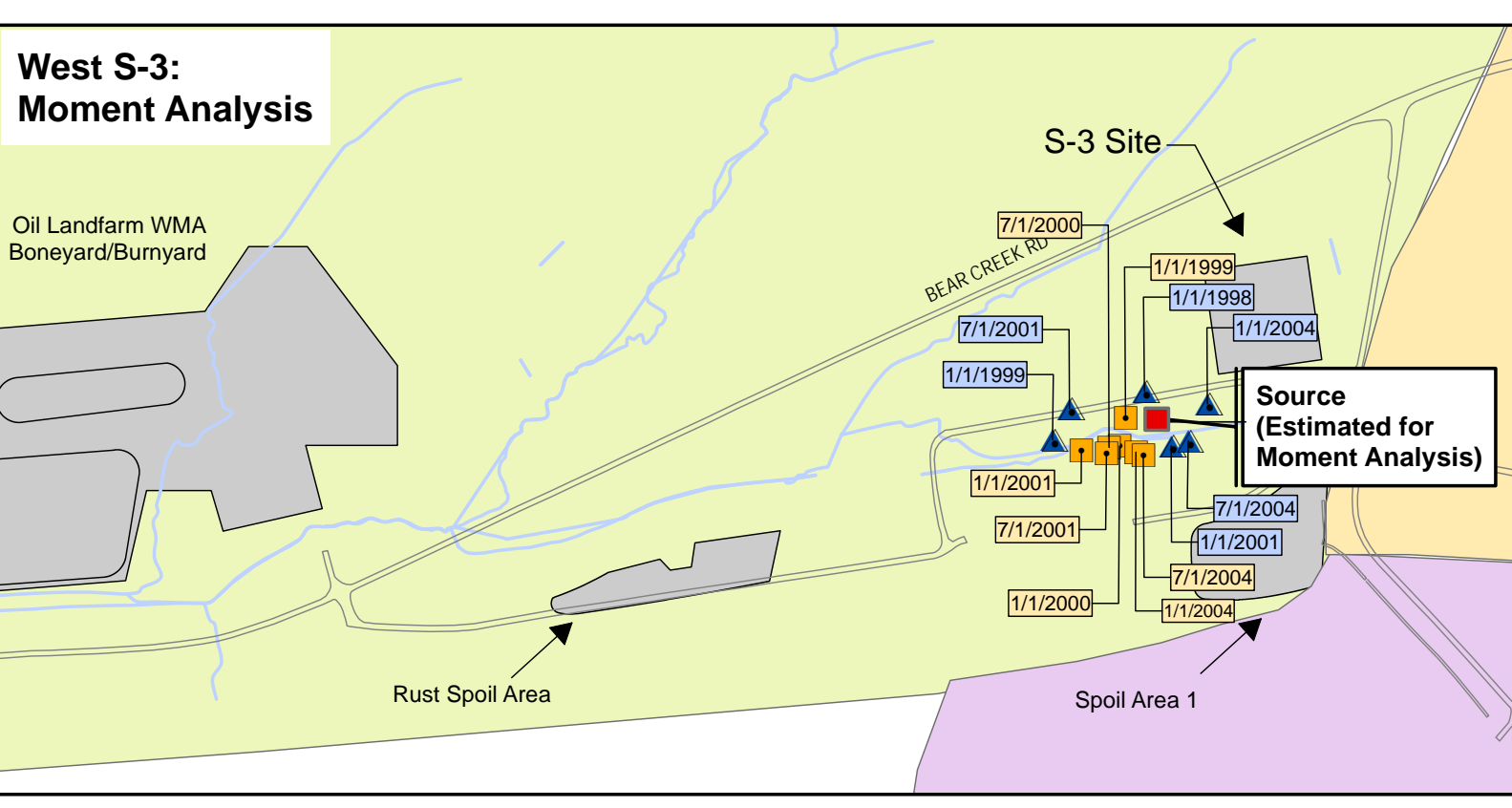

West S-3:

Concentration Trend

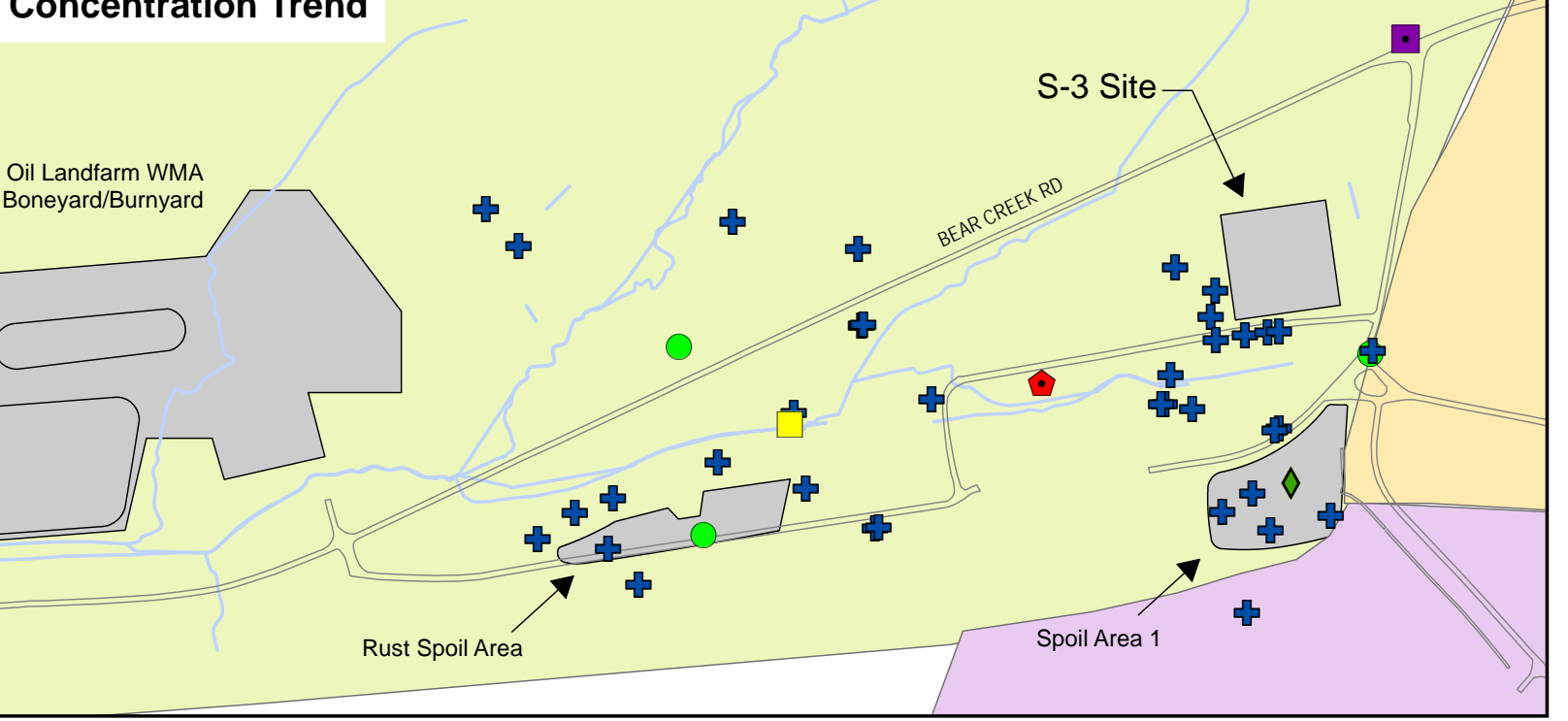

West S-3:

Frequency Recommendation

Oil Landfarm WMA
Boneyard/Burnyard

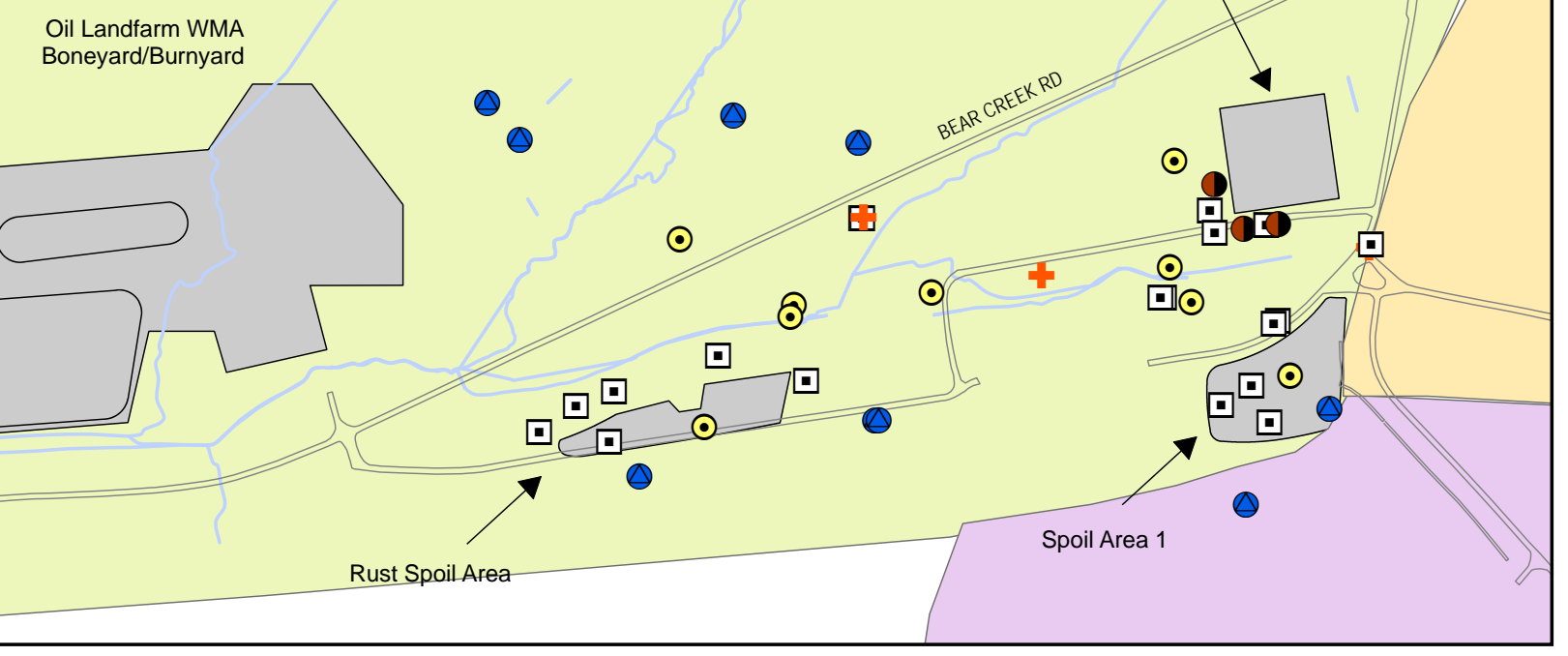

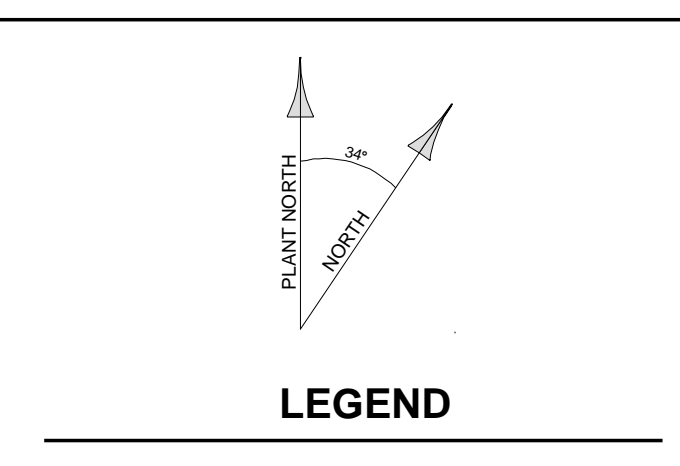

First Moments Analysis

- Nitrate

4 Gross Beta Activity

PCE

- TCE

Concentration Trend

Decreasing

Stable

Probably Increasing

Increasing

Insufficient Da

Non-dretect

Frequency Recommendation

- Biennial

- Seminnuar

- SemiAnnua

+ Regulate

Review

Note:

1) First moments represent the approximate center

2) Concentration trend is for priority constituent at
each location. Well l location ID's are indicated each location. Well location ID's are indicated
on Figure A.2.

3) Detailed results for sample locations shown in

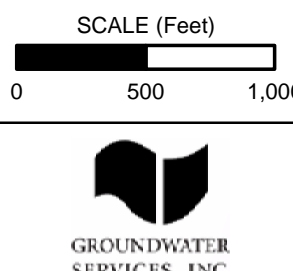

MAROS RESULTS

Bear Creek OLFA/West S-3

Y-12 National Security Complex

\begin{tabular}{|c|c|c|c|}
\hline \multicolumn{4}{|c|}{ Oak Ridge, Tennessee } \\
\hline GSI J Ob No: & G-3038 & Draann by: & CDM \\
\hline Issued: & 12/12/2005 & chked by: & MV \\
\hline Revisedt. & & Appovid & MV \\
\hline Scale: & As Shown & & FIGURE A.5 \\
\hline
\end{tabular}



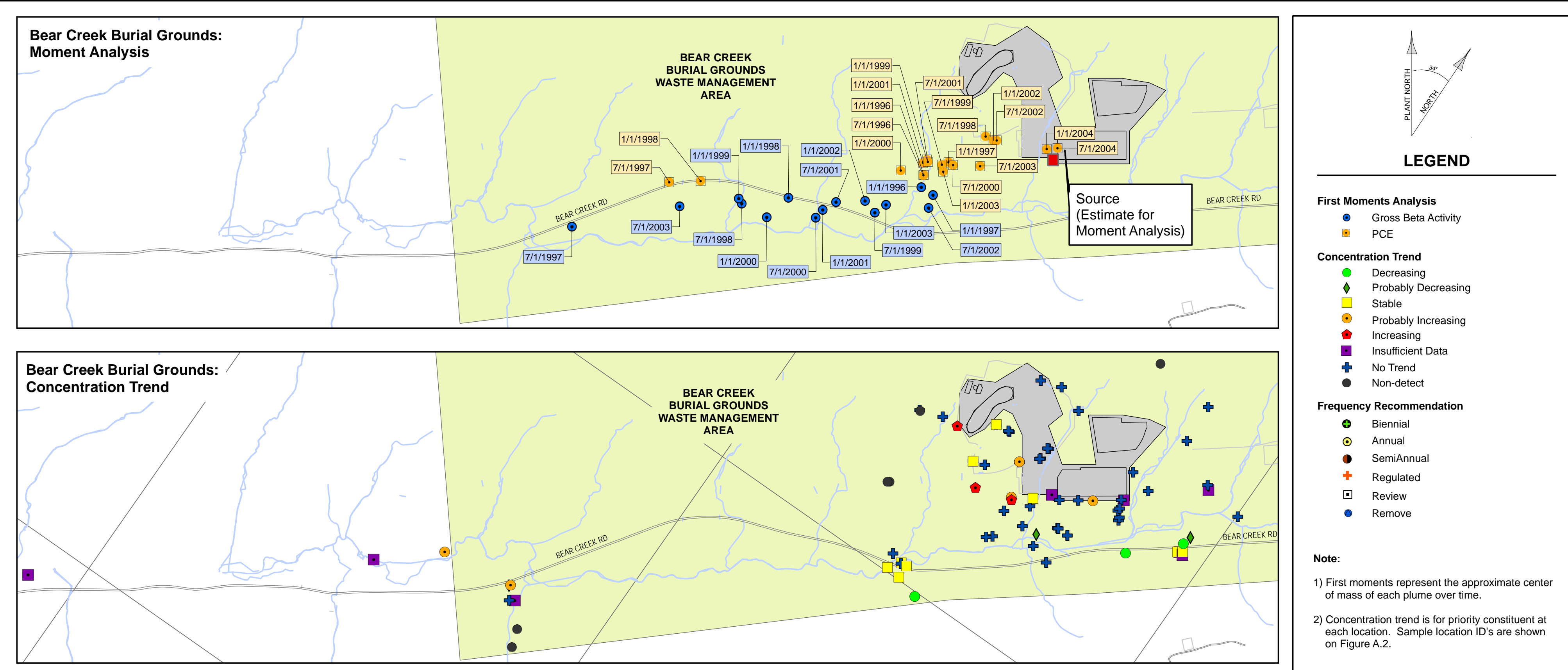

First Moments Analysis

- Gross Beta Activity

- PCE

Concentration Trend

Decreasing

Probably Decreasing

Stable

- Probably Increasing

Increasing

- Insuficient Dar

Non-detect

Fequency Recommendatio

- Biennial

- Annual

- SemiAnnual

- Regulated

$\square$ Review
$-\quad$ Remove

Note

1) First moments represent the approximate center of mass of each plume over time.

2) Concentration trend is for priority consitituent at each location.
on Figure A.2.
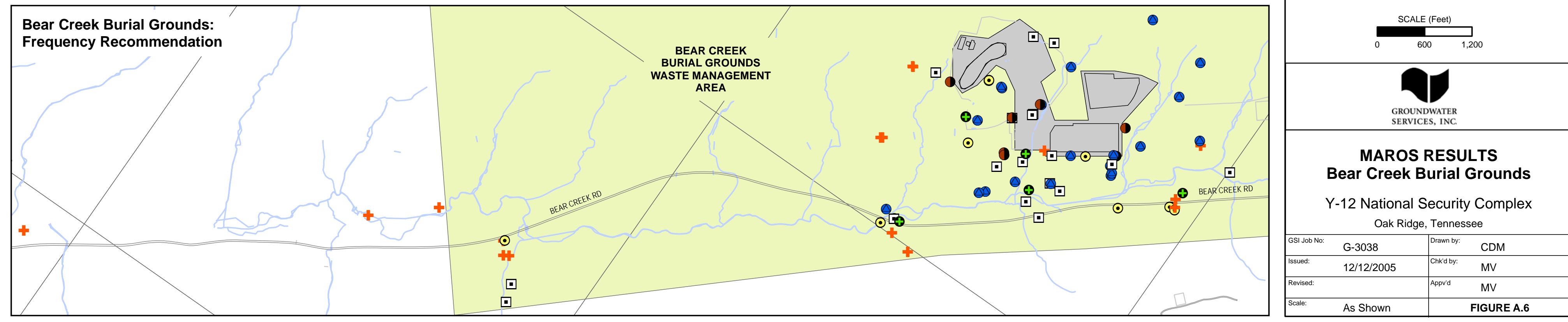

MAROS RESULTS Bear Creek Burial Grounds

Y-12 National Security Complex Oak Ridge, Tennessee

\begin{tabular}{|c|c|c|}
\hline CSIIJ Job No: & G-3038 & Drawn by: \\
\hline | & 12/12/2005 & chked by: \\
\hline Revised: & & Appovid \\
\hline Scale: & As Shown & FIGURE A.6 \\
\hline
\end{tabular}



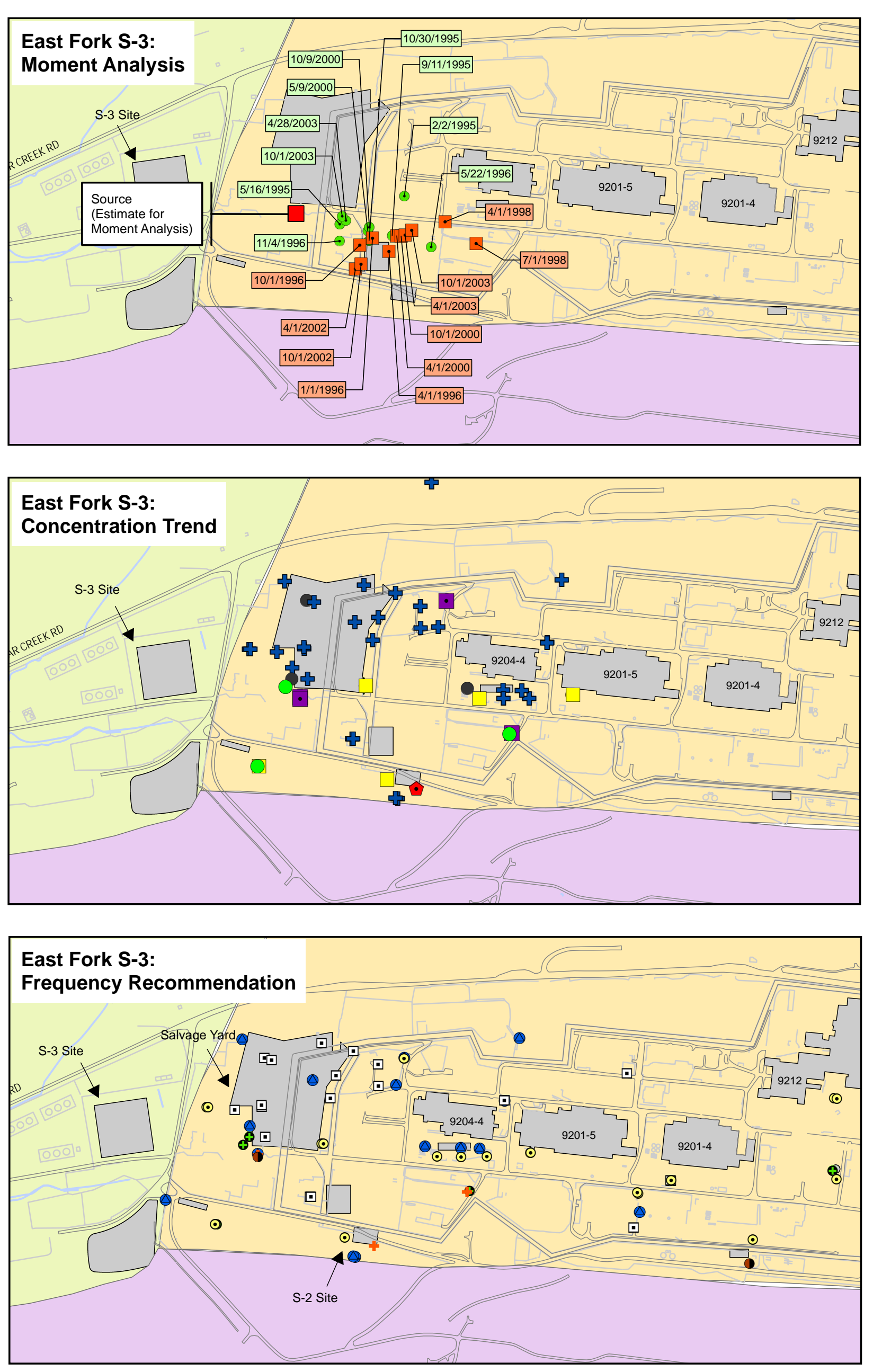
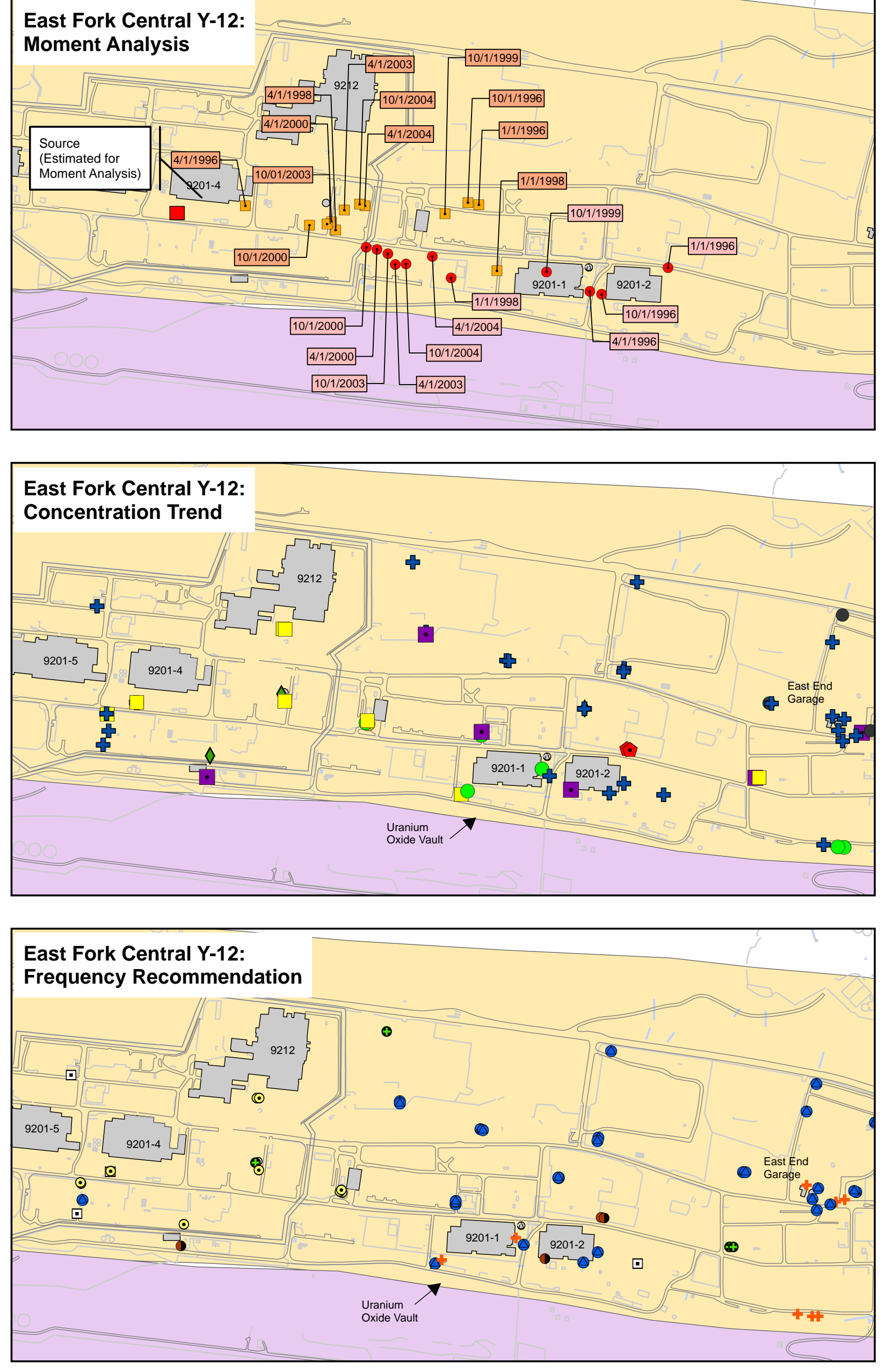

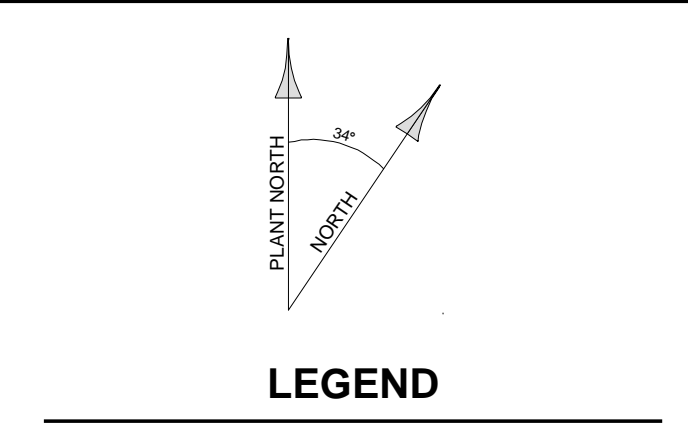

First Moments Analysis

$\begin{array}{ll}- & \text { Nitrate } \\ + \text { PCE } & \text { PCE }\end{array}$

- Uranium

Concentration Trend

$\therefore \quad$ Decreasing

Probably Decreasing

Prob

Probably Increasing

- Increasing

No Trend
Non-detect

equency Recommendation

- Biennial

- Annual

- SemiAnnual

+ Regulated

Review

NOTES:

1) First moments represent the approximate center
of mass of the plume.

2) Concentration trend is for priority constituent at
each location. Sample Location ID's are shown

each location.
on Figure $A$

3) An insufficient number of wells $<6$ ) were sampled
in the East $\mathrm{S}-3$ to determine First Moments for

in the East $\mathrm{S}-3$ to de
2004 sample events.

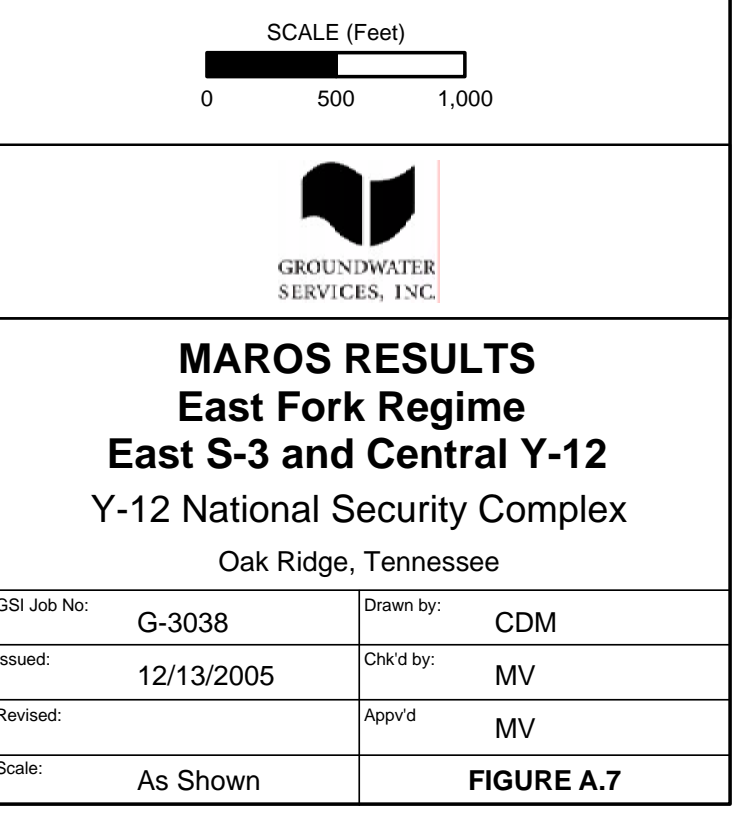



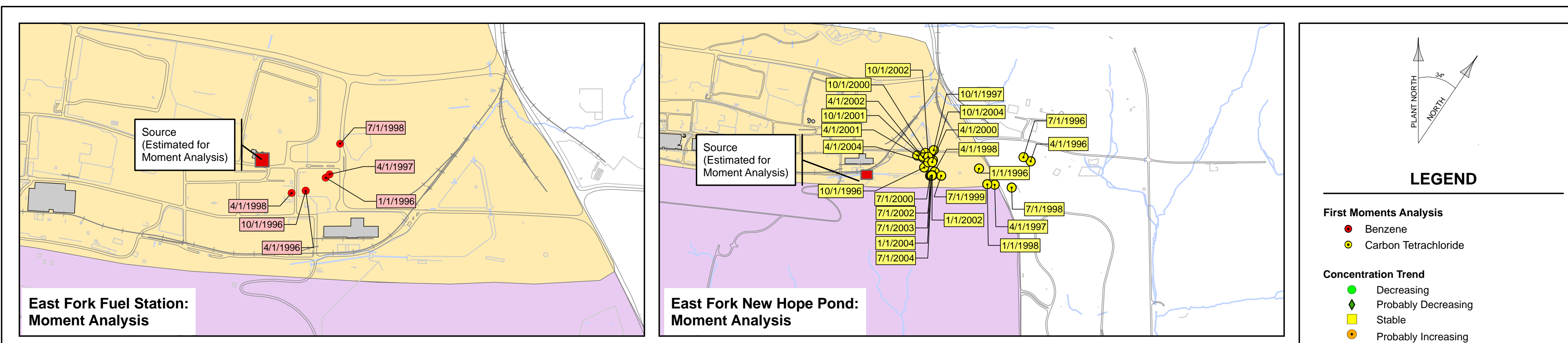

\section{First Moments Analysis \\ - Benzene}

Carbon Tetrachloride

Concentration Trend

$\therefore \quad$ Decreasing

$\diamond \quad$ Probably
$\square \quad$ Stable
$\square \quad$

- Probably Increasing

- Increasing

- Insufficient Data

No Trend
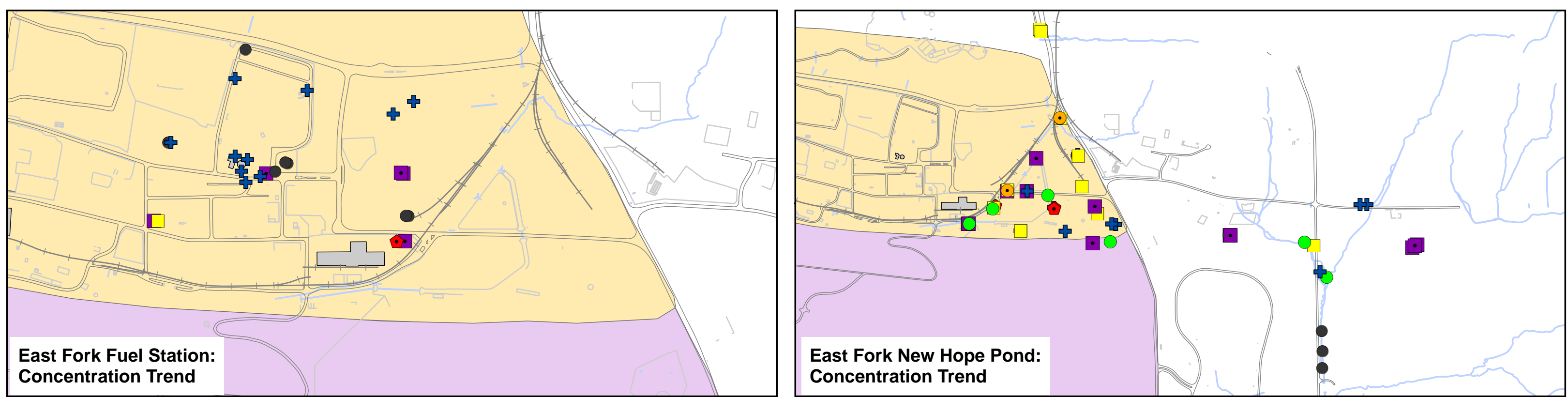

Frequency Recommendation

$\begin{array}{ll}- & \text { Biennial } \\ - & \text { Annual }\end{array}$

- SemiAnnual

- Regulated

(1)

घ Review

$\triangle$ Extraction Well

Note:

Concentration Trend

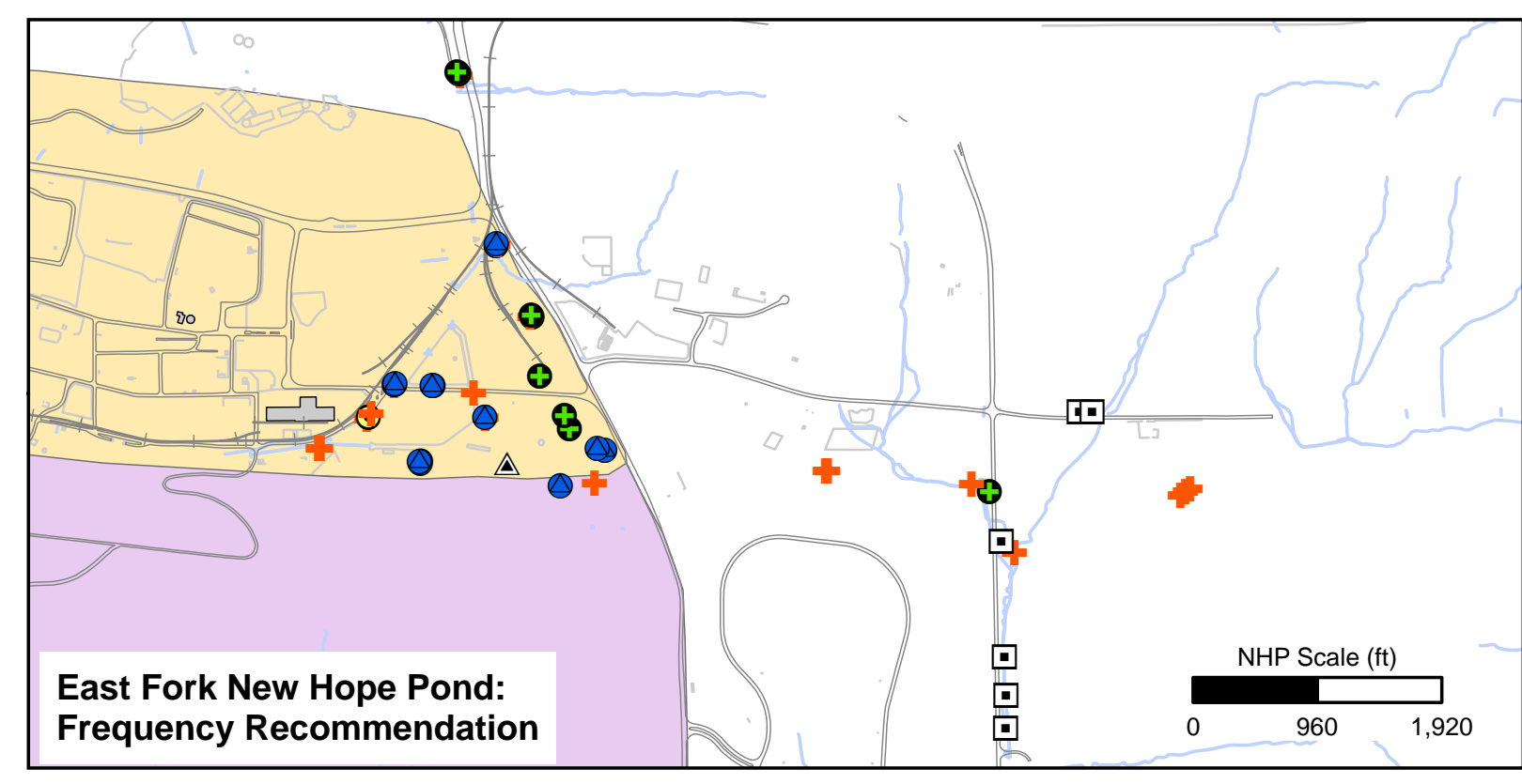

1) First moments represent the approximate center of mass of the plume.

2) Concentration trend is for priority constituent at each location.
Figure A.3.

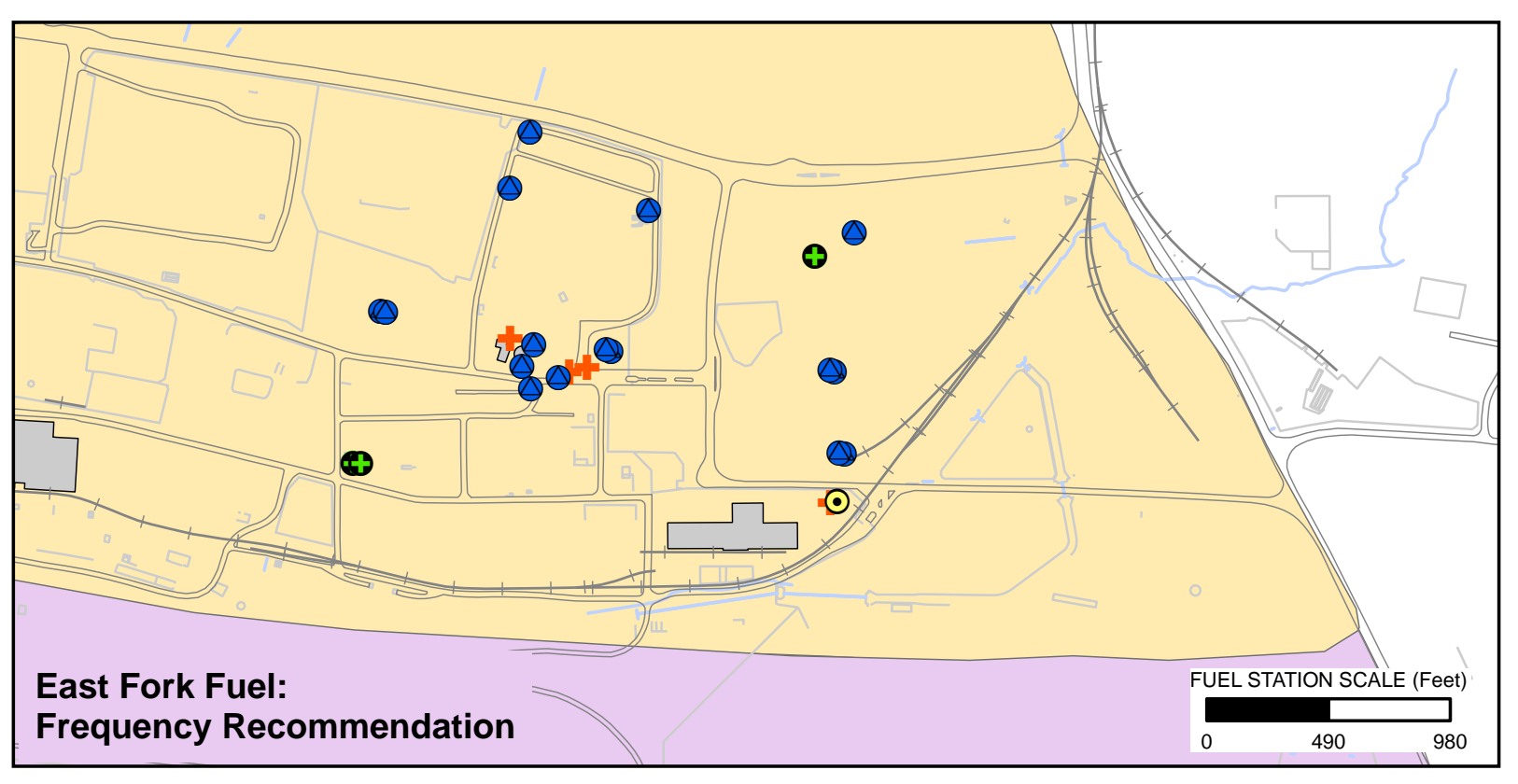

\begin{tabular}{|c|c|c|}
\hline \multirow{2}{*}{\multicolumn{3}{|c|}{$\begin{array}{c}\text { MAROS RESULTS } \\
\text { East ForkRegime } \\
\text { Fuel Station and East y-12 } \\
\text { Y-12 National Security Complex } \\
\text { Oak Ridge, Tennessee }\end{array}$}} \\
\hline & & \\
\hline GSIJ J Dob No: & G-3038 & Drawn by: $\quad$ CDM \\
\hline Issued: & 12/12/2005 & chked by: MV \\
\hline Revised: & & Appvid $\quad$ MV \\
\hline Scale: & As Shown & FIGURE A. 8 \\
\hline
\end{tabular}



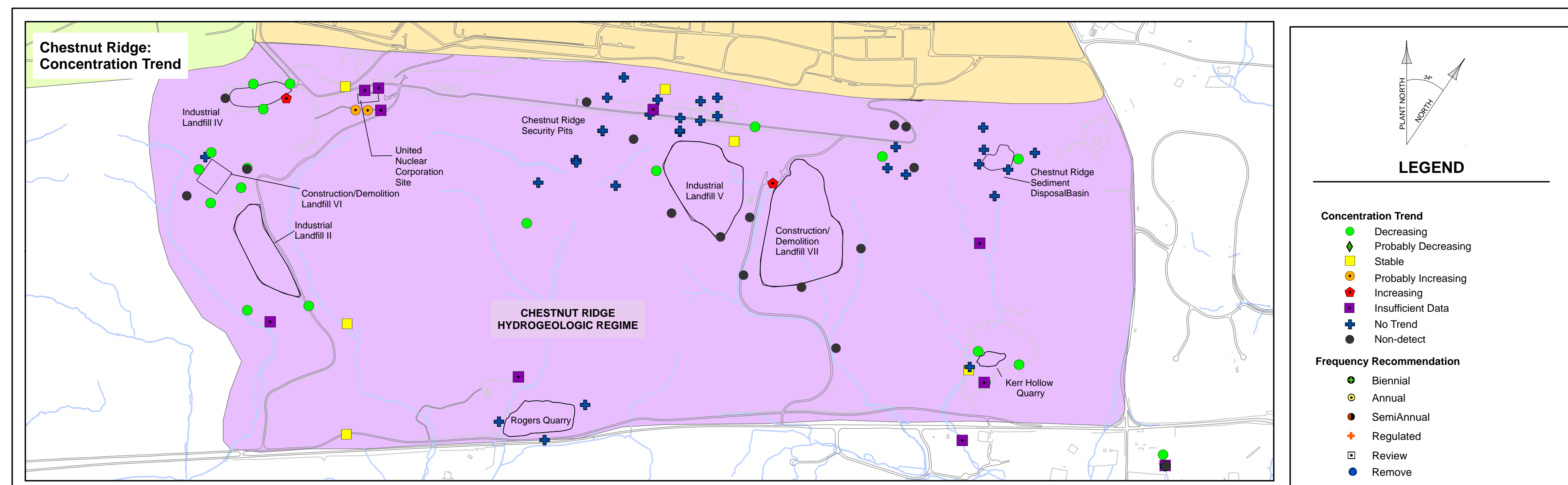

\section{Concentration Trend}

Decreasing

Probably Decreasing

Stable

Probably Increasing

- Increasing

Insufficient
No Trend

No Trend

Frequency Recommendation

- Biennial

$\odot$ Annual

- SemiAnnual

+ Regulated

Review

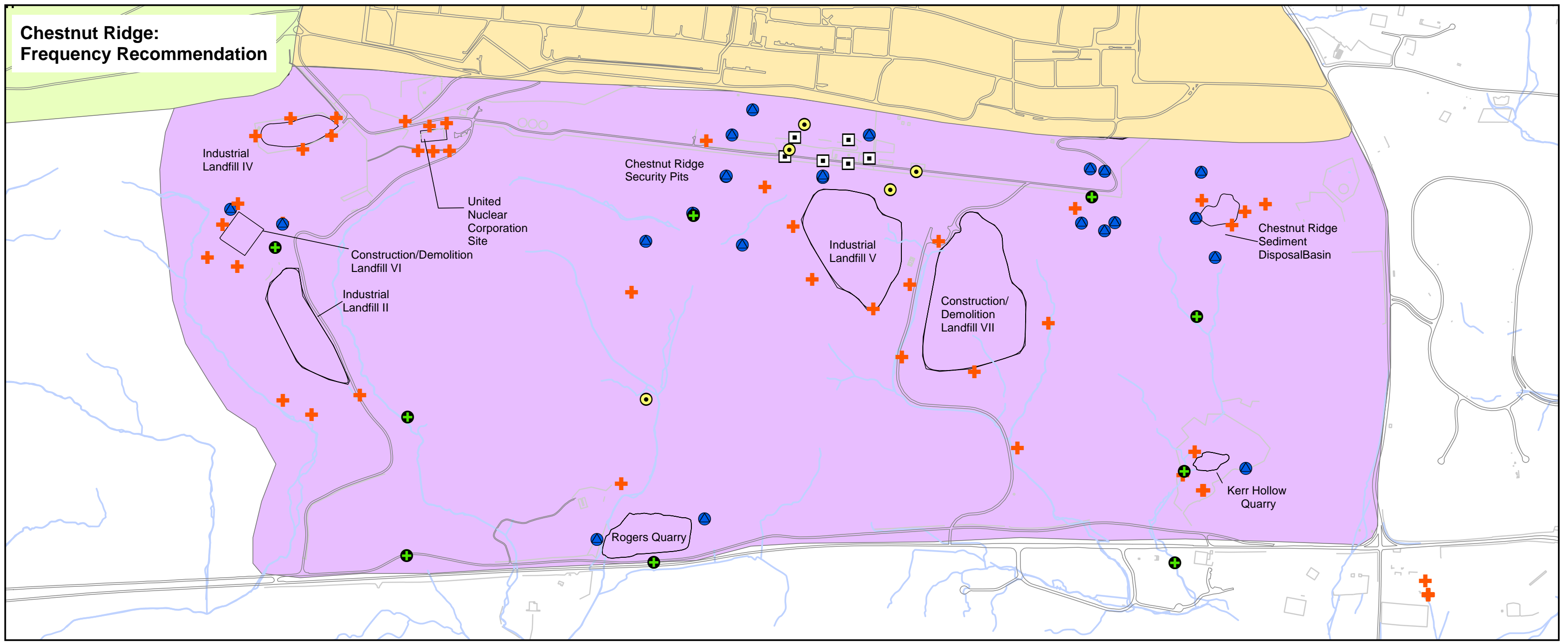

Note:

1) Concentration trend is for priority constituent at

Sample location ID's are shown on Fioure Ad.

First Moment analyses are not included for Chestnut Ridge

concentrations, and large distances between areas

\begin{tabular}{ll|l|}
\multicolumn{1}{c|}{ Scale (ft) } \\
& & \\
&
\end{tabular}




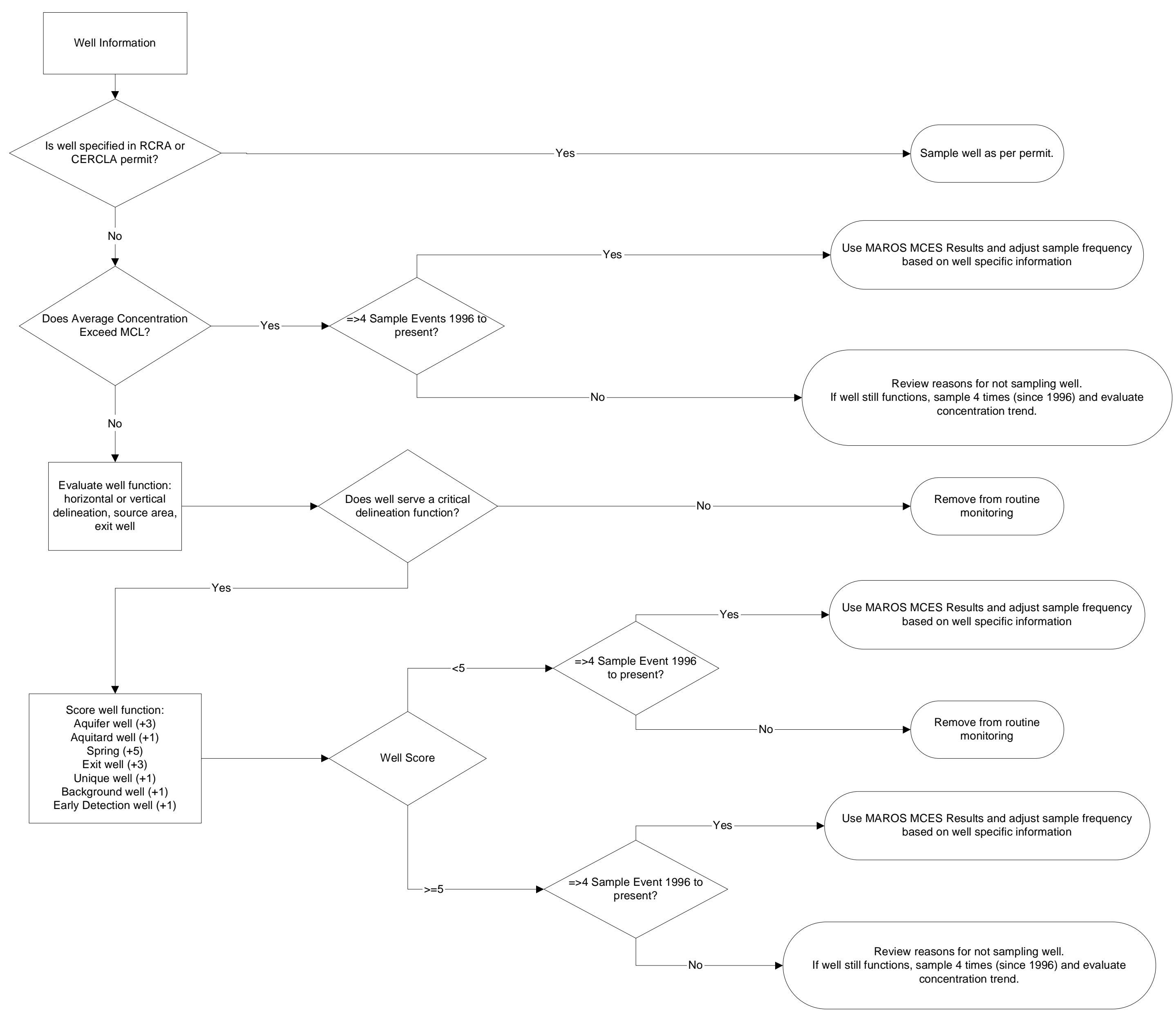

\begin{tabular}{|c|c|}
\hline \multicolumn{2}{|c|}{$\begin{array}{c}\text { GROUNDWATER } \\
\text { SERVICES, INC. }\end{array}$} \\
\hline $\begin{array}{c}\text { Well Network } \\
\text { Decision Logic } \\
\text { Y-12 National Security } \\
\text { Complex }\end{array}$ \\
\hline $12 / 12 / 2005$ & Figure A.10 \\
\hline
\end{tabular}




\section{ASSESSMENT OF THE \\ GROUNDWATER PROTECTION PROGRAM \\ Y-12 NATIONAL SECURITY COMPLEX, OAK RIDGE, TENNESSEE}

Y-12 National Security Complex

Oak Ridge, Tennessee

\section{APPENDIX B: TABLES}

B.1 Major Constituents and Screening Levels

B.2 Location Analysis Groups for MAROS Evaluation

B.3 MAROS Results West S-3 Area

B.4 MAROS Results Oil Landfarm WMA

B.5 MAROS Results Bear Creek Burial Grounds

B.6 MAROS Results East S-3 Area

B.7 MAROS Results Central Y-12 Area

B.8 MAROS Results Fuel Station Area

B.9 MAROS Results East Y-12 Area

B.10 MAROS Results West Chestnut Ridge

B.11 MAROS Results Chestnut Ridge Security Pits

B.12 MAROS Results Landfills V and VII Areas

B.13 MAROS Results East Chestnut Ridge

B.14 Summary Sampling Recommendations Bear Creek Regime

B.15 Summary Sampling Recommendations East Fork Regime

B.16 Summary Sampling Recommendations Chestnut Ridge Regime 
GSI Job No. G-3038

Issued 12/12/2005

Page 1 of 1

TABLE B.1

MAJOR CONSTITUENTS AND SCREENING LEVELS

\author{
Y-12 National Security Complex \\ Oak Ridge, Tennessee
}

\begin{tabular}{|c|l|r|l|l|}
\hline $\begin{array}{c}\text { Constituent } \\
\text { Type }\end{array}$ & \multicolumn{1}{|c|}{ Constituent } & $\begin{array}{r}\text { Screening } \\
\text { Level }\end{array}$ & Units & Source of Screening Level \\
\hline INO & GROSS ALPHA ACTIVITY & 15 & $\mathrm{pCi} / \mathrm{L}$ & $\mathrm{MCL}$ \\
INO & GROSS BETA ACTIVITY & 50 & $\mathrm{pCi} / \mathrm{L}$ & $\mathrm{MCL}$ \\
INO & NITRATE & 10 & $\mathrm{mg} / \mathrm{L}$ & $\mathrm{MCL}$ \\
\hline MET & ALUMINUM & 0.05 & $\mathrm{mg} / \mathrm{L}$ & $\mathrm{Secondary} \mathrm{MCL}$ \\
MET & BARIUM & 2 & $\mathrm{mg} / \mathrm{L}$ & $\mathrm{MCL}$ \\
MET & BERYLLIUM & 0.004 & $\mathrm{mg} / \mathrm{L}$ & $\mathrm{MCL}$ \\
MET & CADMIUM & 0.005 & $\mathrm{mg} / \mathrm{L}$ & $\mathrm{MCL}$ \\
MET & CHROMIUM & 0.1 & $\mathrm{mg} / \mathrm{L}$ & $\mathrm{MCL}$ \\
\hline MET & COPPER & 1 & $\mathrm{mg} / \mathrm{L}$ & $\mathrm{Secondary} \mathrm{MCL}$ \\
MET & LEAD & 0.015 & $\mathrm{mg} / \mathrm{L}$ & $\mathrm{MCL}$ \\
MET & MANGANESE & 0.05 & $\mathrm{mg} / \mathrm{L}$ & $\mathrm{Secondary} \mathrm{MCL}$ \\
MET & MERCURY & 0.002 & $\mathrm{mg} / \mathrm{L}$ & $\mathrm{MCL}$ \\
MET & NICKEL & 0.073 & $\mathrm{mg} / \mathrm{L}$ & $\mathrm{Reg} 9$ PRG \\
\hline MET & STRONTIUM & 22 & $\mathrm{mg} / \mathrm{L}$ & $\mathrm{Reg} 9$ PRG \\
MET & TECHNETIUM-99 & 4000 & $\mathrm{pCi} / \mathrm{L}$ & $\mathrm{DOE}$ Order 5400.5 \\
MET & URANIUM & 0.03 & $\mathrm{mg} / \mathrm{L}$ & $\mathrm{MCL}$ \\
\hline ORG & 1,1,1-TRICHLOROETHANE & 0.2 & $\mathrm{mg} / \mathrm{L}$ & $\mathrm{MCL}$ \\
ORG & 1,1-DICHLOROETHANE & 0.081 & $\mathrm{mg} / \mathrm{L}$ & $\mathrm{Reg} 9$ PRG \\
ORG & 1,1-DICHLOROETHENE & 0.007 & $\mathrm{mg} / \mathrm{L}$ & $\mathrm{MCL}$ \\
ORG & 1,2-DICHLOROETHANE & 0.005 & $\mathrm{mg} / \mathrm{L}$ & $\mathrm{MCL}$ \\
ORG & BENZENE & 0.005 & $\mathrm{mg} / \mathrm{L}$ & $\mathrm{MCL}$ \\
\hline ORG & CARBON TETRACHLORIDE & 0.005 & $\mathrm{mg} / \mathrm{L}$ & $\mathrm{MCL}$ \\
ORG & CHLOROFORM & 0.08 & $\mathrm{mg} / \mathrm{L}$ & $\mathrm{MCL}$ (total trihalomethanes) \\
ORG & Cis-1,2-DICHLOROETHYLENE & 0.07 & $\mathrm{mg} / \mathrm{L}$ & $\mathrm{MCL}$ \\
ORG & TETRACHLOROETHYLENE(PCE) & 0.005 & $\mathrm{mg} / \mathrm{L}$ & $\mathrm{MCL}$ \\
ORG & TRICHLOROETHYLENE (TCE) & 0.005 & $\mathrm{mg} / \mathrm{L}$ & $\mathrm{MCL}$ \\
ORG & VINYL CHLORIDE & 0.002 & $\mathrm{mg} / \mathrm{L}$ & $\mathrm{MCL}$ \\
\hline
\end{tabular}

\title{
Notes
}

1. $\mathrm{MET}=$ Metal; $I N O=$ Inorganic constituent; $\mathrm{ORG}=$ Organic Constituent

2. $\mathrm{MCLS}=$ Maximum Contaminant Levels are USEPA National Primary or Secondary Drinking Water Standards, 2005.

3. Reg 9 PRG = EPA Region IX Preliminary Remediation Goal, 2004 Values.

4. DOE Order $5400.5=$ Radiation Protection of the Public and the Environment, 1/7/1993 
TABLE B.2

LOCATION ANALYSIS GROUPS FOR MAROS EVALUATION

\section{Y-12 National Security Complex}

Oak Ridge, Tennessee

\begin{tabular}{|c|c|c|c|}
\hline Hydrogeologic Regime & MAROS Analysis Group & Location Description & $\begin{array}{c}\text { Number Sample } \\
\text { Locations } \\
\text { Analyzed } \\
\end{array}$ \\
\hline \multirow{3}{*}{ Bear Creek } & Bear Creek Burial Grounds & $\begin{array}{l}\text { Western Bear Creek Regime, Bear Creek } \\
\text { Burial Grounds }\end{array}$ & 80 \\
\hline & Oil Landfarm WMA & $\begin{array}{l}\text { Central Bear Creek Regime includes } \\
\text { EMWMF, Oil Landfarm WMA }\end{array}$ & 59 \\
\hline & West S3 Area & $\begin{array}{l}\text { Eastern Bear Creek Regime, S-3 Site, } \\
\text { Rust Spoil Area, Spoil Area } 1\end{array}$ & 44 \\
\hline \multirow{4}{*}{ Chestnut Ridge } & East Chestnut Ridge & $\begin{array}{l}\text { Eastern Chestnut Ridge Regime, Kerr } \\
\text { Hollow Quarry, Sediment Basin, Borrow } \\
\text { Area Waste Pile, Bethel Valley }\end{array}$ & 26 \\
\hline & Landfills V and VII & $\begin{array}{l}\text { Industrial Landfill V, } \\
\text { Construction/Demolition Landfill VII, South } \\
\text { Side Chestnut Ridge }\end{array}$ & 10 \\
\hline & West Chestnut Ridge & $\begin{array}{l}\text { Industrial Landfill II, Industrial Landfill IV, } \\
\text { United Nuclear Corporation Site, } \\
\text { Construction/Demolition Landfill VI, Oak } \\
\text { Ridge Sludge Farm }\end{array}$ & 24 \\
\hline & Chestnut Ridge Security Pits & $\begin{array}{l}\text { Security Pits, Filled Coal Ash Pond, } \\
\text { Rogers Quarry }\end{array}$ & 29 \\
\hline \multirow{4}{*}{ East Fork Poplar Creek } & East S-3 & $\begin{array}{l}\text { Eastern S-3 and S-2 Sites, Beta-4 } \\
\text { Security Pits, Rust Garage Area, Fire } \\
\text { Training Facility, Y-12 Salvage Yard, } \\
\text { Waste Coolant Processing Facility } \\
\end{array}$ & 49 \\
\hline & Central Y-12 & $\begin{array}{l}\text { Y-12 Industrial Facility, Coal Pile Trench, } \\
\text { Uranium Oxide Vault }\end{array}$ & 44 \\
\hline & Fuel Station & Y-12 Fuel Station, Ravine Disposal Site & 24 \\
\hline & East $Y-12$ & $\begin{array}{l}\text { New Hope Pond, Union Valley, Scarboro } \\
\text { Road }\end{array}$ & 54 \\
\hline
\end{tabular}

Notes:

1. Analysis groups were formed based on geographic proximity and desired sample size.

2. The number of sample locations is the number of locations in the geographic area that had data in the Y-12 Analytical Database (BWXT Y-12, 2005).

3. Sample locations include both monitoring wells and springs. Surface water samples and Westbay wells were not included in the analysis. 
TABLE B.3

MAROS RESULTS WEST S-3 AREA

Bear Creek Hydrogeologic Regime

Y-12 National Security Complex

Oak Ridge, Tennessee

\begin{tabular}{|c|c|c|c|c|c|c|c|c|}
\hline $\begin{array}{c}\text { Location } \\
\text { Name }\end{array}$ & $\begin{array}{c}\text { Earliest } \\
\text { Sample Date }\end{array}$ & $\begin{array}{l}\text { Most Recent } \\
\text { Sample Date }\end{array}$ & Location Type & $\begin{array}{c}\text { Number of } \\
\text { Samples } \\
1996-2005 \\
\end{array}$ & \begin{tabular}{|c|} 
Average \\
Concentration \\
Exceeds Screening \\
Level
\end{tabular} & Historic Priority Constituent & \begin{tabular}{|c|} 
Trend for Priority \\
Constituent 1996- \\
2005
\end{tabular} & $\begin{array}{l}\text { Preliminary } \\
\text { Frequency }\end{array}$ \\
\hline GW-100 & $1 / 22 / 1986$ & $8 / 17 / 2004$ & WL & 2 & Yes & NITRATE & N/A & SemiAnnual \\
\hline GW-101 & 1/23/1986 & $8 / 18 / 2004$ & WL & 2 & Yes & NITRATE & N/A & SemiAnnual \\
\hline GW-115 & $3 / 9 / 1987$ & $1 / 6 / 2004$ & WL & 14 & No & GROSS ALPHA ACTIVITY & NT & Annual \\
\hline GW-122 & 12/12/1986 & 4/13/1991 & WL & 0 & Yes & NITRATE & N/A & Annual \\
\hline GW-123 & $12 / 23 / 1986$ & $8 / 4 / 2003$ & WL & 1 & Yes & GROSS ALPHA ACTIVITY & N/A & Annual \\
\hline GW-124 & $12 / 6 / 1986$ & $8 / 9 / 2001$ & $\mathrm{WL}$ & 2 & Yes & NITRATE & N/A & SemiAnnual \\
\hline GW-125 & $12 / 6 / 1986$ & 9/17/1992 & WL & 0 & Yes & GROSS BETA ACTIVITY & N/A & Annual \\
\hline GW-127 & $12 / 12 / 1986$ & $1 / 18 / 1990$ & WL & 0 & Yes & URANIUM & N/A & Annual \\
\hline GW-236 & $4 / 5 / 1988$ & $8 / 18 / 2004$ & WL & 2 & Yes & NITRATE & N/A & SemiAnnual \\
\hline GW-243 & $12 / 19 / 1986$ & $8 / 13 / 2002$ & WL & 2 & Yes & GROSS BETA ACTIVITY & N/A & SemiAnnual \\
\hline GW-244 & $3 / 13 / 1987$ & $1 / 18 / 1990$ & $\mathrm{WL}$ & 0 & Yes & NITRATE & $\mathrm{N} / \mathrm{A}$ & Biennial \\
\hline GW-245 & 3/10/1987 & $1 / 17 / 1990$ & WL & 0 & Yes & NITRATE & N/A & Biennial \\
\hline GW-246 & 3/16/1987 & $8 / 19 / 2004$ & WL & 2 & Yes & NITRATE & N/A & SemiAnnual \\
\hline GW-247 & 3/16/1987 & $1 / 17 / 1990$ & WL & 0 & Yes & NITRATE & N/A & Biennial \\
\hline GW-276 & $10 / 29 / 1986$ & $7 / 8 / 2004$ & WL & 18 & Yes & URANIUM & D & Annual \\
\hline GW-277 & 10/29/1986 & $1 / 23 / 1990$ & WL & 0 & Yes & NITRATE & N/A & Annual \\
\hline GW-306 & 2/5/1988 & $4 / 26 / 1991$ & WL & 0 & Yes & TRICHLOROETHYLENE (TCE) & N/A & Biennial \\
\hline GW-307 & 2/6/1988 & $4 / 26 / 1991$ & WL & 0 & Yes & TRICHLOROETHYLENE (TCE) & N/A & Biennial \\
\hline GW-308 & 2/5/1988 & $4 / 30 / 1991$ & WL & 0 & Yes & TRICHLOROETHYLENE (TCE) & N/A & Biennial \\
\hline GW-309 & 2/9/1988 & $8 / 30 / 1992$ & WL & 0 & Yes & GROSS BETA ACTIVITY & N/A & Biennial \\
\hline
\end{tabular}

\section{Notes:}

1. Well sample dates taken from $\mathrm{Y}-12$ Analytical Database.

2. $\mathrm{WL}=$ Groundwater Monitoring Well; $\mathrm{SP}=$ Spring.

3. Trend = Insufficient Data (N/A), Decreasing (D), Probably Decreasing (PD), Stable (S), Probably Increasing (PI),

and Increasing (I), No Trend (NT); and non-detect for all sample events (ND).

4. Number of samples between 1996-2005 is the count of analytical samples acquired at the location based on the BWXT Analytical Database (2005).

5. Priority Constituent is the $\mathrm{COC}$ with the highest average concentration from the full dataset normalized by the screening level. (e.g. MAX [Ave Conc./Screening Level]). Priority constituent indicated for wells where average concentrations do not exceed screening levels may be background level.

6. Remove $=$ Do not sample; SemiAnnual $=$ sample once every 6 months; Annual $=$ sample once per year; Biennial $=$ sample once every two years 
TABLE B.3

MAROS RESULTS WEST S-3 AREA

Bear Creek Hydrogeologic Regime

Y-12 National Security Complex

Oak Ridge, Tennessee

\begin{tabular}{|c|c|c|c|c|c|c|c|c|}
\hline $\begin{array}{c}\text { Location } \\
\text { Name }\end{array}$ & $\begin{array}{c}\text { Earliest } \\
\text { Sample Date }\end{array}$ & $\begin{array}{l}\text { Most Recent } \\
\text { Sample Date }\end{array}$ & Location Type & $\begin{array}{c}\text { Number of } \\
\text { Samples } \\
1996-2005\end{array}$ & \begin{tabular}{|c|} 
Average \\
Concentration \\
$\begin{array}{c}\text { Exceeds Screening } \\
\text { Level }\end{array}$ \\
\end{tabular} & Historic Priority Constituent & \begin{tabular}{|c|} 
Trend for Priority \\
Constituent 1996- \\
2005
\end{tabular} & $\begin{array}{l}\text { Preliminary } \\
\text { Frequency }\end{array}$ \\
\hline GW-310 & $2 / 5 / 1988$ & $4 / 30 / 1991$ & WL & 0 & Yes & TRICHLOROETHYLENE (TCE) & $\mathrm{N} / \mathrm{A}$ & Biennial \\
\hline GW-311 & 2/5/1988 & $8 / 2 / 2004$ & WL & 18 & Yes & TRICHLOROETHYLENE (TCE) & D & Annual \\
\hline GW-312 & 2/9/1988 & 8/6/1995 & WL & 0 & Yes & TRICHLOROETHYLENE (TCE) & $\mathrm{N} / \mathrm{A}$ & Biennial \\
\hline GW-313 & $3 / 8 / 1988$ & 8/15/1992 & WL & 0 & Yes & TETRACHLOROETHYLENE(PCE) & $\mathrm{N} / \mathrm{A}$ & Annual \\
\hline GW-314 & $3 / 7 / 1988$ & 8/15/1992 & WL & 0 & Yes & TETRACHLOROETHYLENE(PCE) & N/A & Annual \\
\hline GW-315 & $3 / 7 / 1988$ & $8 / 2 / 2004$ & WL & 18 & Yes & TETRACHLOROETHYLENE(PCE) & PD & Annual \\
\hline GW-316 & 3/7/1988 & 10/24/1993 & WL & 0 & No & GROSS BETA ACTIVITY & $\mathrm{N} / \mathrm{A}$ & Biennial \\
\hline GW-317 & 3/7/1988 & 8/15/1995 & WL & 0 & No & GROSS ALPHA ACTIVITY & $\mathrm{N} / \mathrm{A}$ & Biennial \\
\hline GW-323 & 3/8/1988 & 10/23/1993 & WL & 0 & No & GROSS ALPHA ACTIVITY & $\mathrm{N} / \mathrm{A}$ & Biennial \\
\hline GW-325 & $12 / 8 / 1987$ & 2/15/1994 & WL & 0 & No & TETRACHLOROETHYLENE(PCE) & $\mathrm{N} / \mathrm{A}$ & Biennial \\
\hline GW-345 & 9/28/1988 & $3 / 9 / 1999$ & WL & 1 & Yes & NITRATE & $\mathrm{N} / \mathrm{A}$ & Annual \\
\hline GW-346 & 9/28/1988 & 3/9/1999 & WL & 1 & Yes & NITRATE & $\mathrm{N} / \mathrm{A}$ & SemiAnnual \\
\hline GW-347 & 9/29/1988 & 8/15/1995 & WL & 0 & No & GROSS BETA ACTIVITY & $\mathrm{N} / \mathrm{A}$ & Biennial \\
\hline GW-348 & 9/29/1988 & 8/21/1995 & WL & 0 & No & NITRATE & $\mathrm{N} / \mathrm{A}$ & Biennial \\
\hline GW-526 & 9/28/1988 & $8 / 16 / 2004$ & WL & 1 & Yes & NITRATE & $\mathrm{N} / \mathrm{A}$ & SemiAnnual \\
\hline GW-531 & $12 / 15 / 1988$ & $6 / 24 / 1991$ & WL & 0 & No & GROSS ALPHA ACTIVITY & $\mathrm{N} / \mathrm{A}$ & Biennial \\
\hline GW-613 & 2/9/1990 & 8/11/1997 & WL & 2 & No & GROSS ALPHA ACTIVITY & $\mathrm{N} / \mathrm{A}$ & Biennial \\
\hline GW-615 & $5 / 16 / 1990$ & 8/19/2004 & WL & 2 & Yes & NITRATE & $\mathrm{N} / \mathrm{A}$ & SemiAnnual \\
\hline GW-616 & $5 / 17 / 1990$ & $8 / 9 / 2001$ & WL & 2 & Yes & NITRATE & $\mathrm{N} / \mathrm{A}$ & SemiAnnual \\
\hline GW-630 & $6 / 27 / 1990$ & 8/29/1992 & WL & 0 & No & GROSS BETA ACTIVITY & $\mathrm{N} / \mathrm{A}$ & Biennial \\
\hline GW-648 & $1 / 15 / 1991$ & $7 / 23 / 1992$ & WL & 0 & No & GROSS ALPHA ACTIVITY & $\mathrm{N} / \mathrm{A}$ & Biennial \\
\hline GW-829 & 6/27/1995 & $8 / 1 / 2001$ & WL & 11 & Yes & NITRATE & D & Annual \\
\hline GW-835 & 4/16/1997 & $8 / 21 / 2003$ & WL & 20 & Yes & URANIUM & 1 & SemiAnnual \\
\hline SS-1 & 8/30/1990 & $7 / 20 / 2004$ & $\mathrm{SP}$ & 18 & Yes & NITRATE & $\mathrm{s}$ & Annual \\
\hline
\end{tabular}

Notes:

1. Well sample dates taken from $\mathrm{Y}$-12 Analytical Database.

2. $\mathrm{WL}=$ Groundwater Monitoring Well; $\mathrm{SP}=$ Spring.

3. Trend = Insufficient Data (N/A), Decreasing (D), Probably Decreasing (PD), Stable (S), Probably Increasing (PI),

and Increasing (I), No Trend (NT); and non-detect for all sample events (ND).

4. Number of samples between 1996-2005 is the count of analytical samples acquired at the location based on the BWXT Analytical Database (2005).

5. Priority Constituent is the $\mathrm{COC}$ with the highest average concentration from the full dataset normalized by the screening level. (e.g. MAX [Ave Conc./Screening Level]).

Priority constituent indicated for wells where average concentrations do not exceed screening levels may be background level.

6. Remove = Do not sample; SemiAnnual = sample once every 6 months; Annual = sample once per year; Biennial = sample once every two years 
TABLE B.4
MAROS RESUTLS OIL LANDFARM WMA

Bear Creek Hydrogeologic Regime

Y-12 National Security Complex

\begin{tabular}{|c|c|c|c|c|c|c|c|c|}
\hline $\begin{array}{c}\text { Location } \\
\text { Name }\end{array}$ & $\begin{array}{c}\text { Earliest } \\
\text { Sample Date }\end{array}$ & $\begin{array}{l}\text { Most Recent } \\
\text { Sample Date }\end{array}$ & Location Type & $\begin{array}{c}\text { Number of } \\
\text { Samples 1996- } \\
2005\end{array}$ & $\begin{array}{c}\text { Average } \\
\text { Concentration } \\
\text { Exceeds } \\
\text { Screening Level }\end{array}$ & Historic Priority Constituent & $\begin{array}{c}\text { Trend for } \\
\text { Priority } \\
\text { Constituent } \\
\text { 1996-2005 } \\
\end{array}$ & $\begin{array}{c}\text { Preliminary } \\
\text { Frequency } \\
\end{array}$ \\
\hline GW-006 & $11 / 11 / 1998$ & $8 / 2 / 2000$ & WL & 8 & No & TRICHLOROETHYLENE (TCE) & $\mathrm{s}$ & Annual \\
\hline & $1 / 26 / 1998$ & 7/7/2004 & WL & 14 & Yes & TETRACHLOROETHYLENE(PCE) & NT & Annual \\
\hline GW-010 & $3 / 25 / 1987$ & $2 / 1 / 1990$ & WL & 0 & Yes & TETRACHLOROETHYLENE(PCE) & N/A & Biennial \\
\hline GW-013 & $5 / 21 / 1990$ & 12/19/1993 & WL & 0 & No & CADMIUM & N/A & Remove \\
\hline GW-064 & $11 / 5 / 1987$ & \begin{tabular}{|l|}
$7 / 20 / 1998$ \\
\end{tabular} & WL & 2 & Yes & TRICHLOROETHYLENE (TCE) & N/A & Biennial \\
\hline GW-066 & 9/27/1995 & $8 / 13 / 2002$ & WL & 2 & Yes & TETRACHLOROETHYLENE(PCE) & N/A & SemiAnnua \\
\hline GW-067 & 3/25/1987 & $\begin{array}{l}3 / 133 / 1990 \\
1 / 301990\end{array}$ & WL & 0 & Yes & LEAD & N/A & $\begin{array}{l}\text { Biennial } \\
\text { Bienniai }\end{array}$ \\
\hline GW-073 & $3 / 18 / 1987$ & $1 / 30 / 1990$ & WL & 0 & No & GROSS BETA ACTIVITY & ND & Biennial \\
\hline $\begin{array}{l}\text { GW-074 } \\
\text { GW-075 }\end{array}$ & $5 / 23 / 1990$ & 8/3/1992 & WL & 0 & No & GROSS ALPHA ACTIVITY & ND & Biennial \\
\hline$\frac{\text { GW-075 }}{\text { GW-084 }}$ & $5 / 23 / 1990$ & \begin{tabular}{|l|}
$9 / 8 / 1992$ \\
$813 / 1997$
\end{tabular} & WL & 0 & No & CADMIUM & N/A & Biennial \\
\hline $\begin{array}{l}\text { GW-0084 } \\
\text { GW-085 }\end{array}$ & $\begin{array}{l}3 / 24 / 1987 \\
11 / 5 / 1987\end{array}$ & $\begin{array}{l}8 / 133 / 1997 \\
8 / 3 / 2004\end{array}$ & $\begin{array}{l}\text { WL } \\
\text { WL }\end{array}$ & $\begin{array}{c}4 \\
17\end{array}$ & $\begin{array}{l}\text { No } \\
\text { Yes }\end{array}$ & $\begin{array}{l}\text { LEAD } \\
\text { NITRATE }\end{array}$ & $\begin{array}{l}\mathrm{Pl} \\
\mathrm{Pl}\end{array}$ & $\begin{array}{l}\text { Beiennial } \\
\text { SemiAnnua }\end{array}$ \\
\hline GW-086 & $5 / 22 / 1990$ & $10 / 21 / 1993$ & WL & 0 & No & LEAD & N/A & Remove \\
\hline GW-097 & $3 / 17 / 1987$ & $7 / 15 / 1998$ & WL & 2 & No & LEAD & N/A & Biennial \\
\hline GW-098 & $3 / 17 / 1987$ & $8 / 3 / 2004$ & WL & 4 & Yes & TRICHLOROETHYLENE (TCE) & NT & Annual \\
\hline GW-120 & 3/26/1987 & $1 / 30 / 1990$ & $\overline{W L}$ & 0 & No & GROSS BETA ACTIVITY & ND & Remove \\
\hline GW-225 & 1/12/1986 & 7/29/2004 & WL & 8 & Yes & TRICHLOROETHYLENE (TCE) & NT & SemiAnnua \\
\hline$G W-226$ & 1/12/1986 & 7/28/2004 & WL & 14 & Yes & TRICHLOROETHYLENE (TCE) & s & SemiAnnua \\
\hline$G W-227$ & 1/14/1996 & 9/12/1992 & WL & 0 & Yes & TRICHLOROETHYLENE (TCE) & N/A & Annual \\
\hline GW-228 & $1 / 14 / 1986$ & 9/9/1999 & WL & 1 & Yes & TRICHLOROETHYLENE (TCE) & N/A & Biennial \\
\hline GW-229 & $1 / 14 / 1986$ & \begin{tabular}{|l|}
$7 / 29 / 2004$ \\
\end{tabular} & WL & 6 & Yes & VINYL CHLORIDE & $\mathrm{s}$ & Annual \\
\hline GW-363 & 9/21/1988 & 11/18/2004 & WL & 7 & Yes & GROSS BETA ACTIVITY & PD & Biennial \\
\hline GW-364 & $9 / 22 / 1988$ & $8 / 7 / 2001$ & WL & 2 & Yes & TRICHLOROETHYLENE (TCE) & N/A & Annual \\
\hline $\begin{array}{l}\text { GW-365 } \\
\text { GW-366 }\end{array}$ & $\begin{array}{l}9 / 2 / 1 / 1988 \\
9 / 20 / 1988\end{array}$ & $\begin{array}{l}8 / 7 / 2001 \\
9 / 7 / 1992\end{array}$ & $\begin{array}{l}\text { WL } \\
\text { WL }\end{array}$ & $\begin{array}{l}2 \\
0\end{array}$ & $\begin{array}{l}\text { YYes } \\
\text { No }\end{array}$ & $\begin{array}{l}\text { TRICHLOROETHYLENE (TCE) } \\
\text { LEAD }\end{array}$ & $\begin{array}{l}\text { NA } \\
\text { N/A }\end{array}$ & $\begin{array}{l}\text { Annual } \\
\text { Remove }\end{array}$ \\
\hline GW-367 & $9 / 19 / 1988$ & $5 / 11 / 1991$ & WL & 0 & Yes & TRICHLOROETHYLENE (TCE) & N/A & Biennial \\
\hline GW- 368 & 9/24/1988 & 1/31/1990 & WL & 0 & Yes & TRICHLOROETHYLENE (TCE) & N/A & Biennial \\
\hline GW- 369 & 9/22/1988 & 5/15/1991 & WL & 0 & Yes & TRICHLOROETHYLENE (TCE) & N/A & Biennial \\
\hline GW-520 & 9/20/1988 & 9/7/1992 & WL & 0 & No & LEAD & N/A & Remove \\
\hline GW- 537 & 12/6/1989 & $8 / 3 / 2004$ & WL & 17 & Yes & |NITRATE & D & Annual \\
\hline
\end{tabular}

Woll sample dates taken from $\mathrm{Y}-12$ Analytical Database.

WL = Groundwater Monitoring Well; $\mathrm{SP}=$ Spring

(D), Probably Decreasing (PD), Stable (S), Probably Increasing (PI)

5. Priority Constituent is the $\mathrm{COC}$ with the highest average concentration from the full dataset normalized by the screening level. (e.g. MAX [Ave Conc./Screening Level]).

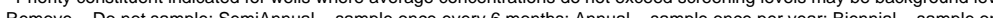


Bear Creek Hydrogeologic Regime

Y-12 National Security Complex
Oak Ridge Tennessee

\begin{tabular}{|c|c|c|c|c|c|c|c|c|}
\hline $\begin{array}{c}\text { Location } \\
\text { Name }\end{array}$ & $\begin{array}{c}\text { Earliest } \\
\text { Sample Date }\end{array}$ & $\begin{array}{l}\text { Most Recent } \\
\text { Sample Date }\end{array}$ & Location Type & $\begin{array}{c}\begin{array}{c}\text { Number of } \\
\text { Samples 1996- } \\
2005\end{array} \\
\end{array}$ & $\begin{array}{c}\text { Average } \\
\text { Concentration } \\
\text { Exceeds } \\
\text { Screening Level }\end{array}$ & Historic Priority Constituent & \begin{tabular}{c|} 
Trend for \\
Priority \\
Constituent \\
$1996-2005$ \\
\end{tabular} & $\begin{array}{c}\text { Preliminary } \\
\text { Frequency }\end{array}$ \\
\hline GW-601 & $3 / 5 / 1990$ & \begin{tabular}{|l|}
$3 / 8 / 1999$ \\
\end{tabular} & WL & 1 & Yes & TRICHLOROETHYLENE (TCE) & N/A & Biennial \\
\hline GW-636 & $2 / 11 / 1991$ & $12 / 18 / 1993$ & WL & 0 & No & GROSS BETA ACTIVITY & N/A & Remove \\
\hline GW-637 & $2 / 12 / 1991$ & 7/19/1995 & WL & 0 & No & GROSS ALPHA ACTIVITY & N/A & Remove \\
\hline GW-638 & $2 / 12 / 1991$ & 12/18/1993 & WL & 0 & No & GROSS ALPHA ACTIVITY & N/A & Remove \\
\hline$\frac{\text { GW- } 645}{\text { GW-646 }}$ & $2 / 12 / 1991$ & $8 / 2 / 1992$ & WL & 0 & No & GROSS ALPHA ACTIVITY & $\mathrm{N} / \mathrm{A}$ & Biennial \\
\hline $\mid \begin{array}{l}\text { GW-646 } \\
\text { GW-723 }\end{array}$ & $\begin{array}{l}1 / 13 / 1991 \\
5 / 27 / 1992\end{array}$ & $\begin{array}{r}8 / 3 / 1992 \\
7 / 23 / 2002\end{array}$ & $\begin{array}{l}\text { WL } \\
\text { WL }\end{array}$ & $\begin{array}{l}0 \\
4\end{array}$ & $\begin{array}{l}\text { No } \\
\text { Yes }\end{array}$ & \begin{tabular}{|l} 
GROSS ALPHA ACTIVITY \\
TRICHOROETHYLNE (TCE)
\end{tabular} & $\begin{array}{l}\text { N/A } \\
\mathrm{PI}\end{array}$ & $\begin{array}{l}\text { Biennial } \\
\text { Annual }\end{array}$ \\
\hline GW-724 & $5 / 20 / 1992$ & $7 / 27 / 2004$ & WL & 18 & Yes & TRICHLOROETHYLENE (TCE) & s & Annual \\
\hline GW-725 & $5 / 21 / 1992$ & 7/27/2004 & WL & 18 & Yes & TRICHLOROETHYLENE (TCE) & $\mathrm{Pl}$ & Annual \\
\hline GW-736 & $5 / 22 / 1992$ & $7 / 17 / 2002$ & WL & 2 & Yes & NITRATE & N/A & SemiAnnual \\
\hline GW-737 & $5 / 22 / 1992$ & $7 / 18 / 2002$ & WL & 2 & Yes & NITRATE & $\mathrm{N} / \mathrm{A}$ & SemiAnnual \\
\hline GW-738 & $5 / 26 / 1992$ & $7 / 26 / 2004$ & WL & 18 & Yes & TRICHLOROETHYLENE (TCE) & $\mathrm{D}$ & Annual \\
\hline GW-739 & $5 / 27 / 1992$ & $7 / 22 / 2002$ & WL & 2 & Yes & TRICHLOROETHYLENE (TCE) & N/A & SemiAnnual \\
\hline GW-740 & 6/2/1992 & $7 / 26 / 2004$ & WL & 18 & Yes & TRICHLOROETHYLENE (TCE) & PD & Annual \\
\hline GW-794 & $12 / 3 / 1992$ & $8 / 26 / 1996$ & WL & 1 & No & GROSS ALPHA ACTIVITY & N/A & Biennial \\
\hline GW-795 & 12/3/1992 & 8/17/2004 & WL & 1 & No & GROSS AL & $N / A$ & Biennial \\
\hline GW-800 & $5 / 11 / 1993$ & 8/5/1995 & WL & 0 & No & GROSS ALPHA ACTIVITY & N/A & Biennial \\
\hline GW-916 & 4/2/2001 & 11/10/2004 & WL & 5 & No & GROSS BETA ACTIVITY & NT & Annual \\
\hline $\begin{array}{l}\text { GW-917 } \\
\text { GW-918 }\end{array}$ & $\begin{array}{l}4 / 3 / 2001 \\
4 / 2 / 2001\end{array}$ & $\begin{array}{l}11 / 4 / 2004 \\
111 / 10 / 2004\end{array}$ & $\begin{array}{l}\text { WL } \\
\text { WL }\end{array}$ & $\begin{array}{l}5 \\
5\end{array}$ & $\begin{array}{l}\text { No } \\
\text { No }\end{array}$ & $\begin{array}{l}\text { GROSS ALPHA ACTIVITY } \\
\text { GROSS BETA ACTIVITY }\end{array}$ & $\begin{array}{l}\mathrm{PI} \\
\mathrm{NT}\end{array}$ & $\begin{array}{l}\text { Biennial } \\
\text { Biennial }\end{array}$ \\
\hline GW-919 & $12 / 4 / 2001$ & \begin{tabular}{|l|}
$1 / 1 / 4 / 2003$ \\
\end{tabular} & WL & 3 & No & GROSS ALPHA ACTIVITY & N/A & Annual \\
\hline GW-920 & $4 / 4 / 2001$ & $11 / 9 / 2004$ & WL & 5 & No & GROSS BETA ACTIVITY & NT & Annual \\
\hline GW-921 & $4 / 3 / 2001$ & $11 / 4 / 2004$ & WL & 5 & No & GROSS BETA ACTIVITY & NT & Biennial \\
\hline GW-922 & $4 / 4 / 2001$ & $11 / 15 / 2004$ & WL & 5 & No & GROSS BETA ACTIVITY & $\mathrm{s}$ & Biennial \\
\hline GW-923 & $4 / 2 / 2001$ & $11 / 16 / 2004$ & WL & 9 & No & LEAD & NT & Biennial \\
\hline GW-924 & $3 / 29 / 2001$ & \begin{tabular}{|l|}
$11 / 17 / 2004$ \\
\end{tabular} & WL & 5 & No & VINYL CHLORIDE & ND & Biennial \\
\hline GW-925 & $4 / 3 / 2001$ & $11 / 8 / 2004$ & WL & 11 & No & LEAD & NT & Biennial \\
\hline GW-926 & $4 / 2 / 2001$ & $11 / 17 / 2004$ & WL & 8 & No & BENZENE & NT & Biennial \\
\hline GW-927 & 4/3/2001 & 11/16/2004 & WL & 5 & No & GROSS ALPHA ACTIVITY & NT & Biennial \\
\hline
\end{tabular}

Well sample dates taken from Y-12 Analytical Database.

WL $=$ Groundwater Monitoring Well; $\mathrm{SP}=\mathrm{Spring}$

Priority constituent indicated for wells where average concentrations do not exceed screening levels may be background level.
Remove $=$ Do not sample; SemiAnnual = sample once every 6 months; Annual = sample once per year; Biennial = sample once every two years. 
Bear Creek Hydrogeologic Regim

Y-12 National Security Complex

Oak Ridge, Tennessee

\begin{tabular}{|c|c|c|c|c|c|c|c|c|}
\hline $\begin{array}{c}\text { Location } \\
\text { Name }\end{array}$ & $\begin{array}{c}\text { Earliest } \\
\text { Sample Date }\end{array}$ & $\begin{array}{l}\text { Most Recent } \\
\text { Sample Date }\end{array}$ & $\begin{array}{c}\text { Location } \\
\text { Type }\end{array}$ & $\begin{array}{c}\text { Number of } \\
\text { Samples } \\
1996-2005 \\
\end{array}$ & $\begin{array}{c}\text { Average } \\
\text { Concentration } \\
\text { Exceeds Screening } \\
\text { Level } \\
\end{array}$ & Historic Priority Constituent & $\begin{array}{c}\text { Trend for } \\
\text { Priority } \\
\text { Constituent } \\
1996-2005 \\
\end{array}$ & $\begin{array}{l}\text { Preliminary } \\
\text { Frequency }\end{array}$ \\
\hline GW-014 & $3 / 14 / 1987$ & $8 / 12 / 2002$ & $\overline{W L}$ & 2 & Yes & TETRACHLOROETHYLENE(PCE) & N/A & SemiAnnual \\
\hline GW-018 & $3 / 21 / 1991$ & 6/20/1991 & WL & 0 & No & VINYL CHLORIDE & N/A & Remove \\
\hline GW-045 & $3 / 14 / 1987$ & 2/8/1990 & WL & 0 & Yes & TETRACHLOROETHYLENE(PCE) & N/A & Biennial \\
\hline GW-046 & $3 / 14 / 1987$ & $7 / 8 / 2004$ & WL & 14 & Yes & TETRACHLOROETHYLENE(PCE) & NT & Annual \\
\hline GW-047 & $3 / 23 / 1987$ & $7 / 24 / 1995$ & WL & 0 & No & 1,1-DICHLOROETHENE & N/A & Remove \\
\hline GW-052 & $6 / 22 / 1990$ & $8 / 16 / 2004$ & $\overline{W L}$ & 2 & Yes & URANIUM & $\mathrm{N} / \mathrm{A}$ & Annual \\
\hline GW-053 & $6 / 22 / 1990$ & $7 / 25 / 2001$ & WL & 12 & Yes & VINYL CHLORIDE & PD & Annual \\
\hline GW-054 & $6 / 13 / 1990$ & 9/2/1992 & WL & 0 & No & VINYL CHLORIDE & N/A & Remove \\
\hline GW-056 & 6/9/1990 & $4 / 27 / 2004$ & WL & 12 & No & URANIUM & $\mathrm{s}$ & Biennial \\
\hline GW-057 & $6 / 13 / 1990$ & $8 / 5 / 1995$ & WL & 0 & No & URANIUM & N/A & Remove \\
\hline GW-058 & $11 / 24 / 1987$ & 9/24/1992 & WL & 0 & Yes & URANIUM & $\mathrm{N} / \mathrm{A}$ & Biennial \\
\hline GW-061 & $8 / 10 / 1989$ & $8 / 5 / 2002$ & WL & 2 & Yes & URANIUM & N/A & Annual \\
\hline GW-068 & $3 / 18 / 1987$ & $3 / 12 / 1990$ & WL & 0 & Yes & TRICHLOROETHYLENE (TCE) & N/A & Biennial \\
\hline GW-069 & 6/22/1990 & $8 / 5 / 2002$ & WL & 6 & No & VINYL CHLORIDE & $\mathrm{PI}$ & Annual \\
\hline GW-071 & $3 / 17 / 1987$ & $8 / 5 / 2004$ & WL & 4 & Yes & TETRACHLOROETHYLENE(PCE) & NT & SemiAnnual \\
\hline GW-072 & $3 / 17 / 1987$ & $8 / 6 / 2002$ & WL & 2 & No & TETRACHLOROETHYLENE(PCE) & N/A & Remove \\
\hline GW-077 & $6 / 13 / 1990$ & $8 / 12 / 2004$ & WL & 15 & No & VINYL CHLORIDE & ND & Biennial \\
\hline GW-078 & $6 / 13 / 1990$ & $8 / 12 / 2004$ & WL & 15 & No & VINYL CHLORIDE & ND & Biennial \\
\hline GW-079 & $5 / 31 / 1990$ & $8 / 12 / 2004$ & WL & 3 & No & URANIUM & N/A & Biennial \\
\hline GW-080 & $5 / 31 / 1990$ & $8 / 12 / 2004$ & WL & 17 & No & VINYL CHLORIDE & ND & Biennial \\
\hline GW-082 & $3 / 23 / 1987$ & $8 / 5 / 2004$ & WL & 13 & Yes & VINYL CHLORIDE & $\mathrm{I}$ & SemiAnnual \\
\hline GW-083 & $6 / 20 / 1990$ & 9/2/1992 & WL & 0 & No & VINYL CHLORIDE & N/A & Biennial \\
\hline GW-089 & $6 / 19 / 1990$ & 8/13/1992 & WL & 0 & Yes & VINYL CHLORIDE & N/A & Biennial \\
\hline GW-091 & $3 / 14 / 2002$ & $8 / 8 / 2002$ & WL & 2 & No & URANIUM & N/A & Annual \\
\hline GW-094 & $3 / 17 / 1987$ & $2 / 15 / 1990$ & WL & 0 & No & URANIUM & N/A & Remove \\
\hline GW-095 & $3 / 17 / 1987$ & $8 / 27 / 1996$ & WL & 2 & No & TRICHLOROETHYLENE (TCE) & $\mathrm{N} / \mathrm{A}$ & Remove \\
\hline GW-126 & $3 / 26 / 1987$ & $3 / 2 / 1999$ & WL & 1 & Yes & TRICHLOROETHYLENE (TCE) & N/A & Biennial \\
\hline GW-237 & 4/15/1988 & 9/20/2004 & WL & 0 & No & URANIUM & N/A & Biennial \\
\hline
\end{tabular}

Notes:

Well sample dates taken from $\mathrm{Y}-12$ Analytical Database.

2. $\mathrm{WL}=$ Groundwater Monitoring Well; $\mathrm{SP}=\mathrm{Spring}$

3. Trend = Insufficient Data (N/A), Decreasing (D), Probably Decreasing (PD), Stable (S), Probably Increasing (PI),

and Increasing (I), No Trend (NT); and non-detect for all sample events (ND).

5. Priority Constituent is the $\mathrm{COC}$ with the highest average concentration from the full dataset normalized by the screening level. (e.g. MAX [Ave Conc./Screening Level]).

Priority constituent indicated for wells where average concentrations do not exceed screening levels may be background level.

6. Remove $=$ Do not sample; SemiAnnual $=$ once every 6 months; Annual $=$ once per year; Biennial $=$ once every two years 
Bear Creek Hydrogeologic Regim

-12 National Security Comp

Oak Ridge, Tennessee

\begin{tabular}{|c|c|c|c|c|c|c|c|c|}
\hline $\begin{array}{c}\text { Location } \\
\text { Name }\end{array}$ & $\begin{array}{c}\text { Earliest } \\
\text { Sample Date }\end{array}$ & $\begin{array}{l}\text { Most Recent } \\
\text { Sample Date }\end{array}$ & $\begin{array}{c}\text { Location } \\
\text { Type }\end{array}$ & $\begin{array}{c}\text { Number of } \\
\text { Samples } \\
1996-2005 \\
\end{array}$ & $\begin{array}{c}\text { Average } \\
\text { Concentration } \\
\text { Exceeds Screening } \\
\text { Level } \\
\end{array}$ & Historic Priority Constituent & $\begin{array}{c}\text { Trend for } \\
\text { Priority } \\
\text { Constituent } \\
1996-2005 \\
\end{array}$ & $\begin{array}{l}\text { Preliminary } \\
\text { Frequency }\end{array}$ \\
\hline GW-242 & $11 / 12 / 1987$ & $3 / 8 / 1999$ & WL & 3 & Yes & VINYL CHLORIDE & N/A & Annual \\
\hline GW-248 & $2 / 10 / 1988$ & $8 / 8 / 1995$ & WL & 0 & No & URANIUM & N/A & Remove \\
\hline GW-249 & $3 / 13 / 1989$ & $2 / 16 / 1990$ & $\mathrm{WL}$ & 0 & No & NITRATE & N/A & Remove \\
\hline GW-250 & 11/9/1987 & 2/16/1990 & WL & 0 & No & URANIUM & N/A & Remove \\
\hline GW-257 & $5 / 9 / 1988$ & $8 / 16 / 2004$ & WL & 2 & Yes & TETRACHLOROETHYLENE(PCE) & N/A & SemiAnnual \\
\hline GW-258 & 2/6/1988 & $3 / 5 / 1990$ & WL & 0 & Yes & TETRACHLOROETHYLENE(PCE) & N/A & Remove \\
\hline GW-259 & 2/9/1988 & $3 / 5 / 1990$ & WL & 0 & Yes & TETRACHLOROETHYLENE(PCE) & N/A & Annual \\
\hline GW-286 & $3 / 27 / 1987$ & $10 / 7 / 1993$ & WL & 0 & No & URANIUM & $\mathrm{N} / \mathrm{A}$ & Remove \\
\hline GW-287 & $3 / 27 / 1987$ & $8 / 21 / 2000$ & WL & 10 & No & TETRACHLOROETHYLENE(PCE) & $\mathrm{s}$ & Annual \\
\hline GW-288 & 2/11/1988 & 8/7/2002 & WL & 2 & Yes & TETRACHLOROETHYLENE(PCE) & N/A & SemiAnnual \\
\hline GW-289 & 2/18/1988 & 8/8/2002 & WL & 4 & Yes & TETRACHLOROETHYLENE(PCE) & $\mathrm{PI}$ & SemiAnnual \\
\hline GW-290 & 2/16/1988 & 8/7/1995 & WL & 0 & No & TETRACHLOROETHYLENE(PCE) & N/A & Biennial \\
\hline GW-291 & $2 / 16 / 1988$ & $8 / 7 / 2002$ & $\overline{\mathrm{WL}}$ & 4 & Yes & TETRACHLOROETHYLENE(PCE) & $\mathrm{s}$ & SemiAnnual \\
\hline GW-370 & 9/9/1988 & 8/8/1995 & WL & 0 & No & URANIUM & N/A & Biennial \\
\hline GW-372 & 9/9/1988 & 8/14/1997 & WL & 0 & No & NITRATE & N/A & Remove \\
\hline GW-375 & 9/12/1988 & $2 / 6 / 1990$ & WL & 0 & No & TRICHLOROETHYLENE (TCE) & N/A & Remove \\
\hline GW-621 & $2 / 9 / 1990$ & $7 / 13 / 2000$ & WL & 9 & No & NITRATE & PD & Annual \\
\hline GW-622 & $2 / 9 / 1990$ & 9/25/1992 & $\overline{\mathrm{WL}}$ & 0 & No & VINYL CHLORIDE & $\mathrm{N} / \mathrm{A}$ & Remove \\
\hline GW-623 & 6/27/1990 & 9/27/1992 & WL & 0 & Yes & TETRACHLOROETHYLENE(PCE) & N/A & Annual \\
\hline GW-624 & 6/28/1990 & 7/15/1998 & WL & 2 & Yes & VINYL CHLORIDE & N/A & SemiAnnual \\
\hline GW-626 & 2/14/1990 & $7 / 25 / 2002$ & WL & 4 & Yes & VINYL CHLORIDE & $\mathrm{PI}$ & SemiAnnual \\
\hline GW-627 & $2 / 13 / 1990$ & $8 / 4 / 2004$ & WL & 19 & Yes & TETRACHLOROETHYLENE(PCE) & i & SemiAnnual \\
\hline GW-629 & $6 / 30 / 1990$ & $7 / 16 / 1998$ & $\overline{\mathrm{WL}}$ & 4 & Yes & TETRACHLOROETHYLENE(PCE) & $\mathrm{s}$ & Biennial \\
\hline GW-639 & 12/6/1990 & $11 / 11 / 2004$ & WL & 8 & No & BENZENE & NT & Biennial \\
\hline GW-641 & 12/7/1990 & 10/14/1993 & WL & 0 & No & VINYL CHLORIDE & N/A & Remove \\
\hline GW-642 & $12 / 1 / 1990$ & $8 / 14 / 1997$ & WL & 4 & No & VINYL CHLORIDE & ND & Remove \\
\hline GW-651 & $3 / 23 / 1991$ & $10 / 10 / 1993$ & $\overline{\mathrm{WL}}$ & 0 & No & VINYL CHLORIDE & $\mathrm{N} / \mathrm{A}$ & Remove \\
\hline GW-652 & $3 / 23 / 1991$ & 9/19/1995 & WL & 0 & No & TRICHLOROETHYLENE (TCE) & N/A & Remove \\
\hline GW-653 & $3 / 23 / 1991$ & $8 / 4 / 2004$ & WL & 18 & No & TETRACHLOROETHYLENE(PCE) & 1 & Annual \\
\hline GW-654 & $3 / 9 / 1991$ & 12/9/1995 & WL & 0 & Yes & URANIUM & N/A & Biennial \\
\hline
\end{tabular}

Notes:

1. Well sample dates taken from $\mathrm{Y}-12$ Analytical Database.

2. $\mathrm{WL}=$ Groundwater Monitoring Well; $\mathrm{SP}=$ Spring.

3. Trend = Insufficient Data (N/A), Decreasing (D), Probably Decreasing (PD), Stable (S), Probably Increasing (PI),

and Increasing (I), No Trend (NT); and non-detect for all sample events (ND)

4. Number of samples between 1996-2005 is the count of analytical samples acquired at the location based on the BWXT Analytical Database (2005).

5. Priority Constituent is the $\mathrm{COC}$ with the highest average concentration from the full dataset normalized by the screening level. (e.g. MAX [Ave Conc./Screening Level]).

Priority constituent indicated for wells where average concentrations do not exceed screening levels may be background level.

6. Remove $=$ Do not sample; SemiAnnual $=$ sample once every 6 months; Annual $=$ sample once per year; Biennial $=$ sample once every two years. 
Bear Creek Hydrogeologic Regim

Y-12 National Security Complex

Oak Ridge, Tennessee

\begin{tabular}{|c|c|c|c|c|c|c|c|c|}
\hline $\begin{array}{c}\text { Location } \\
\text { Name }\end{array}$ & $\begin{array}{c}\text { Earliest } \\
\text { Sample Date }\end{array}$ & $\begin{array}{l}\text { Most Recent } \\
\text { Sample Date }\end{array}$ & $\begin{array}{c}\text { Location } \\
\text { Type }\end{array}$ & $\begin{array}{c}\text { Number of } \\
\text { Samples } \\
1996-2005\end{array}$ & $\begin{array}{c}\text { Average } \\
\text { Concentration } \\
\text { Exceeds Screening } \\
\text { Level }\end{array}$ & Historic Priority Constituent & $\begin{array}{c}\text { Trend for } \\
\text { Priority } \\
\text { Constituent } \\
1996-2005 \\
\end{array}$ & $\begin{array}{l}\text { Preliminary } \\
\text { Frequency }\end{array}$ \\
\hline GW-683 & $5 / 24 / 1991$ & $8 / 16 / 2004$ & WL & 17 & Yes & URANIUM & $\mathrm{D}$ & Annual \\
\hline GW-684 & $5 / 30 / 1991$ & $8 / 16 / 2004$ & WL & 18 & Yes & URANIUM & $\mathrm{s}$ & Annual \\
\hline GW-685 & 6/1/1991 & $3 / 14 / 2001$ & $\overline{W L}$ & 10 & No & NITRATE & $\mathrm{s}$ & Biennial \\
\hline GW-694 & 6/19/1991 & $7 / 17 / 2002$ & WL & 6 & Yes & URANIUM & NT & SemiAnnual \\
\hline GW-695 & 6/18/1991 & $7 / 21 / 2004$ & WL & 18 & No & TRICHLOROETHYLENE (TCE) & $\mathrm{s}$ & Annual \\
\hline GW-703 & 6/18/1991 & $7 / 21 / 2004$ & WL & 18 & Yes & TRICHLOROETHYLENE (TCE) & NT & Annual \\
\hline GW-704 & 6/20/1991 & $7 / 22 / 2004$ & WL & 18 & Yes & TRICHLOROETHYLENE (TCE) & $\mathrm{s}$ & Annual \\
\hline GW-706 & $6 / 20 / 1991$ & $7 / 22 / 2004$ & $\overline{W L}$ & 18 & Yes & URANIUM & $\mathrm{D}$ & Annual \\
\hline GW-710 & 12/5/1991 & $7 / 14 / 2003$ & WL & 0 & Yes & NITRATE & ND & Biennial \\
\hline GW-711 & 12/10/1991 & $7 / 14 / 2003$ & WL & 6 & No & VINYL CHLORIDE & ND & Annual \\
\hline GW-712 & 12/9/1991 & 7/7/2004 & WL & 18 & No & 1,2-DICHLOROETHANE & NT & Biennial \\
\hline GW-713 & $6 / 13 / 1992$ & 7/7/2004 & WL & 3 & No & NITRATE & $\mathrm{N} / \mathrm{A}$ & Annual \\
\hline GW-714 & 9/3/1992 & $7 / 7 / 2004$ & $\overline{W L}$ & 6 & No & NITRATE & PD & Annual \\
\hline GW-715 & 9/4/1992 & $1 / 5 / 2004$ & WL & 17 & No & URANIUM & $\mathrm{PI}$ & Annual \\
\hline SS-4 & 8/30/1990 & $7 / 20 / 2004$ & $\mathrm{SP}$ & 18 & Yes & URANIUM & D & Annual \\
\hline SS-5 & 8/30/1990 & $12 / 8 / 2004$ & SP & 19 & Yes & URANIUM & $\mathrm{s}$ & Annual \\
\hline SS-5_95KM & $5 / 23 / 1995$ & $10 / 26 / 1995$ & $\mathrm{SP}$ & 0 & No & VINYL CHLORIDE & N/A & Annual \\
\hline SS-6_6 & $5 / 23 / 1995$ & $3 / 2 / 2004$ & $\mathrm{SP}$ & 12 & No & URANIUM & NT & Biennial \\
\hline SS $-6 \bar{E}$ & 8/30/1990 & $8 / 1 / 2000$ & $\mathrm{SP}$ & 8 & Yes & URANIUM & $\mathrm{PI}$ & SemiAnnual \\
\hline SS-6W & $5 / 23 / 1995$ & $3 / 4 / 2003$ & SP & 5 & Yes & BENZENE & NT & Annual \\
\hline SS-7 & $5 / 23 / 1995$ & $8 / 19 / 2003$ & SP & 13 & No & URANIUM & I & Annual \\
\hline SS-8 & 8/30/1990 & $8 / 19 / 2003$ & $\mathrm{SP}$ & 2 & No & URANIUM & N/A & Biennial \\
\hline
\end{tabular}

Notes:

1. Well sample dates taken from $\mathrm{Y}$-12 Analytical Database.

2. $\mathrm{WL}=$ Groundwater Monitoring Well; $\mathrm{SP}=$ Spring.

3. Trend = Insufficient Data (N/A), Decreasing (D), Probably Decreasing (PD), Stable (S), Probably Increasing (PI),

and Increasing (I), No Trend (NT); and non-detect for all sample events (ND).

4. Number of samples between 1996-2005 is the count of analytical samples acquired at the location based on the BWXT Analytical Database (2005).

5. Priority Constituent is the $\mathrm{COC}$ with the highest average concentration from the full dataset normalized by the screening level. (e.g. MAX [Ave Conc./Screening Level]). Priority constituent indicated for wells where average concentrations do not exceed screening levels may be background level.

6. Remove = Do not sample; SemiAnnual = sample once every 6 months; Annual $=$ sample once per year; Biennial = sample once every two years. 
TABLE B.6

MAROS RESULTS EAST S-3 AREA

East Fork Poplar Creek Hydrogeologic Regime

Y-12 National Security Complex

\begin{tabular}{|c|c|c|c|c|c|c|c|c|}
\hline $\begin{array}{c}\text { Location } \\
\text { Name }\end{array}$ & $\begin{array}{c}\text { Earliest } \\
\text { Sample Date }\end{array}$ & $\begin{array}{l}\text { Most Recent } \\
\text { Sample Date }\end{array}$ & $\begin{array}{c}\text { Location } \\
\text { Type }\end{array}$ & $\begin{array}{c}\text { Number of } \\
\text { Samples } \\
1996-2005\end{array}$ & $\begin{array}{c}\text { Average } \\
\text { Concentration } \\
\text { Exceeds } \\
\text { Screening Level }\end{array}$ & Historic Priority Constituent & $\begin{array}{c}\text { Trend for } \\
\text { Priority } \\
\text { Constituent } \\
1996-2005 \\
\end{array}$ & $\begin{array}{c}\text { Preliminary } \\
\text { Frequency }\end{array}$ \\
\hline $55-1 \mathrm{~A}$ & $6 / 5 / 1996$ & $11 / 16 / 2004$ & WL & 3 & Yes & CHROMIUM III & $\mathrm{N} / \mathrm{A}$ & Annual \\
\hline $55-1 \mathrm{C}$ & 6/7/1996 & 6/7/1996 & WL & 1 & No & NITRATE & N/A & Biennial \\
\hline $55-2 B$ & 6/10/1996 & $11 / 29 / 2004$ & WL & 5 & Yes & TETRACHLOROETHYLENE(PCE) & $\mathrm{PI}$ & SemiAnnual \\
\hline $55-2 \mathrm{C}$ & 6/9/1996 & $11 / 18 / 2003$ & WL & 8 & Yes & TETRACHLOROETHYLENE(PCE) & $\mathrm{s}$ & Annual \\
\hline GW-105 & $1 / 24 / 1986$ & $10 / 2 / 2003$ & WL & 2 & Yes & NITRATE & $\mathrm{N} / \mathrm{A}$ & SemiAnnual \\
\hline GW-106 & $1 / 24 / 1986$ & $10 / 2 / 2003$ & $\overline{W L}$ & 2 & Yes & NITRATE & N/A & SemiAnnual \\
\hline GW-107 & 6/3/1986 & $1 / 19 / 1990$ & WL & 0 & Yes & CADMIUM & N/A & Remove \\
\hline GW-108 & $1 / 29 / 1986$ & $7 / 8 / 2004$ & WL & 6 & Yes & NITRATE & NT & SemiAnnual \\
\hline GW-109 & $1 / 29 / 1986$ & $10 / 6 / 2003$ & WL & 5 & Yes & NITRATE & NT & SemiAnnual \\
\hline GW-190 & $6 / 20 / 1990$ & $10 / 21 / 2003$ & WL & 2 & Yes & TETRACHLOROETHYLENE(PCE) & ND & Biennial \\
\hline GW-191 & $3 / 17 / 1986$ & $11 / 6 / 1996$ & WL & 3 & No & CADMIUM & ND & Biennial \\
\hline GW-192 & $3 / 17 / 1986$ & 10/17/2001 & WL & 13 & No & CADMIUM & NT & Annual \\
\hline GW-194 & 3/18/1986 & 11/7/1996 & WL & 1 & No & LEAD & N/A & Remove \\
\hline GW-195 & $3 / 18 / 1986$ & $11 / 7 / 1996$ & WL & 2 & Yes & LEAD & N/A & Annual \\
\hline GW-196 & $3 / 17 / 1986$ & 2/21/1990 & WL & 0 & Yes & LEAD & N/A & Biennial \\
\hline GW-197 & $3 / 18 / 1986$ & $2 / 3 / 1990$ & WL & 0 & Yes & LEAD & $\mathrm{N} / \mathrm{A}$ & Biennial \\
\hline GW-251 & 6/10/1986 & $10 / 21 / 2004$ & WL & 19 & Yes & TETRACHLOROETHYLENE(PCE) & $\mathrm{s}$ & Annual \\
\hline GW-252 & 6/11/1986 & $5 / 17 / 1995$ & WL & 0 & No & CADMIUM & N/A & Remove \\
\hline GW-253 & 6/10/1986 & $10 / 21 / 2003$ & WL & 14 & Yes & CADMIUM & 1 & SemiAnnual \\
\hline GW-255 & $6 / 5 / 1986$ & $5 / 17 / 1995$ & WL & 0 & No & LEAD & N/A & Biennial \\
\hline GW-261 & $10 / 28 / 1986$ & $5 / 16 / 1995$ & WL & 0 & No & LEAD & N/A & Remove \\
\hline GW-263 & $1 / 14 / 1987$ & $5 / 16 / 1995$ & WL & 0 & Yes & NITRATE & N/A & Biennial \\
\hline GW-265 & 10/28/1986 & $3 / 13 / 1990$ & WL & 0 & Yes & TETRACHLOROETHYLENE(PCE) & N/A & Biennial \\
\hline GW-268 & 10/28/1986 & 3/13/1990 & WL & 0 & No & LEAD & N/A & Biennial \\
\hline GW-269 & 10/28/1986 & $10 / 23 / 2003$ & WL & 2 & Yes & 1,1-DICHLOROETHENE & N/A & Annual \\
\hline
\end{tabular}

Notes:

Well sample dates taken from $\mathrm{Y}$-12 Analytical Database.

2. $\mathrm{WL}=$ Groundwater Monitoring Well; $\mathrm{SP}=$ Spring.

Trend = Insufficient Data (N/A), Decreasing (D), Probably Decreasing (PD), Stable (S), Probably Increasing (PI)

and Increasing (I), No Trend (NT); and non-detect for all sample events (ND).

(2005).

5. Priority Constituent is his CoC

6. Remove = Do not sample; SemiAnnual = sample once every 6 months; Annual = sample once per year; Biennial = sample once every two years. 
TABLE B.6

MAROS RESULTS EAST S-3 AREA

East Fork Poplar Creek Hydrogeologic Regime

Y-12 National Security Complex

Oak Ridge, Tennessee

\begin{tabular}{|c|c|c|c|c|c|c|c|c|}
\hline $\begin{array}{l}\text { Location } \\
\text { Name }\end{array}$ & $\begin{array}{c}\text { Earliest } \\
\text { Sample Date } \\
\end{array}$ & $\begin{array}{l}\text { Most Recent } \\
\text { Sample Date }\end{array}$ & $\begin{array}{c}\text { Location } \\
\text { Type } \\
\end{array}$ & $\begin{array}{c}\text { Number of } \\
\text { Samples } \\
1996-2005 \\
\end{array}$ & $\begin{array}{c}\text { Average } \\
\text { Concentration } \\
\text { Exceeds } \\
\text { Screening Level } \\
\end{array}$ & Historic Priority Constituent & $\begin{array}{c}\text { Trend for } \\
\text { Priority } \\
\text { Constituent } \\
1996-2005 \\
\end{array}$ & $\begin{array}{l}\text { Preliminary } \\
\text { Frequency }\end{array}$ \\
\hline GW-270 & $10 / 30 / 1986$ & $10 / 20 / 2003$ & $\overline{W L}$ & 2 & Yes & NITRATE & N/A & SemiAnnual \\
\hline GW-271 & 10/30/1986 & $10 / 20 / 2003$ & WL & 2 & No & CADMIUM & N/A & Annual \\
\hline GW-272 & $10 / 30 / 1986$ & $10 / 23 / 2003$ & WL & 2 & Yes & NITRATE & N/A & Annual \\
\hline GW-273 & 10/31/1986 & $10 / 21 / 2003$ & WL & 0 & Yes & NITRATE & N/A & Annual \\
\hline GW-274 & $11 / 4 / 1986$ & $10 / 22 / 2003$ & WL & 5 & Yes & NITRATE & $\mathrm{PD}$ & Annual \\
\hline GW-275 & $11 / 4 / 1986$ & $10 / 22 / 2003$ & $\overline{W L}$ & 5 & Yes & NITRATE & $\mathrm{s}$ & Annual \\
\hline GW-332 & $5 / 24 / 1989$ & 8/4/1998 & WL & 3 & Yes & TETRACHLOROETHYLENE(PCE) & N/A & Annual \\
\hline GW-334 & $5 / 24 / 1989$ & 4/13/1991 & WL & 0 & Yes & TRICHLOROETHYLENE (TCE) & N/A & Remove \\
\hline GW-335 & $6 / 6 / 1989$ & 4/10/1991 & WL & 0 & Yes & CHROMIUM III & N/A & Remove \\
\hline GW-336 & $5 / 25 / 1989$ & $11 / 17 / 2003$ & WL & 2 & Yes & TETRACHLOROETHYLENE(PCE) & N/A & Annual \\
\hline GW-337 & $5 / 25 / 1989$ & $11 / 17 / 2003$ & $\overline{W L}$ & 6 & Yes & TRICHLOROETHYLENE (TCE) & $\mathrm{s}$ & Annual \\
\hline GW-338 & 6/8/1989 & 8/20/1998 & WL & 4 & Yes & CADMIUM & ND & Biennial \\
\hline GW-349 & 9/6/1988 & $10 / 14 / 2002$ & WL & 2 & Yes & CADMIUM & N/A & Annual \\
\hline GW-350 & 9/6/1988 & $10 / 15 / 2002$ & WL & 2 & Yes & CADMIUM & N/A & Annual \\
\hline GW-505 & $12 / 29 / 1988$ & $10 / 6 / 2003$ & WL & 3 & Yes & CHROMIUM III & $\mathrm{N} / \mathrm{A}$ & Annual \\
\hline GW-508 & $12 / 11 / 1990$ & $3 / 2 / 1994$ & WL & 0 & Yes & BENZENE & $\mathrm{N} / \mathrm{A}$ & Remove \\
\hline GW-617 & $5 / 14 / 1990$ & 11/5/1997 & WL & 5 & No & CADMIUM & NT & Annual \\
\hline GW-618 & $5 / 14 / 1990$ & $10 / 22 / 2003$ & WL & 17 & Yes & CADMIUM & D & Annual \\
\hline GW-619 & $5 / 10 / 1990$ & 7/21/1998 & WL & 5 & Yes & TETRACHLOROETHYLENE(PCE) & $\mathrm{s}$ & Annual \\
\hline GW-620 & $5 / 10 / 1990$ & $10 / 21 / 2004$ & WL & 20 & Yes & TETRACHLOROETHYLENE(PCE) & $\mathrm{D}$ & Annual \\
\hline GW-631 & $3 / 11 / 1991$ & $10 / 7 / 2003$ & WL & 2 & Yes & TETRACHLOROETHYLENE(PCE) & ND & Annual \\
\hline GW-633 & 3/11/1991 & $10 / 26 / 2004$ & WL & 7 & Yes & NITRATE & D & Annual \\
\hline GW-778 & $5 / 20 / 1994$ & $5 / 18 / 1995$ & WL & 0 & No & LEAD & N/A & Remove \\
\hline
\end{tabular}

Notes:

Well sample dates taken from $\mathrm{Y}-12$ Analytical Database.

2. $W L=$ Groundwater Monitoring Well; $S P=$ Spring.

Pabably Decreasing (PD), Stable (S), Probably Increasing (PI)

and Increasing (I), No Trend (NT), and non-delect for all sample events (ND).

on the BWXT Analytical Database (2005).

5. Priority Constlue

6. Remove $=$ Do not sample; SemiAnnual = sample once every 6 months; Annual = sample once per year; Biennial = sample once every two years 
TABLE B.7

East Fork Poplar Creek Hydrogeologic Regi

Oak Ridge, Tennessee

\begin{tabular}{|c|c|c|c|c|c|c|c|c|}
\hline Location Name & $\begin{array}{c}\text { Earliest Sample } \\
\text { Date }\end{array}$ & $\begin{array}{l}\text { Most Recent } \\
\text { Sample Date }\end{array}$ & Location Type & \begin{tabular}{|c} 
Number of \\
Samples 1996- \\
2005 \\
\end{tabular} & \begin{tabular}{|c|} 
Average \\
Concentration \\
Exceeds \\
Screening \\
Level \\
\end{tabular} & Historic Priority Constituent & \begin{tabular}{|c|} 
Trend for Priority \\
Constituent 1996. \\
2005
\end{tabular} & $\begin{array}{l}\text { Preliminary } \\
\text { Frequency }\end{array}$ \\
\hline $55-1 \mathrm{~B}$ & $4 / 17 / 2002$ & $10 / 14 / 2002$ & $\overline{W L}$ & 2 & Yes & CHROMIUM III & $\mathrm{N} / \mathrm{A}$ & Annual \\
\hline $55-6 \mathrm{~A}$ & 6/5/1996 & $11 / 16 / 2004$ & WL & 3 & Yes & LEAD & N/A & Annual \\
\hline $56-2 A$ & $3 / 23 / 1998$ & $11 / 18 / 2004$ & WL & 3 & Yes & TETRACHLOROETHYLENE(PCE) & N/A & SemiAnnual \\
\hline $56-2 B$ & $3 / 23 / 1998$ & $11 / 18 / 2004$ & WL & 3 & Yes & TETRACHLOROETHYLENE(PCE) & N/A & SemiAnnual \\
\hline $56-2 \mathrm{C}$ & $3 / 24 / 1998$ & $11 / 18 / 2003$ & WL & 5 & Yes & TETRACHLOROETHYLENE(PCE) & $\mathrm{s}$ & Annual \\
\hline $59-1 \mathrm{~A}$ & $3 / 17 / 1998$ & $10 / 30 / 2003$ & WL & 3 & No & GROSS ALPHA ACTIVITY & $\mathrm{N} / \mathrm{A}$ & Remove \\
\hline $59-1 \mathrm{~B}$ & $3 / 18 / 1998$ & $4 / 28 / 2004$ & WL & 4 & Yes & CHROMIUM III & PD & Annual \\
\hline $59-1 \mathrm{C}$ & 3/18/1998 & $10 / 30 / 2003$ & WL & 3 & No & TRICHLOROETHYLENE (TCE) & N/A & Annual \\
\hline $60-1 \mathrm{~B}$ & $6 / 11 / 2003$ & $10 / 13 / 2003$ & WL & 2 & No & BENZENE & N/A & Remove \\
\hline 9201-3C-4SP & $5 / 18 / 2004$ & $10 / 27 / 2004$ & $\mathrm{SP}$ & 2 & Yes & TETRACHLOROETHYLENE(PCE) & N/A & SemiAnnual \\
\hline GW-193 & $6 / 18 / 1990$ & $7 / 13 / 2004$ & WL & 17 & Yes & BENZENE & $\mathrm{D}$ & Biennial \\
\hline GW-204 & 6/19/1990 & $10 / 25 / 2004$ & WL & 13 & Yes & BENZENE & PD & Biennial \\
\hline GW-218 & 6/7/1996 & $10 / 30 / 2000$ & WL & 4 & No & CHROMIUM III & $\mathrm{s}$ & Annual \\
\hline GW-219 & 9/4/1998 & $11 / 11 / 2004$ & WL & 12 & Yes & URANIUM & D & Annual \\
\hline GW-605 & 8/26/1991 & $7 / 12 / 2004$ & WL & 20 & Yes & CARBON TETRACHLORIDE & $\mathrm{D}$ & Annual \\
\hline GW-606 & $8 / 26 / 1991$ & $7 / 12 / 2004$ & WL & 20 & Yes & CARBON TETRACHLORIDE & $\mathrm{D}$ & Annual \\
\hline GW-656 & 3/7/1991 & $11 / 12 / 2001$ & WL & 4 & Yes & TRICHLOROETHYLENE (TCE) & $\mathrm{s}$ & Annual \\
\hline GW-657 & $3 / 6 / 1991$ & 11/15/1993 & WL & 0 & No & GROSS ALPHA ACTIVITY & $\mathrm{N} / \mathrm{A}$ & Remove \\
\hline GW-686 & 3/23/1998 & $11 / 14 / 2002$ & WL & 2 & Yes & LEAD & N/A & Annual \\
\hline GW-690 & $6 / 8 / 1996$ & $11 / 18 / 2003$ & WL & 5 & Yes & TETRACHLOROETHYLENE(PCE) & $\mathrm{s}$ & Annual \\
\hline
\end{tabular}

\section{Notes:}

1. Well sample dates taken from $\mathrm{Y}-12$ Analytical Database.

2. $\mathrm{WL}=$ Groundwater Monitoring Well; $\mathrm{SP}=$ Spring

3. Trend = Insufficient Data (N/A), Decreasing (D), Probably Decreasing (PD), Stable (S), Probably Increasing (PI),

and Increasing (I), No Trend (NT); and non-detect for all sample events (ND)

(2005)

5. Priority Constituent is the $\mathrm{COC}$ with the highest average concentration from the full dataset normalized by the screening level. (e.g. MAX [Ave Conc./Screening Level]). Priority constituent indicated for wells where average concentrations do not exceed screening levels may be background level.

6. Remove $=$ Do not sample; SemiAnnual $=$ sample once every 6 months; Annual $=$ sample once per year; Biennial $=$ sample once every two years. 
TABLE B.7

East Fork Poplar Creek Hydrogeologic Regime

Y-12 National Security Complex

Oak Ridge, Tennessee

\begin{tabular}{|c|c|c|c|c|c|c|c|c|}
\hline Location Name & $\begin{array}{c}\text { Earliest Sample } \\
\text { Date }\end{array}$ & $\begin{array}{l}\text { Most Recent } \\
\text { Sample Date }\end{array}$ & Location Type & \begin{tabular}{|c|} 
Number of \\
Samples 1996- \\
2005 \\
\end{tabular} & $\begin{array}{c}\text { Average } \\
\text { Concentration } \\
\text { Exceeds } \\
\text { Screening } \\
\text { Level } \\
\end{array}$ & Historic Priority Constituent & \begin{tabular}{|c|} 
Trend for Priority \\
Constituent 1996- \\
2005
\end{tabular} & $\begin{array}{c}\text { Preliminary } \\
\text { Frequency }\end{array}$ \\
\hline GW-691 & $6 / 8 / 1996$ & $11 / 17 / 2004$ & WL & 3 & Yes & TETRACHLOROETHYLENE(PCE) & $\mathrm{N} / \mathrm{A}$ & SemiAnnual \\
\hline GW-692 & $3 / 1 / 1996$ & $11 / 17 / 2004$ & WL & 3 & No & TETRACHLOROETHYLENE(PCE) & N/A & Annual \\
\hline GW-698 & 6/9/1996 & $11 / 3 / 2004$ & WL & 11 & Yes & TRICHLOROETHYLENE (TCE) & NT & SemiAnnual \\
\hline GW-700 & 6/8/1996 & $11 / 10 / 2003$ & WL & 5 & Yes & TETRACHLOROETHYLENE(PCE) & PD & Annual \\
\hline GW-759 & 7/26/1992 & 11/14/1995 & WL & 0 & No & GROSS ALPHA ACTIVITY & N/A & Biennial \\
\hline GW-760 & $7 / 26 / 1992$ & $4 / 27 / 2004$ & WL & 3 & No & CHROMIUM III & N/A & Biennial \\
\hline GW-761 & 7/26/1992 & $10 / 29 / 2003$ & WL & 2 & No & CHROMIUM III & N/A & Biennial \\
\hline GW-765 & 7/28/1992 & $4 / 27 / 2004$ & WL & 2 & No & GROSS ALPHA ACTIVITY & N/A & Biennial \\
\hline GW-769 & $1 / 27 / 1993$ & $10 / 28 / 2004$ & WL & 19 & Yes & CARBON TETRACHLORIDE & I & SemiAnnual \\
\hline GW-770 & $1 / 27 / 1993$ & $10 / 28 / 2004$ & WL & 19 & No & CARBON TETRACHLORIDE & i & SemiAnnual \\
\hline GW-779 & $6 / 9 / 1994$ & $11 / 7 / 1996$ & WL & 1 & No & GROSS ALPHA ACTIVITY & $\mathrm{N} / \mathrm{A}$ & Remove \\
\hline GW-780 & 6/9/1994 & $11 / 7 / 1996$ & WL & 3 & No & CHROMIUM III & N/A & Biennial \\
\hline GW-781 & 6/15/1994 & $10 / 26 / 1999$ & WL & 9 & Yes & TETRACHLOROETHYLENE(PCE) & $\mathrm{PI}$ & Annual \\
\hline GW-782 & 6/16/1994 & $10 / 26 / 2004$ & WL & 20 & Yes & TETRACHLOROETHYLENE(PCE) & D & Annual \\
\hline GW-783 & 6/16/1994 & $4 / 27 / 2004$ & WL & 9 & Yes & TETRACHLOROETHYLENE(PCE) & $\mathrm{s}$ & Annual \\
\hline GW-786 & $6 / 8 / 1994$ & $10 / 28 / 2003$ & $\overline{W L}$ & 0 & No & GROSS ALPHA ACTIVITY & N/A & Remove \\
\hline GW-787 & $6 / 8 / 1994$ & $10 / 28 / 2003$ & WL & 5 & No & CHROMIUM III & NT & Annual \\
\hline GW-788 & 6/10/1994 & $10 / 20 / 1999$ & WL & 4 & No & GROSS ALPHA ACTIVITY & $\mathrm{D}$ & Remove \\
\hline GW-789 & $6 / 15 / 1994$ & $10 / 15 / 2001$ & WL & 9 & No & GROSS ALPHA ACTIVITY & NT & Annual \\
\hline GW-791 & 6/7/1994 & $10 / 25 / 2004$ & WL & 19 & Yes & TETRACHLOROETHYLENE(PCE) & $\mathrm{s}$ & Annual \\
\hline GW-792 & $6 / 7 / 1994$ & $11 / 28 / 1999$ & WL & 9 & Yes & TETRACHLOROETHYLENE(PCE) & $\mathrm{s}$ & Annual \\
\hline GW-819 & 3/26/1998 & $3 / 26 / 1998$ & WL & 1 & No & GROSS ALPHA ACTIVITY & N/A & Remove \\
\hline GW-820 & $3 / 26 / 1998$ & $10 / 13 / 2003$ & WL & 6 & Yes & TETRACHLOROETHYLENE(PCE) & NT & SemiAnnual \\
\hline UEFPC-SP17 & 3/25/1996 & $11 / 5 / 2003$ & $\mathrm{SP}$ & 3 & Yes & NITRATE & N/A & Annual \\
\hline
\end{tabular}

Notes:

Well sample dates taken from $\mathrm{Y}$-12 Analytical Database.

2. $W L=$ Groundwater Monitoring Well; $S P=S$ Spring .

3. Trend = Insufficient Data (N/A), Decreasing (D), Probably Decreasing (PD), Stable (S), Probably Increasing (PI),

and Increasing (I), No Trend (NT); and non-detect for all sample events (ND).

4. Number of samples between 1996-2005 is the count of analytical samples acquired at the location based on the BWXT Analytical Database (2005).

5. Priority Constituent is the $\mathrm{COC}$ with the highest average concentration from the full dataset normalized by the screening level. (e.g. MAX [Ave Conc./Screening Level]).

Priority constituent indicated for wells where average concentrations do not exceed screening levels may be background level.

6. Remove $=$ Do not sample; SemiAnnual $=$ sample once every 6 months; Annual = sample once per year; Biennial = sample once every two years. 
TABLE B.8

East Fork Poplar Creek Hydrogeologic Regim

Y-12 National Security Complex

Oak Ridge, Tennessee

\begin{tabular}{|c|c|c|c|c|c|c|c|c|}
\hline $\begin{array}{l}\text { Location } \\
\text { Name }\end{array}$ & \begin{tabular}{|c|} 
Earliest \\
Sample Date
\end{tabular} & $\begin{array}{l}\text { Most Recent } \\
\text { Sample Date }\end{array}$ & $\begin{array}{c}\text { Location } \\
\text { Type }\end{array}$ & \begin{tabular}{|c} 
Number of \\
Samples 1996- \\
2005 \\
\end{tabular} & $\begin{array}{c}\text { Average } \\
\text { Concentration } \\
\text { Exceeds } \\
\text { Screening Level }\end{array}$ & Historic Priority Constituent & \begin{tabular}{|c} 
Trend for Priority \\
Constituent 1996- \\
2005
\end{tabular} & $\begin{array}{l}\text { Preliminary } \\
\text { Frequency }\end{array}$ \\
\hline GW-183 & $6 / 19 / 1990$ & $11 / 16 / 1993$ & $\overline{W L}$ & 0 & No & BENZENE & $\mathrm{N} / \mathrm{A}$ & Biennial \\
\hline GW-199 & $3 / 17 / 1986$ & 11/16/1995 & WL & 0 & No & LEAD & N/A & Biennial \\
\hline GW-200 & 3/11/1986 & 4/30/1988 & WL & 0 & No & LEAD & N/A & Remove \\
\hline GW-202 & 3/11/1986 & 4/30/1988 & WL & 0 & No & LEAD & ND & Biennial \\
\hline GW-281 & $5 / 4 / 1989$ & $5 / 10 / 2004$ & WL & 0 & No & LEAD & N/A & Remove \\
\hline GW-282 & $5 / 8 / 1989$ & $7 / 29 / 1992$ & $\overline{W L}$ & 0 & No & LEAD & $\mathrm{N} / \mathrm{A}$ & Remove \\
\hline GW-283 & $5 / 4 / 1989$ & 10/14/1993 & WL & 0 & No & LEAD & N/A & Remove \\
\hline GW-658 & 3/9/1991 & $5 / 10 / 2004$ & WL & 6 & Yes & BENZENE & NT & SemiAnnual \\
\hline GW-659 & 3/8/1991 & $7 / 28 / 1998$ & WL & 2 & No & BENZENE & N/A & Biennial \\
\hline GW-751 & 7/30/1992 & 11/18/1996 & WL & 3 & No & BENZENE & ND & Remove \\
\hline GW-752 & $7 / 30 / 1992$ & $11 / 18 / 1996$ & WL & 3 & No & BENZENE & ND & Remove \\
\hline GW-753 & $7 / 31 / 1992$ & 7/29/1998 & WL & 5 & Yes & TRICHLOROETHYLENE (TCE) & NT & Biennial \\
\hline GW-754 & 7/31/1992 & 7/28/1998 & WL & 5 & Yes & TRICHLOROETHYLENE (TCE) & NT & Biennial \\
\hline GW-756 & $7 / 31 / 1992$ & 7/27/1998 & WL & 0 & No & LEAD & N/A & Remove \\
\hline GW-762 & $8 / 11 / 1992$ & $8 / 5 / 2004$ & WL & 12 & Yes & TETRACHLOROETHYLENE(PCE) & 1 & SemiAnnual \\
\hline GW-763 & $8 / 11 / 1992$ & $11 / 1 / 2004$ & $\overline{W L}$ & 20 & Yes & TETRACHLOROETHYLENE(PCE) & NT & Annual \\
\hline GW-766 & 2/1/1993 & 10/9/1996 & WL & 3 & No & TETRACHLOROETHYLENE(PCE) & ND & Biennial \\
\hline GW-767 & 2/2/1993 & 10/9/1996 & WL & 3 & No & TETRACHLOROETHYLENE(PCE) & ND & Biennial \\
\hline GW-773 & 2/3/1993 & 11/18/1995 & WL & 0 & No & BENZENE & ND & Remove \\
\hline GW-774 & 2/4/1993 & 11/18/1995 & WL & 0 & No & LEAD & N/A & Remove \\
\hline GW-775 & $2 / 4 / 1993$ & $4 / 28 / 2004$ & $\mathrm{WL}$ & 11 & No & TRICHLOROETHYLENE (TCE) & NT & Annual \\
\hline GW-776 & 2/4/1993 & 10/31/2002 & WL & 11 & No & TRICHLOROETHYLENE (TCE) & $\mathrm{s}$ & Annual \\
\hline GW-802 & 6/22/1998 & $5 / 10 / 2004$ & WL & 4 & No & BENZENE & ND & Biennial \\
\hline NHPCEMSP & $3 / 11 / 1996$ & 9/3/1996 & SP & 2 & No & BENZENE & N/A & Biennial \\
\hline
\end{tabular}

Notes:

1. Well sample dates taken from $Y$-12 Analytical Database.

2. $\mathrm{WL}=$ Groundwater Monitoring Well; $\mathrm{SP}=$ Spring

3. Trend = Insufficient Data (N/A), Decreasing (D), Probably Decreasing (PD), Stable (S), Probably Increasing (PI), and Increasing (I), No Trend (NT); and non-detect for all sample events (ND)

4. Number of samples between 1996-2005 is the count of analytical samples acquired at the location based on the BWXT Analytical Database (2005).

5. Priority Constituent is the $\mathrm{COC}$ with the highest average concentration from the full dataset normalized by the screening level. (e.g. MAX [Ave Conc./Screening Level]).

Priority constituent indicated for wells where average concentrations do not exceed screening levels may be background level.

6. Remove $=$ Do not sample; SemiAnnual $=$ sample once every 6 months; Annual $=$ sample once per year; Biennial = sample once every two years. 
TABLE B.8

East Fork Poplar Creek Hydrogeologic Regim

Y-12 National Security Complex

Oak Ridge, Tennessee

\begin{tabular}{|c|c|c|c|c|c|c|c|c|}
\hline $\begin{array}{l}\text { Location } \\
\text { Name }\end{array}$ & \begin{tabular}{|c|} 
Earliest \\
Sample Date
\end{tabular} & $\begin{array}{l}\text { Most Recent } \\
\text { Sample Date }\end{array}$ & $\begin{array}{c}\text { Location } \\
\text { Type }\end{array}$ & \begin{tabular}{|c} 
Number of \\
Samples 1996- \\
2005 \\
\end{tabular} & $\begin{array}{c}\text { Average } \\
\text { Concentration } \\
\text { Exceeds } \\
\text { Screening Level }\end{array}$ & Historic Priority Constituent & \begin{tabular}{|c} 
Trend for Priority \\
Constituent 1996- \\
2005
\end{tabular} & $\begin{array}{l}\text { Preliminary } \\
\text { Frequency }\end{array}$ \\
\hline GW-183 & $6 / 19 / 1990$ & $11 / 16 / 1993$ & $\overline{W L}$ & 0 & No & BENZENE & $\mathrm{N} / \mathrm{A}$ & Biennial \\
\hline GW-199 & $3 / 17 / 1986$ & 11/16/1995 & WL & 0 & No & LEAD & N/A & Biennial \\
\hline GW-200 & 3/11/1986 & 4/30/1988 & WL & 0 & No & LEAD & N/A & Remove \\
\hline GW-202 & 3/11/1986 & 4/30/1988 & WL & 0 & No & LEAD & ND & Biennial \\
\hline GW-281 & $5 / 4 / 1989$ & $5 / 10 / 2004$ & WL & 0 & No & LEAD & N/A & Remove \\
\hline GW-282 & $5 / 8 / 1989$ & $7 / 29 / 1992$ & $\overline{W L}$ & 0 & No & LEAD & $\mathrm{N} / \mathrm{A}$ & Remove \\
\hline GW-283 & $5 / 4 / 1989$ & 10/14/1993 & WL & 0 & No & LEAD & N/A & Remove \\
\hline GW-658 & 3/9/1991 & $5 / 10 / 2004$ & WL & 6 & Yes & BENZENE & NT & SemiAnnual \\
\hline GW-659 & 3/8/1991 & $7 / 28 / 1998$ & WL & 2 & No & BENZENE & N/A & Biennial \\
\hline GW-751 & 7/30/1992 & 11/18/1996 & WL & 3 & No & BENZENE & ND & Remove \\
\hline GW-752 & $7 / 30 / 1992$ & $11 / 18 / 1996$ & WL & 3 & No & BENZENE & ND & Remove \\
\hline GW-753 & $7 / 31 / 1992$ & 7/29/1998 & WL & 5 & Yes & TRICHLOROETHYLENE (TCE) & NT & Biennial \\
\hline GW-754 & 7/31/1992 & 7/28/1998 & WL & 5 & Yes & TRICHLOROETHYLENE (TCE) & NT & Biennial \\
\hline GW-756 & $7 / 31 / 1992$ & 7/27/1998 & WL & 0 & No & LEAD & N/A & Remove \\
\hline GW-762 & $8 / 11 / 1992$ & $8 / 5 / 2004$ & WL & 12 & Yes & TETRACHLOROETHYLENE(PCE) & 1 & SemiAnnual \\
\hline GW-763 & $8 / 11 / 1992$ & $11 / 1 / 2004$ & $\overline{W L}$ & 20 & Yes & TETRACHLOROETHYLENE(PCE) & NT & Annual \\
\hline GW-766 & 2/1/1993 & 10/9/1996 & WL & 3 & No & TETRACHLOROETHYLENE(PCE) & ND & Biennial \\
\hline GW-767 & 2/2/1993 & 10/9/1996 & WL & 3 & No & TETRACHLOROETHYLENE(PCE) & ND & Biennial \\
\hline GW-773 & 2/3/1993 & 11/18/1995 & WL & 0 & No & BENZENE & ND & Remove \\
\hline GW-774 & 2/4/1993 & 11/18/1995 & WL & 0 & No & LEAD & N/A & Remove \\
\hline GW-775 & $2 / 4 / 1993$ & $4 / 28 / 2004$ & $\mathrm{WL}$ & 11 & No & TRICHLOROETHYLENE (TCE) & NT & Annual \\
\hline GW-776 & 2/4/1993 & 10/31/2002 & WL & 11 & No & TRICHLOROETHYLENE (TCE) & $\mathrm{s}$ & Annual \\
\hline GW-802 & 6/22/1998 & $5 / 10 / 2004$ & WL & 4 & No & BENZENE & ND & Biennial \\
\hline NHPCEMSP & $3 / 11 / 1996$ & 9/3/1996 & SP & 2 & No & BENZENE & N/A & Biennial \\
\hline
\end{tabular}

Notes:

1. Well sample dates taken from $Y$-12 Analytical Database.

2. $\mathrm{WL}=$ Groundwater Monitoring Well; $\mathrm{SP}=$ Spring

3. Trend = Insufficient Data (N/A), Decreasing (D), Probably Decreasing (PD), Stable (S), Probably Increasing (PI), and Increasing (I), No Trend (NT); and non-detect for all sample events (ND)

4. Number of samples between 1996-2005 is the count of analytical samples acquired at the location based on the BWXT Analytical Database (2005).

5. Priority Constituent is the $\mathrm{COC}$ with the highest average concentration from the full dataset normalized by the screening level. (e.g. MAX [Ave Conc./Screening Level]).

Priority constituent indicated for wells where average concentrations do not exceed screening levels may be background level.

6. Remove $=$ Do not sample; SemiAnnual $=$ sample once every 6 months; Annual $=$ sample once per year; Biennial = sample once every two years. 
TABLE B.9

MAROS RESULTS EAST Y-12 AREA

East Fork Poplar Creek Hydrogeologic Regime

Y-12 National Security Complex

Oak Ridge, Tennessee

\begin{tabular}{|c|c|c|c|c|c|c|c|c|}
\hline $\begin{array}{c}\text { Location } \\
\text { Name }\end{array}$ & $\begin{array}{c}\text { Earliest } \\
\text { Sample Date }\end{array}$ & $\begin{array}{l}\text { Most Recent } \\
\text { Sample Date }\end{array}$ & $\begin{array}{c}\text { Location } \\
\text { Type }\end{array}$ & $\begin{array}{c}\text { Number of } \\
\text { Samples } \\
1996-2005 \\
\end{array}$ & $\begin{array}{c}\text { Average } \\
\begin{array}{c}\text { Concentration } \\
\text { Exceeds Screening } \\
\text { Level }\end{array} \\
\end{array}$ & Historic Priority Constituent & \begin{tabular}{|c|} 
Trend for Priority \\
Constituent 1996 \\
2005
\end{tabular} & $\begin{array}{l}\text { Preliminary } \\
\text { Frequency }\end{array}$ \\
\hline GW-148 & $2 / 23 / 1986$ & $11 / 9 / 1999$ & WL & 10 & No & LEAD & NT & Biennial \\
\hline GW-149 & $2 / 23 / 1986$ & $11 / 14 / 1996$ & WL & 3 & No & GROSS BETA ACTIVITY & N/A & Biennial \\
\hline GW-150 & 2/20/1986 & $3 / 8 / 1988$ & WL & 0 & Yes & LEAD & $\mathrm{N} / \mathrm{A}$ & Remove \\
\hline GW-151 & $2 / 20 / 1986$ & $8 / 10 / 2004$ & WL & 19 & Yes & CARBON TETRACHLORIDE & 1 & SemiAnnual \\
\hline GW-152 & $2 / 25 / 1986$ & $2 / 28 / 1990$ & WL & 0 & Yes & LEAD & N/A & Remove \\
\hline GW-153 & $2 / 24 / 1986$ & $11 / 1 / 2004$ & WL & 20 & Yes & CARBON TETRACHLORIDE & $\frac{S}{S}$ & Annual \\
\hline GW-154 & $2 / 23 / 1986$ & $8 / 11 / 2004$ & WL & 20 & Yes & GROSS ALPHA ACTIVITY & I & SemiAnnual \\
\hline GW-167 & $5 / 30 / 1990$ & 2/14/1996 & WL & 0 & Yes & LEAD & $\mathrm{N} / \mathrm{A}$ & Annual \\
\hline GW-169 & $3 / 1 / 1991$ & $10 / 25 / 2004$ & WL & 13 & Yes & LEAD & NT & Biennial \\
\hline GW-170 & $6 / 4 / 1990$ & $10 / 25 / 2004$ & WL & 27 & Yes & CARBON TETRACHLORIDE & $\mathrm{D}$ & $\begin{array}{l}\text { Annual } \\
\text { Antial }\end{array}$ \\
\hline GW-171 & 9/27/1994 & $8 / 9 / 2004$ & WL & 16 & No & GROSS BETA ACTIVITY & NT & Biennial \\
\hline GW-172 & $6 / 4 / 1990$ & $8 / 9 / 2004$ & WL & 15 & No & GROSS BETA ACTIVITY & NT & Annual \\
\hline GW-207 & $6 / 1 / 1990$ & $11 / 9 / 2004$ & WL & 16 & No & GROSS ALPHA ACTIVITY & s & Biennial \\
\hline GW-208 & $6 / 1 / 1990$ & $11 / 9 / 2004$ & WL & 16 & No & GROSS ALPHA ACTIVITY & $\mathrm{s}$ & Biennial \\
\hline GW-220 & $2 / 20 / 1986$ & $11 / 15 / 2004$ & WL & 22 & Yes & CARBON TETRACHLORIDE & I & SemiAnnual \\
\hline \begin{tabular}{|l|l|l|l|} 
GW-222 \\
\end{tabular} & $2 / 23 / 1986$ & $11 / 30 / 2004$ & WL & 8 & Yes & TETRACHLOROETHYLENE(PCE) & $\mathrm{s}$ & Annual \\
\hline GW-223 & $2 / 25 / 1986$ & $8 / 10 / 2004$ & WL & 15 & Yes & TETRACHLOROETHYLENE(PCE) & $\mathrm{D}$ & Annual \\
\hline GW-230 & $6 / 6 / 1990$ & $8 / 9 / 2004$ & WL & 14 & Yes & GROSS BETA ACTIVITY & NT & Annual \\
\hline GW-232 & $6 / 8 / 1990$ & $10 / 25 / 2004$ & WL & 26 & No & GROSS BETA ACTIVITY & NT & Biennial \\
\hline GW-239 & $6 / 14 / 1990$ & $10 / 30 / 1993$ & WL & 0 & No & GROSS ALPHA ACTIVITY & N/A & Remove \\
\hline \begin{tabular}{|l|} 
GW-240 \\
\end{tabular} & $2 / 25 / 1986$ & $10 / 22 / 2001$ & WL & 8 & Yes & \begin{tabular}{|l} 
CARBON TETRACHLORIDE \\
\end{tabular} & $\mathrm{s}$ & Annual \\
\hline GW-380 & $12 / 22 / 1988$ & $8 / 11 / 2004$ & WL & 17 & Yes & CHROMIUM III & NT & Annual \\
\hline GW-381 & $12 / 17 / 1988$ & $11 / 2 / 2004$ & WL & 10 & Yes & CARBON TETRACHLORIDE & NT & Annual \\
\hline GW-382 & $12 / 10 / 1988$ & $8 / 11 / 2004$ & WL & 11 & Yes & CARBON TETRACHLORIDE & $\mathrm{D}$ & Annual \\
\hline GW-383 & $8 / 23 / 1988$ & $11 / 3 / 2004$ & WL & 21 & Yes & TETRACHLOROETHYLENE(PCE) & NT & SemiAnnual \\
\hline GW-384 & $8 / 18 / 1988$ & $11 / 21 / 1996$ & WL & 4 & No & GROSS ALPHA ACTIVITY & $\mathrm{s}$ & Biennial \\
\hline GW-385 & 8/22/1988 & $11 / 19 / 1996$ & WL & 4 & No & LEAD & $\mathrm{PI}$ & Biennial \\
\hline GW-603 & $2 / 12 / 1990$ & $11 / 25 / 1996$ & WL & 4 & No & LEAD & NT & Biennial \\
\hline GW-733 & $4 / 28 / 1992$ & $7 / 8 / 2004$ & WL & 21 & Yes & CARBON TETRACHLORIDE & D & Annual \\
\hline GW-735 & $4 / 28 / 1992$ & $11 / 15 / 2004$ & WL & 15 & No & GROSS ALPHA ACTIVITY & $\mathrm{s}$ & Annual \\
\hline
\end{tabular}

Notes:

taken from $Y-12$ Analytical Database.

2. $W L=$ Groundwater Monitoring Well; $S P=$ Spring

ufficient Data (N/A), Decreasing (D), Probably Decreasing (PD), Stable (S), Probably Increasing (PI),

and Increasing (I), No Trend (NT); and non-detect for all sample events (ND).

.

5. Priority Constituent is the $\mathrm{COC}$ with the highest average concentration from the full dataset normalized by the screening level. (e.g. MAX [Ave Conc./Screening Level]).

Priority constituent indicated for wells where average concentrations do not exceed screening levels may be background level.

6. Remove $=$ Do not sample: SemiAnnual = sample once every 6 months; Annual = sample once per year; Biennial = sample once every two years. 
TABLE B.9

MAROS RESULTS EAST Y-12 AREA

East Fork Poplar Creek Hydrogeologic Regime

Y-12 National Security Complex

Oak Ridge, Tennessee

\begin{tabular}{|c|c|c|c|c|c|c|c|c|}
\hline $\begin{array}{l}\text { Location } \\
\text { Name }\end{array}$ & $\begin{array}{c}\text { Earliest } \\
\text { Sample Date }\end{array}$ & $\begin{array}{l}\text { Most Recent } \\
\text { Sample Date }\end{array}$ & $\begin{array}{c}\text { Location } \\
\text { Type }\end{array}$ & $\begin{array}{c}\text { Number of } \\
\text { Samples } \\
1996-2005\end{array}$ & $\begin{array}{c}\text { Average } \\
\text { Concentration } \\
\begin{array}{c}\text { Exceeds Screening } \\
\text { Level }\end{array} \\
\end{array}$ & Historic Priority Constituent & \begin{tabular}{|c|} 
Trend for Priority \\
Constituent 1996 \\
2005
\end{tabular} & $\begin{array}{l}\text { Preliminary } \\
\text { Frequency }\end{array}$ \\
\hline GW-744 & $4 / 28 / 1992$ & $11 / 10 / 2004$ & WL & 15 & No & GROSS ALPHA ACTIVITY & $\mathrm{s}$ & Biennial \\
\hline GW-745 & 4/29/1992 & 12/9/1997 & WL & 6 & No & CADMIUM & ND & Biennial \\
\hline GW-746 & 4/29/1992 & $12 / 9 / 1997$ & WL & 6 & No & CHROMIUM III & $\mathrm{PI}$ & Annual \\
\hline GW-747 & $8 / 8 / 1992$ & $11 / 10 / 2004$ & WL & 15 & No & GROSS BETA ACTIVITY & $\mathrm{PI}$ & Biennial \\
\hline GW-748 & 8/8/1992 & $12 / 10 / 1997$ & WL & 3 & No & GROSS ALPHA ACTIVITY & N/A & Biennial \\
\hline GW-749 & $8 / 8 / 1992$ & $12 / 10 / 1997$ & WL & $\frac{6}{6}$ & No & GROSS ALPHA ACTIVITY & $\mathrm{s}$ & Biennial \\
\hline GW-750 & $8 / 9 / 1992$ & $11 / 5 / 2004$ & WL & 18 & No & GROSS BETA ACTIVITY & NT & Biennial \\
\hline GW-816 & 9/21/1994 & $11 / 9 / 2004$ & WL & 20 & No & GROSS BETA ACTIVITY & $\mathrm{s}$ & Biennial \\
\hline GW-817 & 9/21/1994 & 12/4/1997 & WL & 6 & No & GROSS ALPHA ACTIVITY & $\mathrm{s}$ & Biennial \\
\hline GW-832 & $5 / 14 / 1996$ & $8 / 16 / 2004$ & WL & 17 & Yes & CARBON TETRACHLORIDE & $\mathrm{D}$ & Annual \\
\hline GW-845 & $5 / 21 / 1998$ & $5 / 21 / 1998$ & WL & 1 & Yes & CARBON TETRACHLORIDE & $\mathrm{N} / \mathrm{A}$ & Extraction \\
\hline RGQWWSP & 6/19/1996 & $4 / 24 / 1997$ & SP & 2 & No & GROSS BETA ACTIVITY & N/A & Biennial \\
\hline SCR7.10SP & 4/9/1997 & 8/27/1998 & SP & 4 & No & CARBON TETRACHLORIDE & ND & Biennial \\
\hline SCR7.14SP & $3 / 21 / 1996$ & $3 / 21 / 1996$ & SP & 1 & No & GROSS BETA ACTIVITY & N/A & Biennial \\
\hline SCR7.16SP & $3 / 21 / 1996$ & $3 / 21 / 1996$ & $\mathrm{SP}$ & 1 & No & GROSS ALPHA ACTIVITY & $\mathrm{N} / \mathrm{A}$ & Biennial \\
\hline SCR7.18SP & $3 / 21 / 1996$ & $5 / 30 / 2000$ & SP & 10 & No & CARBON TETRACHLORIDE & $\mathrm{s}$ & Biennial \\
\hline SCR7.1SP & 12/13/1995 & 7/19/2004 & SP & 19 & No & CARBON TETRACHLORIDE & D & Biennial \\
\hline SCR7.4SP & 4/9/1997 & 8/27/1998 & SP & 4 & No & CARBON TETRACHLORIDE & ND & Biennial \\
\hline SCR7.6SP & 4/9/1997 & $8 / 27 / 1998$ & $\mathrm{SP}$ & 4 & No & CARBON TETRACHLORIDE & ND & Biennial \\
\hline SCR7.7SP & $4 / 9 / 1997$ & $8 / 27 / 1998$ & $\mathrm{SP}$ & 4 & No & CARBON TETRACHLORIDE & $\mathrm{ND}$ & Biennial \\
\hline SCR7.8SP & 4/9/1997 & 7/19/2004 & SP & 17 & No & TETRACHLOROETHYLENE(PCE) & $\mathrm{D}$ & Biennial \\
\hline SCR7.8SSP & 3/18/1996 & 6/20/1996 & SP & 1 & No & GROSS ALPHA ACTIVITY & N/A & Biennial \\
\hline UV8.5SP & 6/19/1996 & 7/17/1996 & SP & 2 & No & CARBON TETRACHLORIDE & N/A & Biennial \\
\hline UV8.6SP & $7 / 17 / 1996$ & 7/17/1996 & $\mathrm{SP}$ & 1 & No & CARBON TETRACHLORIDE & $\mathrm{N} / \mathrm{A}$ & Biennial \\
\hline
\end{tabular}

Notes:

ample dates taken from $\mathrm{Y}$-12 Analytical Database.

2. $W L=$ Groundwater Monitoring Well; $S P=$ Spring

(Drobably Decreasing (PD), Stable (S), Probably Increasing (PI),

and increasing (I), No Trend (NT); and non-detect for all sample events (ND).

.

5.

. Rionty conothel. 
TABLE B.10

MAROS RESULTS WEST CHESTNUT RIDGE

Chestnut Ridge Hydrogeologic Regime

Y-12 National Security Complex

Oak Ridge, Tennessee

\begin{tabular}{|c|c|c|c|c|c|c|c|c|}
\hline $\begin{array}{l}\text { Location } \\
\text { Name }\end{array}$ & $\begin{array}{c}\text { Earliest } \\
\text { Sample Date }\end{array}$ & $\begin{array}{l}\text { Most Recent } \\
\text { Sample Date }\end{array}$ & Location Type & \begin{tabular}{|c} 
Number of \\
Samples 1996- \\
2005
\end{tabular} & \begin{tabular}{|c|} 
Average \\
Concentration \\
Exceeds Screening \\
Level \\
\end{tabular} & Historic Priority Constituent & $\begin{array}{c}\text { Trend for } \\
\text { Priority } \\
\text { Constituent } \\
1996-2005 \\
\end{array}$ & $\begin{array}{l}\text { Preliminary } \\
\text { Frequency }\end{array}$ \\
\hline 1090 & $2 / 13 / 1986$ & $8 / 4 / 2004$ & WL & 17 & No & LEAD & $\mathrm{S}$ & Biennial \\
\hline GW-141 & 2/18/1988 & $7 / 15 / 2004$ & WL & 17 & No & GROSS ALPHA ACTIVITY & D & Biennial \\
\hline GW-203 & 2/6/1986 & $8 / 3 / 2004$ & WL & 15 & No & GROSS ALPHA ACTIVITY & $\mathrm{PI}$ & Annual \\
\hline GW-205 & $2 / 6 / 1986$ & $8 / 3 / 2004$ & WL & 16 & No & GROSS BETA ACTIVITY & $\mathrm{PI}$ & Annual \\
\hline GW-217 & 2/17/1988 & $7 / 14 / 2004$ & WL & 17 & No & GROSS BETA ACTIVITY & $\mathrm{D}$ & Biennial \\
\hline GW-221 & $2 / 7 / 1986$ & $8 / 4 / 2004$ & $\mathrm{WL}$ & 16 & No & GROSS ALPHA ACTIVITY & NT & Annual \\
\hline GW-302 & $5 / 16 / 1990$ & $8 / 12 / 2003$ & WL & 15 & Yes & CHROMIUM III & NT & Annual \\
\hline GW-305 & 2/18/1988 & $10 / 26 / 2004$ & WL & 26 & Yes & NICKEL & I & SemiAnnual \\
\hline GW-339 & $5 / 15 / 1990$ & $8 / 11 / 2003$ & WL & 15 & No & CHROMIUM III & NT & Biennial \\
\hline GW-521 & 9/8/1989 & $7 / 14 / 2004$ & WL & 20 & No & CADMIUM & ND & Biennial \\
\hline GW-522 & $8 / 30 / 1989$ & $7 / 14 / 2004$ & $\mathrm{WL}$ & 18 & No & LEAD & $\mathrm{D}$ & Biennial \\
\hline GW-539 & 3/16/1991 & $7 / 17 / 2002$ & WL & 10 & Yes & CHROMIUM III & D & Biennial \\
\hline GW-540 & 3/16/1991 & $7 / 21 / 2004$ & WL & 15 & No & GROSS ALPHA ACTIVITY & D & Biennial \\
\hline GW-541 & 3/17/1991 & 4/15/1996 & WL & 1 & No & GROSS ALPHA ACTIVITY & N/A & Remove \\
\hline GW-542 & $3 / 17 / 1991$ & $7 / 21 / 2004$ & WL & 15 & No & GROSS ALPHA ACTIVITY & D & Biennial \\
\hline GW-543 & $3 / 17 / 1991$ & $7 / 21 / 2004$ & $\mathrm{WL}$ & 18 & No & CADMIUM & ND & Biennial \\
\hline GW-544 & 3/17/1991 & $7 / 21 / 2004$ & WL & 18 & No & GROSS ALPHA ACTIVITY & $\mathrm{D}$ & Biennial \\
\hline GW-546 & 3/17/1991 & $4 / 9 / 1996$ & WL & 1 & No & LEAD & ND & Remove \\
\hline GW-709 & 6/19/1991 & $7 / 22 / 2004$ & WL & 15 & No & GROSS ALPHA ACTIVITY & $\mathrm{D}$ & Biennial \\
\hline GW-757 & 6/15/1992 & $7 / 22 / 2004$ & WL & 18 & No & CHROMIUM III & $\mathrm{D}$ & Biennial \\
\hline GW-827 & $4 / 5 / 1995$ & $7 / 20 / 2004$ & WL & 16 & No & GROSS BETA ACTIVITY & $\mathrm{D}$ & Biennial \\
\hline SCR1.25SP & 2/16/1999 & $8 / 17 / 2004$ & $\mathrm{SP}$ & 12 & No & GROSS ALPHA ACTIVITY & NT & Annual \\
\hline SCR2.1SP & $4 / 7 / 1997$ & $7 / 19 / 2004$ & $\mathrm{SP}$ & 14 & No & GROSS ALPHA ACTIVITY & $\mathrm{s}$ & Biennial \\
\hline SCR2.2SP & 3/15/1995 & $7 / 19 / 2004$ & SP & 14 & No & LEAD & $\mathrm{s}$ & Biennial \\
\hline
\end{tabular}

Notes:

1. Well sample dates taken from $\mathrm{Y}-12$ Analytical Database.

2. $\mathrm{WL}=$ Groundwater Monitoring Well; $\mathrm{SP}=$ Spring.

3. Trend = Insufficient Data (N/A), Decreasing (D), Probably Decreasing (PD), Stable (S), Probably Increasing (PI),

and Increasing (I), No Trend (NT); and non-detect for all sample events (ND).

4. Number of samples between 1996-2005 is the count of analytical samples acquired at the location based on the BWXT Analytical Database (2005).

5. Priority Constituent is the $\mathrm{COC}$ with the highest average concentration from the full dataset normalized by the screening level. (e.g. MAX [Ave Conc./Screening Level]).

Priority constituent indicated for wells where average concentrations do not exceed screening levels may be background level.

6. Remove $=$ Do not sample; SemiAnnual = sample once every 6 months; Annual = sample once per year; Biennial = sample once every two years. 
TABLE B.10

MAROS RESULTS WEST CHESTNUT RIDGE

Chestnut Ridge Hydrogeologic Regime

Y-12 National Security Complex

Oak Ridge, Tennessee

\begin{tabular}{|c|c|c|c|c|c|c|c|c|}
\hline $\begin{array}{l}\text { Location } \\
\text { Name }\end{array}$ & $\begin{array}{c}\text { Earliest } \\
\text { Sample Date }\end{array}$ & $\begin{array}{l}\text { Most Recent } \\
\text { Sample Date }\end{array}$ & Location Type & \begin{tabular}{|c} 
Number of \\
Samples 1996- \\
2005
\end{tabular} & \begin{tabular}{|c|} 
Average \\
Concentration \\
Exceeds Screening \\
Level \\
\end{tabular} & Historic Priority Constituent & $\begin{array}{c}\text { Trend for } \\
\text { Priority } \\
\text { Constituent } \\
1996-2005 \\
\end{array}$ & $\begin{array}{l}\text { Preliminary } \\
\text { Frequency }\end{array}$ \\
\hline 1090 & $2 / 13 / 1986$ & $8 / 4 / 2004$ & WL & 17 & No & LEAD & $\mathrm{S}$ & Biennial \\
\hline GW-141 & 2/18/1988 & $7 / 15 / 2004$ & WL & 17 & No & GROSS ALPHA ACTIVITY & D & Biennial \\
\hline GW-203 & 2/6/1986 & $8 / 3 / 2004$ & WL & 15 & No & GROSS ALPHA ACTIVITY & $\mathrm{PI}$ & Annual \\
\hline GW-205 & $2 / 6 / 1986$ & $8 / 3 / 2004$ & WL & 16 & No & GROSS BETA ACTIVITY & $\mathrm{PI}$ & Annual \\
\hline GW-217 & 2/17/1988 & $7 / 14 / 2004$ & WL & 17 & No & GROSS BETA ACTIVITY & $\mathrm{D}$ & Biennial \\
\hline GW-221 & $2 / 7 / 1986$ & $8 / 4 / 2004$ & $\mathrm{WL}$ & 16 & No & GROSS ALPHA ACTIVITY & NT & Annual \\
\hline GW-302 & $5 / 16 / 1990$ & $8 / 12 / 2003$ & WL & 15 & Yes & CHROMIUM III & NT & Annual \\
\hline GW-305 & 2/18/1988 & $10 / 26 / 2004$ & WL & 26 & Yes & NICKEL & I & SemiAnnual \\
\hline GW-339 & $5 / 15 / 1990$ & $8 / 11 / 2003$ & WL & 15 & No & CHROMIUM III & NT & Biennial \\
\hline GW-521 & 9/8/1989 & $7 / 14 / 2004$ & WL & 20 & No & CADMIUM & ND & Biennial \\
\hline GW-522 & $8 / 30 / 1989$ & $7 / 14 / 2004$ & $\mathrm{WL}$ & 18 & No & LEAD & $\mathrm{D}$ & Biennial \\
\hline GW-539 & 3/16/1991 & $7 / 17 / 2002$ & WL & 10 & Yes & CHROMIUM III & D & Biennial \\
\hline GW-540 & 3/16/1991 & $7 / 21 / 2004$ & WL & 15 & No & GROSS ALPHA ACTIVITY & D & Biennial \\
\hline GW-541 & 3/17/1991 & 4/15/1996 & WL & 1 & No & GROSS ALPHA ACTIVITY & N/A & Remove \\
\hline GW-542 & $3 / 17 / 1991$ & $7 / 21 / 2004$ & WL & 15 & No & GROSS ALPHA ACTIVITY & D & Biennial \\
\hline GW-543 & $3 / 17 / 1991$ & $7 / 21 / 2004$ & $\mathrm{WL}$ & 18 & No & CADMIUM & ND & Biennial \\
\hline GW-544 & 3/17/1991 & $7 / 21 / 2004$ & WL & 18 & No & GROSS ALPHA ACTIVITY & $\mathrm{D}$ & Biennial \\
\hline GW-546 & 3/17/1991 & $4 / 9 / 1996$ & WL & 1 & No & LEAD & ND & Remove \\
\hline GW-709 & 6/19/1991 & $7 / 22 / 2004$ & WL & 15 & No & GROSS ALPHA ACTIVITY & $\mathrm{D}$ & Biennial \\
\hline GW-757 & 6/15/1992 & $7 / 22 / 2004$ & WL & 18 & No & CHROMIUM III & $\mathrm{D}$ & Biennial \\
\hline GW-827 & $4 / 5 / 1995$ & $7 / 20 / 2004$ & WL & 16 & No & GROSS BETA ACTIVITY & $\mathrm{D}$ & Biennial \\
\hline SCR1.25SP & 2/16/1999 & $8 / 17 / 2004$ & $\mathrm{SP}$ & 12 & No & GROSS ALPHA ACTIVITY & NT & Annual \\
\hline SCR2.1SP & $4 / 7 / 1997$ & $7 / 19 / 2004$ & $\mathrm{SP}$ & 14 & No & GROSS ALPHA ACTIVITY & $\mathrm{s}$ & Biennial \\
\hline SCR2.2SP & 3/15/1995 & $7 / 19 / 2004$ & SP & 14 & No & LEAD & $\mathrm{s}$ & Biennial \\
\hline
\end{tabular}

Notes:

1. Well sample dates taken from $\mathrm{Y}-12$ Analytical Database.

2. $\mathrm{WL}=$ Groundwater Monitoring Well; $\mathrm{SP}=$ Spring.

3. Trend = Insufficient Data (N/A), Decreasing (D), Probably Decreasing (PD), Stable (S), Probably Increasing (PI),

and Increasing (I), No Trend (NT); and non-detect for all sample events (ND).

4. Number of samples between 1996-2005 is the count of analytical samples acquired at the location based on the BWXT Analytical Database (2005).

5. Priority Constituent is the $\mathrm{COC}$ with the highest average concentration from the full dataset normalized by the screening level. (e.g. MAX [Ave Conc./Screening Level]).

Priority constituent indicated for wells where average concentrations do not exceed screening levels may be background level.

6. Remove $=$ Do not sample; SemiAnnual = sample once every 6 months; Annual = sample once per year; Biennial = sample once every two years. 
TABLE B.11

MAROS RESULTS CHESTNUT RIDGE SECURITY PITS

Chestnut Ridge Hydrogeologic Regime

-12 National Security Complex

\begin{tabular}{|c|c|c|c|c|c|c|c|c|}
\hline $\begin{array}{c}\text { Location } \\
\text { Name }\end{array}$ & \begin{tabular}{|c|} 
Earliest Sample \\
Date
\end{tabular} & $\begin{array}{l}\text { Most Recent } \\
\text { Sample Date }\end{array}$ & Location Type & $\begin{array}{c}\text { Number of } \\
\text { Samples } \\
1996-2005 \\
\end{array}$ & \begin{tabular}{|c|} 
Average \\
Concentration \\
Exceeds \\
Screening Level
\end{tabular} & Historic Priority Constituent & $\begin{array}{c}\text { Trend for } \\
\text { Priority } \\
\text { Constituent } \\
1996-2005 \\
\end{array}$ & $\begin{array}{r}\text { Preliminary } \\
\text { Frequency }\end{array}$ \\
\hline GW-173 & $2 / 4 / 1986$ & $10 / 4 / 2004$ & WL & 2 & Yes & TETRACHLOROETHYLENE(PCE) & $\mathrm{N} / \mathrm{A}$ & SemiAnnual \\
\hline GW-174 & $2 / 4 / 1986$ & $8 / 21 / 2001$ & WL & 3 & Yes & TETRACHLOROETHYLENE(PCE) & N/A & SemiAnnual \\
\hline GW-175 & 8/17/1988 & $10 / 6 / 2004$ & WL & 5 & Yes & TETRACHLOROETHYLENE(PCE) & $\mathrm{s}$ & Annual \\
\hline GW-176 & 2/4/1986 & 10/7/2004 & WL & 2 & Yes & 1,1-DICHLOROETHENE & N/A & Annual \\
\hline GW-177 & $2 / 4 / 1986$ & $7 / 13 / 2004$ & WL & 9 & No & LEAD & ND & Biennial \\
\hline GW-178 & $3 / 12 / 1988$ & $10 / 6 / 2004$ & WL & 2 & No & LEAD & $\mathrm{N} / \mathrm{A}$ & Annual \\
\hline GW-179 & $2 / 10 / 1986$ & $10 / 7 / 2004$ & WL & 2 & Yes & 1,1-DICHLOROETHENE & $\mathrm{N} / \mathrm{A}$ & Annual \\
\hline GW-180 & $3 / 11 / 1988$ & $8 / 21 / 2001$ & WL & 2 & Yes & TETRACHLOROETHYLENE(PCE) & N/A & SemiAnnual \\
\hline GW-181 & $8 / 16 / 1988$ & $11 / 6 / 1995$ & WL & 0 & No & GROSS ALPHA ACTIVITY & $\mathrm{N} / \mathrm{A}$ & Remove \\
\hline GW-184 & $2 / 25 / 1986$ & $4 / 30 / 1996$ & WL & 1 & No & GROSS ALPHA ACTIVITY & $\mathrm{N} / \mathrm{A}$ & Remove \\
\hline GW-186 & $3 / 4 / 1986$ & $5 / 1 / 1996$ & WL & 1 & No & GROSS ALPHA ACTIVITY & $\mathrm{N} / \mathrm{A}$ & Biennial \\
\hline GW-188 & 2/24/1986 & 4/30/1996 & WL & 1 & No & GROSS ALPHA ACTIVITY & N/A & Biennial \\
\hline GW-322 & $3 / 12 / 1988$ & $10 / 11 / 2004$ & WL & 3 & Yes & 1,1-DICHLOROETHENE & N/A & SemiAnnual \\
\hline GW-511 & $8 / 16 / 1988$ & $11 / 7 / 1995$ & WL & 0 & No & LEAD & $\mathrm{N} / \mathrm{A}$ & Biennial \\
\hline GW-512 & $12 / 6 / 1988$ & $5 / 2 / 1996$ & WL & 0 & No & CADMIUM & $\mathrm{N} / \mathrm{A}$ & Biennial \\
\hline GW-513 & $12 / 3 / 1988$ & $10 / 13 / 2004$ & WL & 2 & No & GROSS ALPHA ACTIVITY & $\mathrm{N} / \mathrm{A}$ & Annual \\
\hline GW-514 & $12 / 8 / 1988$ & $8 / 16 / 2001$ & WL & 2 & No & GROSS ALPHA ACTIVITY & N/A & Biennial \\
\hline GW-608 & $2 / 10 / 1990$ & $8 / 20 / 2001$ & WL & 7 & No & TETRACHLOROETHYLENE(PCE) & $\mathrm{s}$ & Annual \\
\hline GW-609 & $2 / 14 / 1990$ & $1 / 10 / 2001$ & WL & 14 & Yes & TETRACHLOROETHYLENE(PCE) & $\mathrm{D}$ & Annual \\
\hline GW-610 & $2 / 9 / 1990$ & $10 / 20 / 2004$ & WL & 1 & No & GROSS ALPHA ACTIVITY & $\mathrm{N} / \mathrm{A}$ & Biennial \\
\hline GW-611 & $2 / 9 / 1990$ & $10 / 20 / 2004$ & WL & 3 & No & 1,1-DICHLOROETHENE & $\mathrm{N} / \mathrm{A}$ & Annual \\
\hline GW-612 & $2 / 10 / 1990$ & $10 / 5 / 2004$ & WL & 5 & Yes & 1,1-DICHLOROETHENE & NT & Annual \\
\hline GW-679 & $4 / 19 / 2004$ & $10 / 12 / 2004$ & WL & 2 & No & GROSS ALPHA ACTIVITY & N/A & Biennial \\
\hline GW-680 & $6 / 7 / 2004$ & $10 / 13 / 2004$ & WL & 2 & No & LEAD & $\mathrm{N} / \mathrm{A}$ & Biennial \\
\hline GW-742 & 4/10/1992 & $10 / 18 / 2004$ & WL & 3 & No & GROSS ALPHA ACTIVITY & N/A & Annual \\
\hline GW-743 & $4 / 7 / 1992$ & $10 / 18 / 2004$ & WL & 2 & No & GROSS ALPHA ACTIVITY & N/A & Biennial \\
\hline GW-831 & $8 / 27 / 1996$ & $7 / 12 / 2004$ & WL & 18 & No & LEAD & $\mathrm{D}$ & Biennial \\
\hline SCR3.4SP & $4 / 8 / 1997$ & 2/20/2001 & SP & 6 & No & GROSS ALPHA ACTIVITY & $\mathrm{PI}$ & Annual \\
\hline SCR3.5SP & $4 / 8 / 1997$ & $8 / 17 / 2004$ & SP & 16 & No & GROSS ALPHA ACTIVITY & NT & Annual \\
\hline
\end{tabular}

Notes:

1. Well sample dates taken from $\mathrm{Y}-12$ Analytical Database.

2. $W L=$ Groundwater Monitoring Well; $S P=$ Spring

3. Trend = Insufficient Data (N/A), Decreasing (D), Probably Decreasing (PD), Stable (S), Probably Increasing (PI),

and Increasing (I), No Trend (NT); and non-detect for all sample events (ND).

Priority Constituent is the $\mathrm{COC}$ with the highest average concentration from the full dataset normalized by the screening level. (e.g. MAX [Ave Conc./Screening Level])

Cecter.

6. Remove $=$ Do not sample; SemiAnnual = sample once every 6 months; Annual = sample once per year; Biennial = sample once every two years 
TABLE B.12

MAROS RESULTS LANDFILLS V AND VII AREAS

Chestnut Ridge Hydrogeologic Regime

Y-12 National Security Complex

Oak Ridge, Tennessee

\begin{tabular}{|c|c|c|c|c|c|c|c|c|}
\hline $\begin{array}{c}\text { Location } \\
\text { Name }\end{array}$ & $\begin{array}{c}\text { Earliest } \\
\text { Sample Date }\end{array}$ & $\begin{array}{l}\text { Most Recent } \\
\text { Sample Date }\end{array}$ & $\begin{array}{c}\text { Location } \\
\text { Type }\end{array}$ & $\begin{array}{c}\text { Number of } \\
\text { Samples 1996- } \\
2005 \\
\end{array}$ & \begin{tabular}{|c|} 
Average \\
Concentration \\
Exceeds Screening \\
Level
\end{tabular} & Historic Priority Constituent & $\begin{array}{c}\text { Trend for } \\
\text { Priority } \\
\text { Constituent } \\
1996-2005 \\
\end{array}$ & $\begin{array}{l}\text { Preliminary } \\
\text { Frequency }\end{array}$ \\
\hline GW-557 & $5 / 26 / 1993$ & $7 / 20 / 2004$ & $\overline{W L}$ & 18 & No & TETRACHLOROETHYLENE(PCE) & ND & Biennial \\
\hline GW-560 & $5 / 26 / 1993$ & $7 / 19 / 2004$ & WL & 15 & No & TETRACHLOROETHYLENE(PCE) & ND & Remove \\
\hline GW-562 & $5 / 26 / 1993$ & $7 / 19 / 2004$ & WL & 15 & No & TETRACHLOROETHYLENE(PCE) & ND & Remove \\
\hline GW-564 & $5 / 26 / 1993$ & $7 / 19 / 2004$ & WL & 15 & No & TETRACHLOROETHYLENE(PCE) & ND & Remove \\
\hline GW-796 & $5 / 27 / 1993$ & $7 / 20 / 2004$ & WL & 18 & No & TETRACHLOROETHYLENE(PCE) & ND & Biennial \\
\hline GW-797 & $5 / 27 / 1993$ & $7 / 15 / 2004$ & WL & 18 & No & LEAD & $\mathrm{D}$ & Biennial \\
\hline GW-798 & $6 / 23 / 1993$ & $7 / 20 / 2004$ & WL & 21 & No & TETRACHLOROETHYLENE(PCE) & I & Annual \\
\hline GW-799 & $5 / 27 / 1993$ & $7 / 19 / 2004$ & WL & 18 & No & TETRACHLOROETHYLENE(PCE) & ND & Biennial \\
\hline GW-801 & $7 / 24 / 1993$ & $7 / 15 / 2004$ & WL & 18 & No & TETRACHLOROETHYLENE(PCE) & ND & Biennial \\
\hline SCR4.3SP & 2/14/1994 & $7 / 22 / 2004$ & SP & 18 & No & TETRACHLOROETHYLENE(PCE) & ND & Biennial \\
\hline
\end{tabular}

Notes:

1. Well sample dates taken from $\mathrm{Y}-12$ Analytical Database

2. $\mathrm{WL}=$ Groundwater Monitoring Well; $\mathrm{SP}=$ Spring.

3. Trend = Insufficient Data (N/A), Decreasing (D), Probably Decreasing (PD), Stable (S), Probably Increasing (PI),

and Increasing (I), No Trend (NT); and non-detect for all sample events (ND).

4. Number of samples between 1996-2005 is the count of analytical samples acquired at the location based on the BWXT Analytical Database (2005).

5. Priority Constituent is the $\mathrm{COC}$ with the highest average concentration from the full dataset normalized by the screening level. (e.g. MAX [Ave Conc./Screening Level]).

Priority constituent indicated for wells where average concentrations do not exceed screening levels may be background level.

6. Remove = Do not sample; SemiAnnual $=$ sample once every 6 months; Annual = sample once per year; Biennial $=$ sample once every two years. 
TABLE B.13

MAROS RESULTS EAST CHESTNUT RIDGE

Chestnut Ridge Hydrogeologic Regim

Y-12 National Security Complex (akn

\begin{tabular}{|c|c|c|c|c|c|c|c|c|}
\hline $\begin{array}{c}\text { Location } \\
\text { Name }\end{array}$ & $\begin{array}{c}\text { Earliest } \\
\text { Sample Date }\end{array}$ & $\begin{array}{l}\text { Most Recent } \\
\text { Sample Date }\end{array}$ & $\begin{array}{c}\text { Location } \\
\text { Type }\end{array}$ & $\begin{array}{c}\text { Number of } \\
\text { Samples 1996- } \\
2005 \\
\end{array}$ & $\begin{array}{c}\text { Average } \\
\text { Concentration } \\
\text { Exceeds Screening } \\
\text { Level } \\
\end{array}$ & Historic Priority Constituent & $\begin{array}{c}\text { Trend for Priority } \\
\text { Constituent 1996- } \\
\quad 2005 \\
\end{array}$ & $\begin{array}{c}\text { Preliminary } \\
\text { Frequency }\end{array}$ \\
\hline GW-142 & $2 / 27 / 1986$ & $10 / 8 / 2003$ & WL & 17 & No & GROSS ALPHA ACTIVITY & $\mathrm{D}$ & Biennial \\
\hline GW-143 & $3 / 6 / 1986$ & $10 / 12 / 2004$ & WL & 19 & No & GROSS ALPHA ACTIVITY & D & Biennial \\
\hline GW-144 & $2 / 28 / 1986$ & $10 / 12 / 2004$ & WL & 19 & No & GROSS ALPHA ACTIVITY & NT & Biennial \\
\hline GW-145 & $3 / 5 / 1986$ & $10 / 12 / 2004$ & WL & 19 & No & GROSS ALPHA ACTIVITY & $\mathrm{s}$ & Annual \\
\hline GW-156 & $2 / 22 / 1986$ & $10 / 14 / 2004$ & WL & 17 & No & URANIUM & $\mathrm{D}$ & Biennial \\
\hline GW-159 & $2 / 19 / 1986$ & $10 / 13 / 2004$ & WL & 2 & Yes & GROSS ALPHA ACTIVITY & $\mathrm{N} / \mathrm{A}$ & Annual \\
\hline GW-160 & $2 / 4 / 1988$ & $4 / 29 / 1996$ & WL & 1 & Yes & LEAD & N/A & Annual \\
\hline GW-231 & $3 / 7 / 1986$ & $10 / 11 / 2004$ & WL & 19 & No & GROSS ALPHA ACTIVITY & $\mathrm{D}$ & Biennial \\
\hline GW-241 & $2 / 28 / 1986$ & $8 / 16 / 2001$ & WL & 2 & No & LEAD & N/A & Annual \\
\hline GW-292 & $12 / 22 / 1987$ & $5 / 8 / 1996$ & WL & 1 & No & CADMIUM & ND & Remove \\
\hline GW-293 & $12 / 22 / 1987$ & $5 / 8 / 1996$ & WL & 1 & No & LEAD & ND & Remove \\
\hline GW-298 & 2/3/1988 & $5 / 1 / 1996$ & WL & 1 & No & LEAD & ND & Remove \\
\hline GW-299 & 2/3/1988 & $4 / 30 / 1996$ & WL & 1 & No & GROSS ALPHA ACTIVITY & $\mathrm{N} / \mathrm{A}$ & Remove \\
\hline GW-300 & $2 / 1 / 1988$ & $10 / 12 / 2004$ & WL & 3 & No & LEAD & N/A & Annual \\
\hline GW-301 & $2 / 3 / 1988$ & $7 / 12 / 2004$ & WL & 18 & No & LEAD & $\mathrm{D}$ & Biennial \\
\hline GW-303 & $12 / 17 / 1988$ & $7 / 16 / 1995$ & WL & 0 & No & GROSS ALPHA ACTIVITY & $\mathrm{N} / \mathrm{A}$ & Remove \\
\hline GW-304 & $12 / 20 / 1988$ & $7 / 12 / 1995$ & WL & 0 & No & GROSS ALPHA ACTIVITY & N/A & Remove \\
\hline GW-731 & 9/27/1991 & $10 / 13 / 2004$ & WL & 2 & No & LEAD & N/A & Biennial \\
\hline GW-732 & 9/27/1991 & $10 / 14 / 2004$ & WL & 2 & No & GROSS ALPHA ACTIVITY & N/A & Biennial \\
\hline GW-841 & 2/6/1997 & $3 / 2 / 2004$ & WL & 8 & Yes & TRICHLOROETHYLENE (TCE) & $\mathrm{D}$ & Annual \\
\hline GW-842 & $2 / 5 / 1997$ & $8 / 18 / 2004$ & WL & 11 & No & TRICHLOROETHYLENE (TCE) & NT & Annual \\
\hline GW-843 & 2/5/1997 & 8/18/2004 & WL & 1 & No & URANIUM & N/A & Annual \\
\hline GW-844 & 2/5/1997 & 2/5/1997 & WL & 1 & No & BENZENE & ND & Annual \\
\hline SCR5.1SP & 4/10/1997 & $8 / 15 / 2001$ & SP & 4 & No & GROSS ALPHA ACTIVITY & NT & Annual \\
\hline SCR5.2SP & 2/19/2001 & 8/19/2002 & SP & 1 & No & GROSS ALPHA ACTIVITY & N/A & Annual \\
\hline SCR5.4SP & $4 / 8 / 1997$ & $8 / 15 / 2001$ & SP & 8 & No & GROSS ALPHA ACTIVITY & NT & Biennial \\
\hline
\end{tabular}

Notes:

1. Well sample dates taken from $Y-12$ Analytical Database.

2. $\mathrm{WL}=$ Groundwater Monitoring Well; $\mathrm{SP}=$ Spring

3. Trend = Insufficient Data (N/A), Decreasing (D), Probably Decreasing (PD), Stable (S), Probably Increasing (PI),

and Increasing (I), No Trend (NT); and non-detect for all sample events (ND).

(1996-2005 is the count of analytical samples acquired at the location based on the BWXT Analytical Database (2005).

5. Priority Constituent is the $\mathrm{COC}$ with the highest average concentration from the full dataset normalized by the screening level. (e.g. MAX [Ave Conc./Screening Level]).

Priority constituent indicated for wells where average concentrations do not exceed screening levels may be background level.

6. Remove $=$ Do not sample; SemiAnnual $=$ sample once every 6 months; Annual = sample once per year; Biennial = sample once every two years. 
TABLE B.14

SUMMARY SAMPLING RECOMMENDATIONS BEAR CREEK REGIME

Bear Creek Hydrogeologic Regime

Y-12 National Security Complex

Oak Ridge, Tennessee

\begin{tabular}{|c|c|c|c|c|c|c|}
\hline Regime & Functional Area & $\begin{array}{l}\text { Location } \\
\text { Name }\end{array}$ & Trend & $\begin{array}{l}\text { Preliminary } \\
\text { Frequency }\end{array}$ & Final Frequency & Recommendation \\
\hline \multicolumn{7}{|c|}{ Regulated Sample Locations } \\
\hline $\mathrm{BC}$ & Oil Landfarm WMA & GW-008 & NT & Annual & Regulated & Sample as per permit \\
\hline $\mathrm{BC}$ & $\begin{array}{l}\text { Bear Creek Burial Grounds } \\
\text { WMA }\end{array}$ & GW-046 & NT & Annual & Regulated & $\begin{array}{l}\text { Sample as per permit, sample all COCs } \\
\text { annually }\end{array}$ \\
\hline $\mathrm{BC}$ & $\begin{array}{l}\text { Bear Creek Burial Grounds } \\
\text { WMA }\end{array}$ & GW-077 & ND & Biennial & Regulated & $\begin{array}{l}\text { Sample as per ROD, largely ND well, } \\
\text { consider reduced effort }\end{array}$ \\
\hline $\mathrm{BC}$ & $\begin{array}{l}\text { Bear Creek Burial Grounds } \\
\text { WMA }\end{array}$ & GW-078 & ND & Biennial & Regulated & $\begin{array}{l}\text { Sample as per ROD, largely ND well, } \\
\text { consider reduced effort }\end{array}$ \\
\hline $\mathrm{BC}$ & $\begin{array}{l}\text { Bear Creek Burial Grounds } \\
\text { WMA }\end{array}$ & GW-079 & N/A & Biennial & Regulated & $\begin{array}{l}\text { Sample as per ROD, concentrations } \\
\text { consistent w/Biennial sampling }\end{array}$ \\
\hline $\mathrm{BC}$ & $\begin{array}{l}\text { Bear Creek Burial Grounds } \\
\text { WMA }\end{array}$ & GW-080 & ND & Biennial & Regulated & $\begin{array}{l}\text { Sample as per ROD, largely ND well, } \\
\text { consider reduced effort }\end{array}$ \\
\hline$B C$ & S-3 Site & GW-276 & $\mathrm{D}$ & Annual & Regulated & $\begin{array}{l}\text { Sample as per permit, consider annual } \\
\text { sampling as trend is Decreasing }\end{array}$ \\
\hline $\mathrm{BC}$ & Oil Landfarm WMA & GW-363 & PD & Biennial & Regulated & $\begin{array}{l}\text { Sample as alternate RCRA well, reduced } \\
\text { frequency for Probably Decreasing trend }\end{array}$ \\
\hline $\mathrm{BC}$ & S-3 Site & GW-526 & $\mathrm{N} / \mathrm{A}$ & SemiAnnual & Regulated & Sample as per ROD \\
\hline $\mathrm{BC}$ & $\begin{array}{l}\text { Bear Creek Burial Grounds } \\
\text { WMA }\end{array}$ & GW-639 & NT & Biennial & Regulated & $\begin{array}{l}\text { Sample as per permit/ROD, consider } \\
\text { reduced effort }\end{array}$ \\
\hline $\mathrm{BC}$ & Exit Pathway - Traverse A & GW-683 & $\mathrm{D}$ & Annual & Regulated & $\begin{array}{l}\text { Sample as per ROD, consider annual } \\
\text { sampling }\end{array}$ \\
\hline $\mathrm{BC}$ & Exit Pathway - Traverse A & GW-684 & $\mathrm{S}$ & Annual & Regulated & $\begin{array}{l}\text { Sample as per ROD, consider annual } \\
\text { sampling }\end{array}$ \\
\hline $\mathrm{BC}$ & Exit Pathway - Traverse B & GW-704 & $\mathrm{S}$ & Annual & Regulated & Sample as per ROD \\
\hline $\mathrm{BC}$ & Exit Pathway - Traverse B & GW-706 & $\mathrm{D}$ & Annual & Regulated & Sample as per ROD \\
\hline $\mathrm{BC}$ & Exit Pathway - Traverse W & GW-712 & NT & Biennial & Regulated & largely non-detect, consider reduced effort \\
\hline $\mathrm{BC}$ & Exit Pathway - Traverse W & GW-713 & $\mathrm{N} / \mathrm{A}$ & Annual & Regulated & sampling \\
\hline $\mathrm{BC}$ & Exit Pathway - Traverse W & GW-714 & $\mathrm{PD}$ & Annual & Regulated & sampling \\
\hline $\mathrm{BC}$ & S-3 Ponds & GW-835 & $\mathrm{I}$ & SemiAnnual & Regulated & above MCL, sample at least annually \\
\hline $\mathrm{BC}$ & EMWMF & GW-916 & NT & Annual & Regulated & $\begin{array}{l}\text { Sample as per permit, low concentrations, } \\
\text { consider reduced effort }\end{array}$ \\
\hline $\mathrm{BC}$ & EMWMF & GW-917 & $\mathrm{Pl}$ & Biennial & Regulated & $\begin{array}{l}\text { Sample as per permit, low concentrations, } \\
\text { consider reduced effort }\end{array}$ \\
\hline $\mathrm{BC}$ & EMWMF & GW-918 & NT & Biennial & Regulated & $\begin{array}{l}\text { Sample as per permit, low concentrations, } \\
\text { consider reduced effort }\end{array}$ \\
\hline $\mathrm{BC}$ & EMWMF & GW-919 & $\mathrm{N} / \mathrm{A}$ & Annual & Regulated & $\begin{array}{l}\text { Sample as per permit, low concentrations, } \\
\text { consider reduced effort }\end{array}$ \\
\hline $\mathrm{BC}$ & EMWMF & GW-920 & NT & Annual & Regulated & $\begin{array}{l}\text { Sample as per permit, low concentrations, } \\
\text { consider reduced effort }\end{array}$ \\
\hline $\mathrm{BC}$ & EMWMF & GW-921 & NT & Biennial & Regulated & $\begin{array}{l}\text { Sample as per permit, low concentrations, } \\
\text { consider reduced effort }\end{array}$ \\
\hline $\mathrm{BC}$ & EMWMF & GW-922 & S & Biennial & Regulated & $\begin{array}{l}\text { Sample as per permit, low concentrations, } \\
\text { consider reduced effort }\end{array}$ \\
\hline $\mathrm{BC}$ & EMWMF & GW-923 & NT & Biennial & Regulated & $\begin{array}{l}\text { Sample as per permit, low concentrations, } \\
\text { consider reduced effort }\end{array}$ \\
\hline $\mathrm{BC}$ & EMWMF & GW-924 & ND & Biennial & Regulated & $\begin{array}{l}\text { Sample as per permit, low concentrations, } \\
\text { consider reduced effort }\end{array}$ \\
\hline $\mathrm{BC}$ & EMWMF & GW-925 & NT & Biennial & Regulated & $\begin{array}{l}\text { Sample as per permit, low concentrations, } \\
\text { consider reduced effort }\end{array}$ \\
\hline$B C$ & EMWMF & GW-926 & NT & Biennial & Regulated & $\begin{array}{l}\text { Sample as per permit, low concentrations, } \\
\text { consider reduced effort }\end{array}$ \\
\hline$B C$ & EMWMF & GW-927 & NT & Biennial & Regulated & $\begin{array}{l}\text { Sample as per permit, low concentrations, } \\
\text { consider reduced effort }\end{array}$ \\
\hline
\end{tabular}


TABLE B.14

SUMMARY SAMPLING RECOMMENDATIONS BEAR CREEK REGIME

Bear Creek Hydrogeologic Regime

Y-12 National Security Complex

Oak Ridge, Tennessee

\begin{tabular}{|c|c|c|c|c|c|c|}
\hline Regime & Functional Area & $\begin{array}{l}\text { Location } \\
\text { Name }\end{array}$ & Trend & $\begin{array}{l}\text { Preliminary } \\
\text { Frequency }\end{array}$ & Final Frequency & Recommendation \\
\hline $\mathrm{BC}$ & $\begin{array}{l}\text { Exit Pathway Spring/Surface } \\
\text { Water }\end{array}$ & SS-6.6 & NT & Biennial & Regulated & Sampled as spring location SS-6 \\
\hline $\mathrm{BC}$ & $\begin{array}{l}\text { Exit Pathway Spring/Surface } \\
\text { Water }\end{array}$ & SS-6E & $\mathrm{PI}$ & SemiAnnual & Regulated & Sampled as spring SS-6 \\
\hline $\mathrm{BC}$ & $\begin{array}{l}\text { Exit Pathway Spring/Surface } \\
\text { Water }\end{array}$ & SS-6W & NT & Annual & Regulated & Sampled as SS-6 \\
\hline $\mathrm{BC}$ & $\begin{array}{l}\text { Exit Pathway Spring/Surface } \\
\text { Water }\end{array}$ & SS-7 & $\mathrm{I}$ & Annual & Regulated & $\begin{array}{l}\text { Sample as per ROD, consider annual } \\
\text { sampling }\end{array}$ \\
\hline $\mathrm{BC}$ & $\begin{array}{l}\text { Exit Pathway Spring/Surface } \\
\text { Water }\end{array}$ & SS-8 & N/A & Biennial & Regulated & Sample as per ROD \\
\hline \multicolumn{7}{|c|}{ Semi-Annual Sampling } \\
\hline $\mathrm{BC}$ & $\begin{array}{l}\text { Bear Creek Burial Grounds } \\
\text { WMA }\end{array}$ & GW-014 & N/A & SemiAnnual & SemiAnnual & $\begin{array}{l}\text { Sample semi-annually to annually as } \\
\text { alternate RCRA location, evaluate trend } \\
\text { with more data }\end{array}$ \\
\hline $\mathrm{BC}$ & $\begin{array}{l}\text { Bear Creek Burial Grounds } \\
\text { WMA }\end{array}$ & GW-071 & NT & SemiAnnual & SemiAnnual & $\begin{array}{l}\text { Sample semi-annually to annually as } \\
\text { alternate RCRA location, evaluate trend } \\
\text { with more data }\end{array}$ \\
\hline $\mathrm{BC}$ & $\begin{array}{l}\text { Bear Creek Burial Grounds } \\
\text { WMA }\end{array}$ & GW-082 & 1 & SemiAnnual & SemiAnnual & $\begin{array}{l}\text { Sample semi-annually to annually as } \\
\text { alternate RCRA location, evaluate trend } \\
\text { with more data }\end{array}$ \\
\hline $\mathrm{BC}$ & Oil Landfarm WMA & GW-085 & $\mathrm{PI}$ & SemiAnnual & SemiAnnual & $\begin{array}{l}\text { Probably Increasing trend for Nitrate, on- } \\
\text { strike with GW-537, GW-829 }\end{array}$ \\
\hline $\mathrm{BC}$ & Oil Landfarm WMA & GW-225 & NT & SemiAnnual & SemiAnnual & $\begin{array}{l}\text { Sanitary Landfill I area, historic high } \\
\text { concentrations }\end{array}$ \\
\hline$B C$ & Oil Landfarm WMA & GW-226 & $\mathrm{S}$ & SemiAnnual & SemiAnnual & $\begin{array}{l}\text { Sanitary Landfill I area, historic high } \\
\text { concentrations }\end{array}$ \\
\hline $\mathrm{BC}$ & S-3 Site & GW-243 & N/A & SemiAnnual & SemiAnnual & $\begin{array}{l}\text { Source area well, may be redundant, } \\
\text { Sample } 4 \mathrm{X} \text { in } 2 \text { to } 4 \text { yrs; historic high } \\
\text { concentrations, consider removing from } \\
\text { program after trend established }\end{array}$ \\
\hline $\mathrm{BC}$ & S-3 Site & GW-246 & N/A & SemiAnnual & SemiAnnual & $\begin{array}{l}\text { Source area well, may be redundant, } \\
\text { Sample } 4 \mathrm{X} \text { in } 2 \text { to } 4 \text { yrs; historic high } \\
\text { concentrations, consider removing from } \\
\text { program after trend established }\end{array}$ \\
\hline $\mathrm{BC}$ & $\begin{array}{l}\text { Bear Creek Burial Grounds } \\
\text { WMA }\end{array}$ & GW-257 & N/A & SemiAnnual & SemiAnnual & $\begin{array}{l}\text { Sample semi-annually to annually as } \\
\text { alternate RCRA location, evaluate trend } \\
\text { with more data }\end{array}$ \\
\hline $\mathrm{BC}$ & $\begin{array}{l}\text { Bear Creek Burial Grounds } \\
\text { WMA }\end{array}$ & GW-289 & $\mathrm{PI}$ & SemiAnnual & SemiAnnual & $\begin{array}{l}\text { Sample semi-annually to annually as } \\
\text { alternate RCRA location, evaluate trend } \\
\text { with more data }\end{array}$ \\
\hline $\mathrm{BC}$ & S-3 Site & GW-615 & N/A & SemiAnnual & SemiAnnual & $\begin{array}{l}\text { Source area well, may be redundant, } \\
\text { Sample } 4 \mathrm{X} \text { in } 2 \text { to } 4 \text { yrs; historic high } \\
\text { concentrations, consider removing from } \\
\text { program after trend established }\end{array}$ \\
\hline $\mathrm{BC}$ & $\begin{array}{l}\text { Bear Creek Burial Grounds } \\
\text { WMA }\end{array}$ & GW-626 & $\mathrm{PI}$ & SemiAnnual & SemiAnnual & Above MCL, Probably Increasing trend \\
\hline $\mathrm{BC}$ & $\begin{array}{l}\text { Bear Creek Burial Grounds } \\
\text { WMA }\end{array}$ & GW-627 & 1 & SemiAnnual & SemiAnnual & Above $\mathrm{MCL}$, Increasing trend \\
\hline $\mathrm{BC}$ & Exit Pathway - Traverse B & GW-694 & NT & SemiAnnual & SemiAnnual & Above MCL, high data variability \\
\hline
\end{tabular}


TABLE B.14

SUMMARY SAMPLING RECOMMENDATIONS BEAR CREEK REGIME

Bear Creek Hydrogeologic Regime

Y-12 National Security Complex

Oak Ridge, Tennessee

\begin{tabular}{|c|c|c|c|c|c|c|}
\hline Regime & Functional Area & $\begin{array}{l}\text { Location } \\
\text { Name }\end{array}$ & Trend & $\begin{array}{l}\text { Preliminary } \\
\text { Frequency }\end{array}$ & Final Frequency & Recommendation \\
\hline \multicolumn{7}{|c|}{ Annual Sampling } \\
\hline $\mathrm{BC}$ & $\begin{array}{l}\text { Bear Creek Burial Grounds } \\
\text { WMA }\end{array}$ & GW-069 & $\mathrm{PI}$ & Annual & Annual & $\begin{array}{l}\text { Sample annually to biennially, possible } \\
\text { increasing VC trend in alternate RCRA well }\end{array}$ \\
\hline $\mathrm{BC}$ & Oil Landfarm WMA & GW-098 & NT & Annual & Annual & Historic exceedance, surveillance well \\
\hline $\mathrm{BC}$ & S-3 Site & GW-100 & $\mathrm{N} / \mathrm{A}$ & SemiAnnual & Annual & $\begin{array}{l}\text { Sample annually and evaluate trend for } \\
\text { nitrate and beta, midpoint between SS-1 } \\
\text { and GW-835 }\end{array}$ \\
\hline $\mathrm{BC}$ & S-3 Site & GW-101 & N/A & SemiAnnual & Annual & $\begin{array}{l}\text { Source area well, may be redundant, } \\
\text { Sample } 4 \mathrm{X} \text { in } 2 \text { to } 4 \text { yrs; historic high } \\
\text { concentrations, consider removing from } \\
\text { program after trend established }\end{array}$ \\
\hline $\mathrm{BC}$ & S-3 Site & GW-127 & N/A & Annual & Annual & $\begin{array}{l}\text { Sample } 4 \mathrm{X} \text { in } 2 \text { to } 4 \mathrm{yrs} \text {; historic high } \\
\text { concentrations of alpha and beta (not } \\
\text { nitrate) }\end{array}$ \\
\hline BC & Oil Landfarm WMA & GW-229 & $\mathrm{S}$ & Annual & Annual & support MNA \\
\hline $\mathrm{BC}$ & S-3 Site & GW-236 & $\mathrm{N} / \mathrm{A}$ & SemiAnnual & Annual & $\begin{array}{l}\text { Sample annually and evaluate trend for } \\
\text { nitrate and beta, may be redundant with SS } \\
1\end{array}$ \\
\hline $\mathrm{BC}$ & $\begin{array}{l}\text { Bear Creek Burial Grounds } \\
\text { WMA }\end{array}$ & GW-291 & $\mathrm{S}$ & SemiAnnual & Annual & $\begin{array}{l}\text { Stable trend, Sample semi-annually to } \\
\text { annually as alternate RCRA location }\end{array}$ \\
\hline $\mathrm{BC}$ & Rust Spoil Area & GW-311 & $\mathrm{D}$ & Annual & Annual & Monitor annually, Decreasing trend \\
\hline $\mathrm{BC}$ & Spoil Area I & GW-315 & PD & Annual & Annual & $\begin{array}{l}\text { Sample annually for VOC in Spoil Area 1, } \\
\text { Probably Decreasing trend }\end{array}$ \\
\hline $\mathrm{BC}$ & Oil Landfarm WMA & GW-537 & $\mathrm{D}$ & Annual & Annual & $\begin{array}{l}\text { Sample as alternate CERCLA well, } \\
\text { Decreasing nitrate trend, on strike with GW- } \\
085 \text { and GW-829 }\end{array}$ \\
\hline $\mathrm{BC}$ & S-3 Site & GW-616 & N/A & SemiAnnual & Annual & Historic nitrate exceedances, possible \\
\hline $\mathrm{BC}$ & Bear Creek Burial Grounds & GW-653 & $\mathrm{I}$ & Annual & Annual & Low concentration Increasing trend \\
\hline $\mathrm{BC}$ & Exit Pathway - Traverse B & GW-695 & $\mathrm{S}$ & Annual & Annual & Low concentration, Stable trend \\
\hline $\mathrm{BC}$ & Exit Pathway - Traverse B & GW-703 & NT & Annual & Annual & Redundant location \\
\hline $\mathrm{BC}$ & Exit Pathway - Traverse W & GW-715 & $\mathrm{PI}$ & Annual & Annual & Potential Exit Pathway \\
\hline $\mathrm{BC}$ & Exit Pathway - Traverse C & GW-724 & $\mathrm{S}$ & Annual & Annual & Historic exceedance, surveillance well \\
\hline $\mathrm{BC}$ & Exit Pathway - Traverse C & GW-725 & $\mathrm{PI}$ & Annual & Annual & Historic exceedance, surveillance well \\
\hline $\mathrm{BC}$ & Exit Pathway - Traverse C & GW-738 & $\mathrm{D}$ & Annual & Annual & Historic exceedance, surveillance well \\
\hline$B C$ & Exit Pathway - Traverse C & GW-740 & PD & Annual & Annual & Historic exceedance, surveillance well \\
\hline $\mathrm{BC}$ & S-3 Site & GW-829 & $\mathrm{D}$ & Annual & Annual & Decreasing trend, sample annually \\
\hline $\mathrm{BC}$ & $\begin{array}{l}\text { Exit Pathway Spring/Surface } \\
\text { Water }\end{array}$ & SS-1 & $\mathrm{S}$ & Annual & Annual & Spring with Stable trend, sample annually \\
\hline $\mathrm{BC}$ & $\begin{array}{l}\text { Exit Pathway Spring/Surface } \\
\text { Water }\end{array}$ & SS-4 & $\mathrm{D}$ & Annual & Annual & Important locations, Decreasing trend \\
\hline $\mathrm{BC}$ & $\begin{array}{l}\text { Exit Pathway Spring/Surface } \\
\text { Water }\end{array}$ & SS-5 & $\mathrm{S}$ & Annual & Annual & Stable trend, priority location \\
\hline
\end{tabular}


TABLE B.14

SUMMARY SAMPLING RECOMMENDATIONS BEAR CREEK REGIME

Bear Creek Hydrogeologic Regime

Y-12 National Security Complex

Oak Ridge, Tennessee

\begin{tabular}{|c|c|c|c|c|c|c|}
\hline Regime & Functional Area & $\begin{array}{l}\text { Location } \\
\text { Name }\end{array}$ & Trend & $\begin{array}{l}\text { Preliminary } \\
\text { Frequency }\end{array}$ & Final Frequency & Recommendation \\
\hline \multicolumn{7}{|c|}{ Biennial Sampling } \\
\hline $\mathrm{BC}$ & Oil Landfarm WMA & GW-006 & $\mathrm{S}$ & Annual & Biennial & $\begin{array}{l}\text { Low concentration, Stable trend, reduced } \\
\text { frequency }\end{array}$ \\
\hline $\mathrm{BC}$ & Oil Landfarm WMA & GW-010 & $\mathrm{N} / \mathrm{A}$ & Biennial & Biennial & Sample as alternate RCRA well \\
\hline $\mathrm{BC}$ & $\begin{array}{l}\text { Bear Creek Burial Grounds } \\
\text { WMA }\end{array}$ & GW-053 & PD & Annual & Biennial & $\begin{array}{l}\text { Probably Decreasing trend, consider } \\
\text { reducing sample frequency }\end{array}$ \\
\hline $\mathrm{BC}$ & Exit Pathway - Traverse A & GW-056 & $\mathrm{S}$ & Biennial & Biennial & Low concentration, Stable trend \\
\hline $\mathrm{BC}$ & Oil Landfarm WMA & GW-075 & N/A & Biennial & Biennial & $\begin{array}{l}\text { Sample as alternate RCRA well, low } \\
\text { concentrations }\end{array}$ \\
\hline $\mathrm{BC}$ & $\begin{array}{l}\text { Bear Creek Burial Grounds } \\
\text { WMA }\end{array}$ & GW-287 & $\mathrm{S}$ & Annual & Biennial & Low concentration, Stable trend \\
\hline $\mathrm{BC}$ & Exit Pathway - Traverse B & GW-621 & PD & Annual & Biennial & $\begin{array}{l}\text { Low concentration, Probably Decreasing } \\
\text { trend }\end{array}$ \\
\hline $\mathrm{BC}$ & $\begin{array}{l}\text { Bear Creek Burial Grounds } \\
\text { WMA }\end{array}$ & GW-629 & $\mathrm{S}$ & Biennial & Biennial & Low concentration, outliers $1991 ?$ \\
\hline $\mathrm{BC}$ & Exit Pathway - Traverse A & GW-685 & $\mathrm{S}$ & Biennial & Biennial & Low concentration, Stable trend \\
\hline $\mathrm{BC}$ & Exit Pathway - Traverse C & GW-723 & $\mathrm{PI}$ & Annual & Biennial & $\begin{array}{l}\text { Low concentrations, may be Increasing, but } \\
\text { redundant location, reduce frequency }\end{array}$ \\
\hline \multicolumn{7}{|l|}{ Review } \\
\hline $\mathrm{BC}$ & $\begin{array}{l}\text { Bear Creek Burial Grounds } \\
\text { WMA }\end{array}$ & GW-045 & $\mathrm{N} / \mathrm{A}$ & Biennial & Review & $\begin{array}{l}\text { Evaluate well construction, if useful sample } \\
\text { to determine trend, consider retaining as } \\
\text { DTW well }\end{array}$ \\
\hline $\mathrm{BC}$ & $\begin{array}{l}\text { Bear Creek Burial Grounds } \\
\text { WMA }\end{array}$ & GW-052 & $\mathrm{N} / \mathrm{A}$ & Annual & Review & $\begin{array}{l}\text { Historic high uranium concentration, sample } \\
2 \mathrm{X} \text { and review trend }\end{array}$ \\
\hline $\mathrm{BC}$ & Exit Pathway - Traverse A & GW-057 & $\mathrm{N} / \mathrm{A}$ & Remove & Review & $\begin{array}{l}\text { Low concentrations, high priority but } \\
\text { redundant location, consider removing from } \\
\text { program }\end{array}$ \\
\hline $\mathrm{BC}$ & $\begin{array}{l}\text { Bear Creek Burial Grounds } \\
\text { WMA }\end{array}$ & GW-058 & $\mathrm{N} / \mathrm{A}$ & Biennial & Review & $\begin{array}{l}\text { Historic uranium hits, review location, if } \\
\text { redundant remove from program, consider } \\
\text { biennial sampling if unique }\end{array}$ \\
\hline BC & $\begin{array}{l}\text { Bear Creek Burial Grounds } \\
\text { WMA }\end{array}$ & GW-061 & $\mathrm{N} / \mathrm{A}$ & Annual & Review & $\begin{array}{l}\text { Historic high uranium concentration, sample } \\
2 \mathrm{X} \text { and review trend }\end{array}$ \\
\hline $\mathrm{BC}$ & Oil Landfarm WMA & GW-064 & $\mathrm{N} / \mathrm{A}$ & Biennial & Review & $\begin{array}{l}\text { Historic exceedance, review location and } \\
\text { sample or remove }\end{array}$ \\
\hline $\mathrm{BC}$ & Oil Landfarm WMA & GW-066 & $\mathrm{N} / \mathrm{A}$ & SemiAnnual & Review & $\begin{array}{l}\text { Historic exceedance, review location and } \\
\text { sample or remove }\end{array}$ \\
\hline $\mathrm{BC}$ & Oil Landfarm WMA & GW-067 & N/A & Biennial & Review & $\begin{array}{l}\text { Historic exceedance, review location and } \\
\text { sample or remove }\end{array}$ \\
\hline$B C$ & $\begin{array}{l}\text { Bear Creek Burial Grounds } \\
\text { WMA }\end{array}$ & GW-068 & $\mathrm{N} / \mathrm{A}$ & Biennial & Review & $\begin{array}{l}\text { Historic high concentrations, review well } \\
\text { construction and function and sample or } \\
\text { remove from program }\end{array}$ \\
\hline $\mathrm{BC}$ & $\begin{array}{l}\text { Bear Creek Burial Grounds } \\
\text { WMA }\end{array}$ & GW-089 & $\mathrm{N} / \mathrm{A}$ & Biennial & Review & $\begin{array}{l}\text { Evaluate location, consider sampling for } \\
\text { MNA support or removing from program }\end{array}$ \\
\hline $\mathrm{BC}$ & S-3 Site & GW-122 & $\mathrm{N} / \mathrm{A}$ & Annual & Review & $\begin{array}{l}\text { Historic high concentrations of nitrate, } \\
\text { review function and sample } 4 \mathrm{X} \text { in } 10 \mathrm{yrs} \text {, } \\
\text { may be redundant w/GW-127 }\end{array}$ \\
\hline
\end{tabular}


TABLE B.14

SUMMARY SAMPLING RECOMMENDATIONS BEAR CREEK REGIME

Bear Creek Hydrogeologic Regime

Y-12 National Security Complex

Oak Ridge, Tennessee

\begin{tabular}{|c|c|c|c|c|c|c|}
\hline Regime & Functional Area & $\begin{array}{l}\text { Location } \\
\text { Name }\end{array}$ & Trend & $\begin{array}{l}\text { Preliminary } \\
\text { Frequency }\end{array}$ & Final Frequency & Recommendation \\
\hline$B C$ & S-3 Site & GW-123 & N/A & Annual & Review & $\begin{array}{l}\text { Historic gross alpha and beta exceedances, } \\
\text { sample } 4 \mathrm{X} \text { in } 10 \text { yrs and consider removing } \\
\text { from program, may be redundant w/GW- } \\
127\end{array}$ \\
\hline$B C$ & S-3 Site & GW-124 & N/A & SemiAnnual & Review & $\begin{array}{l}\text { Historic beta and nitrate exceedances, } \\
\text { review location and function, sample } 4 X \text { in } \\
10 \text { yrs and consider removing from program }\end{array}$ \\
\hline$B C$ & S-3 Site & GW-125 & N/A & Annual & Review & $\begin{array}{l}\text { Review location and function or well, } \\
\text { historic variance in data, consider removing } \\
\text { from program }\end{array}$ \\
\hline$B C$ & $\begin{array}{l}\text { Bear Creek Burial Grounds } \\
\text { WMA }\end{array}$ & GW-126 & $\mathrm{N} / \mathrm{A}$ & Biennial & Review & $\begin{array}{l}\text { Limited use, consider removing from } \\
\text { program }\end{array}$ \\
\hline$B C$ & Oil Landfarm WMA & GW-227 & N/A & Annual & Review & $\begin{array}{l}\text { Historic exceedance, review location and } \\
\text { sample or remove }\end{array}$ \\
\hline$B C$ & Oil Landfarm WMA & GW-228 & $\mathrm{N} / \mathrm{A}$ & Biennial & Review & $\begin{array}{l}\text { Historic exceedance, review location and } \\
\text { sample or remove }\end{array}$ \\
\hline$B C$ & $\begin{array}{l}\text { Bear Creek Burial Grounds } \\
\text { WMA }\end{array}$ & GW-242 & $\mathrm{N} / \mathrm{A}$ & Annual & Review & $\begin{array}{l}\text { Historic concentrations of vinyl chloride, } \\
\text { could support MNA remedy, consider } \\
\text { annual or biennial sampling }\end{array}$ \\
\hline$B C$ & S-3 Site & GW-244 & N/A & Biennial & Review & $\begin{array}{l}\text { Source area well, may be redundant, } \\
\text { Sample } 4 \mathrm{X} \text { in } 2 \text { to } 4 \text { yrs; historic high } \\
\text { concentrations, consider removing from } \\
\text { program after trend established }\end{array}$ \\
\hline$B C$ & S-3 Site & GW-245 & $\mathrm{N} / \mathrm{A}$ & Biennial & Review & $\begin{array}{l}\text { Source area well, may be redundant, } \\
\text { Sample } 4 \mathrm{X} \text { in } 2 \text { to } 4 \text { yrs; historic high } \\
\text { concentrations, consider removing from } \\
\text { program after trend established }\end{array}$ \\
\hline$B C$ & S-3 Site & GW-247 & $\mathrm{N} / \mathrm{A}$ & Biennial & Review & $\begin{array}{l}\text { Source area well, may be redundant, } \\
\text { Sample } 4 \mathrm{X} \text { in } 2 \text { to } 4 \text { yrs; historic high } \\
\text { concentrations, consider removing from } \\
\text { program after trend established }\end{array}$ \\
\hline $\mathrm{BC}$ & $\begin{array}{l}\text { Bear Creek Burial Grounds } \\
\text { WMA }\end{array}$ & GW-258 & N/A & Remove & Review & $\begin{array}{l}\text { Historic high concentrations, review well } \\
\text { construction and function and sample or } \\
\text { remove from program }\end{array}$ \\
\hline$B C$ & $\begin{array}{l}\text { Bear Creek Burial Grounds } \\
\text { WMA }\end{array}$ & GW-259 & N/A & Annual & Review & $\begin{array}{l}\text { Historic high concentrations, review well } \\
\text { construction and function and sample or } \\
\text { remove from program }\end{array}$ \\
\hline$B C$ & S-3 Site & GW-277 & $\mathrm{N} / \mathrm{A}$ & Annual & Review & $\begin{array}{l}\text { Review condition and location, sample } 4 \times \\
\text { in } 10 \text { yrs or consider retaining as DTW in } \\
\text { program }\end{array}$ \\
\hline $\mathrm{BC}$ & $\begin{array}{l}\text { Bear Creek Burial Grounds } \\
\text { WMA }\end{array}$ & GW-288 & N/A & SemiAnnual & Review & $\begin{array}{l}\text { Historic high concentrations, review well } \\
\text { construction and function and sample or } \\
\text { remove from program }\end{array}$ \\
\hline $\mathrm{BC}$ & Rust Spoil Area & GW-306 & $\mathrm{N} / \mathrm{A}$ & Biennial & Review & $\begin{array}{l}\text { Review condition and location, sample } 4 \text { X } \\
\text { in } 10 \text { yrs or consider retaining as } \\
\text { surveillance midpoint between GW-311 and } \\
\text { GW-724 }\end{array}$ \\
\hline$B C$ & Rust Spoil Area & GW-307 & N/A & Biennial & Review & $\begin{array}{l}\text { Review condition and location, sample } 4 \mathrm{X} \\
\text { in } 10 \text { yrs or consider retaining as DTW in } \\
\text { program }\end{array}$ \\
\hline$B C$ & Rust Spoil Area & GW-308 & N/A & Biennial & Review & $\begin{array}{l}\text { Review condition and location, sample } 4 \mathrm{X} \\
\text { in } 10 \text { yrs or consider retaining as DTW in } \\
\text { program }\end{array}$ \\
\hline BC & Rust Spoil Area & GW-309 & N/A & Biennial & Review & $\begin{array}{l}\text { Review condition and location, sample } 4 \times \\
\text { in } 10 \text { yrs or consider retaining as DTW in } \\
\text { program }\end{array}$ \\
\hline
\end{tabular}


TABLE B.14

SUMMARY SAMPLING RECOMMENDATIONS BEAR CREEK REGIME

Bear Creek Hydrogeologic Regime

Y-12 National Security Complex

Oak Ridge, Tennessee

\begin{tabular}{|c|c|c|c|c|c|c|}
\hline Regime & Functional Area & $\begin{array}{l}\text { Location } \\
\text { Name }\end{array}$ & Trend & $\begin{array}{l}\text { Preliminary } \\
\text { Frequency }\end{array}$ & Final Frequency & Recommendation \\
\hline $\mathrm{BC}$ & Rust Spoil Area & GW-310 & $\mathrm{N} / \mathrm{A}$ & Biennial & Review & $\begin{array}{l}\text { Review condition and location, sample } 4 \mathrm{X} \\
\text { in } 10 \text { yrs or consider retaining as DTW in } \\
\text { program }\end{array}$ \\
\hline $\mathrm{BC}$ & Rust Spoil Area & GW-312 & N/A & Biennial & Review & $\begin{array}{l}\text { Review condition and location, sample } 4 \mathrm{X} \\
\text { in } 10 \text { yrs or consider retaining as DTW in } \\
\text { program }\end{array}$ \\
\hline BC & Spoil Area I & GW-313 & N/A & Annual & Review & $\begin{array}{l}\text { Review condition and location, sample } 4 \times \\
\text { in } 10 \text { yrs or consider retaining as DTW in } \\
\text { program }\end{array}$ \\
\hline $\mathrm{BC}$ & Spoil Area I & GW-314 & $\mathrm{N} / \mathrm{A}$ & Annual & Review & $\begin{array}{l}\text { Review condition and location, sample } 4 \mathrm{X} \\
\text { in } 10 \text { yrs or consider retaining as DTW in } \\
\text { program }\end{array}$ \\
\hline $\mathrm{BC}$ & Spoil Area I & GW-317 & $\mathrm{N} / \mathrm{A}$ & Biennial & Review & $\begin{array}{l}\text { Review condition and location, sample } 4 \mathrm{X} \\
\text { in } 10 \text { yrs or consider retaining as DTW in } \\
\text { program }\end{array}$ \\
\hline $\mathrm{BC}$ & S-3 Site & GW-345 & $\mathrm{N} / \mathrm{A}$ & Annual & Review & $\begin{array}{l}\text { Review condition and location, sample } 4 \times \\
\text { in } 10 \text { yrs or consider retaining as DTW in } \\
\text { program, may be redundant w/GW-526 }\end{array}$ \\
\hline $\mathrm{BC}$ & S-3 Site & GW-346 & $\mathrm{N} / \mathrm{A}$ & SemiAnnual & Review & $\begin{array}{l}\text { Review condition and location, sample } 4 \mathrm{X} \\
\text { in } 10 \text { yrs or consider retaining as DTW in } \\
\text { program, may be redundant w/GW-526 }\end{array}$ \\
\hline $\mathrm{BC}$ & Industrial Landfill I & GW-364 & $\mathrm{N} / \mathrm{A}$ & Annual & Review & $\begin{array}{l}\text { Historic exceedance, review location and } \\
\text { sample or remove }\end{array}$ \\
\hline $\mathrm{BC}$ & Industrial Landfill I & GW-365 & N/A & Annual & Review & $\begin{array}{l}\text { Historic exceedance, review location and } \\
\text { sample or remove }\end{array}$ \\
\hline $\mathrm{BC}$ & Industrial Landfill I & GW-367 & N/A & Biennial & Review & $\begin{array}{l}\text { Historic exceedance, review location and } \\
\text { sample or remove }\end{array}$ \\
\hline$B C$ & Industrial Landfill I & GW-368 & $\mathrm{N} / \mathrm{A}$ & Biennial & Review & $\begin{array}{l}\text { Historic exceedance, review location and } \\
\text { sample or remove }\end{array}$ \\
\hline $\mathrm{BC}$ & Industrial Landfill I & GW-369 & $\mathrm{N} / \mathrm{A}$ & Biennial & Review & $\begin{array}{l}\text { Historic exceedance, review location and } \\
\text { sample or remove }\end{array}$ \\
\hline $\mathrm{BC}$ & Oil Landfarm WMA & GW-601 & $\mathrm{N} / \mathrm{A}$ & Biennial & Review & $\begin{array}{l}\text { Historic exceedance, review location and } \\
\text { sample or remove }\end{array}$ \\
\hline$B C$ & $\begin{array}{l}\text { Bear Creek Burial Grounds } \\
\text { WMA }\end{array}$ & GW-623 & $\mathrm{N} / \mathrm{A}$ & Annual & Review & $\begin{array}{l}\text { Sample annually to confirm trend in deep } \\
\text { area }\end{array}$ \\
\hline$B C$ & $\begin{array}{l}\text { Bear Creek Burial Grounds } \\
\text { WMA }\end{array}$ & GW-624 & $\mathrm{N} / \mathrm{A}$ & SemiAnnual & Review & $\begin{array}{l}\text { Historic high concentrations, review well } \\
\text { construction and function and sample or } \\
\text { remove from program }\end{array}$ \\
\hline $\mathrm{BC}$ & $\begin{array}{l}\text { Bear Creek Burial Grounds } \\
\text { WMA }\end{array}$ & GW-654 & $\mathrm{N} / \mathrm{A}$ & Biennial & Review & $\begin{array}{l}\text { Probably redundant, review location and } \\
\text { consider removal }\end{array}$ \\
\hline$B C$ & Exit Pathway - Traverse W & GW-710 & ND & Biennial & Review & $\begin{array}{l}\text { Historic outlier for nitrate, reduced } \\
\text { frequency or remove from program }\end{array}$ \\
\hline $\mathrm{BC}$ & Exit Pathway - Traverse W & GW-711 & ND & Annual & Review & $\begin{array}{l}\text { Largely non-detect well; however has high } \\
\text { function consider removing from program or } \\
\text { sample at reduced frequency }\end{array}$ \\
\hline $\mathrm{BC}$ & Exit Pathway - Traverse C & GW-736 & $\mathrm{N} / \mathrm{A}$ & SemiAnnual & Review & $\begin{array}{l}\text { Historic exceedance, review location and } \\
\text { sample or remove }\end{array}$ \\
\hline $\mathrm{BC}$ & Exit Pathway - Traverse C & GW-737 & $\mathrm{N} / \mathrm{A}$ & SemiAnnual & Review & $\begin{array}{l}\text { Historic exceedance, review location and } \\
\text { sample or remove }\end{array}$ \\
\hline$B C$ & Exit Pathway - Traverse C & GW-739 & $\mathrm{N} / \mathrm{A}$ & SemiAnnual & Review & $\begin{array}{l}\text { Historic exceedance, review location and } \\
\text { sample or remove }\end{array}$ \\
\hline $\mathrm{BC}$ & $\begin{array}{l}\text { Above Grade Low Level } \\
\text { Waste Storage Fac. }\end{array}$ & GW-795 & $\mathrm{N} / \mathrm{A}$ & Biennial & Review & $\begin{array}{l}\text { Low to non-detect, consider removing from } \\
\text { program or reduced frequency }\end{array}$ \\
\hline$B C$ & $\begin{array}{l}\text { Exit Pathway Spring/Surface } \\
\text { Water }\end{array}$ & SS-5.95KM & $\mathrm{N} / \mathrm{A}$ & Annual & Review & $\begin{array}{l}\text { Review location of spring, if sampling is } \\
\text { redundant w/other locations, remove from } \\
\text { program }\end{array}$ \\
\hline
\end{tabular}


TABLE B.14

SUMMARY SAMPLING RECOMMENDATIONS BEAR CREEK REGIME

Bear Creek Hydrogeologic Regime

Y-12 National Security Complex

Oak Ridge, Tennessee

\begin{tabular}{|c|c|c|c|c|c|c|}
\hline Regime & Functional Area & $\begin{array}{l}\text { Location } \\
\text { Name }\end{array}$ & Trend & $\begin{array}{l}\text { Preliminary } \\
\text { Frequency }\end{array}$ & Final Frequency & Recommendation \\
\hline \multicolumn{7}{|c|}{ Remove from Routine Monitoring } \\
\hline $\mathrm{BC}$ & Oil Landfarm WMA & GW-013 & N/A & Remove & Remove & Keep as DTW well \\
\hline$B C$ & $\begin{array}{l}\text { Bear Creek Burial Grounds } \\
\text { WMA }\end{array}$ & GW-018 & N/A & Remove & Remove & Keep as DTW well \\
\hline $\mathrm{BC}$ & $\begin{array}{l}\text { Bear Creek Burial Grounds } \\
\text { WMA }\end{array}$ & GW-047 & N/A & Remove & Remove & Keep as DTW well \\
\hline $\mathrm{BC}$ & $\begin{array}{l}\text { Bear Creek Burial Grounds } \\
\text { WMA }\end{array}$ & GW-054 & N/A & Remove & Remove & Keep as DTW well \\
\hline $\mathrm{BC}$ & $\begin{array}{l}\text { Bear Creek Burial Grounds } \\
\text { WMA }\end{array}$ & GW-072 & N/A & Remove & Remove & Keep as DTW well \\
\hline $\mathrm{BC}$ & Oil Landfarm WMA & GW-073 & ND & Biennial & Remove & Keep as DTW well \\
\hline $\mathrm{BC}$ & Oil Landfarm WMA & GW-074 & ND & Biennial & Remove & Keep as DTW well \\
\hline $\mathrm{BC}$ & $\begin{array}{l}\text { Bear Creek Burial Grounds } \\
\text { WMA }\end{array}$ & GW-083 & N/A & Biennial & Remove & Keep as DTW well \\
\hline $\mathrm{BC}$ & Oil Landfarm WMA & GW-084 & $\mathrm{PI}$ & Biennial & Remove & Keep as DTW well \\
\hline $\mathrm{BC}$ & Oil Landfarm WMA & GW-086 & N/A & Remove & Remove & Keep as DTW well \\
\hline $\mathrm{BC}$ & $\begin{array}{l}\text { Bear Creek Burial Grounds } \\
\text { WMA }\end{array}$ & GW-091 & N/A & Annual & Remove & Keep as DTW well \\
\hline $\mathrm{BC}$ & $\begin{array}{l}\text { Bear Creek Burial Grounds } \\
\text { WMA }\end{array}$ & GW-094 & N/A & Remove & Remove & Keep as DTW well \\
\hline $\mathrm{BC}$ & $\begin{array}{l}\text { Bear Creek Burial Grounds } \\
\text { WMA }\end{array}$ & GW-095 & N/A & Remove & Remove & Keep as DTW well \\
\hline $\mathrm{BC}$ & Oil Landfarm WMA & GW-097 & N/A & Biennial & Remove & Keep as DTW well \\
\hline $\mathrm{BC}$ & S-3 Site & GW-115 & NT & Annual & Remove & Keep as DTW well \\
\hline $\mathrm{BC}$ & Oil Landfarm WMA & GW-120 & ND & Remove & Remove & Keep as DTW well \\
\hline $\mathrm{BC}$ & $\begin{array}{l}\text { Bear Creek Burial Grounds } \\
\text { WMA }\end{array}$ & GW-237 & N/A & Biennial & Remove & Keep as DTW well \\
\hline BC & $\begin{array}{l}\text { Bear Creek Burial Grounds } \\
\text { WMA }\end{array}$ & GW-248 & N/A & Remove & Remove & Keep as DTW well \\
\hline $\mathrm{BC}$ & $\begin{array}{l}\text { Bear Creek Burial Grounds } \\
\text { WMA }\end{array}$ & GW-249 & N/A & Remove & Remove & Keep as DTW well \\
\hline $\mathrm{BC}$ & $\begin{array}{l}\text { Bear Creek Burial Grounds } \\
\text { WMA }\end{array}$ & GW-250 & N/A & Remove & Remove & Keep as DTW well \\
\hline BC & $\begin{array}{l}\text { Bear Creek Burial Grounds } \\
\text { WMA }\end{array}$ & GW-286 & N/A & Remove & Remove & Keep as DTW well \\
\hline $\mathrm{BC}$ & $\begin{array}{l}\text { Bear Creek Burial Grounds } \\
\text { WMA }\end{array}$ & GW-290 & N/A & Biennial & Remove & Keep as DTW well \\
\hline $\mathrm{BC}$ & Spoil Area I & GW-316 & N/A & Biennial & Remove & Keep as DTW well \\
\hline $\mathrm{BC}$ & Spoil Area I & GW-323 & N/A & Biennial & Remove & Keep as DTW well \\
\hline $\mathrm{BC}$ & S-3 Site & GW-325 & N/A & Biennial & Remove & Keep as DTW well \\
\hline$B C$ & S-3 Site & GW-347 & $\mathrm{N} / \mathrm{A}$ & Biennial & Remove & Keep as DTW well \\
\hline $\mathrm{BC}$ & S-3 Site & GW-348 & N/A & Biennial & Remove & Keep as DTW well \\
\hline $\mathrm{BC}$ & Industrial Landfill I & GW-366 & $\mathrm{N} / \mathrm{A}$ & Remove & Remove & Keep as DTW well \\
\hline
\end{tabular}


TABLE B.14

SUMMARY SAMPLING RECOMMENDATIONS BEAR CREEK REGIME

Bear Creek Hydrogeologic Regime

Y-12 National Security Complex

Oak Ridge, Tennessee

\begin{tabular}{|c|c|c|c|c|c|c|}
\hline Regime & Functional Area & $\begin{array}{l}\text { Location } \\
\text { Name }\end{array}$ & Trend & $\begin{array}{l}\text { Preliminary } \\
\text { Frequency }\end{array}$ & Final Frequency & Recommendation \\
\hline $\mathrm{BC}$ & $\begin{array}{l}\text { Bear Creek Burial Grounds } \\
\text { WMA }\end{array}$ & GW-370 & N/A & Biennial & Remove & Keep as DTW well \\
\hline $\mathrm{BC}$ & $\begin{array}{l}\text { Bear Creek Burial Grounds } \\
\text { WMA }\end{array}$ & GW-372 & N/A & Remove & Remove & Keep as DTW well \\
\hline$B C$ & $\begin{array}{l}\text { Bear Creek Burial Grounds } \\
\text { WMA }\end{array}$ & GW-375 & $\mathrm{N} / \mathrm{A}$ & Remove & Remove & Keep as DTW well \\
\hline $\mathrm{BC}$ & Industrial Landfill I & GW-520 & N/A & Remove & Remove & Keep as DTW well \\
\hline $\mathrm{BC}$ & Lysimeter Demo & GW-531 & N/A & Biennial & Remove & Keep as DTW well \\
\hline $\mathrm{BC}$ & S-3 Site & GW-613 & N/A & Biennial & Remove & Keep as DTW well \\
\hline$B C$ & $\begin{array}{l}\text { Bear Creek Burial Grounds } \\
\text { WMA }\end{array}$ & GW-622 & N/A & Remove & Remove & Keep as DTW well \\
\hline $\mathrm{BC}$ & Lysimeter Demo & GW-630 & N/A & Biennial & Remove & Keep as DTW well \\
\hline $\mathrm{BC}$ & Oil Landfarm WMA & GW-636 & $\mathrm{N} / \mathrm{A}$ & Remove & Remove & Keep as DTW well \\
\hline $\mathrm{BC}$ & Oil Landfarm WMA & GW-637 & N/A & Remove & Remove & Keep as DTW well \\
\hline $\mathrm{BC}$ & Oil Landfarm WMA & GW-638 & N/A & Remove & Remove & Keep as DTW well \\
\hline $\mathrm{BC}$ & $\begin{array}{l}\text { Bear Creek Burial Grounds } \\
\text { WMA }\end{array}$ & GW-641 & N/A & Remove & Remove & Keep as DTW well \\
\hline$B C$ & $\begin{array}{l}\text { Bear Creek Burial Grounds } \\
\text { WMA }\end{array}$ & GW-642 & ND & Remove & Remove & Keep as DTW well \\
\hline $\mathrm{BC}$ & Oil Landfarm WMA & GW-645 & N/A & Biennial & Remove & Keep as DTW well \\
\hline $\mathrm{BC}$ & Oil Landfarm WMA & GW-646 & $\mathrm{N} / \mathrm{A}$ & Biennial & Remove & Keep as DTW well \\
\hline $\mathrm{BC}$ & Rust Spoil Area & GW-648 & N/A & Biennial & Remove & Keep as DTW well \\
\hline $\mathrm{BC}$ & $\begin{array}{l}\text { Bear Creek Burial Grounds } \\
\text { WMA }\end{array}$ & GW-651 & N/A & Remove & Remove & Keep as DTW well \\
\hline $\mathrm{BC}$ & $\begin{array}{l}\text { Bear Creek Burial Grounds } \\
\text { WMA }\end{array}$ & GW-652 & N/A & Remove & Remove & Keep as DTW well \\
\hline BC & $\begin{array}{l}\text { Above Grade Low Level } \\
\text { Waste Storage Fac. }\end{array}$ & GW-794 & N/A & Biennial & Remove & Keep as DTW well \\
\hline $\mathrm{BC}$ & Oil Landfarm WMA & GW-800 & N/A & Biennial & Remove & Keep as DTW well \\
\hline
\end{tabular}

Notes

1. Final Frequency for 183 locations:

Regulated $=$ Sampling location and frequency covered under RCRA post-closure permit, CERCLA interim/rinal ROD or other regulatory program

Semi-annual = Locations recommended for semi-annual sampling (every 6 months) based on trend, exceedance of MCL and location in network.

Annual = Locations recommended for annual sampling (once per year) based on trend, exceedance of MCL and location in network.

Biennial = Wells recommended for Biennial sampling (once every 2 years) based on trend, exceedance of MCL and location in network.

Review = Review status of well, if well is in good working order, re-evaluated concentration trend for 4 sample events after 1996.

If concentration trends are Stable to Decreasing, consider removing the well from routine monitoring program.

Remove $=$ Well does not provide significant data in support of monitoring objectives.

2. Well GW-012 is listed as a RCRA permit well (Oil Landfarm WMA), but no data were available in the database to evaluate this location. 
TABLE B.15

SUMMARY SAMPLING RECOMMENDATIONS EAST FORK REGIME

East Fork Poplar Creek Hydrogeologic Regime

Y-12 National Security Complex

Oak Ridge, Tennessee

\begin{tabular}{|c|c|c|c|c|c|c|}
\hline Regime & Functional Area & $\begin{array}{l}\text { Location } \\
\text { Name }\end{array}$ & Trend & $\begin{array}{c}\text { Preliminary } \\
\text { Frequency }\end{array}$ & $\begin{array}{l}\text { Final } \\
\text { Frequency }\end{array}$ & Recommendation \\
\hline \multicolumn{7}{|c|}{ Regulated Sample Locations } \\
\hline EF & S-3 Site & GW-108 & NT & SemiAnnual & Regulated & Sample as per permit \\
\hline EF & New Hope Pond & GW-151 & 1 & SemiAnnual & Regulated & Sample as per ROD \\
\hline EF & New Hope Pond & GW-154 & 1 & SemiAnnual & Regulated & Sample as per ROD \\
\hline EF & Union Valley - Exit Pathway & GW-169 & NT & Biennial & Regulated & Sample as per ROD \\
\hline EF & Union Valley - Exit Pathway & GW-170 & $\mathrm{D}$ & Annual & Regulated & Sample as per ROD \\
\hline EF & Union Valley - Exit Pathway & GW-171 & NT & Biennial & Regulated & Sample as per ROD \\
\hline EF & Union Valley - Exit Pathway & GW-172 & NT & Annual & Regulated & Sample as per ROD \\
\hline EF & Y-12 Plant Site & GW-193 & $\mathrm{D}$ & Biennial & Regulated & Sample as per permit \\
\hline EF & Uranium Oxide Vault & GW-219 & $\mathrm{D}$ & Annual & Regulated & Sample as per ROD \\
\hline EF & New Hope Pond & GW-220 & 1 & SemiAnnual & Regulated & Sample as per ROD \\
\hline EF & New Hope Pond & GW-223 & $\mathrm{D}$ & Annual & Regulated & Sample as per ROD \\
\hline EF & Union Valley - Exit Pathway & GW-230 & NT & Annual & Regulated & Sample as per ROD \\
\hline EF & Union Valley - Exit Pathway & GW-232 & NT & Biennial & Regulated & Sample as per ROD \\
\hline EF & S-2 Site & GW-253 & 1 & SemiAnnual & Regulated & $\begin{array}{l}\text { Semi-annual sampling for Increasing } \\
\text { cadmium trend, or as per ROD }\end{array}$ \\
\hline EF & Y-12 Fuel Station & GW-281 & N/A & Remove & Regulated & Sample as per ROD \\
\hline EF & New Hope Pond & GW-380 & NT & Annual & Regulated & Sample as per ROD \\
\hline EF & New Hope Pond & GW-381 & NT & Annual & Regulated & Sample as per ROD \\
\hline EF & New Hope Pond & GW-382 & $\mathrm{D}$ & Annual & Regulated & Sample as per ROD \\
\hline EF & New Hope Pond & GW-383 & NT & SemiAnnual & Regulated & Sample as per ROD \\
\hline EF & Exit Pathway - Traverse I & GW-605 & $\mathrm{D}$ & Annual & Regulated & Sample as per permit or as per ROD \\
\hline EF & Exit Pathway - Traverse I & GW-606 & $\mathrm{D}$ & Annual & Regulated & Sample as per permit or as per ROD \\
\hline EF & Exit Pathway - Traverse E & GW-618 & $\mathrm{D}$ & Annual & Regulated & Sample as per ROD \\
\hline EF & Y-12 Fuel Station & GW-658 & NT & SemiAnnual & Regulated & Sample as per ROD \\
\hline EF & Exit Pathway - Traverse J & GW-733 & $\mathrm{D}$ & Annual & Regulated & Sample as per permit \\
\hline EF & Y-12 Grid Well K1 & GW-744 & $\mathrm{S}$ & Biennial & Regulated & Sample as per ROD \\
\hline EF & Y-12 Grid Well K2 & GW-747 & $\mathrm{PI}$ & Biennial & Regulated & Sample as per ROD \\
\hline EF & Y-12 Grid Well J-Primary & GW-762 & 1 & SemiAnnual & Regulated & Sample as per ROD \\
\hline EF & Y-12 Fuel Station & GW-802 & ND & Biennial & Regulated & Sample as per ROD \\
\hline EF & Road/Pine Ridge & GW-816 & $\mathrm{S}$ & Biennial & Regulated & Sample as per ROD \\
\hline EF & New Hope Pond & GW-832 & $\mathrm{D}$ & Annual & Regulated & Sample as per ROD \\
\hline EF & Union Valley - Exit Pathway & SCR7.1SP & $\mathrm{D}$ & Biennial & Regulated & Sample as per ROD \\
\hline EF & $\begin{array}{l}\text { Exit Pathway Spring/Surface } \\
\text { Water }\end{array}$ & SCR7.8SP & $\mathrm{D}$ & Biennial & Regulated & Sample as per ROD \\
\hline EF & $\begin{array}{l}\text { Exit Pathway Spring/Surface } \\
\text { Water }\end{array}$ & UEFPC-SP17 & N/A & Annual & Regulated & Sample as per permit \\
\hline
\end{tabular}


TABLE B.15

SUMMARY SAMPLING RECOMMENDATIONS EAST FORK REGIME

East Fork Poplar Creek Hydrogeologic Regime

Y-12 National Security Complex

Oak Ridge, Tennessee

\begin{tabular}{|c|c|c|c|c|c|c|}
\hline Regime & Functional Area & $\begin{array}{l}\text { Location } \\
\text { Name }\end{array}$ & Trend & $\begin{array}{l}\text { Preliminary } \\
\text { Frequency }\end{array}$ & $\begin{array}{l}\text { Final } \\
\text { Frequency }\end{array}$ & Recommendation \\
\hline \multicolumn{7}{|c|}{ Semi-Annual Sampling } \\
\hline EF & Y-12 Grid Well B3 & $55-2 B$ & $\mathrm{PI}$ & SemiAnnual & SemiAnnual & $\begin{array}{l}\text { SemiAnnual sampling due to Increasing VOC } \\
\text { trend in area }\end{array}$ \\
\hline EF & S-3 Site & GW-109 & NT & SemiAnnual & SemiAnnual & $\begin{array}{l}\text { Semi-annual sampling, provides vertical data } \\
\text { near GW-108 }\end{array}$ \\
\hline EF & Building 8110 & GW-698 & NT & SemiAnnual & SemiAnnual & $\begin{array}{l}\text { Centerline of Maynardville Limestone, } \\
\text { monitors VOC plume }\end{array}$ \\
\hline EF & Y-12 Grid Well G3 & GW-769 & $\mathrm{I}$ & SemiAnnual & SemiAnnual & $\begin{array}{l}\text { Increasing trend, Semi-annual monitoring } \\
\text { recommended until trend stabilizes }\end{array}$ \\
\hline EF & Y-12 Grid Well G3 & GW-770 & 1 & SemiAnnual & SemiAnnual & $\begin{array}{l}\text { Increasing trend, Semi-annual monitoring } \\
\text { recommended until trend stabilizes }\end{array}$ \\
\hline EF & Building 9201-2 & GW-820 & NT & SemiAnnual & SemiAnnual & $\begin{array}{l}\text { Centerline of Maynardville Limestone, } \\
\text { monitors VOC plume }\end{array}$ \\
\hline \multicolumn{7}{|c|}{ Annual Sampling } \\
\hline EF & Y-12 Grid Well B3 & $55-2 C$ & $\mathrm{~S}$ & Annual & Annual & Sample Annually for changes in VOC plume \\
\hline EF & Y-12 Grid Well C3 & $56-2 \mathrm{C}$ & $\mathrm{S}$ & Annual & Annual & $\begin{array}{l}\text { Sample Annually to monitor PCE in center of } \\
\text { Complex }\end{array}$ \\
\hline EF & S-3 Site & GW-105 & $\mathrm{N} / \mathrm{A}$ & SemiAnnual & Annual & $\begin{array}{l}\text { Sample Annually until sufficient data to } \\
\text { evaluate trend, consider reducing schedule to } \\
\text { biennial thereafter }\end{array}$ \\
\hline EF & S-3 Site & GW-106 & $\mathrm{N} / \mathrm{A}$ & SemiAnnual & Annual & $\begin{array}{l}\text { Sample Annually until sufficient data to } \\
\text { evaluate trend, consider reducing schedule to } \\
\text { biennial thereafter }\end{array}$ \\
\hline EF & New Hope Pond & GW-153 & $\mathrm{S}$ & Annual & Annual & Historic exceedance, sample Annually \\
\hline EF & Beta-4 Security Pits & GW-192 & NT & Annual & Annual & $\begin{array}{l}\text { Monitor to delineate northern plume, data } \\
\text { support MNA }\end{array}$ \\
\hline EF & New Hope Pond & GW-222 & $\mathrm{S}$ & Annual & Annual & Historic exceedance, sample Annually \\
\hline EF & New Hope Pond & GW-240 & $\mathrm{S}$ & Annual & Annual & Historic exceedance, sample Annually \\
\hline EF & S-2 Site & GW-251 & $\mathrm{S}$ & Annual & Annual & $\begin{array}{l}\text { Centerline of Maynardville Limestone, } \\
\text { supports data from GW-253 on VOC plume }\end{array}$ \\
\hline EF & Y-12 Salvage Yard & GW-274 & PD & Annual & Annual & Annual sampling for high nitrate \\
\hline EF & Y-12 Salvage Yard & GW-275 & $\mathrm{S}$ & Annual & Annual & Annual sampling for high nitrate \\
\hline EF & $\begin{array}{l}\text { Waste Coolant Processing } \\
\text { Facility }\end{array}$ & GW-332 & N/A & Annual & Annual & $\begin{array}{l}\text { Sample Annually until sufficient data to } \\
\text { evaluate trend, consider reducing schedule to } \\
\text { biennial thereafter }\end{array}$ \\
\hline EF & $\begin{array}{l}\text { Waste Coolant Processing } \\
\text { Facility }\end{array}$ & GW-336 & N/A & Annual & Annual & $\begin{array}{l}\text { Sample Annually until sufficient data to } \\
\text { evaluate trend, consider reducing schedule to } \\
\text { biennial thereafter }\end{array}$ \\
\hline EF & $\begin{array}{l}\text { Waste Coolant Processing } \\
\text { Facility }\end{array}$ & GW-337 & $\mathrm{S}$ & Annual & Annual & Historic high TCE concentrations \\
\hline EF & Fire Training Facility & GW-620 & $\mathrm{D}$ & Annual & Annual & $\begin{array}{l}\text { Historic high VOC concentrations, Decreasing } \\
\text { trend, sample Annually }\end{array}$ \\
\hline EF & Y-12 Plant Site & GW-656 & $\mathrm{S}$ & Annual & Annual & $\begin{array}{l}\text { Sample Annually, historic high TCE } \\
\text { concentrations }\end{array}$ \\
\hline EF & Coal Pile Trench & GW-690 & $\mathrm{S}$ & Annual & Annual & $\begin{array}{l}\text { Sample Annually to monitor PCE in center of } \\
\text { Complex }\end{array}$ \\
\hline EF & Coal Pile Trench & GW-691 & $\mathrm{N} / \mathrm{A}$ & SemiAnnual & Annual & $\begin{array}{l}\text { Sample Annually to monitor PCE in center of } \\
\text { Complex }\end{array}$ \\
\hline EF & Building 8110 & GW-700 & PD & Annual & Annual & $\begin{array}{l}\text { Sample Annually to monitor PCE in center of } \\
\text { Complex }\end{array}$ \\
\hline
\end{tabular}


TABLE B.15

SUMMARY SAMPLING RECOMMENDATIONS EAST FORK REGIME

East Fork Poplar Creek Hydrogeologic Regime

Y-12 National Security Complex

Oak Ridge, Tennessee

\begin{tabular}{|c|c|c|c|c|c|c|}
\hline Regime & Functional Area & $\begin{array}{l}\text { Location } \\
\text { Name }\end{array}$ & Trend & $\begin{array}{l}\text { Preliminary } \\
\text { Frequency }\end{array}$ & $\begin{array}{c}\text { Final } \\
\text { Frequency }\end{array}$ & Recommendation \\
\hline EF & Y-12 Grid Well J-Primary & GW-763 & NT & Annual & Annual & Trend consistent with Annual sampling \\
\hline EF & Y-12 Grid Well E3 & GW-781 & $\mathrm{PI}$ & Annual & Annual & $\begin{array}{l}\text { Sample Annually to monitor PCE in center of } \\
\text { Complex }\end{array}$ \\
\hline EF & Y-12 Grid Well E3 & GW-782 & $\mathrm{D}$ & Annual & Annual & $\begin{array}{l}\text { Sample Annually to monitor PCE in center of } \\
\text { Complex }\end{array}$ \\
\hline EF & Y-12 Grid Well E3 & GW-783 & $\mathrm{S}$ & Annual & Annual & $\begin{array}{l}\text { Sample Annually to monitor PCE in center of } \\
\text { Complex }\end{array}$ \\
\hline EF & Y-12 Grid Well D2 & GW-791 & $\mathrm{S}$ & Annual & Annual & $\begin{array}{l}\text { Sample Annually to monitor PCE in center of } \\
\text { Complex }\end{array}$ \\
\hline EF & Y-12 Grid Well D2 & GW-792 & $\mathrm{S}$ & Annual & Annual & $\begin{array}{l}\text { Sample Annually to monitor PCE in center of } \\
\text { Complex }\end{array}$ \\
\hline \multicolumn{7}{|c|}{ Biennial Sampling } \\
\hline EF & Y-12 Plant Site & GW-204 & PD & Biennial & Biennial & $\begin{array}{l}\text { Sample Biennially, monitor trend for Gross } \\
\text { Alpha }\end{array}$ \\
\hline EF & $\begin{array}{l}\text { Exit Pathway Scarboro } \\
\text { Road/Pine Ridge }\end{array}$ & GW-207 & $\mathrm{S}$ & Biennial & Biennial & $\begin{array}{l}\text { Priority location, low to non-detect } \\
\text { concentrations, sample Biennially }\end{array}$ \\
\hline EF & $\begin{array}{l}\text { Exit Pathway Scarboro } \\
\text { Road/Pine Ridge }\end{array}$ & GW-208 & $\mathrm{S}$ & Biennial & Biennial & $\begin{array}{l}\text { Priority location, low to non-detect } \\
\text { concentrations, sample Biennially }\end{array}$ \\
\hline EF & Exit Pathway - Traverse E & GW-617 & NT & Annual & Biennial & $\begin{array}{l}\text { Sample for VOC to confirm Stable to } \\
\text { Decreasing long-term trend }\end{array}$ \\
\hline EF & Fire Training Facility & GW-619 & $\mathrm{S}$ & Annual & Biennial & $\begin{array}{l}\text { Sample occasionally to provide vertical } \\
\text { information near GW-620 }\end{array}$ \\
\hline EF & Rust Garage Area & GW-631 & ND & Annual & Biennial & $\begin{array}{l}\text { Priority location, reduced frequency } \\
\text { appropriate due to low concentrations and } \\
\text { nearby wells }\end{array}$ \\
\hline EF & Rust Garage Area & GW-633 & $\mathrm{D}$ & Annual & Biennial & $\begin{array}{l}\text { Priority location, reduced frequency } \\
\text { appropriate due to Decreasing concentrations } \\
\text { and nearby wells }\end{array}$ \\
\hline EF & Exit Pathway - Traverse J & GW-735 & $\mathrm{S}$ & Annual & Biennial & $\begin{array}{l}\text { Priority location, low to non-detect } \\
\text { concentrations, sample Biennially }\end{array}$ \\
\hline EF & Y-12 Grid Well K2 & GW-748 & N/A & Biennial & Biennial & $\begin{array}{l}\text { Priority location but very low to ND } \\
\text { concentrations }\end{array}$ \\
\hline EF & Y-12 Grid Well K2 & GW-749 & $\mathrm{S}$ & Biennial & Biennial & $\begin{array}{l}\text { Priority location but very low to ND } \\
\text { concentrations }\end{array}$ \\
\hline EF & Exit Pathway - Traverse J & GW-750 & NT & Biennial & Biennial & $\begin{array}{l}\text { Priority location, low to non-detect } \\
\text { concentrations, sample Biennially }\end{array}$ \\
\hline EF & Y-12 Grid Well E1 & GW-765 & $\mathrm{N} / \mathrm{A}$ & Biennial & Biennial & $\begin{array}{l}\text { Occasional sampling to delineate northern } \\
\text { extent of affected groundwater }\end{array}$ \\
\hline EF & Y-12 Grid Well H3 & GW-775 & NT & Annual & Biennial & $\begin{array}{l}\text { Sample Biennially until location statistically } \\
\text { below detection limits for TCE }\end{array}$ \\
\hline EF & Y-12 Grid Well H3 & GW-776 & $\mathrm{S}$ & Annual & Biennial & $\begin{array}{l}\text { Sample Biennially until location statistically } \\
\text { below detection limits for TCE }\end{array}$ \\
\hline EF & Y-12 Grid Well K3 & GW-817 & $\mathrm{S}$ & Biennial & Biennial & $\begin{array}{l}\text { Priority location but very low to ND } \\
\text { concentrations }\end{array}$ \\
\hline EF & $\begin{array}{l}\text { Exit Pathway Spring/Surface } \\
\text { Water }\end{array}$ & NHPCEMSP & N/A & Biennial & Biennial & $\begin{array}{l}\text { Sample Biennially, may reduce frequency } \\
\text { after trend is determined }\end{array}$ \\
\hline EF & Union Valley - Exit Pathway & SCR7.18SP & $\mathrm{S}$ & Biennial & Biennial & $\begin{array}{l}\text { Priority location, low to non-detect } \\
\text { concentrations, limited monitoring schedule } \\
\text { recommended }\end{array}$ \\
\hline
\end{tabular}


TABLE B.15

SUMMARY SAMPLING RECOMMENDATIONS EAST FORK REGIME

East Fork Poplar Creek Hydrogeologic Regime

Y-12 National Security Complex

Oak Ridge, Tennessee

\begin{tabular}{|c|c|c|c|c|c|c|}
\hline Regime & Functional Area & $\begin{array}{l}\text { Location } \\
\text { Name }\end{array}$ & Trend & $\begin{array}{l}\text { Preliminary } \\
\text { Frequency }\end{array}$ & $\begin{array}{l}\text { Final } \\
\text { Frequency }\end{array}$ & Recommendation \\
\hline \multicolumn{7}{|l|}{ Review } \\
\hline EF & Y-12 Grid Well B2 & $55-1 \mathrm{~A}$ & $\mathrm{~N} / \mathrm{A}$ & Annual & Review & $\begin{array}{l}\text { Sample until } 4 \text { recent sample events provide } \\
\text { data to determine trend, consider removing } \\
\text { from program }\end{array}$ \\
\hline EF & Y-12 Plant Site & $55-1 B$ & $\mathrm{~N} / \mathrm{A}$ & Annual & Review & $\begin{array}{l}\text { Sample until } 4 \text { recent sample events provide } \\
\text { data to determine trend, consider removing } \\
\text { from program }\end{array}$ \\
\hline EF & Y-12 Grid Well C2 & $55-6 \mathrm{~A}$ & $\mathrm{~N} / \mathrm{A}$ & Annual & Review & $\begin{array}{l}\text { Sample until } 4 \text { recent sample events provide } \\
\text { data to determine trend, consider removing } \\
\text { from program }\end{array}$ \\
\hline EF & Y-12 Grid Well C3 & $56-2 \mathrm{~A}$ & $\mathrm{~N} / \mathrm{A}$ & SemiAnnual & Review & $\begin{array}{l}\text { Sample until } 4 \text { recent sample events provide } \\
\text { data to determine trend, consider removing } \\
\text { from program }\end{array}$ \\
\hline EF & Y-12 Grid Well C3 & $56-2 \mathrm{~B}$ & $\mathrm{~N} / \mathrm{A}$ & SemiAnnual & Review & $\begin{array}{l}\text { Sample until } 4 \text { recent sample events provide } \\
\text { data to determine trend, consider removing } \\
\text { from program }\end{array}$ \\
\hline EF & Y-12 Plant Site & 9201-3C-4SP & $\mathrm{N} / \mathrm{A}$ & SemiAnnual & Review & $\begin{array}{l}\text { Sample until } 4 \text { recent sample events provide } \\
\text { data to determine trend, consider removing } \\
\text { from program }\end{array}$ \\
\hline EF & Y-12 Plant Site & GW-190 & ND & Biennial & Review & $\begin{array}{l}\text { Sample occasionally (Biennially or less } \\
\text { frequently) to confirm concentrations below } \\
\text { MCLs, monitor metal concentrations }\end{array}$ \\
\hline EF & Beta-4 Security Pits & GW-195 & $\mathrm{N} / \mathrm{A}$ & Annual & Review & $\begin{array}{l}\text { Sample until } 4 \text { recent sample events provide } \\
\text { data to determine trend, consider removing } \\
\text { from program }\end{array}$ \\
\hline EF & Beta-4 Security Pits & GW-196 & $\mathrm{N} / \mathrm{A}$ & Biennial & Review & $\begin{array}{l}\text { Evaluate function in network, consider } \\
\text { removing from program }\end{array}$ \\
\hline EF & Beta-4 Security Pits & GW-197 & $\mathrm{N} / \mathrm{A}$ & Biennial & Review & $\begin{array}{l}\text { Evaluate function in network, consider } \\
\text { removing from program }\end{array}$ \\
\hline EF & Y-12 Grid Well A2 & GW-263 & $\mathrm{N} / \mathrm{A}$ & Biennial & Review & $\begin{array}{l}\text { Sample until } 4 \text { recent sample events provide } \\
\text { data to determine trend, consider removing } \\
\text { from program }\end{array}$ \\
\hline EF & Y-12 Salvage Yard & GW-265 & N/A & Biennial & Review & $\begin{array}{l}\text { Evaluate function in network, consider } \\
\text { removing from program }\end{array}$ \\
\hline EF & Y-12 Salvage Yard & GW-269 & $\mathrm{N} / \mathrm{A}$ & Annual & Review & $\begin{array}{l}\text { Sample until } 4 \text { recent sample events provide } \\
\text { data to determine trend, consider removing } \\
\text { from program }\end{array}$ \\
\hline EF & Y-12 Salvage Yard & GW-270 & $\mathrm{N} / \mathrm{A}$ & SemiAnnual & Review & $\begin{array}{l}\text { Sample until } 4 \text { recent sample events provide } \\
\text { data to determine trend, consider removing } \\
\text { from program }\end{array}$ \\
\hline EF & Y-12 Salvage Yard & GW-271 & $\mathrm{N} / \mathrm{A}$ & Annual & Review & $\begin{array}{l}\text { Evaluate function of well, consider removing } \\
\text { from program }\end{array}$ \\
\hline
\end{tabular}


TABLE B.15

SUMMARY SAMPLING RECOMMENDATIONS EAST FORK REGIME

East Fork Poplar Creek Hydrogeologic Regime

Y-12 National Security Complex

Oak Ridge, Tennessee

\begin{tabular}{|c|c|c|c|c|c|c|}
\hline Regime & Functional Area & $\begin{array}{l}\text { Location } \\
\text { Name }\end{array}$ & Trend & $\begin{array}{c}\text { Preliminary } \\
\text { Frequency }\end{array}$ & $\begin{array}{c}\text { Final } \\
\text { Frequency }\end{array}$ & Recommendation \\
\hline EF & Y-12 Salvage Yard & GW-272 & N/A & Annual & Review & $\begin{array}{l}\text { Sample until } 4 \text { recent sample events provide } \\
\text { data to determine trend, consider removing } \\
\text { from program }\end{array}$ \\
\hline EF & Y-12 Salvage Yard & GW-273 & N/A & Annual & Review & $\begin{array}{l}\text { Sample until } 4 \text { recent sample events (for } \\
\text { nitrate) provide data to determine trend, } \\
\text { consider removing from program }\end{array}$ \\
\hline EF & S-2 Site & GW-349 & N/A & Annual & Review & $\begin{array}{l}\text { Sample until } 4 \text { recent sample events provide } \\
\text { data to determine trend, consider removing } \\
\text { from program }\end{array}$ \\
\hline EF & S-2 Site & GW-350 & N/A & Annual & Review & $\begin{array}{l}\text { Sample until } 4 \text { recent sample events provide } \\
\text { data to determine trend, consider removing } \\
\text { from program }\end{array}$ \\
\hline EF & Rust Garage Area & GW-505 & N/A & Annual & Review & $\begin{array}{l}\text { Sample until } 4 \text { recent sample events provide } \\
\text { data to determine trend, consider removing } \\
\text { from program }\end{array}$ \\
\hline EF & Coal Pile Trench & GW-686 & N/A & Annual & Review & $\begin{array}{l}\text { Sample until } 4 \text { recent sample events provide } \\
\text { data to determine trend, consider removing } \\
\text { from program }\end{array}$ \\
\hline EF & Union Valley - Exit Pathway & RGQWWSP & $\mathrm{N} / \mathrm{A}$ & Biennial & Review & $\begin{array}{l}\text { Priority location, low to non-detect } \\
\text { concentrations, limited monitoring schedule } \\
\text { recommended }\end{array}$ \\
\hline EF & Exit Pathway Spring/Surface $V$ & SCR7.10SP & ND & Biennial & Review & $\begin{array}{l}\text { Priority location, low to non-detect } \\
\text { concentrations, limited monitoring schedule } \\
\text { recommended }\end{array}$ \\
\hline EF & Union Valley - Exit Pathway & SCR7.14SP & N/A & Biennial & Review & $\begin{array}{l}\text { Priority location, low to non-detect } \\
\text { concentrations, limited monitoring schedule } \\
\text { recommended }\end{array}$ \\
\hline EF & Union Valley - Exit Pathway & SCR7.16SP & N/A & Biennial & Review & $\begin{array}{l}\text { Priority location, low to non-detect } \\
\text { concentrations, limited monitoring schedule } \\
\text { recommended }\end{array}$ \\
\hline EF & Exit Pathway Spring/Surface V & SCR7.4SP & ND & Biennial & Review & $\begin{array}{l}\text { Priority location, low to non-detect } \\
\text { concentrations, limited monitoring schedule } \\
\text { recommended }\end{array}$ \\
\hline EF & Exit Pathway Spring/Surface V & SCR7.6SP & ND & Biennial & Review & $\begin{array}{l}\text { Priority location, low to non-detect } \\
\text { concentrations, limited monitoring schedule } \\
\text { recommended }\end{array}$ \\
\hline EF & Exit Pathway Spring/Surface $V$ & SCR7.7SP & ND & Biennial & Review & $\begin{array}{l}\text { Priority location, low to non-detect } \\
\text { concentrations, limited monitoring schedule } \\
\text { recommended }\end{array}$ \\
\hline EF & Union Valley - Exit Pathway & SCR7.8SSP & N/A & Biennial & Review & $\begin{array}{l}\text { Priority location, low to non-detect } \\
\text { concentrations, limited monitoring schedule } \\
\text { recommended }\end{array}$ \\
\hline EF & Union Valley - Exit Pathway & UV8.5SP & N/A & Biennial & Review & $\begin{array}{l}\text { Priority location, low to non-detect } \\
\text { concentrations, limited monitoring schedule } \\
\text { recommended }\end{array}$ \\
\hline EF & Union Valley - Exit Pathway & UV8.6SP & $\mathrm{N} / \mathrm{A}$ & Biennial & Review & $\begin{array}{l}\text { Priority location, low to non-detect } \\
\text { concentrations, limited monitoring schedule } \\
\text { recommended }\end{array}$ \\
\hline
\end{tabular}


TABLE B.15

SUMMARY SAMPLING RECOMMENDATIONS EAST FORK REGIME

East Fork Poplar Creek Hydrogeologic Regime

Y-12 National Security Complex

Oak Ridge, Tennessee

\begin{tabular}{|c|c|c|c|c|c|c|}
\hline Regime & Functional Area & $\begin{array}{l}\text { Location } \\
\text { Name }\end{array}$ & Trend & $\begin{array}{c}\text { Preliminary } \\
\text { Frequency }\end{array}$ & $\begin{array}{c}\text { Final } \\
\text { Frequency }\end{array}$ & Recommendation \\
\hline \multicolumn{7}{|c|}{ Remove from Routine Monitoring } \\
\hline EF & Y-12 Grid Well B2 & $55-1 C$ & $\mathrm{~N} / \mathrm{A}$ & Biennial & Remove & Keep as DTW location \\
\hline EF & Building 9202 & $59-1 \mathrm{~A}$ & $\mathrm{~N} / \mathrm{A}$ & Remove & Remove & Keep as DTW location \\
\hline EF & Building 9202 & $59-1 B$ & $\mathrm{PD}$ & Annual & Remove & Keep as DTW location \\
\hline EF & Building 9202 & $59-1 \mathrm{C}$ & $\mathrm{N} / \mathrm{A}$ & Annual & Remove & Keep as DTW location \\
\hline EF & Y-12 Plant Site & $60-1 \mathrm{~B}$ & $\mathrm{~N} / \mathrm{A}$ & Remove & Remove & Keep as DTW location \\
\hline EF & S-3 Site & GW-107 & $\mathrm{N} / \mathrm{A}$ & Remove & Remove & Keep as DTW location \\
\hline EF & S-3 Site & GW-134 & & & Remove & No data available \\
\hline EF & New Hope Pond & GW-148 & NT & Biennial & Remove & Keep as DTW location \\
\hline EF & New Hope Pond & GW-149 & N/A & Biennial & Remove & Keep as DTW location \\
\hline EF & New Hope Pond & GW-150 & $\mathrm{N} / \mathrm{A}$ & Remove & Remove & Keep as DTW location \\
\hline EF & New Hope Pond & GW-152 & $\mathrm{N} / \mathrm{A}$ & Remove & Remove & Keep as DTW location \\
\hline EF & Scarboro Road & GW-167 & $\mathrm{N} / \mathrm{A}$ & Annual & Remove & Keep as DTW location \\
\hline EF & Y-12 Fuel Station & GW-183 & $\mathrm{N} / \mathrm{A}$ & Biennial & Remove & Keep as DTW location \\
\hline EF & Beta-4 Security Pits & GW-191 & ND & Biennial & Remove & Keep as DTW location \\
\hline EF & Beta-4 Security Pits & GW-194 & $\mathrm{N} / \mathrm{A}$ & Remove & Remove & Keep as DTW location \\
\hline EF & Y-12 Grid Well I1 & GW-199 & $\mathrm{N} / \mathrm{A}$ & Biennial & Remove & Keep as DTW location \\
\hline EF & Ravine Disposal Site & GW-200 & $\mathrm{N} / \mathrm{A}$ & Remove & Remove & Keep as DTW location \\
\hline EF & Ravine Disposal Site & GW-202 & ND & Biennial & Remove & Keep as DTW location \\
\hline EF & Uranium Oxide Vault & GW-218 & $\mathrm{S}$ & Annual & Remove & Keep as DTW location \\
\hline EF & Scarboro Road & GW-239 & $\mathrm{N} / \mathrm{A}$ & Remove & Remove & Keep as DTW location \\
\hline EF & S-2 Site & GW-252 & $\mathrm{N} / \mathrm{A}$ & Remove & Remove & Keep as DTW location \\
\hline EF & S-2 Site & GW-255 & $\mathrm{N} / \mathrm{A}$ & Biennial & Remove & Keep as DTW location \\
\hline EF & Y-12 Grid Well A1 & GW-261 & $\mathrm{N} / \mathrm{A}$ & Remove & Remove & Keep as DTW location \\
\hline EF & Y-12 Salvage Yard & GW-268 & $\mathrm{N} / \mathrm{A}$ & Biennial & Remove & Keep as DTW location \\
\hline EF & Y-12 Fuel Station & GW-282 & $\mathrm{N} / \mathrm{A}$ & Remove & Remove & Keep as DTW location \\
\hline EF & Y-12 Fuel Station & GW-283 & $\mathrm{N} / \mathrm{A}$ & Remove & Remove & Keep as DTW location \\
\hline EF & $\begin{array}{l}\text { Waste Coolant Processing } \\
\text { Facility }\end{array}$ & GW-334 & $\mathrm{N} / \mathrm{A}$ & Remove & Remove & Keep as DTW location \\
\hline EF & $\begin{array}{l}\text { Waste Coolant Processing } \\
\text { Facility }\end{array}$ & GW-335 & $\mathrm{N} / \mathrm{A}$ & Remove & Remove & Keep as DTW location \\
\hline EF & $\begin{array}{l}\text { Waste Coolant Processing } \\
\text { Facility }\end{array}$ & GW-338 & ND & Biennial & Remove & Keep as DTW location \\
\hline EF & New Hope Pond & GW-384 & $\mathrm{S}$ & Biennial & Remove & Keep as DTW location \\
\hline EF & New Hope Pond & GW-385 & $\mathrm{PI}$ & Biennial & Remove & Keep as DTW location \\
\hline EF & Rust Garage Area & GW-508 & $\mathrm{N} / \mathrm{A}$ & Remove & Remove & Keep as DTW location \\
\hline EF & New Hope Pond & GW-603 & NT & Biennial & Remove & Keep as DTW location \\
\hline EF & Y-12 Plant Site & GW-657 & N/A & Remove & Remove & Keep as DTW location \\
\hline EF & Y-12 Fuel Station & GW-659 & $\mathrm{N} / \mathrm{A}$ & Biennial & Remove & Keep as DTW location \\
\hline EF & Coal Pile Trench & GW-692 & $\mathrm{N} / \mathrm{A}$ & Annual & Remove & Keep as DTW location \\
\hline EF & Y-12 Grid Well K1 & GW-745 & ND & Biennial & Remove & Keep as DTW location \\
\hline EF & Y-12 Grid Well K1 & GW-746 & $\mathrm{PI}$ & Annual & Remove & Keep as DTW location \\
\hline EF & Y-12 Grid Well J3 & GW-751 & ND & Remove & Remove & Keep as DTW location \\
\hline EF & Y-12 Grid Well J3 & GW-752 & ND & Remove & Remove & Keep as DTW location \\
\hline EF & Y-12 Grid Well J2 & GW-753 & NT & Biennial & Remove & Keep as DTW location \\
\hline
\end{tabular}


TABLE B.15

SUMMARY SAMPLING RECOMMENDATIONS EAST FORK REGIME

East Fork Poplar Creek Hydrogeologic Regime

Y-12 National Security Complex

Oak Ridge, Tennessee

\begin{tabular}{|c|c|c|c|c|c|c|}
\hline Regime & Functional Area & $\begin{array}{c}\text { Location } \\
\text { Name }\end{array}$ & Trend & $\begin{array}{c}\text { Preliminary } \\
\text { Frequency }\end{array}$ & $\begin{array}{c}\text { Final } \\
\text { Frequency }\end{array}$ & Recommendation \\
\hline EF & Y-12 Grid Well J2 & GW-754 & NT & Biennial & Remove & Keep as DTW location \\
\hline EF & Y-12 Grid Well J1 & GW-756 & $\mathrm{N} / \mathrm{A}$ & Remove & Remove & Keep as DTW location \\
\hline EF & Y-12 Grid Well G1 & GW-759 & $\mathrm{N} / \mathrm{A}$ & Biennial & Remove & Keep as DTW location \\
\hline EF & Y-12 Grid Well G2 & GW-760 & $\mathrm{N} / \mathrm{A}$ & Biennial & Remove & Keep as DTW location \\
\hline EF & Y-12 Grid Well G2 & GW-761 & $\mathrm{N} / \mathrm{A}$ & Biennial & Remove & Keep as DTW location \\
\hline EF & Y-12 Grid Well I2 & GW-766 & ND & Biennial & Remove & Keep as DTW location \\
\hline EF & Y-12 Grid Well I2 & GW-767 & ND & Biennial & Remove & Keep as DTW location \\
\hline EF & Y-12 Grid Well H2 & GW-773 & ND & Remove & Remove & Keep as DTW location \\
\hline EF & Y-12 Grid Well H2 & GW-774 & $\mathrm{N} / \mathrm{A}$ & Remove & Remove & Keep as DTW location \\
\hline EF & Y-12 Grid Well B2 & GW-778 & $\mathrm{N} / \mathrm{A}$ & Remove & Remove & Keep as DTW location \\
\hline EF & Y-12 Grid Well F2 & GW-779 & $\mathrm{N} / \mathrm{A}$ & Remove & Remove & Keep as DTW location \\
\hline EF & Y-12 Grid Well F2 & GW-780 & $\mathrm{N} / \mathrm{A}$ & Biennial & Remove & Keep as DTW location \\
\hline EF & Y-12 Grid Well E2 & GW-786 & $\mathrm{N} / \mathrm{A}$ & Remove & Remove & Keep as DTW location \\
\hline EF & Y-12 Grid Well E2 & GW-787 & NT & Annual & Remove & Keep as DTW location \\
\hline EF & Y-12 Grid Well F3 & GW-788 & $\mathrm{D}$ & Remove & Remove & Keep as DTW location \\
\hline EF & Y-12 Grid Well F3 & GW-789 & NT & Annual & Remove & Keep as DTW location \\
\hline EF & Building 9201-2 & GW-819 & $\mathrm{N} / \mathrm{A}$ & Remove & Remove & Keep as DTW location \\
\hline
\end{tabular}

Notes

1. Final Frequency for 171 locations:

Regulated = Sampling location and frequency covered under RCRA post-closure permit, CERCLA interim/rinal ROD or other regulatory program

Semi-annual = Locations recommended for semi-annual sampling (every 6 months) based on trend, exceedance of MCL and location in network.

Annual = Locations recommended for annual sampling (once per year) based on trend, exceedance of MCL and location in network.

Biennial = Wells recommended for Biennial sampling (once every 2 years) based on trend, exceedance of MCL and location in network.

Review = Review status of well, if well is in good working order, re-evaluated concentration trend for 4 sample events after 1996.

If concentration trends are Stable to Decreasing, consider removing the well from routine monitoring program.

Remove $=$ Well does not provide significant data in support of monitoring objectives.

Extraction well $=$ Sample frequency was not considered the GW-845 extraction well.

2. Westbay well GW-722 was not evaluated, although Annual sampling is recommended. 
TABLE B.16

SUMMARY SAMPLING RECOMMENDATIONS CHESTNUT RIDGE REGIME

Chestnut Ridge Hydrogeologic Regime

Y-12 National Security Complex

Oak Ridge, Tennessee

\begin{tabular}{|c|c|c|c|c|c|c|}
\hline Regime & Functional Area & Location Name & Trend & $\begin{array}{l}\text { Preliminary } \\
\text { Frequency }\end{array}$ & Final Frequency & Recommendation \\
\hline \multicolumn{7}{|c|}{ Regulated Sample Locations } \\
\hline $\mathrm{CR}$ & $\begin{array}{l}\text { United Nuclear Corporation } \\
\text { Site }\end{array}$ & 1090 & $\mathrm{~S}$ & Biennial & Regulated & Sample as per permit \\
\hline $\mathrm{CR}$ & Industrial Landfill IV & GW-141 & $\mathrm{D}$ & Biennial & Regulated & Sample as per permit \\
\hline $\mathrm{CR}$ & Kerr Hollow Quarry & GW-143 & $\mathrm{D}$ & Biennial & Regulated & Sample as per permit or as per ROD \\
\hline $\mathrm{CR}$ & Kerr Hollow Quarry & GW-144 & NT & Biennial & Regulated & Sample as per permit or as per ROD \\
\hline $\mathrm{CR}$ & Kerr Hollow Quarry & GW-145 & $\mathrm{S}$ & Annual & Regulated & Sample as per permit or as per ROD \\
\hline $\mathrm{CR}$ & $\begin{array}{l}\text { Chestnut Ridge Sediment } \\
\text { Disposal Basin }\end{array}$ & GW-156 & $\mathrm{D}$ & Biennial & Regulated & Sample as per permit \\
\hline $\mathrm{CR}$ & $\begin{array}{l}\text { Chestnut Ridge Sediment } \\
\text { Disposal Basin }\end{array}$ & GW-159 & N/A & Annual & Regulated & Sample as per permit \\
\hline $\mathrm{CR}$ & Chestnut Ridge Security Pits & GW-177 & ND & Biennial & Regulated & Sample as per permit \\
\hline $\mathrm{CR}$ & $\begin{array}{l}\text { United Nuclear Corporation } \\
\text { Site }\end{array}$ & GW-203 & $\mathrm{PI}$ & Annual & Regulated & Sample as per permit \\
\hline $\mathrm{CR}$ & $\begin{array}{l}\text { United Nuclear Corporation } \\
\text { Site }\end{array}$ & GW-205 & $\mathrm{PI}$ & Annual & Regulated & Sample as per permit \\
\hline $\mathrm{CR}$ & Industrial Landfill IV & GW-217 & $\mathrm{D}$ & Biennial & Regulated & Sample as per permit \\
\hline $\mathrm{CR}$ & $\begin{array}{l}\text { United Nuclear Corporation } \\
\text { Site }\end{array}$ & GW-221 & NT & Annual & Regulated & Sample as per permit \\
\hline $\mathrm{CR}$ & Kerr Hollow Quarry & GW-231 & $\mathrm{D}$ & Biennial & Regulated & Sample as per permit or as per ROD \\
\hline $\mathrm{CR}$ & $\begin{array}{l}\text { Chestnut Ridge Borrow Area } \\
\text { Waste Pile }\end{array}$ & GW-301 & $\mathrm{D}$ & Biennial & Regulated & Sample as per permit \\
\hline $\mathrm{CR}$ & $\begin{array}{l}\text { United Nuclear Corporation } \\
\text { Site }\end{array}$ & GW-302 & NT & Annual & Regulated & $\begin{array}{l}\text { If dropped from CERCLA, consider Annual } \\
\text { to Biennial sampling, low concentrations }\end{array}$ \\
\hline $\mathrm{CR}$ & Industrial Landfill IV & GW-305 & $\mathrm{I}$ & SemiAnnual & Regulated & Sample as per ROD \\
\hline $\mathrm{CR}$ & \begin{tabular}{|l} 
United Nuclear Corporation \\
Site
\end{tabular} & GW-339 & NT & Biennial & Regulated & Sample as per permit \\
\hline $\mathrm{CR}$ & Industrial Landfill IV & GW-521 & ND & Biennial & Regulated & Sample as per permit \\
\hline $\mathrm{CR}$ & Industrial Landfill IV & GW-522 & $\mathrm{D}$ & Biennial & Regulated & Sample as per permit \\
\hline $\mathrm{CR}$ & Industrial Landfill II & GW-540 & $\mathrm{D}$ & Biennial & Regulated & Sample as per permit \\
\hline $\mathrm{CR}$ & $\begin{array}{l}\text { Construction/Demolition } \\
\text { Landfill VI }\end{array}$ & GW-542 & $\mathrm{D}$ & Biennial & Regulated & Sample as per permit \\
\hline $\mathrm{CR}$ & $\begin{array}{l}\text { Construction/Demolition } \\
\text { Landfill VI }\end{array}$ & GW-543 & ND & Biennial & Regulated & Sample as per permit \\
\hline $\mathrm{CR}$ & $\begin{array}{l}\text { Construction/Demolition } \\
\text { Landfill VI }\end{array}$ & GW-544 & $\mathrm{D}$ & Biennial & Regulated & Sample as per permit \\
\hline $\mathrm{CR}$ & Industrial Landfill V & GW-557 & ND & Biennial & Regulated & Sample as per permit \\
\hline $\mathrm{CR}$ & $\begin{array}{l}\text { Construction/Demolition } \\
\text { Landfill VII }\end{array}$ & GW-560 & ND & Remove & Regulated & Sample as per permit \\
\hline CR & $\begin{array}{l}\text { Construction/Demolition } \\
\text { Landfill VII }\end{array}$ & GW-562 & ND & Remove & Regulated & Sample as per permit \\
\hline
\end{tabular}


TABLE B.16

SUMMARY SAMPLING RECOMMENDATIONS CHESTNUT RIDGE REGIME

Chestnut Ridge Hydrogeologic Regime

Y-12 National Security Complex

Oak Ridge, Tennessee

\begin{tabular}{|c|c|c|c|c|c|c|}
\hline Regime & Functional Area & Location Name & Trend & $\begin{array}{l}\text { Preliminary } \\
\text { Frequency }\end{array}$ & Final Frequency & Recommendation \\
\hline $\mathrm{CR}$ & $\begin{array}{l}\text { Construction/Demolition } \\
\text { Landfill VII }\end{array}$ & GW-564 & ND & Remove & Regulated & Sample as per permit \\
\hline CR & Industrial Landfill II & GW-709 & $\mathrm{D}$ & Biennial & Regulated & Sample as per permit \\
\hline $\mathrm{CR}$ & $\begin{array}{l}\text { Chestnut Ridge Sediment } \\
\text { Disposal Basin }\end{array}$ & GW-731 & $N / A$ & Biennial & Regulated & Sample as per permit \\
\hline CR & $\begin{array}{l}\text { Chestnut Ridge Sediment } \\
\text { Disposal Basin }\end{array}$ & GW-732 & $\mathrm{N} / \mathrm{A}$ & Biennial & Regulated & Sample as per permit \\
\hline $\mathrm{CR}$ & Industrial Landfill II & GW-757 & $\mathrm{D}$ & Biennial & Regulated & Sample as per permit \\
\hline $\mathrm{CR}$ & Industrial Landfill V & GW-796 & ND & Biennial & Regulated & Sample as per permit \\
\hline CR & Industrial Landfill V & GW-797 & $\mathrm{D}$ & Biennial & Regulated & Sample as per permit \\
\hline CR & $\begin{array}{l}\text { Construction/Demolition } \\
\text { Landfill VII }\end{array}$ & GW-798 & 1 & Annual & Regulated & Sample as per permit \\
\hline $\mathrm{CR}$ & Industrial Landfill V & GW-799 & ND & Biennial & Regulated & Sample as per permit \\
\hline CR & Industrial Landfill V & GW-801 & ND & Biennial & Regulated & Sample as per permit \\
\hline CR & $\begin{array}{l}\text { Construction/Demolition } \\
\text { Landfill VI }\end{array}$ & GW-827 & $\mathrm{D}$ & Biennial & Regulated & Sample as per permit \\
\hline $\mathrm{CR}$ & Chestnut Ridge Security Pits & GW-831 & $\mathrm{D}$ & Biennial & Regulated & Sample as per permit \\
\hline $\mathrm{CR}$ & $\begin{array}{l}\text { South Campus Facility, Bethel } \\
\text { Valley }\end{array}$ & GW-841 & $\mathrm{D}$ & Annual & Regulated & Sample as per ROD \\
\hline $\mathrm{CR}$ & $\begin{array}{l}\text { South Campus Facility, Bethel } \\
\text { Valley }\end{array}$ & GW-842 & NT & Annual & Regulated & Sample as per ROD \\
\hline $\mathrm{CR}$ & $\begin{array}{l}\text { South Campus Facility, Bethel } \\
\text { Valley }\end{array}$ & GW-843 & $N / A$ & Annual & Regulated & Sample as per ROD \\
\hline $\mathrm{CR}$ & $\begin{array}{l}\text { South Campus Facility, Bethel } \\
\text { Valley }\end{array}$ & GW-844 & ND & Annual & Regulated & Sample as per ROD \\
\hline $\mathrm{CR}$ & $\begin{array}{l}\text { Exit Pathway Spring/Surface } \\
\text { Water }\end{array}$ & SCR1.25SP & NT & Annual & Regulated & Sample as per permit \\
\hline $\mathrm{CR}$ & $\begin{array}{l}\text { Exit Pathway Spring/Surface } \\
\text { Water }\end{array}$ & SCR3.5SP & NT & Annual & Regulated & Sample as per ROD \\
\hline $\mathrm{CR}$ & Industrial Landfill V & SCR4.3SP & ND & Biennial & Regulated & Sample as per permit \\
\hline
\end{tabular}


TABLE B.16

SUMMARY SAMPLING RECOMMENDATIONS CHESTNUT RIDGE REGIME

Chestnut Ridge Hydrogeologic Regime

Y-12 National Security Complex

Oak Ridge, Tennessee

\begin{tabular}{|c|c|c|c|c|c|c|}
\hline Regime & Functional Area & Location Name & Trend & $\begin{array}{l}\text { Preliminary } \\
\text { Frequency }\end{array}$ & Final Frequency & Recommendation \\
\hline \multicolumn{7}{|c|}{ Annual Sampling } \\
\hline $\mathrm{CR}$ & Chestnut Ridge Security Pits & GW-175 & $\mathrm{S}$ & Annual & Annual & $\begin{array}{l}\text { Stable trend, sample Annually until trend } \\
\text { indicates reduced frequency }\end{array}$ \\
\hline $\mathrm{CR}$ & Chestnut Ridge Security Pits & GW-608 & $\mathrm{S}$ & Annual & Annual & $\begin{array}{l}\text { Stable trend, sample Annually until trend } \\
\text { indicates reduced frequency }\end{array}$ \\
\hline CR & Chestnut Ridge Security Pits & GW-609 & $\mathrm{D}$ & Annual & Annual & $\begin{array}{l}\text { Decreasing trend, sample Annually until } \\
\text { trend indicates reduced frequency }\end{array}$ \\
\hline CR & Chestnut Ridge Security Pits & GW-612 & NT & Annual & Annual & $\begin{array}{l}\text { Monitor Annually until trend indicates } \\
\text { reduced effort is appropriate }\end{array}$ \\
\hline $\mathrm{CR}$ & $\begin{array}{l}\text { Exit Pathway Spring/Surface } \\
\text { Water }\end{array}$ & SCR3.4SP & $\mathrm{PI}$ & Annual & Annual & $\begin{array}{l}\text { Sample annually to biennially as detection } \\
\text { monitoring location }\end{array}$ \\
\hline \multicolumn{7}{|c|}{ Biennial Sampling } \\
\hline $\mathrm{CR}$ & $\begin{array}{l}\text { Chestnut Ridge Borrow Area } \\
\text { Waste Pile }\end{array}$ & GW-160 & N/A & Annual & Biennial & $\begin{array}{l}\text { Historic lead and possible uranium } \\
\text { detections, sample } 4 \mathrm{X} \text { in } 10 \mathrm{yrs} \text { and } \\
\text { reevaluate }\end{array}$ \\
\hline CR & Rogers Quarry & GW-186 & N/A & Biennial & Biennial & $\begin{array}{l}\text { Well monitors property boundary, sample } \\
\text { Biennially for detection monitoring }\end{array}$ \\
\hline $\mathrm{CR}$ & Filled Coal Ash Pond & GW-514 & N/A & Biennial & Biennial & $\begin{array}{l}\text { Low concentrations, sample Biennial until } \\
\text { trend determined }\end{array}$ \\
\hline $\mathrm{CR}$ & Industrial Landfill II & GW-539 & $\mathrm{D}$ & Biennial & Biennial & Removed \\
\hline $\mathrm{CR}$ & $\begin{array}{l}\text { Exit Pathway Spring/Surface } \\
\text { Water }\end{array}$ & SCR2.1SP & $\mathrm{S}$ & Biennial & Biennial & $\begin{array}{l}\text { Largely non-detect for priority COCs, } \\
\text { sample Biennially }\end{array}$ \\
\hline CR & $\begin{array}{l}\text { Exit Pathway Spring/Surface } \\
\text { Water }\end{array}$ & SCR2.2SP & $\mathrm{S}$ & Biennial & Biennial & $\begin{array}{l}\text { Largely non-detect for priority COCs, } \\
\text { sample Biennially }\end{array}$ \\
\hline $\mathrm{CR}$ & $\begin{array}{l}\text { Exit Pathway Spring/Surface } \\
\text { Water }\end{array}$ & SCR5.1SP & NT & Annual & Biennial & $\begin{array}{l}\text { Largely non-detect, but spring is potential } \\
\text { point of exposure, consider biennial } \\
\text { sampling }\end{array}$ \\
\hline $\mathrm{CR}$ & $\begin{array}{l}\text { Exit Pathway Spring/Surface } \\
\text { Water }\end{array}$ & SCR5.2SP & N/A & Annual & Biennial & $\begin{array}{l}\text { Largely non-detect, but spring is potential } \\
\text { point of exposure, consider biennial } \\
\text { sampling }\end{array}$ \\
\hline $\mathrm{CR}$ & $\begin{array}{l}\text { Exit Pathway Spring/Surface } \\
\text { Water }\end{array}$ & SCR5.4SP & NT & Biennial & Biennial & $\begin{array}{l}\text { Largely non-detect, but spring is potential } \\
\text { point of exposure, consider biennial } \\
\text { sampling }\end{array}$ \\
\hline \multicolumn{7}{|l|}{ Review } \\
\hline $\mathrm{CR}$ & Chestnut Ridge Security Pits & GW-173 & $\mathrm{N} / \mathrm{A}$ & SemiAnnual & Review & $\begin{array}{l}\text { Sample } 4 \mathrm{X} \text { in } 10 \text { yrs and consider removing } \\
\text { from program }\end{array}$ \\
\hline $\mathrm{CR}$ & Chestnut Ridge Security Pits & GW-174 & N/A & SemiAnnual & Review & $\begin{array}{l}\text { Sample } 4 \mathrm{X} \text { in } 10 \mathrm{yrs} \text { and consider removing } \\
\text { from program }\end{array}$ \\
\hline CR & Chestnut Ridge Security Pits & GW-176 & N/A & Annual & Review & $\begin{array}{l}\text { Sample } 4 \mathrm{X} \text { in } 10 \mathrm{yrs} \text { and consider removing } \\
\text { from program }\end{array}$ \\
\hline $\mathrm{CR}$ & Chestnut Ridge Security Pits & GW-179 & N/A & Annual & Review & $\begin{array}{l}\text { Sample } 4 \mathrm{X} \text { in } 10 \mathrm{yrs} \text { and consider removing } \\
\text { from program }\end{array}$ \\
\hline $\mathrm{CR}$ & Chestnut Ridge Security Pits & GW-180 & N/A & SemiAnnual & Review & $\begin{array}{l}\text { Decreasing historic trend, sample } 4 \mathrm{X} \text { in } 10 \\
\text { yrs and consider removing from program }\end{array}$ \\
\hline $\mathrm{CR}$ & Chestnut Ridge Security Pits & GW-322 & N/A & SemiAnnual & Review & $\begin{array}{l}\text { Sample } 4 \mathrm{X} \text { in } 10 \text { yrs and consider removing } \\
\text { from program }\end{array}$ \\
\hline
\end{tabular}


TABLE B.16

SUMMARY SAMPLING RECOMMENDATIONS CHESTNUT RIDGE REGIME

Chestnut Ridge Hydrogeologic Regime

Y-12 National Security Complex

Oak Ridge, Tennessee

\begin{tabular}{|c|c|c|c|c|c|c|}
\hline Regime & Functional Area & Location Name & Trend & $\begin{array}{l}\text { Preliminary } \\
\text { Frequency }\end{array}$ & Final Frequency & Recommendation \\
\hline \multicolumn{7}{|c|}{ Remove from Routine Monitoring } \\
\hline $\mathrm{CR}$ & Rogers Quarry & GW-188 & $\mathrm{N} / \mathrm{A}$ & Biennial & Remove & Keep as DTW well \\
\hline $\mathrm{CR}$ & $\begin{array}{l}\text { Chestnut Ridge Sediment } \\
\text { Disposal Basin }\end{array}$ & GW-241 & $\mathrm{N} / \mathrm{A}$ & Annual & Remove & Limited Function Does not exceed MCL \\
\hline $\mathrm{CR}$ & $\begin{array}{l}\text { East Chestnut Ridge Waste } \\
\text { Pile }\end{array}$ & GW-292 & ND & Remove & Remove & $\begin{array}{l}\text { Largely non-detect for COCs, remove from } \\
\text { program }\end{array}$ \\
\hline $\mathrm{CR}$ & $\begin{array}{l}\text { East Chestnut Ridge Waste } \\
\text { Pile }\end{array}$ & GW-293 & ND & Remove & Remove & $\begin{array}{l}\text { Largely non-detect for COCs, remove from } \\
\text { program }\end{array}$ \\
\hline $\mathrm{CR}$ & $\begin{array}{l}\text { Chestnut Ridge Borrow Area } \\
\text { Waste Pile }\end{array}$ & GW-298 & ND & Remove & Remove & $\begin{array}{l}\text { Largely non-detect for COCs, remove from } \\
\text { program }\end{array}$ \\
\hline $\mathrm{CR}$ & $\begin{array}{l}\text { Chestnut Ridge Borrow Area } \\
\text { Waste Pile }\end{array}$ & GW-299 & $\mathrm{N} / \mathrm{A}$ & Remove & Remove & Limited Function Does not exceed MCL \\
\hline $\mathrm{CR}$ & $\begin{array}{l}\text { Chestnut Ridge Borrow Area } \\
\text { Waste Pile }\end{array}$ & GW-300 & $\mathrm{N} / \mathrm{A}$ & Annual & Remove & Limited Function Does not exceed MCL \\
\hline CR & $\begin{array}{l}\text { Chestnut Ridge Sediment } \\
\text { Disposal Basin }\end{array}$ & GW-303 & N/A & Remove & Remove & Limited Function Does not exceed MCL \\
\hline $\mathrm{CR}$ & $\begin{array}{l}\text { Chestnut Ridge Sediment } \\
\text { Disposal Basin }\end{array}$ & GW-304 & $\mathrm{N} / \mathrm{A}$ & Remove & Remove & Limited Function Does not exceed MCL \\
\hline $\mathrm{CR}$ & Chestnut Ridge Security Pits & GW-178 & $\mathrm{N} / \mathrm{A}$ & Annual & Remove & Keep as DTW well \\
\hline $\mathrm{CR}$ & Kerr Hollow Quarry & GW-142 & $\mathrm{D}$ & Biennial & Remove & Removed from RCRA program, Hydro only \\
\hline CR & Rogers Quarry & GW-184 & N/A & Remove & Remove & Keep as DTW well \\
\hline $\mathrm{CR}$ & $\begin{array}{l}\text { Construction/Demolition } \\
\text { Landfill VI }\end{array}$ & GW-546 & ND & Remove & Remove & Keep as DTW well \\
\hline $\mathrm{CR}$ & Chestnut Ridge Security Pits & GW-511 & $\mathrm{N} / \mathrm{A}$ & Biennial & Remove & Keep as DTW well \\
\hline $\mathrm{CR}$ & Filled Coal Ash Pond & GW-512 & $\mathrm{N} / \mathrm{A}$ & Biennial & Remove & Keep as DTW well \\
\hline $\mathrm{CR}$ & Filled Coal Ash Pond & GW-513 & $\mathrm{N} / \mathrm{A}$ & Annual & Remove & Keep as DTW well \\
\hline $\mathrm{CR}$ & Chestnut Ridge Security Pits & GW-610 & $\mathrm{N} / \mathrm{A}$ & Biennial & Remove & Keep as DTW well \\
\hline $\mathrm{CR}$ & Chestnut Ridge Security Pits & GW-611 & $\mathrm{N} / \mathrm{A}$ & Annual & Remove & Keep as DTW well \\
\hline $\mathrm{CR}$ & Filled Coal Ash Pond & GW-679 & $\mathrm{N} / \mathrm{A}$ & Biennial & Remove & Keep as DTW well \\
\hline $\mathrm{CR}$ & Filled Coal Ash Pond & GW-680 & $\mathrm{N} / \mathrm{A}$ & Biennial & Remove & Keep as DTW well \\
\hline CR & Chestnut Ridge Security Pits & GW-742 & $\mathrm{N} / \mathrm{A}$ & Annual & Remove & Keep as DTW well \\
\hline $\mathrm{CR}$ & Chestnut Ridge Security Pits & GW-743 & $\mathrm{N} / \mathrm{A}$ & Biennial & Remove & Keep as DTW well \\
\hline $\mathrm{CR}$ & $\begin{array}{l}\text { Construction/Demolition } \\
\text { Landfill VI }\end{array}$ & GW-541 & N/A & Remove & Remove & Keep as DTW well \\
\hline CR & Chestnut Ridge Security Pits & GW-181 & $\mathrm{N} / \mathrm{A}$ & Remove & Remove & Keep as DTW well \\
\hline
\end{tabular}

Notes

1. Final Frequency for 89 locations:

Regulated $=$ Sampling location and frequency covered under RCRA post-closure permit, CERCLA interim/rinal ROD or other regulatory program.

Semi-annual = Locations recommended for semi-annual sampling (every 6 months) based on trend, exceedance of MCL and location in network.

Annual = Locations recommended for annual sampling (once per year) based on trend, exceedance of $\mathrm{MCL}$ and location in network.

Biennial $=$ Wells recommended for Biennial sampling (once every 2 years) based on trend, exceedance of MCL and location in network.

Review $=$ Review status of well, if well is in good working order, re-evaluated concentration trend for 4 sample events after 1996.

If concentration trends are Stable to Decreasing, consider removing the well from routine monitoring program.

Remove $=$ Well does not provide significant data in support of monitoring objectives.

2. No wells are recommended for Semi-annual sampling (except those under regulatory programs)

3. Well GW-564 is listed as a SWDF regulated well, but no data were available and well information table indicates that the hole is caved. 


\section{ASSESSMENT OF THE GROUNDWATER PROTECTION PROGRAM \\ Y-12 NATIONAL SECURITY COMPLEX, OAK RIDGE, TENNESSEE \\ Y-12 National Security Complex \\ Oak Ridge, Tennessee}




\section{APPENDIX C \\ MAROS 2.1 METHODOLOGY}

\section{Contents}

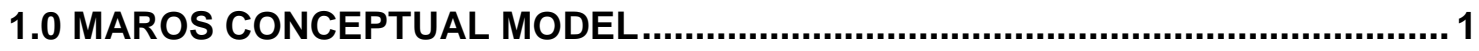

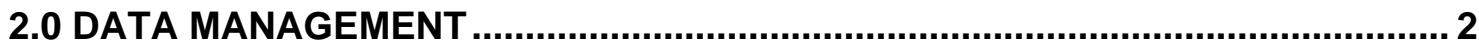

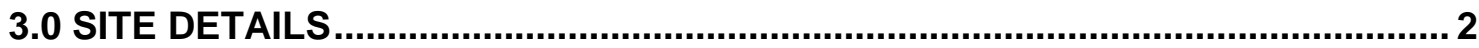

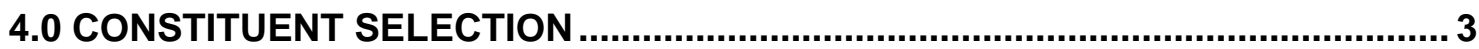

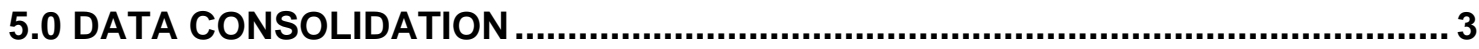

6.0 OVERVIEW STATISTICS: PLUME TREND ANALYSIS ….................................. 3

6.1 Mann-Kendall Analysis ................................................................. 4

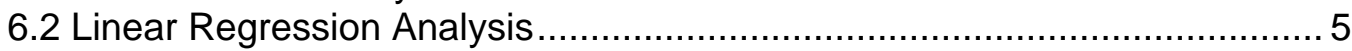

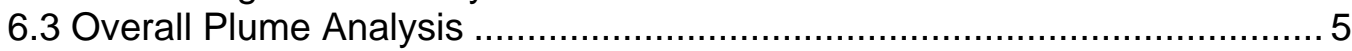

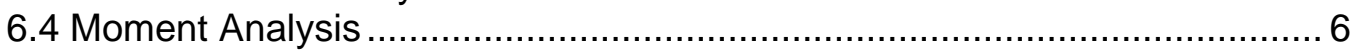

7.0 DETAILED STATISTICS: OPTIMIZATION ANALYSIS ........................................ 8

7.1 Well Redundancy Analysis- Delaunay Method ....................................... 8

7.2 Well Sufficiency Analysis - Delaunay Method …..................................... 9

7.3 Sampling Frequency - Modified CES Method ....................................... 10

7.4 Data Sufficiency - Power Analysis ....................................................... 10

\section{CITED REFERENCES}

Tables

Table C.1 Mann-Kendall Analysis Decision Matrix

Table C.2 Linear Regression Analysis Decision Matrix

Figures

Figure C.1 MAROS Decision Support Tool Flow Chart

Figure C.2 MAROS Overview Statistics Trend Analysis Methodology

Figure C.3 Decision Matrix for Determining Provisional Frequency 


\section{MAROS METHODOLOGY}

MAROS is a collection of tools in one software package that is used in an explanatory, non-linear but linked fashion. The tool includes models, statistics, heuristic rules, and empirical relationships to assist the user in optimizing a groundwater monitoring network system. The final optimized network maintains adequate delineation while providing information on plume dynamics over time. Results generated from the software tool can be used to develop lines of evidence, which, in combination with expert opinion, can be used to inform regulatory decisions for safe and economical long-term monitoring of groundwater plumes. For a detailed description of the structure of the software and further utilities, refer to the MAROS 2.1 Manual (AFCEE, 2003; http://www.gsinet.com/software/MAROS V2 1Manual.pdf) and Aziz et al., 2003.

\subsection{MAROS Conceptual Model}

In MAROS 2.1, two levels of analysis are used for optimizing long-term monitoring plans: 1) an overview statistical evaluation with interpretive trend analysis based on temporal trend analysis and plume stability information; and 2) a more detailed statistical optimization based on spatial and temporal redundancy reduction methods (see Figures C.1 and C.2 for further details). In general, the MAROS method applies to 2-D aquifers that have relatively simple site hydrogeology. However, for a multi-aquifer (3-D) system, the user has the option to apply the statistical analysis layer-by-layer.

The overview statistics or interpretive trend analysis assesses the general monitoring system category by considering individual well concentration trends, overall plume stability, hydrogeologic factors (e.g., seepage velocity, and current plume length), and the location of potential receptors (e.g., property boundaries or drinking water wells). The method relies on temporal trend analysis to assess plume stability, which is then used to determine the general monitoring system category. Since the monitoring system category is evaluated for both source and tail regions of the plume, the site wells are divided into two different zones: the source zone and the tail zone.

Source zone monitoring wells could include areas with non-aqueous phase liquids (NAPLs), contaminated vadose zone soils, and areas where aqueous-phase releases have been introduced into ground water. The source zone generally contains locations with historical high ground water concentrations of the COCs. The tail zone is usually the area downgradient of the contaminant source zone. Although this classification is a simplification of the plume conceptual model, this broadness makes the user aware on an individual well basis that the concentration trend results can have a different interpretation depending on the well location in and around the plume. The location and type of the individual wells allows further interpretation of the trend results, depending on what type of well is being analyzed (e.g., remediation well, leading plume edge well, or monitoring well). General recommendations for the monitoring network frequency and density are suggested based on heuristic rules applied to the source and tail trend results.

The detailed statistics level of analysis or sampling optimization consists of well redundancy and well sufficiency analyses using the Delaunay method, a sampling frequency analysis using the Modified Cost Effective Sampling (MCES) method and a 
data sufficiency analysis including statistical power analysis. The well redundancy analysis is designed to minimize monitoring locations and the Modified CES method is designed to minimize the frequency of sampling. The data sufficiency analysis uses simple statistical methods to assess the sampling record to determine if groundwater concentrations are statistically below target levels and if the current monitoring network and record is sufficient in terms of evaluating concentrations at downgradient locations.

\subsection{Data Management}

In MAROS, ground water monitoring data can be imported from simple database-format Microsoft ${ }^{\circledR}$ Excel spreadsheets, Microsoft Access tables, previously created MAROS database archive files, or entered manually. Monitoring data interpretation in MAROS is based on historical analytical data from a consistent set of wells over a series of sampling events. The analytical data is composed of the well name, coordinate location, constituent, result, detection limit and associated data qualifiers. Statistical validity of the concentration trend analysis requires constraints on the minimum data input of at least four wells (ASTM 1998) in which COCs have been detected. Individual sampling locations need to include data from at least six most-recent sampling events. To ensure a meaningful comparison of $\mathrm{COC}$ concentrations over time and space, both data quality and data quantity need to be considered. Prior to statistical analysis, the user can consolidate irregularly sampled data or smooth data that might result from seasonal fluctuations or a change in site conditions. Because MAROS is a terminal analytical tool designed for long-term planning, impacts of seasonal variation in the water unit are treated on a broad scale, as they relate to multi-year trends.

Imported ground water monitoring data and the site-specific information entered in Site Details can be archived and exported as MAROS archive files. These archive files can be appended as new monitoring data becomes available, resulting in a dynamic longterm monitoring database that reflects the changing conditions at the site (i.e. biodegradation, compliance attainment, completion of remediation phase, etc.). For wells with a limited monitoring history, addition of information as it becomes available can change the frequency or identity of wells in the network.

\subsection{Site Details}

Information needed for the MAROS analysis includes site-specific parameters such as seepage velocity and current plume length and width. Information on the location of potential receptors relative to the source and tail regions of the plume is entered at this point. Part of the trend analysis methodology applied in MAROS focuses on where the monitoring well is located, therefore the user needs to divide site wells into two different zones: the source zone or the tail zone. Although this classification is a simplification of the well function, this broadness makes the user aware on an individual well basis that the concentration trend results can have a different interpretation depending on the well location in and around the plume. It is up to the user to make further interpretation of the trend results, depending on what type of well is being analyzed (e.g., remediation well, leading plume edge well, or monitoring well). The Site Details section of MAROS contains a preliminary map of well locations to confirm well coordinates. 


\subsection{Constituent Selection}

A database with multiple COCs can be entered into the MAROS software. MAROS allows the analysis of up to 5 COCs concurrently and users can pick COCs from a list of compounds existing in the monitoring data. MAROS runs separate optimizations for each compound. For sites with a single source, the suggested strategy is to choose one to three priority COCs for the optimization. If, for example, the site contains multiple chlorinated volatile organic compounds (VOCs), the standard sample chemical analysis will evaluate all VOCs, so the sample locations and frequency should based on the concentration trends of the most prevalent, toxic or mobile compounds. If different chemical classes are present, such as metals and chlorinated VOCs, choose and evaluate the priority constituent in each chemical class.

MAROS includes a short module that provides recommendations on prioritizing COCs based on toxicity, prevalence, and mobility of the compound. The toxicity ranking is determined by examining a representative concentration for each compound for the entire site. The representative concentration is then compared to the screening level (PRG or MCL) for that compound and the COCs are ranked according to the representative concentrations percent exceedence of the screening level. The evaluation of prevalence is performed by determining a representative concentration for each well location and evaluating the total exceedences (values above screening levels) compared to the total number of wells. Compounds found over screening levels are ranked for mobility based on $\mathrm{Kd}$ (sorption partition coefficient). The MAROS COC assessment provides the relative ranking of each $\mathrm{COC}$, but the user must choose which COCs are included in the analysis.

\subsection{Data Consolidation}

Typically, raw data from long-term monitoring have been measured irregularly in time or contain many non-detects, trace level results, and duplicates. Therefore, before the data can be further analyzed, raw data are filtered, consolidated, transformed, and possibly smoothed to allow for a consistent dataset meeting the minimum data requirements for statistical analysis mentioned previously.

MAROS allows users to specify the period of interest in which data will be consolidated (i.e., monthly, bi-monthly, quarterly, semi-annual, yearly, or a biennial basis). In computing the representative value when consolidating, one of four statistics can be used: median, geometric mean, mean, and maximum. Non-detects can be transformed to one half the reporting or method detection limit (DL), the DL, or a fraction of the DL. Trace level results can be represented by their actual values, one half of the DL, the DL, or a fraction of their actual values. Duplicates are reduced in MAROS by one of three ways: assigning the average, maximum, or first value. The reduced data for each COC and each well can be viewed as a time series in a graphical form on a linear or semi-log plot generated by the software.

\subsection{Overview Statistics: Plume Trend Analysis}

Within the MAROS software there are historical data analyses that support a conclusion about plume stability (e.g., increasing plume, etc.) through statistical trend analysis of 
historical monitoring data. Plume stability results are assessed from time-series concentration data with the application of three statistical tools: Mann-Kendall Trend analysis, linear regression trend analysis and moment analysis. The two trend methods are used to estimate the concentration trend for each well and each COC based on a statistical trend analysis of concentrations versus time at each well. These trend analyses are then consolidated to give the user a general plume stability estimate and general monitoring frequency and density recommendations (see Figures C.1 through C.3 for further step-by-step details). Both qualitative and quantitative plume information can be gained by these evaluations of monitoring network historical data trends both spatially and temporally. The MAROS Overview Statistics are the foundation the user needs to make informed optimization decisions at the site. The Overview Statistics are designed to allow site personnel to develop a better understanding of the plume behavior over time and understand how the individual well concentration trends are spatially distributed within the plume. This step allows the user to gain information that will support a more informed decision to be made in the next level or detailed statistics optimization analysis.

\section{$\underline{6.1 \text { Mann-Kendall Analysis }}$}

The Mann-Kendall test is a statistical procedure that is well suited for analyzing trends in data over time. The Mann-Kendall test can be viewed as a non-parametric test for zero slope of the first-order regression of time-ordered concentration data versus time. One advantage of the Mann-Kendall test is that it does not require any assumptions as to the statistical distribution of the data (e.g. normal, lognormal, etc.) and can be used with data sets which include irregular sampling intervals and missing data. The Mann-Kendall test is designed for analyzing a single groundwater constituent, multiple constituents are analyzed separately. The Mann-Kendall S statistic measures the trend in the data: positive values indicate an increase in concentrations over time and negative values indicate a decrease in concentrations over time. The strength of the trend is proportional to the magnitude of the Mann-Kendall statistic (i.e., a large value indicates a strong trend). The confidence in the trend is determined by consulting the $\mathrm{S}$ statistic and the sample size, $\mathrm{n}$, in a Kendall probability table such as the one reported in Hollander and Wolfe (1973).

The concentration trend is determined for each well and each COC based on results of the $S$ statistic, the confidence in the trend, and the Coefficient of Variation (COV). The decision matrix for this evaluation is shown in Table C.1. A Mann-Kendall statistic that is greater than 0 combined with a confidence of greater than $95 \%$ is categorized as an Increasing trend while a Mann-Kendall statistic of less than 0 with a confidence between $90 \%$ and $95 \%$ is defined as a probably Increasing trend, and so on.

Depending on statistical indicators, the concentration trend is classified into six categories:

- Decreasing (D),

- Probably Decreasing (PD),

- Stable (S),

- $\quad$ No Trend (NT),

- Probably Increasing (PI)

- Increasing (I). 
These trend estimates are then analyzed to identify the source and tail region overall stability category (see Figure C.2 for further details).

\section{$\underline{6.2 \text { Linear Regression Analysis }}$}

Linear Regression is a parametric statistical procedure that is typically used for analyzing trends in data over time. Using this type of analysis, a higher degree of scatter simply corresponds to a wider confidence interval about the average log-slope. Assuming the sign (i.e., positive or negative) of the estimated log-slope is correct, a level of confidence that the slope is not zero can be easily determined. Thus, despite a poor goodness of fit, the overall trend in the data may still be ascertained, where low levels of confidence correspond to "Stable" or "No Trend" conditions (depending on the degree of scatter) and higher levels of confidence indicate the stronger likelihood of a trend. The linear regression analysis is based on the first-order linear regression of the logtransformed concentration data versus time. The slope obtained from this logtransformed regression, the confidence level for this log-slope, and the COV of the untransformed data are used to determine the concentration trend. The decision matrix for this evaluation is shown in Table C.2.

To estimate the confidence in the log-slope, the standard error of the log-slope is calculated. The coefficient of variation, defined as the standard deviation divided by the average, is used as a secondary measure of scatter to distinguish between "Stable" or "No Trend" conditions for negative slopes. The Linear Regression Analysis is designed for analyzing a single groundwater constituent; multiple constituents are analyzed separately, (up to five COCs simultaneously). For this evaluation, a decision matrix developed by Groundwater Services, Inc. is also used to determine the "Concentration Trend" category (plume stability) for each well.

Depending on statistical indicators, the concentration trend is classified into six categories:

- Decreasing (D),

- Probably Decreasing (PD),

- Stable (S),

- $\quad$ No Trend (NT),

- Probably Increasing (PI)

- Increasing (I).

The resulting confidence in the trend, together with the log-slope and the COV of the untransformed data, are used in the linear regression analysis decision matrix to determine the concentration trend. For example, a positive log-slope with a confidence of less than $90 \%$ is categorized as having No Trend whereas a negative log-slope is considered Stable if the COV is less than 1 and categorized as No Trend if the COV is greater than 1.

\section{$\underline{6.3 \text { Overall Plume Analysis }}$}

General recommendations for the monitoring network frequency and density are suggested based on heuristic rules applied to the source and tail trend results. 
Individual well trend results are consolidated and weighted by the MAROS according to user input, and the direction and strength of contaminant concentration trends in the source zone and tail zone for each COC are determined. Based on

i) the consolidated trend analysis,

ii) hydrogeologic factors (e.g., seepage velocity), and

iii) location of potential receptors (e.g., wells, discharge points, or property boundaries),

the software suggests a general optimization plan for the current monitoring system in order to efficiently but effectively monitor groundwater in the future. A flow chart utilizing the trend analysis results and other site-specific parameters to form a general sampling frequency and well density recommendation is outlined in Figure C.2. For example, a generic plan for a shrinking petroleum hydrocarbon plume (BTEX) in a slow hydrogeologic environment (silt) with no nearby receptors would entail minimal, low frequency sampling of just a few indicators. On the other hand, the generic plan for a chlorinated solvent plume in a fast hydrogeologic environment that is expanding but has very erratic concentrations over time would entail more extensive, higher frequency sampling. The generic plan is based on a heuristically derived algorithm for assessing future sampling duration, location and density that takes into consideration plume stability. For a detailed description of the heuristic rules used in the MAROS software, refer to the MAROS 2.1Manual (AFCEE, 2003).

\section{$\underline{6.4 \text { Moment Analysis }}$}

An analysis of moments can help resolve plume trends, where the zeroth moment shows change in dissolved mass vs. time, the first moment shows the center of mass location vs. time, and the second moment shows the spread of the plume vs. time. Moment calculations can predict how the plume will change in the future if further statistical analysis is applied to the moments to identify a trend (in this case, Mann Kendall Trend Analysis is applied). The trend analysis of moments can be summarized as:

- Zeroth Moment: An estimate of the total mass of the constituent for each sample event

- First Moment: An estimate of the center of mass for each sample event

- Second Moment: An estimate of the spread of the plume around the center of mass

The role of moment analysis in MAROS is to provide a relative estimate of plume stability and condition within the context of results from other MAROS modules. The Moment analysis algorithms in MAROS are simple approximations of complex calculations and are meant to estimate changes in total mass, center of mass and spread of mass for complex well networks. The Moment Analysis module is sensitive to the number and arrangement of wells in each sampling event, so, changes in the number and identity of wells during monitoring events, and the parameters chosen for data consolidation can cause changes in the estimated moments.

Plume stability may vary by constituent, therefore the MAROS Moment analysis can be used to evaluate multiple COCs simultaneously which can be used to provide a quick way of comparing individual plume parameters to determine the size and movement of constituents relative to one another. Moment analysis in the MAROS software can also 
be used to assist the user in evaluating the impact on plume delineation in future sampling events by removing identified "redundant" wells from a long-term monitoring program (this analysis was not performed as part of this study, for more details on this application of moment analysis refer to the MAROS Users Manual (AFCEE, 2003)).

The zeroth moment is the sum of concentrations for all monitoring wells and is a mass estimate. The zeroth moment calculation can show high variability over time, largely due to the fluctuating concentrations at the most contaminated wells as well as varying monitoring well network. Plume analysis and delineation based exclusively on concentration can exhibit fluctuating temporal and spatial values. The mass estimate is also sensitive to the extent of the site monitoring well network over time. The zeroth moment trend over time is determined by using the Mann-Kendall Trend Methodology. The zeroth Moment trend test allows the user to understand how the plume mass has changed over time. Results for the trend include: Increasing, probably Increasing, no trend, stable, probably decreasing, decreasing or not applicable (N/A) (Insufficient Data). When considering the results of the zeroth moment trend, the following factors should be considered which could effect the calculation and interpretation of the plume mass over time: 1) Change in the spatial distribution of the wells sampled historically 2) Different wells sampled within the well network over time (addition and subtraction of well within the network). 3) Adequate versus inadequate delineation of the plume over time

The first moment estimates the center of mass, coordinates ( $\mathrm{Xc}$ and $\mathrm{Yc}$ ) for each sample event and COC. The changing center of mass locations indicate the movement of the center of mass over time. Whereas, the distance from the original source location to the center of mass locations indicate the movement of the center of mass over time relative to the original source. Calculation of the first moment normalizes the spread by the concentration indicating the center of mass. The first moment trend of the distance to the center of mass over time shows movement of the plume in relation to the original source location over time. Analysis of the movement of mass should be viewed as it relates to 1) the original source location of contamination 2) the direction of groundwater flow and/or 3) source removal or remediation. Spatial and temporal trends in the center of mass can indicate spreading or shrinking or transient movement based on season variation in rainfall or other hydraulic considerations. No appreciable movement or a neutral trend in the center of mass would indicate plume stability. However, changes in the first moment over time do not necessarily completely characterize the changes in the concentration distribution (and the mass) over time. Therefore, in order to fully characterize the plume the First Moment trend should be compared to the zeroth moment trend (mass change over time).

The second moment indicates the spread of the contaminant about the center of mass (Sxx and Syy), or the distance of contamination from the center of mass for a particular COC and sample event. The Second Moment represents the spread of the plume over time in both the $x$ and $y$ directions. The Second Moment trend indicates the spread of the plume about the center of mass. Analysis of the spread of the plume should be viewed as it relates to the direction of groundwater flow. An Increasing trend in the second moment indicates an expanding plume, whereas a declining trend in the second moment indicates a shrinking plume. No appreciable movement or a neutral trend in the center of mass would indicate plume stability. The second moment provides a measure of the spread of the concentration distribution about the plume's center of mass. 
However, changes in the second moment over time do not necessarily completely characterize the changes in the concentration distribution (and the mass) over time. Therefore, in order to fully characterize the plume the Second Moment trend should be compared to the zeroth moment trend (mass change over time).

\subsection{Detailed Statistics: Optimization Analysis}

Although the overall plume analysis shows a general recommendation regarding sampling frequency reduction and a general sampling density, a more detailed analysis is also available with the MAROS 2.1 software in order to allow for further reductions on a well-by-well basis for frequency, well redundancy, well sufficiency and sampling sufficiency. The MAROS Detailed Statistics allows for a quantitative analysis for spatial and temporal optimization of the well network on a well-by-well basis. The results from the Overview Statistics should be considered along with the MAROS optimization recommendations gained from the Detailed Statistical Analysis described previously. The MAROS Detailed Statistics results should be reassessed in view of site knowledge and regulatory requirements as well as in consideration of the Overview Statistics (Figure C.2).

The Detailed Statistics or Sampling Optimization MAROS modules can be used to determine the minimal number of sampling locations and the lowest frequency of sampling that can still meet the requirements of sampling spatially and temporally for an existing monitoring program. It also provides an analysis of the sufficiency of data for the monitoring program.

Sampling optimization in MAROS consists of four parts:

- Well redundancy analysis using the Delaunay method

- Well sufficiency analysis using the Delaunay method

- Sampling frequency determination using the Modified CES method

- Data sufficiency analysis using statistical power analysis.

The well redundancy analysis using the Delaunay method identifies and eliminates redundant locations from the monitoring network. The well sufficiency analysis can determine the areas where new sampling locations might be needed. The Modified CES method determines the optimal sampling frequency for a sampling location based on the direction, magnitude, and uncertainty in its concentration trend. The data sufficiency analysis examines the risk-based site cleanup status and power and expected sample size associated with the cleanup status evaluation.

\subsection{Well Redundancy Analysis - Delaunay Method}

The well redundancy analysis using the Delaunay method is designed to select the minimum number of sampling locations based on the spatial analysis of the relative importance of each sampling location in the monitoring network. The approach allows elimination of sampling locations that have little impact on the historical characterization of a contaminant plume. An extended method or wells sufficiency analysis, based on the Delaunay method, can also be used for recommending new sampling locations. 
Details about the Delaunay method can be found in Appendix A.2 of the MAROS Manual (AFCEE, 2003).

Sampling Location determination uses the Delaunay triangulation method to determine the significance of the current sampling locations relative to the overall monitoring network. The Delaunay method calculates the network Area and Average concentration of the plume using data from multiple monitoring wells. A slope factor (SF) is calculated for each well to indicate the significance of this well in the system (i.e. how removing a well changes the average concentration.)

The Sampling Location optimization process is performed in a stepwise fashion. Step one involves assessing the significance of the well in the system, if a well has a small SF (little significance to the network), the well may be removed from the monitoring network. Step two involves evaluating the information loss of removing a well from the network. If one well has a small SF, it may or may not be eliminated depending on whether the information loss is significant. If the information loss is not significant, the well can be eliminated from the monitoring network and the process of optimization continues with fewer wells. However if the well information loss is significant then the optimization terminates. This sampling optimization process allows the user to assess "redundant" wells that will not incur significant information loss on a constituent-by-constituent basis for individual sampling events.

\section{$\underline{7.2 \text { Well Sufficiency Analysis - Delaunay Method }}$}

The well sufficiency analysis, using the Delaunay method, is designed to recommend new sampling locations in areas within the existing monitoring network where there is a high level of uncertainty in contaminant concentration. Details about the well sufficiency analysis can be found in Appendix A.2 of the MAROS Manual (AFCEE, 2003).

In many cases, new sampling locations need to be added to the existing network to enhance the spatial plume characterization. If the MAROS algorithm calculates a high level of uncertainty in predicting the constituent concentration for a particular area, a new sampling location is recommended. The Slope Factor (SF) values obtained from the redundancy evaluation described above are used to calculate the concentration estimation error for each triangle area formed in the Delaunay triangulation. The estimated SF value for each area is then classified into four levels: Small, Moderate, Large, or Extremely large (S, M, L, E) because the larger the estimated SF value, the higher the estimation error at this area. Therefore, the triangular areas with the estimated SF value at the Extremely large or Large level can be candidate regions for new sampling locations.

The results from the Delaunay method and the method for determining new sampling locations are derived solely from the spatial configuration of the monitoring network and the spatial pattern of the contaminant plume. No parameters such as the hydrogeologic conditions are considered in the analysis. Therefore, professional judgment and regulatory considerations must be used to make final decisions. 


\section{$\underline{7.3 \text { Sampling Frequency Determination - Modified CES Method }}$}

The Modified CES method optimizes sampling frequency for each sampling location based on the magnitude, direction, and uncertainty of its concentration trend derived from its recent and historical monitoring records. The Modified Cost Effective Sampling (MCES) estimates a conservative lowest-frequency sampling schedule for a given groundwater monitoring location that still provides needed information for regulatory and remedial decision-making. The MCES method was developed on the basis of the Cost Effective Sampling (CES) method developed by Ridley et al (1995). Details about the MCES method can be found in Appendix A.9 of the MAROS Manual (AFCEE, 2003).

In order to estimate the least frequent sampling schedule for a monitoring location that still provides enough information for regulatory and remedial decision-making, MCES employs three steps to determine the sampling frequency. The first step involves analyzing frequency based on recent trends. A preliminary location sampling frequency (PLSF) is developed based on the rate of change of well concentrations calculated by linear regression along with the Mann-Kendall trend analysis of the most recent monitoring data (see Figure C.3). The variability within the sequential sampling data is accounted for by the Mann-Kendall analysis. The rate of change vs. trend result matrix categorizes wells as requiring annual, semi-annual or quarterly sampling. The PLSF is then reevaluated and adjusted based on overall trends. If the long-term history of change is significantly greater than the recent trend, the frequency may be reduced by one level.

The final step in the analysis involves reducing frequency based on risk, site-specific conditions, regulatory requirements or other external issues. Since not all compounds in the target being assessed are equally harmful, frequency is reduced by one level if recent maximum concentration for a compound of high risk is less than $1 / 2$ of the Maximum Concentration Limit (MCL). The result of applying this method is a suggested sampling frequency based on recent sampling data trends and overall sampling data trends and expert judgment.

The final sampling frequency determined from the MCES method can be Quarterly, Semiannual, Annual, or Biennial. Users can further reduce the sampling frequency to, for example, once every three years, if the trend estimated from Biennial data (i.e., data drawn once every two years from the original data) is the same as that estimated from the original data.

\subsection{Data Sufficiency Analysis - Power Analysis}

The MAROS Data Sufficiency module employs simple statistical methods to evaluate whether the collected data are adequate both in quantity and in quality for revealing changes in constituent concentrations. The first section of the module evaluates individual well concentrations to determine if they are statistically below a target screening level. The second section includes a simple calculation for estimating projected groundwater concentrations at a specified point downgradient of the plume. A statistical Power analysis is then applied to the projected concentrations to determine if the downgradient concentrations are statistically below the cleanup standard. If the number of projected concentrations is below the level to provide statistical significance, 
then the number of sample events required to statistically confirm concentrations below standards is estimated from the Power analysis.

Before testing the cleanup status for individual wells, the stability or trend of the contaminant plume should be evaluated. Only after the plume has reached stability or is reliably diminishing can we conduct a test to examine the cleanup status of wells. Applying the analysis to wells in an expanding plume may cause incorrect conclusions and is less meaningful.

Statistical power analysis is a technique for interpreting the results of statistical tests. The Power of a statistical test is a measure of the ability of the test to detect an effect given that the effect actually exists. The method provides additional information about a statistical test: 1 ) the power of the statistical test, i.e., the probability of finding a difference in the variable of interest when a difference truly exists; and 2) the expected sample size of a future sampling plan given the minimum detectable difference it is supposed to detect. For example, if the mean concentration is lower than the cleanup goal but a statistical test cannot prove this, the power and expected sample size can tell the reason and how many more samples are needed to result in a significant test. The additional samples can be obtained by a longer period of sampling or an increased sampling frequency. Details about the data sufficiency analysis can be found in Appendix A.6 of the MAROS Manual (AFCEE, 2003).

When applying the MAROS power analysis method, a hypothetical statistical compliance boundary (HSCB) is assigned to be a line perpendicular to the groundwater flow direction (see figure below). Monitoring well concentrations are projected onto the HSCB using the distance from each well to the compliance boundary along with a decay coefficient. The projected concentrations from each well and each sampling event are then used in the risk-based power analysis. Since there may be more than one sampling event selected by the user, the risk-based power analysis results are given on an eventby-event basis. This power analysis can then indicate if target are statistically achieved at the HSCB. For instance, at a site where the historical monitoring record is short with few wells, the HSCB would be distant; whereas, at a site with longer duration of sampling with many wells, the HSCB would be close. Ultimately, at a site the goal would be to have the HSCB coincide with or be within the actual compliance boundary (typically the site property line). 


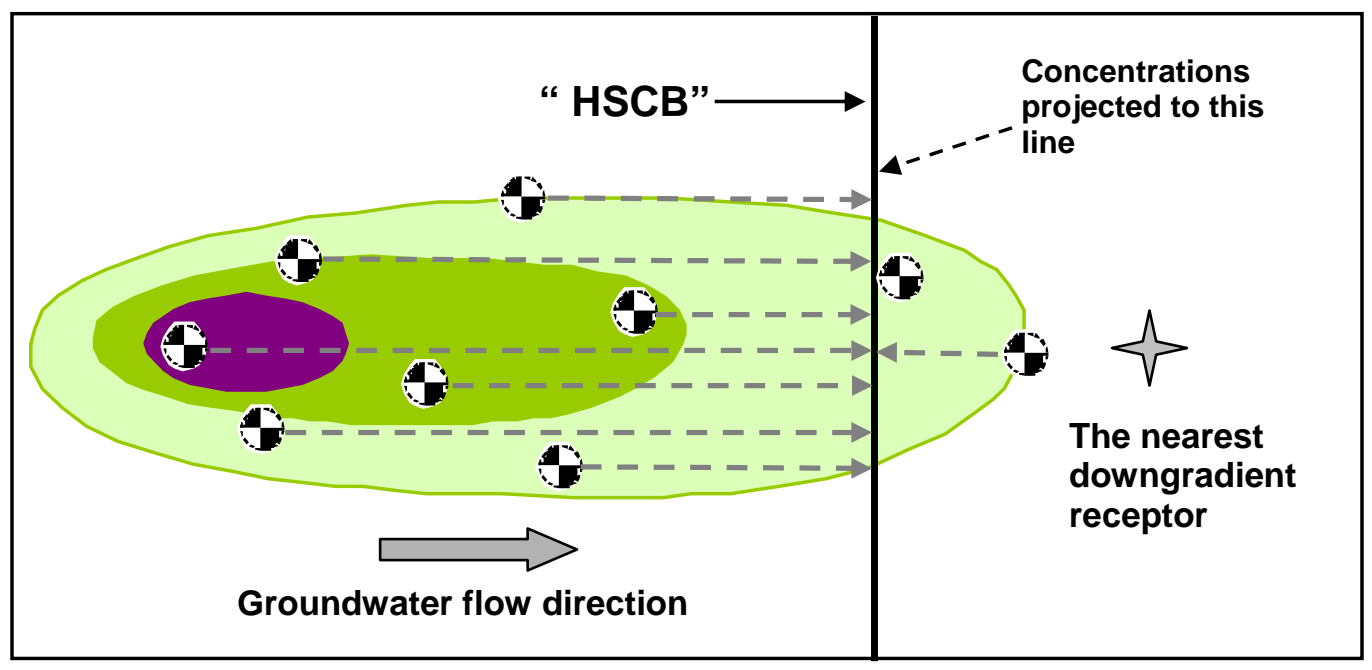

In order to perform a risk-based cleanup status evaluation for the whole site, a strategy was developed as follows.

- Estimate concentration versus distance decay coefficient from plume centerline wells.

- Extrapolate concentration versus distance for each well using this decay coefficient.

- Comparing the extrapolated concentrations with the compliance concentration using power analysis.

Results from this analysis can be Attained or Not Attained, providing a statistical interpretation of whether the cleanup goal has been met on the site-scale from the riskbased point of view. The results as a function of time can be used to evaluate if the monitoring system has enough power at each step in the sampling record to indicate certainty of compliance by the plume location and condition relative to the compliance boundary. For example, if results are Not Attained at early sampling events but are Attained in recent sampling events, it indicates that the recent sampling record provides a powerful enough result to indicate compliance of the plume relative to the location of the receptor or compliance boundary. 


\section{CITED REFERENCES}

AFCEE 2003. Monitoring and Remediation Optimization System (MAROS) 2.1 Software Users Guide. Air Force Center for Environmental Excellence. http://www.gsinet.com/software/MAROS V2 1Manual.pdf

AFCEE. 1997. Air Force Center for Environmental Excellence, AFCEE Long-Term Monitoring Optimization Guide, http://www.afcee.brooks.af.mil.

Aziz, J. A., C. J. Newell, M. Ling, H. S. Rifai and J. R. Gonzales (2003). "MAROS: A Decision Support System for Optimizing Monitoring Plans." Ground Water 41(3): 355367.

Gilbert, R. O., 1987, Statistical Methods for Environmental Pollution Monitoring, Van Nostrand Reinhold, New York, NY, ISBN 0-442-23050-8.

Hollander, M. and Wolfe, D. A. (1973). Nonparametric Statistical Methods, Wiley, New York, NY.

Ridley, M.N. et al., 1995. Cost-Effective Sampling of Groundwater Monitoring Wells, the Regents of UC/LLNL, Lawrence Livermore National Laboratory.

U.S. Environmental Protection Agency, 1992. Methods for Evaluating the Attainment of Cleanup Standards Volume 2: Ground Water.

Weight, W. D. and J. L. Sonderegger (2001). Manual of Applied Field Hydrogeology.

New York, NY, McGraw-Hill. 
TABLE C.1

Mann-Kendall Analysis Decision Matrix (Aziz, et. al., 2003)

\begin{tabular}{ccc}
$\begin{array}{c}\text { Mann-Kendall } \\
\text { Statistic }\end{array}$ & $\begin{array}{c}\text { Confidence in the } \\
\text { Trend }\end{array}$ & Concentration Trend \\
\hline $\mathrm{S}>0$ & $>95 \%$ & Increasing \\
$\mathrm{S}>0$ & $90-95 \%$ & Probably Increasing \\
$\mathrm{S}>0$ & $<90 \%$ & No Trend \\
$\mathrm{S} \leq 0$ & $<90 \%$ and COV $\geq 1$ & No Trend \\
$\mathrm{S} \leq 0$ & $<90 \%$ and COV $<1$ & Stable \\
$\mathrm{S}<0$ & $90-95 \%$ & Probably Decreasing \\
$\mathrm{S}<0$ & $>95 \%$ & Decreasing
\end{tabular}

TABLE C.2

Linear Regression Analysis Decision Matrix (Aziz, et. al., 2003)

\begin{tabular}{ccc|} 
Confidence in the & \multicolumn{2}{c}{ Log-slope } \\
\cline { 2 - 3 } Trend & Positive & Negative \\
\hline$<90 \%$ & No Trend & COV $<1$ Stable \\
& COV $>1$ No Trend \\
$90-95 \%$ & Probably Increasing & Probably Decreasing \\
$>95 \%$ & Increasing & Decreasing \\
\hline
\end{tabular}




\section{MAROS: Decision Support Tool}

MAROS is a collection of tools in one software package that is used in an explanatory, non-linear fashion. The tool includes models, geostatistics, heuristic rules, and empirical relationships to assist the user in optimizing a groundwater monitoring network system while maintaining adequate delineation of the plume as well as knowledge of the plume state over time. Different users utilize the tool in different ways and interpret the results from a different viewpoint.

\section{Overview Statistics}

What it is: Simple, qualitative and quantitative plume information can be gained through evaluation of monitoring network historical data trends both spatially and temporally. The MAROS Overview Statistics are the foundation the user needs to make informed optimization decisions at the site.

What it does: The Overview Statistics are designed to allow site personnel to develop a better understanding of the plume behavior over time and understand how the individual well concentration trends are spatially distributed within the plume. This step allows the user to gain information that will support a more informed decision to be made in the next level of optimization analysis.

What are the tools: Overview Statistics includes two analytical tools:

1) Trend Analysis: includes Mann-Kendall and Linear Regression statistics for individual wells and results in general heuristically-derived monitoring categories with a suggested sampling density and monitoring frequency.

2) Moment Analysis: includes dissolved mass estimation ( $0^{\text {th }}$ Moment), center of mass ( $1^{\text {st }}$ Moment), and plume spread $\left(2^{\text {nd }}\right.$ Moment) over time. Trends of these moments show the user another piece of information about the plume stability over time.

What is the product: A first-cut blueprint for a future long-term monitoring program that is intended to be a foundation for more detailed statistical analysis.

\section{Detailed Statistics}

What it is: The MAROS Detailed Statistics allows for a quantitative analysis for spatial and temporal optimization of the well network on a well-by-well basis.

What it does: The results from the Overview Statistics should be considered along side the MAROS optimization recommendations gained from the Detailed Statistical Analysis. The MAROS Detailed Statistics results should be reassessed in view of site knowledge and regulatory requirements as well as the Overview Statistics.

What are the tools: Detailed Statistics includes four analytical tools:

1) Sampling Frequency Optimization: uses the Modified CES method to establish a recommended future sampling frequency.

2) Well Redundancy Analysis: uses the Delaunay Method to evaluate if any wells within the monitoring network are redundant and can be eliminated without any significant loss of plume information.

3) Well Sufficiency Analysis: uses the Delaunay Method to evaluate areas where new wells are recommended within the monitoring network due to high levels of concentration uncertainty.

4) Data Sufficiency Analysis: uses Power Analysis to assess if the historical monitoring data record has sufficient power to accurately reflect the location of the plume relative to the nearest receptor or compliance point.

What is the product: List of wells to remove from the monitoring program, locations where monitoring wells may need to be added, recommended frequency of sampling for each well, analysis if the overall system is statistically powerful to monitor the plume.

Figure C.1. MAROS Decision Support Tool Flow Chart 
GSI Job No. G-3038

Issued: 12/12/2005

Page 1 of 1
GROUNDWATER SERVICES, INC.

\section{Select Representative Wells in "Source" and "Plume" Zone}

1

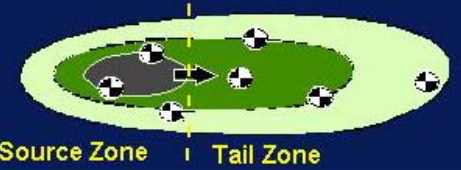

2 Identify Site Constituents of Concern (COCs).

(Assistance provided by software.)

Analyze Lines of Evidence (LOEs)

for Plume Stability (by well and by coc)

Categorize concentrations of $\mathrm{COC}$ in each well as:

3

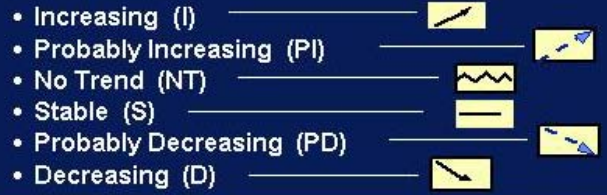

\section{Determine General Trend} for Each Well Based On All

(4) LOE's

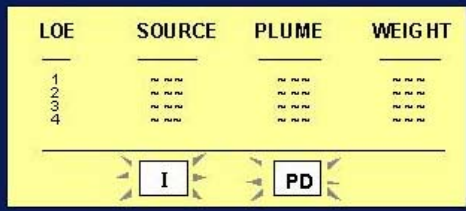

"Lump Lines of Evidence"

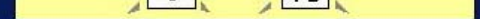

Determine General Trend for Source and Tail Zones
5 - Increasing (I)
- Probably Increasing $(\mathrm{P})$
- No Trend (NT)
- Stable (S)
- Probably Decreasing (PD)
- Decreasing (D)

"Lump Wells" in Source and Tail Zone
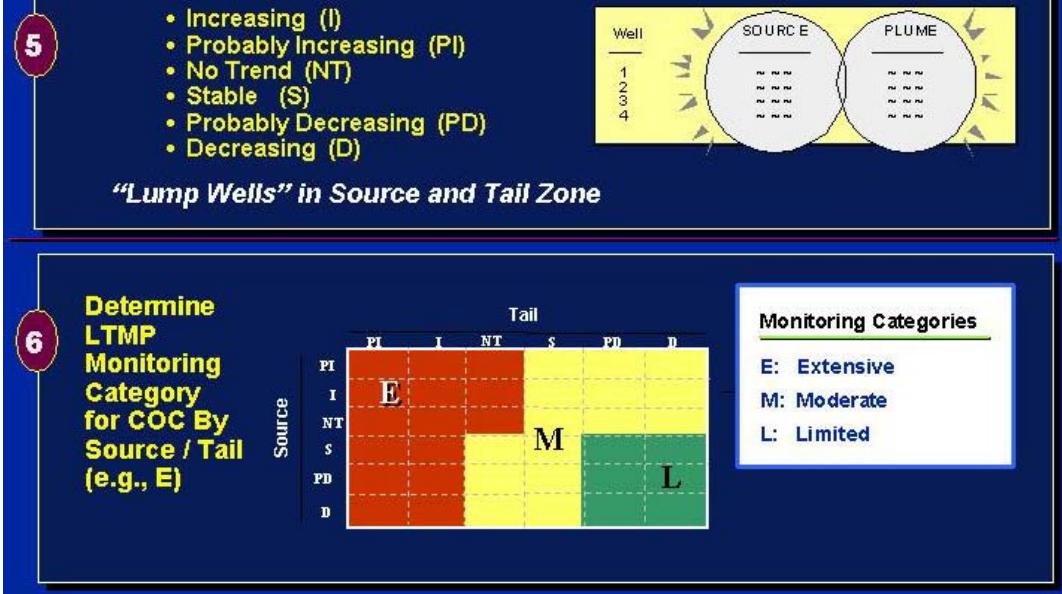

Specify Preliminary Monitoring

7 System Optimization Results based on Monitoring category and site-specific parameters.

- Well Density

- Sampling Frequency

Sampling Duration

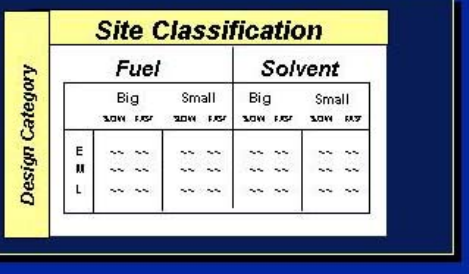

Figure C.2:

MAROS Overview Statistics Trend Analysis Methodology 


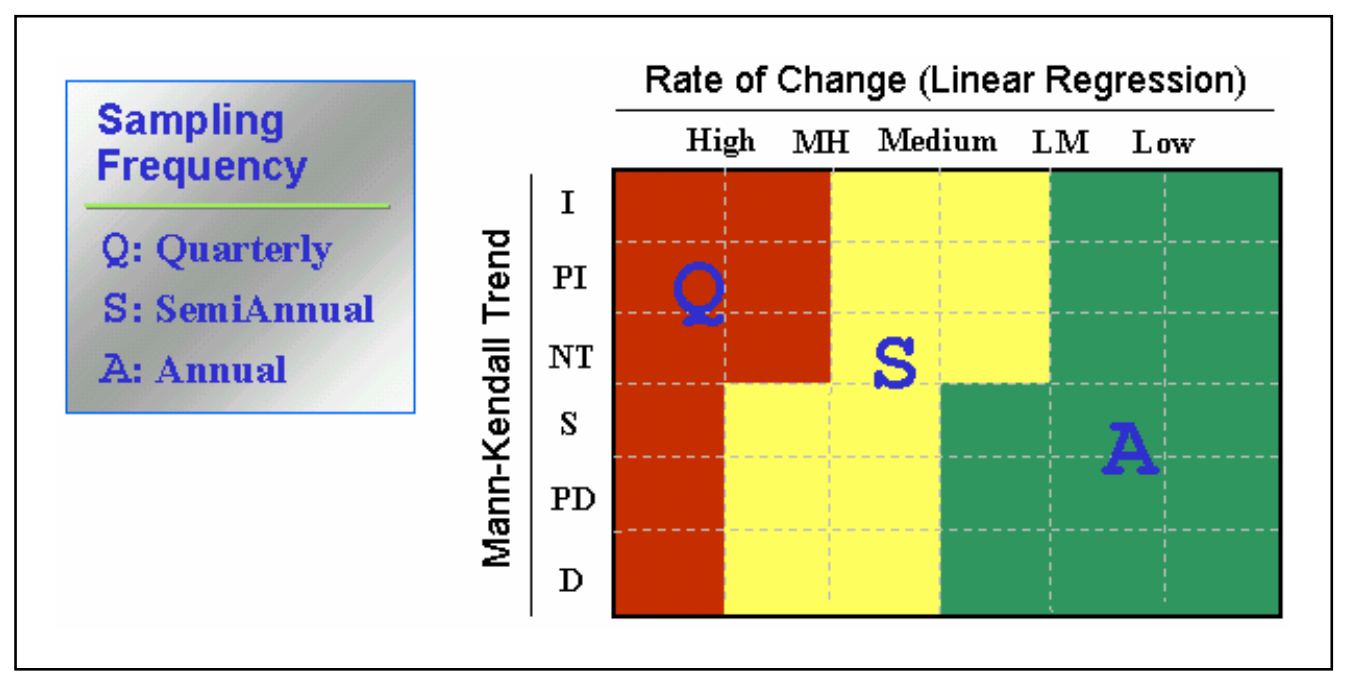

Figure C.3. Decision Matrix for Determining Provisional Frequency (Figure A.3.1 of the MAROS Manual (AFCEE 2003) 


\section{ASSESSMENT OF THE \\ GROUNDWATER PROTECTION PROGRAM \\ Y-12 NATIONAL SECURITY COMPLEX, OAK RIDGE, TENNESSEE}

Y-12 National Security Complex

Oak Ridge, Tennessee

APPENDIX D: QUALITATIVE REVIEW AND MAROS REPORTS

Appendix D.1 Bear Creek Regime West S-3

Appendix D.2 Bear Creek Regime Oil Landfarm WMA

Appendix D.3 Bear Creek Regime Bear Creek Burial Grounds

Appendix D.4 East Fork Regime East S-3 Area

Appendix D.5 East Fork Regime Central Y-12 Area

Appendix D.6 East Fork Regime Fuel Station Area

Appendix D.7 East Fork Regime East Y-12

Appendix D.8 Chestnut Ridge Regime West Chestnut Ridge

Appendix D.9 Chestnut Ridge Regime Security Pits Area

Appendix D.10 Chestnut Ridge Regime Landfills V and VII Area

Appendix D.11 Chestnut Ridge Regime East Kerr Hollow Area 


\section{ASSESSMENT OF THE GROUNDWATER PROTECTION PROGRAM \\ Y-12 NATIONAL SECURITY COMPLEX, OAK RIDGE, TENNESSEE}

Y-12 National Security Complex

Oak Ridge, Tennessee

\section{Appendix D.1 Bear Creek Regime West S-3}

Table D.1.1

Qualitative Analysis West S-3 Area

Table D.1.2

Aquifer Input Parameters

MAROS Report

COC Assessment

MAROS Report Plume Summary

MAROS Report Spatial Moment Analysis Summary

MAROS Chart New Location Analysis PCE West S-3 
TABLE D.1.1

QUALITATIVE ANALYSIS WEST S-3 AREA

Bear Creek Hydrogeologic Regime

Y-12 National Security Complex

Oak Ridge, Tennessee

\begin{tabular}{|c|c|c|c|c|c|c|c|c|c|c|c|c|}
\hline $\begin{array}{c}\text { Location } \\
\text { Name }\end{array}$ & Location Type & $\begin{array}{c}\text { Average } \\
\text { Concentration } \\
\text { Exceeds } \\
\text { Screening } \\
\end{array}$ & $\begin{array}{c}\text { Formation } \\
\text { Type }\end{array}$ & $\begin{array}{l}\text { Horizontal } \\
\text { Delineation }\end{array}$ & $\begin{array}{c}\text { Vertical } \\
\text { Delineation }\end{array}$ & $\begin{array}{c}\text { Exit } \\
\text { Location }\end{array}$ & RCRA & CERCLA & Unique & $\begin{array}{c}\text { Monitors } \\
\text { Background } \\
\text { Water Quality }\end{array}$ & $\begin{array}{c}\text { Early } \\
\text { Detection }\end{array}$ & $\begin{array}{c}\text { Monitor } \\
\text { Source }\end{array}$ \\
\hline \begin{tabular}{|l|}
$G W-100$ \\
$G W-101$ \\
$G W-115$ \\
$G W-122$ \\
$G W-123$ \\
\end{tabular} & $\begin{array}{l}\text { WL } \\
W L \\
W L \\
W L \\
W L\end{array}$ & $\begin{array}{l}X \\
X \\
X \\
X \\
\end{array}$ & $\begin{array}{l}\text { Aquifer } \\
\text { Aquitard } \\
\text { Aquitard } \\
\text { Aquifer } \\
\text { Aquifer }\end{array}$ & & $x$ & $\begin{array}{l}X \\
X\end{array}$ & & $\begin{array}{l}X \\
X\end{array}$ & & & $\begin{array}{l}X \\
X\end{array}$ & \\
\hline GW-124 & WL & $\mathrm{X}$ & Aquifer & & $\bar{x}$ & & & & & & $x$ & \\
\hline GW-125 & WL & $x$ & Aquifer & $x$ & $x$ & & & $x$ & & & $x$ & \\
\hline GW-127 & WL & $x$ & Aquifer & & & $x$ & & & & & & \\
\hline GW-236 & WL & $x$ & Aquifer & & & & & & & & & \\
\hline$G W-243$ & WL & $x$ & Aquitard & & & $\mathrm{x}$ & & & & & & $x$ \\
\hline GW-244 & WL & $\mathrm{X}$ & Aquitard & & & $\mathrm{X}$ & & & & & & $\mathrm{X}$ \\
\hline GW-245 & WL & $\mathrm{x}$ & Aquitard & & & $\mathrm{x}$ & & & & & & $\mathrm{x}$ \\
\hline GW-246 & WL & $x$ & Aquitard & & & $x$ & & & & & & $\mathrm{x}$ \\
\hline GW-247 & WL & $x$ & Aquitard & & & $\mathrm{x}$ & & & & & & $\mathrm{x}$ \\
\hline GW-276 & WL & $x$ & Aquitard & & & $\mathrm{x}$ & & & & & & \\
\hline GW-277 & $\overline{W L}$ & $\mathrm{X}$ & Aquitard & & & & & & & & & \\
\hline GW-306 & WL & $x$ & Aquifer & $x$ & & & & & & $x$ & & \\
\hline GW-307 & WL & $\mathrm{x}$ & Aquifer & $\mathrm{x}$ & & & & & & & & \\
\hline GW-308 & WL & $x$ & Aquifer & $\mathrm{x}$ & & & & & & & & \\
\hline GW-309 & WL & $x$ & Aquifer & $x$ & & & & & & & & \\
\hline
\end{tabular}

1. $\mathrm{WL}=$ Monitoring Well; $\mathrm{SP}=$ Spring

2. Well data taken from BWXT Y-12 Analytical Database. Sample locations shown on Figure A.1 and A.2.

3. RCRA indicates wells monitored as part of compliance with RCRA Post-Closure Corrective Action Monitoring or designated Alternate location;

CERCLA indicates locations monitored as part of compliance with CERCLA ROD or backup location. Data from BWXT, 2003a and BWXT 2004a.

4. Average Concentration Exceeds Screening = The average concentration over the entire sampling record for the priority constituent is above the MCL or other designated screening level as defined in Table B.1.

5. Aquifer and aquitard formations identified in Fig. A.2 from BWXT Y12, 2003 Groundwater Monitoring Report, (12/01/2003).

6. Details of the decision criteria for each category are presented in the text. 
TABLE D.1.1

QUALITATIVE ANALYSIS WEST S-3 AREA

Bear Creek Hydrogeologic Regime

Y-12 National Security Complex

Oak Ridge, Tennessee

\begin{tabular}{|c|c|c|c|c|c|c|c|c|c|c|c|c|}
\hline $\begin{array}{c}\text { Location } \\
\text { Name }\end{array}$ & Location Type & $\begin{array}{c}\text { Average } \\
\text { Concentration } \\
\text { Exceeds } \\
\text { Screening } \\
\end{array}$ & $\begin{array}{c}\text { Formation } \\
\text { Type }\end{array}$ & $\begin{array}{l}\text { Horizontal } \\
\text { Delineation }\end{array}$ & $\begin{array}{c}\text { Vertical } \\
\text { Delineation }\end{array}$ & $\begin{array}{c}\text { Exit } \\
\text { Location }\end{array}$ & RCRA & CERCLA & Unique & $\begin{array}{c}\text { Monitors } \\
\text { Background } \\
\text { Water Quality }\end{array}$ & $\begin{array}{c}\text { Early } \\
\text { Detection }\end{array}$ & $\begin{array}{c}\text { Monitor } \\
\text { Source }\end{array}$ \\
\hline GW-310 & $\overline{W L}$ & $\bar{x}$ & Aquifer & & & & & & & & & \\
\hline GW-311 & WL & $x$ & Aquifer & $\mathrm{x}$ & & & & & & & & \\
\hline GW-312 & WL & $x$ & Aquifer & & & & & & & & & \\
\hline GW-313 & WL & $x$ & Aquifer & $x$ & & & & & & & & $x$ \\
\hline GW-314 & WL & $\mathrm{x}$ & Aquifer & $x$ & & & & & & & & $x$ \\
\hline GW-315 & WL & $\mathrm{X}$ & Aquifer & $\mathrm{x}$ & & & & & & & & $\mathrm{x}$ \\
\hline GW-316 & WL & & Aquifer & & & & & & & & & $x$ \\
\hline GW-317 & WL & & Aquifer & $x$ & & & & $\mathrm{x}$ & & & & $\mathrm{x}$ \\
\hline GW-323 & WL & & Aquifer & $x$ & & & & & & $x$ & & \\
\hline GW-325 & WL & & Aquitard & & & & & & & & & \\
\hline GW-345 & WL & $\mathrm{x}$ & Aquitard & & & & & & & & & \\
\hline GW-346 & WL & $\mathrm{x}$ & Aquitard & & & & & $\mathrm{x}$ & & & & \\
\hline GW-347 & WL & & Aquifer & & & & & & & & & \\
\hline GW-348 & WL & & Aquifer & & & & & $\mathrm{x}$ & & $\mathrm{x}$ & & \\
\hline GW-526 & WL & $x$ & Aquitard & & & & $\mathrm{x}$ & & & & & \\
\hline GW-531 & $\overline{W L}$ & & Aquitard & & & & & & & & & \\
\hline GW-613 & WL & & Aquitard & $x$ & & & & & & $x$ & & \\
\hline GW-615 & WL & $x$ & Aquitard & & & $x$ & & & & & & \\
\hline GW-616 & WL & $x$ & Aquifer & & & & & & & & & \\
\hline GW-630 & WL & & Aquifer & $x$ & & & & & & & & \\
\hline GW-648 & $\overline{W L}$ & & Aquifer & $\bar{x}$ & & & & & & $\bar{x}$ & & \\
\hline GW-829 & WL & $x$ & Aquitard & $x$ & & & & & & & & \\
\hline GW-835 & WL & $x$ & & $x$ & & & $x$ & & & & $\mathrm{x}$ & \\
\hline SS-1 & SP & $\mathrm{x}$ & Spring & $\mathrm{x}$ & $\mathrm{x}$ & $x$ & & & & & & \\
\hline
\end{tabular}

\section{Notes:}

2. Well data taken from BWXT Y-12 Analytical Database. Sample locations shown on Figure A.1 and A.2.

3. RCRA indicates wells monitored as part of compliance with RCRA Post-Closure Corrective Action Monitoring or designated Alternate location;

CERCLA indicates locations monitored as part of compliance with CERCLA ROD or backup location. Data from BWXT, 2003a and BWXT 2004a.

4. Average Concentration Exceeds Screening $=$ The average concentration over the entire sampling record for the priority constituent is above the MCL or other designated screening level

as defined in Table B.1.

5. Aquifer and aquitard formations identified in Fig. A.2 from BWXT Y12, 2003 Groundwater Monitoring Report, (12/01/2003)

6. Details of the decision criteria for each category are presented in the text. 
GSI Job No. G-3038

Issued $12 / 12 / 2005$

Page 1 of 1

GROUNDWATER

SERVICES, INC

TABLE D.1.2

AQUIFER INPUT PARAMETERS

West S3 Area

Bear Creek Regime

Y-12 National Security Complex

\begin{tabular}{|c|c|c|}
\hline Parameter & Value & Units \\
\hline Current Plume Length & 3000 & $\mathrm{ft}$ \\
\hline Maximum Plume Length & 3000 & $\mathrm{ft}$ \\
\hline PlumeWidth & 1400 & $\mathrm{ft}$ \\
\hline SeepageVelocity (ft/yr) & 200 & $\mathrm{ft} / \mathrm{yr}$ \\
\hline Distance to Receptors & 5000 & $\mathrm{ft}$ \\
\hline GWFluctuations & Yes & -- \\
\hline SourceTreatment & None & -- \\
\hline PlumeType & Nitrate/GB/GA/VOC & -- \\
\hline Free NAPL Present & Yes & -- \\
\hline Priority COCs & Screening Levels & \\
\hline Trichloroethene (TCE) & 0.005 & $\overline{\mathrm{mg} / \mathrm{L}}$ \\
\hline 1,1-Dichloroethene & 0.007 & $\mathrm{mg} / \mathrm{L}$ \\
\hline Vinyl Chloride & 0.002 & \\
\hline Benzene & 0.006 & $\mathrm{mg} / \mathrm{L}$ \\
\hline Tetrachloroethene (PCE) & 0.005 & $\mathrm{mg} / \mathrm{L}$ \\
\hline Parameter & Value & \\
\hline Groundwater flow direction & W/SW & 200 \\
\hline Effective Porosity & 0.1 & -- \\
\hline Source Location near Well & GW-243 & -- \\
\hline Source X-Coordinate & 51990.37 & $\mathrm{ft}^{\star}$ \\
\hline Source Y-Coordinate & 30154.79 & $\mathrm{ft}^{*}$ \\
\hline Saturated Thickness & 50 & $\mathrm{ft}$ \\
\hline Source Wells & Value & \\
\hline
\end{tabular}

Notes:

1. Aquifer data are general values for the hydrologic regime.

2. Priority COCs defined by prevalence, toxicty and mobility.

3. $\mathrm{ft}^{\star}=$ Coordinates in $\mathrm{Y}-12$ Plant coordinates, feet.

4. Screening Levels are USEPA MCLs, except in the case of compounds without MCLs where the level is the Region 9 PRG for tap water.

5. Effective Porosity estimated based on average high and low values for aquifer and aquitard suburfaces. 


\section{MAROS COC Assessment}

Project: Y-12 Security Complex

Location: West S-3

Toxicity:

\begin{tabular}{lccc}
\hline Contaminant of Concern & $\begin{array}{c}\text { Representative } \\
\text { Concentration } \\
\text { (mg/L) }\end{array}$ & $\begin{array}{c}\text { PRG } \\
\text { (mg/L) }\end{array}$ & $\begin{array}{c}\text { Percent } \\
\text { Above } \\
\text { PRG }\end{array}$ \\
\hline GROSS BETA ACTIVITY & $1.8 \mathrm{E}+04$ & $5.0 \mathrm{E}+01$ & $36781.2 \%$ \\
NITRATE & $9.8 \mathrm{E}+02$ & $1.0 \mathrm{E}+01$ & $9716.6 \%$ \\
URANIUM & $9.7 \mathrm{E}-01$ & $3.0 \mathrm{E}-02$ & $3122.9 \%$ \\
GROSS ALPHA ACTIVITY & $3.7 \mathrm{E}+02$ & $1.5 \mathrm{E}+01$ & $2343.3 \%$ \\
TETRACHLOROETHYLENE(PCE) & $1.0 \mathrm{E}-01$ & $5.0 \mathrm{E}-03$ & $1952.1 \%$ \\
VINYL CHLORIDE & $6.5 \mathrm{E}-03$ & $2.0 \mathrm{E}-03$ & $223.9 \%$ \\
TRICHLOROETHYLENE (TCE) & $1.0 \mathrm{E}-02$ & $5.0 \mathrm{E}-03$ & $108.4 \%$ \\
TECHNETIUM-99 & $4.2 \mathrm{E}+03$ & $4.0 \mathrm{E}+03$ & $4.1 \%$
\end{tabular}

Note: Top COCs by toxicity were determined by examining a representative concentration for each compound over the entire site. The compound representative concentrations are then compared with the chosen PRG for that compound, with the percentage excedence from the PRG determining the compound's toxicity. All compounds above exceed the PRG.

\section{Prevalence:}

\begin{tabular}{|c|c|c|c|c|c|}
\hline Contaminant of Concern & Class & $\begin{array}{l}\text { Total } \\
\text { Wells }\end{array}$ & $\begin{array}{c}\text { Total } \\
\text { Excedences }\end{array}$ & $\begin{array}{c}\text { Percent } \\
\text { Excedences }\end{array}$ & $\begin{array}{l}\text { Total } \\
\text { detects }\end{array}$ \\
\hline NITRATE & INO & 44 & 28 & $63.6 \%$ & 40 \\
\hline GROSS BETA ACTIVITY & INO & 44 & 20 & $45.5 \%$ & 44 \\
\hline GROSS ALPHA ACTIVITY & INO & 44 & 20 & $45.5 \%$ & 44 \\
\hline TRICHLOROETHYLENE (TCE) & ORG & 44 & 11 & $25.0 \%$ & 27 \\
\hline TETRACHLOROETHYLENE(PCE) & ORG & 44 & 10 & $22.7 \%$ & 32 \\
\hline URANIUM & MET & 44 & 9 & $20.5 \%$ & 43 \\
\hline TECHNETIUM-99 & MET & 28 & 5 & $17.9 \%$ & 28 \\
\hline VINYL CHLORIDE & ORG & 44 & 1 & $2.3 \%$ & 1 \\
\hline
\end{tabular}

Note: Top COCs by prevalence were determined by examining a representative concentration for each well location at the site. The total excedences (values above the chosen PRGs) are compared to the total number of wells to determine the prevalence of the compound.

\section{Mobility:}

TECHNETIUM-99

NITRATE

GROSS BETA ACTIVITY

GROSS ALPHA ACTIVITY

VINYL CHLORIDE 0.042

TRICHLOROETHYLENE (TCE) 0.297

TETRACHLOROETHYLENE(PCE) 0.923

URANIUM

2960

Note: Top COCs by mobility were determined by examining each detected compound in the dataset and comparing their mobilities (Koc's for organics, assume foc $=0.001$, and Kd's for metals). 
Project: Y-12 Security Complex

User Name: MV

Location: West S-3

State: Tennessee

Contaminants of Concern (COC's)

GROSS ALPHA ACTIVITY

GROSS BETA ACTIVITY

NITRATE

TETRACHLOROETHYLENE(PCE)

VINYL CHLORIDE 


\section{MAROS Plume Analysis Summary}

Project: Y-12 Security Complex

Location: West S-3

Time Period: 1/1/1996

to $1 / 1 / 2005$

Consolidation Period: No Time Consolidation

Consolidation Type: Median

Duplicate Consolidation: Average

ND Values: Specified Detection Limit

J Flag Values : Actual Value

\begin{tabular}{|c|c|c|c|c|c|c|c|c|c|c|c|}
\hline Constituent & Well & $\begin{array}{c}\text { Sourcel } \\
\text { Tail }\end{array}$ & $\begin{array}{c}\text { Number } \\
\text { of } \\
\text { Samples }\end{array}$ & $\begin{array}{c}\text { Number } \\
\text { of } \\
\text { Detects }\end{array}$ & $\begin{array}{c}\text { Average } \\
\text { (mg/L) }\end{array}$ & $\begin{array}{r}\text { Median } \\
\text { (mg/L) }\end{array}$ & $\begin{array}{c}\text { All } \\
\text { Samples } \\
\text { "ND" ? }\end{array}$ & $\begin{array}{l}\text { Mann- } \\
\text { Kendall }\end{array}$ & $\begin{array}{c}\text { Linear } \\
\text { Regression }\end{array}$ & Modeling & Empirica \\
\hline \multicolumn{12}{|c|}{ GROSS ALPHA ACTIVITY } \\
\hline & GW-243 & $\mathrm{S}$ & 2 & 2 & $5.1 \mathrm{E}+02$ & $5.1 \mathrm{E}+02$ & No & N/A & N/A & N/A & N/A \\
\hline & GW-246 & s & 2 & 2 & $2.8 \mathrm{E}+02$ & $2.8 \mathrm{E}+02$ & No & $\mathrm{N} / \mathrm{A}$ & N/A & N/A & N/A \\
\hline & GW-276 & $\mathrm{s}$ & 18 & 18 & $3.2 \mathrm{E}+02$ & $3.4 \mathrm{E}+02$ & No & $\mathrm{s}$ & NT & N/A & N/A \\
\hline & GW-615 & $\mathrm{s}$ & 2 & 2 & $2.8 \mathrm{E}+02$ & $2.8 \mathrm{E}+02$ & No & N/A & N/A & $N / A$ & N/A \\
\hline & GW-829 & $\mathrm{T}$ & 10 & 10 & $2.2 \mathrm{E}+00$ & 7.7E-01 & No & $\mathrm{D}$ & $\mathrm{D}$ & N/A & N/A \\
\hline & GW-613 & $\mathrm{T}$ & 2 & 2 & $1.0 \mathrm{E}+00$ & $1.0 \mathrm{E}+00$ & No & $N / A$ & $N / A$ & N/A & N/A \\
\hline & GW-315 & $\mathrm{T}$ & 15 & 15 & $1.9 \mathrm{E}+00$ & $1.7 \mathrm{E}+00$ & No & $\mathrm{D}$ & $\mathrm{PD}$ & $N / A$ & N/A \\
\hline & GW-124 & $\mathrm{T}$ & 2 & 2 & $4.0 \mathrm{E}+00$ & $4.0 \mathrm{E}+00$ & No & N/A & N/A & $\mathrm{N} / \mathrm{A}$ & $\mathrm{N} / \mathrm{A}$ \\
\hline & GW-616 & $\mathrm{T}$ & 1 & 1 & $3.1 \mathrm{E}+00$ & $3.1 \mathrm{E}+00$ & No & N/A & N/A & $N / A$ & N/A \\
\hline & GW-345 & $\mathrm{T}$ & 1 & 1 & $1.4 \mathrm{E}+00$ & $1.4 \mathrm{E}+00$ & No & N/A & N/A & $\mathrm{N} / \mathrm{A}$ & $\mathrm{N} / \mathrm{A}$ \\
\hline & GW-123 & $\mathrm{T}$ & 1 & 1 & 1. $2 \mathrm{E}+01$ & $1.2 \mathrm{E}+01$ & No & N/A & $N / A$ & $N / A$ & N/A \\
\hline & GW-115 & $\mathrm{T}$ & 14 & 7 & $1.2 \mathrm{E}+00$ & 2.0E-01 & No & NT & NT & $N / A$ & N/A \\
\hline & GW-526 & $\mathrm{T}$ & 14 & 8 & $4.0 \mathrm{E}+01$ & $8.2 \mathrm{E}+00$ & No & PD & $\mathrm{D}$ & $\mathrm{N} / \mathrm{A}$ & N/A \\
\hline & SS-1 & $\mathrm{T}$ & 18 & 18 & $1.9 \mathrm{E}+01$ & $1.9 \mathrm{E}+01$ & No & NT & NT & $N / A$ & N/A \\
\hline & GW-835 & $\mathrm{T}$ & 4 & 4 & $1.8 \mathrm{E}+02$ & $1.6 \mathrm{E}+02$ & No & $\mathrm{s}$ & PD & N/A & N/A \\
\hline & GW-101 & $\mathrm{T}$ & 2 & 2 & $5.3 \mathrm{E}+00$ & $5.3 E+00$ & No & $\mathrm{N} / \mathrm{A}$ & $\mathrm{N} / \mathrm{A}$ & N/A & N/A \\
\hline & GW-311 & $\mathrm{T}$ & 11 & 11 & $3.6 \mathrm{E}+00$ & $2.2 \mathrm{E}+00$ & No & NT & NT & $N / A$ & $N / A$ \\
\hline & GW-236 & $\mathrm{T}$ & 3 & 3 & $4.1 E+00$ & $3.9 \mathrm{E}+00$ & No & $N / A$ & $N / A$ & $N / A$ & $N / A$ \\
\hline \multicolumn{12}{|c|}{ GROSS BETA ACTIVITY } \\
\hline & GW-276 & $\mathrm{S}$ & 17 & 17 & $5.0 \mathrm{E}+02$ & $4.7 \mathrm{E}+02$ & No & $\mathrm{D}$ & $\mathrm{D}$ & $\mathrm{N} / \mathrm{A}$ & N/A \\
\hline & GW-246 & S & 2 & 2 & $1.7 \mathrm{E}+04$ & $1.7 \mathrm{E}+04$ & No & N/A & N/A & $\mathrm{N} / \mathrm{A}$ & N/A \\
\hline
\end{tabular}

User Name: MV

State: Tennessee 
Project: Y-12 Security Complex

Location: West S-3
User Name: MV

State: Tennessee

\begin{tabular}{|c|c|c|c|c|c|c|c|c|c|c|c|}
\hline Constituent & Well & $\begin{array}{c}\text { Sourcel } \\
\text { Tail }\end{array}$ & $\begin{array}{c}\text { Number } \\
\text { of } \\
\text { Samples }\end{array}$ & $\begin{array}{c}\text { Number } \\
\text { of } \\
\text { Detects }\end{array}$ & $\begin{array}{c}\text { Average } \\
\text { (mg/L) }\end{array}$ & $\begin{array}{r}\text { Median } \\
(\mathrm{mg} / \mathrm{L})\end{array}$ & $\begin{array}{c}\text { All } \\
\text { Samples } \\
\text { "ND" ? }\end{array}$ & $\begin{array}{l}\text { Mann- } \\
\text { Kendall }\end{array}$ & $\begin{array}{c}\text { Linear } \\
\text { Regression }\end{array}$ & Modeling & Empirical \\
\hline \multicolumn{12}{|c|}{ GROSS BETA ACTIVITY } \\
\hline & GW-243 & S & 2 & 2 & $1.5 \mathrm{E}+04$ & $1.5 \mathrm{E}+04$ & No & N/A & N/A & N/A & N/A \\
\hline & GW-615 & s & 2 & 2 & $6.1 \mathrm{E}+02$ & $6.1 \mathrm{E}+02$ & No & $\mathrm{N} / \mathrm{A}$ & $\mathrm{N} / \mathrm{A}$ & N/A & N/A \\
\hline & GW-124 & $\mathrm{T}$ & 2 & 2 & $8.7 \mathrm{E}+01$ & $8.7 \mathrm{E}+01$ & No & $N / A$ & $N / A$ & N/A & N/A \\
\hline & GW-315 & $\mathrm{T}$ & 18 & 18 & $2.8 \mathrm{E}+01$ & $3.0 \mathrm{E}+01$ & No & $\mathrm{PI}$ & NT & N/A & N/A \\
\hline & GW-345 & $\mathrm{T}$ & 1 & 1 & $1.8 \mathrm{E}+00$ & $1.8 \mathrm{E}+00$ & No & N/A & N/A & N/A & N/A \\
\hline & GW-311 & $\mathrm{T}$ & 13 & 13 & $7.0 \mathrm{E}+00$ & $4.4 \mathrm{E}+00$ & No & $\mathrm{PD}$ & $\mathrm{D}$ & N/A & N/A \\
\hline & GW-526 & $\mathrm{T}$ & 14 & 8 & $9.0 \mathrm{E}+01$ & $1.3 \mathrm{E}+01$ & No & NT & NT & N/A & N/A \\
\hline & GW-100 & $\mathrm{T}$ & 2 & 2 & $2.1 \mathrm{E}+00$ & $2.1 \mathrm{E}+00$ & No & $\mathrm{N} / \mathrm{A}$ & N/A & $\mathrm{N} / \mathrm{A}$ & N/A \\
\hline & GW-123 & $\mathrm{T}$ & 2 & 2 & $9.5 E+00$ & $9.5 \mathrm{E}+00$ & No & $N / A$ & $N / A$ & N/A & N/A \\
\hline & GW-236 & $\mathrm{T}$ & 3 & 3 & $7.0 \mathrm{E}+01$ & 7.3E+01 & No & $N / A$ & $N / A$ & N/A & N/A \\
\hline & GW-346 & $\mathrm{T}$ & 1 & 1 & $3.8 \mathrm{E}+01$ & $3.8 \mathrm{E}+01$ & No & $N / A$ & $N / A$ & $\mathrm{~N} / \mathrm{A}$ & N/A \\
\hline & GW-115 & $\mathrm{T}$ & 15 & 13 & $2.6 \mathrm{E}+00$ & $1.5 \mathrm{E}+00$ & No & NT & NT & N/A & N/A \\
\hline & GW-101 & $\mathrm{T}$ & 2 & 2 & $4.2 \mathrm{E}+00$ & $4.2 \mathrm{E}+00$ & No & $N / A$ & $N / A$ & N/A & N/A \\
\hline & GW-835 & $\mathrm{T}$ & 4 & 4 & $2.3 \mathrm{E}+02$ & $2.1 \mathrm{E}+02$ & No & $\mathrm{s}$ & $\mathrm{s}$ & $N / A$ & N/A \\
\hline & GW-616 & $\mathrm{T}$ & 1 & 1 & $6.1 \mathrm{E}-01$ & 6.1E-01 & No & N/A & N/A & N/A & N/A \\
\hline & SS-1 & $\mathrm{T}$ & 18 & 18 & $4.1 \mathrm{E}+01$ & $4.2 \mathrm{E}+01$ & No & 1 & 1 & N/A & N/A \\
\hline & GW-613 & $\mathrm{T}$ & 2 & 2 & 4. $4 \mathrm{E}+00$ & 4.4E+00 & No & $\mathrm{N} / \mathrm{A}$ & N/A & $\mathrm{N} / \mathrm{A}$ & N/A \\
\hline & GW-829 & $\mathrm{T}$ & 12 & 12 & $5.3 \mathrm{E}+01$ & $5.3 \mathrm{E}+00$ & No & NT & NT & $N / A$ & N/A \\
\hline \multicolumn{12}{|l|}{ NITRATE } \\
\hline & GW-276 & $\mathrm{s}$ & 6 & 6 & $1.3 \mathrm{E}+02$ & $1.3 \mathrm{E}+02$ & No & NT & NT & $\mathrm{N} / \mathrm{A}$ & $\mathrm{N} / \mathrm{A}$ \\
\hline & GW-615 & $\mathrm{s}$ & 2 & 2 & $1.1 \mathrm{E}+04$ & $1.1 \mathrm{E}+04$ & No & $\mathrm{N} / \mathrm{A}$ & $\mathrm{N} / \mathrm{A}$ & N/A & N/A \\
\hline & GW-246 & $\mathrm{s}$ & 2 & 2 & $2.9 \mathrm{E}+03$ & $2.9 \mathrm{E}+03$ & No & $N / A$ & $N / A$ & $N / A$ & N/A \\
\hline & GW-243 & s & 2 & 2 & $7.9 \mathrm{E}+03$ & $7.9 \mathrm{E}+03$ & No & $N / A$ & $N / A$ & $N / A$ & N/A \\
\hline & GW-835 & $\mathrm{T}$ & 1 & 1 & $9.8 \mathrm{E}+01$ & $9.8 \mathrm{E}+01$ & No & $N / A$ & $N / A$ & N/A & N/A \\
\hline & SS-1 & $\mathrm{T}$ & 18 & 18 & $1.6 \mathrm{E}+01$ & $1.6 \mathrm{E}+01$ & No & $\mathrm{s}$ & NT & $N / A$ & N/A \\
\hline & GW-100 & $T$ & 2 & 2 & $5.3 \mathrm{E}+01$ & $5.3 \mathrm{E}+01$ & No & $\mathrm{N} / \mathrm{A}$ & N/A & N/A & N/A \\
\hline & GW-311 & $\mathrm{T}$ & 17 & 17 & 3.5E-01 & 3.3E-01 & No & $\mathrm{S}$ & $\mathrm{S}$ & $\mathrm{N} / \mathrm{A}$ & N/A \\
\hline & GW-123 & $\mathrm{T}$ & 2 & 0 & $2.0 \mathrm{E}-02$ & $2.0 \mathrm{E}-02$ & Yes & $N / A$ & $N / A$ & N/A & N/A \\
\hline & GW-829 & $T$ & 11 & 11 & $2.7 E+01$ & $2.8 \mathrm{E}+01$ & No & $\mathrm{D}$ & PD & N/A & N/A \\
\hline & GW-236 & $\mathrm{T}$ & 2 & 2 & $5.3 \mathrm{E}+01$ & $5.3 \mathrm{E}+01$ & No & $N / A$ & $\mathrm{~N} / \mathrm{A}$ & $\mathrm{N} / \mathrm{A}$ & N/A \\
\hline & GW-346 & $\mathrm{T}$ & 1 & 1 & $1.0 \mathrm{E}+03$ & $1.0 \mathrm{E}+03$ & No & $\mathrm{N} / \mathrm{A}$ & $N / A$ & N/A & N/A \\
\hline & GW-315 & $\mathrm{T}$ & 17 & 17 & $8.0 \mathrm{E}+00$ & $8.0 \mathrm{E}+00$ & No & D & I & $\mathrm{N} / \mathrm{A}$ & N/A \\
\hline & GW-526 & $\mathrm{T}$ & 1 & 1 & $1.2 \mathrm{E}+03$ & $1.2 \mathrm{E}+03$ & No & $N / A$ & $N / A$ & $N / A$ & N/A \\
\hline
\end{tabular}


Project: Y-12 Security Complex

Location: West S-3
User Name: MV

State: Tennessee

\begin{tabular}{|c|c|c|c|c|c|c|c|c|c|c|c|}
\hline Constituent & Well & $\begin{array}{c}\text { Sourcel } \\
\text { Tail }\end{array}$ & $\begin{array}{c}\text { Number } \\
\text { of } \\
\text { Samples }\end{array}$ & $\begin{array}{c}\text { Number } \\
\text { of } \\
\text { Detects }\end{array}$ & $\begin{array}{c}\text { Average } \\
\text { (mg/L) }\end{array}$ & $\begin{array}{r}\text { Median } \\
(\mathrm{mg} / \mathrm{L})\end{array}$ & $\begin{array}{c}\text { All } \\
\text { Samples } \\
\text { "ND" ? }\end{array}$ & $\begin{array}{l}\text { Mann- } \\
\text { Kendall }\end{array}$ & $\begin{array}{l}\text { Linear } \\
\text { Regression }\end{array}$ & Modeling & Empirical \\
\hline \multicolumn{12}{|l|}{ NITRATE } \\
\hline & GW-124 & $T$ & 2 & 2 & $1.9 \mathrm{E}+01$ & $1.9 \mathrm{E}+01$ & No & $\mathrm{N} / \mathrm{A}$ & $\mathrm{N} / \mathrm{A}$ & $\mathrm{N} / \mathrm{A}$ & N/A \\
\hline & GW-616 & $\mathrm{T}$ & 2 & 2 & $2.7 \mathrm{E}+02$ & $2.7 \mathrm{E}+02$ & No & N/A & N/A & N/A & N/A \\
\hline & GW-345 & $\mathrm{T}$ & 1 & 1 & $4.8 \mathrm{E}-01$ & $4.8 \mathrm{E}-01$ & No & $\mathrm{N} / \mathrm{A}$ & $\mathrm{N} / \mathrm{A}$ & $N / A$ & $N / A$ \\
\hline & GW-613 & $\mathrm{T}$ & 4 & 2 & 2.6E-02 & $2.5 \mathrm{E}-02$ & No & NT & $\mathrm{PI}$ & N/A & N/A \\
\hline & GW-101 & $\mathrm{T}$ & 2 & 2 & $7.3 \mathrm{E}+01$ & $7.3 \mathrm{E}+01$ & No & N/A & N/A & N/A & N/A \\
\hline & GW-115 & $\mathrm{T}$ & 7 & 2 & 5.7E-02 & $2.0 \mathrm{E}-02$ & No & NT & PD & N/A & N/A \\
\hline \multicolumn{12}{|c|}{ TETRACHLOROETHYLENE(PCE) } \\
\hline & GW-276 & $\mathrm{s}$ & 18 & 18 & $1.3 \mathrm{E}-02$ & $1.0 \mathrm{E}-02$ & No & $\mathrm{D}$ & $\mathrm{D}$ & $\mathrm{N} / \mathrm{A}$ & $\mathrm{N} / \mathrm{A}$ \\
\hline & GW-615 & s & 6 & 0 & $5.0 \mathrm{E}-05$ & $5.0 \mathrm{E}-05$ & Yes & $\mathrm{s}$ & s & N/A & N/A \\
\hline & GW-243 & s & 2 & 2 & $4.0 \mathrm{E}+00$ & $4.0 \mathrm{E}+00$ & No & $\mathrm{N} / \mathrm{A}$ & N/A & N/A & $N / A$ \\
\hline & GW-246 & s & 2 & 2 & $1.0 \mathrm{E}-01$ & $1.0 \mathrm{E}-01$ & No & $\mathrm{N} / \mathrm{A}$ & $N / A$ & $N / A$ & $N / A$ \\
\hline & GW-115 & $\mathrm{T}$ & 18 & 0 & $5.0 \mathrm{E}-05$ & $5.0 \mathrm{E}-05$ & Yes & $\mathrm{s}$ & $\mathrm{s}$ & N/A & N/A \\
\hline & GW-123 & $\mathrm{T}$ & 2 & 0 & $5.0 \mathrm{E}-05$ & 5.0E-05 & Yes & N/A & N/A & $N / A$ & $N / A$ \\
\hline & GW-100 & $\mathrm{T}$ & 2 & 0 & $5.0 \mathrm{E}-05$ & 5.0E-05 & Yes & $N / A$ & $N / A$ & $N / A$ & $N / A$ \\
\hline & GW-101 & $\mathrm{T}$ & 2 & 0 & $5.0 \mathrm{E}-05$ & $5.0 \mathrm{E}-05$ & Yes & $\mathrm{N} / \mathrm{A}$ & $\mathrm{N} / \mathrm{A}$ & N/A & N/A \\
\hline & GW-124 & $\mathrm{T}$ & 2 & 1 & $5.2 \mathrm{E}-04$ & $5.2 \mathrm{E}-04$ & No & $\mathrm{N} / \mathrm{A}$ & N/A & $N / A$ & $N / A$ \\
\hline & GW-236 & $\mathrm{T}$ & 3 & 2 & $5.2 \mathrm{E}-04$ & $5.0 \mathrm{E}-04$ & No & $N / A$ & $N / A$ & $N / A$ & N/A \\
\hline & GW-835 & $\mathrm{T}$ & 20 & 16 & $1.8 \mathrm{E}-03$ & $2.0 \mathrm{E}-03$ & No & 1 & 1 & $N / A$ & $N / A$ \\
\hline & GW-613 & $\mathrm{T}$ & 4 & 0 & $5.0 \mathrm{E}-05$ & $5.0 \mathrm{E}-05$ & Yes & s & s & $N / A$ & $N / A$ \\
\hline & GW-616 & $\mathrm{T}$ & 2 & 0 & $5.0 \mathrm{E}-05$ & $5.0 \mathrm{E}-05$ & Yes & $N / A$ & $N / A$ & $N / A$ & $N / A$ \\
\hline & GW-346 & $\mathrm{T}$ & 1 & 0 & $5.0 \mathrm{E}-05$ & $5.0 \mathrm{E}-05$ & Yes & $\mathrm{N} / \mathrm{A}$ & $\mathrm{N} / \mathrm{A}$ & $N / A$ & $N / A$ \\
\hline & GW-829 & $\mathrm{T}$ & 12 & 0 & $5.0 \mathrm{E}-05$ & $5.0 \mathrm{E}-05$ & Yes & $\mathrm{s}$ & s & $N / A$ & $N / A$ \\
\hline & GW-345 & $\mathrm{T}$ & 1 & 0 & $5.0 \mathrm{E}-05$ & $5.0 \mathrm{E}-05$ & Yes & $N / A$ & $N / A$ & $N / A$ & $N / A$ \\
\hline & GW-526 & $\mathrm{T}$ & 11 & 0 & $5.0 \mathrm{E}-05$ & $5.0 \mathrm{E}-05$ & Yes & $\mathrm{s}$ & 1 & $N / A$ & $N / A$ \\
\hline & GW-315 & $\mathrm{T}$ & 18 & 18 & $1.1 \mathrm{E}-02$ & 1.1E-02 & No & $\mathrm{D}$ & $S$ & N/A & N/A \\
\hline & GW-311 & $\mathrm{T}$ & 18 & 1 & 7.5E-05 & 5.0E-05 & No & NT & PI & $N / A$ & N/A \\
\hline & SS-1 & $\mathrm{T}$ & 18 & 4 & $2.1 \mathrm{E}-04$ & $5.0 \mathrm{E}-05$ & No & D & D & N/A & N/A \\
\hline \multicolumn{12}{|c|}{ VINYL CHLORIDE } \\
\hline & GW-615 & $\mathrm{s}$ & 6 & 0 & $5.0 \mathrm{E}-04$ & $5.0 \mathrm{E}-04$ & Yes & $\mathrm{s}$ & $\mathrm{s}$ & $N / A$ & $N / A$ \\
\hline & GW-276 & s & 18 & 0 & $5.0 \mathrm{E}-04$ & $5.0 \mathrm{E}-04$ & Yes & $\mathrm{s}$ & s & $N / A$ & $N / A$ \\
\hline & GW-243 & s & 2 & 0 & $5.0 \mathrm{E}-04$ & $5.0 \mathrm{E}-04$ & Yes & $N / A$ & $N / A$ & $N / A$ & $N / A$ \\
\hline & GW-246 & s & 2 & 0 & $5.0 \mathrm{E}-04$ & $5.0 \mathrm{E}-04$ & Yes & $\mathrm{N} / \mathrm{A}$ & $\mathrm{N} / \mathrm{A}$ & $N / A$ & $N / A$ \\
\hline
\end{tabular}


Project: Y-12 Security Complex

Location: West S-3
User Name: $\quad$ MV

State: Tennessee

\begin{tabular}{|c|c|c|c|c|c|c|c|c|c|c|c|}
\hline Constituent & Well & $\begin{array}{c}\text { Sourcel } \\
\text { Tail }\end{array}$ & $\begin{array}{c}\text { Number } \\
\text { of } \\
\text { Samples }\end{array}$ & $\begin{array}{c}\text { Number } \\
\text { of } \\
\text { Detects }\end{array}$ & $\begin{array}{c}\text { Average } \\
\text { (mg/L) }\end{array}$ & $\begin{array}{r}\text { Median } \\
(\mathrm{mg} / \mathrm{L})\end{array}$ & $\begin{array}{c}\text { All } \\
\text { Samples } \\
\text { "ND" ? }\end{array}$ & $\begin{array}{l}\text { Mann- } \\
\text { Kendall }\end{array}$ & $\begin{array}{l}\text { Linear } \\
\text { Regression }\end{array}$ & Modeling & Empirical \\
\hline \multicolumn{12}{|c|}{ VINYL CHLORIDE } \\
\hline & GW-100 & $T$ & 2 & 0 & 5.0E-04 & $5.0 \mathrm{E}-04$ & Yes & $\mathrm{N} / \mathrm{A}$ & $\mathrm{N} / \mathrm{A}$ & $\mathrm{N} / \mathrm{A}$ & $\mathrm{N} / \mathrm{A}$ \\
\hline & GW-835 & $\mathrm{T}$ & 20 & 0 & $5.0 \mathrm{E}-04$ & 5.0E-04 & Yes & s & 1 & $N / A$ & N/A \\
\hline & GW-616 & $\mathrm{T}$ & 2 & 0 & $5.0 \mathrm{E}-04$ & 5.0E-04 & Yes & $\mathrm{N} / \mathrm{A}$ & N/A & N/A & $N / A$ \\
\hline & GW-101 & $\mathrm{T}$ & 2 & 0 & $5.0 \mathrm{E}-04$ & $5.0 \mathrm{E}-04$ & Yes & N/A & N/A & N/A & $N / A$ \\
\hline & GW-829 & $\mathrm{T}$ & 12 & 0 & $5.0 \mathrm{E}-04$ & $5.0 \mathrm{E}-04$ & Yes & s & s & N/A & N/A \\
\hline & GW-613 & $\mathrm{T}$ & 4 & 0 & $5.0 \mathrm{E}-04$ & $5.0 \mathrm{E}-04$ & Yes & s & s & $\mathrm{N} / \mathrm{A}$ & $N / A$ \\
\hline & GW-311 & $\mathrm{T}$ & 18 & 0 & $5.0 \mathrm{E}-04$ & 5.0E-04 & Yes & S & S & N/A & $N / A$ \\
\hline & GW-526 & $\mathrm{T}$ & 11 & 0 & $5.0 \mathrm{E}-04$ & $5.0 \mathrm{E}-04$ & Yes & $\mathrm{s}$ & I & N/A & N/A \\
\hline & GW-346 & $\mathrm{T}$ & 1 & 0 & $5.0 \mathrm{E}-04$ & $5.0 \mathrm{E}-04$ & Yes & $\mathrm{N} / \mathrm{A}$ & N/A & $\mathrm{N} / \mathrm{A}$ & $N / A$ \\
\hline & GW-123 & $\mathrm{T}$ & 2 & 0 & $5.0 \mathrm{E}-04$ & $5.0 \mathrm{E}-04$ & Yes & N/A & N/A & N/A & $N / A$ \\
\hline & GW-345 & $\mathrm{T}$ & 1 & 0 & $5.0 \mathrm{E}-04$ & $5.0 \mathrm{E}-04$ & Yes & $\mathrm{N} / \mathrm{A}$ & $\mathrm{N} / \mathrm{A}$ & $\mathrm{N} / \mathrm{A}$ & $N / A$ \\
\hline & GW-315 & $T$ & 18 & 0 & $5.0 \mathrm{E}-04$ & $5.0 \mathrm{E}-04$ & Yes & $\mathrm{s}$ & $\mathrm{s}$ & $\mathrm{N} / \mathrm{A}$ & $N / A$ \\
\hline & GW-124 & $\mathrm{T}$ & 2 & 0 & $5.0 \mathrm{E}-04$ & 5.0E-04 & Yes & N/A & N/A & N/A & $N / A$ \\
\hline & SS-1 & $\mathrm{T}$ & 18 & 0 & $5.0 \mathrm{E}-04$ & $5.0 \mathrm{E}-04$ & Yes & $\mathrm{s}$ & $\mathrm{s}$ & N/A & N/A \\
\hline & GW-236 & $\mathrm{T}$ & 3 & 0 & $5.0 \mathrm{E}-04$ & $5.0 \mathrm{E}-04$ & Yes & $\mathrm{N} / \mathrm{A}$ & $\mathrm{N} / \mathrm{A}$ & $\mathrm{N} / \mathrm{A}$ & $N / A$ \\
\hline & GW-115 & $\mathrm{T}$ & 18 & 0 & $5.0 \mathrm{E}-04$ & 5.0E-04 & Yes & $\mathrm{S}$ & $\mathrm{s}$ & N/A & $N / A$ \\
\hline
\end{tabular}

Note: Increasing (I); Probably Increasing (PI); Stable (S); Probably Decreasing (PD); Decreasing (D); No Trend (NT); Not Applicable (N/A) - Due to insufficient Data (< 4 sampling events); Source/Tail (S/T)

The Number of Samples and Number of Detects shown above are post-consolidation values. 


\section{MAROS Spatial Moment Analysis Summary}

Project: Y-12 Security Complex

Location: West S-3
User Name: MV

State: Tennessee 2nd Moment (Spread)

\section{Estimated}

Mass $(\mathrm{Kg})$

$\underline{1 \text { st Moment (Center of Mass) }}$

Effective Date

(1)

GROSS ALPHA ACTIVITY

\begin{tabular}{|c|c|c|c|c|c|c|c|}
\hline 1/1/1996 & $5.3 E+03$ & 51,519 & 30,036 & 486 & 134,370 & 98,255 & 6 \\
\hline 7/1/1996 & $0.0 \mathrm{E}+00$ & & & & & & 5 \\
\hline 10/1/1996 & $0.0 \mathrm{E}+00$ & & & & & & 1 \\
\hline $1 / 1 / 1997$ & $0.0 \mathrm{E}+00$ & & & & & & 5 \\
\hline 4/1/1997 & $0.0 \mathrm{E}+00$ & & & & & & 1 \\
\hline $7 / 1 / 1997$ & $0.0 \mathrm{E}+00$ & & & & & & 5 \\
\hline 10/1/1997 & $0.0 \mathrm{E}+00$ & & & & & & 2 \\
\hline 1/1/1998 & $6.2 \mathrm{E}+03$ & 51,784 & 30,021 & 246 & 294,961 & 93,795 & 8 \\
\hline $7 / 1 / 1998$ & $1.5 E+03$ & 51,033 & 29,772 & 1,031 & 241,620 & 18,320 & 7 \\
\hline 1/1/1999 & $2.7 E+03$ & 51,706 & 29,843 & 422 & 91,621 & 31,065 & 6 \\
\hline 7/1/1999 & $1.5 \mathrm{E}+03$ & 51,756 & 29,789 & 434 & 25,457 & 42,246 & 6 \\
\hline $1 / 1 / 2000$ & $2.4 \mathrm{E}+03$ & 51,395 & 29,752 & 719 & 271,722 & 48,920 & 7 \\
\hline $7 / 1 / 2000$ & $3.7 E+02$ & 50,999 & 29,571 & 1,150 & 215,604 & 33,804 & 7 \\
\hline $1 / 1 / 2001$ & $9.4 \mathrm{E}+02$ & 51,656 & 30,070 & 345 & 362,488 & 62,017 & 7 \\
\hline $7 / 1 / 2001$ & $1.8 \mathrm{E}+03$ & 51,174 & 29,808 & 887 & 369,037 & 35,419 & 9 \\
\hline $1 / 1 / 2002$ & $8.1 E+03$ & 51,560 & 29,875 & 513 & 84,013 & 21,865 & 6 \\
\hline $7 / 1 / 2002$ & $1.7 \mathrm{E}+03$ & 52,068 & 29,862 & 303 & 237,943 & 59,021 & 7 \\
\hline $1 / 1 / 2003$ & $2.6 \mathrm{E}+02$ & 51,544 & 29,908 & 509 & 234,326 & 154,978 & 6 \\
\hline $7 / 1 / 2003$ & $0.0 \mathrm{E}+00$ & & & & & & 5 \\
\hline $1 / 1 / 2004$ & $4.8 \mathrm{E}+03$ & 51,808 & 29,975 & 256 & 157,126 & 52,717 & 9 \\
\hline $7 / 1 / 2004$ & $2.4 \mathrm{E}+03$ & 51,864 & 29,926 & 261 & 72,759 & 7,616 & 7 \\
\hline
\end{tabular}

GROSS BETA ACTIVITY

\begin{tabular}{|c|c|c|c|c|c|c|c|}
\hline 1/1/1996 & $1.2 \mathrm{E}+04$ & 51,530 & 29,907 & 522 & 135,003 & 106,171 & 7 \\
\hline $7 / 1 / 1996$ & $0.0 \mathrm{E}+00$ & & & & & & 4 \\
\hline $1 / 1 / 1997$ & $0.0 \mathrm{E}+00$ & & & & & & 5 \\
\hline 4/1/1997 & $0.0 \mathrm{E}+00$ & & & & & & 1 \\
\hline 7/1/1997 & $2.4 \mathrm{E}+04$ & 51,647 & 29,923 & 413 & 203,090 & 81,442 & 7 \\
\hline 10/1/1997 & $0.0 \mathrm{E}+00$ & & & & & & 2 \\
\hline 1/1/1998 & $1.8 \mathrm{E}+04$ & 51,791 & 29,985 & 262 & 264,505 & 92,320 & 8 \\
\hline 7/1/1998 & $7.6 \mathrm{E}+03$ & 51,606 & 30,023 & 406 & 216,012 & 60,441 & 6 \\
\hline 1/1/1999 & $1.1 \mathrm{E}+04$ & 51,450 & 29,807 & 643 & 325,318 & 45,611 & 9 \\
\hline 7/1/1999 & $1.0 \mathrm{E}+04$ & 51,629 & 29,746 & 545 & 128,787 & 44,150 & 7 \\
\hline $1 / 1 / 2000$ & $3.1 E+03$ & 51,020 & 29,616 & 1,110 & 234,910 & 73,189 & 7 \\
\hline $7 / 1 / 2000$ & $2.4 \mathrm{E}+03$ & 51,127 & 29,655 & 997 & 271,940 & 80,718 & 7 \\
\hline $1 / 1 / 2001$ & $1.7 \mathrm{E}+03$ & 51,890 & 29,784 & 385 & 517,626 & 66,425 & 9 \\
\hline $7 / 1 / 2001$ & $2.2 \mathrm{E}+04$ & 51,513 & 29,922 & 531 & 250,696 & 79,907 & 8 \\
\hline $1 / 1 / 2002$ & $2.2 \mathrm{E}+04$ & 52,282 & 30,065 & 305 & 36,783 & 70,389 & 6 \\
\hline $7 / 1 / 2002$ & $3.5 E+04$ & 52,258 & 30,106 & 273 & 90,897 & 84,496 & 7 \\
\hline
\end{tabular}


Project: Y-12 Security Complex

Location: West S-3

\begin{tabular}{|c|c|c|c|c|c|c|c|}
\hline \multirow[b]{2}{*}{ Effective Date } & \multirow{2}{*}{$\begin{array}{l}\text { Esth Moment } \\
\text { Eass (kg) }\end{array}$} & \multicolumn{3}{|c|}{ 1st Moment (Center of Mass) } & \multicolumn{2}{|c|}{ 2nd Moment (Spread) } & \multirow[b]{2}{*}{$\begin{array}{c}\text { Number of } \\
\text { Wells }\end{array}$} \\
\hline & & $\mathrm{Xc}(\mathrm{ft})$ & Yc (ft) & $\begin{array}{c}\text { Source } \\
\text { Distance (ft) }\end{array}$ & $\begin{array}{l}\text { Sigma } X X \\
(\mathrm{sq} \mathrm{ft})\end{array}$ & $\begin{array}{l}\text { Sigma YY } \\
(\mathrm{sq} f t)\end{array}$ & \\
\hline BETA ACTIVITY & & & & & & & \\
\hline $1 / 1 / 2003$ & $5.3 \mathrm{E}+03$ & 51,870 & 30,009 & 189 & 222,051 & 76,801 & 6 \\
\hline $7 / 1 / 2003$ & $4.6 \mathrm{E}+03$ & 51,870 & 30,009 & 189 & 277,214 & 89,482 & 7 \\
\hline $1 / 1 / 2004$ & $1.6 \mathrm{E}+04$ & 52,023 & 29,940 & 218 & 118,570 & 56,113 & 10 \\
\hline $7 / 1 / 2004$ & $9.1 \mathrm{E}+03$ & 51,953 & 29,803 & 354 & 111,916 & 13,656 & 10 \\
\hline
\end{tabular}

NITRATE

User Name: MV

State: Tennessee

2nd Moment (Spread)

\begin{tabular}{|c|c|c|c|c|c|c|c|}
\hline 1/1/1996 & $2.7 \mathrm{E}+03$ & 51,507 & 29,767 & 619 & 191,119 & 52,565 & 7 \\
\hline 7/1/1996 & $2.3 E+03$ & 51,504 & 29,745 & 636 & 190,559 & 37,204 & 7 \\
\hline $10 / 1 / 1996$ & $0.0 \mathrm{E}+00$ & & & & & & 1 \\
\hline 1/1/1997 & $1.7 \mathrm{E}+03$ & 51,419 & 29,792 & 676 & 230,021 & 52,475 & 7 \\
\hline $7 / 1 / 1997$ & $4.9 \mathrm{E}+02$ & 51,521 & 29,976 & 501 & 154,560 & 55,290 & 6 \\
\hline 10/1/1997 & $0.0 \mathrm{E}+00$ & & & & & & 1 \\
\hline 1/1/1998 & $2.3 E+03$ & 51,624 & 29,811 & 502 & 152,202 & 66,757 & 6 \\
\hline $7 / 1 / 1998$ & $4.4 \mathrm{E}+03$ & 51,581 & 29,855 & 507 & 397,572 & 68,321 & 7 \\
\hline 1/1/1999 & $3.3 E+03$ & 51,091 & 29,701 & 1,007 & 27,386 & 5,792 & 7 \\
\hline $7 / 1 / 1999$ & $0.0 \mathrm{E}+00$ & & & & & & 1 \\
\hline $1 / 1 / 2000$ & $0.0 \mathrm{E}+00$ & & & & & & 4 \\
\hline $7 / 1 / 2000$ & $0.0 \mathrm{E}+00$ & & & & & & 4 \\
\hline $1 / 1 / 2001$ & $2.5 E+03$ & 50,965 & 29,675 & 1,132 & 153,568 & 15,779 & 6 \\
\hline $7 / 1 / 2001$ & $2.6 \mathrm{E}+03$ & 50,975 & 29,676 & 1,122 & 167,525 & 15,402 & 6 \\
\hline $1 / 1 / 2002$ & $0.0 \mathrm{E}+00$ & & & & & & 4 \\
\hline $7 / 1 / 2002$ & $0.0 \mathrm{E}+00$ & & & & & & 4 \\
\hline $1 / 1 / 2003$ & $0.0 \mathrm{E}+00$ & & & & & & 4 \\
\hline $7 / 1 / 2003$ & $0.0 \mathrm{E}+00$ & & & & & & 4 \\
\hline $1 / 1 / 2004$ & $1.7 \mathrm{E}+04$ & 51,799 & 29,895 & 322 & 93,692 & 21,036 & 8 \\
\hline $7 / 1 / 2004$ & $1.8 \mathrm{E}+04$ & 51,804 & 29,902 & 314 & 89,417 & 20,674 & 8 \\
\hline
\end{tabular}

TETRACHLOROETHYLENE(PCE)

\begin{tabular}{|c|c|c|c|c|c|c|c|}
\hline 1/1/1996 & 2.3E-01 & 51,598 & 29,965 & 435 & 113,664 & 127,422 & 7 \\
\hline 7/1/1996 & 6.9E-01 & 51,576 & 29,806 & 541 & 111,931 & 71,904 & 7 \\
\hline 10/1/1996 & $0.0 \mathrm{E}+00$ & & & & & & 1 \\
\hline 1/1/1997 & 7.1E-01 & 51,578 & 29,807 & 539 & 110,983 & 71,815 & 7 \\
\hline 4/1/1997 & $0.0 \mathrm{E}+00$ & & & & & & 1 \\
\hline 7/1/1997 & 3.8E-01 & 51,579 & 29,999 & 440 & 103,130 & 59,922 & 6 \\
\hline 10/1/1997 & $0.0 \mathrm{E}+00$ & & & & & & 2 \\
\hline 1/1/1998 & $1.4 \mathrm{E}-01$ & 51,857 & 29,841 & 341 & 208,359 & 74,247 & 7 \\
\hline $7 / 1 / 1998$ & 1.3E-01 & 51,851 & 29,843 & 341 & 213,391 & 75,322 & 7 \\
\hline 1/1/1999 & 2.1E-01 & 51,720 & 29,889 & 379 & 123,680 & 69,663 & 9 \\
\hline $7 / 1 / 1999$ & 2.2E-01 & 51,701 & 29,824 & 439 & 80,694 & 68,068 & 7 \\
\hline 10/1/1999 & $0.0 \mathrm{E}+00$ & & & & & & 2 \\
\hline $1 / 1 / 2000$ & 1.2E-01 & 51,698 & 29,783 & 473 & 281,426 & 84,902 & 9 \\
\hline $4 / 1 / 2000$ & $0.0 \mathrm{E}+00$ & & & & & & 2 \\
\hline $7 / 1 / 2000$ & 1.7E-01 & 51,659 & 29,778 & 501 & 259,842 & 87,384 & 9 \\
\hline
\end{tabular}


Project: Y-12 Security Complex

Location: West S-3
User Name: MV

State: Tennessee

\begin{tabular}{|c|c|c|c|c|c|c|c|}
\hline \multirow[b]{2}{*}{ Effective Date } & \multirow{2}{*}{$\begin{array}{l}\text { 0th Moment } \\
\text { Estimated } \\
\text { Mass (kg) }\end{array}$} & \multicolumn{3}{|c|}{ 1st Moment (Center of Mass) } & \multicolumn{2}{|c|}{ 2nd Moment (Spread) } & \multirow[b]{2}{*}{$\begin{array}{l}\text { Number of } \\
\text { Wells }\end{array}$} \\
\hline & & $\mathrm{Xc}(\mathrm{ft})$ & Yc (ft) & $\begin{array}{l}\text { Source } \\
\text { Distance (ft) }\end{array}$ & $\underset{\text { (sq ft) }}{\text { Sigma XX }}$ & $\begin{array}{c}\text { Sigma } Y Y \\
(s q \mathrm{ft})\end{array}$ & \\
\hline \multicolumn{8}{|c|}{ TETRACHLOROETHYLENE(PCE) } \\
\hline 10/1/2000 & $0.0 \mathrm{E}+00$ & & & & & & 1 \\
\hline $1 / 1 / 2001$ & $1.3 \mathrm{E}-01$ & 51,557 & 29,768 & 580 & 255,370 & 104,467 & 10 \\
\hline 4/1/2001 & $0.0 \mathrm{E}+00$ & & & & & & 1 \\
\hline 7/1/2001 & $1.6 \mathrm{E}-01$ & 51,650 & 29,757 & 524 & 283,590 & 91,574 & 10 \\
\hline 10/1/2001 & $0.0 \mathrm{E}+00$ & & & & & & 1 \\
\hline $1 / 1 / 2002$ & $4.1 \mathrm{E}+00$ & 52,071 & 29,935 & 234 & 84,437 & 50,473 & 8 \\
\hline 4/1/2002 & $0.0 \mathrm{E}+00$ & & & & & & 1 \\
\hline 7/1/2002 & $4.4 \mathrm{E}+00$ & 52,068 & 29,927 & 241 & 83,876 & 47,938 & 8 \\
\hline 10/1/2002 & $0.0 \mathrm{E}+00$ & & & & & & 1 \\
\hline $1 / 1 / 2003$ & $1.6 \mathrm{E}-01$ & 51,652 & 29,758 & 521 & 240,656 & 90,251 & 8 \\
\hline 4/1/2003 & $0.0 \mathrm{E}+00$ & & & & & & 1 \\
\hline 7/1/2003 & $1.7 \mathrm{E}-01$ & 51,655 & 29,749 & 526 & 239,710 & 86,842 & 8 \\
\hline $1 / 1 / 2004$ & $2.8 \mathrm{E}-01$ & 51,759 & 29,760 & 457 & 127,403 & 31,995 & 11 \\
\hline 7/1/2004 & $2.3 \mathrm{E}-01$ & 51,786 & 29,751 & 453 & 93,874 & 11,342 & 10 \\
\hline
\end{tabular}

VINYL CHLORIDE

\begin{tabular}{|c|c|c|c|c|c|c|c|}
\hline $1 / 1 / 1996$ & 2.3E-01 & 51,403 & 30,071 & 593 & 248,611 & 140,935 & 7 \\
\hline 7/1/1996 & 2.3E-01 & 51,403 & 30,071 & 593 & 248,611 & 140,935 & 7 \\
\hline 10/1/1996 & $0.0 \mathrm{E}+00$ & & & & & & 1 \\
\hline 1/1/1997 & 2.3E-01 & 51,403 & 30,071 & 593 & 248,611 & 140,935 & 7 \\
\hline 4/1/1997 & $0.0 \mathrm{E}+00$ & & & & & & 1 \\
\hline 7/1/1997 & 2.3E-01 & 51,403 & 30,071 & 593 & 235,146 & 118,852 & 6 \\
\hline 10/1/1997 & $0.0 \mathrm{E}+00$ & & & & & & 2 \\
\hline 1/1/1998 & 2.0E-01 & 51,477 & 29,999 & 536 & 330,897 & 109,789 & 7 \\
\hline 7/1/1998 & 2.0E-01 & 51,477 & 29,999 & 536 & 330,897 & 109,789 & 7 \\
\hline 1/1/1999 & 2.0E-01 & 51,477 & 29,999 & 536 & 306,919 & 121,944 & 9 \\
\hline $7 / 1 / 1999$ & 2.0E-01 & 51,477 & 29,999 & 536 & 246,220 & 101,260 & 7 \\
\hline 10/1/1999 & $0.0 \mathrm{E}+00$ & & & & & & 2 \\
\hline $1 / 1 / 2000$ & 2.0E-01 & 51,477 & 29,999 & 536 & 376,694 & 117,115 & 9 \\
\hline $4 / 1 / 2000$ & $0.0 \mathrm{E}+00$ & & & & & & 2 \\
\hline $7 / 1 / 2000$ & 2.0E-01 & 51,477 & 29,999 & 536 & 376,694 & 117,115 & 9 \\
\hline $10 / 1 / 2000$ & $0.0 \mathrm{E}+00$ & & & & & & 1 \\
\hline $1 / 1 / 2001$ & 2.0E-01 & 51,477 & 29,999 & 536 & 376,899 & 109,355 & 10 \\
\hline 4/1/2001 & $0.0 \mathrm{E}+00$ & & & & & & 1 \\
\hline $7 / 1 / 2001$ & 2.0E-01 & 51,477 & 29,999 & 536 & 376,899 & 109,355 & 10 \\
\hline 10/1/2001 & $0.0 \mathrm{E}+00$ & & & & & & 1 \\
\hline $1 / 1 / 2002$ & 1.6E-01 & 51,638 & 29,983 & 392 & 281,443 & 133,199 & 8 \\
\hline $4 / 1 / 2002$ & $0.0 \mathrm{E}+00$ & & & & & & 1 \\
\hline $7 / 1 / 2002$ & 1.6E-01 & 51,638 & 29,983 & 392 & 281,443 & 133,199 & 8 \\
\hline 10/1/2002 & $0.0 \mathrm{E}+00$ & & & & & & 1 \\
\hline $1 / 1 / 2003$ & 1.6E-01 & 51,638 & 29,983 & 392 & 274,513 & 111,455 & 8 \\
\hline $4 / 1 / 2003$ & $0.0 \mathrm{E}+00$ & & & & & & 1 \\
\hline $7 / 1 / 2003$ & 1.6E-01 & 51,638 & 29,983 & 392 & 274,513 & 111,455 & 8 \\
\hline
\end{tabular}


Project: Y-12 Security Complex

Location: West S-3

\begin{tabular}{|c|c|c|c|c|c|c|c|}
\hline \multirow[b]{2}{*}{ Effective Date } & \multirow{2}{*}{$\begin{array}{l}\text { 0th Moment } \\
\text { Estimated } \\
\text { Mass (kg) }\end{array}$} & \multicolumn{3}{|c|}{ 1st Moment (Center of Mass) } & \multicolumn{2}{|c|}{ 2nd Moment (Spread) } & \multirow[b]{2}{*}{$\begin{array}{c}\text { Number of } \\
\text { Wells }\end{array}$} \\
\hline & & Xc (ft) & Yc (ft) & $\begin{array}{c}\text { Source } \\
\text { Distance (ft) }\end{array}$ & $\underset{(\text { Sq ft) }}{\text { Sigma } X X}$ & $\begin{array}{l}\text { Sigma YY } \\
\text { (sq ft) }\end{array}$ & \\
\hline \multicolumn{8}{|l|}{ VINYL CHLORIDE } \\
\hline $1 / 1 / 2004$ & $1.6 \mathrm{E}-01$ & 51,636 & 29,982 & 394 & 292,017 & 136,676 & 11 \\
\hline $7 / 1 / 2004$ & $1.0 \mathrm{E}-01$ & 51,395 & 29,735 & 728 & 234,693 & 40,970 & 10 \\
\hline
\end{tabular}

User Name: MV

State: Tennessee 
Project: Y-12 Security Complex

Location: West S-3
User Name: $\mathrm{MV}$

State: Tennessee

\begin{tabular}{|c|c|c|c|c|c|}
\hline Moment Type & Constituent & $\begin{array}{l}\text { Coefficient } \\
\text { of Variation }\end{array}$ & $\begin{array}{l}\text { Mann-Kendall } \\
\text { S Statistic }\end{array}$ & $\begin{array}{l}\text { Confidence } \\
\text { in Trend }\end{array}$ & $\begin{array}{c}\text { Moment } \\
\text { Trend }\end{array}$ \\
\hline \multicolumn{6}{|c|}{ Zeroth Moment: Mass } \\
\hline & GROSS ALPHA ACTIVITY & 1.23 & 49 & $92.6 \%$ & $\mathrm{PI}$ \\
\hline & GROSS BETA ACTIVITY & 0.97 & 38 & $88.3 \%$ & NT \\
\hline & NITRATE & 1.82 & 0 & $48.7 \%$ & NT \\
\hline & TETRACHLOROETHYLENE(PCE) & 2.46 & -11 & $57.4 \%$ & NT \\
\hline & VINYL CHLORIDE & 0.83 & -121 & $98.9 \%$ & $\mathrm{D}$ \\
\hline \multicolumn{6}{|c|}{ 1st Moment: Distance to Source } \\
\hline & GROSS ALPHA ACTIVITY & 0.55 & -17 & $80.6 \%$ & $\mathrm{~S}$ \\
\hline & GROSS BETA ACTIVITY & 0.59 & -46 & $97.9 \%$ & $\mathrm{D}$ \\
\hline & NITRATE & 0.44 & -3 & $56.0 \%$ & $\mathrm{~S}$ \\
\hline & TETRACHLOROETHYLENE(PCE) & 0.23 & 7 & $58.9 \%$ & NT \\
\hline & VINYL CHLORIDE & 0.18 & -73 & $99.7 \%$ & $\mathrm{D}$ \\
\hline \multicolumn{6}{|c|}{ 2nd Moment: Sigma XX } \\
\hline & GROSS ALPHA ACTIVITY & 0.54 & -11 & $70.5 \%$ & $\mathrm{~S}$ \\
\hline & GROSS BETA ACTIVITY & 0.54 & -12 & $68.7 \%$ & $\mathrm{~S}$ \\
\hline & NITRATE & 0.56 & -25 & $97.0 \%$ & $\mathrm{D}$ \\
\hline & TETRACHLOROETHYLENE(PCE) & 0.46 & 9 & $61.7 \%$ & NT \\
\hline & VINYL CHLORIDE & 0.18 & 11 & $64.6 \%$ & NT \\
\hline \multicolumn{6}{|c|}{ 2nd Moment: Sigma YY } \\
\hline & GROSS ALPHA ACTIVITY & 0.72 & -7 & $62.6 \%$ & $\mathrm{~S}$ \\
\hline & GROSS BETA ACTIVITY & 0.32 & -24 & $84.7 \%$ & $\mathrm{~S}$ \\
\hline & NITRATE & 0.60 & -13 & $82.1 \%$ & $\mathrm{~S}$ \\
\hline & TETRACHLOROETHYLENE(PCE) & 0.37 & -27 & $83.5 \%$ & $\mathrm{~S}$ \\
\hline & VINYL CHLORIDE & 0.20 & -43 & $94.4 \%$ & PD \\
\hline
\end{tabular}

Note: The following assumptions were applied for the calculation of the Zeroth Moment:

Porosity: $0.10 \quad$ Saturated Thickness: Uniform: $50 \mathrm{ft}$

Mann-Kendall Trend test performed on all sample events for each constituent. Increasing (I); Probably Increasing (PI); Stable (S); Probably Decreasing (PD); Decreasing (D); No Trend (NT); Not Applicable (N/A)-Due to insufficient Data (< 4 sampling events). estimated groundwater flow direction. Moments are not calculated for sample events with less than 6 wells. 


\section{ASSESSMENT OF THE GROUNDWATER PROTECTION PROGRAM \\ Y-12 NATIONAL SECURITY COMPLEX, OAK RIDGE, TENNESSEE}

Y-12 National Security Complex

Oak Ridge, Tennessee

\section{Appendix D.2 Bear Creek Regime Oil Landfarm WMA}

Table D.2.1 Qualitative Analysis Oil Landfarm Area

Table D.2.2 Aquifer Input Parameters

MAROS Report COC Assessment

MAROS Report Plume Summary

MAROS Report Spatial Moment Analysis Summary

MAROS Chart New Location Analysis Nitrate OLFA 
TABLE D.2.1

QUALITATIVE ANALYSIS OIL LANDFARM AREA

Bear Creek Hydrogeologic Regime

-12 National Security Complex

Oak Ridge, Tennessee

\begin{tabular}{|c|c|c|c|c|c|c|c|c|c|c|c|c|}
\hline $\begin{array}{c}\text { Location } \\
\text { Name }\end{array}$ & $\begin{array}{c}\text { Location } \\
\text { Type }\end{array}$ & \begin{tabular}{|c|} 
Average \\
Concentration \\
Exceeds \\
Screening Level \\
\end{tabular} & Formation Type & $\begin{array}{c}\text { Horizontal } \\
\text { Delineation }\end{array}$ & $\begin{array}{c}\text { Vertical } \\
\text { Delineation }\end{array}$ & Exit Location & RCRA & CERCLA & Unique & $\begin{array}{c}\text { Monitors } \\
\text { Background } \\
\text { Water Quality }\end{array}$ & $\begin{array}{c}\text { Early } \\
\text { Detection }\end{array}$ & $\begin{array}{l}\text { Monitor } \\
\text { Source }\end{array}$ \\
\hline GW-006 & WL & & Aquitard & & & & & & & & & $\mathrm{x}$ \\
\hline GW-008 & WL & $x$ & Aquitard & & & & $x$ & & & & & $x$ \\
\hline GW-010 & WL & $\mathrm{x}$ & Aquitard & & & & $\mathrm{x}$ & & & & & $x$ \\
\hline GW-013 & WL & & Aquitard & & & & & & & & & \\
\hline GW-064 & WL & $\mathrm{x}$ & Aquifer & & & & & & & & & \\
\hline GW-066 & WL & $x$ & Aquifer & $\mathrm{x}$ & & & & & & & & $x$ \\
\hline GW-067 & WL & $x$ & Aquifer & & & & & & & & & \\
\hline GW-073 & WL & & Aquitard & & & & & & $\mathrm{x}$ & $\mathrm{x}$ & & \\
\hline GW-074 & WL & & Aquitard & & $x$ & & & & $x$ & $x$ & & \\
\hline GW-075 & WL & & Aquitard & & $\mathrm{x}$ & & $\mathrm{x}$ & & & & & $\mathrm{x}$ \\
\hline GW-084 & WL & & Aquitard & & & & & & & $x$ & & \\
\hline GW-085 & WL & $\mathrm{x}$ & Aquitard & & & & & & & & $\mathrm{x}$ & $\mathrm{x}$ \\
\hline GW-086 & WL & & Aquitard & & & & & & & & & \\
\hline GW-097 & WL & & Aquitard & & & & & & & & & \\
\hline GW-098 & WL & $x$ & Aquitard & & $x$ & & & & & & & $x$ \\
\hline GW-120 & WL & & Aquitard & & & & & & $x$ & $x$ & & \\
\hline GW-225 & WL & $x$ & Aquifer & & $x$ & & & & & & $x$ & \\
\hline GW-226 & WL & $x$ & Aquifer & $x$ & & & & & & & $x$ & \\
\hline GW-227 & WL & $\mathrm{x}$ & Aquifer & & & & & & & & & \\
\hline GW-228 & WL & $x$ & Aquifer & & & & & & & & & \\
\hline GW-229 & WL & $x$ & Aquifer & & & & & & & & & \\
\hline GW-363 & WL & $x$ & Aquitard & & & & $x$ & $x$ & & $\mathrm{x}$ & & \\
\hline GW-364 & WL & $x$ & Aquifer & $x$ & & & & & & & $\mathrm{x}$ & \\
\hline GW-365 & WL & $x$ & Aquifer & $x$ & $x$ & & & & & & $x$ & \\
\hline GW-366 & WL & & Aquifer & & $x$ & & & & $\mathrm{x}$ & & & \\
\hline GW-367 & WL & $x$ & Aquifer & & & & & & & $\mathrm{X}$ & & \\
\hline GW-368 & WL & $x$ & Aquifer & & $x$ & & & & & $x$ & & \\
\hline GW-369 & WL & $x$ & Aquifer & & $x$ & & & & & & & \\
\hline GW-520 & WL & & Aquifer & & & & & & & & & \\
\hline GW-537 & WL & $x$ & Aquitard & $\mathrm{x}$ & & & & $\mathrm{x}$ & & & $\mathrm{x}$ & $\mathrm{x}$ \\
\hline
\end{tabular}

1. $\mathrm{WL}=$ Monitoring Well; $\mathrm{SP}=$ Spring

2. Well data taken from BWXT Y-12 Analytical Database. Sample locations shown on Figure A.1 and A.2.

3. RCRA indicates wells monitored as part of compliance with RCRA Post-Closure Corrective Action Monitoring or designated Alternate location;

4. Average Concentration Exceeds Screening = The average concentration over the entire sampling record for the priority constituent is above the MCL or other designated screening level

as defined in Table B.1.

fintions identified in Fig. A.2 from BWXT Y12,2003 Groundwater Monitoring Report, (12/01/2003).

6. Details of the decision criteria for each category are presented in the text. 
TABLE D.2.1

QUALITATIVE ANALYSIS OIL LANDFARM AREA

Bear Creek Hydrogeologic Regime

-12 National Security Complex

Oak Ridge, Tennessee

\begin{tabular}{|c|c|c|c|c|c|c|c|c|c|c|c|c|}
\hline $\begin{array}{c}\text { Location } \\
\text { Name }\end{array}$ & $\begin{array}{c}\text { Location } \\
\text { Type }\end{array}$ & \begin{tabular}{|c|} 
Average \\
Concentration \\
Exceeds \\
Screening Level
\end{tabular} & Formation Type & $\begin{array}{l}\text { Horizontal } \\
\text { Delineation }\end{array}$ & $\begin{array}{c}\text { Vertical } \\
\text { Delineation }\end{array}$ & Exit Location & RCRA & CERCLA & Unique & $\begin{array}{c}\text { Monitors } \\
\text { Background } \\
\text { Water Quality }\end{array}$ & $\begin{array}{c}\text { Early } \\
\text { Detection }\end{array}$ & $\begin{array}{l}\text { Monitor } \\
\text { Source }\end{array}$ \\
\hline GW-601 & WL & $\mathrm{x}$ & Aquifer & $\mathrm{x}$ & $\mathrm{x}$ & & & & & & & \\
\hline GW-636 & WL & & Aquitard & & $x$ & & & & $x$ & & & \\
\hline GW-637 & WL & & Aquitard & $x$ & & & & & $x$ & $\mathrm{x}$ & & \\
\hline GW-638 & WL & & Aquitard & & & & & & & & & \\
\hline GW-645 & WL & & Aquifer & $\mathrm{x}$ & & & & & & & & \\
\hline GW-646 & WL & & Aquifer & & & & & & & & & \\
\hline GW-723 & WL & $\mathrm{x}$ & Aquifer & & & $x$ & & & & & $x$ & \\
\hline GW-724 & WL & $x$ & Aquifer & & & $x$ & & & & & $x$ & \\
\hline GW-725 & WL & $x$ & Aquifer & $\mathrm{x}$ & & $x$ & & & & & $x$ & \\
\hline GW-736 & WL & $x$ & Aquifer & & $\mathrm{x}$ & $\mathrm{x}$ & & & & & & $\mathrm{x}$ \\
\hline GW-737 & WL & $x$ & Aquifer & & & $x$ & & & & & & $x$ \\
\hline GW-738 & WL & $\mathrm{x}$ & Aquifer & & & $x$ & & & & & & \\
\hline GW-739 & WL & $x$ & Aquifer & & & $x$ & & & & & & \\
\hline GW-740 & WL & $\mathrm{x}$ & Aquifer & & & $x$ & & & & & & \\
\hline GW-794 & WL & & Aquifer & $\mathrm{x}$ & & & & & $x$ & $\mathrm{x}$ & & \\
\hline GW-795 & WL & & Aquifer & $x$ & & & & & & $\mathrm{x}$ & & \\
\hline GW-800 & WL & & Aquifer & $x$ & & & & & & $\mathrm{x}$ & & \\
\hline GW-916 & WL & & Aquitard & $\mathrm{x}$ & & & & $x$ & & & & \\
\hline GW-917 & WL & & Aquitard & $x$ & & & & $x$ & & $\mathrm{x}$ & & \\
\hline GW-918 & WL & & Aquitard & $x$ & & & & $x$ & & $x$ & & \\
\hline GW-919 & WL & & Aquitard & & & & & $\mathrm{x}$ & & $\mathrm{x}$ & & \\
\hline GW-920 & WL & & Aquitard & & & & & $x$ & & $\mathrm{x}$ & & \\
\hline GW-921 & WL & & Aquitard & & & & & $x$ & & & & \\
\hline GW-922 & WL & & Aquitard & & & & & $x$ & & $\mathrm{x}$ & & \\
\hline GW-923 & WL & & Aquitard & & & & & $\mathrm{x}$ & & & & \\
\hline $\begin{array}{l}\text { GW-924 } \\
\end{array}$ & $\overline{W L}$ & & Aquitard & & & & & $x$ & & & & \\
\hline GW-925 & WL & & Aquitard & & $x$ & & & $\mathrm{x}$ & & & & \\
\hline GW-926 & WL & & Aquitard & & $x$ & & & $x$ & & & & \\
\hline GW-927 & WL & & Aquitard & & & & & $\mathrm{x}$ & & $\mathrm{x}$ & & \\
\hline
\end{tabular}

1. $\mathrm{WL}=$ Monitoring Well; $\mathrm{SP}=$ Spring

2. Well data taken from BWXT Y-12 Analytical Database. Sample locations shown on Figure A.1 and A.2.

3. RCRA indicates wells monitored as part of compliance with RCRA Post-Closure Corrective Action Monitoring or designated Alternate location;

4. Average Concentration Exceeds Screening = The average concentration over the entire sampling record for the priority constituent is above the MCL or other designated screening level

as defined in Table B.1.

formations identified in Fig. A.2 from BWXT Y12, 2003 Groundwater Monitoring Report, (12/01/2003).

6. Details of the decision criteria for each category are presented in the text. 
GSI Job No. G-3038

Issued 12/12/2005

Page 1 of 1

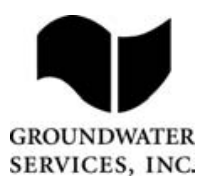

TABLE D.2.2

AQUIFER INPUT PARAMETERS

Oil Landfarm WMA

Bear Creek Regime

Y-12 National Security Complex

\begin{tabular}{|c|c|c|}
\hline Parameter & Value & Units \\
\hline Current Plume Length & 4000 & $\mathrm{ft}$ \\
\hline Maximum Plume Length & 4000 & $\mathrm{ft}$ \\
\hline PlumeWidth & 2200 & $\mathrm{ft}$ \\
\hline SeepageVelocity (ft/yr) & 200 & $\mathrm{ft} / \mathrm{yr}$ \\
\hline Distance to Receptors & 5000 & $\mathrm{ft}$ \\
\hline GWFluctuations & Yes & -- \\
\hline SourceTreatment & None & -- \\
\hline PlumeType & Nitrate/GA/GB/VOC & -- \\
\hline Free NAPL Present & Yes & -- \\
\hline Parameter & Value & \\
\hline Groundwater flow direction & W/SW & 200 \\
\hline Effective Porosity & 0.1 & -- \\
\hline Source Location near Well & GW-064 & -- \\
\hline Source X-Coordinate & 49169 & $\mathrm{ft}^{*}$ \\
\hline Source Y-Coordinate & 29195 & $\mathrm{ft}^{*}$ \\
\hline Saturated Thickness & 50 & $\mathrm{ft}$ \\
\hline \multicolumn{3}{|l|}{ Source Wells } \\
\hline$\overline{\mathrm{GW}}$ & $-064, G W-725$ & \\
\hline
\end{tabular}

\section{Notes:}

1. Aquifer data are general values for the hydrologic regime.

2. Priority COCs defined by prevalence, toxicty and mobility.

3. $\mathrm{ft}^{\star}=$ Coordinates in $\mathrm{Y}-12$ Plant coordinates, feet.

4. Screening Levels are USEPA MCLs, except in the case of compounds without MCLs where the level is the Region 9 PRG for tap water.

5. Effective Porosity estimated based on average high and low values for aquifer and aquitard suburfaces. 


\section{MAROS COC Assessment}

Project: $\quad$ Y-12 Security Complex

Location: OLFA

Toxicity:

\begin{tabular}{lccc} 
Toxicity: & $\begin{array}{c}\text { Representative } \\
\text { Concentration } \\
\text { (mg/L) }\end{array}$ & $\begin{array}{c}\text { PRG } \\
\text { (mg/L) }\end{array}$ & $\begin{array}{c}\text { Percent } \\
\text { Above } \\
\text { PRG }\end{array}$ \\
\hline TRICHLOROETHYLENE (TCE) & $2.5 \mathrm{E}-02$ & $5.0 \mathrm{E}-03$ & $403.7 \%$ \\
NITRATE & $2.8 \mathrm{E}+01$ & $1.0 \mathrm{E}+01$ & $184.1 \%$ \\
VINYL CHLORIDE & $4.2 \mathrm{E}-03$ & $2.0 \mathrm{E}-03$ & $110.4 \%$
\end{tabular}

Note: Top COCs by toxicity were determined by examining a representative concentration for each compound over the entire site. The compound representative concentrations are then compared with the chosen PRG for that compound, with the percentage excedence from the PRG determining the compound's toxicity. All compounds above exceed the PRG.

\section{Prevalence:}

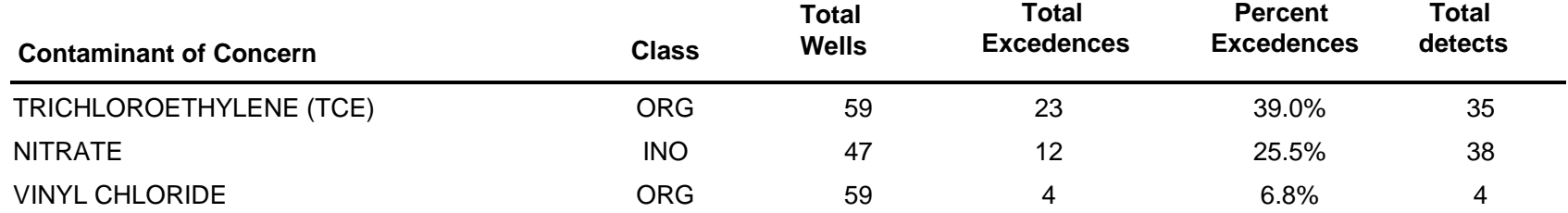

Note: Top COCs by prevalence were determined by examining a representative concentration for each well location at the site. The total excedences (values above the chosen PRGs) are compared to the total number of wells to determine the prevalence of the compound.

\section{Mobility:}

Contaminant of Concern

Kd

\begin{tabular}{ll}
\hline NITRATE & \\
VINYL CHLORIDE & 0.042 \\
TRICHLOROETHYLENE (TCE) & 0.297
\end{tabular}

Note: Top COCs by mobility were determined by examining each detected compound in the dataset and comparing their mobilities (Koc's for organics, assume foc $=0.001$, and Kd's for metals).

\section{Contaminants of Concern (COC's)}

VINYL CHLORIDE

TETRACHLOROETHYLENE(PCE)

NITRATE 


\section{MAROS Plume Analysis Summary}

Project: $\quad \mathrm{Y}-12$ Security Complex

Location: OLFA

Time Period: 1/1/1996

to $1 / 1 / 2005$

Consolidation Period: No Time Consolidation

Consolidation Type: Median

Duplicate Consolidation: Average

ND Values: Specified Detection Limit

J Flag Values : Actual Value

\begin{tabular}{|c|c|c|c|c|c|c|c|c|c|c|c|}
\hline Constituent & Well & $\begin{array}{c}\text { Sourcel } \\
\text { Tail }\end{array}$ & $\begin{array}{c}\text { Number } \\
\text { of } \\
\text { Samples }\end{array}$ & $\begin{array}{c}\text { Number } \\
\text { of } \\
\text { Detects }\end{array}$ & $\begin{array}{c}\text { Average } \\
\text { (mg/L) }\end{array}$ & $\begin{array}{r}\text { Median } \\
(\mathrm{mg} / \mathrm{L})\end{array}$ & $\begin{array}{c}\text { All } \\
\text { Samples } \\
\text { "ND" ? }\end{array}$ & $\begin{array}{l}\text { Mann- } \\
\text { Kendall }\end{array}$ & $\begin{array}{c}\text { Linear } \\
\text { Regression }\end{array}$ & Modeling & Empirical \\
\hline \multicolumn{12}{|l|}{ NITRATE } \\
\hline & GW-725 & $S$ & 17 & 17 & $3.4 \mathrm{E}+01$ & $2.5 \mathrm{E}+01$ & No & D & D & $\mathrm{N} / \mathrm{A}$ & N/A \\
\hline & GW-064 & $\mathrm{s}$ & 1 & 1 & $3.7 \mathrm{E}+00$ & $3.7 \mathrm{E}+00$ & No & N/A & N/A & N/A & N/A \\
\hline & GW-229 & S & 6 & 0 & 2.0E-02 & 2.0E-02 & Yes & S & D & $\mathrm{N} / \mathrm{A}$ & N/A \\
\hline & GW-225 & S & 8 & 8 & $3.4 E+01$ & $3.6 \mathrm{E}+01$ & No & NT & S & $\mathrm{N} / \mathrm{A}$ & N/A \\
\hline & GW-739 & $\mathrm{T}$ & 2 & 2 & $1.5 \mathrm{E}+00$ & $1.5 \mathrm{E}+00$ & No & N/A & $\mathrm{N} / \mathrm{A}$ & $\mathrm{N} / \mathrm{A}$ & N/A \\
\hline & GW-364 & $\mathrm{T}$ & 2 & 2 & $5.3 \mathrm{E}-01$ & 5.3E-01 & No & N/A & N/A & N/A & N/A \\
\hline & GW-736 & $\mathrm{T}$ & 2 & 2 & $1.7 \mathrm{E}+01$ & 1.7E+01 & No & N/A & $N / A$ & $\mathrm{~N} / \mathrm{A}$ & N/A \\
\hline & GW-365 & T & 2 & 0 & 2.0E-02 & $2.0 \mathrm{E}-02$ & Yes & N/A & N/A & N/A & N/A \\
\hline & GW-724 & $\mathrm{T}$ & 17 & 17 & $2.4 E+01$ & $2.4 \mathrm{E}+01$ & No & D & D & N/A & N/A \\
\hline & GW-794 & $\mathrm{T}$ & 2 & 2 & 4.4E-01 & 4.4E-01 & No & N/A & N/A & $\mathrm{N} / \mathrm{A}$ & N/A \\
\hline & GW-537 & T & 17 & 17 & $6.6 \mathrm{E}+02$ & $6.6 \mathrm{E}+02$ & No & D & D & N/A & N/A \\
\hline & GW-226 & $\mathrm{T}$ & 13 & 13 & $1.6 \mathrm{E}+01$ & 1.7E+01 & No & NT & NT & N/A & N/A \\
\hline & GW-740 & $\mathrm{T}$ & 17 & 17 & $2.7 \mathrm{E}+00$ & $2.4 \mathrm{E}+00$ & No & D & D & N/A & N/A \\
\hline & GW-795 & T & 4 & 0 & 2.0E-02 & $2.0 \mathrm{E}-02$ & Yes & $\mathrm{s}$ & $\mathrm{s}$ & N/A & N/A \\
\hline & GW-098 & $\mathrm{T}$ & 4 & 0 & 2.0E-02 & 2.0E-02 & Yes & S & S & N/A & N/A \\
\hline & GW-363 & $\mathrm{T}$ & 4 & 1 & 2.2E-02 & 2.0E-02 & No & NT & NT & N/A & N/A \\
\hline & GW-723 & T & 4 & 2 & $2.3 E+00$ & $1.7 \mathrm{E}+00$ & No & NT & D & N/A & N/A \\
\hline & GW-085 & $\mathrm{T}$ & 17 & 17 & $1.6 \mathrm{E}+02$ & $1.8 \mathrm{E}+02$ & No & NT & PI & N/A & N/A \\
\hline & GW-006 & $\mathrm{T}$ & 1 & 1 & $2.0 \mathrm{E}+00$ & $2.0 \mathrm{E}+00$ & No & N/A & $\mathrm{N} / \mathrm{A}$ & N/A & N/A \\
\hline & GW-084 & T & 4 & 1 & $5.0 \mathrm{E}-02$ & $2.0 \mathrm{E}-02$ & No & NT & NT & N/A & N/A \\
\hline & GW-738 & $\mathrm{T}$ & 17 & 17 & 1. $4 \mathrm{E}+01$ & $1.4 \mathrm{E}+01$ & No & $S$ & S & $\mathrm{N} / \mathrm{A}$ & N/A \\
\hline & GW-066 & T & 2 & 2 & $2.6 \mathrm{E}+00$ & $2.6 \mathrm{E}+00$ & No & N/A & N/A & N/A & N/A \\
\hline
\end{tabular}

MAROS Version 2.1, 2004, AFCEE
User Name: MV

State: Tennessee 
Project: Y-12 Security Complex

Location: OLFA
User Name: MV

State: Tennessee

\begin{tabular}{|c|c|c|c|c|c|c|c|c|c|c|c|}
\hline Constituent & Well & $\begin{array}{c}\text { Sourcel } \\
\text { Tail }\end{array}$ & $\begin{array}{c}\text { Number } \\
\text { of } \\
\text { Samples }\end{array}$ & $\begin{array}{c}\text { Number } \\
\text { of } \\
\text { Detects }\end{array}$ & $\begin{array}{c}\text { Average } \\
\text { (mg/L) }\end{array}$ & $\begin{array}{r}\text { Median } \\
(\mathrm{mg} / \mathrm{L})\end{array}$ & $\begin{array}{c}\text { All } \\
\text { Samples } \\
\text { "ND" ? }\end{array}$ & $\begin{array}{l}\text { Mann- } \\
\text { Kendall }\end{array}$ & $\begin{array}{l}\text { Linear } \\
\text { Regression }\end{array}$ & Modeling & Empirical \\
\hline \multicolumn{12}{|l|}{ NITRATE } \\
\hline & GW-737 & $T$ & 2 & 2 & $1.3 \mathrm{E}+01$ & $1.3 \mathrm{E}+01$ & No & N/A & $\mathrm{N} / \mathrm{A}$ & $\mathrm{N} / \mathrm{A}$ & N/A \\
\hline & GW-008 & $\mathrm{T}$ & 2 & 0 & $2.0 \mathrm{E}-02$ & $2.0 \mathrm{E}-02$ & Yes & N/A & N/A & N/A & N/A \\
\hline & GW-601 & $\mathrm{T}$ & 1 & 1 & $1.4 \mathrm{E}+01$ & $1.4 \mathrm{E}+01$ & No & $\mathrm{N} / \mathrm{A}$ & $\mathrm{N} / \mathrm{A}$ & $N / A$ & $N / A$ \\
\hline \multicolumn{12}{|c|}{ TETRACHLOROETHYLENE(PCE) } \\
\hline & GW-725 & $\mathrm{s}$ & 18 & 9 & $5.7 \mathrm{E}-04$ & $2.8 \mathrm{E}-04$ & No & NT & NT & $\mathrm{N} / \mathrm{A}$ & $\mathrm{N} / \mathrm{A}$ \\
\hline & GW-229 & s & 6 & 1 & $1.3 \mathrm{E}-04$ & 5.0E-05 & No & NT & $\mathrm{PI}$ & N/A & N/A \\
\hline & GW-064 & s & 2 & 1 & $2.8 \mathrm{E}-04$ & $2.8 \mathrm{E}-04$ & No & $N / A$ & $N / A$ & $N / A$ & $N / A$ \\
\hline & GW-225 & s & 8 & 4 & 4.0E-04 & $2.8 \mathrm{E}-04$ & No & 1 & 1 & $N / A$ & $N / A$ \\
\hline & GW-601 & $\mathrm{T}$ & 1 & 0 & $5.0 \mathrm{E}-05$ & $5.0 \mathrm{E}-05$ & Yes & $\mathrm{N} / \mathrm{A}$ & N/A & N/A & N/A \\
\hline & GW-006 & $\mathrm{T}$ & 8 & 4 & $2.4 \mathrm{E}-04$ & $2.0 \mathrm{E}-04$ & No & $\mathrm{s}$ & $\mathrm{s}$ & $N / A$ & $N / A$ \\
\hline & GW-008 & $\mathrm{T}$ & 14 & 14 & $3.4 \mathrm{E}-02$ & $2.4 \mathrm{E}-02$ & No & NT & NT & $N / A$ & $N / A$ \\
\hline & GW-724 & $\mathrm{T}$ & 18 & 12 & $1.1 \mathrm{E}-03$ & $1.5 \mathrm{E}-03$ & No & $\mathrm{PI}$ & $\mathrm{PI}$ & N/A & N/A \\
\hline & GW-723 & $\mathrm{T}$ & 4 & 0 & 5.0E-05 & $5.0 \mathrm{E}-05$ & Yes & s & s & $\mathrm{N} / \mathrm{A}$ & $N / A$ \\
\hline & GW-066 & $\mathrm{T}$ & 2 & 2 & 7.0E-03 & 7.0E-03 & No & $N / A$ & $N / A$ & $N / A$ & $N / A$ \\
\hline & GW-084 & $\mathrm{T}$ & 4 & 0 & $5.0 \mathrm{E}-05$ & $5.0 \mathrm{E}-05$ & Yes & $\mathrm{s}$ & $\mathrm{s}$ & N/A & N/A \\
\hline & GW-363 & $\mathrm{T}$ & 15 & 0 & 5.0E-05 & $5.0 \mathrm{E}-05$ & Yes & S & s & $N / A$ & $N / A$ \\
\hline & GW-097 & $\mathrm{T}$ & 2 & 1 & 7.7E-04 & $7.8 \mathrm{E}-04$ & No & $N / A$ & $\mathrm{~N} / \mathrm{A}$ & $N / A$ & N/A \\
\hline & GW-364 & $\mathrm{T}$ & 2 & 0 & 5.0E-05 & 5.0E-05 & Yes & $\mathrm{N} / \mathrm{A}$ & $N / A$ & $N / A$ & $N / A$ \\
\hline & GW-098 & $\mathrm{T}$ & 4 & 0 & 5.0E-05 & $5.0 \mathrm{E}-05$ & Yes & s & s & $N / A$ & $N / A$ \\
\hline & GW-537 & $\mathrm{T}$ & 18 & 1 & 7.5E-05 & $5.0 \mathrm{E}-05$ & No & NT & PD & $N / A$ & $N / A$ \\
\hline & GW-226 & $\mathrm{T}$ & 14 & 2 & $1.1 \mathrm{E}-04$ & 5.0E-05 & No & NT & 1 & $N / A$ & $N / A$ \\
\hline & GW-228 & $\mathrm{T}$ & 1 & 0 & $5.0 \mathrm{E}-05$ & $5.0 \mathrm{E}-05$ & Yes & $\mathrm{N} / \mathrm{A}$ & $\mathrm{N} / \mathrm{A}$ & N/A & N/A \\
\hline & GW-365 & $\mathrm{T}$ & 2 & 0 & $5.0 \mathrm{E}-05$ & $5.0 \mathrm{E}-05$ & Yes & $N / A$ & $N / A$ & $N / A$ & $N / A$ \\
\hline & GW-085 & $\mathrm{T}$ & 18 & 0 & $5.0 \mathrm{E}-05$ & 5.0E-05 & Yes & s & s & $N / A$ & $N / A$ \\
\hline & GW-919 & $\mathrm{T}$ & 5 & 0 & $5.0 \mathrm{E}-05$ & 5.0E-05 & Yes & $\mathrm{s}$ & $\mathrm{D}$ & $N / A$ & $N / A$ \\
\hline & GW-916 & $\mathrm{T}$ & 11 & 1 & 2.3E-04 & 5.0E-05 & No & NT & NT & $N / A$ & N/A \\
\hline & GW-918 & $\mathrm{T}$ & 11 & 0 & $5.0 \mathrm{E}-05$ & 5.0E-05 & Yes & $\mathrm{S}$ & $\mathrm{D}$ & $N / A$ & $N / A$ \\
\hline & GW-794 & $\mathrm{T}$ & 2 & 0 & $5.0 \mathrm{E}-05$ & $5.0 \mathrm{E}-05$ & Yes & $N / A$ & $N / A$ & $N / A$ & $N / A$ \\
\hline & GW-924 & $\mathrm{T}$ & 12 & 0 & $5.0 \mathrm{E}-05$ & $5.0 \mathrm{E}-05$ & Yes & $\mathrm{s}$ & $\mathrm{s}$ & $N / A$ & N/A \\
\hline & GW-917 & $\mathrm{T}$ & 11 & 0 & 5.0E-05 & 5.0E-05 & Yes & S & D & $N / A$ & $N / A$ \\
\hline & GW-922 & $\mathrm{T}$ & 11 & 0 & 5.0E-05 & $5.0 \mathrm{E}-05$ & Yes & s & D & $\mathrm{N} / \mathrm{A}$ & $N / A$ \\
\hline & GW-795 & $\mathrm{T}$ & 4 & 0 & $5.0 \mathrm{E}-05$ & $5.0 \mathrm{E}-05$ & Yes & s & s & $N / A$ & $N / A$ \\
\hline & GW-923 & $\mathrm{T}$ & 9 & 0 & $5.0 \mathrm{E}-05$ & 5.0E-05 & Yes & s & s & $N / A$ & $N / A$ \\
\hline
\end{tabular}


Project: Y-12 Security Complex

Location: OLFA
User Name: MV

State: Tennessee

\begin{tabular}{|c|c|c|c|c|c|c|c|c|c|c|c|}
\hline Constituent & Well & $\begin{array}{l}\text { Sourcel } \\
\text { Tail }\end{array}$ & $\begin{array}{l}\text { Number } \\
\text { of } \\
\text { Samples }\end{array}$ & $\begin{array}{c}\text { Number } \\
\text { of } \\
\text { Detects }\end{array}$ & $\begin{array}{c}\text { Average } \\
\text { (mg/L) }\end{array}$ & $\begin{array}{r}\text { Median } \\
(\mathrm{mg} / \mathrm{L})\end{array}$ & $\begin{array}{c}\text { All } \\
\text { Samples } \\
\text { "ND" ? }\end{array}$ & $\begin{array}{l}\text { Mann- } \\
\text { Kendall }\end{array}$ & $\begin{array}{c}\text { Linear } \\
\text { Regression }\end{array}$ & Modeling & Empirical \\
\hline \multicolumn{12}{|c|}{ TETRACHLOROETHYLENE(PCE) } \\
\hline & GW-739 & $\mathrm{T}$ & 2 & 0 & $5.0 \mathrm{E}-05$ & $5.0 \mathrm{E}-05$ & Yes & N/A & $\mathrm{N} / \mathrm{A}$ & $\mathrm{N} / \mathrm{A}$ & $\mathrm{N} / \mathrm{A}$ \\
\hline & GW-740 & $\mathrm{T}$ & 18 & 0 & $5.0 \mathrm{E}-05$ & 5.0E-05 & Yes & $\mathrm{s}$ & s & N/A & $N / A$ \\
\hline & GW-738 & $\mathrm{T}$ & 18 & 0 & $5.0 \mathrm{E}-05$ & 5.0E-05 & Yes & S & s & N/A & $N / A$ \\
\hline & GW-926 & T & 11 & 1 & $5.0 \mathrm{E}-05$ & $5.0 \mathrm{E}-05$ & No & $\mathrm{s}$ & D & N/A & N/A \\
\hline & GW-921 & $\mathrm{T}$ & 11 & 0 & 5.0E-05 & 5.0E-05 & Yes & $\mathrm{s}$ & $\mathrm{D}$ & N/A & $N / A$ \\
\hline & GW-737 & $\mathrm{T}$ & 2 & 2 & $1.3 \mathrm{E}-03$ & 1.3E-03 & No & N/A & $\mathrm{N} / \mathrm{A}$ & N/A & $N / A$ \\
\hline & GW-920 & $\mathrm{T}$ & 11 & 0 & $5.0 \mathrm{E}-05$ & 5.0E-05 & Yes & S & D & N/A & $N / A$ \\
\hline & GW-927 & $\mathrm{T}$ & 11 & 0 & $5.0 \mathrm{E}-05$ & 5.0E-05 & Yes & s & D & N/A & $N / A$ \\
\hline & GW-736 & $\mathrm{T}$ & 2 & 1 & $5.2 \mathrm{E}-04$ & $5.2 \mathrm{E}-04$ & No & N/A & N/A & N/A & N/A \\
\hline & GW-925 & $\mathrm{T}$ & 11 & 0 & $5.0 \mathrm{E}-05$ & 5.0E-05 & Yes & $\mathrm{s}$ & $\mathrm{D}$ & N/A & $N / A$ \\
\hline \multicolumn{12}{|c|}{ VINYL CHLORIDE } \\
\hline & GW-229 & $\mathrm{S}$ & 6 & 6 & $3.1 \mathrm{E}-02$ & $3.1 \mathrm{E}-02$ & No & $\mathrm{s}$ & PD & $\mathrm{N} / \mathrm{A}$ & N/A \\
\hline & GW-725 & s & 18 & 0 & $5.0 \mathrm{E}-04$ & $5.0 \mathrm{E}-04$ & Yes & S & s & N/A & $N / A$ \\
\hline & GW-064 & s & 2 & 0 & $5.0 \mathrm{E}-04$ & $5.0 \mathrm{E}-04$ & Yes & N/A & $\mathrm{N} / \mathrm{A}$ & N/A & $N / A$ \\
\hline & GW-225 & s & 8 & 0 & $5.0 \mathrm{E}-04$ & 5.0E-04 & Yes & s & s & $\mathrm{N} / \mathrm{A}$ & $N / A$ \\
\hline & GW-085 & $\mathrm{T}$ & 18 & 0 & $5.0 \mathrm{E}-04$ & $5.0 \mathrm{E}-04$ & Yes & S & s & N/A & $N / A$ \\
\hline & GW-921 & $\mathrm{T}$ & 4 & 0 & $5.0 \mathrm{E}-04$ & $5.0 \mathrm{E}-04$ & Yes & s & s & $\mathrm{N} / \mathrm{A}$ & $N / A$ \\
\hline & GW-097 & $\mathrm{T}$ & 2 & 0 & $5.0 \mathrm{E}-04$ & 5.0E-04 & Yes & N/A & N/A & $\mathrm{N} / \mathrm{A}$ & N/A \\
\hline & GW-922 & $T$ & 4 & 0 & $5.0 \mathrm{E}-04$ & $5.0 \mathrm{E}-04$ & Yes & s & s & N/A & $N / A$ \\
\hline & GW-084 & $\mathrm{T}$ & 4 & 0 & $5.0 \mathrm{E}-04$ & $5.0 \mathrm{E}-04$ & Yes & $\mathrm{s}$ & $\mathrm{s}$ & N/A & $\mathrm{N} / \mathrm{A}$ \\
\hline & GW-920 & $\mathrm{T}$ & 4 & 0 & $5.0 \mathrm{E}-04$ & 5.0E-04 & Yes & s & s & N/A & $N / A$ \\
\hline & GW-066 & $\mathrm{T}$ & 2 & 0 & $5.0 \mathrm{E}-04$ & $5.0 \mathrm{E}-04$ & Yes & N/A & $\mathrm{N} / \mathrm{A}$ & $\mathrm{N} / \mathrm{A}$ & $N / A$ \\
\hline & GW-924 & $\mathrm{T}$ & 5 & 0 & $5.0 \mathrm{E}-04$ & $5.0 \mathrm{E}-04$ & Yes & $\mathrm{s}$ & $\mathrm{D}$ & $\mathrm{N} / \mathrm{A}$ & $N / A$ \\
\hline & GW-925 & $T$ & 4 & 0 & $5.0 \mathrm{E}-04$ & $5.0 \mathrm{E}-04$ & Yes & s & s & N/A & $N / A$ \\
\hline & GW-008 & $\mathrm{T}$ & 14 & 0 & $5.0 \mathrm{E}-04$ & 5.0E-04 & Yes & $\mathrm{s}$ & I & $\mathrm{N} / \mathrm{A}$ & $N / A$ \\
\hline & GW-926 & $\mathrm{T}$ & 4 & 0 & $5.0 \mathrm{E}-04$ & $5.0 \mathrm{E}-04$ & Yes & S & s & N/A & $N / A$ \\
\hline & GW-006 & $T$ & 8 & 0 & $5.0 \mathrm{E}-04$ & $5.0 \mathrm{E}-04$ & Yes & $\mathrm{s}$ & s & N/A & $N / A$ \\
\hline & GW-923 & $\mathrm{T}$ & 4 & 0 & $5.0 \mathrm{E}-04$ & 5.0E-04 & Yes & $\mathrm{s}$ & s & N/A & $N / A$ \\
\hline & GW-794 & $\mathrm{T}$ & 2 & 0 & $5.0 \mathrm{E}-04$ & $5.0 \mathrm{E}-04$ & Yes & N/A & N/A & N/A & $N / A$ \\
\hline & GW-736 & $T$ & 2 & 0 & $5.0 \mathrm{E}-04$ & $5.0 \mathrm{E}-04$ & Yes & N/A & $\mathrm{N} / \mathrm{A}$ & N/A & $\mathrm{N} / \mathrm{A}$ \\
\hline & GW-724 & $T$ & 18 & 0 & $5.0 \mathrm{E}-04$ & 5.0E-04 & Yes & $\mathrm{s}$ & $\mathrm{s}$ & $\mathrm{N} / \mathrm{A}$ & $N / A$ \\
\hline & GW-927 & $\mathrm{T}$ & 4 & 0 & $5.0 \mathrm{E}-04$ & $5.0 \mathrm{E}-04$ & Yes & $\mathrm{s}$ & $\mathrm{s}$ & N/A & $N / A$ \\
\hline & GW-737 & $T$ & 2 & 0 & $5.0 \mathrm{E}-04$ & $5.0 \mathrm{E}-04$ & Yes & N/A & N/A & N/A & $\mathrm{N} / \mathrm{A}$ \\
\hline
\end{tabular}


Project: Y-12 Security Complex

Location: OLFA
User Name: $\quad$ MV

State: Tennessee

\begin{tabular}{|c|c|c|c|c|c|c|c|c|c|c|c|}
\hline Constituent & Well & $\begin{array}{l}\text { Sourcel } \\
\text { Tail }\end{array}$ & $\begin{array}{c}\text { Number } \\
\text { of } \\
\text { Samples }\end{array}$ & $\begin{array}{c}\text { Number } \\
\text { of } \\
\text { Detects }\end{array}$ & $\begin{array}{c}\text { Average } \\
\text { (mg/L) }\end{array}$ & $\begin{array}{r}\text { Median } \\
(\mathrm{mg} / \mathrm{L})\end{array}$ & $\begin{array}{c}\text { All } \\
\text { Samples } \\
\text { "ND" ? }\end{array}$ & $\begin{array}{l}\text { Mann- } \\
\text { Kendall }\end{array}$ & $\begin{array}{c}\text { Linear } \\
\text { Regression }\end{array}$ & Modeling & Empirical \\
\hline \multicolumn{12}{|c|}{ VINYL CHLORIDE } \\
\hline & GW-738 & $T$ & 18 & 0 & $5.0 \mathrm{E}-04$ & $5.0 \mathrm{E}-04$ & Yes & $\mathrm{s}$ & $\mathrm{S}$ & $\mathrm{N} / \mathrm{A}$ & $\mathrm{N} / \mathrm{A}$ \\
\hline & GW-601 & $\mathrm{T}$ & 1 & 0 & $5.0 \mathrm{E}-04$ & $5.0 \mathrm{E}-04$ & Yes & $\mathrm{N} / \mathrm{A}$ & N/A & N/A & N/A \\
\hline & GW-739 & $\mathrm{T}$ & 2 & 0 & $5.0 \mathrm{E}-04$ & $5.0 \mathrm{E}-04$ & Yes & $\mathrm{N} / \mathrm{A}$ & $N / A$ & $N / A$ & $N / A$ \\
\hline & GW-537 & $\mathrm{T}$ & 18 & 0 & $5.0 \mathrm{E}-04$ & 5.0E-04 & Yes & s & S & N/A & N/A \\
\hline & GW-918 & $\mathrm{T}$ & 4 & 0 & $5.0 \mathrm{E}-04$ & $5.0 \mathrm{E}-04$ & Yes & s & s & N/A & $\mathrm{N} / \mathrm{A}$ \\
\hline & GW-365 & $\mathrm{T}$ & 2 & 1 & 7.5E-04 & 7.5E-04 & No & N/A & N/A & N/A & $\mathrm{N} / \mathrm{A}$ \\
\hline & GW-098 & $\mathrm{T}$ & 4 & 0 & $5.0 \mathrm{E}-04$ & 5.0E-04 & Yes & s & s & N/A & $N / A$ \\
\hline & GW-364 & $\mathrm{T}$ & 2 & 0 & $5.0 \mathrm{E}-04$ & $5.0 \mathrm{E}-04$ & Yes & N/A & N/A & N/A & $\mathrm{N} / \mathrm{A}$ \\
\hline & GW-795 & $\mathrm{T}$ & 4 & 0 & $5.0 \mathrm{E}-04$ & 5.0E-04 & Yes & s & s & $N / A$ & $\mathrm{~N} / \mathrm{A}$ \\
\hline & GW-363 & $\mathrm{T}$ & 8 & 0 & $5.0 \mathrm{E}-04$ & 5.0E-04 & Yes & s & S & $N / A$ & $N / A$ \\
\hline & GW-916 & $\mathrm{T}$ & 4 & 0 & $5.0 \mathrm{E}-04$ & $5.0 \mathrm{E}-04$ & Yes & s & S & $\mathrm{N} / \mathrm{A}$ & $\mathrm{N} / \mathrm{A}$ \\
\hline & GW-917 & $\mathrm{T}$ & 4 & 0 & $5.0 \mathrm{E}-04$ & $5.0 \mathrm{E}-04$ & Yes & $\mathrm{s}$ & s & N/A & N/A \\
\hline & GW-228 & $\mathrm{T}$ & 1 & 0 & $5.0 \mathrm{E}-04$ & 5.0E-04 & Yes & N/A & $N / A$ & $N / A$ & $N / A$ \\
\hline & GW-226 & $\mathrm{T}$ & 14 & 0 & $5.0 \mathrm{E}-04$ & $5.0 \mathrm{E}-04$ & Yes & $S$ & 1 & N/A & $N / A$ \\
\hline & GW-723 & $\mathrm{T}$ & 4 & 0 & $5.0 \mathrm{E}-04$ & $5.0 \mathrm{E}-04$ & Yes & s & s & $N / A$ & $N / A$ \\
\hline & GW-919 & $\mathrm{T}$ & 2 & 0 & $5.0 \mathrm{E}-04$ & 5.0E-04 & Yes & $N / A$ & N/A & $N / A$ & $N / A$ \\
\hline & GW-740 & $\mathrm{T}$ & 18 & 0 & $5.0 \mathrm{E}-04$ & $5.0 \mathrm{E}-04$ & Yes & $\mathrm{s}$ & $\mathrm{s}$ & N/A & $N / A$ \\
\hline
\end{tabular}

Note: Increasing (I); Probably Increasing (PI); Stable (S); Probably Decreasing (PD); Decreasing (D); No Trend (NT); Not Applicable (N/A) - Due to insufficient Data (< 4 sampling events); Source/Tail (S/T)

The Number of Samples and Number of Detects shown above are post-consolidation values. 


\section{MAROS Spatial Moment Analysis Summary}

Project: Y-12 Security Complex

Location: OLFA
User Name: MV

State: Tennessee

\begin{tabular}{|c|c|c|c|c|c|c|c|}
\hline \multirow[b]{2}{*}{ Effective Date } & \multirow{2}{*}{$\begin{array}{l}\text { 0th Moment } \\
\text { Estimated } \\
\text { Mass }(\mathrm{Kg})\end{array}$} & \multicolumn{3}{|c|}{$\underline{1 s t}$ Moment (Center of Mass) } & \multicolumn{2}{|c|}{ 2nd Moment (Spread) } & \multirow[b]{2}{*}{$\begin{array}{c}\text { Number of } \\
\text { Wells }\end{array}$} \\
\hline & & $X c(f t)$ & Yc (ft) & $\begin{array}{c}\text { Source } \\
\text { Distance (ft) }\end{array}$ & $\underset{(\mathrm{sq} \mathrm{ft})}{\operatorname{Sigma} X X}$ & $\begin{array}{c}\text { Sigma } Y Y \\
(s q \mathrm{ft})\end{array}$ & \\
\hline
\end{tabular}

NITRATE

$\begin{array}{rrrrrrrr}1 / 1 / 1996 & 6.4 \mathrm{E}+03 & 49,115 & 29,744 & 552 & 90,052 & 34,278 & 11 \\ 7 / 1 / 1996 & 5.4 \mathrm{E}+03 & 49,109 & 29,736 & 544 & 98,171 & 37,142 & 11 \\ 1 / 1 / 1997 & 4.9 \mathrm{E}+03 & 49,150 & 29,750 & 555 & 33,090 & 27,347 & 8 \\ 7 / 1 / 1997 & 4.2 \mathrm{E}+03 & 49,129 & 29,766 & 572 & 46,860 & 34,575 & 8 \\ 1 / 1 / 1998 & 3.7 \mathrm{E}+03 & 49,001 & 29,616 & 454 & 115,759 & 54,016 & 8 \\ 7 / 1 / 1998 & 3.3 \mathrm{E}+03 & 49,045 & 29,677 & 498 & 103,683 & 43,907 & 9 \\ 10 / 1 / 1998 & 0.0 \mathrm{E}+00 & & & & & & 1 \\ 1 / 1 / 1999 & 6.9 \mathrm{E}+03 & 48,779 & 29,549 & 527 & 160,519 & 54,216 & 8 \\ 1 / 1 / 2000 & 7.4 \mathrm{E}+03 & 48,867 & 29,609 & 512 & 118,633 & 37,793 & 7 \\ 7 / 1 / 2000 & 7.0 \mathrm{E}+03 & 48,898 & 29,634 & 516 & 116,577 & 35,825 & 7 \\ 1 / 1 / 2001 & 6.4 \mathrm{E}+03 & 48,874 & 29,625 & 522 & 159,826 & 36,540 & 11 \\ 7 / 1 / 2001 & 6.6 \mathrm{E}+03 & 48,866 & 29,628 & 528 & 158,008 & 36,622 & 11 \\ 1 / 1 / 2002 & 6.3 \mathrm{E}+03 & 49,039 & 29,706 & 527 & 106,418 & 28,555 & 14 \\ 7 / 1 / 2002 & 4.7 \mathrm{E}+03 & 48,994 & 29,689 & 524 & 133,575 & 31,180 & 14 \\ 1 / 1 / 2003 & 7.0 \mathrm{E}+03 & 48,841 & 29,608 & 528 & 140,310 & 36,825 & 9 \\ 7 / 1 / 2003 & 6.9 \mathrm{E}+03 & 48,847 & 29,617 & 531 & 139,620 & 34,734 & 9 \\ 1 / 1 / 2004 & 9.1 \mathrm{E}+03 & 48,785 & 29,593 & 553 & 148,055 & 36,415 & 11 \\ 7 / 1 / 2004 & 4.7 \mathrm{E}+03 & 48,796 & 29,585 & 539 & 164,008 & 39,176 & 11\end{array}$

TETRACHLOROETHYLENE(PCE)

\begin{tabular}{|c|c|c|c|c|c|c|c|}
\hline 1/1/1996 & $5.0 \mathrm{E}-02$ & 47,907 & 29,561 & 1,314 & 465,707 & 63,305 & 11 \\
\hline 7/1/1996 & 3.4E-02 & 47,864 & 29,583 & 1,362 & 448,571 & 67,437 & 11 \\
\hline 1/1/1997 & 1.4E-02 & 48,460 & 29,823 & 947 & 154,611 & 49,616 & 8 \\
\hline $7 / 1 / 1997$ & 1.4E-02 & 48,460 & 29,823 & 947 & 154,611 & 49,616 & 8 \\
\hline 1/1/1998 & 1.4E-01 & 48,344 & 29,550 & 898 & 102,213 & 31,358 & 9 \\
\hline 4/1/1998 & $0.0 \mathrm{E}+00$ & & & & & & 1 \\
\hline 7/1/1998 & 1.7E-01 & 48,190 & 29,537 & 1,037 & 235,391 & 26,161 & 10 \\
\hline 10/1/1998 & $0.0 \mathrm{E}+00$ & & & & & & 1 \\
\hline 1/1/1999 & 8.4E-02 & 48,378 & 29,477 & 840 & 157,856 & 39,421 & 10 \\
\hline 4/1/1999 & $0.0 \mathrm{E}+00$ & & & & & & 1 \\
\hline 7/1/1999 & 1.7E-02 & 48,189 & 29,521 & 1,033 & 267,658 & 34,855 & 10 \\
\hline 10/1/1999 & $0.0 \mathrm{E}+00$ & & & & & & 1 \\
\hline $1 / 1 / 2000$ & 2.7E-02 & 48,305 & 29,600 & 954 & 303,162 & 27,620 & 9 \\
\hline $4 / 1 / 2000$ & $0.0 \mathrm{E}+00$ & & & & & & 1 \\
\hline $7 / 1 / 2000$ & $3.5 \mathrm{E}-02$ & 48,104 & 29,571 & 1,130 & 209,280 & 24,714 & 9 \\
\hline $1 / 1 / 2001$ & 1.1E-01 & 47,803 & 29,693 & 1,454 & 356,038 & 50,364 & 13 \\
\hline $4 / 1 / 2001$ & 1.2E-02 & 47,370 & 30,742 & 2,373 & 157,502 & 85,700 & 12 \\
\hline $7 / 1 / 2001$ & 3.0E-01 & 48,114 & 29,719 & 1,178 & 220,928 & 117,764 & 24 \\
\hline $10 / 1 / 2001$ & 1.2E-02 & 47,370 & 30,742 & 2,373 & 150,226 & 121,117 & 13 \\
\hline
\end{tabular}


Project: Y-12 Security Complex

Location: OLFA
User Name: MV

State: Tennessee

\begin{tabular}{|c|c|c|c|c|c|c|c|}
\hline \multirow[b]{2}{*}{ Effective Date } & \multirow{2}{*}{$\begin{array}{l}\text { 0th Moment } \\
\text { Estimated } \\
\text { Mass (kg) }\end{array}$} & \multicolumn{3}{|c|}{ 1st Moment (Center of Mass) } & \multicolumn{2}{|c|}{ 2nd Moment (Spread) } & \multirow[b]{2}{*}{$\begin{array}{c}\text { Number of } \\
\text { Wells }\end{array}$} \\
\hline & & $\mathrm{Xc}(\mathrm{ft})$ & Yc (ft) & $\begin{array}{c}\text { Source } \\
\text { Distance (ft) }\end{array}$ & $\begin{array}{c}\text { Sigma } X X \\
(s q \mathrm{ft})\end{array}$ & $\begin{array}{c}\text { Sigma YY } \\
(\mathrm{sq} \mathrm{ft})\end{array}$ & \\
\hline \multicolumn{8}{|c|}{ TETRACHLOROETHYLENE(PCE) } \\
\hline $1 / 1 / 2002$ & 3.0E-01 & 48,180 & 29,585 & 1,063 & 113,254 & 27,331 & 15 \\
\hline $7 / 1 / 2002$ & 3.3E-01 & 48,202 & 29,587 & 1,044 & 134,010 & 26,355 & 15 \\
\hline $1 / 1 / 2003$ & 2.2E-01 & 48,095 & 29,723 & 1,197 & 241,619 & 145,143 & 23 \\
\hline $4 / 1 / 2003$ & $1.2 \mathrm{E}-02$ & 47,370 & 30,742 & 2,373 & 150,226 & 121,117 & 13 \\
\hline $7 / 1 / 2003$ & 3.3E-01 & 48,107 & 29,756 & 1,201 & 228,978 & 186,099 & 23 \\
\hline $10 / 1 / 2003$ & $1.2 \mathrm{E}-02$ & 47,370 & 30,742 & 2,373 & 150,226 & 121,117 & 13 \\
\hline $1 / 1 / 2004$ & 3.0E-01 & 48,020 & 29,644 & 1,234 & 250,390 & 128,151 & 24 \\
\hline $4 / 1 / 2004$ & $1.2 \mathrm{E}-02$ & 47,355 & 30,740 & 2,383 & 149,308 & 85,476 & 11 \\
\hline $7 / 1 / 2004$ & 1.6E-01 & 47,905 & 29,725 & 1,371 & 353,310 & 192,891 & 23 \\
\hline $10 / 1 / 2004$ & $1.2 \mathrm{E}-02$ & 47,370 & 30,742 & 2,373 & 157,502 & 85,700 & 12 \\
\hline
\end{tabular}

VINYL CHLORIDE

\begin{tabular}{|c|c|c|c|c|c|c|c|}
\hline 1/1/1996 & 2.3E-01 & 47,950 & 29,654 & 1,302 & 569,631 & 77,017 & 11 \\
\hline $7 / 1 / 1996$ & 2.3E-01 & 47,950 & 29,654 & 1,302 & 569,631 & 77,017 & 11 \\
\hline $1 / 1 / 1997$ & $1.4 \mathrm{E}-01$ & 48,460 & 29,823 & 947 & 154,611 & 49,616 & 8 \\
\hline $7 / 1 / 1997$ & $1.4 \mathrm{E}-01$ & 48,460 & 29,823 & 947 & 154,611 & 49,616 & 8 \\
\hline 1/1/1998 & $1.0 \mathrm{E}-01$ & 48,500 & 29,520 & 743 & 169,195 & 47,128 & 9 \\
\hline 4/1/1998 & $0.0 \mathrm{E}+00$ & & & & & & 1 \\
\hline $7 / 1 / 1998$ & $1.2 \mathrm{E}-01$ & 48,374 & 29,514 & 857 & 280,905 & 42,274 & 10 \\
\hline 10/1/1998 & $0.0 \mathrm{E}+00$ & & & & & & 1 \\
\hline 1/1/1999 & $1.2 \mathrm{E}-01$ & 48,440 & 29,464 & 777 & 209,984 & 53,535 & 10 \\
\hline 4/1/1999 & $0.0 \mathrm{E}+00$ & & & & & & 1 \\
\hline 7/1/1999 & $1.0 \mathrm{E}-01$ & 48,491 & 29,521 & 752 & 213,161 & 44,644 & 10 \\
\hline 10/1/1999 & $0.0 \mathrm{E}+00$ & & & & & & 1 \\
\hline $1 / 1 / 2000$ & $1.0 \mathrm{E}-01$ & 48,491 & 29,521 & 752 & 195,809 & 41,832 & 9 \\
\hline $4 / 1 / 2000$ & $0.0 \mathrm{E}+00$ & & & & & & 1 \\
\hline $7 / 1 / 2000$ & $1.0 \mathrm{E}-01$ & 48,491 & 29,521 & 752 & 195,809 & 41,832 & 9 \\
\hline $1 / 1 / 2001$ & 2.1E-01 & 47,854 & 29,624 & 1,383 & 580,376 & 72,890 & 13 \\
\hline $4 / 1 / 2001$ & $1.2 \mathrm{E}-01$ & 47,370 & 30,742 & 2,373 & 157,502 & 85,700 & 12 \\
\hline $7 / 1 / 2001$ & 4.0E-01 & 47,820 & 30,092 & 1,620 & 547,502 & 332,981 & 24 \\
\hline $10 / 1 / 2001$ & $1.2 \mathrm{E}-01$ & 47,370 & 30,742 & 2,373 & 150,226 & 121,117 & 13 \\
\hline $1 / 1 / 2002$ & $1.5 \mathrm{E}-01$ & 48,176 & 29,479 & 1,032 & 416,380 & 36,419 & 15 \\
\hline $7 / 1 / 2002$ & $1.5 \mathrm{E}-01$ & 48,170 & 29,478 & 1,038 & 418,254 & 36,193 & 15 \\
\hline $1 / 1 / 2003$ & $1.4 \mathrm{E}-01$ & 48,172 & 29,486 & 1,039 & 376,809 & 36,201 & 10 \\
\hline $4 / 1 / 2003$ & $1.2 \mathrm{E}-01$ & 47,370 & 30,742 & 2,373 & 150,226 & 121,117 & 13 \\
\hline $7 / 1 / 2003$ & $1.4 \mathrm{E}-01$ & 48,196 & 29,489 & 1,017 & 367,850 & 37,030 & 10 \\
\hline $1 / 1 / 2004$ & 2.0E-01 & 47,679 & 29,437 & 1,510 & 853,159 & 35,845 & 12 \\
\hline $7 / 1 / 2004$ & $1.9 \mathrm{E}-01$ & 47,711 & 29,442 & 1,479 & 846,147 & 36,295 & 12 \\
\hline
\end{tabular}


Project: Y-12 Security Complex

Location: OLFA
User Name: $\mathrm{MV}$

State: Tennessee

\begin{tabular}{|c|c|c|c|c|c|}
\hline Moment Type & Constituent & $\begin{array}{l}\text { Coefficient } \\
\text { of Variation }\end{array}$ & $\begin{array}{l}\text { Mann-Kendall } \\
\text { S Statistic }\end{array}$ & $\begin{array}{l}\text { Confidence } \\
\text { in Trend }\end{array}$ & $\begin{array}{c}\text { Moment } \\
\text { Trend }\end{array}$ \\
\hline \multicolumn{6}{|c|}{ Zeroth Moment: Mass } \\
\hline & NITRATE & 0.36 & 31 & $87.0 \%$ & NT \\
\hline & TETRACHLOROETHYLENE(PCE) & 1.26 & 54 & $83.8 \%$ & NT \\
\hline & VINYL CHLORIDE & 0.70 & 59 & $89.8 \%$ & NT \\
\hline \multicolumn{6}{|c|}{ 1st Moment: Distance to Source } \\
\hline & NITRATE & 0.05 & 16 & $72.9 \%$ & NT \\
\hline & TETRACHLOROETHYLENE(PCE) & 0.40 & 130 & $100.0 \%$ & 1 \\
\hline & VINYL CHLORIDE & 0.43 & 62 & $96.8 \%$ & 1 \\
\hline \multicolumn{6}{|c|}{ 2nd Moment: Sigma XX } \\
\hline & NITRATE & 0.32 & 76 & $99.9 \%$ & 1 \\
\hline & TETRACHLOROETHYLENE(PCE) & 0.45 & -38 & $81.9 \%$ & $\mathrm{~S}$ \\
\hline & VINYL CHLORIDE & 0.62 & 28 & $79.0 \%$ & NT \\
\hline \multicolumn{6}{|c|}{ 2nd Moment: Sigma YY } \\
\hline & NITRATE & 0.19 & 2 & $51.6 \%$ & NT \\
\hline & TETRACHLOROETHYLENE(PCE) & 0.65 & 100 & $99.4 \%$ & 1 \\
\hline & VINYL CHLORIDE & 0.93 & -66 & $97.6 \%$ & $\mathrm{D}$ \\
\hline
\end{tabular}

Note: The following assumptions were applied for the calculation of the Zeroth Moment:

Porosity: $0.10 \quad$ Saturated Thickness: Uniform: $50 \mathrm{ft}$

Mann-Kendall Trend test performed on all sample events for each constituent. Increasing (I); Probably Increasing (PI); Stable (S);

Probably Decreasing (PD); Decreasing (D); No Trend (NT); Not Applicable (N/A)-Due to insufficient Data (< 4 sampling events).

Note: The Sigma $X X$ and Sigma $Y Y$ components are estimated using the given field coordinate system and then rotated to align with the estimated groundwater flow direction. Moments are not calculated for sample events with less than 6 wells. 


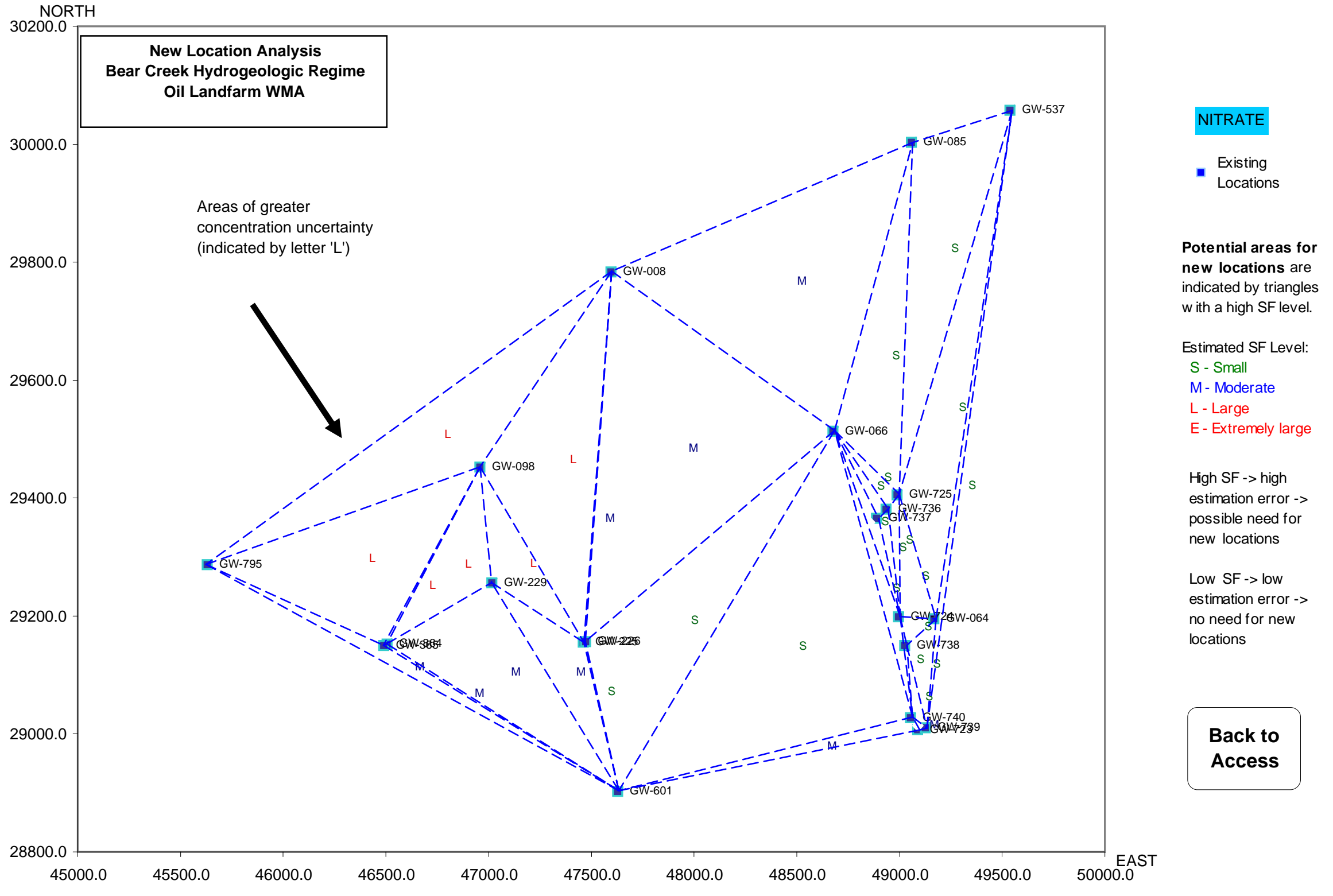




\section{ASSESSMENT OF THE GROUNDWATER PROTECTION PROGRAM \\ Y-12 NATIONAL SECURITY COMPLEX, OAK RIDGE, TENNESSEE}

Y-12 National Security Complex

Oak Ridge, Tennessee

Appendix D.3 Bear Creek Regime Bear Creek Burial Grounds

Table D.3.1 Qualitative Analysis Bear Creek Burial Grounds

Table D.3.2 Aquifer Input Parameters

MAROS Report COC Assessment

MAROS Report Plume Summary

MAROS Report Spatial Moment Analysis Summary

(No Well Sufficiency areas of interest identified) 
Bear Creek Hydrogeologic Regime

Y-12 National Security Complex

Oak Ridge, Tennessee

\begin{tabular}{|c|c|c|c|c|c|c|c|c|c|c|c|c|}
\hline $\begin{array}{l}\text { Location Name } \\
\end{array}$ & Location Type & \begin{tabular}{|c|} 
Average \\
Concentration \\
Exceeds \\
Screening \\
\end{tabular} & Formation Type & $\begin{array}{c}\text { Horizontal } \\
\text { Delineation } \\
\end{array}$ & $\begin{array}{c}\text { Vertical } \\
\text { Delineation } \\
\end{array}$ & $\begin{array}{c}\begin{array}{c}\text { Exit } \\
\text { Location }\end{array} \\
\end{array}$ & RCRA & CERCLA & Unique & $\begin{array}{c}\text { Monitors } \\
\text { Background } \\
\text { Water Quality } \\
\end{array}$ & $\begin{array}{c}\text { Early } \\
\text { Detection } \\
\end{array}$ & $\begin{array}{c}\text { Monitor } \\
\text { Source }\end{array}$ \\
\hline GW-014 & $\mathrm{WL}$ & $x$ & Aquitard & $\mathrm{x}$ & & & $x$ & & & & & \\
\hline GW-018 & WL & & Aquitard & & & & & & & & & $x$ \\
\hline GW-045 & WL & $\mathrm{x}$ & Aquifer & & & & & & & & & \\
\hline GW-046 & WL & $x$ & Aquitard & & & & $x$ & & & & & \\
\hline GW-047 & WL & & Aquitard & & & & & & & & & \\
\hline GW-052 & $\mathrm{WL}$ & $\mathrm{x}$ & Aquifer & & & & & & & & & \\
\hline GW-053 & WL & $x$ & Aquifer & & & & & & & & & \\
\hline GW-054 & WL & & Aquifer & & & & & & $x$ & & & \\
\hline GW-056 & WL & & Aquifer & & & $\mathrm{x}$ & & & $\mathrm{x}$ & & $x$ & \\
\hline GW-057 & WL & & Aquifer & & & $\mathrm{x}$ & & & & & & \\
\hline GW-058 & $\overline{W L}$ & $x$ & Aquifer & $\bar{x}$ & & & & & $x$ & & & \\
\hline GW-061 & WL & $x$ & Aquifer & & & & & & & & & \\
\hline GW-068 & WL & $x$ & Aquitard & & & & & & & & & \\
\hline GW-069 & WL & & Aquitard & & & & $\mathrm{x}$ & & & & & \\
\hline GW-071 & WL & $\mathrm{x}$ & Aquitard & & $\mathrm{x}$ & & $\mathrm{x}$ & & & & & \\
\hline GW-072 & WL & & Aquitard & & & & & & & & & \\
\hline GW-077 & WL & & Aquitard & & $\mathrm{x}$ & & & $\mathrm{x}$ & & & & \\
\hline GW-078 & WL & & Aquitard & & & & & $\mathrm{x}$ & & & & \\
\hline GW-079 & WL & & Aquitard & & & & & $\mathrm{x}$ & & & & \\
\hline GW-080 & WL & & Aquitard & & & & & $\mathrm{x}$ & & $\mathrm{x}$ & & \\
\hline GW-082 & $\mathrm{WL}$ & $\mathrm{x}$ & Aquitard & & & & $\mathrm{X}$ & & & & $\mathrm{x}$ & \\
\hline GW-083 & WL & & Aquitard & & & & & & $\mathrm{x}$ & & & \\
\hline GW-089 & WL & $x$ & Aquitard & & & & & & & & & \\
\hline GW-091 & WL & & Aquitard & & & & & & & & & \\
\hline GW-094 & WL & & Aquifer & & $\mathrm{x}$ & & & & $\mathrm{x}$ & & & \\
\hline GW-095 & WL & & Aquifer & & $x$ & & & & $x$ & & & \\
\hline GW-126 & WL & $\mathrm{x}$ & Aquitard & & & & & & $\mathrm{x}$ & & & \\
\hline GW-237 & WL & & Aquifer & & & & & & & & & \\
\hline GW-242 & WL & $x$ & Aquitard & & & & & & & & & \\
\hline GW-248 & WL & & Aquitard & & & & & & $\mathrm{x}$ & & & \\
\hline
\end{tabular}

Notes:

1. $\mathrm{WL}=$ Monitoring Well; $\mathrm{SP}=$ Spring

2. Well data taken from BWXT Y-12 Analytical Database. Sample locations shown on Figure A.1 and A.2.

3. RCRA indicates wells monitored as part of compliance with RCRA Post-Closure Corrective Action Monitoring or designated Alternate location;

CERCLA indicates locations monitored as part of compliance with CERCLA ROD or backup location. Data from BWXT, 2003a and BWXT 2004a.

4. Average Concentration Exceeds Screening = The average concentration over the entire sampling record for the priority constituent is above the MCL or other designated screening level

as defined in Table B.1.

5. Aquifer and aquitard formations identified in Fig. A.2 from BWXT Y12, 2003 Groundwater Monitoring Report, (12/01/2003).

6 . Details of the decision criteria for each category are presented in the text. 
Bear Creek Hydrogeologic Regime

Y-12 National Security Complex

Oak Ridge, Tennessee

\begin{tabular}{|c|c|c|c|c|c|c|c|c|c|c|c|c|}
\hline Location Name & $\begin{array}{l}\text { Location Type } \\
\end{array}$ & \begin{tabular}{|c|} 
Average \\
Concentration \\
Exceeds \\
Screening \\
\end{tabular} & Formation Type & $\begin{array}{c}\text { Horizontal } \\
\text { Delineation } \\
\end{array}$ & $\begin{array}{c}\text { Vertical } \\
\text { Delineation }\end{array}$ & $\begin{array}{c}\text { Exit } \\
\text { Location } \\
\end{array}$ & RCRA & CERCLA & Unique & $\begin{array}{c}\text { Monitors } \\
\text { Background } \\
\text { Water Quality } \\
\end{array}$ & $\begin{array}{c}\text { Early } \\
\text { Detection } \\
\end{array}$ & $\begin{array}{l}\text { Monitor } \\
\text { Source } \\
\end{array}$ \\
\hline $\begin{array}{l}\text { GW-249 } \\
\text { GW-250 } \\
\text { GW-257 } \\
\text { GW-258 } \\
\text { GW-259 }\end{array}$ & $\begin{array}{l}\text { WL } \\
W L \\
W L \\
W L \\
W L\end{array}$ & $\begin{array}{l}X \\
X \\
X \\
\end{array}$ & $\begin{array}{l}\text { Aquitard } \\
\text { Aquitard } \\
\text { Aquitard } \\
\text { Aquitard } \\
\text { Aquitard } \\
\end{array}$ & & & & $\mathrm{x}$ & & $\mathrm{x}$ & & & \\
\hline $\begin{array}{l}\text { GW-286 } \\
\text { GW-287 } \\
\text { GW-288 } \\
\text { GW-289 } \\
\text { GW-290 } \\
\end{array}$ & $\begin{array}{l}\text { WL } \\
W L \\
W L \\
W L \\
W L \\
\end{array}$ & $\begin{array}{l}X \\
X\end{array}$ & $\begin{array}{l}\text { Aquitard } \\
\text { Aquitard } \\
\text { Aquitard } \\
\text { Aquitard } \\
\text { Aquitard } \\
\end{array}$ & & & & $\mathrm{x}$ & & $\begin{array}{r}x \\
x \\
\end{array}$ & & $\begin{array}{l}x \\
x\end{array}$ & $\begin{array}{l}x \\
x\end{array}$ \\
\hline $\begin{array}{l}\text { GW-291 } \\
\text { GW-370 } \\
\text { GW-372 } \\
\text { GW-375 } \\
\text { GW-621 } \\
\end{array}$ & $\begin{array}{l}\text { WL } \\
W L \\
W L \\
W L \\
W L\end{array}$ & $\mathrm{X}$ & $\begin{array}{l}\text { Aquitard } \\
\text { Aquitard } \\
\text { Aquitard } \\
\text { Aquifer } \\
\text { Aquifer } \\
\end{array}$ & $\mathrm{x}$ & & $\mathrm{x}$ & $\mathrm{X}$ & & $\mathrm{x}$ & & $\mathrm{X}$ & \\
\hline $\begin{array}{l}\text { GW-622 } \\
\text { GW-623 } \\
\text { GW-624 } \\
\text { GW-626 } \\
\text { GW-627 }\end{array}$ & $\begin{array}{l}\text { WL } \\
W L \\
W L \\
W L \\
W L\end{array}$ & $\begin{array}{l}X \\
X \\
X \\
X\end{array}$ & $\begin{array}{l}\text { Aquitard } \\
\text { Aquitard } \\
\text { Aquitard } \\
\text { Aquitard } \\
\text { Aquitard }\end{array}$ & & $\mathrm{X}$ & & & & & & & \\
\hline $\begin{array}{l}\text { GW-629 } \\
\text { GW-639 } \\
\text { GW-641 } \\
\text { GW-642 } \\
\text { GW-651 } \\
\end{array}$ & $\begin{array}{l}\text { WL } \\
W L \\
W L \\
W L \\
W L\end{array}$ & $\mathrm{X}$ & $\begin{array}{l}\text { Aquitard } \\
\text { Aquitard } \\
\text { Aquitard } \\
\text { Aquitard } \\
\text { Aquifer } \\
\end{array}$ & $\mathrm{x}$ & & & & $\mathrm{X}$ & $\mathrm{x}$ & & & \\
\hline $\begin{array}{l}\text { GW-652 } \\
\text { GW-653 } \\
\text { GW-654 } \\
\text { GW-683 } \\
\text { GW-684 }\end{array}$ & $\begin{array}{l}\text { WL } \\
W L \\
W L \\
W L \\
W L\end{array}$ & $\begin{array}{l}X \\
X \\
X \\
\end{array}$ & $\begin{array}{l}\text { Aquifer } \\
\text { Aquitard } \\
\text { Aquitard } \\
\text { Aquifer } \\
\text { Aquifer } \\
\end{array}$ & $x$ & $\begin{array}{l}X \\
X \\
\end{array}$ & $\begin{array}{l}X \\
X \\
\end{array}$ & & $\begin{array}{l}X \\
X \\
\end{array}$ & & & & \\
\hline
\end{tabular}

Notes:

1. $\mathrm{WL}=$ Monitoring Well; $\mathrm{SP}=$ Spring

2. Well data taken from BWXT Y-12 Analytical Database. Sample locations shown on Figure A.1 and A.2.

3. RCRA indicates wells monitored as part of compliance with RCRA Post-Closure Corrective Action Monitoring or designated Alternate location;

CERCLA indicates locations monitored as part of compliance with CERCLA ROD or backup location. Data from BWXT, 2003a and BWXT 2004a.

4. Average Concentration Exceeds Screening = The average concentration over the entire sampling record for the priority constituent is above the MCL or other designated screening level

as defined in Table B.1.

5. Aquifer and aquitard formations identified in Fig. A.2 from BWXT Y12, 2003 Groundwater Monitoring Report, (12/01/2003).

6. Details of the decision criteria for each category are presented in the text. 
Bear Creek Hydrogeologic Regime

Y-12 National Security Complex

Oak Ridge, Tennessee

\begin{tabular}{|c|c|c|c|c|c|c|c|c|c|c|c|c|}
\hline Location Name & Location Type & \begin{tabular}{|c|} 
Average \\
Concentration \\
Exceeds \\
Screening \\
\end{tabular} & Formation Type & $\begin{array}{c}\text { Horizontal } \\
\text { Delineation } \\
\end{array}$ & $\begin{array}{c}\text { Vertical } \\
\text { Delineation } \\
\end{array}$ & $\begin{array}{c}\begin{array}{c}\text { Exit } \\
\text { Location }\end{array} \\
\end{array}$ & RCRA & CERCLA & Unique & 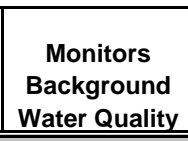 & $\begin{array}{c}\text { Early } \\
\text { Detection } \\
\end{array}$ & $\begin{array}{c}\text { Monitor } \\
\text { Source } \\
\end{array}$ \\
\hline $\begin{array}{l}\text { GW-685 } \\
\text { GW-694 } \\
\text { GW-695 } \\
\text { GW-703 } \\
\text { GW-704 } \\
\end{array}$ & $\begin{array}{l}\text { WL } \\
W L \\
W L \\
W L \\
W L\end{array}$ & $\begin{array}{l}X \\
X \\
X\end{array}$ & $\begin{array}{l}\text { Aquifer } \\
\text { Aquifer } \\
\text { Aquifer } \\
\text { Aquifer } \\
\text { Aquifer } \\
\end{array}$ & & & $\begin{array}{l}X \\
X \\
X \\
X \\
X \\
\end{array}$ & & $\mathrm{x}$ & & & $\begin{array}{l}x \\
x \\
\end{array}$ & \\
\hline $\begin{array}{l}\text { GW-706 } \\
\text { GW-710 } \\
\text { GW-711 } \\
\text { GW-712 } \\
\text { GW-713 }\end{array}$ & $\begin{array}{l}\text { WL } \\
W L \\
W L \\
W L \\
W L\end{array}$ & $\begin{array}{l}X \\
x\end{array}$ & $\begin{array}{l}\text { Aquifer } \\
\text { Aquifer } \\
\text { Aquifer } \\
\text { Aquifer } \\
\text { Aquifer }\end{array}$ & $\begin{array}{l}x \\
x \\
X \\
x\end{array}$ & $\begin{array}{l}x \\
x \\
X \\
x\end{array}$ & $\begin{array}{l}X \\
X \\
X \\
X \\
X\end{array}$ & $\begin{array}{l}X \\
X\end{array}$ & $\begin{array}{l}X \\
X \\
X\end{array}$ & & & $\mathrm{x}$ & \\
\hline $\begin{array}{c}\text { GW-714 } \\
\text { GW-715 } \\
\text { SS-4 } \\
\text { SS-5 } \\
\text { SS-5_95KM }\end{array}$ & $\begin{array}{l}\text { WL } \\
\text { WL } \\
\text { SP } \\
\text { SP } \\
\text { SP }\end{array}$ & $\begin{array}{l}x \\
x\end{array}$ & $\begin{array}{l}\text { Aquifer } \\
\text { Aquifer } \\
\text { Spring } \\
\text { Spring } \\
\text { Spring }\end{array}$ & $\begin{array}{l}x \\
x \\
x\end{array}$ & & $\begin{array}{l}X \\
X \\
x \\
x \\
x\end{array}$ & $\begin{array}{l}X \\
x\end{array}$ & $x$ & & & & $\begin{array}{l}x \\
x \\
x\end{array}$ \\
\hline $\begin{array}{l}\text { SS-6_6 } \\
\text { SS-6E } \\
\text { SS-6W } \\
\text { SS-7 } \\
\text { SS-8 }\end{array}$ & $\begin{array}{l}\text { SP } \\
\text { SP } \\
\text { SP } \\
\text { SP } \\
\text { SP }\end{array}$ & $\begin{array}{l}x \\
x\end{array}$ & $\begin{array}{l}\text { Spring } \\
\text { Spring } \\
\text { Spring } \\
\text { Spring } \\
\text { Spring }\end{array}$ & & & $\begin{array}{l}X \\
x \\
x \\
x \\
x\end{array}$ & & $\begin{array}{l}X \\
X \\
x \\
X \\
x\end{array}$ & & & & $\begin{array}{l}X \\
X \\
X \\
X \\
X\end{array}$ \\
\hline
\end{tabular}

Notes:

1. $\mathrm{WL}=$ Monitoring Well; $\mathrm{SP}=$ Spring

2. Well data taken from BWXT Y-12 Analytical Database. Sample locations shown on Figure A.1 and A.2.

3. RCRA indicates wells monitored as part of compliance with RCRA Post-Closure Corrective Action Monitoring or designated Alternate location;

CERCLA indicates locations monitored as part of compliance with CERCLA ROD or backup location. Data from BWXT, 2003a and BWXT 2004a.

4. Average Concentration Exceeds Screening = The average concentration over the entire sampling record for the priority constituent is above the MCL or other designated screening level

as defined in Table B.1.

5. Aquifer and aquitard formations identified in Fig. A.2 from BWXT Y12, 2003 Groundwater Monitoring Report, (12/01/2003).

6. Details of the decision criteria for each category are presented in the text. 
GSI Job No. G-3038

Issued $12 / 12 / 2005$

Page 1 of 1

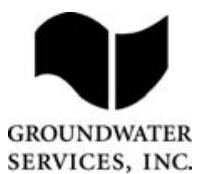

TABLE D.3.2

AQUIFER INPUT PARAMETERS

\author{
Bear Creek Burial Grounds \\ Bear Creek Hydrogeologic Regime \\ Y-12 National Security Complex
}

\begin{tabular}{|c|c|c|}
\hline Parameter & Value & Units \\
\hline Current Plume Length & 5000 & $\mathrm{ft}$ \\
\hline Maximum Plume Length & 8000 & $\mathrm{ft}$ \\
\hline PlumeWidth & 2500 & $\mathrm{ft}$ \\
\hline SeepageVelocity (ft/yr) & 200 & $\mathrm{ft} / \mathrm{yr}$ \\
\hline Distance to Receptors & 9000 & $\mathrm{ft}$ \\
\hline GWFluctuations & Yes & -- \\
\hline SourceTreatment & None & -- \\
\hline PlumeType & Chlorinated Solvent/Nitrate/GA/GB & -- \\
\hline Free NAPL Present & Yes & -- \\
\hline Parameter & Value & \\
\hline Groundwater flow direction & WISW & 200 \\
\hline Effective Porosity & 0.1 & -- \\
\hline Source Location near Well & GW-046 & -- \\
\hline Source X-Coordinate & 43283.53 & $\mathrm{ft}^{*}$ \\
\hline Source Y-Coordinate & 29562.34 & $\mathrm{ft}^{*}$ \\
\hline Saturated Thickness & 50 & $\mathrm{ft}$ \\
\hline \multicolumn{3}{|l|}{ Source Wells } \\
\hline $0+0$ & $N-6$ & \\
\hline
\end{tabular}

\title{
Notes:
}

1. Aquifer data are general values for the hydrologic regime.

2. Priority COCs defined by prevalence, toxicty and mobility.

3. $\mathrm{ft}^{*}=$ Coordinates in $\mathrm{Y}-12$ Plant coordinates, feet.

4. Screening Levels are USEPA MCLs, except in the case of compounds without MCLs where the level is the Region 9 PRG for tap water.

5. Effective Porosity estimated based on average high and low values for aquifer and aquitard suburfaces. 


\section{MAROS COC Assessment}

Project: Y-12 Security Complex

Location: Bear Creek Burial Grounds

Toxicity:

\begin{tabular}{lccc}
\hline Contaminant of Concern & $\begin{array}{c}\text { Representative } \\
\text { Concentration } \\
\text { (mg/L) }\end{array}$ & $\begin{array}{c}\text { PRG } \\
\text { (mg/L) }\end{array}$ & $\begin{array}{c}\text { Percent } \\
\text { Above } \\
\text { PRG }\end{array}$ \\
\hline TETRACHLOROETHYLENE(PCE) & $3.4 \mathrm{E}-01$ & $5.0 \mathrm{E}-03$ & $6770.2 \%$ \\
TRICHLOROETHYLENE (TCE) & $2.0 \mathrm{E}-01$ & $5.0 \mathrm{E}-03$ & $3946.9 \%$ \\
VINYL CHLORIDE & $3.9 \mathrm{E}-02$ & $2.0 \mathrm{E}-03$ & $1831.2 \%$ \\
BENZENE & $1.3 \mathrm{E}-02$ & $5.0 \mathrm{E}-03$ & $152.6 \%$ \\
CIS-1,2-DICHLOROETHYLENE & $1.3 \mathrm{E}-01$ & $7.0 \mathrm{E}-02$ & $83.3 \%$ \\
1,1-DICHLOROETHENE & $1.3 \mathrm{E}-02$ & $7.0 \mathrm{E}-03$ & $79.6 \%$ \\
CARBON TETRACHLORIDE & $7.8 \mathrm{E}-03$ & $5.0 \mathrm{E}-03$ & $55.1 \%$ \\
1,2-DICHLOROETHANE & $7.7 \mathrm{E}-03$ & $5.0 \mathrm{E}-03$ & $54.9 \%$
\end{tabular}

Note: Top COCs by toxicity were determined by examining a representative concentration for each compound over the entire site. The compound representative concentrations are then compared with the chosen PRG for that compound, with the percentage excedence from the PRG determining the compound's toxicity. All compounds above exceed the PRG.

Prevalence:

\begin{tabular}{|c|c|c|c|c|c|}
\hline Contaminant of Concern & Class & $\begin{array}{l}\text { Total } \\
\text { Wells }\end{array}$ & $\begin{array}{c}\text { Total } \\
\text { Excedences }\end{array}$ & $\begin{array}{c}\text { Percent } \\
\text { Excedences }\end{array}$ & $\begin{array}{l}\text { Total } \\
\text { detects }\end{array}$ \\
\hline TRICHLOROETHYLENE (TCE) & ORG & 80 & 19 & $23.8 \%$ & 44 \\
\hline TETRACHLOROETHYLENE(PCE) & ORG & 80 & 16 & $20.0 \%$ & 37 \\
\hline VINYL CHLORIDE & ORG & 80 & 15 & $18.8 \%$ & 15 \\
\hline BENZENE & ORG & 80 & 10 & $12.5 \%$ & 29 \\
\hline 1,1-DICHLOROETHENE & ORG & 80 & 9 & $11.3 \%$ & 20 \\
\hline cis-1,2-DICHLOROETHYLENE & ORG & 80 & 8 & $10.0 \%$ & 32 \\
\hline 1,2-DICHLOROETHANE & ORG & 80 & 6 & $7.5 \%$ & 13 \\
\hline CARBON TETRACHLORIDE & ORG & 80 & 5 & $6.3 \%$ & 12 \\
\hline
\end{tabular}

Note: Top COCs by prevalence were determined by examining a representative concentration for each well location at the site. The total excedences (values above the chosen PRGs) are compared to the total number of wells to determine the prevalence of the compound.

\section{Mobility:}

Contaminant of Concern

VINYL CHLORIDE

1,2-DICHLOROETHANE

cIS-1,2-DICHLOROETHYLENE

BENZENE

1,1-DICHLOROETHENE

CARBON TETRACHLORIDE

TRICHLOROETHYLENE (TCE)

TETRACHLOROETHYLENE(PCE)
Kd
0.042
0.0679
0.0724
0.0984
0.13
0.277
0.297
0.923

Note: Top COCs by mobility were determined by examining each detected compound in the dataset and comparing their mobilities (Koc's for organics, assume foc $=0.001$, and Kd's for metals). 
Project: Y-12 Security Complex

Location: Bear Creek Burial Grounds

Contaminants of Concern (COC's)

TETRACHLOROETHYLENE(PCE)

TRICHLOROETHYLENE (TCE)

VINYL CHLORIDE

GROSS BETA ACTIVITY

NITRATE
User Name: MV

State: Tennessee 


\section{MAROS Plume Analysis Summary}

Project: Y-12 Security Complex

Location: Bear Creek Burial Grounds

Time Period: 1/1/1996

Consolidation Period: No Time Consolidation

Consolidation Type: Median

Duplicate Consolidation: Average

ND Values: Specified Detection Limit

J Flag Values : Actual Value

\begin{tabular}{|c|c|c|c|c|c|c|c|c|c|c|c|}
\hline Constituent & Well & $\begin{array}{l}\text { Sourcel } \\
\text { Tail }\end{array}$ & $\begin{array}{c}\text { Number } \\
\text { of } \\
\text { Samples }\end{array}$ & $\begin{array}{c}\text { Number } \\
\text { of } \\
\text { Detects }\end{array}$ & $\begin{array}{c}\text { Average } \\
\text { (mg/L) }\end{array}$ & $\begin{array}{l}\text { Median } \\
(\mathrm{mg} / \mathrm{L})\end{array}$ & $\begin{array}{c}\text { All } \\
\text { Samples } \\
\text { "ND" ? }\end{array}$ & $\begin{array}{l}\text { Mann- } \\
\text { Kendall }\end{array}$ & $\begin{array}{c}\text { Linear } \\
\text { Regression }\end{array}$ & Modeling & Empirical \\
\hline \multicolumn{12}{|c|}{ GROSS BETA ACTIVITY } \\
\hline & GW-624 & $\mathrm{S}$ & 2 & 2 & $3.4 \mathrm{E}+00$ & $3.4 \mathrm{E}+00$ & No & $\mathrm{N} / \mathrm{A}$ & $\mathrm{N} / \mathrm{A}$ & $\mathrm{N} / \mathrm{A}$ & $\mathrm{N} / \mathrm{A}$ \\
\hline & GW-071 & s & 1 & 1 & $3.0 \mathrm{E}+00$ & $3.0 \mathrm{E}+00$ & No & $\mathrm{N} / \mathrm{A}$ & $\mathrm{N} / \mathrm{A}$ & $\mathrm{N} / \mathrm{A}$ & N/A \\
\hline & GW-082 & S & 11 & 10 & $5.1 \mathrm{E}+00$ & $5.2 \mathrm{E}+00$ & No & 1 & $\mathrm{PI}$ & $N / A$ & $N / A$ \\
\hline & GW-014 & s & 1 & 1 & $1.8 \mathrm{E}+00$ & $1.8 \mathrm{E}+00$ & No & $N / A$ & $\mathrm{~N} / \mathrm{A}$ & $N / A$ & $\mathrm{~N} / \mathrm{A}$ \\
\hline & GW-046 & s & 14 & 13 & $3.3 \mathrm{E}+00$ & $3.3 \mathrm{E}+00$ & No & $\mathrm{s}$ & $\mathrm{s}$ & $\mathrm{N} / \mathrm{A}$ & N/A \\
\hline & GW-626 & s & 2 & 2 & $3.8 \mathrm{E}+00$ & $3.8 \mathrm{E}+00$ & No & N/A & N/A & N/A & N/A \\
\hline & GW-372 & $\mathrm{T}$ & 3 & 3 & $2.0 \mathrm{E}+00$ & $2.3 \mathrm{E}+00$ & No & $N / A$ & $\mathrm{~N} / \mathrm{A}$ & $N / A$ & $\mathrm{~N} / \mathrm{A}$ \\
\hline & SS-4 & $\mathrm{T}$ & 18 & 18 & $9.6 \mathrm{E}+01$ & $8.2 E+01$ & No & NT & NT & $\mathrm{N} / \mathrm{A}$ & N/A \\
\hline & GW-621 & $T$ & 10 & 10 & $7.4 \mathrm{E}+00$ & $6.4 \mathrm{E}+00$ & No & s & $\mathrm{s}$ & N/A & $\mathrm{N} / \mathrm{A}$ \\
\hline & GW-653 & $T$ & 13 & 13 & $2.8 \mathrm{E}+00$ & $3.1 E+00$ & No & NT & NT & $\mathrm{N} / \mathrm{A}$ & $\mathrm{N} / \mathrm{A}$ \\
\hline & GW-291 & $T$ & 4 & 3 & $1.4 \mathrm{E}+00$ & $1.6 \mathrm{E}+00$ & No & NT & NT & $N / A$ & $\mathrm{~N} / \mathrm{A}$ \\
\hline & GW-289 & $\mathrm{T}$ & 3 & 3 & $4.0 \mathrm{E}+00$ & $2.3 E+00$ & No & $\mathrm{N} / \mathrm{A}$ & $\mathrm{N} / \mathrm{A}$ & $N / A$ & $\mathrm{~N} / \mathrm{A}$ \\
\hline & GW-627 & $\mathrm{T}$ & 10 & 10 & $8.1 \mathrm{E}+00$ & $6.7 \mathrm{E}+00$ & No & $\mathrm{PI}$ & $\mathrm{PI}$ & $N / A$ & $\mathrm{~N} / \mathrm{A}$ \\
\hline & GW-629 & $T$ & 4 & 0 & $1.0 \mathrm{E}-02$ & 1.0E-02 & Yes & $\mathrm{s}$ & $\mathrm{s}$ & $N / A$ & $N / A$ \\
\hline & GW-639 & $T$ & 5 & 1 & $9.5 \mathrm{E}-01$ & 1.0E-02 & No & NT & NT & $\mathrm{N} / \mathrm{A}$ & N/A \\
\hline & GW-715 & $\mathrm{T}$ & 16 & 16 & $1.7 \mathrm{E}+01$ & $3.9 \mathrm{E}+00$ & No & NT & NT & $N / A$ & $\mathrm{~N} / \mathrm{A}$ \\
\hline & GW-714 & $T$ & 18 & 18 & $4.1 \mathrm{E}+00$ & $4.2 \mathrm{E}+00$ & No & $\mathrm{s}$ & $\mathrm{D}$ & $N / A$ & $\mathrm{~N} / \mathrm{A}$ \\
\hline & GW-257 & $T$ & 2 & 2 & $2.5 \mathrm{E}+00$ & $2.5 \mathrm{E}+00$ & No & N/A & $\mathrm{N} / \mathrm{A}$ & $\mathrm{N} / \mathrm{A}$ & N/A \\
\hline & GW-683 & $\mathrm{T}$ & 17 & 17 & $2.3 \mathrm{E}+01$ & $2.0 \mathrm{E}+01$ & No & $\mathrm{s}$ & $\mathrm{S}$ & $\mathrm{N} / \mathrm{A}$ & $\mathrm{N} / \mathrm{A}$ \\
\hline & GW-684 & $\mathrm{T}$ & 18 & 18 & 2.7E+01 & $2.6 \mathrm{E}+01$ & No & s & s & $N / A$ & $\mathrm{~N} / \mathrm{A}$ \\
\hline & GW-685 & $\mathrm{T}$ & 9 & 9 & $9.4 \mathrm{E}+00$ & $8.0 \mathrm{E}+00$ & No & s & s & $N / A$ & $\mathrm{~N} / \mathrm{A}$ \\
\hline & GW-713 & $T$ & 17 & 16 & $7.5 \mathrm{E}+00$ & $2.6 \mathrm{E}+00$ & No & NT & NT & $\mathrm{N} / \mathrm{A}$ & $\mathrm{N} / \mathrm{A}$ \\
\hline
\end{tabular}

User Name: MV

State: Tennessee 
Project: Y-12 Security Complex

Location: Bear Creek Burial Grounds
User Name: MV

State: Tennessee

\begin{tabular}{|c|c|c|c|c|c|c|c|c|c|c|c|}
\hline Constituent & Well & $\begin{array}{c}\text { Sourcel } \\
\text { Tail }\end{array}$ & $\begin{array}{l}\text { Number } \\
\text { of } \\
\text { Samples }\end{array}$ & $\begin{array}{c}\text { Number } \\
\text { of } \\
\text { Detects }\end{array}$ & $\begin{array}{c}\text { Average } \\
\text { (mg/L) }\end{array}$ & $\begin{array}{r}\text { Median } \\
(\mathrm{mg} / \mathrm{L})\end{array}$ & $\begin{array}{c}\text { All } \\
\text { Samples } \\
\text { "ND" ? }\end{array}$ & $\begin{array}{l}\text { Mann- } \\
\text { Kendall }\end{array}$ & $\begin{array}{c}\text { Linear } \\
\text { Regression }\end{array}$ & Modeling & Empirical \\
\hline \multicolumn{12}{|c|}{ GROSS BETA ACTIVITY } \\
\hline & GW-694 & $\mathrm{T}$ & 5 & 5 & $1.7 \mathrm{E}+01$ & $1.4 \mathrm{E}+01$ & No & $\mathrm{S}$ & $\mathrm{s}$ & N/A & $\mathrm{N} / \mathrm{A}$ \\
\hline & GW-695 & $\mathrm{T}$ & 18 & 18 & $3.2 \mathrm{E}+01$ & $3.0 \mathrm{E}+01$ & No & 1 & 1 & N/A & $\mathrm{N} / \mathrm{A}$ \\
\hline & GW-703 & $\mathrm{T}$ & 18 & 18 & $4.2 \mathrm{E}+01$ & $4.6 \mathrm{E}+01$ & No & 1 & 1 & N/A & $\mathrm{N} / \mathrm{A}$ \\
\hline & GW-704 & $\mathrm{T}$ & 18 & 18 & $2.1 \mathrm{E}+01$ & $1.6 \mathrm{E}+01$ & No & 1 & 1 & N/A & N/A \\
\hline & GW-712 & $\mathrm{T}$ & 16 & 14 & $4.1 \mathrm{E}+00$ & $3.0 \mathrm{E}+00$ & No & NT & NT & N/A & N/A \\
\hline & GW-706 & $\mathrm{T}$ & 19 & 19 & $1.1 \mathrm{E}+02$ & $8.9 \mathrm{E}+01$ & No & NT & $\mathrm{PI}$ & N/A & $\mathrm{N} / \mathrm{A}$ \\
\hline & GW-710 & $\mathrm{T}$ & 4 & 4 & $2.4 \mathrm{E}+01$ & $1.4 \mathrm{E}+01$ & No & NT & NT & N/A & N/A \\
\hline & GW-642 & $\mathrm{T}$ & 2 & 2 & $4.6 \mathrm{E}+00$ & $4.6 \mathrm{E}+00$ & No & $\mathrm{N} / \mathrm{A}$ & N/A & N/A & $\mathrm{N} / \mathrm{A}$ \\
\hline & GW-078 & $T$ & 11 & 7 & $2.0 \mathrm{E}+00$ & 6.9E-01 & No & NT & NT & N/A & N/A \\
\hline & SS-8 & $\mathrm{T}$ & 14 & 7 & 8.0E-01 & 2.4E-01 & No & NT & NT & N/A & $\mathrm{N} / \mathrm{A}$ \\
\hline & GW-052 & $\mathrm{T}$ & 2 & 2 & $1.8 \mathrm{E}+01$ & $1.8 \mathrm{E}+01$ & No & $N / A$ & N/A & N/A & $\mathrm{N} / \mathrm{A}$ \\
\hline & GW-053 & $T$ & 11 & 11 & $2.8 \mathrm{E}+00$ & $2.7 \mathrm{E}+00$ & No & $\mathrm{s}$ & PD & N/A & $\mathrm{N} / \mathrm{A}$ \\
\hline & GW-056 & $\mathrm{T}$ & 8 & 8 & $1.1 \mathrm{E}+01$ & 1.1E+01 & No & $\mathrm{PI}$ & 1 & N/A & $\mathrm{N} / \mathrm{A}$ \\
\hline & GW-061 & $\mathrm{T}$ & 2 & 2 & $6.8 \mathrm{E}+01$ & $6.8 \mathrm{E}+01$ & No & $N / A$ & N/A & N/A & $\mathrm{N} / \mathrm{A}$ \\
\hline & SS-7 & $T$ & 13 & 9 & $4.6 \mathrm{E}+00$ & 4.4E+00 & No & $\mathrm{PI}$ & NT & N/A & N/A \\
\hline & GW-069 & $\mathrm{T}$ & 5 & 5 & $6.0 \mathrm{E}+00$ & $6.5 \mathrm{E}+00$ & No & NT & $\mathrm{PI}$ & N/A & $\mathrm{N} / \mathrm{A}$ \\
\hline & GW-072 & $T$ & 2 & 2 & $2.0 \mathrm{E}+00$ & $2.0 \mathrm{E}+00$ & No & $\mathrm{N} / \mathrm{A}$ & N/A & N/A & $\mathrm{N} / \mathrm{A}$ \\
\hline & GW-287 & $\mathrm{T}$ & 9 & 9 & 3.7E+00 & $3.1 \mathrm{E}+00$ & No & $\mathrm{D}$ & $\mathrm{D}$ & N/A & $\mathrm{N} / \mathrm{A}$ \\
\hline & GW-077 & $\mathrm{T}$ & 11 & 11 & $5.7 \mathrm{E}+00$ & $3.0 \mathrm{E}+00$ & No & $\mathrm{PD}$ & D & N/A & $\mathrm{N} / \mathrm{A}$ \\
\hline & GW-711 & $T$ & 1 & 1 & $1.1 \mathrm{E}+01$ & $1.1 \mathrm{E}+01$ & No & $\mathrm{N} / \mathrm{A}$ & $\mathrm{N} / \mathrm{A}$ & N/A & $\mathrm{N} / \mathrm{A}$ \\
\hline & GW-095 & $\mathrm{T}$ & 2 & 2 & $3.5 \mathrm{E}+00$ & $3.5 \mathrm{E}+00$ & No & $\mathrm{N} / \mathrm{A}$ & $N / A$ & N/A & $\mathrm{N} / \mathrm{A}$ \\
\hline & SS-5 & $\mathrm{T}$ & 19 & 19 & $3.6 \mathrm{E}+01$ & $3.0 \mathrm{E}+01$ & No & NT & NT & N/A & $\mathrm{N} / \mathrm{A}$ \\
\hline & GW-242 & $\mathrm{T}$ & 3 & 3 & $1.8 \mathrm{E}+01$ & $7.4 \mathrm{E}+00$ & No & $N / A$ & $N / A$ & $N / A$ & N/A \\
\hline & GW-237 & $\mathrm{T}$ & 2 & 2 & $4.7 \mathrm{E}+00$ & $4.7 \mathrm{E}+00$ & No & $\mathrm{N} / \mathrm{A}$ & $\mathrm{N} / \mathrm{A}$ & N/A & $\mathrm{N} / \mathrm{A}$ \\
\hline & SS-6W & $\mathrm{T}$ & 5 & 5 & $1.9 \mathrm{E}+01$ & $1.2 \mathrm{E}+01$ & No & $\mathrm{s}$ & PD & N/A & $\mathrm{N} / \mathrm{A}$ \\
\hline & SS-6_6 & $T$ & 12 & 11 & $9.1 \mathrm{E}+00$ & $4.5 \mathrm{E}+00$ & No & NT & NT & N/A & $\mathrm{N} / \mathrm{A}$ \\
\hline & GW-079 & $\mathrm{T}$ & 13 & 12 & $2.8 \mathrm{E}+00$ & $1.8 \mathrm{E}+00$ & No & $\mathrm{S}$ & s & N/A & $\mathrm{N} / \mathrm{A}$ \\
\hline & GW-091 & $\mathrm{T}$ & 2 & 2 & $3.5 \mathrm{E}+00$ & $3.5 \mathrm{E}+00$ & No & $N / A$ & N/A & N/A & $\mathrm{N} / \mathrm{A}$ \\
\hline & SS-6E & $T$ & 7 & 7 & $1.4 \mathrm{E}+01$ & $9.2 \mathrm{E}+00$ & No & NT & NT & N/A & $\mathrm{N} / \mathrm{A}$ \\
\hline & GW-080 & $\mathrm{T}$ & 13 & 10 & $6.1 \mathrm{E}+00$ & $1.3 \mathrm{E}+00$ & No & NT & NT & $N / A$ & $\mathrm{~N} / \mathrm{A}$ \\
\hline & GW-126 & $\mathrm{T}$ & 1 & 1 & $7.8 \mathrm{E}+00$ & $7.8 \mathrm{E}+00$ & No & $N / A$ & $N / A$ & N/A & $\mathrm{N} / \mathrm{A}$ \\
\hline \multicolumn{12}{|l|}{ NITRATE } \\
\hline & GW-071 & $\mathrm{s}$ & 4 & 1 & $2.4 \mathrm{E}-02$ & $2.0 \mathrm{E}-02$ & No & $\mathrm{s}$ & $\mathrm{S}$ & $\mathrm{N} / \mathrm{A}$ & $\mathrm{N} / \mathrm{A}$ \\
\hline
\end{tabular}


Project: Y-12 Security Complex

Location: Bear Creek Burial Grounds
User Name: MV

State: Tennessee

\begin{tabular}{|c|c|c|c|c|c|c|c|c|c|c|c|}
\hline Constituent & Well & $\begin{array}{c}\text { Sourcel } \\
\text { Tail }\end{array}$ & $\begin{array}{c}\text { Number } \\
\text { of } \\
\text { Samples }\end{array}$ & $\begin{array}{c}\text { Number } \\
\text { of } \\
\text { Detects }\end{array}$ & $\begin{array}{c}\text { Average } \\
\text { (mg/L) }\end{array}$ & $\begin{array}{r}\text { Median } \\
(\mathrm{mg} / \mathrm{L})\end{array}$ & $\begin{array}{c}\text { All } \\
\text { Samples } \\
\text { "ND" ? }\end{array}$ & $\begin{array}{l}\text { Mann- } \\
\text { Kendall }\end{array}$ & $\begin{array}{c}\text { Linear } \\
\text { Regression }\end{array}$ & Modeling & Empirical \\
\hline \multicolumn{12}{|l|}{ NITRATE } \\
\hline & GW-626 & S & 2 & 1 & $2.5 \mathrm{E}-02$ & $2.5 \mathrm{E}-02$ & No & $\mathrm{N} / \mathrm{A}$ & $\mathrm{N} / \mathrm{A}$ & $\mathrm{N} / \mathrm{A}$ & N/A \\
\hline & GW-082 & s & 9 & 2 & 3.3E-02 & 2.0E-02 & No & $\mathrm{s}$ & s & N/A & N/A \\
\hline & GW-046 & s & 2 & 2 & $7.2 \mathrm{E}-01$ & $7.2 \mathrm{E}-01$ & No & $\mathrm{N} / \mathrm{A}$ & $\mathrm{N} / \mathrm{A}$ & $N / A$ & $N / A$ \\
\hline & GW-014 & s & 2 & 0 & $2.0 \mathrm{E}-02$ & 2.0E-02 & Yes & N/A & $\mathrm{N} / \mathrm{A}$ & N/A & N/A \\
\hline & GW-653 & $\mathrm{T}$ & 17 & 0 & $2.0 \mathrm{E}-02$ & 2.0E-02 & Yes & s & s & N/A & N/A \\
\hline & GW-694 & $\mathrm{T}$ & 4 & 4 & $6.3 \mathrm{E}+00$ & $4.2 \mathrm{E}+00$ & No & s & s & $N / A$ & $N / A$ \\
\hline & GW-056 & $\mathrm{T}$ & 10 & 9 & 3.4E-01 & 3.2E-01 & No & 1 & I & N/A & $N / A$ \\
\hline & GW-289 & $\mathrm{T}$ & 2 & 2 & $2.6 \mathrm{E}-01$ & 2.6E-01 & No & N/A & N/A & N/A & N/A \\
\hline & GW-072 & $\mathrm{T}$ & 2 & 1 & $3.2 \mathrm{E}-02$ & $3.2 \mathrm{E}-02$ & No & $N / A$ & $N / A$ & $N / A$ & $N / A$ \\
\hline & GW-713 & $\mathrm{T}$ & 6 & 3 & $2.1 \mathrm{E}-01$ & $6.0 \mathrm{E}-02$ & No & NT & NT & $N / A$ & $N / A$ \\
\hline & SS-7 & $\mathrm{T}$ & 1 & 1 & $3.1 \mathrm{E}-01$ & 3.1E-01 & No & $\mathrm{N} / \mathrm{A}$ & $\mathrm{N} / \mathrm{A}$ & N/A & N/A \\
\hline & GW-061 & $\mathrm{T}$ & 2 & 2 & $1.1 \mathrm{E}+01$ & $1.1 \mathrm{E}+01$ & No & $N / A$ & $N / A$ & $N / A$ & $N / A$ \\
\hline & GW-684 & $\mathrm{T}$ & 13 & 13 & $5.7 \mathrm{E}+00$ & $5.2 \mathrm{E}+00$ & No & NT & NT & $N / A$ & $N / A$ \\
\hline & GW-291 & $\mathrm{T}$ & 2 & 2 & $2.4 \mathrm{E}-01$ & 2.4E-01 & No & N/A & N/A & N/A & N/A \\
\hline & GW-683 & $\mathrm{T}$ & 12 & 12 & $7.3 E+00$ & $7.4 \mathrm{E}+00$ & No & $\mathrm{s}$ & $\mathrm{s}$ & $N / A$ & N/A \\
\hline & GW-069 & $\mathrm{T}$ & 6 & 1 & $2.2 \mathrm{E}-02$ & 2.0E-02 & No & NT & $\mathrm{PI}$ & $N / A$ & $N / A$ \\
\hline & GW-091 & $\mathrm{T}$ & 2 & 1 & $2.4 \mathrm{E}-02$ & 2.4E-02 & No & N/A & $\mathrm{N} / \mathrm{A}$ & N/A & $N / A$ \\
\hline & GW-372 & $\mathrm{T}$ & 4 & 0 & 2.0E-02 & 2.0E-02 & Yes & $\mathrm{s}$ & $\mathrm{s}$ & N/A & $N / A$ \\
\hline & GW-685 & $\mathrm{T}$ & 10 & 10 & $2.8 \mathrm{E}+00$ & $2.5 \mathrm{E}+00$ & No & S & s & $N / A$ & $N / A$ \\
\hline & GW-080 & $\mathrm{T}$ & 4 & 0 & 2.0E-02 & 2.0E-02 & Yes & $\mathrm{s}$ & s & $\mathrm{N} / \mathrm{A}$ & $N / A$ \\
\hline & SS-6E & $\mathrm{T}$ & 6 & 6 & $1.6 \mathrm{E}+00$ & $1.6 \mathrm{E}+00$ & No & NT & s & $N / A$ & $N / A$ \\
\hline & GW-621 & $\mathrm{T}$ & 9 & 9 & $3.2 \mathrm{E}+00$ & $2.4 \mathrm{E}+00$ & No & S & D & $N / A$ & $N / A$ \\
\hline & GW-642 & $\mathrm{T}$ & 4 & 1 & $3.2 \mathrm{E}-02$ & 2.0E-02 & No & NT & NT & N/A & $N / A$ \\
\hline & GW-714 & $\mathrm{T}$ & 6 & 4 & $1.8 \mathrm{E}+00$ & $2.0 \mathrm{E}+00$ & No & S & D & $N / A$ & $N / A$ \\
\hline & SS-6W & $\mathrm{T}$ & 1 & 1 & $3.4 \mathrm{E}+00$ & $3.4 \mathrm{E}+00$ & No & $N / A$ & $N / A$ & $N / A$ & $N / A$ \\
\hline & GW-288 & $\mathrm{T}$ & 2 & 1 & $5.2 \mathrm{E}-02$ & 5.2E-02 & No & $\mathrm{N} / \mathrm{A}$ & $\mathrm{N} / \mathrm{A}$ & N/A & $N / A$ \\
\hline & GW-079 & $\mathrm{T}$ & 4 & 0 & $2.0 \mathrm{E}-02$ & 2.0E-02 & Yes & $\mathrm{s}$ & $\mathrm{s}$ & N/A & N/A \\
\hline & GW-237 & $\mathrm{T}$ & 2 & 2 & $1.8 \mathrm{E}-01$ & $1.8 \mathrm{E}-01$ & No & $N / A$ & $N / A$ & $N / A$ & $N / A$ \\
\hline & GW-715 & $\mathrm{T}$ & 6 & 6 & $1.1 \mathrm{E}+00$ & 5.5E-01 & No & NT & NT & N/A & $N / A$ \\
\hline & GW-627 & $\mathrm{T}$ & 17 & 0 & $2.0 \mathrm{E}-02$ & 2.0E-02 & Yes & $\mathrm{s}$ & $\mathrm{s}$ & N/A & N/A \\
\hline & SS-4 & $\mathrm{T}$ & 18 & 18 & $2.6 \mathrm{E}+01$ & $2.2 \mathrm{E}+01$ & No & NT & NT & N/A & $N / A$ \\
\hline & GW-257 & $\mathrm{T}$ & 2 & 2 & 2.6E-01 & 2.6E-01 & No & $N / A$ & $\mathrm{~N} / \mathrm{A}$ & N/A & $N / A$ \\
\hline & GW-710 & $\mathrm{T}$ & 6 & 0 & $2.0 \mathrm{E}-02$ & 2.0E-02 & Yes & $\mathrm{s}$ & $\mathrm{s}$ & N/A & N/A \\
\hline & GW-242 & $\mathrm{T}$ & 1 & 0 & $2.0 \mathrm{E}-02$ & $2.0 \mathrm{E}-02$ & Yes & $N / A$ & $N / A$ & $N / A$ & $N / A$ \\
\hline
\end{tabular}


Project: Y-12 Security Complex

Location: Bear Creek Burial Grounds
User Name: MV

State: Tennessee

\begin{tabular}{|c|c|c|c|c|c|c|c|c|c|c|c|}
\hline Constituent & Well & $\begin{array}{c}\text { Sourcel } \\
\text { Tail }\end{array}$ & $\begin{array}{c}\text { Number } \\
\text { of } \\
\text { Samples }\end{array}$ & $\begin{array}{c}\text { Number } \\
\text { of } \\
\text { Detects }\end{array}$ & $\begin{array}{c}\text { Average } \\
\text { (mg/L) }\end{array}$ & $\begin{array}{c}\text { Median } \\
(\mathrm{mg} / \mathrm{L})\end{array}$ & $\begin{array}{c}\text { All } \\
\text { Samples } \\
\text { "ND" ? }\end{array}$ & $\begin{array}{l}\text { Mann- } \\
\text { Kendall }\end{array}$ & $\begin{array}{c}\text { Linear } \\
\text { Regression }\end{array}$ & Modeling & Empirical \\
\hline \multicolumn{12}{|l|}{ NITRATE } \\
\hline & SS-8 & $T$ & 1 & 1 & $2.8 \mathrm{E}-01$ & $2.8 \mathrm{E}-01$ & No & N/A & N/A & N/A & $\mathrm{N} / \mathrm{A}$ \\
\hline & GW-126 & $\mathrm{T}$ & 1 & 0 & 2.0E-02 & 2.0E-02 & Yes & N/A & N/A & N/A & $N / A$ \\
\hline & GW-706 & $\mathrm{T}$ & 17 & 17 & $3.3 \mathrm{E}+01$ & $2.7 \mathrm{E}+01$ & No & $\mathrm{s}$ & NT & N/A & $\mathrm{N} / \mathrm{A}$ \\
\hline & SS-5 & $\mathrm{T}$ & 18 & 18 & $8.4 \mathrm{E}+00$ & $7.9 \mathrm{E}+00$ & No & NT & S & N/A & N/A \\
\hline & GW-052 & $\mathrm{T}$ & 2 & 2 & $2.9 \mathrm{E}+00$ & $2.9 \mathrm{E}+00$ & No & N/A & N/A & N/A & N/A \\
\hline & GW-711 & $\mathrm{T}$ & 6 & 0 & $2.0 \mathrm{E}-02$ & 2.0E-02 & Yes & $\mathrm{s}$ & $\mathrm{s}$ & N/A & N/A \\
\hline & GW-704 & $\mathrm{T}$ & 17 & 16 & $1.2 \mathrm{E}+01$ & $1.5 \mathrm{E}+01$ & No & D & S & N/A & $N / A$ \\
\hline & GW-712 & $\mathrm{T}$ & 6 & 4 & $1.2 \mathrm{E}-01$ & 6.1E-02 & No & NT & NT & N/A & $\mathrm{N} / \mathrm{A}$ \\
\hline & GW-695 & $T$ & 17 & 17 & $9.6 \mathrm{E}+00$ & $9.9 \mathrm{E}+00$ & No & 1 & $\mathrm{PI}$ & N/A & N/A \\
\hline & GW-095 & $\mathrm{T}$ & 2 & 1 & $2.5 \mathrm{E}-02$ & 2.5E-02 & No & N/A & N/A & N/A & $N / A$ \\
\hline & GW-703 & $T$ & 17 & 17 & $1.6 \mathrm{E}+01$ & $1.8 \mathrm{E}+01$ & No & 1 & NT & N/A & N/A \\
\hline & GW-053 & $\mathrm{T}$ & 11 & 1 & 2.3E-02 & 2.0E-02 & No & s & $\mathrm{s}$ & N/A & N/A \\
\hline & SS-6_6 & $\mathrm{T}$ & 1 & 1 & $3.8 \mathrm{E}-01$ & 3.8E-01 & No & N/A & N/A & N/A & $N / A$ \\
\hline & GW-287 & $T$ & 9 & 5 & 7.7E-02 & 3.0E-02 & No & NT & NT & N/A & N/A \\
\hline \multicolumn{12}{|c|}{ TETRACHLOROETHYLENE(PCE) } \\
\hline & GW-626 & $\mathrm{S}$ & 4 & 4 & $3.1 \mathrm{E}-01$ & 1.7E-01 & No & NT & 1 & $\mathrm{~N} / \mathrm{A}$ & N/A \\
\hline & GW-624 & s & 2 & 2 & $3.8 \mathrm{E}-01$ & 3.8E-01 & No & N/A & $\mathrm{N} / \mathrm{A}$ & $\mathrm{N} / \mathrm{A}$ & N/A \\
\hline & GW-014 & s & 2 & 2 & 2.0E-02 & 2.0E-02 & No & N/A & N/A & N/A & $\mathrm{N} / \mathrm{A}$ \\
\hline & GW-071 & s & 4 & 4 & 4.0E-01 & 3.6E-01 & No & NT & NT & N/A & $\mathrm{N} / \mathrm{A}$ \\
\hline & GW-046 & s & 14 & 13 & $2.3 \mathrm{E}+00$ & $1.4 \mathrm{E}+00$ & No & NT & NT & N/A & $\mathrm{N} / \mathrm{A}$ \\
\hline & GW-082 & s & 13 & 1 & $5.8 \mathrm{E}-04$ & 5.0E-04 & No & NT & NT & N/A & $\mathrm{N} / \mathrm{A}$ \\
\hline & GW-091 & $\mathrm{T}$ & 2 & 0 & $5.0 \mathrm{E}-04$ & 5.0E-04 & Yes & N/A & N/A & N/A & $\mathrm{N} / \mathrm{A}$ \\
\hline & GW-237 & $\mathrm{T}$ & 2 & 0 & $5.0 \mathrm{E}-04$ & 5.0E-04 & Yes & N/A & $\mathrm{N} / \mathrm{A}$ & N/A & N/A \\
\hline & GW-126 & $\mathrm{T}$ & 1 & 0 & $5.0 \mathrm{E}-04$ & $5.0 \mathrm{E}-04$ & Yes & N/A & N/A & N/A & $N / A$ \\
\hline & GW-715 & $\mathrm{T}$ & 17 & 0 & $5.0 \mathrm{E}-04$ & $5.0 \mathrm{E}-04$ & Yes & $\mathrm{s}$ & $\mathrm{s}$ & N/A & N/A \\
\hline & GW-095 & $\mathrm{T}$ & 2 & 0 & $5.0 \mathrm{E}-04$ & 5.0E-04 & Yes & N/A & N/A & N/A & $\mathrm{N} / \mathrm{A}$ \\
\hline & SS-6E & $\mathrm{T}$ & 8 & 0 & $5.0 \mathrm{E}-04$ & $5.0 \mathrm{E}-04$ & Yes & s & s & N/A & $\mathrm{N} / \mathrm{A}$ \\
\hline & SS-4 & $\mathrm{T}$ & 18 & 0 & $5.0 \mathrm{E}-04$ & $5.0 \mathrm{E}-04$ & Yes & S & s & N/A & N/A \\
\hline & SS-5 & $T$ & 19 & 0 & $5.0 \mathrm{E}-04$ & 5.0E-04 & Yes & s & s & $\mathrm{N} / \mathrm{A}$ & $\mathrm{N} / \mathrm{A}$ \\
\hline & GW-287 & $\mathrm{T}$ & 10 & 6 & $1.9 \mathrm{E}-03$ & $1.5 \mathrm{E}-03$ & No & s & NT & N/A & $N / A$ \\
\hline & GW-079 & $\mathrm{T}$ & 18 & 0 & $5.0 \mathrm{E}-04$ & $5.0 \mathrm{E}-04$ & Yes & $\mathrm{s}$ & $\mathrm{s}$ & $\mathrm{N} / \mathrm{A}$ & $\mathrm{N} / \mathrm{A}$ \\
\hline & GW-289 & $T$ & 4 & 4 & $5.3 \mathrm{E}-01$ & 5.3E-01 & No & NT & I & $\mathrm{N} / \mathrm{A}$ & $N / A$ \\
\hline & GW-621 & $\mathrm{T}$ & 10 & 0 & $5.0 \mathrm{E}-04$ & $5.0 \mathrm{E}-04$ & Yes & $\mathrm{s}$ & s & N/A & $N / A$ \\
\hline
\end{tabular}


Project: Y-12 Security Complex

Location: Bear Creek Burial Grounds
User Name: MV

State: Tennessee

\begin{tabular}{|c|c|c|c|c|c|c|c|c|c|c|c|}
\hline Constituent & Well & $\begin{array}{l}\text { Sourcel } \\
\text { Tail }\end{array}$ & $\begin{array}{c}\text { Number } \\
\text { of } \\
\text { Samples }\end{array}$ & $\begin{array}{c}\text { Number } \\
\text { of } \\
\text { Detects }\end{array}$ & $\begin{array}{c}\text { Average } \\
\text { (mg/L) }\end{array}$ & $\begin{array}{r}\text { Median } \\
(\mathrm{mg} / \mathrm{L})\end{array}$ & $\begin{array}{c}\text { All } \\
\text { Samples } \\
\text { "ND" ? }\end{array}$ & $\begin{array}{l}\text { Mann- } \\
\text { Kendall }\end{array}$ & $\begin{array}{c}\text { Linear } \\
\text { Regression }\end{array}$ & Modeling & Empirical \\
\hline \multicolumn{12}{|c|}{ TETRACHLOROETHYLENE(PCE) } \\
\hline & GW-242 & $T$ & 3 & 0 & $5.0 \mathrm{E}-04$ & $5.0 \mathrm{E}-04$ & Yes & N/A & N/A & N/A & N/A \\
\hline & GW-372 & $\mathrm{T}$ & 4 & 0 & $5.0 \mathrm{E}-04$ & $5.0 \mathrm{E}-04$ & Yes & $\mathrm{s}$ & s & N/A & N/A \\
\hline & GW-257 & $\mathrm{T}$ & 2 & 2 & 2.1E-01 & $2.1 \mathrm{E}-01$ & No & $\mathrm{N} / \mathrm{A}$ & $\mathrm{N} / \mathrm{A}$ & $N / A$ & $N / A$ \\
\hline & GW-291 & $\mathrm{T}$ & 4 & 4 & 4.6E-01 & 4.1E-01 & No & S & s & N/A & N/A \\
\hline & SS-6_6 & $\mathrm{T}$ & 12 & 0 & $5.0 \mathrm{E}-04$ & $5.0 \mathrm{E}-04$ & Yes & s & s & N/A & N/A \\
\hline & GW-080 & $\mathrm{T}$ & 17 & 0 & $5.0 \mathrm{E}-04$ & $5.0 \mathrm{E}-04$ & Yes & s & s & $N / A$ & $N / A$ \\
\hline & GW-078 & $\mathrm{T}$ & 15 & 0 & $5.0 \mathrm{E}-04$ & 5.0E-04 & Yes & S & S & $N / A$ & $N / A$ \\
\hline & GW-288 & $\mathrm{T}$ & 2 & 2 & $2.2 \mathrm{E}-01$ & $2.2 \mathrm{E}-01$ & No & N/A & N/A & N/A & N/A \\
\hline & GW-712 & $\mathrm{T}$ & 18 & 0 & $5.0 \mathrm{E}-04$ & $5.0 \mathrm{E}-04$ & Yes & $\mathrm{s}$ & $\mathrm{s}$ & $N / A$ & $N / A$ \\
\hline & GW-061 & $\mathrm{T}$ & 2 & 0 & $5.0 \mathrm{E}-04$ & 5.0E-04 & Yes & $\mathrm{N} / \mathrm{A}$ & $N / A$ & $N / A$ & $N / A$ \\
\hline & GW-713 & $\mathrm{T}$ & 18 & 0 & $5.0 \mathrm{E}-04$ & $5.0 \mathrm{E}-04$ & Yes & s & $S$ & N/A & N/A \\
\hline & GW-685 & $\mathrm{T}$ & 11 & 0 & $5.0 \mathrm{E}-04$ & $5.0 \mathrm{E}-04$ & Yes & s & D & $N / A$ & $N / A$ \\
\hline & GW-072 & $\mathrm{T}$ & 2 & 0 & $5.0 \mathrm{E}-04$ & $5.0 \mathrm{E}-04$ & Yes & $N / A$ & $N / A$ & $N / A$ & $N / A$ \\
\hline & GW-056 & $\mathrm{T}$ & 11 & 1 & $9.1 \mathrm{E}-04$ & $5.0 \mathrm{E}-04$ & No & NT & D & N/A & N/A \\
\hline & GW-694 & $\mathrm{T}$ & 6 & 1 & $5.8 \mathrm{E}-04$ & 5.0E-04 & No & $\mathrm{s}$ & s & $N / A$ & N/A \\
\hline & SS-7 & $\mathrm{T}$ & 13 & 0 & $5.0 \mathrm{E}-04$ & $5.0 \mathrm{E}-04$ & Yes & S & s & $N / A$ & $N / A$ \\
\hline & GW-683 & $\mathrm{T}$ & 17 & 0 & $5.0 \mathrm{E}-04$ & $5.0 \mathrm{E}-04$ & Yes & s & s & N/A & $N / A$ \\
\hline & GW-695 & $\mathrm{T}$ & 18 & 0 & $5.0 \mathrm{E}-04$ & 5.0E-04 & Yes & s & s & $N / A$ & N/A \\
\hline & GW-684 & $\mathrm{T}$ & 18 & 0 & $5.0 \mathrm{E}-04$ & 5.0E-04 & Yes & S & s & $N / A$ & $N / A$ \\
\hline & GW-703 & $\mathrm{T}$ & 18 & 0 & $5.0 \mathrm{E}-04$ & $5.0 \mathrm{E}-04$ & Yes & $\mathrm{s}$ & $S$ & $\mathrm{~N} / \mathrm{A}$ & $N / A$ \\
\hline & GW-052 & $\mathrm{T}$ & 2 & 0 & $5.0 \mathrm{E}-04$ & $5.0 \mathrm{E}-04$ & Yes & $N / A$ & $N / A$ & $N / A$ & $N / A$ \\
\hline & GW-704 & $\mathrm{T}$ & 18 & 0 & $5.0 \mathrm{E}-04$ & $5.0 \mathrm{E}-04$ & Yes & $\mathrm{s}$ & $\mathrm{s}$ & $N / A$ & $N / A$ \\
\hline & GW-706 & $\mathrm{T}$ & 18 & 0 & $5.0 \mathrm{E}-04$ & $5.0 \mathrm{E}-04$ & Yes & s & s & N/A & $N / A$ \\
\hline & SS-8 & $\mathrm{T}$ & 14 & 0 & $5.0 \mathrm{E}-04$ & 5.0E-04 & Yes & s & 1 & $N / A$ & $N / A$ \\
\hline & GW-710 & $\mathrm{T}$ & 6 & 0 & $5.0 \mathrm{E}-04$ & $5.0 \mathrm{E}-04$ & Yes & s & s & $N / A$ & $N / A$ \\
\hline & GW-711 & $\mathrm{T}$ & 6 & 0 & $5.0 \mathrm{E}-04$ & $5.0 \mathrm{E}-04$ & Yes & s & $S$ & N/A & $N / A$ \\
\hline & GW-053 & $\mathrm{T}$ & 12 & 3 & $5.8 \mathrm{E}-04$ & $5.0 \mathrm{E}-04$ & No & s & s & N/A & N/A \\
\hline & GW-642 & $\mathrm{T}$ & 4 & 0 & $5.0 \mathrm{E}-04$ & 5.0E-04 & Yes & s & s & $N / A$ & $N / A$ \\
\hline & GW-629 & $\mathrm{T}$ & 4 & 1 & $4.8 \mathrm{E}-04$ & $5.0 \mathrm{E}-04$ & No & s & $S$ & N/A & $N / A$ \\
\hline & GW-077 & $\mathrm{T}$ & 15 & 1 & $5.0 \mathrm{E}-04$ & $5.0 \mathrm{E}-04$ & No & s & s & N/A & N/A \\
\hline & GW-653 & $\mathrm{T}$ & 18 & 17 & 4.1E-03 & $2.0 \mathrm{E}-03$ & No & 1 & 1 & $N / A$ & $N / A$ \\
\hline & GW-627 & $\mathrm{T}$ & 19 & 19 & 3.6E-01 & $3.5 \mathrm{E}-01$ & No & 1 & 1 & N/A & $N / A$ \\
\hline & GW-069 & $\mathrm{T}$ & 6 & 0 & $5.0 \mathrm{E}-04$ & $5.0 \mathrm{E}-04$ & Yes & s & s & N/A & N/A \\
\hline & GW-639 & $\mathrm{T}$ & 11 & 0 & $5.0 \mathrm{E}-04$ & $5.0 \mathrm{E}-04$ & Yes & s & D & $N / A$ & $N / A$ \\
\hline
\end{tabular}


Project: Y-12 Security Complex

Location: Bear Creek Burial Grounds
User Name: MV

State: Tennessee

\begin{tabular}{|c|c|c|c|c|c|c|c|c|c|c|c|}
\hline Constituent & Well & $\begin{array}{c}\text { Sourcel } \\
\text { Tail }\end{array}$ & $\begin{array}{c}\text { Number } \\
\text { of } \\
\text { Samples }\end{array}$ & $\begin{array}{c}\text { Number } \\
\text { of } \\
\text { Detects }\end{array}$ & $\begin{array}{c}\text { Average } \\
\text { (mg/L) }\end{array}$ & $\begin{array}{c}\text { Median } \\
(\mathrm{mg} / \mathrm{L})\end{array}$ & $\begin{array}{c}\text { All } \\
\text { Samples } \\
\text { "ND" ? }\end{array}$ & $\begin{array}{l}\text { Mann- } \\
\text { Kendall }\end{array}$ & $\begin{array}{c}\text { Linear } \\
\text { Regression }\end{array}$ & Modeling & Empirical \\
\hline \multicolumn{12}{|c|}{ TETRACHLOROETHYLENE(PCE) } \\
\hline & SS-6W & $T$ & 5 & 0 & $5.0 \mathrm{E}-04$ & $5.0 \mathrm{E}-04$ & Yes & S & 1 & N/A & N/A \\
\hline & GW-714 & $\mathrm{T}$ & 18 & 0 & $5.0 \mathrm{E}-04$ & 5.0E-04 & Yes & s & s & N/A & $N / A$ \\
\hline \multicolumn{12}{|c|}{ TRICHLOROETHYLENE (TCE) } \\
\hline & GW-046 & $\mathrm{S}$ & 14 & 14 & $2.0 \mathrm{E}+00$ & $1.6 \mathrm{E}+00$ & No & $\mathrm{s}$ & $\mathrm{S}$ & N/A & N/A \\
\hline & GW-071 & s & 4 & 4 & 8.7E-02 & 8.6E-02 & No & s & D & N/A & N/A \\
\hline & GW-624 & s & 2 & 2 & 7.2E-01 & 7.2E-01 & No & N/A & N/A & N/A & N/A \\
\hline & GW-082 & s & 13 & 0 & $5.0 \mathrm{E}-05$ & $5.0 \mathrm{E}-05$ & Yes & $\mathrm{s}$ & 1 & $\mathrm{~N} / \mathrm{A}$ & $N / A$ \\
\hline & GW-014 & S & 2 & 2 & $1.5 \mathrm{E}-01$ & 1.5E-01 & No & N/A & N/A & N/A & $N / A$ \\
\hline & GW-626 & s & 4 & 4 & 2.3E-01 & 1.0E-01 & No & NT & 1 & N/A & N/A \\
\hline & GW-095 & $\mathrm{T}$ & 2 & 0 & $5.0 \mathrm{E}-05$ & 5.0E-05 & Yes & N/A & N/A & N/A & $N / A$ \\
\hline & GW-078 & $T$ & 15 & 0 & $5.0 \mathrm{E}-05$ & 5.0E-05 & Yes & $\mathrm{s}$ & $\mathrm{s}$ & N/A & N/A \\
\hline & SS-8 & $T$ & 14 & 0 & $5.0 \mathrm{E}-05$ & 5.0E-05 & Yes & $\mathrm{s}$ & s & N/A & N/A \\
\hline & GW-126 & $\mathrm{T}$ & 1 & 0 & $5.0 \mathrm{E}-05$ & 5.0E-05 & Yes & N/A & N/A & N/A & $\mathrm{N} / \mathrm{A}$ \\
\hline & GW-077 & $\mathrm{T}$ & 15 & 0 & $5.0 \mathrm{E}-05$ & 5.0E-05 & Yes & $\mathrm{s}$ & $\mathrm{s}$ & N/A & N/A \\
\hline & GW-052 & $\mathrm{T}$ & 2 & 0 & $5.0 \mathrm{E}-05$ & 5.0E-05 & Yes & N/A & N/A & N/A & $N / A$ \\
\hline & GW-072 & $\mathrm{T}$ & 2 & 0 & $5.0 \mathrm{E}-05$ & 5.0E-05 & Yes & N/A & N/A & N/A & $N / A$ \\
\hline & GW-056 & $\mathrm{T}$ & 11 & 1 & $1.4 \mathrm{E}-04$ & 5.0E-05 & No & NT & $\mathrm{D}$ & N/A & N/A \\
\hline & GW-091 & $\mathrm{T}$ & 2 & 0 & $5.0 \mathrm{E}-05$ & 5.0E-05 & Yes & N/A & N/A & N/A & $N / A$ \\
\hline & GW-053 & $\mathrm{T}$ & 12 & 12 & $1.4 \mathrm{E}-03$ & 1.5E-03 & No & 1 & 1 & N/A & $N / A$ \\
\hline & GW-079 & $T$ & 18 & 0 & $5.0 \mathrm{E}-05$ & 5.0E-05 & Yes & s & s & N/A & N/A \\
\hline & SS-6W & $\mathrm{T}$ & 5 & 0 & $5.0 \mathrm{E}-05$ & 5.0E-05 & Yes & s & 1 & N/A & $N / A$ \\
\hline & SS-6E & $\mathrm{T}$ & 8 & 0 & 5.0E-05 & 5.0E-05 & Yes & $\mathrm{s}$ & s & N/A & $N / A$ \\
\hline & SS-6_6 & $\mathrm{T}$ & 12 & 0 & $5.0 \mathrm{E}-05$ & 5.0E-05 & Yes & $\mathrm{s}$ & s & N/A & N/A \\
\hline & GW-069 & $\mathrm{T}$ & 6 & 0 & $5.0 \mathrm{E}-05$ & 5.0E-05 & Yes & $\mathrm{s}$ & s & N/A & $N / A$ \\
\hline & GW-061 & $\mathrm{T}$ & 2 & 0 & $5.0 \mathrm{E}-05$ & $5.0 \mathrm{E}-05$ & Yes & N/A & N/A & N/A & N/A \\
\hline & GW-080 & $\mathrm{T}$ & 17 & 0 & $5.0 \mathrm{E}-05$ & 5.0E-05 & Yes & $\mathrm{s}$ & 1 & N/A & $\mathrm{N} / \mathrm{A}$ \\
\hline & SS-7 & $\mathrm{T}$ & 13 & 0 & 5.0E-05 & 5.0E-05 & Yes & s & 1 & N/A & $N / A$ \\
\hline & GW-684 & $\mathrm{T}$ & 18 & 0 & $5.0 \mathrm{E}-05$ & $5.0 \mathrm{E}-05$ & Yes & $\mathrm{s}$ & s & N/A & $N / A$ \\
\hline & GW-621 & $T$ & 10 & 1 & $9.5 \mathrm{E}-05$ & 5.0E-05 & No & NT & NT & $\mathrm{N} / \mathrm{A}$ & $N / A$ \\
\hline & GW-715 & $\mathrm{T}$ & 17 & 0 & 5.0E-05 & 5.0E-05 & Yes & $\mathrm{s}$ & $D$ & N/A & $N / A$ \\
\hline & GW-627 & $\mathrm{T}$ & 19 & 18 & $1.1 \mathrm{E}-01$ & $9.2 \mathrm{E}-02$ & No & 1 & 1 & N/A & $\mathrm{N} / \mathrm{A}$ \\
\hline & GW-714 & $\mathrm{T}$ & 18 & 0 & 5.0E-05 & 5.0E-05 & Yes & s & s & N/A & $N / A$ \\
\hline & SS-5 & $\mathrm{T}$ & 19 & 3 & 2.3E-04 & 5.0E-05 & No & NT & NT & N/A & $N / A$ \\
\hline
\end{tabular}


Project: Y-12 Security Complex

Location: Bear Creek Burial Grounds
User Name: MV

State: Tennessee

\begin{tabular}{|c|c|c|c|c|c|c|c|c|c|c|c|}
\hline Constituent & Well & $\begin{array}{c}\text { Sourcel } \\
\text { Tail }\end{array}$ & $\begin{array}{l}\text { Number } \\
\text { of } \\
\text { Samples }\end{array}$ & $\begin{array}{c}\text { Number } \\
\text { of } \\
\text { Detects }\end{array}$ & $\begin{array}{c}\text { Average } \\
\text { (mg/L) }\end{array}$ & $\begin{array}{r}\text { Median } \\
(\mathrm{mg} / \mathrm{L})\end{array}$ & $\begin{array}{c}\text { All } \\
\text { Samples } \\
\text { "ND" ? }\end{array}$ & $\begin{array}{l}\text { Mann- } \\
\text { Kendall }\end{array}$ & $\begin{array}{c}\text { Linear } \\
\text { Regression }\end{array}$ & Modeling & Empirical \\
\hline \multicolumn{12}{|c|}{ TRICHLOROETHYLENE (TCE) } \\
\hline & GW-639 & $T$ & 11 & 0 & $5.0 \mathrm{E}-05$ & $5.0 \mathrm{E}-05$ & Yes & $\mathrm{s}$ & $\mathrm{D}$ & N/A & N/A \\
\hline & GW-642 & $\mathrm{T}$ & 4 & 0 & $5.0 \mathrm{E}-05$ & 5.0E-05 & Yes & s & s & N/A & N/A \\
\hline & GW-653 & $\mathrm{T}$ & 18 & 13 & $2.4 \mathrm{E}-03$ & 1.0E-03 & No & 1 & $\mathrm{PI}$ & N/A & N/A \\
\hline & GW-372 & $\mathrm{T}$ & 4 & 0 & $5.0 \mathrm{E}-05$ & 5.0E-05 & Yes & s & s & N/A & N/A \\
\hline & GW-683 & $\mathrm{T}$ & 17 & 0 & $5.0 \mathrm{E}-05$ & 5.0E-05 & Yes & $\mathrm{s}$ & 1 & N/A & N/A \\
\hline & GW-629 & $\mathrm{T}$ & 4 & 3 & $1.6 \mathrm{E}-03$ & 2.0E-03 & No & NT & NT & N/A & $N / A$ \\
\hline & GW-685 & $\mathrm{T}$ & 11 & 1 & $9.1 \mathrm{E}-05$ & 5.0E-05 & No & NT & NT & N/A & $N / A$ \\
\hline & GW-694 & $T$ & 6 & 5 & $1.8 \mathrm{E}-03$ & $1.5 \mathrm{E}-03$ & No & $\mathrm{s}$ & $P D$ & N/A & $\mathrm{N} / \mathrm{A}$ \\
\hline & GW-712 & $T$ & 18 & 0 & $5.0 \mathrm{E}-05$ & 5.0E-05 & Yes & S & S & N/A & $N / A$ \\
\hline & GW-695 & $\mathrm{T}$ & 18 & 18 & $5.1 \mathrm{E}-03$ & 5.5E-03 & No & s & NT & N/A & $N / A$ \\
\hline & GW-703 & $\mathrm{T}$ & 18 & 17 & $1.5 \mathrm{E}-02$ & 1.6E-02 & No & s & $\mathrm{PI}$ & N/A & $\mathrm{N} / \mathrm{A}$ \\
\hline & GW-704 & $\mathrm{T}$ & 18 & 17 & $5.5 \mathrm{E}-02$ & 5.3E-02 & No & D & NT & N/A & $N / A$ \\
\hline & GW-706 & $\mathrm{T}$ & 18 & 17 & $1.1 \mathrm{E}-02$ & 1.1E-02 & No & NT & $\mathrm{PI}$ & N/A & N/A \\
\hline & GW-711 & $\mathrm{T}$ & 6 & 0 & $5.0 \mathrm{E}-05$ & 5.0E-05 & Yes & $\mathrm{s}$ & s & N/A & $\mathrm{N} / \mathrm{A}$ \\
\hline & GW-710 & $T$ & 6 & 0 & $5.0 \mathrm{E}-05$ & 5.0E-05 & Yes & s & s & N/A & $N / A$ \\
\hline & GW-713 & $\mathrm{T}$ & 18 & 0 & $5.0 \mathrm{E}-05$ & $5.0 \mathrm{E}-05$ & Yes & $\mathrm{s}$ & s & N/A & $N / A$ \\
\hline & GW-288 & $\mathrm{T}$ & 2 & 2 & $1.3 \mathrm{E}-02$ & 1.3E-02 & No & N/A & N/A & N/A & $\mathrm{N} / \mathrm{A}$ \\
\hline & GW-237 & $\mathrm{T}$ & 2 & 0 & $5.0 \mathrm{E}-05$ & 5.0E-05 & Yes & N/A & N/A & N/A & $N / A$ \\
\hline & GW-289 & $\mathrm{T}$ & 4 & 4 & $9.6 \mathrm{E}-03$ & $1.0 \mathrm{E}-02$ & No & 1 & $\mathrm{PI}$ & $\mathrm{N} / \mathrm{A}$ & $N / A$ \\
\hline & GW-291 & $T$ & 4 & 4 & $5.1 \mathrm{E}-02$ & 4.7E-02 & No & D & PD & N/A & N/A \\
\hline & GW-257 & $T$ & 2 & 0 & $5.0 \mathrm{E}-05$ & 5.0E-05 & Yes & N/A & N/A & N/A & N/A \\
\hline & GW-287 & $\mathrm{T}$ & 10 & 0 & $5.0 \mathrm{E}-05$ & $5.0 \mathrm{E}-05$ & Yes & $\mathrm{s}$ & $\mathrm{s}$ & $\mathrm{N} / \mathrm{A}$ & $N / A$ \\
\hline & SS-4 & $T$ & 18 & 18 & $8.1 \mathrm{E}-03$ & 7.5E-03 & No & NT & NT & $\mathrm{N} / \mathrm{A}$ & N/A \\
\hline & GW-242 & $\mathrm{T}$ & 3 & 2 & $6.8 \mathrm{E}-04$ & 5.0E-04 & No & N/A & $\mathrm{N} / \mathrm{A}$ & N/A & N/A \\
\hline \multicolumn{12}{|c|}{ VINYL CHLORIDE } \\
\hline & GW-046 & $\mathrm{s}$ & 14 & 14 & $5.5 \mathrm{E}-01$ & $5.2 \mathrm{E}-01$ & No & $\mathrm{s}$ & $\mathrm{S}$ & $\mathrm{N} / \mathrm{A}$ & $\mathrm{N} / \mathrm{A}$ \\
\hline & GW-082 & s & 13 & 13 & $1.6 \mathrm{E}-01$ & $1.5 \mathrm{E}-01$ & No & 1 & 1 & N/A & N/A \\
\hline & GW-624 & s & 2 & 2 & 4.6E-01 & 4.6E-01 & No & N/A & N/A & N/A & $\mathrm{N} / \mathrm{A}$ \\
\hline & GW-071 & s & 4 & 4 & $1.5 \mathrm{E}-03$ & 1.0E-03 & No & NT & NT & N/A & N/A \\
\hline & GW-626 & s & 4 & 2 & $3.1 \mathrm{E}-01$ & 2.1E-02 & No & NT & $\mathrm{PI}$ & N/A & $N / A$ \\
\hline & GW-014 & s & 2 & 2 & $8.1 \mathrm{E}-02$ & 8.1E-02 & No & N/A & N/A & N/A & $N / A$ \\
\hline & GW-685 & $T$ & 11 & 0 & $5.0 \mathrm{E}-04$ & 5.0E-04 & Yes & $\mathrm{s}$ & $\mathrm{D}$ & N/A & N/A \\
\hline & GW-257 & $\mathrm{T}$ & 2 & 0 & $5.0 \mathrm{E}-04$ & $5.0 \mathrm{E}-04$ & Yes & N/A & $\mathrm{N} / \mathrm{A}$ & $\mathrm{N} / \mathrm{A}$ & $N / A$ \\
\hline
\end{tabular}


Project: Y-12 Security Complex

Location: Bear Creek Burial Grounds
User Name: MV

State: Tennessee

\begin{tabular}{|c|c|c|c|c|c|c|c|c|c|c|c|}
\hline Constituent & Well & $\begin{array}{l}\text { Sourcel } \\
\text { Tail }\end{array}$ & $\begin{array}{c}\text { Number } \\
\text { of } \\
\text { Samples }\end{array}$ & $\begin{array}{c}\text { Number } \\
\text { of } \\
\text { Detects }\end{array}$ & $\begin{array}{c}\text { Average } \\
\text { (mg/L) }\end{array}$ & $\begin{array}{r}\text { Median } \\
(\mathrm{mg} / \mathrm{L})\end{array}$ & $\begin{array}{c}\text { All } \\
\text { Samples } \\
\text { "ND" ? }\end{array}$ & $\begin{array}{l}\text { Mann- } \\
\text { Kendall }\end{array}$ & $\begin{array}{c}\text { Linear } \\
\text { Regression }\end{array}$ & Modeling & Empirical \\
\hline \multicolumn{12}{|c|}{ VINYL CHLORIDE } \\
\hline & GW-053 & $T$ & 12 & 12 & $2.4 \mathrm{E}-03$ & $2.5 \mathrm{E}-03$ & No & PD & PD & N/A & N/A \\
\hline & GW-056 & $\mathrm{T}$ & 11 & 0 & $5.0 \mathrm{E}-04$ & $5.0 \mathrm{E}-04$ & Yes & s & $\mathrm{D}$ & N/A & N/A \\
\hline & GW-694 & $\mathrm{T}$ & 6 & 0 & $5.0 \mathrm{E}-04$ & $5.0 \mathrm{E}-04$ & Yes & s & s & $N / A$ & $N / A$ \\
\hline & GW-684 & $\mathrm{T}$ & 18 & 0 & $5.0 \mathrm{E}-04$ & 5.0E-04 & Yes & S & s & N/A & N/A \\
\hline & GW-712 & $\mathrm{T}$ & 18 & 0 & $5.0 \mathrm{E}-04$ & $5.0 \mathrm{E}-04$ & Yes & $\mathrm{s}$ & $\mathrm{s}$ & N/A & N/A \\
\hline & GW-242 & $\mathrm{T}$ & 3 & 3 & 3.7E-02 & $4.2 \mathrm{E}-02$ & No & $N / A$ & $N / A$ & $N / A$ & $N / A$ \\
\hline & GW-095 & $\mathrm{T}$ & 2 & 0 & $5.0 \mathrm{E}-04$ & 5.0E-04 & Yes & $\mathrm{N} / \mathrm{A}$ & $\mathrm{N} / \mathrm{A}$ & N/A & $N / A$ \\
\hline & SS-4 & $\mathrm{T}$ & 18 & 0 & $5.0 \mathrm{E}-04$ & $5.0 \mathrm{E}-04$ & Yes & $\mathrm{s}$ & s & N/A & N/A \\
\hline & GW-052 & $\mathrm{T}$ & 2 & 0 & $5.0 \mathrm{E}-04$ & $5.0 \mathrm{E}-04$ & Yes & $N / A$ & $N / A$ & $N / A$ & $N / A$ \\
\hline & SS-5 & $\mathrm{T}$ & 19 & 0 & $5.0 \mathrm{E}-04$ & $5.0 \mathrm{E}-04$ & Yes & s & $\mathrm{S}$ & $N / A$ & $N / A$ \\
\hline & GW-703 & $\mathrm{T}$ & 18 & 0 & $5.0 \mathrm{E}-04$ & $5.0 \mathrm{E}-04$ & Yes & s & s & N/A & N/A \\
\hline & SS-7 & $\mathrm{T}$ & 13 & 0 & $5.0 \mathrm{E}-04$ & $5.0 \mathrm{E}-04$ & Yes & s & s & $N / A$ & $N / A$ \\
\hline & GW-704 & $\mathrm{T}$ & 18 & 0 & $5.0 \mathrm{E}-04$ & $5.0 \mathrm{E}-04$ & Yes & S & s & $N / A$ & $N / A$ \\
\hline & GW-711 & $\mathrm{T}$ & 6 & 0 & $5.0 \mathrm{E}-04$ & $5.0 \mathrm{E}-04$ & Yes & s & s & N/A & N/A \\
\hline & GW-237 & $\mathrm{T}$ & 2 & 0 & $5.0 \mathrm{E}-04$ & 5.0E-04 & Yes & $N / A$ & $N / A$ & $N / A$ & N/A \\
\hline & GW-706 & $\mathrm{T}$ & 18 & 0 & $5.0 \mathrm{E}-04$ & $5.0 \mathrm{E}-04$ & Yes & $\mathrm{s}$ & $\mathrm{s}$ & $N / A$ & $N / A$ \\
\hline & GW-126 & $\mathrm{T}$ & 1 & 0 & $5.0 \mathrm{E}-04$ & $5.0 \mathrm{E}-04$ & Yes & $\mathrm{N} / \mathrm{A}$ & $\mathrm{N} / \mathrm{A}$ & N/A & $N / A$ \\
\hline & GW-710 & $\mathrm{T}$ & 6 & 0 & $5.0 \mathrm{E}-04$ & 5.0E-04 & Yes & $\mathrm{s}$ & $\mathrm{s}$ & $N / A$ & N/A \\
\hline & GW-695 & $\mathrm{T}$ & 18 & 0 & $5.0 \mathrm{E}-04$ & 5.0E-04 & Yes & S & s & $N / A$ & $N / A$ \\
\hline & SS-6E & $\mathrm{T}$ & 8 & 0 & $5.0 \mathrm{E}-04$ & $5.0 \mathrm{E}-04$ & Yes & s & s & $\mathrm{N} / \mathrm{A}$ & $N / A$ \\
\hline & GW-291 & $\mathrm{T}$ & 4 & 0 & $5.0 \mathrm{E}-04$ & $5.0 \mathrm{E}-04$ & Yes & s & s & $N / A$ & $N / A$ \\
\hline & GW-080 & $\mathrm{T}$ & 17 & 0 & $5.0 \mathrm{E}-04$ & 5.0E-04 & Yes & s & s & $N / A$ & $N / A$ \\
\hline & GW-372 & $\mathrm{T}$ & 4 & 0 & $5.0 \mathrm{E}-04$ & $5.0 \mathrm{E}-04$ & Yes & s & s & N/A & $N / A$ \\
\hline & GW-079 & $\mathrm{T}$ & 18 & 0 & $5.0 \mathrm{E}-04$ & $5.0 \mathrm{E}-04$ & Yes & s & s & $N / A$ & N/A \\
\hline & GW-715 & $\mathrm{T}$ & 17 & 0 & $5.0 \mathrm{E}-04$ & $5.0 \mathrm{E}-04$ & Yes & s & s & $N / A$ & $N / A$ \\
\hline & SS-6_6 & $\mathrm{T}$ & 12 & 0 & $5.0 \mathrm{E}-04$ & $5.0 \mathrm{E}-04$ & Yes & s & $S$ & N/A & $N / A$ \\
\hline & GW-621 & $\mathrm{T}$ & 10 & 0 & $5.0 \mathrm{E}-04$ & $5.0 \mathrm{E}-04$ & Yes & $\mathrm{s}$ & s & N/A & N/A \\
\hline & GW-289 & $\mathrm{T}$ & 4 & 2 & $1.0 \mathrm{E}-03$ & 7.5E-04 & No & NT & 1 & $N / A$ & $N / A$ \\
\hline & GW-078 & $\mathrm{T}$ & 15 & 0 & $5.0 \mathrm{E}-04$ & $5.0 \mathrm{E}-04$ & Yes & $\mathrm{s}$ & s & N/A & $N / A$ \\
\hline & SS-8 & $\mathrm{T}$ & 14 & 0 & $5.0 \mathrm{E}-04$ & $5.0 \mathrm{E}-04$ & Yes & $\mathrm{s}$ & 1 & N/A & N/A \\
\hline & GW-714 & $\mathrm{T}$ & 18 & 0 & $5.0 \mathrm{E}-04$ & 5.0E-04 & Yes & $\mathrm{s}$ & s & $N / A$ & $N / A$ \\
\hline & GW-061 & $\mathrm{T}$ & 2 & 0 & $5.0 \mathrm{E}-04$ & $5.0 \mathrm{E}-04$ & Yes & $N / A$ & $\mathrm{~N} / \mathrm{A}$ & N/A & $N / A$ \\
\hline & GW-627 & $\mathrm{T}$ & 19 & 16 & $1.1 \mathrm{E}-02$ & 1.1E-02 & No & 1 & 1 & N/A & N/A \\
\hline & GW-683 & $\mathrm{T}$ & 17 & 0 & $5.0 \mathrm{E}-04$ & $5.0 \mathrm{E}-04$ & Yes & s & s & $N / A$ & $N / A$ \\
\hline
\end{tabular}


Project: Y-12 Security Complex

Location: Bear Creek Burial Grounds
User Name: $\quad$ MV

State: Tennessee

\begin{tabular}{|c|c|c|c|c|c|c|c|c|c|c|c|}
\hline Constituent & Well & $\begin{array}{l}\text { Sourcel } \\
\text { Tail }\end{array}$ & $\begin{array}{l}\text { Number } \\
\text { of } \\
\text { Samples }\end{array}$ & $\begin{array}{c}\text { Number } \\
\text { of } \\
\text { Detects }\end{array}$ & $\begin{array}{c}\text { Average } \\
\text { (mg/L) }\end{array}$ & $\begin{array}{r}\text { Median } \\
(\mathrm{mg} / \mathrm{L})\end{array}$ & $\begin{array}{c}\text { All } \\
\text { Samples } \\
\text { "ND" ? }\end{array}$ & $\begin{array}{l}\text { Mann- } \\
\text { Kendall }\end{array}$ & $\begin{array}{c}\text { Linear } \\
\text { Regression }\end{array}$ & Modeling & Empirical \\
\hline \multicolumn{12}{|c|}{ VINYL CHLORIDE } \\
\hline & GW-072 & $T$ & 2 & 0 & $5.0 \mathrm{E}-04$ & $5.0 \mathrm{E}-04$ & Yes & N/A & N/A & N/A & $\mathrm{N} / \mathrm{A}$ \\
\hline & GW-288 & $\mathrm{T}$ & 2 & 2 & 3.5E-03 & $3.5 \mathrm{E}-03$ & No & N/A & N/A & N/A & N/A \\
\hline & GW-287 & $\mathrm{T}$ & 10 & 0 & $5.0 \mathrm{E}-04$ & $5.0 \mathrm{E}-04$ & Yes & $\mathrm{s}$ & $\mathrm{s}$ & $N / A$ & $N / A$ \\
\hline & GW-639 & $\mathrm{T}$ & 4 & 0 & $5.0 \mathrm{E}-04$ & 5.0E-04 & Yes & s & s & N/A & N/A \\
\hline & GW-642 & $\mathrm{T}$ & 4 & 0 & $5.0 \mathrm{E}-04$ & $5.0 \mathrm{E}-04$ & Yes & s & s & N/A & N/A \\
\hline & GW-713 & $\mathrm{T}$ & 18 & 0 & $5.0 \mathrm{E}-04$ & $5.0 \mathrm{E}-04$ & Yes & s & s & $N / A$ & $N / A$ \\
\hline & GW-069 & $\mathrm{T}$ & 6 & 3 & 8.3E-04 & $7.5 \mathrm{E}-04$ & No & NT & 1 & N/A & N/A \\
\hline & GW-653 & $\mathrm{T}$ & 18 & 0 & $5.0 \mathrm{E}-04$ & $5.0 \mathrm{E}-04$ & Yes & S & s & N/A & N/A \\
\hline & SS-6W & $\mathrm{T}$ & 5 & 0 & $5.0 \mathrm{E}-04$ & $5.0 \mathrm{E}-04$ & Yes & s & I & $N / A$ & $N / A$ \\
\hline & GW-629 & $\mathrm{T}$ & 4 & 0 & $5.0 \mathrm{E}-04$ & 5.0E-04 & Yes & s & S & $N / A$ & $N / A$ \\
\hline & GW-091 & $\mathrm{T}$ & 2 & 0 & $5.0 \mathrm{E}-04$ & $5.0 \mathrm{E}-04$ & Yes & $\mathrm{N} / \mathrm{A}$ & N/A & N/A & N/A \\
\hline & GW-077 & $\mathrm{T}$ & 15 & 0 & $5.0 \mathrm{E}-04$ & 5.0E-04 & Yes & s & s & $N / A$ & $N / A$ \\
\hline
\end{tabular}

Note: Increasing (I); Probably Increasing (PI); Stable (S); Probably Decreasing (PD); Decreasing (D); No Trend (NT); Not Applicable (N/A) - Due to insufficient Data (< 4 sampling events); Source/Tail (S/T)

The Number of Samples and Number of Detects shown above are post-consolidation values. 


\section{MAROS Spatial Moment Analysis Summary}

Project: Y-12 Security Complex

Location: Bear Creek Burial Grounds
User Name: MV

State: Tennessee

\begin{tabular}{|c|c|c|c|c|c|c|c|}
\hline \multirow[b]{2}{*}{ Effective Date } & \multirow{2}{*}{$\frac{\text { 0th Moment }}{\text { Estimated }}$} & \multicolumn{3}{|c|}{ 1st Moment (Center of Mass) } & \multicolumn{2}{|c|}{ 2nd Moment (Spread) } & \multirow[b]{2}{*}{$\begin{array}{c}\text { Number of } \\
\text { Wells }\end{array}$} \\
\hline & & $X c(f t)$ & Yc (ft) & $\begin{array}{c}\text { Source } \\
\text { Distance (ft) }\end{array}$ & $\underset{(s q \mathrm{ft})}{\operatorname{Sigma} x}$ & $\underset{\text { (sq ft) }}{\text { Sigma YY }}$ & \\
\hline
\end{tabular}

GROSS BETA ACTIVITY

\begin{tabular}{|c|c|c|c|c|c|c|c|}
\hline 1/1/1996 & 1.7E+04 & 41,637 & 29,196 & 1,687 & $4,881,980$ & 562,033 & 25 \\
\hline 7/1/1996 & $1.8 \mathrm{E}+04$ & 40,841 & 28,784 & 2,564 & $3,925,575$ & 295,080 & 18 \\
\hline 1/1/1997 & $1.1 \mathrm{E}+04$ & 41,785 & 29,095 & 1,570 & $3,385,632$ & 262,211 & 21 \\
\hline 4/1/1997 & $1.2 \mathrm{E}+03$ & 36,076 & 29,360 & 7,211 & $6,971,796$ & 58,044 & 9 \\
\hline $7 / 1 / 1997$ & $4.8 \mathrm{E}+04$ & 37,228 & 28,702 & 6,117 & $13,589,404$ & 240,779 & 29 \\
\hline 1/1/1998 & $1.3 \mathrm{E}+04$ & 39,960 & 29,065 & 3,361 & $7,262,733$ & 186,390 & 29 \\
\hline 4/1/1998 & $1.5 \mathrm{E}+03$ & 43,213 & 30,062 & 504 & 266,509 & 105,378 & 8 \\
\hline 7/1/1998 & $2.3 \mathrm{E}+04$ & 39,371 & 28,989 & 3,955 & $9,757,693$ & 196,321 & 36 \\
\hline 1/1/1999 & $3.8 \mathrm{E}+04$ & 39,333 & 29,054 & 3,983 & $5,414,673$ & 131,814 & 25 \\
\hline 7/1/1999 & $1.0 \mathrm{E}+04$ & 41,051 & 28,874 & 2,336 & $6,163,086$ & 83,796 & 23 \\
\hline $1 / 1 / 2000$ & $1.8 \mathrm{E}+04$ & 39,686 & 28,820 & 3,674 & $14,301,235$ & 146,380 & 28 \\
\hline $7 / 1 / 2000$ & $9.7 \mathrm{E}+03$ & 40,305 & 28,811 & 3,073 & $13,354,995$ & 97,363 & 27 \\
\hline $1 / 1 / 2001$ & $2.3 \mathrm{E}+04$ & 40,394 & 28,910 & 2,963 & $9,906,867$ & 152,169 & 25 \\
\hline 4/1/2001 & $0.0 \mathrm{E}+00$ & & & & & & 1 \\
\hline $7 / 1 / 2001$ & $1.6 \mathrm{E}+04$ & 40,562 & 29,009 & 2,778 & $11,652,889$ & 141,269 & 23 \\
\hline $10 / 1 / 2001$ & $0.0 \mathrm{E}+00$ & & & & & & 1 \\
\hline $1 / 1 / 2002$ & $2.4 \mathrm{E}+04$ & 40,928 & 29,024 & 2,417 & $12,683,266$ & 115,526 & 28 \\
\hline $7 / 1 / 2002$ & $1.9 \mathrm{E}+04$ & 41,729 & 28,935 & 1,677 & $4,575,214$ & 204,153 & 24 \\
\hline $1 / 1 / 2003$ & $2.1 \mathrm{E}+04$ & 41,192 & 28,975 & 2,173 & $7,143,977$ & 143,175 & 23 \\
\hline 4/1/2003 & $0.0 \mathrm{E}+00$ & & & & & & 1 \\
\hline $7 / 1 / 2003$ & $2.4 \mathrm{E}+04$ & 38,589 & 28,956 & 4,734 & $16,043,403$ & 160,922 & 24 \\
\hline $1 / 1 / 2004$ & $1.6 \mathrm{E}+04$ & 40,278 & 28,988 & 3,060 & $8,630,375$ & 108,076 & 18 \\
\hline $7 / 1 / 2004$ & $1.3 \mathrm{E}+04$ & 41,606 & 29,022 & 1,762 & $2,853,420$ & 127,148 & 19 \\
\hline $10 / 1 / 2004$ & $0.0 \mathrm{E}+00$ & & & & & & 1 \\
\hline
\end{tabular}

NITRATE

\begin{tabular}{|c|c|c|c|c|c|c|c|}
\hline 1/1/1996 & $1.4 \mathrm{E}+03$ & 42,076 & 28,749 & 1,457 & $4,469,858$ & 134,609 & 28 \\
\hline $7 / 1 / 1996$ & $1.4 \mathrm{E}+03$ & 41,995 & 28,789 & 1,503 & $4,171,569$ & 152,849 & 28 \\
\hline 1/1/1997 & $1.0 \mathrm{E}+03$ & 42,274 & 28,860 & 1,231 & $4,191,581$ & 199,242 & 26 \\
\hline 7/1/1997 & $1.8 \mathrm{E}+03$ & 42,148 & 28,759 & 1,391 & $4,148,107$ & 138,582 & 26 \\
\hline 1/1/1998 & $1.0 \mathrm{E}+03$ & 41,750 & 28,838 & 1,696 & $5,311,619$ & 64,843 & 21 \\
\hline 7/1/1998 & $2.2 \mathrm{E}+03$ & 40,437 & 28,848 & 2,936 & $14,990,231$ & 64,988 & 25 \\
\hline 1/1/1999 & $1.3 \mathrm{E}+03$ & 41,503 & 28,957 & 1,881 & $4,574,699$ & 155,938 & 19 \\
\hline 7/1/1999 & $0.0 \mathrm{E}+00$ & & & & & & 3 \\
\hline $1 / 1 / 2000$ & $2.3 \mathrm{E}+03$ & 41,339 & 28,764 & 2,103 & $4,263,888$ & 52,154 & 16 \\
\hline $7 / 1 / 2000$ & $1.6 \mathrm{E}+03$ & 41,555 & 28,839 & 1,875 & $4,424,060$ & 62,011 & 16 \\
\hline $1 / 1 / 2001$ & $1.5 \mathrm{E}+03$ & 43,546 & 28,802 & 804 & 535,369 & 62,078 & 14 \\
\hline $7 / 1 / 2001$ & $1.2 \mathrm{E}+03$ & 43,515 & 28,785 & 811 & 496,247 & 48,999 & 12 \\
\hline $1 / 1 / 2002$ & $1.9 \mathrm{E}+03$ & 43,278 & 28,858 & 705 & 539,764 & 41,916 & 21 \\
\hline
\end{tabular}


Project: Y-12 Security Complex

Location: Bear Creek Burial Grounds

th Moment

Estimated

Effective Date 1st Moment (Center of Mass)

Source $\mathrm{Xc}(\mathrm{ft}) \quad \mathrm{Yc}(\mathrm{ft})$

\begin{abstract}
Distance (ft)
\end{abstract}
User Name: MV

State: Tennessee 2nd Moment (Spread)

Sigma XX Sigma YY Number of $(\mathrm{sq} f \mathrm{ft}) \quad(\mathrm{sq} \mathrm{ft})$
Wells

NITRATE

$\begin{array}{llllrrrr}7 / 1 / 2002 & 9.7 \mathrm{E}+02 & 43,274 & 28,803 & 759 & 527,316 & 61,102 & 22 \\ 1 / 1 / 2003 & 1.3 \mathrm{E}+03 & 42,539 & 28,794 & 1,070 & 2,184,029 & 91,010 & 11 \\ 7 / 1 / 2003 & 7.1 \mathrm{E}+02 & 42,783 & 28,833 & 885 & 2,359,172 & 98,340 & 11 \\ 1 / 1 / 2004 & 6.4 \mathrm{E}+02 & 43,377 & 28,928 & 641 & 467,102 & 43,240 & 13 \\ 7 / 1 / 2004 & 9.4 \mathrm{E}+02 & 43,287 & 28,909 & 653 & 374,094 & 35,603 & 13\end{array}$

TETRACHLOROETHYLENE(PCE)

\begin{tabular}{|c|c|c|c|c|c|c|c|}
\hline 1/1/1996 & $1.4 \mathrm{E}+00$ & 41,669 & 29,352 & 1,628 & $4,060,693$ & 448,338 & 28 \\
\hline 7/1/1996 & $1.4 \mathrm{E}+00$ & 41,663 & 29,349 & 1,635 & $4,177,494$ & 457,896 & 28 \\
\hline 1/1/1997 & $1.8 \mathrm{E}+00$ & 41,980 & 29,514 & 1,305 & $3,528,668$ & 470,550 & 26 \\
\hline 4/1/1997 & 8.4E-01 & 36,215 & 29,371 & 7,072 & $9,545,364$ & 68,256 & 9 \\
\hline 7/1/1997 & $2.4 \mathrm{E}+00$ & 38,456 & 29,263 & 4,837 & $14,905,657$ & 418,237 & 33 \\
\hline 1/1/1998 & $2.2 \mathrm{E}+00$ & 38,852 & 29,276 & 4,441 & $13,885,816$ & 175,409 & 30 \\
\hline 4/1/1998 & $3.1 \mathrm{E}+00$ & 42,735 & 30,329 & 943 & 29,269 & 51,710 & 8 \\
\hline 7/1/1998 & $1.4 \mathrm{E}+01$ & 42,449 & 29,838 & 880 & $5,078,223$ & 224,670 & 37 \\
\hline 1/1/1999 & $6.4 \mathrm{E}+00$ & 41,708 & 29,546 & 1,576 & $9,541,868$ & 173,057 & 31 \\
\hline 7/1/1999 & $7.9 \mathrm{E}+00$ & 41,897 & 29,482 & 1,389 & $7,424,966$ & 105,016 & 28 \\
\hline $1 / 1 / 2000$ & $5.3 E+00$ & 41,378 & 29,408 & 1,913 & $10,167,236$ & 119,014 & 27 \\
\hline $7 / 1 / 2000$ & 8.7E+00 & 42,038 & 29,478 & 1,249 & $6,953,004$ & 105,734 & 28 \\
\hline $1 / 1 / 2001$ & $6.8 \mathrm{E}+00$ & 41,658 & 29,505 & 1,627 & $8,122,165$ & 120,317 & 26 \\
\hline $4 / 1 / 2001$ & $0.0 \mathrm{E}+00$ & & & & & & 1 \\
\hline $7 / 1 / 2001$ & $6.5 \mathrm{E}+00$ & 41,719 & 29,519 & 1,565 & $8,317,844$ & 127,533 & 24 \\
\hline $10 / 1 / 2001$ & $0.0 \mathrm{E}+00$ & & & & & & 1 \\
\hline $1 / 1 / 2002$ & $2.2 \mathrm{E}+01$ & 42,549 & 29,785 & 768 & $2,779,875$ & 110,516 & 32 \\
\hline $7 / 1 / 2002$ & $2.2 \mathrm{E}+01$ & 42,590 & 29,784 & 729 & $2,573,110$ & 119,491 & 32 \\
\hline $1 / 1 / 2003$ & $9.6 \mathrm{E}+00$ & 41,913 & 29,395 & 1,381 & $7,781,523$ & 185,104 & 27 \\
\hline $4 / 1 / 2003$ & $0.0 \mathrm{E}+00$ & & & & & & 1 \\
\hline 7/1/2003 & $1.5 \mathrm{E}+01$ & 42,380 & 29,464 & 910 & $5,461,027$ & 152,626 & 26 \\
\hline $10 / 1 / 2003$ & $0.0 \mathrm{E}+00$ & & & & & & 1 \\
\hline $1 / 1 / 2004$ & $3.9 \mathrm{E}+01$ & 43,219 & 29,678 & 133 & $1,038,181$ & 37,054 & 26 \\
\hline $4 / 1 / 2004$ & $0.0 \mathrm{E}+00$ & & & & & & 1 \\
\hline $7 / 1 / 2004$ & $4.8 \mathrm{E}+01$ & 43,356 & 29,690 & 147 & 269,327 & 27,694 & 24 \\
\hline $10 / 1 / 2004$ & $0.0 \mathrm{E}+00$ & & & & & & 2 \\
\hline
\end{tabular}

TRICHLOROETHYLENE (TCE)

\begin{tabular}{|c|c|c|c|c|c|c|c|}
\hline $1 / 1 / 1996$ & 3.5E-01 & 42,709 & 29,109 & 732 & $2,830,510$ & 267,991 & 28 \\
\hline $7 / 1 / 1996$ & 3.0E-01 & 42,778 & 29,110 & 678 & $3,323,005$ & 295,148 & 28 \\
\hline $1 / 1 / 1997$ & 3.3E-01 & 42,390 & 29,227 & 955 & $2,381,818$ & 263,366 & 26 \\
\hline 4/1/1997 & 8.4E-02 & 36,215 & 29,371 & 7,072 & $9,545,364$ & 68,256 & 9 \\
\hline $7 / 1 / 1997$ & 7.3E-01 & 41,272 & 29,079 & 2,069 & $11,522,169$ & 307,474 & 33 \\
\hline $1 / 1 / 1998$ & $2.5 \mathrm{E}+00$ & 42,987 & 29,280 & 410 & $3,060,235$ & 75,876 & 30 \\
\hline 4/1/1998 & $2.8 \mathrm{E}-01$ & 42,804 & 30,010 & 657 & 29,045 & 169,019 & 8 \\
\hline $7 / 1 / 1998$ & $7.1 \mathrm{E}+00$ & 43,214 & 29,474 & 113 & $1,404,643$ & 183,283 & 37 \\
\hline
\end{tabular}


Project: Y-12 Security Complex

Location: Bear Creek Burial Grounds
User Name: MV

State: Tennessee

\begin{tabular}{|c|c|c|c|c|c|c|c|}
\hline \multirow[b]{2}{*}{ Effective Date } & th Moment & \multicolumn{3}{|c|}{ 1st Moment (Center of Mass) } & \multicolumn{2}{|c|}{ 2nd Moment (Spread) } & \multirow[b]{2}{*}{$\begin{array}{c}\text { Number of } \\
\text { Wells }\end{array}$} \\
\hline & $\begin{array}{c}\text { Estimated } \\
\text { Mass (kg) }\end{array}$ & $\mathrm{Xc}(\mathrm{ft})$ & Yc (ft) & $\begin{array}{c}\text { Source } \\
\text { Distance (ft) }\end{array}$ & $\begin{array}{c}\text { Sigma } X X \\
(\mathrm{sq} f t)\end{array}$ & $\begin{array}{c}\text { Sigma YY } \\
\text { (sq ft) }\end{array}$ & \\
\hline
\end{tabular}

TRICHLOROETHYLENE (TCE)

\begin{tabular}{|c|c|c|c|c|c|c|c|}
\hline 1/1/1999 & $4.8 \mathrm{E}+00$ & 43,166 & 29,468 & 151 & $1,928,273$ & 153,786 & 31 \\
\hline 7/1/1999 & $4.4 \mathrm{E}+00$ & 43,142 & 29,287 & 310 & $1,849,598$ & 62,160 & 28 \\
\hline $1 / 1 / 2000$ & $4.5 \mathrm{E}+00$ & 43,149 & 29,281 & 312 & $1,817,442$ & 62,129 & 27 \\
\hline $7 / 1 / 2000$ & $4.6 \mathrm{E}+00$ & 43,171 & 29,281 & 303 & $1,793,908$ & 61,982 & 28 \\
\hline $1 / 1 / 2001$ & $9.4 \mathrm{E}+00$ & 43,510 & 29,268 & 372 & $1,117,204$ & 67,656 & 26 \\
\hline 4/1/2001 & $0.0 \mathrm{E}+00$ & & & & & & 1 \\
\hline 7/1/2001 & $5.0 \mathrm{E}+00$ & 43,241 & 29,355 & 211 & $1,749,441$ & 84,301 & 24 \\
\hline 10/1/2001 & $0.0 \mathrm{E}+00$ & & & & & & 1 \\
\hline $1 / 1 / 2002$ & 8.7E+00 & 43,274 & 29,579 & 20 & $1,303,266$ & 82,856 & 32 \\
\hline $7 / 1 / 2002$ & $7.6 \mathrm{E}+00$ & 43,331 & 29,528 & 58 & $1,537,825$ & 111,213 & 32 \\
\hline $1 / 1 / 2003$ & $5.1 \mathrm{E}+00$ & 43,051 & 29,353 & 313 & $2,025,570$ & 100,086 & 27 \\
\hline $4 / 1 / 2003$ & $0.0 \mathrm{E}+00$ & & & & & & 1 \\
\hline $7 / 1 / 2003$ & $9.5 \mathrm{E}+00$ & 43,264 & 29,337 & 226 & $1,172,656$ & 71,356 & 26 \\
\hline $10 / 1 / 2003$ & $0.0 \mathrm{E}+00$ & & & & & & 1 \\
\hline $1 / 1 / 2004$ & 2.7E+00 & 43,199 & 29,495 & 108 & $1,648,492$ & 78,726 & 26 \\
\hline $4 / 1 / 2004$ & $0.0 \mathrm{E}+00$ & & & & & & 1 \\
\hline $7 / 1 / 2004$ & $2.6 \mathrm{E}+00$ & 43,399 & 29,509 & 126 & 514,065 & 77,892 & 24 \\
\hline $10 / 1 / 2004$ & $0.0 \mathrm{E}+00$ & & & & & & 2 \\
\hline
\end{tabular}

VINYL CHLORIDE

\begin{tabular}{|c|c|c|c|c|c|c|c|}
\hline 1/1/1996 & $1.3 \mathrm{E}+00$ & 41,526 & 29,290 & 1,779 & $4,383,054$ & 507,140 & 28 \\
\hline $7 / 1 / 1996$ & $1.3 \mathrm{E}+00$ & 41,686 & 29,325 & 1,616 & $4,463,749$ & 508,741 & 28 \\
\hline 1/1/1997 & $1.3 \mathrm{E}+00$ & 41,574 & 29,286 & 1,733 & $4,344,204$ & 494,734 & 26 \\
\hline 4/1/1997 & $3.3 E+00$ & 36,287 & 29,431 & 6,999 & $7,093,230$ & 41,450 & 9 \\
\hline $7 / 1 / 1997$ & $3.0 \mathrm{E}+00$ & 39,399 & 29,437 & 3,887 & $15,785,796$ & 542,595 & 33 \\
\hline 1/1/1998 & $3.1 E+00$ & 40,282 & 29,296 & 3,013 & $14,603,734$ & 152,718 & 30 \\
\hline 4/1/1998 & 3.9E-01 & 42,684 & 30,347 & 988 & 26,595 & 100,618 & 8 \\
\hline 7/1/1998 & $9.8 \mathrm{E}+00$ & 42,275 & 29,718 & 1,021 & $7,990,774$ & 200,766 & 37 \\
\hline 1/1/1999 & $1.1 \mathrm{E}+01$ & 42,227 & 29,848 & 1,094 & $7,014,696$ & 218,719 & 31 \\
\hline 7/1/1999 & $3.6 \mathrm{E}+00$ & 40,696 & 29,318 & 2,600 & $13,410,930$ & 144,634 & 28 \\
\hline $1 / 1 / 2000$ & $3.7 E+00$ & 40,733 & 29,319 & 2,563 & $13,380,470$ & 145,252 & 27 \\
\hline $7 / 1 / 2000$ & $3.0 \mathrm{E}+00$ & 40,163 & 29,297 & 3,132 & $14,731,076$ & 158,131 & 28 \\
\hline $1 / 1 / 2001$ & $9.2 \mathrm{E}+00$ & 41,999 & 29,627 & 1,286 & $6,359,486$ & 103,613 & 26 \\
\hline 4/1/2001 & $0.0 \mathrm{E}+00$ & & & & & & 1 \\
\hline $7 / 1 / 2001$ & $1.1 \mathrm{E}+01$ & 42,330 & 29,700 & 964 & $5,701,897$ & 106,669 & 24 \\
\hline 10/1/2001 & $0.0 \mathrm{E}+00$ & & & & & & 1 \\
\hline $1 / 1 / 2002$ & $4.9 \mathrm{E}+00$ & 41,233 & 29,580 & 2,051 & $9,855,553$ & 140,276 & 32 \\
\hline $7 / 1 / 2002$ & $3.9 \mathrm{E}+00$ & 40,926 & 29,541 & 2,359 & $10,730,797$ & 191,058 & 32 \\
\hline $1 / 1 / 2003$ & $1.0 \mathrm{E}+01$ & 41,840 & 29,592 & 1,445 & $7,176,760$ & 169,840 & 26 \\
\hline 4/1/2003 & $0.0 \mathrm{E}+00$ & & & & & & 1 \\
\hline $7 / 1 / 2003$ & $1.4 \mathrm{E}+01$ & 42,174 & 29,633 & 1,112 & $5,318,518$ & 129,970 & 25 \\
\hline $1 / 1 / 2004$ & $3.2 \mathrm{E}+00$ & 41,295 & 29,423 & 1,994 & $7,258,094$ & 189,537 & 25 \\
\hline $7 / 1 / 2004$ & $2.9 \mathrm{E}+00$ & 42,473 & 29,489 & 814 & $1,834,809$ & 183,248 & 23 \\
\hline
\end{tabular}


Project: Y-12 Security Complex

Location: Bear Creek Burial Grounds

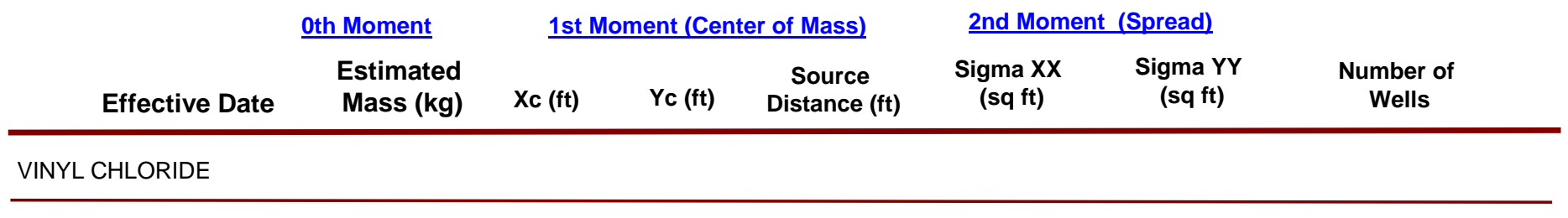

User Name: $\quad$ MV

State: Tennessee

2nd Moment (Spread) 
Project: Y-12 Security Complex

Location: Bear Creek Burial Grounds
User Name: $\mathrm{MV}$

State: Tennessee

\begin{tabular}{|c|c|c|c|c|c|}
\hline Moment Type & Constituent & $\begin{array}{l}\text { Coefficient } \\
\text { of Variation }\end{array}$ & $\begin{array}{l}\text { Mann-Kendall } \\
\text { S Statistic }\end{array}$ & $\begin{array}{l}\text { Confidence } \\
\text { in Trend }\end{array}$ & $\begin{array}{c}\text { Moment } \\
\text { Trend }\end{array}$ \\
\hline \multicolumn{6}{|c|}{ Zeroth Moment: Mass } \\
\hline & GROSS BETA ACTIVITY & 0.79 & -26 & $73.0 \%$ & S \\
\hline & NITRATE & 0.44 & -43 & $94.4 \%$ & PD \\
\hline & TETRACHLOROETHYLENE(PCE) & 1.41 & 62 & $91.0 \%$ & $\mathrm{PI}$ \\
\hline & TRICHLOROETHYLENE (TCE) & 1.06 & 10 & $57.8 \%$ & NT \\
\hline & VINYL CHLORIDE & 0.96 & 22 & $69.7 \%$ & NT \\
\hline \multicolumn{6}{|c|}{ 1st Moment: Distance to Source } \\
\hline & GROSS BETA ACTIVITY & 0.51 & -22 & $75.0 \%$ & $\mathrm{~S}$ \\
\hline & NITRATE & 0.48 & -60 & $99.3 \%$ & $\mathrm{D}$ \\
\hline & TETRACHLOROETHYLENE(PCE) & 0.94 & -98 & $99.9 \%$ & $\mathrm{D}$ \\
\hline & TRICHLOROETHYLENE (TCE) & 2.05 & -102 & $100.0 \%$ & $\mathrm{D}$ \\
\hline & VINYL CHLORIDE & 0.67 & -44 & $91.8 \%$ & PD \\
\hline \multicolumn{6}{|c|}{ 2nd Moment: Sigma XX } \\
\hline & GROSS BETA ACTIVITY & 0.54 & 38 & $88.3 \%$ & NT \\
\hline & NITRATE & 1.03 & -70 & $99.8 \%$ & $\mathrm{D}$ \\
\hline & TETRACHLOROETHYLENE(PCE) & 0.66 & -42 & $90.7 \%$ & PD \\
\hline & TRICHLOROETHYLENE (TCE) & 1.08 & -94 & $99.9 \%$ & $\mathrm{D}$ \\
\hline & VINYL CHLORIDE & 0.55 & 0 & $48.7 \%$ & $\mathrm{~S}$ \\
\hline \multicolumn{6}{|c|}{ 2nd Moment: Sigma YY } \\
\hline & GROSS BETA ACTIVITY & 0.62 & -54 & $95.7 \%$ & $\mathrm{D}$ \\
\hline & NITRATE & 0.56 & -68 & $99.8 \%$ & $\mathrm{D}$ \\
\hline & TETRACHLOROETHYLENE(PCE) & 0.78 & -72 & $99.0 \%$ & $\mathrm{D}$ \\
\hline & TRICHLOROETHYLENE (TCE) & 0.65 & -64 & $98.0 \%$ & $\mathrm{D}$ \\
\hline & VINYL CHLORIDE & 0.70 & -36 & $87.0 \%$ & $\mathrm{~S}$ \\
\hline
\end{tabular}

Note: The following assumptions were applied for the calculation of the Zeroth Moment:

Porosity: $0.10 \quad$ Saturated Thickness: Uniform: $50 \mathrm{ft}$

Mann-Kendall Trend test performed on all sample events for each constituent. Increasing (I); Probably Increasing (PI); Stable (S); Probably Decreasing (PD); Decreasing (D); No Trend (NT); Not Applicable (N/A)-Due to insufficient Data (< 4 sampling events).

Note: The Sigma $X X$ and Sigma $Y Y$ components are estimated using the given field coordinate system and then rotated to align with the estimated groundwater flow direction. Moments are not calculated for sample events with less than 6 wells. 


\section{ASSESSMENT OF THE GROUNDWATER PROTECTION PROGRAM \\ Y-12 NATIONAL SECURITY COMPLEX, OAK RIDGE, TENNESSEE}

Y-12 National Security Complex

Oak Ridge, Tennessee

Appendix D.4 East Fork Regime East S-3 Area

Table D.4.1

Qualitative Analysis East S-3 Area

Table D.4.2

Aquifer Input Parameters

MAROS Report

COC Assessment

MAROS Report Plume Summary

MAROS Report Spatial Moment Analysis Summary

MAROS Chart New Location Analysis PCE East S-3 
TABLE D.4.1

QUALITATIVE ANALYSIS EAST FORK REGIME EAST S-3 AREA

East Fork Poplar Creek Hydrogeologic Regime

Y-12 National Security Complex

Oak Ridge, Tennessee

\begin{tabular}{|c|c|c|c|c|c|c|c|c|c|c|c|c|}
\hline $\begin{array}{l}\text { Location } \\
\text { Name }\end{array}$ & $\begin{array}{c}\text { Location } \\
\text { Type }\end{array}$ & $\begin{array}{c}\text { Average Concentration } \\
\text { Exceeds Screening Level }\end{array}$ & $\begin{array}{c}\text { Formation } \\
\text { Type }\end{array}$ & $\begin{array}{c}\text { Horizontal } \\
\text { Delineation } \\
\end{array}$ & $\begin{array}{c}\text { Vertical } \\
\text { Delineation }\end{array}$ & Exit Location & RCRA & CERCLA & Unique & $\begin{array}{c}\text { Monitors } \\
\text { Background } \\
\text { Water Quality }\end{array}$ & $\begin{array}{c}\text { Early } \\
\text { Detection }\end{array}$ & $\begin{array}{c}\text { Monitor } \\
\text { Source }\end{array}$ \\
\hline $55-1 \mathrm{~A}$ & WL & $x$ & Aquitard & $\mathrm{x}$ & & & & & & & & \\
\hline $55-1 C$ & WL & & Aquitard & & & & & & $x$ & $x$ & & \\
\hline $55-2 B$ & WL & $x$ & Aquitard & $x$ & & & & & & & & \\
\hline $55-2 C$ & WL & $x$ & Aquitard & $x$ & & & & & & & & \\
\hline GW-105 & WL & $\mathrm{x}$ & Aquitard & $\mathrm{x}$ & & & & & & & & $x$ \\
\hline GW-106 & WL & $\mathrm{x}$ & Aquitard & $x$ & & & & & & & & $\mathrm{x}$ \\
\hline GW-107 & WL & $x$ & Aquitard & & & & & & & & & $x$ \\
\hline GW-108 & WL & $x$ & Aquitard & & $x$ & & $x$ & & & & $x$ & $x$ \\
\hline GW-109 & WL & $x$ & Aquitard & & $x$ & & $x$ & & & & & $x$ \\
\hline GW-190 & WL & $\mathrm{x}$ & Aquifer & & & & & & & $\mathrm{x}$ & & \\
\hline GW-191 & WL & & Aquitard & $x$ & & & & & $\mathrm{X}$ & & & \\
\hline GW-192 & WL & & Aquitard & $x$ & & & & & & & & \\
\hline GW-194 & WL & & Aquitard & & & & & & $x$ & & & \\
\hline GW-195 & WL & $x$ & Aquitard & & & & & & & & & \\
\hline GW-196 & WL & $\mathrm{x}$ & Aquitard & & & & & & $\mathrm{x}$ & & & \\
\hline GW-197 & WL & $\mathrm{x}$ & Aquitard & & & & & & $x$ & $x$ & & \\
\hline GW-251 & WL & $\mathrm{x}$ & Aquifer & $x$ & & & & $x$ & & & & \\
\hline GW-252 & WL & & Aquifer & $x$ & & & & & $x$ & & & \\
\hline GW-253 & WL & $x$ & Aquifer & $x$ & & & $x$ & $x$ & & & & \\
\hline GW-255 & WL & & Aquifer & $x$ & & & & & & & & \\
\hline GW-261 & WL & & Aquitard & & & & & & & & & \\
\hline GW-263 & WL & $x$ & Aquitard & & & & & & & & & \\
\hline GW-265 & WL & $x$ & Aquitard & & & & & & & & & \\
\hline GW-268 & WL & & Aquitard & & & & & & $x$ & $x$ & & \\
\hline GW-269 & WL & $\mathrm{x}$ & Aquitard & & & & & & & & & \\
\hline
\end{tabular}

Notes:

1. $\mathrm{WL}=$ Monitoring Well; $\mathrm{SP}=$ Spring

2. Well data taken from BWXT Y-12 Analytical Database. Sample locations shown on Figures A.1 and A.3.

3. RCRA indicates wells monitored as part of compliance with RCRA Post-Closure Corrective Action Monitoring or designated Alternate location;

CERCLA indicates locations monitored as part of compliance with CERCLA ROD or backup location. Data from BWXT, 2003a and BWXT 2004a.

4. Average Concentration Exceeds Screening = The average concentration over the entire sampling record for the priority constituent is above the MCL or other designated screening level

as defined in Table B.1.

5. Aquifer and aquitard formations identified in Fig. A.2 from BWXT Y12,2003 Groundwater Monitoring Report, (12/01/2003).

6. Details of the decision criteria for each category are presented in the text. 
TABLE D.4.1

QUALITATIVE ANALYSIS EAST FORK REGIME EAST S-3 AREA

East Fork Poplar Creek Hydrogeologic Regime

Y-12 National Security Complex

Oak Ridge, Tennessee

\begin{tabular}{|c|c|c|c|c|c|c|c|c|c|c|c|c|}
\hline $\begin{array}{l}\text { Location } \\
\text { Name }\end{array}$ & $\begin{array}{c}\text { Location } \\
\text { Type }\end{array}$ & $\begin{array}{c}\text { Average Concentration } \\
\text { Exceeds Screening Level }\end{array}$ & $\begin{array}{c}\text { Formation } \\
\text { Type }\end{array}$ & $\begin{array}{l}\text { Horizontal } \\
\text { Delineation }\end{array}$ & $\begin{array}{c}\text { Vertical } \\
\text { Delineation }\end{array}$ & Exit Location & RCRA & CERCLA & Unique & $\begin{array}{c}\text { Monitors } \\
\text { Background } \\
\text { Water Quality }\end{array}$ & $\begin{array}{c}\text { Early } \\
\text { Detection }\end{array}$ & $\begin{array}{c}\text { Monitor } \\
\text { Source }\end{array}$ \\
\hline GW-270 & WL & $\mathrm{x}$ & Aquitard & & & & & & & & & \\
\hline GW-271 & WL & & Aquitard & & & & & & $\mathrm{x}$ & & & \\
\hline GW-272 & WL & $\mathrm{x}$ & Aquitard & & & & & & $x$ & & & \\
\hline GW-273 & WL & $\mathrm{x}$ & Aquitard & & & & & & & & & \\
\hline GW-274 & WL & $x$ & Aquitard & & & & $x$ & & & & & \\
\hline GW-275 & WL & $\mathrm{X}$ & Aquitard & & & & $\mathrm{X}$ & & & & & \\
\hline GW-332 & WL & $x$ & Aquitard & & & & & & & & & \\
\hline GW-334 & WL & $\mathrm{x}$ & Aquitard & & & & & & & $\mathrm{x}$ & & \\
\hline GW-335 & WL & $\mathrm{x}$ & Aquitard & & & & & & & & & \\
\hline GW-336 & WL & $x$ & Aquitard & & & & & & & & & \\
\hline GW-337 & WL & $x$ & Aquitard & & & & & & & & & \\
\hline GW-338 & WL & $x$ & Aquitard & & & & & & $\mathrm{x}$ & & & \\
\hline GW-349 & WL & $\mathrm{x}$ & Aquifer & & & & & & & & & \\
\hline GW-350 & WL & $\mathrm{x}$ & Aquifer & & & & & & $\mathrm{x}$ & & & \\
\hline GW-505 & WL & $\mathrm{x}$ & Aquitard & & & & & & & & & \\
\hline GW-508 & WL & $x$ & Aquitard & & & & & & & & & \\
\hline GW-617 & WL & & Aquifer & $x$ & & $x$ & & & & & & \\
\hline GW-618 & WL & $\mathrm{x}$ & Aquifer & $x$ & & $x$ & & $x$ & & & & \\
\hline GW-619 & WL & $\mathrm{x}$ & Aquifer & $x$ & & & & & & & $\mathrm{x}$ & \\
\hline GW-620 & WL & $x$ & Aquifer & $\mathrm{x}$ & & & & $x$ & & & $x$ & \\
\hline GW-631 & WL & $x$ & Aquitard & & & & & & $x$ & & & $\mathrm{X}$ \\
\hline GW-633 & WL & $x$ & Aquitard & & & & & & & & & $x$ \\
\hline GW-778 & WL & & Aquitard & & & & & & & & & \\
\hline SPR14.0SP & $\mathrm{SP}$ & & Spring & $\mathrm{x}$ & & $\mathrm{x}$ & & & & $x$ & & \\
\hline
\end{tabular}

\section{Notes:}

1. $\mathrm{WL}=$ Monitoring Well; $\mathrm{SP}=$ Spring

2. Well data taken from BWXT Y-12 Analytical Database. Sample locations shown on Figures A.1 and A.3.

3. RCRA indicates wells monitored as part of compliance with RCRA Post-Closure Corrective Action Monitoring or designated Alternate location;

CERCLA indicates locations monitored as part of compliance with CERCLA ROD or backup location. Data from BWXT, 2003a and BWXT 2004a.

4. Average Concentration Exceeds Screening = The average concentration over the entire sampling record for the priority constituent is above the MCL or other designated screening level

as defined in Table B.1.

5. Aquifer and aquitard formations identified in Fig. A.2 from BWXT Y12,2003 Groundwater Monitoring Report, (12/01/2003).

6. Details of the decision criteria for each category are presented in the text. 
TABLE D.4.2

AQUIFER INPUT PARAMETERS

\author{
East S-3
}

East Fork Poplar Creek Regime

Y-12 National Security Complex

\begin{tabular}{|c|c|c|}
\hline Parameter & Value & Units \\
\hline Current Plume Length & 2500 & $\mathrm{ft}$ \\
\hline Maximum Plume Length & 2500 & $\mathrm{ft}$ \\
\hline PlumeWidth & 1500 & $\mathrm{ft}$ \\
\hline SeepageVelocity (ft/yr) & 200 & $\mathrm{ft} / \mathrm{yr}$ \\
\hline Distance to Receptors & 5000 & $\mathrm{ft}$ \\
\hline GWFluctuations & Yes & -- \\
\hline SourceTreatment & None & -- \\
\hline PlumeType & Chlorinated Solvent/BTEX & -- \\
\hline Free NAPL Present & Yes & -- \\
\hline Parameter & Value & \\
\hline Groundwater flow direction & E/SE & 0 \\
\hline Effective Porosity & 0.1 & -- \\
\hline Source Location near Well & GW-108 & -- \\
\hline Source X-Coordinate & 53207 & $\mathrm{ft}^{*}$ \\
\hline Source Y-Coordinate & 30070 & $\mathrm{ft}^{\star}$ \\
\hline Saturated Thickness & 50 & $\mathrm{ft}$ \\
\hline Source Wells & Value & \\
\hline \multicolumn{3}{|c|}{ GW-108, GW-109, GW-633, GW-274, GW-275, GW-107 } \\
\hline
\end{tabular}

Notes:

1. Aquifer data are general values for the hydrologic regime.

2. Priority COCs defined by prevalence, toxicty and mobility.

3. $\mathrm{ft}^{*}=$ Coordinates in $\mathrm{Y}-12$ Plant coordinates, feet.

4. Screening Levels are USEPA MCLs, except in the case of compounds without MCLs where the level is the Region 9 PRG for tap water.

5. Effective Porosity estimated based on average high and low values for aquifer and aquitard suburfaces. 


\section{MAROS COC Assessment}

Project: $\quad$ Y-12 Security Complex

Location: East S-3

Toxicity:

\begin{tabular}{lccc}
\hline Contaminant of Concern & $\begin{array}{c}\text { Representative } \\
\text { Concentration } \\
\text { (mg/L) }\end{array}$ & $\begin{array}{c}\text { PRG } \\
\text { (mg/L) }\end{array}$ & $\begin{array}{c}\text { Percent } \\
\text { Above } \\
\text { PRG }\end{array}$ \\
\hline NITRATE & $1.2 \mathrm{E}+03$ & $1.0 \mathrm{E}+01$ & $11811.7 \%$ \\
BENZENE & $2.5 \mathrm{E}-01$ & $5.0 \mathrm{E}-03$ & $4951.9 \%$ \\
CADMIUM & $2.5 \mathrm{E}-01$ & $5.0 \mathrm{E}-03$ & $4912.7 \%$ \\
TETRACHLOROETHYLENE(PCE) & $1.3 \mathrm{E}-01$ & $5.0 \mathrm{E}-03$ & $2593.0 \%$ \\
VINYL CHLORIDE & $4.9 \mathrm{E}-02$ & $2.0 \mathrm{E}-03$ & $2344.1 \%$ \\
TRICHLOROETHYLENE (TCE) & $9.2 \mathrm{E}-02$ & $5.0 \mathrm{E}-03$ & $1739.2 \%$ \\
Cis-1,2-DICHLOROETHYLENE & $4.3 \mathrm{E}-01$ & $7.0 \mathrm{E}-02$ & $517.5 \%$ \\
1,2-DICHLOROETHANE & $2.7 \mathrm{E}-02$ & $5.0 \mathrm{E}-03$ & $430.2 \%$ \\
CARBON TETRACHLORIDE & $2.6 \mathrm{E}-02$ & $5.0 \mathrm{E}-03$ & $419.8 \%$ \\
1,1-DICHLOROETHENE & $3.3 \mathrm{E}-02$ & $7.0 \mathrm{E}-03$ & $376.7 \%$ \\
NICKEL & $2.5 \mathrm{E}-01$ & $7.3 \mathrm{E}-02$ & $241.8 \%$ \\
MERCURY & $4.1 \mathrm{E}-03$ & $2.0 \mathrm{E}-03$ & $103.8 \%$ \\
COPPER & $2.1 \mathrm{E}+00$ & $1.3 \mathrm{E}+00$ & $65.0 \%$ \\
LEAD & $2.4 \mathrm{E}-02$ & $1.5 \mathrm{E}-02$ & $57.4 \%$ \\
CHROMIUM III & $1.1 \mathrm{E}-01$ & $1.0 \mathrm{E}-01$ & $8.6 \%$
\end{tabular}

Note: Top COCs by toxicity were determined by examining a representative concentration for each compound over the entire site. The compound representative concentrations are then compared with the chosen PRG for that compound, with the percentage excedence from the PRG determining the compound's toxicity. All compounds above exceed the PRG.

\section{Prevalence:}

\begin{tabular}{|c|c|c|c|c|c|}
\hline Contaminant of Concern & Class & $\begin{array}{l}\text { Total } \\
\text { Wells }\end{array}$ & $\begin{array}{c}\text { Total } \\
\text { Excedences }\end{array}$ & $\begin{array}{c}\text { Percent } \\
\text { Excedences }\end{array}$ & $\begin{array}{c}\text { Total } \\
\text { detects }\end{array}$ \\
\hline CADMIUM & MET & 39 & 27 & $69.2 \%$ & 39 \\
\hline TETRACHLOROETHYLENE(PCE) & ORG & 49 & 22 & $44.9 \%$ & 39 \\
\hline NITRATE & INO & 46 & 17 & $37.0 \%$ & 46 \\
\hline TRICHLOROETHYLENE (TCE) & ORG & 49 & 17 & $34.7 \%$ & 35 \\
\hline LEAD & MET & 43 & 13 & $30.2 \%$ & 43 \\
\hline NICKEL & MET & 45 & 11 & $24.4 \%$ & 45 \\
\hline VINYL CHLORIDE & ORG & 49 & 11 & $22.4 \%$ & 11 \\
\hline cis-1,2-DICHLOROETHYLENE & ORG & 47 & 10 & $21.3 \%$ & 23 \\
\hline CHROMIUM III & MET & 44 & 9 & $20.5 \%$ & 44 \\
\hline 1,1-DICHLOROETHENE & ORG & 49 & 9 & $18.4 \%$ & 20 \\
\hline BENZENE & ORG & 49 & 8 & $16.3 \%$ & 19 \\
\hline 1,2-DICHLOROETHANE & ORG & 49 & 5 & $10.2 \%$ & 8 \\
\hline CARBON TETRACHLORIDE & ORG & 49 & 4 & $8.2 \%$ & 5 \\
\hline MERCURY & MET & 29 & 2 & $6.9 \%$ & 29 \\
\hline COPPER & MET & 40 & 1 & $2.5 \%$ & 40 \\
\hline
\end{tabular}

Note: Top COCs by prevalence were determined by examining a representative concentration for each well location at the site. The total excedences (values above the chosen PRGs) are compared to the total number of wells to determine the prevalence of the compound. 
Project: Y-12 Security Complex

User Name: MV

Location: East S-3

State: Tennessee

\section{Mobility:}

\begin{tabular}{lr} 
Contaminant of Concern & Kd \\
\hline NITRATE & \\
VINYL CHLORIDE & 0.042 \\
1,2-DICHLOROETHANE & 0.0679 \\
CIS-1,2-DICHLOROETHYLENE & 0.0724 \\
BENZENE & 0.0984 \\
1,1-DICHLOROETHENE & 0.13 \\
CARBON TETRACHLORIDE & 0.277 \\
TRICHLOROETHYLENE (TCE) & 0.297 \\
TETRACHLOROETHYLENE(PCE) & 0.923 \\
LEAD & 10 \\
CADMIUM & 15 \\
NICKEL & 16 \\
COPPER & 40 \\
MERCURY & 52 \\
CHROMIUM III & 1200
\end{tabular}

Note: Top COCs by mobility were determined by examining each detected compound in the dataset and comparing their mobilities (Koc's for organics, assume foc $=0.001$, and Kd's for metals).

Contaminants of Concern (COC's)

GROSS BETA ACTIVITY

NITRATE

TRICHLOROETHYLENE (TCE)

TETRACHLOROETHYLENE(PCE)

GROSS ALPHA ACTIVITY 


\section{MAROS Plume Analysis Summary}

Project: $\quad$ Y-12 National Security Complex

Location: East S-3

Time Period: 1/1/1996

to $12 / 1 / 2004$

Consolidation Period: No Time Consolidation

Consolidation Type: Median

Duplicate Consolidation: Average

ND Values: Specified Detection Limit

J Flag Values : Actual Value

\begin{tabular}{|c|c|c|c|c|c|c|c|c|c|c|c|}
\hline Constituent & Well & $\begin{array}{c}\text { Sourcel } \\
\text { Tail }\end{array}$ & $\begin{array}{l}\text { Number } \\
\text { of } \\
\text { Samples }\end{array}$ & $\begin{array}{c}\text { Number } \\
\text { of } \\
\text { Detects }\end{array}$ & $\begin{array}{c}\text { Average } \\
\text { (mg/L) }\end{array}$ & $\begin{array}{r}\text { Median } \\
\text { (mg/L) }\end{array}$ & $\begin{array}{c}\text { All } \\
\text { Samples } \\
\text { "ND" ? }\end{array}$ & $\begin{array}{l}\text { Mann- } \\
\text { Kendall }\end{array}$ & $\begin{array}{c}\text { Linear } \\
\text { Regression }\end{array}$ & Modeling & Empirical \\
\hline \multicolumn{12}{|c|}{ GROSS ALPHA ACTIVITY } \\
\hline & GW-633 & $\mathrm{S}$ & 8 & 8 & $3.6 \mathrm{E}+01$ & 1.7E+01 & No & NT & NT & N/A & N/A \\
\hline & GW-275 & s & 4 & 4 & $1.1 \mathrm{E}+02$ & $1.1 \mathrm{E}+02$ & No & $\mathrm{s}$ & NT & N/A & N/A \\
\hline & GW-109 & S & 5 & 5 & $1.6 \mathrm{E}+02$ & $1.1 \mathrm{E}+02$ & No & s & NT & N/A & N/A \\
\hline & GW-108 & $\mathrm{s}$ & 17 & 13 & $2.4 \mathrm{E}+02$ & $7.3 \mathrm{E}+01$ & No & NT & NT & $N / A$ & N/A \\
\hline & GW-274 & $\mathrm{s}$ & 3 & 3 & $6.7 \mathrm{E}+01$ & $5.9 \mathrm{E}+01$ & No & N/A & N/A & N/A & N/A \\
\hline & $55-2 B$ & $\mathrm{~T}$ & 4 & 2 & $4.1 \mathrm{E}+00$ & 1.2E-01 & No & NT & NT & $\mathrm{N} / \mathrm{A}$ & N/A \\
\hline & GW-505 & $\mathrm{T}$ & 3 & 3 & $3.5 \mathrm{E}+01$ & $2.8 \mathrm{E}+01$ & No & N/A & N/A & $N / A$ & N/A \\
\hline & GW-619 & $\mathrm{T}$ & 3 & 3 & $2.9 \mathrm{E}+00$ & $2.9 \mathrm{E}+00$ & No & $\mathrm{N} / \mathrm{A}$ & N/A & $\mathrm{N} / \mathrm{A}$ & $\mathrm{N} / \mathrm{A}$ \\
\hline & GW-332 & $\mathrm{T}$ & 1 & 1 & $9.9 \mathrm{E}-01$ & 9.9E-01 & No & N/A & N/A & $N / A$ & N/A \\
\hline & GW-336 & $\mathrm{T}$ & 1 & 1 & $1.9 \mathrm{E}+00$ & $1.9 \mathrm{E}+00$ & No & N/A & N/A & N/A & N/A \\
\hline & $55-2 C$ & $\mathrm{~T}$ & 8 & 6 & $2.4 \mathrm{E}+00$ & $1.4 \mathrm{E}+00$ & No & NT & NT & $N / A$ & $N / A$ \\
\hline & GW-620 & $\mathrm{T}$ & 15 & 15 & $1.8 \mathrm{E}+00$ & $1.0 \mathrm{E}+00$ & No & NT & $\mathrm{D}$ & $N / A$ & N/A \\
\hline & GW-338 & $\mathrm{T}$ & 2 & 2 & $3.7 \mathrm{E}+00$ & $3.7 \mathrm{E}+00$ & No & N/A & $N / A$ & $\mathrm{~N} / \mathrm{A}$ & N/A \\
\hline & GW-617 & $\mathrm{T}$ & 3 & 3 & $8.9 \mathrm{E}-01$ & 7.6E-01 & No & N/A & N/A & $N / A$ & N/A \\
\hline & GW-106 & $\mathrm{T}$ & 2 & 2 & $8.1 \mathrm{E}+01$ & $8.1 \mathrm{E}+01$ & No & N/A & N/A & N/A & N/A \\
\hline & GW-349 & $\mathrm{T}$ & 1 & 1 & $5.9 E+00$ & $5.9 \mathrm{E}+00$ & No & N/A & $\mathrm{N} / \mathrm{A}$ & N/A & N/A \\
\hline & GW-350 & $\mathrm{T}$ & 2 & 2 & $2.4 \mathrm{E}+00$ & $2.4 \mathrm{E}+00$ & No & $N / A$ & N/A & $N / A$ & $N / A$ \\
\hline & GW-105 & $\mathrm{T}$ & 2 & 2 & $7.7 \mathrm{E}+01$ & 7.7E+01 & No & N/A & N/A & $N / A$ & N/A \\
\hline & GW-618 & $\mathrm{T}$ & 15 & 11 & $1.3 \mathrm{E}+00$ & 8.6E-01 & No & PD & $\mathrm{D}$ & N/A & N/A \\
\hline & $55-1 C$ & $\mathrm{~T}$ & 1 & 1 & $3.3 \mathrm{E}+00$ & $3.3 E+00$ & No & N/A & $N / A$ & $N / A$ & $N / A$ \\
\hline & GW-195 & $\mathrm{T}$ & 2 & 2 & $3.2 \mathrm{E}+00$ & $3.2 \mathrm{E}+00$ & No & $\mathrm{N} / \mathrm{A}$ & $N / A$ & $N / A$ & $\mathrm{~N} / \mathrm{A}$ \\
\hline & GW-251 & $\mathrm{T}$ & 19 & 19 & $7.4 \mathrm{E}+00$ & $8.0 \mathrm{E}+00$ & No & $\mathrm{D}$ & $\mathrm{PD}$ & $\mathrm{N} / \mathrm{A}$ & $\mathrm{N} / \mathrm{A}$ \\
\hline
\end{tabular}

User Name:

State: Tennessee 
Project: Y-12 National Security Complex

Location: East S-3
User Name:

State: Tennessee

\begin{tabular}{|c|c|c|c|c|c|c|c|c|c|c|c|}
\hline Constituent & Well & $\begin{array}{c}\text { Sourcel } \\
\text { Tail }\end{array}$ & $\begin{array}{c}\text { Number } \\
\text { of } \\
\text { Samples }\end{array}$ & $\begin{array}{c}\text { Number } \\
\text { of } \\
\text { Detects }\end{array}$ & $\begin{array}{c}\text { Average } \\
\text { (mg/L) }\end{array}$ & $\begin{array}{c}\text { Median } \\
(\mathrm{mg} / \mathrm{L})\end{array}$ & $\begin{array}{c}\text { All } \\
\text { Samples } \\
\text { "ND" ? }\end{array}$ & $\begin{array}{l}\text { Mann- } \\
\text { Kendall }\end{array}$ & $\begin{array}{c}\text { Linear } \\
\text { Regression }\end{array}$ & Modeling & Empirical \\
\hline \multicolumn{12}{|c|}{ GROSS ALPHA ACTIVITY } \\
\hline & GW-194 & $T$ & 1 & 1 & $3.1 \mathrm{E}+00$ & $3.1 \mathrm{E}+00$ & No & N/A & N/A & $\mathrm{N} / \mathrm{A}$ & N/A \\
\hline & SPR14_0SP & $\mathrm{T}$ & 1 & 1 & $2.6 \mathrm{E}+00$ & $2.6 \mathrm{E}+00$ & No & N/A & $\mathrm{N} / \mathrm{A}$ & N/A & N/A \\
\hline & GW-253 & $\mathrm{T}$ & 15 & 15 & $4.7 \mathrm{E}+01$ & $4.2 \mathrm{E}+01$ & No & $\mathrm{s}$ & $\mathrm{s}$ & $N / A$ & N/A \\
\hline & GW-269 & $\mathrm{T}$ & 1 & 1 & $5.2 \mathrm{E}-01$ & $5.2 \mathrm{E}-01$ & No & N/A & N/A & N/A & N/A \\
\hline & GW-192 & $\mathrm{T}$ & 8 & 8 & $1.4 \mathrm{E}+00$ & $1.1 \mathrm{E}+00$ & No & PD & $\mathrm{D}$ & N/A & N/A \\
\hline & GW-270 & $\mathrm{T}$ & 2 & 2 & $4.3 \mathrm{E}+00$ & $4.3 \mathrm{E}+00$ & No & $N / A$ & N/A & $N / A$ & $N / A$ \\
\hline & GW-271 & $T$ & 2 & 2 & $2.2 \mathrm{E}+00$ & $2.2 \mathrm{E}+00$ & No & N/A & N/A & $N / A$ & N/A \\
\hline & GW-337 & $\mathrm{T}$ & 4 & 4 & $1.0 \mathrm{E}+00$ & 9.6E-01 & No & $\mathrm{D}$ & $D$ & $\mathrm{~N} / \mathrm{A}$ & N/A \\
\hline & $55-1 A$ & $T$ & 2 & 2 & $6.5 \mathrm{E}-01$ & $6.5 \mathrm{E}-01$ & No & $N / A$ & $N / A$ & $N / A$ & N/A \\
\hline & GW-191 & $T$ & 2 & 2 & $1.2 \mathrm{E}+00$ & $1.2 \mathrm{E}+00$ & No & $\mathrm{N} / \mathrm{A}$ & $\mathrm{N} / \mathrm{A}$ & $N / A$ & N/A \\
\hline & GW-631 & $\mathrm{T}$ & 2 & 2 & $2.9 \mathrm{E}+00$ & $2.9 \mathrm{E}+00$ & No & $\mathrm{N} / \mathrm{A}$ & N/A & $\mathrm{N} / \mathrm{A}$ & N/A \\
\hline & GW-272 & $\mathrm{T}$ & 2 & 2 & $1.6 \mathrm{E}+01$ & $1.6 \mathrm{E}+01$ & No & $\mathrm{N} / \mathrm{A}$ & $\mathrm{N} / \mathrm{A}$ & $N / A$ & $N / A$ \\
\hline & GW-273 & $\mathrm{T}$ & 1 & 1 & $1.1 \mathrm{E}+00$ & $1.1 \mathrm{E}+00$ & No & N/A & $N / A$ & $N / A$ & $N / A$ \\
\hline & GW-190 & $\mathrm{T}$ & 2 & 2 & $5.2 \mathrm{E}-01$ & 5.2E-01 & No & N/A & N/A & $\mathrm{N} / \mathrm{A}$ & N/A \\
\hline \multicolumn{12}{|c|}{ GROSS BETA ACTIVITY } \\
\hline & GW-274 & $\mathrm{S}$ & 5 & 5 & $7.2 \mathrm{E}+03$ & $7.8 \mathrm{E}+03$ & No & NT & NT & $\mathrm{N} / \mathrm{A}$ & N/A \\
\hline & GW-109 & s & 5 & 5 & $1.2 \mathrm{E}+04$ & $1.1 \mathrm{E}+04$ & No & NT & 1 & $N / A$ & N/A \\
\hline & GW-275 & S & 4 & 4 & $3.5 \mathrm{E}+02$ & $4.0 \mathrm{E}+02$ & No & $\mathrm{s}$ & s & $N / A$ & N/A \\
\hline & GW-108 & S & 18 & 18 & $1.0 \mathrm{E}+04$ & $1.0 \mathrm{E}+04$ & No & 1 & 1 & $N / A$ & N/A \\
\hline & GW-633 & s & 9 & 9 & $3.3 \mathrm{E}+03$ & $3.4 \mathrm{E}+03$ & No & s & I & $\mathrm{N} / \mathrm{A}$ & N/A \\
\hline & SPR14_0SP & $T$ & 1 & 1 & $2.6 \mathrm{E}+00$ & $2.6 \mathrm{E}+00$ & No & N/A & N/A & $N / A$ & N/A \\
\hline & GW-619 & $T$ & 3 & 3 & $1.2 \mathrm{E}+01$ & $9.4 \mathrm{E}+00$ & No & $N / A$ & $N / A$ & $N / A$ & N/A \\
\hline & $55-2 B$ & $\mathrm{~T}$ & 5 & 5 & $1.2 \mathrm{E}+01$ & $5.6 \mathrm{E}+00$ & No & NT & I & $N / A$ & $N / A$ \\
\hline & GW-269 & $\mathrm{T}$ & 2 & 2 & $1.2 \mathrm{E}+01$ & $1.2 \mathrm{E}+01$ & No & N/A & $N / A$ & $N / A$ & $N / A$ \\
\hline & GW-332 & $\mathrm{T}$ & 1 & 1 & $3.9 \mathrm{E}+00$ & $3.9 \mathrm{E}+00$ & No & N/A & N/A & $N / A$ & $N / A$ \\
\hline & GW-192 & $\mathrm{T}$ & 11 & 11 & $4.3 \mathrm{E}+00$ & $3.8 \mathrm{E}+00$ & No & $\mathrm{s}$ & NT & $N / A$ & $\mathrm{~N} / \mathrm{A}$ \\
\hline & $55-1 C$ & $T$ & 1 & 1 & $4.5 \mathrm{E}+00$ & $4.5 \mathrm{E}+00$ & No & N/A & N/A & $N / A$ & $N / A$ \\
\hline & GW-620 & $\mathrm{T}$ & 17 & 17 & $1.1 \mathrm{E}+01$ & $1.2 \mathrm{E}+01$ & No & NT & NT & $N / A$ & $N / A$ \\
\hline & GW-336 & $T$ & 2 & 2 & $2.3 \mathrm{E}+00$ & $2.3 \mathrm{E}+00$ & No & N/A & $\mathrm{N} / \mathrm{A}$ & $N / A$ & $\mathrm{~N} / \mathrm{A}$ \\
\hline & GW-270 & $T$ & 2 & 2 & $1.1 \mathrm{E}+01$ & $1.1 \mathrm{E}+01$ & No & $\mathrm{N} / \mathrm{A}$ & $N / A$ & $N / A$ & N/A \\
\hline & GW-253 & $\mathrm{T}$ & 15 & 15 & $3.0 \mathrm{E}+01$ & $2.9 \mathrm{E}+01$ & No & NT & $\mathrm{D}$ & $N / A$ & $N / A$ \\
\hline & GW-618 & $T$ & 16 & 15 & $4.2 \mathrm{E}+00$ & $4.6 \mathrm{E}+00$ & No & NT & s & $N / A$ & $N / A$ \\
\hline & GW-271 & $\mathrm{T}$ & 2 & 2 & $2.5 \mathrm{E}+01$ & $2.5 \mathrm{E}+01$ & No & N/A & $N / A$ & $N / A$ & $N / A$ \\
\hline
\end{tabular}


Project: Y-12 National Security Complex

Location: East S-3
User Name:

State: Tennessee

\begin{tabular}{|c|c|c|c|c|c|c|c|c|c|c|c|}
\hline Constituent & Well & $\begin{array}{c}\text { Sourcel } \\
\text { Tail }\end{array}$ & $\begin{array}{c}\text { Number } \\
\text { of } \\
\text { Samples }\end{array}$ & $\begin{array}{c}\text { Number } \\
\text { of } \\
\text { Detects }\end{array}$ & $\begin{array}{c}\text { Average } \\
\text { (mg/L) }\end{array}$ & $\begin{array}{c}\text { Median } \\
(\mathrm{mg} / \mathrm{L})\end{array}$ & $\begin{array}{c}\text { All } \\
\text { Samples } \\
\text { "ND" ? }\end{array}$ & $\begin{array}{l}\text { Mann- } \\
\text { Kendall }\end{array}$ & $\begin{array}{c}\text { Linear } \\
\text { Regression }\end{array}$ & Modeling & Empirical \\
\hline \multicolumn{12}{|c|}{ GROSS BETA ACTIVITY } \\
\hline & GW-337 & $T$ & 5 & 5 & $4.7 \mathrm{E}+00$ & $3.5 \mathrm{E}+00$ & No & NT & $\mathrm{PI}$ & $\mathrm{N} / \mathrm{A}$ & N/A \\
\hline & GW-349 & $\mathrm{T}$ & 2 & 2 & $2.7 \mathrm{E}+00$ & $2.7 \mathrm{E}+00$ & No & N/A & $\mathrm{N} / \mathrm{A}$ & N/A & N/A \\
\hline & GW-505 & $\mathrm{T}$ & 3 & 3 & $1.4 \mathrm{E}+01$ & $1.7 \mathrm{E}+01$ & No & N/A & N/A & $N / A$ & N/A \\
\hline & GW-350 & $\mathrm{T}$ & 1 & 1 & $1.7 \mathrm{E}+00$ & $1.7 \mathrm{E}+00$ & No & N/A & N/A & N/A & N/A \\
\hline & $55-1 \mathrm{~A}$ & $\mathrm{~T}$ & 2 & 2 & $9.9 \mathrm{E}+00$ & $9.9 \mathrm{E}+00$ & No & N/A & N/A & N/A & N/A \\
\hline & GW-631 & $\mathrm{T}$ & 1 & 1 & $3.7 \mathrm{E}+00$ & $3.7 \mathrm{E}+00$ & No & $\mathrm{N} / \mathrm{A}$ & $\mathrm{N} / \mathrm{A}$ & $N / A$ & N/A \\
\hline & GW-191 & $\mathrm{T}$ & 3 & 3 & $6.5 \mathrm{E}+00$ & $5.7 \mathrm{E}+00$ & No & N/A & N/A & N/A & N/A \\
\hline & $55-2 C$ & $\mathrm{~T}$ & 8 & 8 & $1.5 \mathrm{E}+01$ & $1.1 \mathrm{E}+01$ & No & 1 & $\mathrm{PI}$ & $\mathrm{N} / \mathrm{A}$ & N/A \\
\hline & GW-194 & $T$ & 3 & 3 & $4.8 \mathrm{E}+00$ & $6.0 \mathrm{E}+00$ & No & $N / A$ & $N / A$ & $N / A$ & N/A \\
\hline & GW-190 & $\mathrm{T}$ & 1 & 1 & $2.8 \mathrm{E}-01$ & 2.8E-01 & No & $\mathrm{N} / \mathrm{A}$ & $\mathrm{N} / \mathrm{A}$ & $N / A$ & $N / A$ \\
\hline & GW-338 & $\mathrm{T}$ & 1 & 1 & $2.0 \mathrm{E}+00$ & $2.0 \mathrm{E}+00$ & No & $N / A$ & $N / A$ & $N / A$ & N/A \\
\hline & GW-617 & $\mathrm{T}$ & 2 & 2 & $2.9 \mathrm{E}+00$ & $2.9 \mathrm{E}+00$ & No & $\mathrm{N} / \mathrm{A}$ & $\mathrm{N} / \mathrm{A}$ & $N / A$ & $N / A$ \\
\hline & GW-251 & $\mathrm{T}$ & 18 & 18 & $6.9 \mathrm{E}+00$ & $5.6 \mathrm{E}+00$ & No & NT & NT & $N / A$ & $N / A$ \\
\hline & GW-195 & $\mathrm{T}$ & 3 & 3 & $1.5 \mathrm{E}+01$ & $1.0 \mathrm{E}+01$ & No & N/A & N/A & N/A & N/A \\
\hline \multicolumn{12}{|l|}{ NITRATE } \\
\hline & GW-108 & $\mathrm{S}$ & 5 & 5 & $1.3 \mathrm{E}+04$ & $1.1 \mathrm{E}+04$ & No & $\mathrm{s}$ & NT & $\mathrm{N} / \mathrm{A}$ & N/A \\
\hline & GW-275 & S & 4 & 4 & $7.2 \mathrm{E}+03$ & $7.2 \mathrm{E}+03$ & No & s & $\mathrm{D}$ & $N / A$ & $N / A$ \\
\hline & GW-633 & S & 7 & 7 & $1.6 \mathrm{E}+03$ & $1.5 \mathrm{E}+03$ & No & $\mathrm{D}$ & $\mathrm{D}$ & $N / A$ & N/A \\
\hline & GW-109 & S & 4 & 4 & $9.4 \mathrm{E}+03$ & $9.3 \mathrm{E}+03$ & No & NT & NT & $N / A$ & N/A \\
\hline & GW-274 & s & 4 & 4 & $3.8 \mathrm{E}+03$ & $3.5 \mathrm{E}+03$ & No & $\mathrm{D}$ & $\mathrm{D}$ & $\mathrm{N} / \mathrm{A}$ & N/A \\
\hline & GW-191 & $\mathrm{T}$ & 1 & 1 & 3.0E-02 & 3.0E-02 & No & N/A & N/A & $N / A$ & N/A \\
\hline & GW-631 & $T$ & 2 & 2 & $6.1 \mathrm{E}-01$ & 6.1E-01 & No & $N / A$ & $N / A$ & $N / A$ & N/A \\
\hline & GW-270 & $\mathrm{T}$ & 2 & 2 & $7.3 \mathrm{E}+01$ & $7.3 \mathrm{E}+01$ & No & $\mathrm{N} / \mathrm{A}$ & $\mathrm{N} / \mathrm{A}$ & $N / A$ & $N / A$ \\
\hline & GW-269 & $\mathrm{T}$ & 2 & 2 & $5.3 \mathrm{E}-01$ & 5.3E-01 & No & N/A & $N / A$ & $N / A$ & $N / A$ \\
\hline & GW-253 & $\mathrm{T}$ & 2 & 2 & $7.3 \mathrm{E}+02$ & $7.3 \mathrm{E}+02$ & No & N/A & N/A & $N / A$ & $N / A$ \\
\hline & $55-1 A$ & $\mathrm{~T}$ & 3 & 3 & $1.1 \mathrm{E}+01$ & $1.2 \mathrm{E}+01$ & No & $\mathrm{N} / \mathrm{A}$ & $N / A$ & $N / A$ & $\mathrm{~N} / \mathrm{A}$ \\
\hline & GW-251 & $T$ & 19 & 19 & $5.5 \mathrm{E}+01$ & $5.6 \mathrm{E}+01$ & No & PD & $\mathrm{s}$ & $N / A$ & $N / A$ \\
\hline & SPR14_0SP & $\mathrm{T}$ & 1 & 1 & 3.2E-02 & $3.2 \mathrm{E}-02$ & No & N/A & N/A & $N / A$ & $N / A$ \\
\hline & GW-194 & $T$ & 1 & 1 & 3.0E-02 & 3.0E-02 & No & N/A & $\mathrm{N} / \mathrm{A}$ & $N / A$ & $\mathrm{~N} / \mathrm{A}$ \\
\hline & GW-195 & $T$ & 2 & 2 & $2.2 \mathrm{E}-01$ & $2.2 \mathrm{E}-01$ & No & $\mathrm{N} / \mathrm{A}$ & $\mathrm{N} / \mathrm{A}$ & $N / A$ & $N / A$ \\
\hline & GW-192 & $\mathrm{T}$ & 4 & 4 & 4.5E-02 & $3.9 \mathrm{E}-02$ & No & $\mathrm{s}$ & $\mathrm{s}$ & $N / A$ & $N / A$ \\
\hline & GW-332 & $\mathrm{T}$ & 1 & 1 & $1.8 \mathrm{E}+00$ & $1.8 \mathrm{E}+00$ & No & $\mathrm{N} / \mathrm{A}$ & $N / A$ & $N / A$ & $N / A$ \\
\hline & GW-505 & $\mathrm{T}$ & 2 & 2 & $1.4 \mathrm{E}-01$ & 1.4E-01 & No & N/A & $N / A$ & $N / A$ & $N / A$ \\
\hline
\end{tabular}


Project: Y-12 National Security Complex

Location: East S-3
User Name:

State: Tennessee

\begin{tabular}{|c|c|c|c|c|c|c|c|c|c|c|c|}
\hline Constituent & Well & $\begin{array}{c}\text { Sourcel } \\
\text { Tail }\end{array}$ & $\begin{array}{c}\text { Number } \\
\text { of } \\
\text { Samples }\end{array}$ & $\begin{array}{c}\text { Number } \\
\text { of } \\
\text { Detects }\end{array}$ & $\begin{array}{c}\text { Average } \\
\text { (mg/L) }\end{array}$ & $\begin{array}{c}\text { Median } \\
(\mathrm{mg} / \mathrm{L})\end{array}$ & $\begin{array}{c}\text { All } \\
\text { Samples } \\
\text { "ND" ? }\end{array}$ & $\begin{array}{l}\text { Mann- } \\
\text { Kendall }\end{array}$ & $\begin{array}{c}\text { Linear } \\
\text { Regression }\end{array}$ & Modeling & Empirical \\
\hline \multicolumn{12}{|l|}{ NITRATE } \\
\hline & GW-105 & $T$ & 2 & 2 & $5.5 \mathrm{E}+02$ & $5.5 \mathrm{E}+02$ & No & $\mathrm{N} / \mathrm{A}$ & N/A & $\mathrm{N} / \mathrm{A}$ & N/A \\
\hline & $55-2 C$ & $\mathrm{~T}$ & 8 & 8 & $1.5 \mathrm{E}+02$ & $1.4 \mathrm{E}+02$ & No & 1 & 1 & $\mathrm{~N} / \mathrm{A}$ & N/A \\
\hline & GW-106 & $T$ & 2 & 2 & $7.3 \mathrm{E}+02$ & $7.3 \mathrm{E}+02$ & No & $\mathrm{N} / \mathrm{A}$ & $N / A$ & $N / A$ & $N / A$ \\
\hline & GW-338 & $\mathrm{T}$ & 3 & 3 & $8.8 \mathrm{E}-01$ & 8.3E-01 & No & N/A & N/A & N/A & N/A \\
\hline & GW-617 & $\mathrm{T}$ & 5 & 5 & $1.5 \mathrm{E}+00$ & $2.1 \mathrm{E}+00$ & No & $\mathrm{D}$ & PD & $\mathrm{N} / \mathrm{A}$ & N/A \\
\hline & GW-336 & $\mathrm{T}$ & 2 & 2 & $1.7 \mathrm{E}+00$ & $1.7 \mathrm{E}+00$ & No & $N / A$ & $N / A$ & $N / A$ & $N / A$ \\
\hline & GW-618 & $\mathrm{T}$ & 8 & 8 & $1.1 \mathrm{E}+00$ & 9.3E-01 & No & $\mathrm{D}$ & D & $N / A$ & N/A \\
\hline & $55-2 B$ & $\mathrm{~T}$ & 5 & 5 & $1.7 \mathrm{E}+02$ & $1.5 \mathrm{E}+02$ & No & 1 & I & $\mathrm{N} / \mathrm{A}$ & N/A \\
\hline & GW-272 & $T$ & 2 & 2 & $3.8 \mathrm{E}+02$ & $3.8 \mathrm{E}+02$ & No & $N / A$ & $N / A$ & $N / A$ & N/A \\
\hline & GW-190 & $\mathrm{T}$ & 2 & 2 & $5.5 \mathrm{E}-02$ & 5.5E-02 & No & $\mathrm{N} / \mathrm{A}$ & $\mathrm{N} / \mathrm{A}$ & $N / A$ & $N / A$ \\
\hline & GW-619 & $T$ & 3 & 3 & $9.8 \mathrm{E}-01$ & 8.3E-01 & No & $\mathrm{N} / \mathrm{A}$ & N/A & $\mathrm{N} / \mathrm{A}$ & N/A \\
\hline & $55-1 C$ & $\mathrm{~T}$ & 1 & 1 & $2.9 \mathrm{E}+00$ & $2.9 \mathrm{E}+00$ & No & $\mathrm{N} / \mathrm{A}$ & $\mathrm{N} / \mathrm{A}$ & $N / A$ & $N / A$ \\
\hline & GW-620 & $\mathrm{T}$ & 19 & 19 & $1.7 \mathrm{E}+00$ & $1.3 \mathrm{E}+00$ & No & D & D & N/A & N/A \\
\hline \multicolumn{12}{|c|}{ TETRACHLOROETHYLENE(PCE) } \\
\hline & GW-275 & $\mathrm{s}$ & 4 & 2 & $2.5 \mathrm{E}-03$ & $5.0 \mathrm{E}-04$ & No & NT & NT & $\mathrm{N} / \mathrm{A}$ & $\mathrm{N} / \mathrm{A}$ \\
\hline & GW-633 & S & 8 & 8 & 2.0E-01 & 1.9E-01 & No & NT & NT & $N / A$ & N/A \\
\hline & GW-274 & S & 4 & 4 & $8.6 \mathrm{E}-01$ & 8.8E-01 & No & 1 & 1 & $N / A$ & $N / A$ \\
\hline & GW-109 & S & 5 & 5 & $1.6 \mathrm{E}-01$ & 1.6E-01 & No & s & s & $N / A$ & N/A \\
\hline & GW-108 & S & 17 & 11 & $5.9 \mathrm{E}-04$ & 5.0E-04 & No & 1 & 1 & $N / A$ & N/A \\
\hline & GW-192 & $T$ & 13 & 8 & $1.2 \mathrm{E}-03$ & $1.0 \mathrm{E}-03$ & No & NT & NT & $N / A$ & N/A \\
\hline & GW-105 & $T$ & 2 & 0 & $6.0 \mathrm{E}-06$ & $6.0 \mathrm{E}-06$ & Yes & N/A & N/A & $N / A$ & N/A \\
\hline & $55-1 \mathrm{~A}$ & $T$ & 3 & 0 & $6.0 \mathrm{E}-06$ & 6.0E-06 & Yes & $N / A$ & $N / A$ & $N / A$ & N/A \\
\hline & GW-106 & $\mathrm{T}$ & 2 & 0 & $6.0 \mathrm{E}-06$ & $6.0 \mathrm{E}-06$ & Yes & $\mathrm{N} / \mathrm{A}$ & $\mathrm{N} / \mathrm{A}$ & $N / A$ & $N / A$ \\
\hline & $55-2 B$ & $\mathrm{~T}$ & 5 & 5 & $5.7 \mathrm{E}-01$ & 5.5E-01 & No & NT & 1 & $N / A$ & $N / A$ \\
\hline & GW-194 & $\mathrm{T}$ & 3 & 0 & $6.0 \mathrm{E}-06$ & $6.0 \mathrm{E}-06$ & Yes & N/A & N/A & $N / A$ & $N / A$ \\
\hline & $55-1 C$ & $T$ & 1 & 0 & $6.0 \mathrm{E}-06$ & $6.0 \mathrm{E}-06$ & Yes & $\mathrm{N} / \mathrm{A}$ & $\mathrm{N} / \mathrm{A}$ & $\mathrm{N} / \mathrm{A}$ & N/A \\
\hline & GW-190 & $T$ & 2 & 0 & $6.0 \mathrm{E}-06$ & $6.0 \mathrm{E}-06$ & Yes & N/A & N/A & $N / A$ & $N / A$ \\
\hline & GW-191 & $\mathrm{T}$ & 3 & 0 & $6.0 \mathrm{E}-06$ & $6.0 \mathrm{E}-06$ & Yes & N/A & N/A & $N / A$ & $N / A$ \\
\hline & $55-2 C$ & $T$ & 8 & 8 & $4.8 \mathrm{E}-01$ & 5.1E-01 & No & $\mathrm{PD}$ & $\mathrm{s}$ & $N / A$ & $\mathrm{~N} / \mathrm{A}$ \\
\hline & GW-619 & $T$ & 5 & 5 & 3.6E-02 & 3.3E-02 & No & $\mathrm{s}$ & $P D$ & $N / A$ & $N / A$ \\
\hline & GW-349 & $T$ & 2 & 0 & $6.0 \mathrm{E}-06$ & $6.0 \mathrm{E}-06$ & Yes & $\mathrm{N} / \mathrm{A}$ & N/A & $\mathrm{N} / \mathrm{A}$ & N/A \\
\hline & GW-505 & $\mathrm{T}$ & 2 & 0 & $6.0 \mathrm{E}-06$ & $6.0 \mathrm{E}-06$ & Yes & $\mathrm{N} / \mathrm{A}$ & $N / A$ & $N / A$ & $N / A$ \\
\hline & GW-338 & $\mathrm{T}$ & 4 & 0 & $6.0 \mathrm{E}-06$ & $6.0 \mathrm{E}-06$ & Yes & $\mathrm{s}$ & $\mathrm{s}$ & $N / A$ & $N / A$ \\
\hline
\end{tabular}


Project: Y-12 National Security Complex

Location: East S-3
User Name:

State: Tennessee

\begin{tabular}{|c|c|c|c|c|c|c|c|c|c|c|c|}
\hline Constituent & Well & $\begin{array}{c}\text { Sourcel } \\
\text { Tail }\end{array}$ & $\begin{array}{l}\text { Number } \\
\text { of } \\
\text { Samples }\end{array}$ & $\begin{array}{c}\text { Number } \\
\text { of } \\
\text { Detects }\end{array}$ & $\begin{array}{c}\text { Average } \\
\text { (mg/L) }\end{array}$ & $\begin{array}{r}\text { Median } \\
(\mathrm{mg} / \mathrm{L})\end{array}$ & $\begin{array}{c}\text { All } \\
\text { Samples } \\
\text { "ND" ? }\end{array}$ & $\begin{array}{l}\text { Mann- } \\
\text { Kendall }\end{array}$ & $\begin{array}{c}\text { Linear } \\
\text { Regression }\end{array}$ & Modeling & Empirical \\
\hline \multicolumn{12}{|c|}{ TETRACHLOROETHYLENE(PCE) } \\
\hline & GW-617 & $T$ & 5 & 1 & $7.0 \mathrm{E}-04$ & $6.0 \mathrm{E}-06$ & No & NT & NT & $\mathrm{N} / \mathrm{A}$ & N/A \\
\hline & GW-336 & $\mathrm{T}$ & 2 & 2 & $4.8 \mathrm{E}-01$ & 4.8E-01 & No & N/A & N/A & N/A & N/A \\
\hline & GW-618 & $\mathrm{T}$ & 17 & 17 & 7.1E-03 & $5.0 \mathrm{E}-03$ & No & $\mathrm{s}$ & $\mathrm{s}$ & $N / A$ & N/A \\
\hline & GW-332 & $\mathrm{T}$ & 3 & 3 & $1.0 \mathrm{E}+00$ & 9.3E-01 & No & N/A & N/A & $\mathrm{N} / \mathrm{A}$ & N/A \\
\hline & GW-350 & $\mathrm{T}$ & 2 & 0 & $6.0 \mathrm{E}-06$ & $6.0 \mathrm{E}-06$ & Yes & N/A & N/A & N/A & N/A \\
\hline & GW-273 & $\mathrm{T}$ & 2 & 0 & $6.0 \mathrm{E}-06$ & $6.0 \mathrm{E}-06$ & Yes & $N / A$ & $N / A$ & $N / A$ & $N / A$ \\
\hline & GW-337 & $T$ & 6 & 6 & 7.3E-01 & 7.1E-01 & No & NT & NT & $N / A$ & N/A \\
\hline & GW-272 & $\mathrm{T}$ & 2 & 0 & $6.0 \mathrm{E}-06$ & $6.0 \mathrm{E}-06$ & Yes & $\mathrm{N} / \mathrm{A}$ & N/A & $\mathrm{N} / \mathrm{A}$ & N/A \\
\hline & GW-271 & $T$ & 2 & 0 & $6.0 \mathrm{E}-06$ & $6.0 \mathrm{E}-06$ & Yes & $N / A$ & $N / A$ & $N / A$ & N/A \\
\hline & GW-253 & $\mathrm{T}$ & 14 & 13 & $5.6 \mathrm{E}-01$ & 5.8E-01 & No & NT & s & $N / A$ & N/A \\
\hline & GW-195 & $\mathrm{T}$ & 3 & 0 & $6.0 \mathrm{E}-06$ & $6.0 \mathrm{E}-06$ & Yes & $\mathrm{N} / \mathrm{A}$ & N/A & $\mathrm{N} / \mathrm{A}$ & N/A \\
\hline & SPR14_0SP & $\mathrm{T}$ & 1 & 0 & $6.0 \mathrm{E}-06$ & $6.0 \mathrm{E}-06$ & Yes & $\mathrm{N} / \mathrm{A}$ & $\mathrm{N} / \mathrm{A}$ & $N / A$ & $N / A$ \\
\hline & GW-270 & $\mathrm{T}$ & 2 & 0 & $6.0 \mathrm{E}-06$ & 6.0E-06 & Yes & N/A & $N / A$ & $N / A$ & $N / A$ \\
\hline & GW-251 & $\mathrm{T}$ & 19 & 19 & $2.0 \mathrm{E}-01$ & 1.6E-01 & No & s & s & $\mathrm{N} / \mathrm{A}$ & N/A \\
\hline & GW-631 & $\mathrm{T}$ & 2 & 0 & $6.0 \mathrm{E}-06$ & $6.0 \mathrm{E}-06$ & Yes & $\mathrm{N} / \mathrm{A}$ & $N / A$ & $N / A$ & $\mathrm{~N} / \mathrm{A}$ \\
\hline & GW-269 & $T$ & 2 & 2 & 3.3E-02 & 3.3E-02 & No & N/A & $\mathrm{N} / \mathrm{A}$ & $N / A$ & N/A \\
\hline & GW-620 & $T$ & 20 & 20 & $5.1 \mathrm{E}-02$ & $1.6 \mathrm{E}-02$ & No & $\mathrm{D}$ & D & N/A & N/A \\
\hline \multicolumn{12}{|c|}{ TRICHLOROETHYLENE (TCE) } \\
\hline & GW-633 & $\mathrm{S}$ & 8 & 8 & $7.1 \mathrm{E}-03$ & $8.0 \mathrm{E}-03$ & No & PD & PD & $\mathrm{N} / \mathrm{A}$ & N/A \\
\hline & GW-109 & s & 5 & 5 & 3.3E-03 & $2.0 \mathrm{E}-03$ & No & $\mathrm{D}$ & $\mathrm{D}$ & $N / A$ & N/A \\
\hline & GW-108 & s & 17 & 16 & $1.4 \mathrm{E}-03$ & $1.5 \mathrm{E}-03$ & No & 1 & 1 & $\mathrm{~N} / \mathrm{A}$ & N/A \\
\hline & GW-275 & s & 4 & 0 & $5.0 \mathrm{E}-06$ & $5.0 \mathrm{E}-06$ & Yes & s & s & $N / A$ & N/A \\
\hline & GW-274 & s & 4 & 4 & $1.1 \mathrm{E}-02$ & $1.1 \mathrm{E}-02$ & No & s & I & $N / A$ & N/A \\
\hline & $55-1 \mathrm{~A}$ & $\mathrm{~T}$ & 3 & 0 & $5.0 \mathrm{E}-06$ & $5.0 \mathrm{E}-06$ & Yes & N/A & $N / A$ & $N / A$ & $N / A$ \\
\hline & GW-617 & $\mathrm{T}$ & 5 & 1 & $6.0 \mathrm{E}-04$ & 5.0E-06 & No & NT & NT & $N / A$ & $N / A$ \\
\hline & $55-2 B$ & $T$ & 5 & 5 & 2.6E-01 & $2.5 \mathrm{E}-01$ & No & $P D$ & $\mathrm{D}$ & $N / A$ & N/A \\
\hline & GW-618 & $T$ & 17 & 17 & $1.2 \mathrm{E}-02$ & 1.1E-02 & No & s & $\mathrm{s}$ & $N / A$ & $N / A$ \\
\hline & GW-505 & $T$ & 2 & 0 & $5.0 \mathrm{E}-06$ & $5.0 \mathrm{E}-06$ & Yes & $\mathrm{N} / \mathrm{A}$ & N/A & $\mathrm{N} / \mathrm{A}$ & N/A \\
\hline & GW-620 & $T$ & 20 & 17 & 1.6E-02 & 7.0E-03 & No & $\mathrm{D}$ & $\mathrm{D}$ & $N / A$ & $\mathrm{~N} / \mathrm{A}$ \\
\hline & $55-1 C$ & $T$ & 1 & 0 & $5.0 \mathrm{E}-06$ & $5.0 \mathrm{E}-06$ & Yes & $\mathrm{N} / \mathrm{A}$ & $\mathrm{N} / \mathrm{A}$ & $N / A$ & $N / A$ \\
\hline & GW-350 & $T$ & 2 & 1 & $5.0 \mathrm{E}-04$ & $5.0 \mathrm{E}-04$ & No & $\mathrm{N} / \mathrm{A}$ & N/A & $\mathrm{N} / \mathrm{A}$ & N/A \\
\hline & GW-619 & $T$ & 5 & 5 & $8.5 \mathrm{E}-03$ & $9.0 \mathrm{E}-03$ & No & $\mathrm{s}$ & s & $N / A$ & $N / A$ \\
\hline & $55-2 C$ & $\mathrm{~T}$ & 8 & 8 & $2.7 \mathrm{E}-01$ & $2.8 \mathrm{E}-01$ & No & $\mathrm{PD}$ & s & $N / A$ & $N / A$ \\
\hline
\end{tabular}


Project: Y-12 National Security Complex

Location: East S-3
User Name:

State: Tennessee

\begin{tabular}{|c|c|c|c|c|c|c|c|c|c|c|c|}
\hline Constituent & Well & $\begin{array}{c}\text { Sourcel } \\
\text { Tail }\end{array}$ & $\begin{array}{c}\text { Number } \\
\text { of } \\
\text { Samples }\end{array}$ & $\begin{array}{c}\text { Number } \\
\text { of } \\
\text { Detects }\end{array}$ & $\begin{array}{c}\text { Average } \\
\text { (mg/L) }\end{array}$ & $\begin{array}{r}\text { Median } \\
(\mathrm{mg} / \mathrm{L})\end{array}$ & $\begin{array}{c}\text { All } \\
\text { Samples } \\
\text { "ND" ? }\end{array}$ & $\begin{array}{l}\text { Mann- } \\
\text { Kendall }\end{array}$ & $\begin{array}{l}\text { Linear } \\
\text { Regression }\end{array}$ & Modeling & Empirical \\
\hline \multicolumn{12}{|c|}{ TRICHLOROETHYLENE (TCE) } \\
\hline & GW-631 & $T$ & 2 & 0 & 5.0E-06 & $5.0 \mathrm{E}-06$ & Yes & $\mathrm{N} / \mathrm{A}$ & N/A & N/A & N/A \\
\hline & GW-272 & $\mathrm{T}$ & 2 & 0 & $5.0 \mathrm{E}-06$ & $5.0 \mathrm{E}-06$ & Yes & N/A & N/A & N/A & N/A \\
\hline & GW-195 & $\mathrm{T}$ & 3 & 0 & $5.0 \mathrm{E}-06$ & 5.0E-06 & Yes & $\mathrm{N} / \mathrm{A}$ & N/A & N/A & $N / A$ \\
\hline & GW-192 & $\mathrm{T}$ & 13 & 11 & $2.8 \mathrm{E}-03$ & $1.5 \mathrm{E}-03$ & No & NT & NT & N/A & N/A \\
\hline & GW-251 & $\mathrm{T}$ & 19 & 19 & $8.8 \mathrm{E}-02$ & $7.6 \mathrm{E}-02$ & No & s & s & N/A & N/A \\
\hline & GW-253 & $\mathrm{T}$ & 14 & 13 & $5.0 \mathrm{E}-01$ & $5.0 \mathrm{E}-01$ & No & NT & s & $\mathrm{N} / \mathrm{A}$ & $N / A$ \\
\hline & GW-191 & $\mathrm{T}$ & 3 & 0 & $5.0 \mathrm{E}-06$ & 5.0E-06 & Yes & N/A & N/A & N/A & $N / A$ \\
\hline & GW-269 & $\mathrm{T}$ & 2 & 2 & 2.0E-03 & $2.0 \mathrm{E}-03$ & No & N/A & N/A & N/A & N/A \\
\hline & GW-270 & $\mathrm{T}$ & 2 & 0 & $5.0 \mathrm{E}-06$ & $5.0 \mathrm{E}-06$ & Yes & $\mathrm{N} / \mathrm{A}$ & N/A & $\mathrm{N} / \mathrm{A}$ & N/A \\
\hline & GW-106 & $\mathrm{T}$ & 2 & 0 & $5.0 \mathrm{E}-06$ & 5.0E-06 & Yes & $N / A$ & $N / A$ & N/A & $N / A$ \\
\hline & SPR14_0SP & $\mathrm{T}$ & 1 & 0 & $5.0 \mathrm{E}-06$ & $5.0 \mathrm{E}-06$ & Yes & $\mathrm{N} / \mathrm{A}$ & N/A & N/A & $N / A$ \\
\hline & GW-349 & $\mathrm{T}$ & 2 & 1 & $5.0 \mathrm{E}-04$ & $5.0 \mathrm{E}-04$ & No & $\mathrm{N} / \mathrm{A}$ & N/A & $\mathrm{N} / \mathrm{A}$ & $N / A$ \\
\hline & GW-273 & $\mathrm{T}$ & 2 & 0 & $5.0 \mathrm{E}-06$ & 5.0E-06 & Yes & N/A & $N / A$ & N/A & $N / A$ \\
\hline & GW-332 & $\mathrm{T}$ & 3 & 3 & 2.6E-01 & $2.6 \mathrm{E}-01$ & No & N/A & N/A & N/A & N/A \\
\hline & GW-194 & $\mathrm{T}$ & 3 & 0 & $5.0 \mathrm{E}-06$ & $5.0 \mathrm{E}-06$ & Yes & $\mathrm{N} / \mathrm{A}$ & $\mathrm{N} / \mathrm{A}$ & $\mathrm{N} / \mathrm{A}$ & $N / A$ \\
\hline & GW-336 & $\mathrm{T}$ & 2 & 2 & $4.9 \mathrm{E}-01$ & 4.9E-01 & No & $\mathrm{N} / \mathrm{A}$ & N/A & N/A & $N / A$ \\
\hline & GW-271 & $\mathrm{T}$ & 2 & 0 & $5.0 \mathrm{E}-06$ & $5.0 \mathrm{E}-06$ & Yes & N/A & N/A & N/A & N/A \\
\hline & GW-337 & $\mathrm{T}$ & 6 & 6 & $8.1 \mathrm{E}-01$ & $7.8 \mathrm{E}-01$ & No & NT & $\mathrm{D}$ & $\mathrm{N} / \mathrm{A}$ & $N / A$ \\
\hline & GW-338 & $\mathrm{T}$ & 4 & 0 & $5.0 \mathrm{E}-06$ & 5.0E-06 & Yes & $\mathrm{s}$ & s & N/A & $N / A$ \\
\hline & GW-105 & $\mathrm{T}$ & 2 & 0 & $5.0 \mathrm{E}-06$ & $5.0 \mathrm{E}-06$ & Yes & $\mathrm{N} / \mathrm{A}$ & N/A & N/A & $N / A$ \\
\hline & GW-190 & $\mathrm{T}$ & 2 & 0 & $5.0 \mathrm{E}-06$ & 5.0E-06 & Yes & $\mathrm{N} / \mathrm{A}$ & N/A & N/A & $\mathrm{N} / \mathrm{A}$ \\
\hline
\end{tabular}

Note: Increasing (I); Probably Increasing (PI); Stable (S); Probably Decreasing (PD); Decreasing (D); No Trend (NT); Not Applicable (N/A) - Due to insufficient Data (< 4 sampling events); Source/Tail (S/T)

The Number of Samples and Number of Detects shown above are post-consolidation values. 


\section{MAROS Spatial Moment Analysis Summary}

Project: Y-12 National Security Complex

Location: East S-3
User Name:

State: Tennessee

2nd Moment (Spread)

Oth Moment

$\underline{1 \text { st Moment (Center of Mass) }}$

Estimated

Effective Date

Mass (Kg)
Source

Distance (ft) (sq ft)
53,554

54,007

53,676

10/1/1996

$1 / 1 / 1997$

$4 / 1 / 1997$

7/1/1997

10/1/1997

$1 / 1 / 1998$

$4 / 1 / 1998$

$7 / 1 / 1998$

10/1/1998

1/1/1999

4/1/1999

$7 / 1 / 1999$

10/1/1999

$1 / 1 / 2000$

$4 / 1 / 2000$

$7 / 1 / 2000$

$10 / 1 / 2000$

$1 / 1 / 2001$

$4 / 1 / 2001$

$7 / 1 / 2001$

10/1/2001

$1 / 1 / 2002$

$4 / 1 / 2002$

$7 / 1 / 2002$

10/1/2002

$1 / 1 / 2003$

4/1/2003

$7 / 1 / 2003$

$10 / 1 / 2003$

$1 / 1 / 2004$

$4 / 1 / 2004$

7/1/2004

$10 / 1 / 2004$
$6.2 \mathrm{E}+03$

$9.6 \mathrm{E}+02$

$2.5 \mathrm{E}+03$

$0.0 \mathrm{E}+00$

$0.0 \mathrm{E}+00$

$0.0 \mathrm{E}+00$

$0.0 \mathrm{E}+00$

$0.0 \mathrm{E}+00$

2.3E+02

$0.0 \mathrm{E}+00$

$0.0 \mathrm{E}+00$

$0.0 \mathrm{E}+00$

$0.0 \mathrm{E}+00$

$0.0 \mathrm{E}+00$

$0.0 \mathrm{E}+00$

$0.0 \mathrm{E}+00$

$2.7 \mathrm{E}+03$

$0.0 \mathrm{E}+00$

$1.1 \mathrm{E}+03$

$0.0 \mathrm{E}+00$

$0.0 \mathrm{E}+00$

$0.0 \mathrm{E}+00$

$0.0 \mathrm{E}+00$

$0.0 \mathrm{E}+00$

$4.9 \mathrm{E}+02$

$0.0 \mathrm{E}+00$

$0.0 \mathrm{E}+00$

$0.0 \mathrm{E}+00$

2. $2 \mathrm{E}+03$

$0.0 \mathrm{E}+00$

$6.7 \mathrm{E}+03$

$0.0 \mathrm{E}+00$

$0.0 \mathrm{E}+00$

$0.0 \mathrm{E}+00$

$0.0 \mathrm{E}+00$
54,106

53,744

53,695

53,784

53,627

53,393

\section{0,010}

29,914

29,974

29,850

926

81,187

29,939

29,841

553

125,717

141,675

117,132

675

424

203

104,821

\section{3}

815

479

99,704

152,811

75,649

(sq ft)
Number of

Wells
49,660

43,447

34,085

45,824

43,477

18,180

17,041
14

11

7

2

4

5

2

7

3

3

2

4

3

4

1

11

1

10

4

1

4

1

7

1

5

1

20

1

17

2

3

1

2

GROSS BETA ACTIVITY

$\begin{array}{lrrrrrrr}1 / 1 / 1996 & 3.1 \mathrm{E}+04 & 53,581 & 30,010 & 379 & 98,437 & 45,161 & 12 \\ 4 / 1 / 1996 & 1.8 \mathrm{E}+03 & 54,010 & 29,973 & 808 & 189,080 & 55,372 & 12\end{array}$


Project: Y-12 National Security Complex

Location: East S-3
User Name:

State: Tennessee

\begin{tabular}{|c|c|c|c|c|c|c|c|}
\hline \multirow[b]{2}{*}{ Effective Date } & \multirow{2}{*}{$\begin{array}{r}\text { 0th Moment } \\
\text { Estimated } \\
\text { Mass (kg) }\end{array}$} & \multicolumn{3}{|c|}{ 1st Moment (Center of Mass) } & \multicolumn{2}{|c|}{ 2nd Moment (Spread) } & \multirow[b]{2}{*}{$\begin{array}{c}\text { Number of } \\
\text { Wells }\end{array}$} \\
\hline & & $\mathrm{Xc}(\mathrm{ft})$ & Yc (ft) & $\begin{array}{c}\text { Source } \\
\text { Distance (ft) }\end{array}$ & $\begin{array}{c}\text { Sigma } X X \\
(s q \mathrm{ft})\end{array}$ & $\begin{array}{c}\text { Sigma YY } \\
(\mathrm{sq} f t)\end{array}$ & \\
\hline \multicolumn{8}{|l|}{ GROSS BETA ACTIVITY } \\
\hline 10/1/1996 & $5.1 \mathrm{E}+03$ & 53,584 & 29,942 & 398 & 56,835 & 50,616 & 10 \\
\hline $1 / 1 / 1997$ & $0.0 \mathrm{E}+00$ & & & & & & 2 \\
\hline $4 / 1 / 1997$ & $0.0 \mathrm{E}+00$ & & & & & & 5 \\
\hline $7 / 1 / 1997$ & $0.0 \mathrm{E}+00$ & & & & & & 1 \\
\hline 10/1/1997 & $0.0 \mathrm{E}+00$ & & & & & & 4 \\
\hline 1/1/1998 & $0.0 \mathrm{E}+00$ & & & & & & 2 \\
\hline $4 / 1 / 1998$ & $1.1 \mathrm{E}+03$ & 54,002 & 29,932 & 807 & 141,383 & 31,586 & 7 \\
\hline $7 / 1 / 1998$ & $0.0 \mathrm{E}+00$ & & & & & & 4 \\
\hline 10/1/1998 & $0.0 \mathrm{E}+00$ & & & & & & 4 \\
\hline 1/1/1999 & $0.0 \mathrm{E}+00$ & & & & & & 2 \\
\hline 4/1/1999 & $0.0 \mathrm{E}+00$ & & & & & & 4 \\
\hline $7 / 1 / 1999$ & $0.0 \mathrm{E}+00$ & & & & & & 4 \\
\hline 10/1/1999 & $0.0 \mathrm{E}+00$ & & & & & & 5 \\
\hline $1 / 1 / 2000$ & $0.0 \mathrm{E}+00$ & & & & & & 1 \\
\hline $4 / 1 / 2000$ & $6.4 \mathrm{E}+04$ & 53,463 & 30,025 & 259 & 43,319 & 29,157 & 11 \\
\hline $7 / 1 / 2000$ & $0.0 \mathrm{E}+00$ & & & & & & 1 \\
\hline $10 / 1 / 2000$ & $2.9 \mathrm{E}+04$ & 53,437 & 30,009 & 238 & 74,506 & 36,942 & 11 \\
\hline $1 / 1 / 2001$ & $0.0 \mathrm{E}+00$ & & & & & & 1 \\
\hline $4 / 1 / 2001$ & $0.0 \mathrm{E}+00$ & & & & & & 5 \\
\hline $7 / 1 / 2001$ & $0.0 \mathrm{E}+00$ & & & & & & 1 \\
\hline $10 / 1 / 2001$ & $0.0 \mathrm{E}+00$ & & & & & & 5 \\
\hline $1 / 1 / 2002$ & $0.0 \mathrm{E}+00$ & & & & & & 1 \\
\hline $4 / 1 / 2002$ & $2.3 \mathrm{E}+03$ & 53,589 & 29,832 & 450 & 110,900 & 9,061 & 7 \\
\hline $7 / 1 / 2002$ & $0.0 \mathrm{E}+00$ & & & & & & 1 \\
\hline $10 / 1 / 2002$ & $0.0 \mathrm{E}+00$ & & & & & & 5 \\
\hline $1 / 1 / 2003$ & $0.0 \mathrm{E}+00$ & & & & & & 1 \\
\hline $4 / 1 / 2003$ & $8.0 \mathrm{E}+04$ & 53,591 & 29,986 & 393 & 36,195 & 30,621 & 14 \\
\hline $7 / 1 / 2003$ & $0.0 \mathrm{E}+00$ & & & & & & 1 \\
\hline $10 / 1 / 2003$ & $7.3 \mathrm{E}+04$ & 53,482 & 29,948 & 301 & 37,184 & 26,325 & 16 \\
\hline $1 / 1 / 2004$ & $0.0 \mathrm{E}+00$ & & & & & & 2 \\
\hline $4 / 1 / 2004$ & $0.0 \mathrm{E}+00$ & & & & & & 5 \\
\hline $7 / 1 / 2004$ & $0.0 \mathrm{E}+00$ & & & & & & 1 \\
\hline $10 / 1 / 2004$ & $0.0 \mathrm{E}+00$ & & & & & & 4 \\
\hline \multicolumn{8}{|l|}{ NITRATE } \\
\hline 1/1/1996 & $3.0 \mathrm{E}+02$ & 53,996 & 29,756 & 849 & 93,367 & 1,584 & 6 \\
\hline 4/1/1996 & $1.2 \mathrm{E}+03$ & 54,198 & 29,817 & 1,023 & 176,803 & 40,514 & 12 \\
\hline 10/1/1996 & $6.1 \mathrm{E}+03$ & 53,527 & 29,861 & 382 & 56,472 & 29,381 & 10 \\
\hline 1/1/1997 & $0.0 \mathrm{E}+00$ & & & & & & 2 \\
\hline 4/1/1997 & $0.0 \mathrm{E}+00$ & & & & & & 4 \\
\hline 7/1/1997 & $0.0 \mathrm{E}+00$ & & & & & & 1 \\
\hline 10/1/1997 & $0.0 \mathrm{E}+00$ & & & & & & 3 \\
\hline 1/1/1998 & $0.0 \mathrm{E}+00$ & & & & & & 2 \\
\hline
\end{tabular}


Project: Y-12 National Security Complex

Location: East S-3
User Name:

State: Tennessee

\begin{tabular}{|c|c|c|c|c|c|c|c|}
\hline \multirow[b]{2}{*}{ Effective Date } & \multirow{2}{*}{$\begin{array}{l}\text { 0th Moment } \\
\begin{array}{r}\text { Estimated } \\
\text { Mass (kg) }\end{array}\end{array}$} & \multicolumn{3}{|c|}{ 1st Moment (Center of Mass) } & \multicolumn{2}{|c|}{ 2nd Moment (Spread) } & \multirow[b]{2}{*}{$\begin{array}{c}\text { Number of } \\
\text { Wells }\end{array}$} \\
\hline & & $X c(f t)$ & Yc (ft) & $\begin{array}{c}\text { Source } \\
\text { Distance (ft) }\end{array}$ & $\begin{array}{c}\text { Sigma } X X \\
(s q \mathrm{ft})\end{array}$ & $\begin{array}{c}\text { Sigma } Y Y \\
(s q \mathrm{ft})\end{array}$ & \\
\hline \multicolumn{8}{|l|}{ NITRATE } \\
\hline 4/1/1998 & $4.8 \mathrm{E}+02$ & 53,936 & 29,988 & 733 & 160,427 & 9,339 & 6 \\
\hline 7/1/1998 & $0.0 \mathrm{E}+00$ & & & & & & 4 \\
\hline 10/1/1998 & $0.0 \mathrm{E}+00$ & & & & & & 4 \\
\hline 4/1/1999 & $0.0 \mathrm{E}+00$ & & & & & & 3 \\
\hline 10/1/1999 & $0.0 \mathrm{E}+00$ & & & & & & 4 \\
\hline $4 / 1 / 2000$ & $8.7 \mathrm{E}+04$ & 53,734 & 29,934 & 544 & 145,402 & 9,791 & 7 \\
\hline $10 / 1 / 2000$ & $6.9 \mathrm{E}+04$ & 53,745 & 29,962 & 549 & 158,843 & 18,602 & 8 \\
\hline $4 / 1 / 2001$ & $0.0 \mathrm{E}+00$ & & & & & & 2 \\
\hline $10 / 1 / 2001$ & $0.0 \mathrm{E}+00$ & & & & & & 2 \\
\hline $4 / 1 / 2002$ & $0.0 \mathrm{E}+00$ & & & & & & 3 \\
\hline $10 / 1 / 2002$ & $0.0 \mathrm{E}+00$ & & & & & & 3 \\
\hline $4 / 1 / 2003$ & $6.0 \mathrm{E}+04$ & 53,574 & 30,009 & 372 & 72,950 & 33,644 & 16 \\
\hline $10 / 1 / 2003$ & $6.9 \mathrm{E}+04$ & 53,547 & 30,041 & 341 & 67,450 & 36,303 & 16 \\
\hline $1 / 1 / 2004$ & $0.0 \mathrm{E}+00$ & & & & & & 1 \\
\hline $4 / 1 / 2004$ & $0.0 \mathrm{E}+00$ & & & & & & 5 \\
\hline $10 / 1 / 2004$ & $0.0 \mathrm{E}+00$ & & & & & & 4 \\
\hline
\end{tabular}

TETRACHLOROETHYLENE(PCE)

\begin{tabular}{|c|c|c|c|c|c|c|c|}
\hline 1/1/1996 & 3.0E-01 & 53,738 & 29,862 & 570 & 76,401 & 2,106 & 10 \\
\hline 4/1/1996 & $9.7 \mathrm{E}-01$ & 53,758 & 29,657 & 689 & 88,725 & 33,432 & 15 \\
\hline 10/1/1996 & 1.8E-01 & 53,608 & 29,756 & 510 & 197,355 & 10,784 & 11 \\
\hline 1/1/1997 & $0.0 \mathrm{E}+00$ & & & & & & 2 \\
\hline 4/1/1997 & $0.0 \mathrm{E}+00$ & & & & & & 5 \\
\hline 7/1/1997 & $0.0 \mathrm{E}+00$ & & & & & & 1 \\
\hline 10/1/1997 & $0.0 \mathrm{E}+00$ & & & & & & 5 \\
\hline 1/1/1998 & $0.0 \mathrm{E}+00$ & & & & & & 2 \\
\hline 4/1/1998 & $8.9 \mathrm{E}+00$ & 54,300 & 30,000 & 1,095 & 242,035 & 54,364 & 9 \\
\hline 7/1/1998 & $2.0 \mathrm{E}+01$ & 54,572 & 29,843 & 1,383 & 26,621 & 480 & 9 \\
\hline 10/1/1998 & $0.0 \mathrm{E}+00$ & & & & & & 4 \\
\hline 1/1/1999 & $0.0 \mathrm{E}+00$ & & & & & & 2 \\
\hline 4/1/1999 & $0.0 \mathrm{E}+00$ & & & & & & 4 \\
\hline 7/1/1999 & $0.0 \mathrm{E}+00$ & & & & & & 4 \\
\hline 10/1/1999 & $0.0 \mathrm{E}+00$ & & & & & & 5 \\
\hline $1 / 1 / 2000$ & $0.0 \mathrm{E}+00$ & & & & & & 1 \\
\hline $4 / 1 / 2000$ & $3.3 \mathrm{E}+01$ & 53,946 & 29,898 & 759 & 217,086 & 54,068 & 11 \\
\hline $7 / 1 / 2000$ & $0.0 \mathrm{E}+00$ & & & & & & 1 \\
\hline $10 / 1 / 2000$ & $1.5 \mathrm{E}+01$ & 53,913 & 29,825 & 747 & 229,781 & 32,536 & 11 \\
\hline $1 / 1 / 2001$ & $0.0 \mathrm{E}+00$ & & & & & & 1 \\
\hline $4 / 1 / 2001$ & $0.0 \mathrm{E}+00$ & & & & & & 5 \\
\hline $7 / 1 / 2001$ & $0.0 \mathrm{E}+00$ & & & & & & 1 \\
\hline $10 / 1 / 2001$ & $0.0 \mathrm{E}+00$ & & & & & & 5 \\
\hline $1 / 1 / 2002$ & $0.0 \mathrm{E}+00$ & & & & & & 1 \\
\hline $4 / 1 / 2002$ & $6.2 \mathrm{E}-01$ & 53,623 & 29,574 & 647 & 52,463 & 20,013 & 7 \\
\hline
\end{tabular}


Project: Y-12 National Security Complex

Location: East S-3
User Name:

State: Tennessee

\begin{tabular}{|c|c|c|c|c|c|c|c|}
\hline \multirow[b]{2}{*}{ Effective Date } & \multirow{2}{*}{$\begin{array}{l}\text { Esth Moment } \\
\text { Mass (kg) }\end{array}$} & \multicolumn{3}{|c|}{ 1st Moment (Center of Mass) } & \multicolumn{2}{|c|}{ 2nd Moment (Spread) } & \multirow[b]{2}{*}{$\begin{array}{c}\text { Number of } \\
\text { Wells }\end{array}$} \\
\hline & & $X c(f t)$ & $Y c(f t)$ & $\begin{array}{c}\text { Source } \\
\text { Distance (ft) }\end{array}$ & $\begin{array}{c}\text { Sigma } X X \\
(\mathrm{sq} \mathrm{ft})\end{array}$ & $\begin{array}{c}\text { Sigma YY } \\
(\mathrm{sq} f t)\end{array}$ & \\
\hline \multicolumn{8}{|c|}{ TETRACHLOROETHYLENE(PCE) } \\
\hline $7 / 1 / 2002$ & $0.0 \mathrm{E}+00$ & & & & & & 1 \\
\hline $10 / 1 / 2002$ & $5.0 \mathrm{E}-01$ & 53,653 & 29,615 & 637 & 72,922 & 25,019 & 7 \\
\hline $1 / 1 / 2003$ & $0.0 \mathrm{E}+00$ & & & & & & 1 \\
\hline $4 / 1 / 2003$ & $2.5 \mathrm{E}+01$ & 54,019 & 29,893 & 831 & 196,369 & 44,513 & 21 \\
\hline $7 / 1 / 2003$ & $0.0 \mathrm{E}+00$ & & & & & & 1 \\
\hline $10 / 1 / 2003$ & $2.4 \mathrm{E}+01$ & 54,070 & 29,931 & 874 & 207,172 & 55,870 & 21 \\
\hline $1 / 1 / 2004$ & $0.0 \mathrm{E}+00$ & & & & & & 2 \\
\hline $4 / 1 / 2004$ & $0.0 \mathrm{E}+00$ & & & & & & 5 \\
\hline $7 / 1 / 2004$ & $0.0 \mathrm{E}+00$ & & & & & & 1 \\
\hline $10 / 1 / 2004$ & $0.0 \mathrm{E}+00$ & & & & & & 5 \\
\hline
\end{tabular}

TRICHLOROETHYLENE (TCE)

\begin{tabular}{|c|c|c|c|c|c|c|c|}
\hline 1/1/1996 & 1.5E-01 & 53,879 & 29,852 & 707 & 121,197 & 3,635 & 10 \\
\hline 4/1/1996 & 4.6E-01 & 53,837 & 29,678 & 742 & 119,742 & 32,070 & 15 \\
\hline 10/1/1996 & 1.1E-01 & 53,811 & 29,777 & 671 & 244,598 & 10,759 & 11 \\
\hline $1 / 1 / 1997$ & $0.0 \mathrm{E}+00$ & & & & & & 2 \\
\hline $4 / 1 / 1997$ & $0.0 \mathrm{E}+00$ & & & & & & 5 \\
\hline $7 / 1 / 1997$ & $0.0 \mathrm{E}+00$ & & & & & & 1 \\
\hline 10/1/1997 & $0.0 \mathrm{E}+00$ & & & & & & 5 \\
\hline 1/1/1998 & $0.0 \mathrm{E}+00$ & & & & & & 2 \\
\hline 4/1/1998 & $7.1 \mathrm{E}+00$ & 54,265 & 30,013 & 1,059 & 218,580 & 48,443 & 9 \\
\hline 7/1/1998 & $1.2 \mathrm{E}+01$ & 54,511 & 29,837 & 1,325 & 73,382 & 1,372 & 9 \\
\hline 10/1/1998 & $0.0 \mathrm{E}+00$ & & & & & & 4 \\
\hline 1/1/1999 & $0.0 \mathrm{E}+00$ & & & & & & 2 \\
\hline 4/1/1999 & $0.0 \mathrm{E}+00$ & & & & & & 4 \\
\hline 7/1/1999 & $0.0 \mathrm{E}+00$ & & & & & & 4 \\
\hline 10/1/1999 & $0.0 \mathrm{E}+00$ & & & & & & 5 \\
\hline $1 / 1 / 2000$ & $0.0 \mathrm{E}+00$ & & & & & & 1 \\
\hline $4 / 1 / 2000$ & $1.5 \mathrm{E}+01$ & 54,326 & 29,904 & 1,131 & 154,361 & 78,746 & 11 \\
\hline $7 / 1 / 2000$ & $0.0 \mathrm{E}+00$ & & & & & & 1 \\
\hline $10 / 1 / 2000$ & $9.8 \mathrm{E}+00$ & 54,405 & 29,915 & 1,208 & 93,663 & 75,707 & 11 \\
\hline $1 / 1 / 2001$ & $0.0 \mathrm{E}+00$ & & & & & & 1 \\
\hline 4/1/2001 & $0.0 \mathrm{E}+00$ & & & & & & 5 \\
\hline $7 / 1 / 2001$ & $0.0 \mathrm{E}+00$ & & & & & & 1 \\
\hline $10 / 1 / 2001$ & $0.0 \mathrm{E}+00$ & & & & & & 5 \\
\hline $1 / 1 / 2002$ & $0.0 \mathrm{E}+00$ & & & & & & 1 \\
\hline $4 / 1 / 2002$ & 4.2E-01 & 53,720 & 29,571 & 716 & 64,878 & 15,324 & 7 \\
\hline 7/1/2002 & $0.0 \mathrm{E}+00$ & & & & & & 1 \\
\hline $10 / 1 / 2002$ & $1.2 \mathrm{E}+00$ & 53,867 & 29,665 & 775 & 95,961 & 14,615 & 7 \\
\hline $1 / 1 / 2003$ & $0.0 \mathrm{E}+00$ & & & & & & 1 \\
\hline $4 / 1 / 2003$ & $1.1 \mathrm{E}+01$ & 54,347 & 29,839 & 1,163 & 77,143 & 49,349 & 21 \\
\hline $7 / 1 / 2003$ & $0.0 \mathrm{E}+00$ & & & & & & 1 \\
\hline $10 / 1 / 2003$ & $1.0 \mathrm{E}+01$ & 54,347 & 29,865 & 1,158 & 90,041 & 57,489 & 21 \\
\hline
\end{tabular}


Project: Y-12 National Security Complex

Location: East S-3

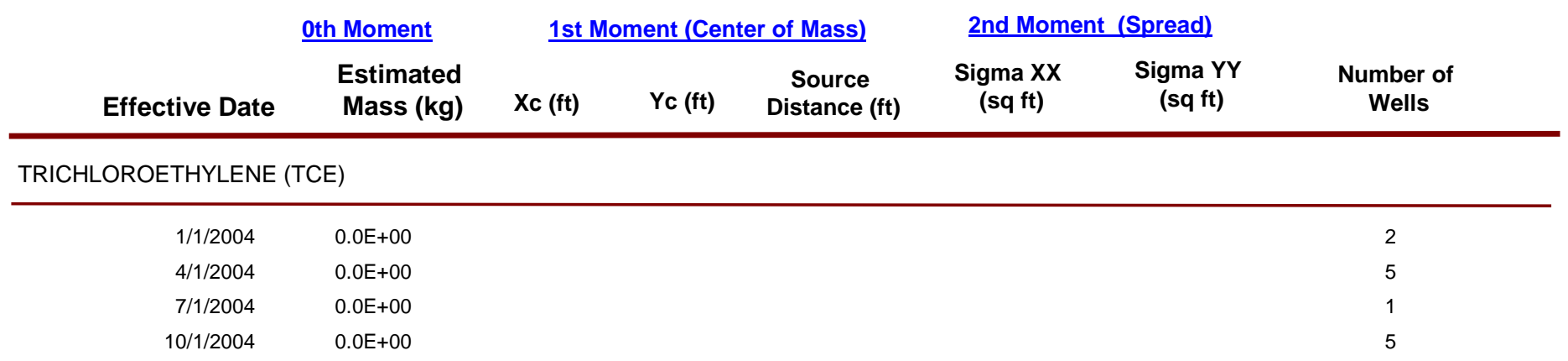

User Name:

State: Tennessee

2nd Moment (Spread) 
Project: Y-12 National Security Complex

Location: East S-3
User Name:

State: Tennessee

\begin{tabular}{|c|c|c|c|c|c|}
\hline Moment Type & Constituent & $\begin{array}{l}\text { Coefficient } \\
\text { of Variation }\end{array}$ & $\begin{array}{l}\text { Mann-Kendall } \\
\text { S Statistic }\end{array}$ & $\begin{array}{c}\text { Confidence } \\
\text { in Trend }\end{array}$ & $\begin{array}{c}\text { Moment } \\
\text { Trend }\end{array}$ \\
\hline
\end{tabular}

Zeroth Moment: Mass

GROSS ALPHA ACTIVITY
GROSS BETA ACTIVITY
NITRATE
TETRACHLOROETHYLENE(PCE)
TRICHLOROETHYLENE (TCE)

$\begin{array}{llll}2.45 & -50 & 75.6 \% & \text { NT } \\ 2.59 & -40 & 70.9 \% & \text { NT } \\ 2.24 & -16 & 64.4 \% & \text { NT } \\ 2.34 & -31 & 66.4 \% & \text { NT } \\ 2.20 & -31 & 66.4 \% & \text { NT }\end{array}$

1st Moment: Distance to Source

GROSS ALPHA ACTIVITY
GROSS BETA ACTIVITY
NITRATE
TETRACHLOROETHYLENE(PCE)
TRICHLOROETHYLENE (TCE)

$\begin{array}{lccc}0.41 & -8 & 76.2 \% & \mathrm{~S} \\ 0.48 & -10 & 82.1 \% & \mathrm{~S} \\ 0.41 & -18 & 98.4 \% & \mathrm{D} \\ 0.32 & 11 & 77.7 \% & \mathrm{NT} \\ 0.25 & 19 & 91.8 \% & \mathrm{PI}\end{array}$

2nd Moment: Sigma XX

GROSS ALPHA ACTIVITY
GROSS BETA ACTIVITY
NITRATE
TETRACHLOROETHYLENE(PCE)
TRICHLOROETHYLENE (TCE)

$\begin{array}{lccc}0.23 & 0 & 46.0 \% & \mathrm{~S} \\ 0.60 & -16 & 94.0 \% & \mathrm{PD} \\ 0.42 & -8 & 80.1 \% & \mathrm{~S} \\ 0.56 & 5 & 61.9 \% & \mathrm{NT} \\ 0.48 & -23 & 95.7 \% & \mathrm{D}\end{array}$

2nd Moment: Sigma YY

\begin{tabular}{lcccc} 
GROSS ALPHA ACTIVITY & 0.35 & -4 & $61.9 \%$ & $\mathrm{~S}$ \\
GROSS BETA ACTIVITY & 0.40 & -22 & $98.8 \%$ & $\mathrm{D}$ \\
NITRATE & 0.65 & 10 & $86.2 \%$ & NT \\
TETRACHLOROETHYLENE(PCE) & 0.68 & 17 & $89.1 \%$ & NT \\
TRICHLOROETHYLENE (TCE) & 0.80 & 17 & $89.1 \%$ & NT \\
\hline
\end{tabular}

Note: The following assumptions were applied for the calculation of the Zeroth Moment:

Porosity: $0.10 \quad$ Saturated Thickness: Uniform: $50 \mathrm{ft}$

Mann-Kendall Trend test performed on all sample events for each constituent. Increasing (I); Probably Increasing (PI); Stable (S); Probably Decreasing (PD); Decreasing (D); No Trend (NT); Not Applicable (N/A)-Due to insufficient Data (< 4 sampling events).

Note: The Sigma $X X$ and Sigma $Y Y$ components are estimated using the given field coordinate system and then rotated to align with the estimated groundwater flow direction. Moments are not calculated for sample events with less than 6 wells. 


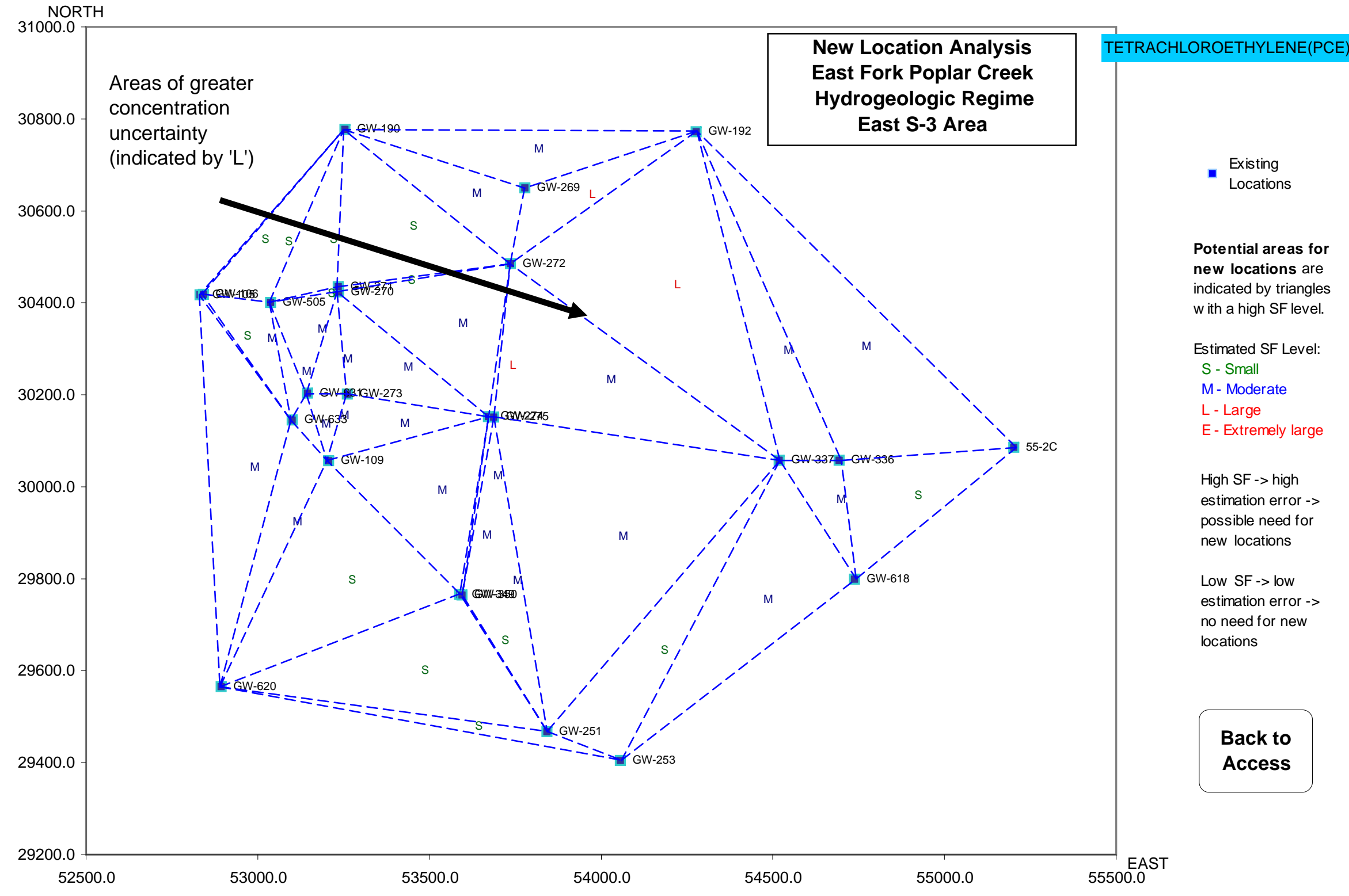




\section{ASSESSMENT OF THE GROUNDWATER PROTECTION PROGRAM \\ Y-12 NATIONAL SECURITY COMPLEX, OAK RIDGE, TENNESSEE}

Y-12 National Security Complex

Oak Ridge, Tennessee

\section{Appendix D.5 East Fork Regime Central Y-12 Area}

Table D.5.1 Qualitative Analysis East S-3 Area

Table D.5.2 Aquifer Input Parameters

MAROS Report COC Assessment

MAROS Report Plume Summary

MAROS Report Spatial Moment Analysis Summary

(No Well Sufficiency areas of interest identified) 
TABLE D.5.1

QUALITATIVE ANALYSIS EAST FORK REGIME CENTRAL Y-12 AREA

East Fork Poplar Creek Hydrogeologic Regime

-12 National Security Complex

Oak Ridge, Tennessee

\begin{tabular}{|c|c|c|c|c|c|c|c|c|c|c|c|c|}
\hline $\begin{array}{l}\text { Location } \\
\text { Name }\end{array}$ & \begin{tabular}{|c} 
Location \\
Type
\end{tabular} & $\begin{array}{c}\text { Average } \\
\text { Concentration } \\
\text { Exceeds Screening } \\
\text { Level } \\
\end{array}$ & $\begin{array}{c}\text { Formation } \\
\text { Type }\end{array}$ & $\begin{array}{c}\text { Horizontal } \\
\text { Delineation }\end{array}$ & $\begin{array}{c}\text { Vertical } \\
\text { Delineation }\end{array}$ & $\begin{array}{c}\text { Exit } \\
\text { Location }\end{array}$ & RCRA & CERCLA & Unique & $\begin{array}{c}\text { Monitors } \\
\text { Background } \\
\text { Water Quality }\end{array}$ & Early Detection & $\begin{array}{c}\text { Monitor } \\
\text { Source }\end{array}$ \\
\hline $55-1 \mathrm{~B}$ & WL & $\mathrm{X}$ & Aquitard & $\mathrm{X}$ & & & & & $\mathrm{X}$ & & & \\
\hline $55-6 \mathrm{~A}$ & WL & $x$ & Aquitard & & & & & & & & & \\
\hline $56-2 A$ & WL & $x$ & Aquitard & & & & & & & & & \\
\hline $56-2 B$ & WL & $x$ & Aquitard & & & & & & & & & \\
\hline $56-2 C$ & WL & $\mathrm{x}$ & Aquitard & & & & & & & & & \\
\hline $59-1 \mathrm{~A}$ & $\overline{W L}$ & & Aquitard & & & & & & $\bar{x}$ & $\bar{x}$ & & \\
\hline $59-1 B$ & WL & & Aquitard & & & & & & $x$ & & & \\
\hline $59-1 \mathrm{C}$ & WL & & Aquitard & & & & & & & & & \\
\hline $60-1 B$ & WL & & Aquifer & & & & & & $x$ & $x$ & & \\
\hline $9201-3 C-4 S P$ & $\mathrm{SP}$ & $\mathrm{x}$ & & & & & & & & & & \\
\hline GW-193 & $\overline{W L}$ & $\bar{x}$ & Aquifer & & & & $\bar{x}$ & & & & & \\
\hline GW-204 & WL & $\mathrm{x}$ & Aquifer & $x$ & & & & & & & & \\
\hline GW-218 & WL & & Aquifer & & & & & & $x$ & & & \\
\hline GW-219 & WL & $x$ & Aquifer & & & & & $x$ & & & & \\
\hline GW-605 & WL & $x$ & Aquifer & $x$ & & $x$ & $\mathrm{x}$ & $x$ & & & $\mathrm{x}$ & \\
\hline GW-606 & WL & $\mathrm{X}$ & Aquifer & & $x$ & $\mathrm{X}$ & $x$ & $x$ & & & & \\
\hline GW-656 & WL & $x$ & Aquitard & & & & & & & & & \\
\hline GW-657 & WL & & Aquifer & & & & & & $x$ & $x$ & & \\
\hline GW-686 & WL & $\mathrm{x}$ & Aquifer & & & & & & & & & \\
\hline GW-690 & WL & $x$ & Aquifer & & & & & & & & & \\
\hline GW-691 & WL & $\mathrm{X}$ & Aquifer & & & & & & & & & \\
\hline GW-692 & WL & & Aquifer & & & & & & & & & \\
\hline GW-698 & WL & $x$ & Aquifer & $x$ & & & & & & & $x$ & \\
\hline GW-700 & WL & $x$ & Aquifer & & & & & & & & & \\
\hline GW-759 & WL & & Aquitard & $\mathrm{x}$ & & & & & & $x$ & & \\
\hline
\end{tabular}

Notes:

1. $\mathrm{WL}=$ Monitoring Well; $\mathrm{SP}=\mathrm{Spring}$

2. Well data taken from BWXT Y-12 Analytical Database, Sample locations shown on Figures A.1 and A.3.

3. RCRA indicates wells monitored as part of compliance with RCRA Post-Closure Corrective Action Monitoring or designated Alternate location;

CERCLA indicates locations monitored as part of compliance with CERCLA ROD or backup location. Data from BWXT, 2003a and BWXT 2004a.

4. Average Concentration Exceeds Screening = The average concentration over the entire sampling record for the priority constituent is above the MCL or other designated screening leve as defined in Table B.1.

5. Aquifer and aquitard formations identified in Fig. A.2 from BWXT Y12, 2003 Groundwater Monitoring Report, (12/01/2003).

6. Details of the decision criteria for each category are presented in the text. 
TABLE D.5.1

QUALITATIVE ANALYSIS EAST FORK REGIME CENTRAL Y-12 AREA

East Fork Poplar Creek Hydrogeologic Regime

-12 National Security Complex

Oak Ridge, Tennessee

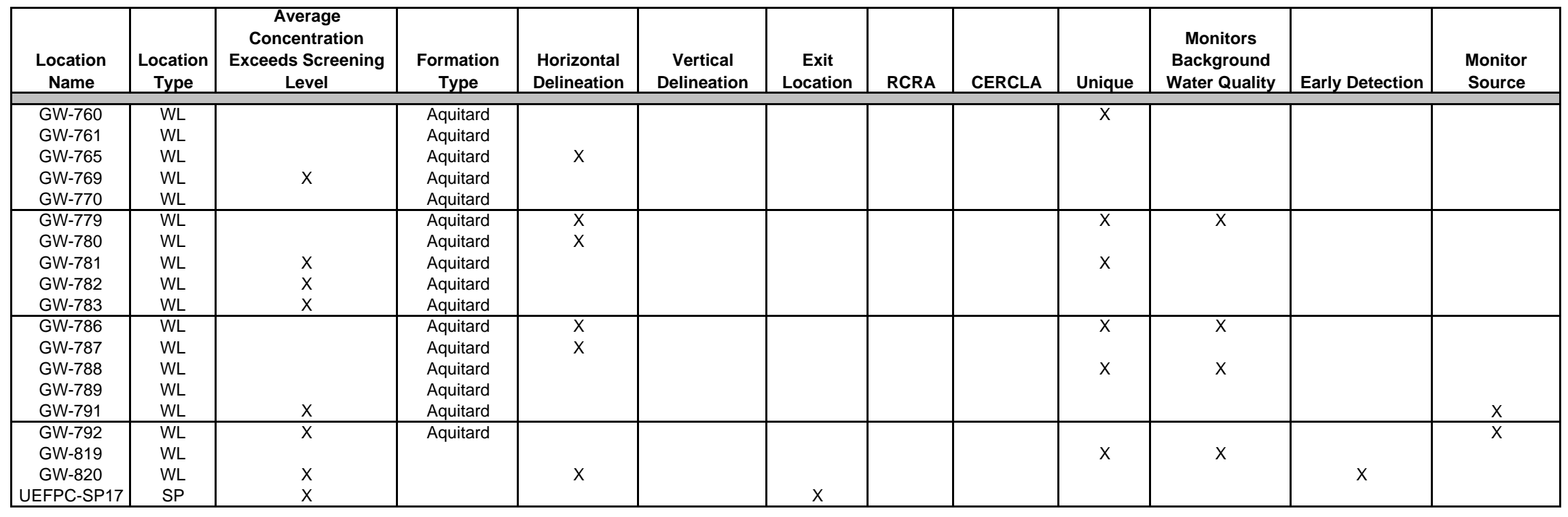

Notes:

1. $\mathrm{WL}=$ Monitoring Well; $\mathrm{SP}=$ Spring

2. Well data taken from BWXT Y-12 Analytical Database. Sample locations shown on Figures A.1 and A.3.

3. RCRA indicates wells monitored as part of compliance with RCRA Post-Closure Corrective Action Monitoring or designated Alternate location;

CERCLA indicates locations monitored as part of compliance with CERCLA ROD or backup location. Data from BWXT, 2003a and BWXT 2004a.

4. Average Concentration Exceeds Screening = The average concentration over the entire sampling record for the priority constituent is above the $\mathrm{MCL}$ or other designated screening level as defined in Table B.1.

5. Aquifer and aquitard formations identified in Fig. A.2 from BWXT Y12, 2003 Groundwater Monitoring Report, (12/01/2003).

6 . Details of the decision criteria for each category are presented in the text. 
TABLE D.5.2

AQUIFER INPUT PARAMETERS

Central Y-12

East Fork Poplar Creek

Y-12 National Security Complex

\begin{tabular}{|c|c|c|}
\hline Parameter & Value & Units \\
\hline Current Plume Length & 5300 & $\mathrm{ft}$ \\
\hline Maximum Plume Length & 5300 & $\mathrm{ft}$ \\
\hline PlumeWidth & 1500 & $\mathrm{ft}$ \\
\hline SeepageVelocity (ft/yr) & 200 & $\mathrm{ft} / \mathrm{yr}$ \\
\hline Distance to Receptors & 5000 & $\mathrm{ft}$ \\
\hline GWFluctuations & Yes & -- \\
\hline SourceTreatment & None & -- \\
\hline PlumeType & Chlorinated Solvent & -- \\
\hline Free NAPL Present & Yes & -- \\
\hline Parameter & Value & \\
\hline Groundwater flow direction & $\mathrm{E}$ & 0 \\
\hline Effective Porosity & 0.1 & -- \\
\hline Source Location near Well & $56-2 B$ & -- \\
\hline Source X-Coordinate & 56225.6 & $\mathrm{ft}^{*}$ \\
\hline Source Y-Coordinate & 29883.61 & $\mathrm{ft}^{*}$ \\
\hline Saturated Thickness & 50 & $\mathrm{ft}$ \\
\hline \multicolumn{3}{|l|}{ Source Wells } \\
\hline -2B, GW-820, GW-791, & GW-656,C & GW-204, GW-60 \\
\hline
\end{tabular}

Notes:

1. Aquifer data are general values for the hydrologic regime.

2. Priority COCs defined by prevalence, toxicty and mobility.

3. $\mathrm{ft}^{\star}=$ Coordinates in $\mathrm{Y}-12$ Plant coordinates, feet.

4. Screening Levels are USEPA MCLs, except in the case of compounds without MCLs where the level is the Region 9 PRG for tap water.

5. Effective Porosity estimated based on average high and low values for aquifer and aquitard suburfaces. 


\section{MAROS COC Assessment}

Project: $\quad$ Y-12 National Security Complex

Location: Central Y-12 Facility

Toxicity:

\begin{tabular}{lccc} 
Toxicity: & $\begin{array}{c}\text { Representative } \\
\text { Concentration } \\
\text { (mg/L) }\end{array}$ & $\begin{array}{c}\text { PRG } \\
\text { (mg/L) }\end{array}$ & $\begin{array}{c}\text { Percent } \\
\text { Above } \\
\text { PRG }\end{array}$ \\
\hline TRICHLOROETHYLENE (TCE) & $2.8 \mathrm{E}-01$ & $5.0 \mathrm{E}-03$ & $5489.4 \%$ \\
TETRACHLOROETHYLENE(PCE) & $2.1 \mathrm{E}-01$ & $5.0 \mathrm{E}-03$ & $4188.9 \%$ \\
BENZENE & $3.5 \mathrm{E}-02$ & $5.0 \mathrm{E}-03$ & $607.7 \%$ \\
URANIUM & $1.7 \mathrm{E}-01$ & $3.0 \mathrm{E}-02$ & $460.8 \%$ \\
CARBON TETRACHLORIDE & $2.5 \mathrm{E}-02$ & $5.0 \mathrm{E}-03$ & $395.1 \%$ \\
NITRATE & $1.2 \mathrm{E}+01$ & $1.0 \mathrm{E}+01$ & $23.7 \%$ \\
LEAD & $1.6 \mathrm{E}-02$ & $1.5 \mathrm{E}-02$ & $4.1 \%$
\end{tabular}

Note: Top COCs by toxicity were determined by examining a representative concentration for each compound over the entire site. The compound representative concentrations are then compared with the chosen PRG for that compound, with the percentage excedence from the PRG determining the compound's toxicity. All compounds above exceed the PRG.

\section{Prevalence:}

\begin{tabular}{|c|c|c|c|c|c|}
\hline Contaminant of Concern & Class & $\begin{array}{l}\text { Total } \\
\text { Wells }\end{array}$ & $\begin{array}{c}\text { Total } \\
\text { Excedences }\end{array}$ & $\begin{array}{c}\text { Percent } \\
\text { Excedences }\end{array}$ & $\begin{array}{l}\text { Total } \\
\text { detects }\end{array}$ \\
\hline NITRATE & INO & 3 & 2 & $66.7 \%$ & 2 \\
\hline URANIUM & MET & 44 & 21 & $47.7 \%$ & 30 \\
\hline TETRACHLOROETHYLENE(PCE) & ORG & 45 & 20 & $44.4 \%$ & 23 \\
\hline TRICHLOROETHYLENE (TCE) & ORG & 45 & 15 & $33.3 \%$ & 23 \\
\hline CARBON TETRACHLORIDE & ORG & 45 & 4 & $8.9 \%$ & 8 \\
\hline BENZENE & ORG & 45 & 4 & $8.9 \%$ & 7 \\
\hline
\end{tabular}

Note: Top COCs by prevalence were determined by examining a representative concentration for each well location at the site. The total excedences (values above the chosen PRGs) are compared to the total number of wells to determine the prevalence of the compound.

\section{Mobility:}

\section{Contaminant of Concern}

\section{NITRATE}

BENZENE

CARBON TETRACHLORIDE

TRICHLOROETHYLENE (TCE)

TETRACHLOROETHYLENE(PCE)

LEAD

URANIUM
Kd

$$
\begin{array}{r}
0.0984 \\
0.277 \\
0.297 \\
0.923 \\
10 \\
2960
\end{array}
$$

Note: Top COCs by mobility were determined by examining each detected compound in the dataset and comparing their mobilities (Koc's for organics, assume foc $=0.001$, and Kd's for metals). 
Project: Y-12 National Security Complex Location: Central Y-12 Facility
User Name:

State: Tennessee

TETRACHLOROETHYLENE(PCE)

NITRATE

BENZENE

URANIUM 


\section{MAROS Plume Analysis Summary}

Project: Y-12 National Security Complex

Location: Central Y-12

Time Period: 1/1/1996

to $1 / 1 / 2005$

Consolidation Period: No Time Consolidation

Consolidation Type: Median

Duplicate Consolidation: Average

ND Values: Specified Detection Limit

J Flag Values : Actual Value

\begin{tabular}{|c|c|c|c|c|c|c|c|c|c|c|c|}
\hline Constituent & Well & $\begin{array}{c}\text { Sourcel } \\
\text { Tail }\end{array}$ & $\begin{array}{l}\text { Number } \\
\text { of } \\
\text { Samples }\end{array}$ & $\begin{array}{c}\text { Number } \\
\text { of } \\
\text { Detects }\end{array}$ & $\begin{array}{c}\text { Average } \\
\text { (mg/L) }\end{array}$ & $\begin{array}{r}\text { Median } \\
\text { (mg/L) }\end{array}$ & $\begin{array}{c}\text { All } \\
\text { Samples } \\
\text { "ND" ? }\end{array}$ & $\begin{array}{l}\text { Mann- } \\
\text { Kendall }\end{array}$ & $\begin{array}{c}\text { Linear } \\
\text { Regression }\end{array}$ & Modeling & Empirica \\
\hline \multicolumn{12}{|l|}{ BENZENE } \\
\hline & GW-820 & $\mathrm{S}$ & 6 & 0 & $5.0 \mathrm{E}-04$ & 5.0E-04 & Yes & $\mathrm{s}$ & $\mathrm{s}$ & $\mathrm{N} / \mathrm{A}$ & $\mathrm{N} / \mathrm{A}$ \\
\hline & GW-791 & s & 19 & 0 & $5.0 \mathrm{E}-04$ & 5.0E-04 & Yes & $\mathrm{s}$ & s & $N / A$ & N/A \\
\hline & $56-2 B$ & $\mathrm{~s}$ & 3 & 0 & $5.0 \mathrm{E}-04$ & 5.0E-04 & Yes & $\mathrm{N} / \mathrm{A}$ & $N / A$ & $N / A$ & $N / A$ \\
\hline & GW-698 & $\mathrm{s}$ & 11 & 0 & $5.0 \mathrm{E}-04$ & 5.0E-04 & Yes & $\mathrm{s}$ & $\mathrm{D}$ & $N / A$ & $N / A$ \\
\hline & GW-691 & s & 3 & 0 & $5.0 \mathrm{E}-04$ & 5.0E-04 & Yes & N/A & N/A & $N / A$ & N/A \\
\hline & $56-2 C$ & $\mathrm{~s}$ & 5 & 0 & $5.0 \mathrm{E}-04$ & 5.0E-04 & Yes & $\mathrm{s}$ & $s$ & N/A & N/A \\
\hline & GW-605 & $\mathrm{s}$ & 20 & 0 & $5.0 \mathrm{E}-04$ & 5.0E-04 & Yes & $\mathrm{s}$ & I & $N / A$ & $\mathrm{~N} / \mathrm{A}$ \\
\hline & GW-219 & s & 12 & 0 & $5.0 \mathrm{E}-04$ & 5.0E-04 & Yes & $\mathrm{s}$ & s & $N / A$ & N/A \\
\hline & GW-656 & $\mathrm{s}$ & 4 & 2 & $7.5 \mathrm{E}-04$ & 7.5E-04 & No & NT & $\mathrm{PI}$ & N/A & N/A \\
\hline & GW-204 & S & 12 & 0 & $5.0 \mathrm{E}-04$ & 5.0E-04 & Yes & $\mathrm{s}$ & $\mathrm{s}$ & $\mathrm{N} / \mathrm{A}$ & N/A \\
\hline & GW-690 & $\mathrm{T}$ & 5 & 0 & $5.0 \mathrm{E}-04$ & 5.0E-04 & Yes & $\mathrm{s}$ & $\mathrm{s}$ & $\mathrm{N} / \mathrm{A}$ & $\mathrm{N} / \mathrm{A}$ \\
\hline & GW-692 & $\mathrm{T}$ & 3 & 0 & $5.0 \mathrm{E}-04$ & 5.0E-04 & Yes & N/A & N/A & $\mathrm{N} / \mathrm{A}$ & N/A \\
\hline & GW-789 & $\mathrm{T}$ & 13 & 0 & $5.0 \mathrm{E}-04$ & $5.0 \mathrm{E}-04$ & Yes & $\mathrm{s}$ & $\mathrm{s}$ & $N / A$ & N/A \\
\hline & $55-1 B$ & $\mathrm{~T}$ & 2 & 0 & $5.0 \mathrm{E}-04$ & 5.0E-04 & Yes & $\mathrm{N} / \mathrm{A}$ & $\mathrm{N} / \mathrm{A}$ & $\mathrm{N} / \mathrm{A}$ & N/A \\
\hline & GW-700 & $\mathrm{T}$ & 5 & 0 & $5.0 \mathrm{E}-04$ & 5.0E-04 & Yes & $\mathrm{S}$ & S & $\mathrm{N} / \mathrm{A}$ & N/A \\
\hline & GW-686 & $\mathrm{T}$ & 2 & 0 & $5.0 \mathrm{E}-04$ & 5.0E-04 & Yes & N/A & N/A & $N / A$ & N/A \\
\hline & GW-788 & $\mathrm{T}$ & 9 & 0 & $5.0 \mathrm{E}-04$ & 5.0E-04 & Yes & $\mathrm{S}$ & $\mathrm{S}$ & N/A & $\mathrm{N} / \mathrm{A}$ \\
\hline & GW-761 & $\mathrm{T}$ & 2 & 0 & $5.0 \mathrm{E}-04$ & 5.0E-04 & Yes & N/A & N/A & $N / A$ & N/A \\
\hline & GW-765 & $\mathrm{T}$ & 2 & 0 & $5.0 \mathrm{E}-04$ & 5.0E-04 & Yes & $N / A$ & N/A & $N / A$ & N/A \\
\hline & GW-769 & $\mathrm{T}$ & 19 & 0 & $5.0 \mathrm{E}-04$ & 5.0E-04 & Yes & $\mathrm{S}$ & $\mathrm{s}$ & $N / A$ & $N / A$ \\
\hline & GW-770 & $\mathrm{T}$ & 19 & 0 & $5.0 \mathrm{E}-04$ & 5.0E-04 & Yes & $\mathrm{s}$ & $\mathrm{s}$ & $\mathrm{N} / \mathrm{A}$ & $\mathrm{N} / \mathrm{A}$ \\
\hline & GW-787 & $\mathrm{T}$ & 5 & 0 & $5.0 \mathrm{E}-04$ & 5.0E-04 & Yes & $\mathrm{s}$ & D & $N / A$ & N/A \\
\hline
\end{tabular}

User Name: MV

State: Tennessee 
Project: Y-12 National Security Complex

Location: Central Y-12
User Name: MV

State: Tennessee

\begin{tabular}{|c|c|c|c|c|c|c|c|c|c|c|c|}
\hline Constituent & Well & $\begin{array}{c}\text { Sourcel } \\
\text { Tail }\end{array}$ & $\begin{array}{c}\text { Number } \\
\text { of } \\
\text { Samples }\end{array}$ & $\begin{array}{c}\text { Number } \\
\text { of } \\
\text { Detects }\end{array}$ & $\begin{array}{c}\text { Average } \\
\text { (mg/L) }\end{array}$ & $\begin{array}{c}\text { Median } \\
(\mathrm{mg} / \mathrm{L})\end{array}$ & $\begin{array}{c}\text { All } \\
\text { Samples } \\
\text { "ND" ? }\end{array}$ & $\begin{array}{l}\text { Mann- } \\
\text { Kendall }\end{array}$ & $\begin{array}{c}\text { Linear } \\
\text { Regression }\end{array}$ & Modeling & Empirical \\
\hline \multicolumn{12}{|l|}{ BENZENE } \\
\hline & GW-779 & $T$ & 3 & 0 & $5.0 \mathrm{E}-04$ & $5.0 \mathrm{E}-04$ & Yes & N/A & N/A & N/A & $\mathrm{N} / \mathrm{A}$ \\
\hline & GW-780 & $\mathrm{T}$ & 3 & 0 & $5.0 \mathrm{E}-04$ & 5.0E-04 & Yes & N/A & N/A & N/A & N/A \\
\hline & GW-781 & $\mathrm{T}$ & 9 & 0 & $5.0 \mathrm{E}-04$ & $5.0 \mathrm{E}-04$ & Yes & $\mathrm{s}$ & $\mathrm{s}$ & N/A & $\mathrm{N} / \mathrm{A}$ \\
\hline & GW-786 & $\mathrm{T}$ & 5 & 0 & $5.0 \mathrm{E}-04$ & 5.0E-04 & Yes & S & D & N/A & N/A \\
\hline & GW-782 & $\mathrm{T}$ & 20 & 2 & $5.5 \mathrm{E}-04$ & 5.0E-04 & No & $\mathrm{S}$ & $\mathrm{S}$ & N/A & N/A \\
\hline & GW-760 & $\mathrm{T}$ & 2 & 0 & $5.0 \mathrm{E}-04$ & $5.0 \mathrm{E}-04$ & Yes & N/A & $\mathrm{N} / \mathrm{A}$ & N/A & N/A \\
\hline & GW-193 & $\mathrm{T}$ & 17 & 12 & $6.1 \mathrm{E}-02$ & 2.5E-02 & No & D & D & N/A & $N / A$ \\
\hline & $55-6 \mathrm{~A}$ & $\mathrm{~T}$ & 3 & 0 & $5.0 \mathrm{E}-04$ & 5.0E-04 & Yes & N/A & N/A & N/A & $\mathrm{N} / \mathrm{A}$ \\
\hline & $56-2 A$ & $T$ & 3 & 0 & $5.0 \mathrm{E}-04$ & 5.0E-04 & Yes & N/A & $\mathrm{N} / \mathrm{A}$ & N/A & N/A \\
\hline & UEFPC-SP17 & $\mathrm{T}$ & 8 & 0 & $5.0 \mathrm{E}-04$ & 5.0E-04 & Yes & $\mathrm{s}$ & $\mathrm{S}$ & N/A & $N / A$ \\
\hline & $59-1 \mathrm{~A}$ & T & 3 & 0 & $5.0 \mathrm{E}-04$ & 5.0E-04 & Yes & N/A & N/A & N/A & N/A \\
\hline & N-S PIPE & $\mathrm{T}$ & 6 & 0 & $5.0 \mathrm{E}-04$ & 5.0E-04 & Yes & $\mathrm{s}$ & $\mathrm{s}$ & N/A & $\mathrm{N} / \mathrm{A}$ \\
\hline & $59-1 \mathrm{~B}$ & $\mathrm{~T}$ & 3 & 0 & $5.0 \mathrm{E}-04$ & $5.0 \mathrm{E}-04$ & Yes & N/A & N/A & N/A & $N / A$ \\
\hline & $59-1 C$ & $\mathrm{~T}$ & 3 & 0 & $5.0 \mathrm{E}-04$ & $5.0 \mathrm{E}-04$ & Yes & N/A & $\mathrm{N} / \mathrm{A}$ & N/A & N/A \\
\hline & GW-606 & $\mathrm{T}$ & 20 & 5 & $6.3 \mathrm{E}-04$ & 5.0E-04 & No & $\mathrm{s}$ & $\mathrm{s}$ & N/A & $\mathrm{N} / \mathrm{A}$ \\
\hline & 9201-3C-4SP & $\mathrm{T}$ & 2 & 0 & $5.0 \mathrm{E}-04$ & $5.0 \mathrm{E}-04$ & Yes & N/A & N/A & N/A & $\mathrm{N} / \mathrm{A}$ \\
\hline & GW-218 & $\mathrm{T}$ & 4 & 0 & $5.0 \mathrm{E}-04$ & $5.0 \mathrm{E}-04$ & Yes & $\mathrm{s}$ & s & N/A & $\mathrm{N} / \mathrm{A}$ \\
\hline & GW-819 & $\mathrm{T}$ & 1 & 0 & $5.0 \mathrm{E}-04$ & $5.0 \mathrm{E}-04$ & Yes & N/A & N/A & N/A & $\mathrm{N} / \mathrm{A}$ \\
\hline & GW-792 & $\mathrm{T}$ & 9 & 1 & $5.6 \mathrm{E}-04$ & 5.0E-04 & No & $\mathrm{s}$ & D & N/A & $N / A$ \\
\hline & GW-783 & $\mathrm{T}$ & 9 & 0 & $5.0 \mathrm{E}-04$ & $5.0 \mathrm{E}-04$ & Yes & s & s & N/A & $\mathrm{N} / \mathrm{A}$ \\
\hline & $60-1 B$ & $\mathrm{~T}$ & 2 & 0 & $5.0 \mathrm{E}-04$ & 5.0E-04 & Yes & N/A & $\mathrm{N} / \mathrm{A}$ & $\mathrm{N} / \mathrm{A}$ & $N / A$ \\
\hline \multicolumn{12}{|c|}{ GROSS ALPHA ACTIVITY } \\
\hline & GW-656 & $\mathrm{s}$ & 2 & 2 & $1.9 \mathrm{E}+00$ & $1.9 \mathrm{E}+00$ & No & N/A & $\mathrm{N} / \mathrm{A}$ & $\mathrm{N} / \mathrm{A}$ & N/A \\
\hline & GW-204 & s & 13 & 13 & $4.7 \mathrm{E}+01$ & $3.7 \mathrm{E}+01$ & No & $\mathrm{PD}$ & $\mathrm{PD}$ & N/A & $\mathrm{N} / \mathrm{A}$ \\
\hline & GW-698 & s & 10 & 10 & $3.1 \mathrm{E}+00$ & $2.8 \mathrm{E}+00$ & No & NT & NT & N/A & N/A \\
\hline & GW-219 & s & 12 & 12 & $1.2 \mathrm{E}+02$ & $1.2 \mathrm{E}+02$ & No & $\mathrm{s}$ & NT & N/A & $\mathrm{N} / \mathrm{A}$ \\
\hline & GW-691 & s & 2 & 2 & $2.1 \mathrm{E}+00$ & $2.1 \mathrm{E}+00$ & No & N/A & N/A & N/A & $\mathrm{N} / \mathrm{A}$ \\
\hline & $56-2 C$ & s & 4 & 4 & $1.8 \mathrm{E}+00$ & $1.6 \mathrm{E}+00$ & No & NT & NT & N/A & N/A \\
\hline & $56-2 B$ & s & 3 & 3 & $1.2 \mathrm{E}+00$ & $1.2 \mathrm{E}+00$ & No & N/A & $\mathrm{N} / \mathrm{A}$ & $\mathrm{N} / \mathrm{A}$ & $\mathrm{N} / \mathrm{A}$ \\
\hline & GW-605 & s & 19 & 19 & $7.0 \mathrm{E}+01$ & $5.2 \mathrm{E}+01$ & No & $\mathrm{s}$ & $\mathrm{PD}$ & N/A & $\mathrm{N} / \mathrm{A}$ \\
\hline & GW-820 & s & 5 & 5 & $1.7 \mathrm{E}+00$ & $9.0 \mathrm{E}-01$ & No & s & NT & N/A & $\mathrm{N} / \mathrm{A}$ \\
\hline & GW-791 & s & 11 & 11 & $2.0 \mathrm{E}+00$ & $1.8 \mathrm{E}+00$ & No & s & s & N/A & N/A \\
\hline & 9201-3C-4SP & $\mathrm{T}$ & 1 & 1 & $6.2 \mathrm{E}-01$ & $6.2 \mathrm{E}-01$ & No & N/A & N/A & N/A & N/A \\
\hline
\end{tabular}


Project: Y-12 National Security Complex

Location: Central Y-12
User Name: MV

State: Tennessee

\begin{tabular}{|c|c|c|c|c|c|c|c|c|c|c|c|}
\hline Constituent & Well & $\begin{array}{l}\text { Sourcel } \\
\text { Tail }\end{array}$ & $\begin{array}{c}\text { Number } \\
\text { of } \\
\text { Samples }\end{array}$ & $\begin{array}{c}\text { Number } \\
\text { of } \\
\text { Detects }\end{array}$ & $\begin{array}{c}\text { Average } \\
\text { (mg/L) }\end{array}$ & $\begin{array}{r}\text { Median } \\
(\mathrm{mg} / \mathrm{L})\end{array}$ & $\begin{array}{c}\text { All } \\
\text { Samples } \\
\text { "ND" ? }\end{array}$ & $\begin{array}{l}\text { Mann- } \\
\text { Kendall }\end{array}$ & $\begin{array}{c}\text { Linear } \\
\text { Regression }\end{array}$ & Modeling & Empirical \\
\hline \multicolumn{12}{|c|}{ GROSS ALPHA ACTIVITY } \\
\hline & GW-692 & $T$ & 4 & 4 & $7.3 \mathrm{E}+00$ & $6.0 \mathrm{E}+00$ & No & NT & $\mathrm{PI}$ & N/A & $\mathrm{N} / \mathrm{A}$ \\
\hline & N-S PIPE & $\mathrm{T}$ & 10 & 10 & $1.7 \mathrm{E}+01$ & $2.0 \mathrm{E}+01$ & No & s & s & N/A & N/A \\
\hline & GW-770 & $\mathrm{T}$ & 14 & 14 & $1.7 \mathrm{E}+00$ & $1.5 \mathrm{E}+00$ & No & S & s & $N / A$ & $N / A$ \\
\hline & GW-787 & $\mathrm{T}$ & 4 & 4 & $2.4 \mathrm{E}+00$ & $5.8 \mathrm{E}-01$ & No & NT & NT & N/A & N/A \\
\hline & $59-1 \mathrm{~A}$ & $\mathrm{~T}$ & 3 & 3 & $8.1 \mathrm{E}+00$ & $6.9 \mathrm{E}+00$ & No & N/A & N/A & N/A & N/A \\
\hline & GW-193 & $\mathrm{T}$ & 18 & 15 & $7.6 \mathrm{E}+00$ & $2.9 \mathrm{E}+00$ & No & $\mathrm{D}$ & $\mathrm{D}$ & $N / A$ & $N / A$ \\
\hline & GW-789 & $\mathrm{T}$ & 9 & 9 & $3.8 \mathrm{E}+00$ & $1.5 \mathrm{E}+00$ & No & NT & NT & N/A & $N / A$ \\
\hline & GW-769 & $\mathrm{T}$ & 15 & 15 & $1.8 \mathrm{E}+00$ & $1.3 \mathrm{E}+00$ & No & $\mathrm{s}$ & NT & N/A & N/A \\
\hline & GW-819 & $\mathrm{T}$ & 1 & 1 & $3.8 \mathrm{E}-01$ & $3.8 \mathrm{E}-01$ & No & $N / A$ & $N / A$ & $N / A$ & $N / A$ \\
\hline & $59-1 B$ & $\mathrm{~T}$ & 3 & 3 & $4.5 \mathrm{E}+00$ & $5.2 \mathrm{E}+00$ & No & $\mathrm{N} / \mathrm{A}$ & $\mathrm{N} / \mathrm{A}$ & $N / A$ & $N / A$ \\
\hline & GW-788 & $\mathrm{T}$ & 4 & 4 & $3.4 \mathrm{E}+00$ & $2.7 \mathrm{E}+00$ & No & $D$ & $D$ & N/A & N/A \\
\hline & GW-765 & $\mathrm{T}$ & 2 & 2 & $1.6 \mathrm{E}+00$ & $1.6 \mathrm{E}+00$ & No & $N / A$ & $N / A$ & $N / A$ & $N / A$ \\
\hline & GW-760 & $\mathrm{T}$ & 2 & 2 & $9.7 \mathrm{E}-01$ & $9.7 \mathrm{E}-01$ & No & $N / A$ & $N / A$ & $N / A$ & $N / A$ \\
\hline & $59-1 C$ & $\mathrm{~T}$ & 2 & 2 & $1.1 \mathrm{E}+00$ & $1.1 \mathrm{E}+00$ & No & $\mathrm{N} / \mathrm{A}$ & N/A & N/A & N/A \\
\hline & GW-700 & $\mathrm{T}$ & 5 & 5 & $2.0 \mathrm{E}+00$ & $1.7 \mathrm{E}+00$ & No & $\mathrm{s}$ & $\mathrm{s}$ & $N / A$ & N/A \\
\hline & GW-792 & $\mathrm{T}$ & 4 & 4 & $1.9 \mathrm{E}+00$ & $1.6 \mathrm{E}+00$ & No & s & s & $N / A$ & $N / A$ \\
\hline & GW-783 & $\mathrm{T}$ & 8 & 8 & $2.7 \mathrm{E}+00$ & $2.9 \mathrm{E}+00$ & No & NT & NT & N/A & $N / A$ \\
\hline & $55-1 B$ & $\mathrm{~T}$ & 2 & 2 & $3.4 \mathrm{E}+00$ & $3.4 \mathrm{E}+00$ & No & $\mathrm{N} / \mathrm{A}$ & $\mathrm{N} / \mathrm{A}$ & N/A & $N / A$ \\
\hline & $55-6 \mathrm{~A}$ & $\mathrm{~T}$ & 2 & 2 & $4.1 \mathrm{E}+00$ & $4.1 \mathrm{E}+00$ & No & $N / A$ & $N / A$ & $N / A$ & $N / A$ \\
\hline & GW-606 & $\mathrm{T}$ & 20 & 19 & $5.9 \mathrm{E}+00$ & $5.7 \mathrm{E}+00$ & No & NT & $\mathrm{s}$ & $\mathrm{N} / \mathrm{A}$ & $N / A$ \\
\hline & GW-782 & $\mathrm{T}$ & 20 & 20 & $4.2 \mathrm{E}+01$ & $4.4 \mathrm{E}+01$ & No & $D$ & $D$ & $N / A$ & $N / A$ \\
\hline & GW-686 & $\mathrm{T}$ & 1 & 1 & $1.6 \mathrm{E}+01$ & $1.6 \mathrm{E}+01$ & No & $N / A$ & $N / A$ & $N / A$ & $N / A$ \\
\hline & $56-2 A$ & $\mathrm{~T}$ & 2 & 2 & $1.2 \mathrm{E}+00$ & $1.2 \mathrm{E}+00$ & No & $\mathrm{N} / \mathrm{A}$ & $\mathrm{N} / \mathrm{A}$ & N/A & $N / A$ \\
\hline & GW-779 & $\mathrm{T}$ & 1 & 1 & $2.1 \mathrm{E}+00$ & $2.1 \mathrm{E}+00$ & No & N/A & N/A & N/A & N/A \\
\hline & GW-781 & $\mathrm{T}$ & 4 & 4 & $2.2 \mathrm{E}+00$ & $1.6 \mathrm{E}+00$ & No & NT & NT & $N / A$ & $N / A$ \\
\hline & $60-1 B$ & $\mathrm{~T}$ & 1 & 1 & 7.4E-02 & 7.4E-02 & No & $\mathrm{N} / \mathrm{A}$ & $\mathrm{N} / \mathrm{A}$ & N/A & $N / A$ \\
\hline & GW-690 & $\mathrm{T}$ & 4 & 4 & $8.1 \mathrm{E}+00$ & $5.1 \mathrm{E}+00$ & No & $\mathrm{s}$ & $\mathrm{D}$ & N/A & N/A \\
\hline & UEFPC-SP17 & $\mathrm{T}$ & 8 & 8 & $2.4 \mathrm{E}+00$ & $1.9 \mathrm{E}+00$ & No & $\mathrm{PD}$ & s & $N / A$ & $N / A$ \\
\hline & GW-218 & $\mathrm{T}$ & 4 & 4 & $2.7 \mathrm{E}+00$ & $2.7 \mathrm{E}+00$ & No & NT & NT & N/A & $N / A$ \\
\hline \multicolumn{12}{|c|}{ TETRACHLOROETHYLENE(PCE) } \\
\hline & GW-698 & $\mathrm{s}$ & 11 & 10 & $1.1 \mathrm{E}-01$ & $1.3 \mathrm{E}-01$ & No & NT & NT & $\mathrm{N} / \mathrm{A}$ & $\mathrm{N} / \mathrm{A}$ \\
\hline & GW-219 & s & 12 & 0 & $5.0 \mathrm{E}-04$ & $5.0 \mathrm{E}-04$ & Yes & $\mathrm{s}$ & $\mathrm{s}$ & $N / A$ & $N / A$ \\
\hline & GW-204 & s & 12 & 1 & $1.8 \mathrm{E}-03$ & 5.0E-04 & No & NT & D & $N / A$ & $N / A$ \\
\hline
\end{tabular}


Project: Y-12 National Security Complex

Location: Central Y-12
User Name: MV

State: Tennessee

\begin{tabular}{|c|c|c|c|c|c|c|c|c|c|c|c|}
\hline Constituent & Well & $\begin{array}{c}\text { Sourcel } \\
\text { Tail }\end{array}$ & $\begin{array}{c}\text { Number } \\
\text { of } \\
\text { Samples }\end{array}$ & $\begin{array}{c}\text { Number } \\
\text { of } \\
\text { Detects }\end{array}$ & $\begin{array}{c}\text { Average } \\
\text { (mg/L) }\end{array}$ & $\begin{array}{c}\text { Median } \\
(\mathrm{mg} / \mathrm{L})\end{array}$ & $\begin{array}{c}\text { All } \\
\text { Samples } \\
\text { "ND" ? }\end{array}$ & $\begin{array}{l}\text { Mann- } \\
\text { Kendall }\end{array}$ & $\begin{array}{l}\text { Linear } \\
\text { Regression }\end{array}$ & Modeling & Empirical \\
\hline \multicolumn{12}{|c|}{ TETRACHLOROETHYLENE(PCE) } \\
\hline & GW-691 & S & 3 & 3 & $8.0 \mathrm{E}-01$ & $9.8 \mathrm{E}-01$ & No & N/A & N/A & N/A & N/A \\
\hline & GW-656 & s & 4 & 4 & $5.8 \mathrm{E}-02$ & 5.7E-02 & No & s & s & $N / A$ & N/A \\
\hline & GW-820 & s & 6 & 6 & $4.4 \mathrm{E}+00$ & $4.3 \mathrm{E}+00$ & No & NT & NT & $N / A$ & $N / A$ \\
\hline & GW-605 & s & 20 & 19 & 2.6E-02 & 1.5E-02 & No & 1 & 1 & N/A & N/A \\
\hline & $56-2 B$ & s & 3 & 3 & $1.0 \mathrm{E}+00$ & 8.7E-01 & No & $N / A$ & $N / A$ & N/A & N/A \\
\hline & $56-2 \mathrm{C}$ & s & 5 & 5 & $1.4 \mathrm{E}+00$ & $1.4 \mathrm{E}+00$ & No & $\mathrm{s}$ & PD & $N / A$ & $N / A$ \\
\hline & GW-791 & s & 19 & 19 & $6.0 \mathrm{E}-01$ & 4.1E-01 & No & s & s & $N / A$ & $N / A$ \\
\hline & GW-786 & $T$ & 5 & 0 & $5.0 \mathrm{E}-04$ & 5.0E-04 & Yes & s & D & N/A & $N / A$ \\
\hline & GW-779 & $\mathrm{T}$ & 3 & 0 & $5.0 \mathrm{E}-04$ & $5.0 \mathrm{E}-04$ & Yes & $N / A$ & $N / A$ & $N / A$ & $N / A$ \\
\hline & GW-761 & $\mathrm{T}$ & 2 & 0 & $5.0 \mathrm{E}-04$ & 5.0E-04 & Yes & $\mathrm{N} / \mathrm{A}$ & N/A & $N / A$ & $N / A$ \\
\hline & GW-789 & $\mathrm{T}$ & 13 & 2 & $5.8 \mathrm{E}-04$ & 5.0E-04 & No & $\mathrm{s}$ & $\mathrm{D}$ & $N / A$ & $N / A$ \\
\hline & GW-218 & $\mathrm{T}$ & 4 & 0 & $5.0 \mathrm{E}-04$ & 5.0E-04 & Yes & s & s & $N / A$ & N/A \\
\hline & GW-780 & $\mathrm{T}$ & 3 & 0 & $5.0 \mathrm{E}-04$ & $5.0 \mathrm{E}-04$ & Yes & $N / A$ & $N / A$ & $N / A$ & $N / A$ \\
\hline & GW-690 & $\mathrm{T}$ & 5 & 5 & $2.1 \mathrm{E}-01$ & $6.6 \mathrm{E}-02$ & No & NT & $\mathrm{D}$ & $N / A$ & $N / A$ \\
\hline & GW-792 & $\mathrm{T}$ & 9 & 9 & $6.3 \mathrm{E}-03$ & $6.0 \mathrm{E}-03$ & No & $\mathrm{s}$ & NT & N/A & $N / A$ \\
\hline & GW-781 & $\mathrm{T}$ & 9 & 6 & $1.2 \mathrm{E}-02$ & $5.0 \mathrm{E}-03$ & No & PI & 1 & $N / A$ & $N / A$ \\
\hline & GW-686 & $\mathrm{T}$ & 2 & 0 & $5.0 \mathrm{E}-04$ & $5.0 \mathrm{E}-04$ & Yes & $N / A$ & $\mathrm{~N} / \mathrm{A}$ & $N / A$ & $N / A$ \\
\hline & UEFPC-SP17 & $\mathrm{T}$ & 8 & 0 & $5.0 \mathrm{E}-04$ & 5.0E-04 & Yes & $\mathrm{s}$ & $\mathrm{s}$ & $N / A$ & $N / A$ \\
\hline & $55-6 \mathrm{~A}$ & $\mathrm{~T}$ & 3 & 0 & $5.0 \mathrm{E}-04$ & $5.0 \mathrm{E}-04$ & Yes & $N / A$ & $\mathrm{~N} / \mathrm{A}$ & $N / A$ & $N / A$ \\
\hline & GW-782 & $\mathrm{T}$ & 20 & 20 & $1.8 \mathrm{E}-01$ & $1.9 \mathrm{E}-01$ & No & $\mathrm{D}$ & $\mathrm{D}$ & N/A & $N / A$ \\
\hline & $55-1 B$ & $\mathrm{~T}$ & 2 & 0 & $5.0 \mathrm{E}-04$ & $5.0 \mathrm{E}-04$ & Yes & $N / A$ & $N / A$ & $N / A$ & $N / A$ \\
\hline & GW-783 & $\mathrm{T}$ & 9 & 9 & $2.4 \mathrm{E}-02$ & $2.4 \mathrm{E}-02$ & No & $\mathrm{s}$ & $\mathrm{s}$ & $N / A$ & $N / A$ \\
\hline & $56-2 A$ & $\mathrm{~T}$ & 3 & 3 & $2.4 \mathrm{E}-02$ & 1.3E-02 & No & $\mathrm{N} / \mathrm{A}$ & $\mathrm{N} / \mathrm{A}$ & N/A & $N / A$ \\
\hline & GW-788 & $\mathrm{T}$ & 9 & 0 & $5.0 \mathrm{E}-04$ & 5.0E-04 & Yes & $\mathrm{s}$ & $\mathrm{s}$ & $N / A$ & N/A \\
\hline & $59-1 C$ & $\mathrm{~T}$ & 3 & 0 & $5.0 \mathrm{E}-04$ & $5.0 \mathrm{E}-04$ & Yes & $N / A$ & $N / A$ & $N / A$ & $N / A$ \\
\hline & GW-606 & $\mathrm{T}$ & 20 & 17 & $4.8 \mathrm{E}-03$ & $5.0 \mathrm{E}-03$ & No & $\mathrm{PI}$ & 1 & N/A & $N / A$ \\
\hline & $60-1 B$ & $\mathrm{~T}$ & 2 & 0 & $5.0 \mathrm{E}-04$ & $5.0 \mathrm{E}-04$ & Yes & $\mathrm{N} / \mathrm{A}$ & $\mathrm{N} / \mathrm{A}$ & $N / A$ & $N / A$ \\
\hline & GW-760 & $\mathrm{T}$ & 2 & 0 & $5.0 \mathrm{E}-04$ & $5.0 \mathrm{E}-04$ & Yes & $N / A$ & $N / A$ & $N / A$ & $N / A$ \\
\hline & GW-765 & $\mathrm{T}$ & 2 & 0 & $5.0 \mathrm{E}-04$ & 5.0E-04 & Yes & $\mathrm{N} / \mathrm{A}$ & N/A & N/A & $N / A$ \\
\hline & $59-1 B$ & $\mathrm{~T}$ & 3 & 0 & $5.0 \mathrm{E}-04$ & $5.0 \mathrm{E}-04$ & Yes & $N / A$ & $N / A$ & $N / A$ & $N / A$ \\
\hline & GW-787 & $\mathrm{T}$ & 5 & 0 & $5.0 \mathrm{E}-04$ & $5.0 \mathrm{E}-04$ & Yes & $\mathrm{s}$ & D & $N / A$ & $N / A$ \\
\hline & N-S PIPE & $\mathrm{T}$ & 6 & 5 & $2.2 \mathrm{E}-03$ & $1.5 \mathrm{E}-03$ & No & NT & NT & N/A & $N / A$ \\
\hline & GW-769 & $\mathrm{T}$ & 19 & 18 & $8.9 \mathrm{E}-03$ & 8.0E-03 & No & 1 & 1 & N/A & N/A \\
\hline & GW-692 & $\mathrm{T}$ & 3 & 2 & 3.5E-03 & $2.0 \mathrm{E}-03$ & No & $N / A$ & $N / A$ & $N / A$ & $N / A$ \\
\hline
\end{tabular}


Project: Y-12 National Security Complex

Location: Central Y-12
User Name: MV

State: Tennessee

\begin{tabular}{|c|c|c|c|c|c|c|c|c|c|c|c|}
\hline Constituent & Well & $\begin{array}{l}\text { Sourcel } \\
\text { Tail }\end{array}$ & $\begin{array}{l}\text { Number } \\
\text { of } \\
\text { Samples }\end{array}$ & $\begin{array}{c}\text { Number } \\
\text { of } \\
\text { Detects }\end{array}$ & $\begin{array}{c}\text { Average } \\
\text { (mg/L) }\end{array}$ & $\begin{array}{r}\text { Median } \\
(\mathrm{mg} / \mathrm{L})\end{array}$ & $\begin{array}{c}\text { All } \\
\text { Samples } \\
\text { "ND" ? }\end{array}$ & $\begin{array}{l}\text { Mann- } \\
\text { Kendall }\end{array}$ & $\begin{array}{c}\text { Linear } \\
\text { Regression }\end{array}$ & Modeling & Empirical \\
\hline \multicolumn{12}{|c|}{ TETRACHLOROETHYLENE(PCE) } \\
\hline & 9201-3C-4SP & $\mathrm{T}$ & 2 & 1 & $5.7 \mathrm{E}-03$ & 5.7E-03 & No & N/A & $\mathrm{N} / \mathrm{A}$ & $\mathrm{N} / \mathrm{A}$ & $\mathrm{N} / \mathrm{A}$ \\
\hline & $59-1 \mathrm{~A}$ & $\mathrm{~T}$ & 3 & 0 & $5.0 \mathrm{E}-04$ & $5.0 \mathrm{E}-04$ & Yes & N/A & N/A & N/A & $N / A$ \\
\hline & GW-770 & $\mathrm{T}$ & 19 & 0 & $5.0 \mathrm{E}-04$ & 5.0E-04 & Yes & s & s & N/A & $N / A$ \\
\hline & GW-193 & T & 17 & 0 & $5.0 \mathrm{E}-04$ & $5.0 \mathrm{E}-04$ & Yes & $\mathrm{s}$ & $\mathrm{s}$ & N/A & N/A \\
\hline & GW-819 & $\mathrm{T}$ & 1 & 0 & $5.0 \mathrm{E}-04$ & 5.0E-04 & Yes & N/A & N/A & N/A & $N / A$ \\
\hline & GW-700 & $\mathrm{T}$ & 5 & 5 & $1.9 \mathrm{E}-01$ & 1.5E-01 & No & s & D & $\mathrm{N} / \mathrm{A}$ & $N / A$ \\
\hline \multicolumn{12}{|c|}{ TRICHLOROETHYLENE (TCE) } \\
\hline & GW-219 & $\mathrm{S}$ & 12 & 1 & $5.4 \mathrm{E}-04$ & $5.0 \mathrm{E}-04$ & No & $\mathrm{S}$ & $\mathrm{S}$ & $\mathrm{N} / \mathrm{A}$ & $\mathrm{N} / \mathrm{A}$ \\
\hline & GW-820 & S & 6 & 6 & $6.6 \mathrm{E}-01$ & 5.7E-01 & No & NT & $\mathrm{PI}$ & $\mathrm{N} / \mathrm{A}$ & $N / A$ \\
\hline & $56-2 B$ & s & 3 & 3 & $6.5 \mathrm{E}-02$ & 6.1E-02 & No & $\mathrm{N} / \mathrm{A}$ & N/A & N/A & $N / A$ \\
\hline & GW-605 & s & 20 & 19 & 2.5E-02 & $1.9 \mathrm{E}-02$ & No & 1 & 1 & N/A & $N / A$ \\
\hline & $56-2 C$ & s & 5 & 5 & $5.9 \mathrm{E}-01$ & 3.8E-01 & No & S & s & $\mathrm{N} / \mathrm{A}$ & $N / A$ \\
\hline & GW-204 & s & 12 & 1 & $5.4 \mathrm{E}-04$ & $5.0 \mathrm{E}-04$ & No & S & D & N/A & $N / A$ \\
\hline & GW-791 & s & 19 & 10 & $1.4 \mathrm{E}-03$ & $1.0 \mathrm{E}-03$ & No & NT & NT & N/A & $N / A$ \\
\hline & GW-656 & s & 4 & 4 & $4.3 \mathrm{E}+00$ & $4.4 \mathrm{E}+00$ & No & S & s & $\mathrm{N} / \mathrm{A}$ & $N / A$ \\
\hline & GW-691 & s & 3 & 3 & 7.2E-03 & 8.0E-03 & No & N/A & N/A & N/A & $N / A$ \\
\hline & GW-698 & s & 11 & 11 & $2.8 \mathrm{E}-01$ & 3.1E-01 & No & NT & NT & N/A & $N / A$ \\
\hline & GW-786 & $\mathrm{T}$ & 5 & 0 & $5.0 \mathrm{E}-04$ & 5.0E-04 & Yes & s & D & $\mathrm{N} / \mathrm{A}$ & N/A \\
\hline & $59-1 C$ & $T$ & 3 & 2 & 4.5E-03 & $6.0 \mathrm{E}-03$ & No & N/A & N/A & N/A & $N / A$ \\
\hline & $55-6 \mathrm{~A}$ & $\mathrm{~T}$ & 3 & 0 & $5.0 \mathrm{E}-04$ & 5.0E-04 & Yes & N/A & N/A & N/A & $N / A$ \\
\hline & 9201-3C-4SP & $\mathrm{T}$ & 2 & 1 & $1.0 \mathrm{E}-03$ & $1.0 \mathrm{E}-03$ & No & $\mathrm{N} / \mathrm{A}$ & $\mathrm{N} / \mathrm{A}$ & $\mathrm{N} / \mathrm{A}$ & $N / A$ \\
\hline & GW-700 & $\mathrm{T}$ & 5 & 5 & $1.2 \mathrm{E}-02$ & $1.0 \mathrm{E}-02$ & No & NT & NT & $\mathrm{N} / \mathrm{A}$ & $N / A$ \\
\hline & GW-781 & $\mathrm{T}$ & 9 & 3 & $1.9 \mathrm{E}-03$ & 5.0E-04 & No & NT & NT & $\mathrm{N} / \mathrm{A}$ & $N / A$ \\
\hline & $60-1 B$ & $\mathrm{~T}$ & 2 & 0 & $5.0 \mathrm{E}-04$ & $5.0 \mathrm{E}-04$ & Yes & N/A & $\mathrm{N} / \mathrm{A}$ & N/A & $N / A$ \\
\hline & GW-760 & $\mathrm{T}$ & 2 & 0 & $5.0 \mathrm{E}-04$ & 5.0E-04 & Yes & N/A & $\mathrm{N} / \mathrm{A}$ & $\mathrm{N} / \mathrm{A}$ & $N / A$ \\
\hline & $56-2 A$ & $\mathrm{~T}$ & 3 & 3 & $3.2 \mathrm{E}-03$ & $1.5 \mathrm{E}-03$ & No & $\mathrm{N} / \mathrm{A}$ & $N / A$ & $\mathrm{~N} / \mathrm{A}$ & $N / A$ \\
\hline & GW-193 & $\mathrm{T}$ & 17 & 0 & $5.0 \mathrm{E}-04$ & $5.0 \mathrm{E}-04$ & Yes & $\mathrm{s}$ & $\mathrm{s}$ & N/A & $N / A$ \\
\hline & GW-761 & $\mathrm{T}$ & 2 & 0 & $5.0 \mathrm{E}-04$ & $5.0 \mathrm{E}-04$ & Yes & N/A & $\mathrm{N} / \mathrm{A}$ & N/A & $N / A$ \\
\hline & GW-787 & $\mathrm{T}$ & 5 & 0 & $5.0 \mathrm{E}-04$ & 5.0E-04 & Yes & s & D & N/A & $N / A$ \\
\hline & $59-1 B$ & $T$ & 3 & 0 & $5.0 \mathrm{E}-04$ & $5.0 \mathrm{E}-04$ & Yes & N/A & $\mathrm{N} / \mathrm{A}$ & N/A & $N / A$ \\
\hline & GW-765 & $\mathrm{T}$ & 2 & 0 & $5.0 \mathrm{E}-04$ & 5.0E-04 & Yes & $\mathrm{N} / \mathrm{A}$ & $\mathrm{N} / \mathrm{A}$ & $\mathrm{N} / \mathrm{A}$ & $N / A$ \\
\hline & N-S PIPE & $\mathrm{T}$ & 6 & 1 & $5.8 \mathrm{E}-04$ & $5.0 \mathrm{E}-04$ & No & NT & NT & N/A & $N / A$ \\
\hline & $59-1 \mathrm{~A}$ & $T$ & 3 & 0 & $5.0 \mathrm{E}-04$ & $5.0 \mathrm{E}-04$ & Yes & N/A & N/A & N/A & $\mathrm{N} / \mathrm{A}$ \\
\hline
\end{tabular}


Project: Y-12 National Security Complex

Location: Central Y-12
User Name: MV

State: Tennessee

\begin{tabular}{|c|c|c|c|c|c|c|c|c|c|c|c|}
\hline Constituent & Well & $\begin{array}{c}\text { Sourcel } \\
\text { Tail }\end{array}$ & $\begin{array}{c}\text { Number } \\
\text { of } \\
\text { Samples }\end{array}$ & $\begin{array}{c}\text { Number } \\
\text { of } \\
\text { Detects }\end{array}$ & $\begin{array}{c}\text { Average } \\
\text { (mg/L) }\end{array}$ & $\begin{array}{c}\text { Median } \\
(\mathrm{mg} / \mathrm{L})\end{array}$ & $\begin{array}{c}\text { All } \\
\text { Samples } \\
\text { "ND" ? }\end{array}$ & $\begin{array}{l}\text { Mann- } \\
\text { Kendall }\end{array}$ & $\begin{array}{c}\text { Linear } \\
\text { Regression }\end{array}$ & Modeling & Empirical \\
\hline \multicolumn{12}{|c|}{ TRICHLOROETHYLENE (TCE) } \\
\hline & GW-769 & $T$ & 19 & 14 & $1.3 \mathrm{E}-03$ & $1.0 \mathrm{E}-03$ & No & 1 & 1 & N/A & $\mathrm{N} / \mathrm{A}$ \\
\hline & GW-779 & $\mathrm{T}$ & 3 & 0 & $5.0 \mathrm{E}-04$ & 5.0E-04 & Yes & N/A & N/A & N/A & N/A \\
\hline & GW-770 & $\mathrm{T}$ & 19 & 0 & $5.0 \mathrm{E}-04$ & $5.0 \mathrm{E}-04$ & Yes & $\mathrm{s}$ & $\mathrm{s}$ & N/A & $\mathrm{N} / \mathrm{A}$ \\
\hline & GW-780 & $\mathrm{T}$ & 3 & 0 & $5.0 \mathrm{E}-04$ & 5.0E-04 & Yes & N/A & N/A & N/A & N/A \\
\hline & GW-789 & $\mathrm{T}$ & 13 & 6 & $7.3 \mathrm{E}-04$ & 5.0E-04 & No & $\mathrm{s}$ & s & N/A & N/A \\
\hline & GW-690 & T & 5 & 5 & $1.2 \mathrm{E}-02$ & 7.0E-03 & No & NT & NT & N/A & N/A \\
\hline & $55-1 \mathrm{~B}$ & $\mathrm{~T}$ & 2 & 0 & $5.0 \mathrm{E}-04$ & 5.0E-04 & Yes & N/A & N/A & N/A & $N / A$ \\
\hline & GW-686 & $\mathrm{T}$ & 2 & 0 & $5.0 \mathrm{E}-04$ & 5.0E-04 & Yes & N/A & N/A & N/A & $\mathrm{N} / \mathrm{A}$ \\
\hline & GW-819 & $T$ & 1 & 0 & $5.0 \mathrm{E}-04$ & 5.0E-04 & Yes & N/A & $\mathrm{N} / \mathrm{A}$ & N/A & N/A \\
\hline & UEFPC-SP17 & $\mathrm{T}$ & 8 & 0 & $5.0 \mathrm{E}-04$ & 5.0E-04 & Yes & s & s & N/A & $N / A$ \\
\hline & GW-783 & T & 9 & 8 & $9.5 \mathrm{E}-03$ & 1.1E-02 & No & NT & s & N/A & N/A \\
\hline & GW-218 & $\mathrm{T}$ & 4 & 0 & $5.0 \mathrm{E}-04$ & 5.0E-04 & Yes & $\mathrm{s}$ & s & N/A & $\mathrm{N} / \mathrm{A}$ \\
\hline & GW-792 & $\mathrm{T}$ & 9 & 0 & $5.0 \mathrm{E}-04$ & 5.0E-04 & Yes & s & s & N/A & $N / A$ \\
\hline & GW-606 & T & 20 & 1 & $8.8 \mathrm{E}-04$ & $5.0 \mathrm{E}-04$ & No & NT & NT & N/A & N/A \\
\hline & GW-692 & $\mathrm{T}$ & 3 & 2 & $6.7 \mathrm{E}-04$ & 5.0E-04 & No & N/A & $\mathrm{N} / \mathrm{A}$ & $\mathrm{N} / \mathrm{A}$ & N/A \\
\hline & GW-782 & $\mathrm{T}$ & 20 & 20 & $5.4 \mathrm{E}-02$ & 5.7E-02 & No & D & S & N/A & $N / A$ \\
\hline & GW-788 & $\mathrm{T}$ & 9 & 0 & $5.0 \mathrm{E}-04$ & $5.0 \mathrm{E}-04$ & Yes & s & s & N/A & $\mathrm{N} / \mathrm{A}$ \\
\hline \multicolumn{12}{|l|}{ URANIUM } \\
\hline & GW-691 & $\mathrm{s}$ & 3 & 3 & $7.2 \mathrm{E}-04$ & 4.7E-04 & No & $\mathrm{N} / \mathrm{A}$ & $\mathrm{N} / \mathrm{A}$ & $\mathrm{N} / \mathrm{A}$ & N/A \\
\hline & GW-698 & S & 9 & 9 & $1.2 \mathrm{E}-03$ & $1.2 \mathrm{E}-03$ & No & $\mathrm{s}$ & PD & $\mathrm{N} / \mathrm{A}$ & N/A \\
\hline & GW-204 & s & 12 & 12 & $5.9 \mathrm{E}-02$ & 4.6E-02 & No & D & $\mathrm{D}$ & N/A & $N / A$ \\
\hline & GW-791 & s & 19 & 0 & $1.0 \mathrm{E}-05$ & 1.0E-05 & Yes & $\mathrm{s}$ & s & N/A & $\mathrm{N} / \mathrm{A}$ \\
\hline & GW-656 & s & 4 & 1 & $1.9 \mathrm{E}-04$ & $1.0 \mathrm{E}-05$ & No & NT & NT & N/A & $\mathrm{N} / \mathrm{A}$ \\
\hline & GW-820 & s & 6 & 0 & $1.0 \mathrm{E}-05$ & 1.0E-05 & Yes & $\mathrm{s}$ & $D$ & N/A & $N / A$ \\
\hline & $56-2 C$ & s & 5 & 0 & $1.0 \mathrm{E}-05$ & $1.0 \mathrm{E}-05$ & Yes & $\mathrm{s}$ & s & N/A & N/A \\
\hline & GW-219 & s & 12 & 12 & $3.7 \mathrm{E}-01$ & 3.3E-01 & No & D & D & $\mathrm{N} / \mathrm{A}$ & N/A \\
\hline & $56-2 B$ & s & 3 & 0 & 1.0E-05 & 1.0E-05 & Yes & N/A & N/A & N/A & $N / A$ \\
\hline & GW-605 & s & 20 & 20 & $1.4 \mathrm{E}-01$ & $9.8 \mathrm{E}-02$ & No & $\mathrm{s}$ & $\mathrm{D}$ & N/A & N/A \\
\hline & GW-782 & $T$ & 20 & 20 & $1.3 \mathrm{E}-03$ & 1.3E-03 & No & D & D & $\mathrm{N} / \mathrm{A}$ & $\mathrm{N} / \mathrm{A}$ \\
\hline & GW-779 & $\mathrm{T}$ & 3 & 1 & 3.3E-03 & 1.0E-05 & No & N/A & N/A & N/A & $N / A$ \\
\hline & $55-6 A$ & $\mathrm{~T}$ & 3 & 2 & $1.5 \mathrm{E}-03$ & $6.8 \mathrm{E}-04$ & No & N/A & $\mathrm{N} / \mathrm{A}$ & $\mathrm{N} / \mathrm{A}$ & $\mathrm{N} / \mathrm{A}$ \\
\hline & $56-2 A$ & $T$ & 3 & 1 & $1.9 \mathrm{E}-04$ & 1.0E-05 & No & N/A & $\mathrm{N} / \mathrm{A}$ & $\mathrm{N} / \mathrm{A}$ & $N / A$ \\
\hline & $55-1 B$ & $\mathrm{~T}$ & 2 & 2 & 8.3E-04 & 8.3E-04 & No & N/A & $\mathrm{N} / \mathrm{A}$ & N/A & $N / A$ \\
\hline
\end{tabular}


Project: Y-12 National Security Complex

Location: Central Y-12
User Name: MV

State: Tennessee

\begin{tabular}{|c|c|c|c|c|c|c|c|c|c|c|c|}
\hline Constituent & Well & $\begin{array}{l}\text { Sourcel } \\
\text { Tail }\end{array}$ & $\begin{array}{l}\text { Number } \\
\text { of } \\
\text { Samples }\end{array}$ & $\begin{array}{c}\text { Number } \\
\text { of } \\
\text { Detects }\end{array}$ & $\begin{array}{c}\text { Average } \\
\text { (mg/L) }\end{array}$ & $\begin{array}{r}\text { Median } \\
(\mathrm{mg} / \mathrm{L})\end{array}$ & $\begin{array}{c}\text { All } \\
\text { Samples } \\
\text { "ND" ? }\end{array}$ & $\begin{array}{l}\text { Mann- } \\
\text { Kendall }\end{array}$ & $\begin{array}{c}\text { Linear } \\
\text { Regression }\end{array}$ & Modeling & Empirical \\
\hline \multicolumn{12}{|l|}{ URANIUM } \\
\hline & GW-780 & $T$ & 3 & 0 & $1.0 \mathrm{E}-05$ & $1.0 \mathrm{E}-05$ & Yes & $\mathrm{N} / \mathrm{A}$ & N/A & N/A & N/A \\
\hline & GW-781 & $\mathrm{T}$ & 9 & 0 & $1.0 \mathrm{E}-05$ & $1.0 \mathrm{E}-05$ & Yes & s & I & $\mathrm{N} / \mathrm{A}$ & N/A \\
\hline & GW-786 & $\mathrm{T}$ & 5 & 0 & $1.0 \mathrm{E}-05$ & 1.0E-05 & Yes & s & D & N/A & N/A \\
\hline & GW-783 & $\mathrm{T}$ & 10 & 10 & $1.0 \mathrm{E}-03$ & 1.1E-03 & No & NT & D & N/A & N/A \\
\hline & N-S PIPE & $\mathrm{T}$ & 10 & 10 & 7.1E-02 & 5.7E-02 & No & NT & S & N/A & N/A \\
\hline & $60-1 B$ & $\mathrm{~T}$ & 2 & 0 & $1.0 \mathrm{E}-05$ & $1.0 \mathrm{E}-05$ & Yes & N/A & N/A & N/A & $N / A$ \\
\hline & GW-686 & $\mathrm{T}$ & 2 & 2 & 7.3E-04 & 7.3E-04 & No & N/A & N/A & N/A & N/A \\
\hline & GW-789 & $\mathrm{T}$ & 13 & 1 & $2.9 \mathrm{E}-05$ & $1.0 \mathrm{E}-05$ & No & NT & NT & N/A & N/A \\
\hline & GW-690 & $\mathrm{T}$ & 5 & 1 & $1.8 \mathrm{E}-04$ & $1.0 \mathrm{E}-05$ & No & NT & $\mathrm{D}$ & N/A & N/A \\
\hline & GW-792 & $\mathrm{T}$ & 9 & 0 & $1.0 \mathrm{E}-05$ & $1.0 \mathrm{E}-05$ & Yes & $\mathrm{s}$ & I & N/A & $N / A$ \\
\hline & GW-218 & $\mathrm{T}$ & 4 & 4 & $5.9 \mathrm{E}-03$ & $6.0 \mathrm{E}-03$ & No & s & s & N/A & N/A \\
\hline & GW-788 & $\mathrm{T}$ & 9 & 9 & $1.3 \mathrm{E}-03$ & $1.3 \mathrm{E}-03$ & No & NT & NT & N/A & N/A \\
\hline & GW-193 & $\mathrm{T}$ & 17 & 11 & $1.3 \mathrm{E}-02$ & $3.9 \mathrm{E}-03$ & No & $\mathrm{D}$ & $\mathrm{D}$ & N/A & N/A \\
\hline & UEFPC-SP17 & $\mathrm{T}$ & 6 & 4 & $1.1 \mathrm{E}-03$ & $1.4 \mathrm{E}-03$ & No & PD & s & N/A & N/A \\
\hline & GW-819 & $\mathrm{T}$ & 1 & 0 & $1.0 \mathrm{E}-05$ & $1.0 \mathrm{E}-05$ & Yes & $\mathrm{N} / \mathrm{A}$ & N/A & N/A & N/A \\
\hline & $59-1 C$ & $\mathrm{~T}$ & 3 & 0 & $1.0 \mathrm{E}-05$ & $1.0 \mathrm{E}-05$ & Yes & N/A & N/A & N/A & N/A \\
\hline & GW-700 & $\mathrm{T}$ & 5 & 4 & $5.6 \mathrm{E}-04$ & 4.3E-04 & No & $\mathrm{s}$ & $\mathrm{s}$ & N/A & N/A \\
\hline & GW-770 & $\mathrm{T}$ & 19 & 16 & 7.5E-04 & 7.3E-04 & No & s & NT & $\mathrm{N} / \mathrm{A}$ & N/A \\
\hline & GW-760 & $\mathrm{T}$ & 3 & 1 & $9.3 \mathrm{E}-05$ & $1.0 \mathrm{E}-05$ & No & $\mathrm{N} / \mathrm{A}$ & N/A & N/A & $N / A$ \\
\hline & GW-692 & $\mathrm{T}$ & 3 & 3 & 3.7E-04 & 3.3E-04 & No & $\mathrm{N} / \mathrm{A}$ & N/A & $N / A$ & $N / A$ \\
\hline & GW-787 & $\mathrm{T}$ & 5 & 1 & $1.9 \mathrm{E}-04$ & $1.0 \mathrm{E}-05$ & No & NT & NT & $\mathrm{N} / \mathrm{A}$ & N/A \\
\hline & GW-606 & $\mathrm{T}$ & 20 & 15 & $3.9 \mathrm{E}-03$ & $5.3 \mathrm{E}-03$ & No & $\mathrm{PD}$ & $\mathrm{s}$ & N/A & $N / A$ \\
\hline & GW-761 & $\mathrm{T}$ & 2 & 0 & $1.0 \mathrm{E}-05$ & $1.0 \mathrm{E}-05$ & Yes & N/A & N/A & $N / A$ & $N / A$ \\
\hline & $59-1 B$ & $\mathrm{~T}$ & 4 & 4 & 4.5E-04 & 4.3E-04 & No & $\mathrm{S}$ & $\mathrm{s}$ & N/A & N/A \\
\hline & GW-765 & $\mathrm{T}$ & 3 & 0 & $1.0 \mathrm{E}-05$ & $1.0 \mathrm{E}-05$ & Yes & N/A & N/A & N/A & N/A \\
\hline & $59-1 \mathrm{~A}$ & $\mathrm{~T}$ & 3 & 3 & $6.6 \mathrm{E}-03$ & 8.3E-03 & No & N/A & N/A & N/A & $N / A$ \\
\hline & GW-769 & $\mathrm{T}$ & 19 & 0 & $1.0 \mathrm{E}-05$ & 1.0E-05 & Yes & s & $\mathrm{S}$ & N/A & N/A \\
\hline & 9201-3C-4SP & $\mathrm{T}$ & 2 & 2 & $1.0 \mathrm{E}-03$ & $1.0 \mathrm{E}-03$ & No & $\mathrm{N} / \mathrm{A}$ & N/A & N/A & N/A \\
\hline
\end{tabular}

Note: Increasing (I); Probably Increasing (PI); Stable (S); Probably Decreasing (PD); Decreasing (D); No Trend (NT); Not Applicable (N/A) - Due to insufficient Data (< 4 sampling events); Source/Tail (S/T)

The Number of Samples and Number of Detects shown above are post-consolidation values. 


\section{MAROS Spatial Moment Analysis Summary}

Project: Y-12 National Security Complex

Location: Central Y-12
User Name: MV

State: Tennessee 2nd Moment (Spread)

Oth Moment

$\underline{\text { 1st Moment (Center of Mass) }}$

Mass (Kg)
Sigma XX Sigma YY

(sq ft)

(sq ft)
Estimated

Source

Effective Date
Number of

Wells

\begin{tabular}{|c|c|c|c|c|c|c|c|}
\hline 1/1/1996 & 1.7E-01 & 59,322 & 29,745 & 3,099 & 805,250 & 129,340 & 15 \\
\hline 4/1/1996 & 4.2E-01 & 58,364 & 29,710 & 2,145 & $1,850,792$ & 176,296 & 22 \\
\hline 7/1/1996 & $0.0 \mathrm{E}+00$ & & & & & & 2 \\
\hline 10/1/1996 & 7.0E-01 & 59,761 & 29,541 & 3,551 & 555,264 & 91,215 & 16 \\
\hline 1/1/1997 & $0.0 \mathrm{E}+00$ & & & & & & 3 \\
\hline 4/1/1997 & 1.0E-01 & 59,282 & 29,627 & 3,067 & 682,355 & 90,089 & 10 \\
\hline $7 / 1 / 1997$ & $0.0 \mathrm{E}+00$ & & & & & & 4 \\
\hline $10 / 1 / 1997$ & 6.8E-02 & 58,586 & 29,905 & 2,360 & 150,613 & 5,786 & 9 \\
\hline 1/1/1998 & 9.3E-01 & 58,328 & 29,557 & 2,127 & 794,815 & 26,623 & 13 \\
\hline 4/1/1998 & 1.4E-01 & 58,161 & 29,756 & 1,940 & 407,203 & 28,381 & 11 \\
\hline 7/1/1998 & 2.8E-01 & 59,608 & 29,137 & 3,464 & 609,913 & 12,800 & 7 \\
\hline 10/1/1998 & 5.4E-02 & 58,595 & 29,901 & 2,369 & 150,242 & 6,309 & 9 \\
\hline 1/1/1999 & $0.0 \mathrm{E}+00$ & & & & & & 4 \\
\hline 4/1/1999 & 5.5E-02 & 58,591 & 29,902 & 2,365 & 153,085 & 6,534 & 9 \\
\hline 7/1/1999 & 3.0E-01 & 58,924 & 29,396 & 2,742 & 792,264 & 42,396 & 7 \\
\hline 10/1/1999 & $9.2 \mathrm{E}-02$ & 58,794 & 29,716 & 2,573 & 199,562 & 53,680 & 11 \\
\hline $1 / 1 / 2000$ & $0.0 \mathrm{E}+00$ & & & & & & 4 \\
\hline $4 / 1 / 2000$ & 2.6E-01 & 58,524 & 29,600 & 2,315 & $1,549,382$ & 114,348 & 15 \\
\hline $7 / 1 / 2000$ & $0.0 \mathrm{E}+00$ & & & & & & 5 \\
\hline $10 / 1 / 2000$ & 2.1E-01 & 58,081 & 29,716 & 1,863 & 819,863 & 66,952 & 13 \\
\hline $1 / 1 / 2001$ & $0.0 \mathrm{E}+00$ & & & & & & 3 \\
\hline $4 / 1 / 2001$ & 1.8E-01 & 58,225 & 29,675 & 2,010 & 460,069 & 57,660 & 9 \\
\hline $7 / 1 / 2001$ & $0.0 \mathrm{E}+00$ & & & & & & 5 \\
\hline 10/1/2001 & 1.8E-01 & 58,225 & 29,675 & 2,010 & 460,069 & 57,660 & 9 \\
\hline $1 / 1 / 2002$ & $0.0 \mathrm{E}+00$ & & & & & & 3 \\
\hline $4 / 1 / 2002$ & 2.8E-01 & 57,567 & 29,825 & 1,342 & $1,032,405$ & 73,835 & 8 \\
\hline $7 / 1 / 2002$ & $0.0 \mathrm{E}+00$ & & & & & & 3 \\
\hline $10 / 1 / 2002$ & 2.9E-01 & 57,460 & 29,821 & 1,236 & $1,144,100$ & 85,313 & 9 \\
\hline $1 / 1 / 2003$ & $0.0 \mathrm{E}+00$ & & & & & & 3 \\
\hline $4 / 1 / 2003$ & 3.6E-01 & 58,380 & 29,910 & 2,154 & 944,249 & 151,528 & 20 \\
\hline $7 / 1 / 2003$ & $0.0 \mathrm{E}+00$ & & & & & & 3 \\
\hline $10 / 1 / 2003$ & 3.6E-01 & 58,380 & 29,910 & 2,154 & 944,249 & 151,528 & 20 \\
\hline $1 / 1 / 2004$ & $0.0 \mathrm{E}+00$ & & & & & & 3 \\
\hline $4 / 1 / 2004$ & 2.9E-01 & 57,812 & 29,775 & 1,590 & $1,182,721$ & 115,712 & 13 \\
\hline $7 / 1 / 2004$ & $0.0 \mathrm{E}+00$ & & & & & & 3 \\
\hline $10 / 1 / 2004$ & 2.9E-01 & 57,812 & 29,775 & 1,590 & $1,182,721$ & 115,712 & 13 \\
\hline
\end{tabular}

GROSS ALPHA ACTIVITY

$1 / 1 / 1996$

$5.2 \mathrm{E}+03$

58,173

29,686

1,957

$1,497,105$

82,574

18 
Project: Y-12 National Security Complex

Location: Central Y-12
User Name: $\quad$ MV

State: Tennessee

\begin{tabular}{|c|c|c|c|c|c|c|c|}
\hline \multirow[b]{2}{*}{ Effective Date } & \multirow{2}{*}{$\begin{array}{l}\text { Oth Moment } \\
\text { Estimated } \\
\text { Mass (kg) }\end{array}$} & \multicolumn{3}{|c|}{ 1st Moment (Center of Mass) } & \multicolumn{2}{|c|}{ 2nd Moment (Spread) } & \multirow[b]{2}{*}{$\begin{array}{c}\text { Number of } \\
\text { Wells }\end{array}$} \\
\hline & & $\mathrm{Xc}(\mathrm{ft})$ & Yc (ft) & $\begin{array}{c}\text { Source } \\
\text { Distance (ft) }\end{array}$ & $\begin{array}{c}\text { Sigma } X X \\
(\mathrm{sq} \mathrm{ft})\end{array}$ & $\begin{array}{c}\text { Sigma YY } \\
(\mathrm{sq} f t)\end{array}$ & \\
\hline \multicolumn{8}{|l|}{ GROSS ALPHA ACTIVITY } \\
\hline $4 / 1 / 1996$ & $2.5 \mathrm{E}+03$ & 58,118 & 29,743 & 1,897 & $1,467,993$ & 130,967 & 14 \\
\hline 7/1/1996 & $0.0 \mathrm{E}+00$ & & & & & & 2 \\
\hline 10/1/1996 & $2.0 \mathrm{E}+03$ & 59,340 & 29,303 & 3,168 & 693,538 & 28,420 & 8 \\
\hline $1 / 1 / 1997$ & $0.0 \mathrm{E}+00$ & & & & & & 4 \\
\hline $4 / 1 / 1997$ & $0.0 \mathrm{E}+00$ & & & & & & 5 \\
\hline 7/1/1997 & $0.0 \mathrm{E}+00$ & & & & & & 4 \\
\hline 10/1/1997 & $9.2 \mathrm{E}+02$ & 58,175 & 29,775 & 1,952 & 209,391 & 24,025 & 7 \\
\hline $1 / 1 / 1998$ & $1.1 \mathrm{E}+03$ & 59,175 & 29,449 & 2,981 & $1,193,804$ & 54,552 & 11 \\
\hline $4 / 1 / 1998$ & $1.4 \mathrm{E}+03$ & 57,936 & 29,765 & 1,715 & 238,565 & 22,046 & 8 \\
\hline $7 / 1 / 1998$ & $1.6 \mathrm{E}+03$ & 59,062 & 29,145 & 2,930 & 734,513 & 12,743 & 6 \\
\hline 10/1/1998 & $1.3 \mathrm{E}+02$ & 58,355 & 29,930 & 2,130 & 143,697 & 10,194 & 9 \\
\hline 1/1/1999 & $0.0 \mathrm{E}+00$ & & & & & & 4 \\
\hline 4/1/1999 & $4.2 \mathrm{E}+02$ & 58,505 & 29,912 & 2,280 & 141,990 & 4,424 & 8 \\
\hline 7/1/1999 & $7.0 \mathrm{E}+02$ & 59,534 & 29,347 & 3,351 & 314,929 & 21,093 & 6 \\
\hline 10/1/1999 & $4.7 \mathrm{E}+02$ & 59,098 & 29,512 & 2,896 & 185,035 & 33,343 & 9 \\
\hline $1 / 1 / 2000$ & $0.0 \mathrm{E}+00$ & & & & & & 3 \\
\hline $4 / 1 / 2000$ & $2.1 \mathrm{E}+03$ & 58,169 & 29,657 & 1,956 & 873,822 & 89,945 & 13 \\
\hline $7 / 1 / 2000$ & $0.0 \mathrm{E}+00$ & & & & & & 4 \\
\hline $10 / 1 / 2000$ & $2.4 \mathrm{E}+03$ & 57,892 & 29,764 & 1,670 & 451,509 & 64,794 & 11 \\
\hline $1 / 1 / 2001$ & $0.0 \mathrm{E}+00$ & & & & & & 3 \\
\hline $4 / 1 / 2001$ & $4.2 \mathrm{E}+03$ & 58,282 & 29,451 & 2,101 & 300,171 & 11,587 & 7 \\
\hline $7 / 1 / 2001$ & $0.0 \mathrm{E}+00$ & & & & & & 5 \\
\hline $10 / 1 / 2001$ & $0.0 \mathrm{E}+00$ & & & & & & 4 \\
\hline $1 / 1 / 2002$ & $0.0 \mathrm{E}+00$ & & & & & & 3 \\
\hline $4 / 1 / 2002$ & $6.4 \mathrm{E}+03$ & 58,070 & 29,630 & 1,861 & 712,299 & 69,864 & 8 \\
\hline $7 / 1 / 2002$ & $0.0 \mathrm{E}+00$ & & & & & & 4 \\
\hline $10 / 1 / 2002$ & $0.0 \mathrm{E}+00$ & & & & & & 5 \\
\hline $1 / 1 / 2003$ & $0.0 \mathrm{E}+00$ & & & & & & 5 \\
\hline $4 / 1 / 2003$ & $5.6 \mathrm{E}+03$ & 58,148 & 29,631 & 1,938 & 559,228 & 68,470 & 17 \\
\hline $7 / 1 / 2003$ & $0.0 \mathrm{E}+00$ & & & & & & 3 \\
\hline $10 / 1 / 2003$ & $7.7 \mathrm{E}+03$ & 58,202 & 29,713 & 1,983 & 475,243 & 54,476 & 15 \\
\hline $1 / 1 / 2004$ & $0.0 \mathrm{E}+00$ & & & & & & 3 \\
\hline $4 / 1 / 2004$ & $3.3 \mathrm{E}+03$ & 58,074 & 29,497 & 1,888 & 539,282 & 15,902 & 8 \\
\hline $7 / 1 / 2004$ & $0.0 \mathrm{E}+00$ & & & & & & 3 \\
\hline $10 / 1 / 2004$ & $3.7 \mathrm{E}+03$ & 57,991 & 29,626 & 1,784 & 766,162 & 85,099 & 12 \\
\hline \multicolumn{8}{|c|}{ TETRACHLOROETHYLENE(PCE) } \\
\hline 1/1/1996 & 5.1E-01 & 58,682 & 29,951 & 2,456 & $1,012,919$ & 109,623 & 15 \\
\hline 4/1/1996 & $6.7 \mathrm{E}+01$ & 56,778 & 29,944 & 555 & 93,328 & 22,189 & 22 \\
\hline $7 / 1 / 1996$ & $0.0 \mathrm{E}+00$ & & & & & & 2 \\
\hline 10/1/1996 & $9.5 \mathrm{E}-01$ & 58,592 & 29,970 & 2,368 & 992,301 & 123,284 & 16 \\
\hline 1/1/1997 & $0.0 \mathrm{E}+00$ & & & & & & 3 \\
\hline 4/1/1997 & 5.9E-01 & 58,359 & 29,934 & 2,134 & 523,071 & 64,773 & 10 \\
\hline
\end{tabular}


Project: Y-12 National Security Complex

Location: Central Y-12
User Name: MV

State: Tennessee

\begin{tabular}{|c|c|c|c|c|c|c|c|}
\hline \multirow[b]{2}{*}{ Effective Date } & th Moment & \multicolumn{3}{|c|}{ 1st Moment (Center of Mass) } & \multicolumn{2}{|c|}{ 2nd Moment (Spread) } & \multirow[b]{2}{*}{$\begin{array}{c}\text { Number of } \\
\text { Wells }\end{array}$} \\
\hline & $\begin{array}{c}\text { Estimated } \\
\text { Mass (kg) }\end{array}$ & $\mathrm{Xc}(\mathrm{ft})$ & Yc (ft) & $\begin{array}{c}\text { Source } \\
\text { Distance (ft) }\end{array}$ & $\begin{array}{c}\text { Sigma } X X \\
(\mathrm{sq} f t)\end{array}$ & $\begin{array}{c}\text { Sigma YY } \\
\text { (sq ft) }\end{array}$ & \\
\hline
\end{tabular}

TETRACHLOROETHYLENE(PCE)

\begin{tabular}{|c|c|c|c|c|c|c|c|}
\hline 7/1/1997 & $0.0 \mathrm{E}+00$ & & & & & & 4 \\
\hline 10/1/1997 & 3.6E-01 & 58,174 & 30,007 & 1,952 & 202,677 & 22,312 & 9 \\
\hline $1 / 1 / 1998$ & $2.9 \mathrm{E}+00$ & 58,829 & 29,416 & 2,645 & 541,967 & 52,206 & 13 \\
\hline 4/1/1998 & 3.7E+00 & 57,514 & 29,745 & 1,296 & 171,402 & 8,841 & 11 \\
\hline 7/1/1998 & 3.0E-01 & 58,872 & 29,138 & 2,749 & 788,065 & 15,134 & 7 \\
\hline 10/1/1998 & 5.6E-01 & 58,416 & 29,937 & 2,191 & 182,008 & 10,105 & 9 \\
\hline 1/1/1999 & $0.0 \mathrm{E}+00$ & & & & & & 4 \\
\hline 4/1/1999 & 4.0E-01 & 58,361 & 29,963 & 2,136 & 225,870 & 16,003 & 9 \\
\hline 7/1/1999 & 9.2E-01 & 59,400 & 29,244 & 3,238 & 496,643 & 32,114 & 7 \\
\hline 10/1/1999 & 4.6E-01 & 58,405 & 29,880 & 2,179 & 225,979 & 41,628 & 11 \\
\hline $1 / 1 / 2000$ & $0.0 \mathrm{E}+00$ & & & & & & 4 \\
\hline $4 / 1 / 2000$ & $1.8 \mathrm{E}+01$ & 57,479 & 29,813 & 1,255 & $1,257,100$ & 72,768 & 15 \\
\hline $7 / 1 / 2000$ & $0.0 \mathrm{E}+00$ & & & & & & 5 \\
\hline $10 / 1 / 2000$ & $2.1 \mathrm{E}+01$ & 57,300 & 29,786 & 1,078 & 746,686 & 45,149 & 13 \\
\hline $1 / 1 / 2001$ & $0.0 \mathrm{E}+00$ & & & & & & 3 \\
\hline $4 / 1 / 2001$ & 8.7E+00 & 57,724 & 29,649 & 1,517 & 177,849 & 38,561 & 9 \\
\hline $7 / 1 / 2001$ & $0.0 \mathrm{E}+00$ & & & & & & 5 \\
\hline $10 / 1 / 2001$ & $9.5 \mathrm{E}+00$ & 57,740 & 29,661 & 1,530 & 190,938 & 40,915 & 9 \\
\hline $1 / 1 / 2002$ & $0.0 \mathrm{E}+00$ & & & & & & 3 \\
\hline $4 / 1 / 2002$ & $1.9 \mathrm{E}+01$ & 57,755 & 29,881 & 1,529 & 926,358 & 45,719 & 8 \\
\hline $7 / 1 / 2002$ & $0.0 \mathrm{E}+00$ & & & & & & 3 \\
\hline $10 / 1 / 2002$ & 1.7E+01 & 58,383 & 29,894 & 2,158 & 394,210 & 19,126 & 9 \\
\hline $1 / 1 / 2003$ & $0.0 \mathrm{E}+00$ & & & & & & 3 \\
\hline $4 / 1 / 2003$ & $1.5 \mathrm{E}+01$ & 57,585 & 29,910 & 1,359 & 955,299 & 102,081 & 20 \\
\hline $7 / 1 / 2003$ & $0.0 \mathrm{E}+00$ & & & & & & 3 \\
\hline $10 / 1 / 2003$ & $1.5 \mathrm{E}+01$ & 57,444 & 29,797 & 1,221 & 971,408 & 100,340 & 20 \\
\hline $1 / 1 / 2004$ & $0.0 \mathrm{E}+00$ & & & & & & 3 \\
\hline $4 / 1 / 2004$ & $2.0 \mathrm{E}+01$ & 57,752 & 29,943 & 1,527 & 970,186 & 78,097 & 13 \\
\hline $7 / 1 / 2004$ & $0.0 \mathrm{E}+00$ & & & & & & 3 \\
\hline $10 / 1 / 2004$ & $2.4 \mathrm{E}+01$ & 57,710 & 29,959 & 1,485 & $1,030,524$ & 79,806 & 13 \\
\hline
\end{tabular}

TRICHLOROETHYLENE (TCE)

\begin{tabular}{|c|c|c|c|c|c|c|c|}
\hline 1/1/1996 & 2.6E-01 & 59,146 & 29,808 & 2,921 & 883,461 & 116,606 & 15 \\
\hline 4/1/1996 & $1.6 \mathrm{E}+00$ & 57,619 & 29,773 & 1,397 & $1,097,124$ & 109,399 & 22 \\
\hline $7 / 1 / 1996$ & $0.0 \mathrm{E}+00$ & & & & & & 2 \\
\hline 10/1/1996 & 3.4E-01 & 58,873 & 29,888 & 2,647 & 888,268 & 111,023 & 16 \\
\hline 1/1/1997 & $0.0 \mathrm{E}+00$ & & & & & & 3 \\
\hline 4/1/1997 & 1.7E-01 & 58,855 & 29,747 & 2,633 & 748,489 & 88,035 & 10 \\
\hline 7/1/1997 & $0.0 \mathrm{E}+00$ & & & & & & 4 \\
\hline $10 / 1 / 1997$ & 8.6E-02 & 58,553 & 29,900 & 2,327 & 166,626 & 10,081 & 9 \\
\hline 1/1/1998 & $2.3 \mathrm{E}+00$ & 58,716 & 29,534 & 2,515 & 369,248 & 47,434 & 13 \\
\hline 4/1/1998 & 6.4E-01 & 57,722 & 29,724 & 1,504 & 295,681 & 20,645 & 11 \\
\hline 7/1/1998 & 3.5E-01 & 58,881 & 29,131 & 2,760 & 703,094 & 13,438 & 7 \\
\hline
\end{tabular}


Project: Y-12 National Security Complex

Location: Central Y-12
User Name: $\quad$ MV

State: Tennessee

\begin{tabular}{ccccccccc} 
& \multicolumn{2}{c}{ 0th Moment } & & \multicolumn{2}{c}{ 1st Moment (Center of Mass) } & & \multicolumn{2}{c}{ 2nd Moment (Spread) } \\
Effective Date & $\begin{array}{c}\text { Estimated } \\
\text { Mass (kg) }\end{array}$ & Xc (ft) & Yc (ft) & $\begin{array}{c}\text { Source } \\
\text { Distance (ft) }\end{array}$ & $\begin{array}{c}\text { Sigma XX } \\
\text { (sq ft) }\end{array}$ & $\begin{array}{c}\text { Sigma YY } \\
\text { (sq ft) }\end{array}$ & $\begin{array}{c}\text { Number of } \\
\text { Wells }\end{array}$
\end{tabular}

TRICHLOROETHYLENE (TCE)

\begin{tabular}{|c|c|c|c|c|c|c|c|}
\hline $10 / 1 / 1998$ & 1.6E-01 & 58,415 & 29,923 & 2,190 & 144,477 & 5,819 & 9 \\
\hline 1/1/1999 & $0.0 \mathrm{E}+00$ & & & & & & 4 \\
\hline 4/1/1999 & 9.1E-02 & 58,399 & 29,928 & 2,174 & 160,022 & 7,643 & 9 \\
\hline 7/1/1999 & 3.6E-01 & 59,540 & 29,215 & 3,381 & 495,379 & 28,413 & 7 \\
\hline 10/1/1999 & 1.5E-01 & 58,536 & 29,810 & 2,311 & 230,263 & 48,149 & 11 \\
\hline $1 / 1 / 2000$ & $0.0 \mathrm{E}+00$ & & & & & & 4 \\
\hline $4 / 1 / 2000$ & $5.0 \mathrm{E}+00$ & 57,767 & 29,644 & 1,560 & $1,376,946$ & 64,802 & 15 \\
\hline $7 / 1 / 2000$ & $0.0 \mathrm{E}+00$ & & & & & & 5 \\
\hline $10 / 1 / 2000$ & $8.1 E+00$ & 57,399 & 29,693 & 1,188 & 449,030 & 18,594 & 13 \\
\hline $1 / 1 / 2001$ & $0.0 \mathrm{E}+00$ & & & & & & 3 \\
\hline $4 / 1 / 2001$ & $2.3 \mathrm{E}+01$ & 57,511 & 29,611 & 1,314 & 43,240 & 7,934 & 9 \\
\hline $7 / 1 / 2001$ & $0.0 \mathrm{E}+00$ & & & & & & 5 \\
\hline $10 / 1 / 2001$ & $1.9 \mathrm{E}+01$ & 57,505 & 29,615 & 1,307 & 42,039 & 7,496 & 9 \\
\hline $1 / 1 / 2002$ & $0.0 \mathrm{E}+00$ & & & & & & 3 \\
\hline $4 / 1 / 2002$ & 4.7E+00 & 57,573 & 29,689 & 1,361 & 613,257 & 80,424 & 8 \\
\hline $7 / 1 / 2002$ & $0.0 \mathrm{E}+00$ & & & & & & 3 \\
\hline $10 / 1 / 2002$ & 3.7E+00 & 57,975 & 29,620 & 1,768 & 470,859 & 55,411 & 9 \\
\hline $1 / 1 / 2003$ & $0.0 \mathrm{E}+00$ & & & & & & 3 \\
\hline $4 / 1 / 2003$ & $4.1 \mathrm{E}+00$ & 57,891 & 29,666 & 1,679 & $1,324,567$ & 85,475 & 20 \\
\hline $7 / 1 / 2003$ & $0.0 \mathrm{E}+00$ & & & & & & 3 \\
\hline $10 / 1 / 2003$ & $6.6 \mathrm{E}+00$ & 57,400 & 29,619 & 1,203 & 948,917 & 53,699 & 20 \\
\hline $1 / 1 / 2004$ & $0.0 \mathrm{E}+00$ & & & & & & 3 \\
\hline $4 / 1 / 2004$ & $3.4 \mathrm{E}+00$ & 57,688 & 29,623 & 1,485 & 550,788 & 75,985 & 13 \\
\hline $7 / 1 / 2004$ & $0.0 \mathrm{E}+00$ & & & & & & 3 \\
\hline $10 / 1 / 2004$ & $4.1 \mathrm{E}+00$ & 57,666 & 29,666 & 1,456 & 621,377 & 88,998 & 13 \\
\hline
\end{tabular}

URANIUM

\begin{tabular}{|c|c|c|c|c|c|c|c|}
\hline 1/1/1996 & $1.5 \mathrm{E}-01$ & 60,224 & 29,438 & 4,023 & $1,199,997$ & 182,117 & 16 \\
\hline 4/1/1996 & $6.0 \mathrm{E}-01$ & 59,586 & 29,249 & 3,419 & $1,694,685$ & 88,386 & 23 \\
\hline $7 / 1 / 1996$ & $0.0 \mathrm{E}+00$ & & & & & & 2 \\
\hline 10/1/1996 & $2.3 \mathrm{E}+00$ & 59,683 & 29,224 & 3,519 & 357,965 & 23,194 & 17 \\
\hline 1/1/1997 & $0.0 \mathrm{E}+00$ & & & & & & 4 \\
\hline 4/1/1997 & $9.6 \mathrm{E}-03$ & 58,375 & 29,915 & 2,149 & 135,589 & 8,807 & 9 \\
\hline $7 / 1 / 1997$ & $0.0 \mathrm{E}+00$ & & & & & & 4 \\
\hline 10/1/1997 & $1.7 \mathrm{E}-01$ & 58,700 & 29,584 & 2,492 & 241,321 & 20,267 & 10 \\
\hline $1 / 1 / 1998$ & $6.1 \mathrm{E}-01$ & 58,455 & 29,358 & 2,290 & $1,544,166$ & 39,985 & 13 \\
\hline 4/1/1998 & $9.9 \mathrm{E}-02$ & 58,292 & 29,684 & 2,075 & 282,319 & 28,567 & 10 \\
\hline 7/1/1998 & $0.0 \mathrm{E}+00$ & & & & & & 4 \\
\hline 10/1/1998 & 3.5E-03 & 58,340 & 29,920 & 2,114 & 147,546 & 9,810 & 9 \\
\hline 1/1/1999 & $0.0 \mathrm{E}+00$ & & & & & & 4 \\
\hline 4/1/1999 & $3.4 \mathrm{E}-03$ & 58,339 & 29,921 & 2,113 & 148,095 & 9,781 & 9 \\
\hline 7/1/1999 & $4.5 \mathrm{E}+00$ & 58,388 & 29,537 & 2,190 & 220,927 & 6,844 & 7 \\
\hline 10/1/1999 & $1.4 \mathrm{E}-01$ & 59,233 & 29,405 & 3,045 & 88,112 & 11,834 & 11 \\
\hline
\end{tabular}


Project: Y-12 National Security Complex

Location: Central Y-12
User Name: $\quad$ MV

State: Tennessee

\begin{tabular}{|c|c|c|c|c|c|c|c|}
\hline \multirow[b]{2}{*}{ Effective Date } & \multirow{2}{*}{$\begin{array}{r}\text { 0th Moment } \\
\text { Estimated } \\
\text { Mass }(\mathrm{kg})\end{array}$} & \multicolumn{3}{|c|}{ 1st Moment (Center of Mass) } & \multicolumn{2}{|c|}{$\underline{\text { 2nd Moment (Spread) }}$} & \multirow[b]{2}{*}{$\begin{array}{c}\text { Number of } \\
\text { Wells }\end{array}$} \\
\hline & & $\mathrm{Xc}(\mathrm{ft})$ & Yc (ft) & $\begin{array}{c}\text { Source } \\
\text { Distance (ft) }\end{array}$ & $\begin{array}{l}\text { Sigma } X X \\
(s q \mathrm{ft})\end{array}$ & $\begin{array}{l}\text { Sigma } Y Y \\
(\mathrm{sq} \mathrm{ft})\end{array}$ & \\
\hline \multicolumn{8}{|l|}{ URANIUM } \\
\hline $1 / 1 / 2000$ & $0.0 \mathrm{E}+00$ & & & & & & 4 \\
\hline $4 / 1 / 2000$ & 2.4E-01 & 57,850 & 29,588 & 1,651 & 369,422 & 61,389 & 13 \\
\hline $7 / 1 / 2000$ & $0.0 \mathrm{E}+00$ & & & & & & 3 \\
\hline $10 / 1 / 2000$ & 3.4E-01 & 57,763 & 29,606 & 1,561 & 335,427 & 54,091 & 13 \\
\hline $1 / 1 / 2001$ & $0.0 \mathrm{E}+00$ & & & & & & 3 \\
\hline $4 / 1 / 2001$ & $1.0 \mathrm{E}+00$ & 57,989 & 29,436 & 1,819 & 98,240 & 21,746 & 9 \\
\hline $7 / 1 / 2001$ & $0.0 \mathrm{E}+00$ & & & & & & 5 \\
\hline 10/1/2001 & 8.4E-01 & 58,003 & 29,427 & 1,834 & 102,327 & 16,597 & 9 \\
\hline $1 / 1 / 2002$ & $0.0 \mathrm{E}+00$ & & & & & & 3 \\
\hline $4 / 1 / 2002$ & $1.2 \mathrm{E}+00$ & 57,912 & 29,493 & 1,731 & 299,189 & 34,758 & 8 \\
\hline $7 / 1 / 2002$ & $0.0 \mathrm{E}+00$ & & & & & & 4 \\
\hline $10 / 1 / 2002$ & $7.9 \mathrm{E}-01$ & 57,748 & 29,515 & 1,566 & 504,944 & 39,902 & 9 \\
\hline $1 / 1 / 2003$ & $0.0 \mathrm{E}+00$ & & & & & & 5 \\
\hline $4 / 1 / 2003$ & $1.4 \mathrm{E}+00$ & 58,000 & 29,465 & 1,822 & 147,237 & 32,486 & 21 \\
\hline $7 / 1 / 2003$ & $0.0 \mathrm{E}+00$ & & & & & & 3 \\
\hline $10 / 1 / 2003$ & 5.7E-01 & 57,940 & 29,555 & 1,745 & 322,797 & 44,983 & 20 \\
\hline $1 / 1 / 2004$ & $0.0 \mathrm{E}+00$ & & & & & & 3 \\
\hline $4 / 1 / 2004$ & 8.7E-01 & 58,303 & 29,530 & 2,106 & 606,120 & 38,178 & 17 \\
\hline $7 / 1 / 2004$ & $0.0 \mathrm{E}+00$ & & & & & & 3 \\
\hline $10 / 1 / 2004$ & $6.9 \mathrm{E}-01$ & 58,087 & 29,472 & 1,906 & 658,124 & 34,850 & 13 \\
\hline
\end{tabular}


Project: Y-12 National Security Complex

Location: Central Y-12
User Name: MV

State: Tennessee

\begin{tabular}{llllll} 
Moment Type & Constituent & $\begin{array}{l}\text { Coefficient } \\
\text { of Variation }\end{array}$ & $\begin{array}{c}\text { Mann-Kendall } \\
\text { S Statistic }\end{array}$ & $\begin{array}{c}\text { Confidence } \\
\text { in Trend }\end{array}$ & $\begin{array}{c}\text { Moment } \\
\text { Trend }\end{array}$ \\
\hline
\end{tabular}

Zeroth Moment: Mass

$\begin{array}{lllll}\text { BENZENE } & 1.26 & -21 & 60.7 \% & \text { NT } \\ \text { GROSS ALPHA ACTIVITY } & 1.46 & -12 & 55.9 \% & \text { NT } \\ \text { TETRACHLOROETHYLENE(PCE) } & 1.89 & 39 & 69.7 \% & \text { NT } \\ \text { TRICHLOROETHYLENE (TCE) } & 2.08 & 27 & 63.7 \% & \text { NT } \\ \text { URANIUM } & 1.89 & 15 & 57.5 \% & \text { NT }\end{array}$

1st Moment: Distance to Source

BENZENE
GROSS ALPHA ACTIVITY
TETRACHLOROETHYLENE(PCE)
TRICHLOROETHYLENE (TCE)
URANIUM

$\begin{array}{lccc}0.27 & -113 & 99.9 \% & \mathrm{D} \\ 0.24 & -47 & 94.6 \% & \mathrm{PD} \\ 0.35 & -63 & 96.0 \% & \mathrm{D} \\ 0.33 & -103 & 99.8 \% & \mathrm{D} \\ 0.31 & -110 & 100.0 \% & \mathrm{D}\end{array}$

2nd Moment: Sigma XX

BENZENE
GROSS ALPHA ACTIVITY
TETRACHLOROETHYLENE(PCE)
TRICHLOROETHYLENE (TCE)
URANIUM

$\begin{array}{cccc}0.59 & 65 & 96.5 \% & \mathrm{I} \\ 0.69 & -13 & 66.1 \% & \mathrm{~S} \\ 0.63 & 55 & 93.6 \% & \mathrm{PI} \\ 0.68 & -11 & 61.0 \% & \mathrm{~S} \\ 1.03 & -6 & 55.9 \% & \mathrm{NT}\end{array}$

2nd Moment: Sigma YY

\begin{tabular}{llllc} 
BENZENE & 0.68 & 59 & $94.9 \%$ & PI \\
GROSS ALPHA ACTIVITY & 0.75 & -1 & $50.0 \%$ & $\mathrm{~S}$ \\
TETRACHLOROETHYLENE(PCE) & 0.67 & 43 & $88.0 \%$ & NT \\
TRICHLOROETHYLENE (TCE) & 0.73 & -7 & $56.6 \%$ & $\mathrm{~S}$ \\
URANIUM & 1.00 & 12 & $62.9 \%$ & NT \\
\hline
\end{tabular}

Note: The following assumptions were applied for the calculation of the Zeroth Moment:

Porosity: $0.10 \quad$ Saturated Thickness: Uniform: $50 \mathrm{ft}$

Mann-Kendall Trend test performed on all sample events for each constituent. Increasing (I); Probably Increasing (PI); Stable (S); Probably Decreasing (PD); Decreasing (D); No Trend (NT); Not Applicable (N/A)-Due to insufficient Data (< 4 sampling events).

Note: The Sigma XX and Sigma YY components are estimated using the given field coordinate system and then rotated to align with the estimated groundwater flow direction. Moments are not calculated for sample events with less than 6 wells. 


\section{ASSESSMENT OF THE GROUNDWATER PROTECTION PROGRAM \\ Y-12 NATIONAL SECURITY COMPLEX, OAK RIDGE, TENNESSEE}

Y-12 National Security Complex

Oak Ridge, Tennessee

Appendix D.6 East Fork Regime Fuel Station Area

Table D.6.1 Qualitative Analysis Fuel Station Area

Table D.6.2 $\quad$ Aquifer Input Parameters

MAROS Report COC Assessment

MAROS Report Plume Summary

MAROS Report Spatial Moment Analysis Summary

(No Well Sufficiency areas of interest identified) 
TABLE D.6.1

QUALITATIVE ANALYSIS EAST FORK POPLAR CREEK FUEL STATION AREA

East Fork Poplar Creek Hydrogeologic Regime

Y-12 National Security Complex

Oak Ridge, Tennessee

\begin{tabular}{|c|c|c|c|c|c|c|c|c|c|c|c|c|}
\hline $\begin{array}{l}\text { Location } \\
\text { Name }\end{array}$ & $\begin{array}{c}\text { Location } \\
\text { Type }\end{array}$ & $\begin{array}{c}\text { Average Concentration } \\
\text { Exceeds Screening } \\
\text { Level }\end{array}$ & $\begin{array}{c}\text { Formation } \\
\text { Type }\end{array}$ & $\begin{array}{c}\text { Horizontal } \\
\text { Delineation }\end{array}$ & $\begin{array}{c}\text { Vertical } \\
\text { Delineation }\end{array}$ & $\begin{array}{c}\text { Exit } \\
\text { Location }\end{array}$ & RCRA & CERCLA & Unique & $\begin{array}{c}\text { Monitors } \\
\text { Background } \\
\text { Water Quality } \\
\end{array}$ & $\begin{array}{c}\text { Early } \\
\text { Detection }\end{array}$ & $\begin{array}{c}\text { Monitor } \\
\text { Source }\end{array}$ \\
\hline GW-183 & $\overline{W L}$ & & Aquifer & & & & & & & & & $\bar{X}$ \\
\hline GW-199 & WL & & Aquitard & $\mathrm{X}$ & & & & & & & & \\
\hline GW-200 & WL & & Aquitard & & & & & & & & & \\
\hline GW-202 & WL & & Aquitard & $x$ & & & & & & & & \\
\hline GW-281 & WL & & Aquitard & & & & & $\mathrm{x}$ & & & & $x$ \\
\hline GW-282 & $\mathrm{WL}$ & & Aquitard & & & & & & & & & $\mathrm{X}$ \\
\hline GW-283 & WL & & Aquitard & & & & & & $x$ & & & $x$ \\
\hline GW-658 & WL & $x$ & Aquitard & & & & & $\mathrm{x}$ & & & & $\mathrm{x}$ \\
\hline GW-659 & WL & & Aquitard & & & & & & & & & $x$ \\
\hline GW-751 & WL & & Aquitard & $x$ & & & & & $x$ & $x$ & & \\
\hline GW-752 & WL & & Aquitard & $x$ & & & & & & $x$ & & \\
\hline GW-753 & WL & $x$ & Aquitard & $x$ & & & & & $x$ & & & \\
\hline GW-754 & WL & $\mathrm{x}$ & Aquitard & $\mathrm{x}$ & & & & & & & & \\
\hline GW-756 & WL & & Aquitard & $x$ & & & & & & & & \\
\hline GW-762 & WL & $x$ & Aquitard & & & & & $x$ & & & & \\
\hline GW-763 & WL & $\mathrm{X}$ & Aquitard & $x$ & & & & & & & & \\
\hline GW-766 & WL & & Aquitard & & & & & & $x$ & & & \\
\hline GW-767 & WL & & Aquitard & & & & & & & & & \\
\hline GW-773 & WL & & Aquitard & $x$ & & & & & $\mathrm{x}$ & $x$ & & \\
\hline GW-774 & WL & & Aquitard & $x$ & & & & & & & & \\
\hline GW-775 & WL & & Aquitard & & & & & & & & & \\
\hline GW-776 & WL & & Aquitard & & & & & & & & & \\
\hline GW-802 & WL & & Aquitard & $x$ & & & & $x$ & & & & \\
\hline NHPCEMSP & SP & & & & & $x$ & & & & & & \\
\hline
\end{tabular}

1. $\mathrm{WL}=$ Monitoring Well; $\mathrm{SP}=$ Spring

2. Well data taken from BWXT Y-12 Analytical Database. Sample locations shown on Figures A.1 and A.3.

3. RCRA indicates wells monitored as part of compliance with RCRA Post-Closure Corrective Action Monitoring or designated Alternate location

CERCLA indicates locations monitored as part of compliance with CERCLA ROD or backup location. Data from BWXT, 2003a and BWXT 2004a.

4. Average Concentration Exceeds Screening = The average concentration over the entire sampling record for the priority constituent is above the MCL or other designated screening level

as defined in Table B.1.

5. Aquifer and aquitard formations identified in Fig. A.2 from BWXT Y12, 2003 Groundwater Monitoring Report , (12/01/2003).

6. Details of the decision criteria for each category are presented in the text. 
TABLE D.6.2

AQUIFER INPUT PARAMETERS

\author{
Fuel Station \\ East Fork Poplar Creek Regime \\ Y-12 National Security Complex
}

\begin{tabular}{|l|c|c|}
\hline \multicolumn{1}{|c|}{ Parameter } & Value & Units \\
\hline Current Plume Length & 1500 & $\mathrm{ft}$ \\
Maximum Plume Length & 1500 & $\mathrm{ft}$ \\
PlumeWidth & 500 & $\mathrm{ft}$ \\
SeepageVelocity (ft/yr) & 200 & $\mathrm{ft} / \mathrm{yr}$ \\
Distance to Receptors & 5000 & $\mathrm{ft}$ \\
GWFluctuations & Yes & -- \\
SourceTreatment & None & -- \\
PlumeType & BTEX & -- \\
Free NAPL Present & Yes & -- \\
\hline \multicolumn{1}{|c|}{ Parameter } & Value & 0 \\
\hline Groundwater flow direction & E/SE & -- \\
Effective Porosity & 0.1 & -- \\
Source Location near Well & GW-183 & $\mathrm{ft}$ \\
Source X-Coordinate & 61954.52 & $\mathrm{ft}$ \\
Source Y-Coordinate & 29658.59 & $\mathrm{ft}$ \\
Saturated Thickness & 50 & \\
\hline \multicolumn{2}{|c|}{ Source Wells } & GW-183, GW-281, GW-282 \\
\hline \multicolumn{2}{|c|}{} \\
\hline
\end{tabular}

Notes:

1. Aquifer data are general values for the hydrologic regime.

2. Priority COCs defined by prevalence, toxicty and mobility.

3. $\mathrm{ft}^{*}=$ Coordinates in $\mathrm{Y}-12$ Plant coordinates, feet.

4. Screening Levels are USEPA MCLs, except in the case of compounds without MCLs where the level is the Region 9 PRG for tap water.

5. Effective Porosity estimated based on average high and low values for aquifer and aquitard suburfaces. 


\section{MAROS COC Assessment}

Project: $\quad$ Y-12 National Security Complex

Location: Fuel Station

Toxicity:

\begin{tabular}{lccc}
\hline Toxicity: & $\begin{array}{c}\text { Representative } \\
\text { Concentration } \\
\text { (mg/L) }\end{array}$ & $\begin{array}{c}\text { PRG } \\
\text { (mg/L) }\end{array}$ & $\begin{array}{c}\text { Percent } \\
\text { Above } \\
\text { PRG }\end{array}$ \\
\hline BENZENE & $4.4 \mathrm{E}-01$ & $5.0 \mathrm{E}-03$ & $8764.7 \%$ \\
TETRACHLOROETHYLENE(PCE) & $8.1 \mathrm{E}-02$ & $5.0 \mathrm{E}-03$ & $1516.8 \%$ \\
TRICHLOROETHYLENE (TCE) & $4.5 \mathrm{E}-02$ & $5.0 \mathrm{E}-03$ & $795.3 \%$
\end{tabular}

Note: Top COCs by toxicity were determined by examining a representative concentration for each compound over the entire site. The compound representative concentrations are then compared with the chosen PRG for that compound, with the percentage excedence from the PRG determining the compound's toxicity. All compounds above exceed the PRG.

\section{Prevalence:}

\begin{tabular}{|c|c|c|c|c|c|}
\hline Contaminant of Concern & Class & $\begin{array}{l}\text { Total } \\
\text { Wells }\end{array}$ & $\begin{array}{c}\text { Total } \\
\text { Excedences }\end{array}$ & $\begin{array}{c}\text { Percent } \\
\text { Excedences }\end{array}$ & $\begin{array}{l}\text { Total } \\
\text { detects }\end{array}$ \\
\hline TRICHLOROETHYLENE (TCE) & ORG & 22 & 6 & $27.3 \%$ & 9 \\
\hline BENZENE & ORG & 22 & 3 & $13.6 \%$ & 3 \\
\hline TETRACHLOROETHYLENE(PCE) & ORG & 22 & 2 & $9.1 \%$ & 7 \\
\hline
\end{tabular}

Note: Top COCs by prevalence were determined by examining a representative concentration for each well location at the site. The total excedences (values above the chosen PRGs) are compared to the total number of wells to determine the prevalence of the compound.

\section{Mobility:}

Contaminant of Concern

BENZENE

TRICHLOROETHYLENE (TCE)

TETRACHLOROETHYLENE(PCE)
Kd

0.0984
0.297
0.923

0.0984

0.923

Note: Top COCs by mobility were determined by examining each detected compound in the dataset and comparing their mobilities (Koc's for organics, assume foc $=0.001$, and Kd's for metals).

\section{Contaminants of Concern (COC's)}

MANGANESE

TETRACHLOROETHYLENE(PCE)

TRICHLOROETHYLENE (TCE)

BENZENE

CHROMIUM III 


\section{MAROS Plume Analysis Summary}

Project: $\quad$ Y-12 National Security Complex

Location: Fuel Station

Time Period: 1/1/1996

to $1 / 1 / 2005$

Consolidation Period: No Time Consolidation

Consolidation Type: Median

Duplicate Consolidation: Average

ND Values: Specified Detection Limit

J Flag Values : Actual Value

\begin{tabular}{|c|c|c|c|c|c|c|c|c|c|c|c|}
\hline Constituent & Well & $\begin{array}{c}\text { Sourcel } \\
\text { Tail }\end{array}$ & $\begin{array}{c}\text { Number } \\
\text { of } \\
\text { Samples }\end{array}$ & $\begin{array}{c}\text { Number } \\
\text { of } \\
\text { Detects }\end{array}$ & $\begin{array}{c}\text { Average } \\
\text { (mg/L) }\end{array}$ & $\begin{array}{r}\text { Median } \\
(\mathrm{mg} / \mathrm{L})\end{array}$ & $\begin{array}{c}\text { All } \\
\text { Samples } \\
\text { "ND" ? }\end{array}$ & $\begin{array}{l}\text { Mann- } \\
\text { Kendall }\end{array}$ & $\begin{array}{c}\text { Linear } \\
\text { Regression }\end{array}$ & Modeling & Empirical \\
\hline \multicolumn{12}{|l|}{ BENZENE } \\
\hline & GW-281 & $\mathrm{S}$ & 2 & 0 & $5.0 \mathrm{E}-04$ & 5.0E-04 & Yes & $\mathrm{N} / \mathrm{A}$ & $\mathrm{N} / \mathrm{A}$ & $\mathrm{N} / \mathrm{A}$ & N/A \\
\hline & GW-752 & $\mathrm{T}$ & 3 & 0 & $5.0 \mathrm{E}-04$ & 5.0E-04 & Yes & N/A & N/A & N/A & N/A \\
\hline & GW-659 & $\mathrm{T}$ & 2 & 0 & $5.0 \mathrm{E}-04$ & 5.0E-04 & Yes & N/A & $N / A$ & $N / A$ & $\mathrm{~N} / \mathrm{A}$ \\
\hline & GW-776 & $\mathrm{T}$ & 11 & 0 & $5.0 \mathrm{E}-04$ & 5.0E-04 & Yes & S & D & N/A & N/A \\
\hline & GW-775 & $\mathrm{T}$ & 11 & 0 & $5.0 \mathrm{E}-04$ & 5.0E-04 & Yes & $\mathrm{s}$ & D & $N / A$ & $N / A$ \\
\hline & GW-751 & $\mathrm{T}$ & 3 & 0 & $5.0 \mathrm{E}-04$ & 5.0E-04 & Yes & N/A & N/A & N/A & N/A \\
\hline & GW-766 & $\mathrm{T}$ & 3 & 0 & $5.0 \mathrm{E}-04$ & 5.0E-04 & Yes & N/A & N/A & N/A & N/A \\
\hline & GW-802 & $\mathrm{T}$ & 4 & 0 & $5.0 \mathrm{E}-04$ & 5.0E-04 & Yes & $\mathrm{s}$ & $\mathrm{s}$ & $N / A$ & $N / A$ \\
\hline & GW-763 & $\mathrm{T}$ & 20 & 0 & $5.0 \mathrm{E}-04$ & 5.0E-04 & Yes & S & D & N/A & N/A \\
\hline & GW-754 & $\mathrm{T}$ & 5 & 0 & $5.0 \mathrm{E}-04$ & 5.0E-04 & Yes & $\mathrm{s}$ & $\mathrm{s}$ & N/A & N/A \\
\hline & GW-658 & $\mathrm{T}$ & 6 & 5 & $7.6 \mathrm{E}+00$ & $7.8 \mathrm{E}+00$ & No & NT & NT & $N / A$ & N/A \\
\hline & NHPCEMSP & $\mathrm{T}$ & 2 & 0 & $5.0 \mathrm{E}-04$ & 5.0E-04 & Yes & N/A & $N / A$ & $N / A$ & N/A \\
\hline & GW-762 & $\mathrm{T}$ & 12 & 0 & $5.0 \mathrm{E}-04$ & 5.0E-04 & Yes & $\mathrm{s}$ & s & N/A & N/A \\
\hline & GW-753 & $\mathrm{T}$ & 5 & 0 & $5.0 \mathrm{E}-04$ & 5.0E-04 & Yes & $\mathrm{s}$ & s & $N / A$ & N/A \\
\hline & GW-756 & $\mathrm{T}$ & 4 & 0 & $5.0 \mathrm{E}-04$ & 5.0E-04 & Yes & S & s & N/A & N/A \\
\hline & GW-767 & $\mathrm{T}$ & 3 & 0 & $5.0 \mathrm{E}-04$ & $5.0 \mathrm{E}-04$ & Yes & N/A & N/A & N/A & $\mathrm{N} / \mathrm{A}$ \\
\hline \multicolumn{12}{|l|}{ CHROMIUM III } \\
\hline & GW-766 & $\mathrm{T}$ & 3 & 0 & $1.5 \mathrm{E}-04$ & 1.5E-04 & Yes & $\mathrm{N} / \mathrm{A}$ & $\mathrm{N} / \mathrm{A}$ & $\mathrm{N} / \mathrm{A}$ & $\mathrm{N} / \mathrm{A}$ \\
\hline & GW-767 & $\mathrm{T}$ & 3 & 0 & $1.5 \mathrm{E}-04$ & 1.5E-04 & Yes & N/A & $\mathrm{N} / \mathrm{A}$ & N/A & N/A \\
\hline & GW-763 & $\mathrm{T}$ & 20 & 2 & $1.1 \mathrm{E}-03$ & 1.5E-04 & No & NT & NT & $\mathrm{N} / \mathrm{A}$ & N/A \\
\hline & GW-762 & $\mathrm{T}$ & 12 & 0 & $1.5 \mathrm{E}-04$ & 1.5E-04 & Yes & $\mathrm{s}$ & $\mathrm{s}$ & N/A & N/A \\
\hline
\end{tabular}

User Name: MV

State: Tennessee 
Project: Y-12 National Security Complex

Location: Fuel Station
User Name: MV

State: Tennessee

\begin{tabular}{|c|c|c|c|c|c|c|c|c|c|c|c|}
\hline Constituent & Well & $\begin{array}{l}\text { Sourcel } \\
\text { Tail }\end{array}$ & $\begin{array}{c}\text { Number } \\
\text { of } \\
\text { Samples }\end{array}$ & $\begin{array}{c}\text { Number } \\
\text { of } \\
\text { Detects }\end{array}$ & $\begin{array}{c}\text { Average } \\
\text { (mg/L) }\end{array}$ & $\begin{array}{r}\text { Median } \\
(\mathrm{mg} / \mathrm{L})\end{array}$ & $\begin{array}{c}\text { All } \\
\text { Samples } \\
\text { "ND" ? }\end{array}$ & $\begin{array}{l}\text { Mann- } \\
\text { Kendall }\end{array}$ & $\begin{array}{c}\text { Linear } \\
\text { Regression }\end{array}$ & Modeling & Empirical \\
\hline \multicolumn{12}{|l|}{ CHROMIUM III } \\
\hline & GW-752 & $T$ & 3 & 1 & $2.1 \mathrm{E}-03$ & $1.5 \mathrm{E}-04$ & No & N/A & N/A & N/A & $\mathrm{N} / \mathrm{A}$ \\
\hline & GW-751 & $\mathrm{T}$ & 3 & 1 & $1.8 \mathrm{E}-03$ & $1.5 \mathrm{E}-04$ & No & N/A & N/A & N/A & N/A \\
\hline & NHPCEMSP & $\mathrm{T}$ & 2 & 0 & $1.5 \mathrm{E}-04$ & $1.5 \mathrm{E}-04$ & Yes & N/A & N/A & N/A & $\mathrm{N} / \mathrm{A}$ \\
\hline & GW-658 & $\mathrm{T}$ & 2 & 0 & $1.5 \mathrm{E}-04$ & 1.5E-04 & Yes & N/A & N/A & N/A & N/A \\
\hline & GW-775 & $\mathrm{T}$ & 12 & 0 & $1.5 \mathrm{E}-04$ & $1.5 \mathrm{E}-04$ & Yes & $\mathrm{S}$ & s & N/A & N/A \\
\hline & GW-776 & $\mathrm{T}$ & 11 & 11 & 3.5E-01 & 8.8E-02 & No & $P D$ & $P D$ & N/A & N/A \\
\hline \multicolumn{12}{|l|}{ LEAD } \\
\hline & GW-763 & $\bar{T}$ & 20 & 7 & $8.4 \mathrm{E}-04$ & $4.5 \mathrm{E}-04$ & No & NT & NT & $\mathrm{N} / \mathrm{A}$ & N/A \\
\hline & NHPCEMSP & $\mathrm{T}$ & 2 & 0 & 4.5E-04 & 4.5E-04 & Yes & N/A & N/A & N/A & N/A \\
\hline & GW-658 & $\mathrm{T}$ & 2 & 1 & 2.6E-03 & 2.6E-03 & No & N/A & N/A & N/A & $\mathrm{N} / \mathrm{A}$ \\
\hline & GW-752 & $\mathrm{T}$ & 3 & 0 & 4.5E-04 & 4.5E-04 & Yes & N/A & $\mathrm{N} / \mathrm{A}$ & N/A & N/A \\
\hline & GW-767 & T & 3 & 1 & $5.2 \mathrm{E}-04$ & 4.5E-04 & No & N/A & N/A & N/A & N/A \\
\hline & GW-776 & $\mathrm{T}$ & 11 & 3 & $5.6 \mathrm{E}-04$ & 4.5E-04 & No & $S$ & $\mathrm{~s}$ & N/A & $\mathrm{N} / \mathrm{A}$ \\
\hline & GW-762 & $\mathrm{T}$ & 12 & 0 & 4.5E-04 & 4.5E-04 & Yes & s & s & N/A & N/A \\
\hline & GW-775 & $\mathrm{T}$ & 12 & 3 & $9.1 \mathrm{E}-04$ & 4.5E-04 & No & NT & $\mathrm{PI}$ & N/A & $N / A$ \\
\hline & GW-766 & $\mathrm{T}$ & 3 & 0 & 4.5E-04 & 4.5E-04 & Yes & N/A & N/A & N/A & $\mathrm{N} / \mathrm{A}$ \\
\hline & GW-751 & $T$ & 3 & 0 & 4.5E-04 & 4.5E-04 & Yes & N/A & N/A & N/A & $\mathrm{N} / \mathrm{A}$ \\
\hline \multicolumn{12}{|c|}{ TETRACHLOROETHYLENE(PCE) } \\
\hline & GW-281 & $\mathrm{S}$ & 2 & 0 & $5.0 \mathrm{E}-04$ & 5.0E-04 & Yes & N/A & $\mathrm{N} / \mathrm{A}$ & $\mathrm{N} / \mathrm{A}$ & $N / A$ \\
\hline & GW-659 & $T$ & 2 & 0 & $5.0 \mathrm{E}-04$ & 5.0E-04 & Yes & N/A & N/A & N/A & N/A \\
\hline & GW-752 & $\mathrm{T}$ & 3 & 0 & $5.0 \mathrm{E}-04$ & 5.0E-04 & Yes & N/A & N/A & N/A & $\mathrm{N} / \mathrm{A}$ \\
\hline & GW-751 & $T$ & 3 & 0 & $5.0 \mathrm{E}-04$ & 5.0E-04 & Yes & N/A & $\mathrm{N} / \mathrm{A}$ & N/A & N/A \\
\hline & GW-753 & $T$ & 5 & 0 & $5.0 \mathrm{E}-04$ & $5.0 \mathrm{E}-04$ & Yes & $\mathrm{s}$ & s & N/A & N/A \\
\hline & GW-658 & $\mathrm{T}$ & 6 & 0 & $5.0 \mathrm{E}-04$ & 5.0E-04 & Yes & $\mathrm{s}$ & s & N/A & $\mathrm{N} / \mathrm{A}$ \\
\hline & GW-767 & $\mathrm{T}$ & 3 & 0 & $5.0 \mathrm{E}-04$ & 5.0E-04 & Yes & N/A & $\mathrm{N} / \mathrm{A}$ & N/A & N/A \\
\hline & NHPCEMSP & $\mathrm{T}$ & 2 & 0 & $5.0 \mathrm{E}-04$ & 5.0E-04 & Yes & N/A & N/A & N/A & $N / A$ \\
\hline & GW-756 & $\mathrm{T}$ & 4 & 0 & $5.0 \mathrm{E}-04$ & 5.0E-04 & Yes & $\mathrm{S}$ & $\mathrm{S}$ & N/A & $\mathrm{N} / \mathrm{A}$ \\
\hline & GW-802 & $\mathrm{T}$ & 4 & 0 & $5.0 \mathrm{E}-04$ & 5.0E-04 & Yes & s & s & $\mathrm{N} / \mathrm{A}$ & $\mathrm{N} / \mathrm{A}$ \\
\hline & GW-762 & $\mathrm{T}$ & 12 & 12 & $2.1 \mathrm{E}+00$ & $2.2 E+00$ & No & 1 & 1 & N/A & $N / A$ \\
\hline & GW-763 & $\mathrm{T}$ & 20 & 13 & $1.9 \mathrm{E}-02$ & 2.0E-02 & No & NT & NT & N/A & $\mathrm{N} / \mathrm{A}$ \\
\hline & GW-776 & $T$ & 11 & 4 & $6.4 \mathrm{E}-04$ & 5.0E-04 & No & $\mathrm{PD}$ & $\mathrm{D}$ & N/A & N/A \\
\hline & GW-766 & $\mathrm{T}$ & 3 & 0 & $5.0 \mathrm{E}-04$ & 5.0E-04 & Yes & N/A & N/A & N/A & $N / A$ \\
\hline
\end{tabular}


Project: Y-12 National Security Complex

Location: Fuel Station
User Name: $\quad$ MV

State: Tennessee

\begin{tabular}{|c|c|c|c|c|c|c|c|c|c|c|c|}
\hline Constituent & Well & $\begin{array}{c}\text { Sourcel } \\
\text { Tail }\end{array}$ & $\begin{array}{c}\text { Number } \\
\text { of } \\
\text { Samples }\end{array}$ & $\begin{array}{c}\text { Number } \\
\text { of } \\
\text { Detects }\end{array}$ & $\begin{array}{c}\text { Average } \\
\text { (mg/L) }\end{array}$ & $\begin{array}{r}\text { Median } \\
(\mathrm{mg} / \mathrm{L})\end{array}$ & $\begin{array}{c}\text { All } \\
\text { Samples } \\
\text { "ND" ? }\end{array}$ & $\begin{array}{l}\text { Mann- } \\
\text { Kendall }\end{array}$ & $\begin{array}{c}\text { Linear } \\
\text { Regression }\end{array}$ & Modeling & Empirical \\
\hline \multicolumn{12}{|c|}{ TETRACHLOROETHYLENE(PCE) } \\
\hline & GW-754 & $T$ & 5 & 0 & $5.0 \mathrm{E}-04$ & $5.0 \mathrm{E}-04$ & Yes & S & S & N/A & N/A \\
\hline & GW-775 & $\mathrm{T}$ & 11 & 0 & $5.0 \mathrm{E}-04$ & $5.0 \mathrm{E}-04$ & Yes & s & $\mathrm{D}$ & N/A & N/A \\
\hline \multicolumn{12}{|c|}{ TRICHLOROETHYLENE (TCE) } \\
\hline & GW-281 & $\mathrm{S}$ & 2 & 0 & $5.0 \mathrm{E}-04$ & $5.0 \mathrm{E}-04$ & Yes & $\mathrm{N} / \mathrm{A}$ & N/A & $\mathrm{N} / \mathrm{A}$ & $\mathrm{N} / \mathrm{A}$ \\
\hline & GW-802 & $\mathrm{T}$ & 4 & 0 & $5.0 \mathrm{E}-04$ & $5.0 \mathrm{E}-04$ & Yes & $\mathrm{s}$ & $\mathrm{s}$ & N/A & N/A \\
\hline & GW-776 & $\mathrm{T}$ & 11 & 10 & $1.7 \mathrm{E}-03$ & $1.5 \mathrm{E}-03$ & No & s & $\mathrm{PD}$ & N/A & N/A \\
\hline & GW-658 & $\mathrm{T}$ & 6 & 0 & $5.0 \mathrm{E}-04$ & $5.0 \mathrm{E}-04$ & Yes & s & $\mathrm{s}$ & N/A & $N / A$ \\
\hline & GW-775 & $\mathrm{T}$ & 11 & 11 & 3.4E-03 & $2.5 \mathrm{E}-03$ & No & NT & NT & $N / A$ & $N / A$ \\
\hline & GW-659 & $\mathrm{T}$ & 2 & 0 & $5.0 \mathrm{E}-04$ & $5.0 \mathrm{E}-04$ & Yes & N/A & N/A & N/A & N/A \\
\hline & GW-762 & $\mathrm{T}$ & 12 & 12 & $1.3 \mathrm{E}-01$ & 1.4E-01 & No & NT & NT & N/A & $N / A$ \\
\hline & GW-766 & $\mathrm{T}$ & 3 & 0 & $5.0 \mathrm{E}-04$ & $5.0 \mathrm{E}-04$ & Yes & $N / A$ & N/A & $N / A$ & $N / A$ \\
\hline & GW-751 & $\mathrm{T}$ & 3 & 0 & $5.0 \mathrm{E}-04$ & $5.0 \mathrm{E}-04$ & Yes & N/A & N/A & N/A & N/A \\
\hline & GW-763 & $\mathrm{T}$ & 20 & 13 & $5.1 \mathrm{E}-03$ & 2.7E-03 & No & NT & NT & N/A & N/A \\
\hline & NHPCEMSP & $\mathrm{T}$ & 2 & 0 & $5.0 \mathrm{E}-04$ & $5.0 \mathrm{E}-04$ & Yes & $\mathrm{N} / \mathrm{A}$ & N/A & $N / A$ & $N / A$ \\
\hline & GW-752 & $\mathrm{T}$ & 3 & 0 & $5.0 \mathrm{E}-04$ & $5.0 \mathrm{E}-04$ & Yes & N/A & N/A & N/A & N/A \\
\hline & GW-756 & $\mathrm{T}$ & 4 & 0 & $5.0 \mathrm{E}-04$ & $5.0 \mathrm{E}-04$ & Yes & $\mathrm{s}$ & $\mathrm{s}$ & N/A & N/A \\
\hline & GW-753 & $\mathrm{T}$ & 5 & 1 & $2.2 \mathrm{E}-01$ & $5.0 \mathrm{E}-04$ & No & NT & NT & N/A & $N / A$ \\
\hline & GW-754 & $\mathrm{T}$ & 5 & 1 & $1.2 \mathrm{E}+00$ & 5.0E-04 & No & NT & NT & N/A & N/A \\
\hline & GW-767 & $\mathrm{T}$ & 3 & 0 & $5.0 \mathrm{E}-04$ & $5.0 \mathrm{E}-04$ & Yes & N/A & N/A & N/A & $\mathrm{N} / \mathrm{A}$ \\
\hline
\end{tabular}

Note: Increasing (I); Probably Increasing (PI); Stable (S); Probably Decreasing (PD); Decreasing (D); No Trend (NT); Not Applicable (N/A) - Due to insufficient Data (< 4 sampling events); Source/Tail (S/T)

The Number of Samples and Number of Detects shown above are post-consolidation values. 


\section{MAROS Spatial Moment Analysis Summary}

Project: Y-12 National Security Complex

Location: Fuel Station
User Name: $\mathrm{MV}$

State: Tennessee

\begin{tabular}{|c|c|c|c|c|c|c|c|}
\hline \multirow[b]{2}{*}{ Effective Date } & \multirow{2}{*}{$\begin{array}{l}\text { Oth Moment } \\
\text { Estimated } \\
\text { Mass (Kq) }\end{array}$} & \multicolumn{3}{|c|}{ 1st Moment (Center of Mass) } & \multicolumn{2}{|c|}{ 2nd Moment (Spread) } & \multirow[b]{2}{*}{$\begin{array}{c}\text { Number of } \\
\text { Wells }\end{array}$} \\
\hline & & $\mathrm{Xc}(\mathrm{ft})$ & Yc (ft) & $\begin{array}{c}\text { Source } \\
\text { Distance (ft) }\end{array}$ & $\underset{(s q \mathrm{ft})}{\operatorname{Sigma} X X}$ & $\underset{\text { (sq ft) }}{\text { Sigma YY }}$ & \\
\hline
\end{tabular}

BENZENE

\begin{tabular}{|c|c|c|c|c|c|c|}
\hline 1/1/1996 & 6.9E-02 & 62,558 & 29,496 & 624 & 105,764 & 26,911 \\
\hline 4/1/1996 & 4.4E-02 & 62,368 & 29,374 & 502 & 64,581 & 726 \\
\hline 7/1/1996 & $0.0 \mathrm{E}+00$ & & & & & \\
\hline 10/1/1996 & 4.4E-02 & 62,368 & 29,374 & 502 & 64,581 & 726 \\
\hline 4/1/1997 & 7.4E-02 & 62,595 & 29,528 & 653 & 19,853 & 31,727 \\
\hline 7/1/1997 & $0.0 \mathrm{E}+00$ & & & & & \\
\hline 10/1/1997 & $0.0 \mathrm{E}+00$ & & & & & \\
\hline 1/1/1998 & $0.0 \mathrm{E}+00$ & & & & & \\
\hline 4/1/1998 & $3.2 \mathrm{E}-02$ & 62,234 & 29,350 & 415 & 16,089 & 1,910 \\
\hline 7/1/1998 & 4.5E-02 & 62,695 & 29,819 & 758 & 38,503 & 2,135 \\
\hline 10/1/1998 & $0.0 \mathrm{E}+00$ & & & & & \\
\hline 1/1/1999 & $0.0 \mathrm{E}+00$ & & & & & \\
\hline 4/1/1999 & $0.0 \mathrm{E}+00$ & & & & & \\
\hline 7/1/1999 & $0.0 \mathrm{E}+00$ & & & & & \\
\hline 10/1/1999 & $0.0 \mathrm{E}+00$ & & & & & \\
\hline $4 / 1 / 2000$ & $0.0 \mathrm{E}+00$ & & & & & \\
\hline $7 / 1 / 2000$ & $0.0 \mathrm{E}+00$ & & & & & \\
\hline $10 / 1 / 2000$ & $0.0 \mathrm{E}+00$ & & & & & \\
\hline $1 / 1 / 2001$ & $0.0 \mathrm{E}+00$ & & & & & \\
\hline $4 / 1 / 2001$ & $0.0 \mathrm{E}+00$ & & & & & \\
\hline $7 / 1 / 2001$ & $0.0 \mathrm{E}+00$ & & & & & \\
\hline $10 / 1 / 2001$ & $0.0 \mathrm{E}+00$ & & & & & \\
\hline $1 / 1 / 2002$ & $0.0 \mathrm{E}+00$ & & & & & \\
\hline $4 / 1 / 2002$ & $0.0 \mathrm{E}+00$ & & & & & \\
\hline $7 / 1 / 2002$ & $0.0 \mathrm{E}+00$ & & & & & \\
\hline $10 / 1 / 2002$ & $0.0 \mathrm{E}+00$ & & & & & \\
\hline $1 / 1 / 2003$ & $0.0 \mathrm{E}+00$ & & & & & \\
\hline $4 / 1 / 2003$ & $0.0 \mathrm{E}+00$ & & & & & \\
\hline $7 / 1 / 2003$ & $0.0 \mathrm{E}+00$ & & & & & \\
\hline $10 / 1 / 2003$ & $0.0 \mathrm{E}+00$ & & & & & \\
\hline $1 / 1 / 2004$ & $0.0 \mathrm{E}+00$ & & & & & \\
\hline $4 / 1 / 2004$ & $0.0 \mathrm{E}+00$ & & & & & \\
\hline $7 / 1 / 2004$ & $0.0 \mathrm{E}+00$ & & & & & \\
\hline $10 / 1 / 2004$ & $0.0 \mathrm{E}+00$ & & & & & \\
\hline
\end{tabular}

CHROMIUM III

$\begin{array}{lrllllrr}1 / 1 / 1996 & 1.6 \mathrm{E}-01 & 62,304 & 29,389 & 441 & 30,614 & 5,968 & 8 \\ 4 / 1 / 1996 & 1.4 \mathrm{E}-01 & 62,299 & 29,373 & 447 & 28,934 & 728 & 7 \\ 7 / 1 / 1996 & 0.0 \mathrm{E}+00 & & & & & & 2\end{array}$


Project: Y-12 National Security Complex

Location: Fuel Station
User Name: $\quad$ MV

State: Tennessee

\begin{tabular}{|c|c|c|c|c|c|c|c|}
\hline \multirow[b]{2}{*}{ Effective Date } & \multirow{2}{*}{$\begin{array}{l}\text { 0th Moment } \\
\text { Estimated } \\
\text { Mass (kg) }\end{array}$} & \multicolumn{3}{|c|}{ 1st Moment (Center of Mass) } & \multicolumn{2}{|c|}{ 2nd Moment (Spread) } & \multirow[b]{2}{*}{$\begin{array}{c}\text { Number of } \\
\text { Wells }\end{array}$} \\
\hline & & Xc (ft) & Yc (ft) & $\begin{array}{c}\text { Source } \\
\text { Distance (ft) }\end{array}$ & $\begin{array}{l}\text { Sigma } X X \\
(s q \mathrm{ft})\end{array}$ & $\begin{array}{l}\text { Sigma } Y Y \\
(s q \mathrm{ft})\end{array}$ & \\
\hline \multicolumn{8}{|l|}{ CHROMIUM III } \\
\hline 10/1/1996 & $1.3 \mathrm{E}-01$ & 62,277 & 29,372 & 431 & 16,025 & 752 & 7 \\
\hline $4 / 1 / 1997$ & $0.0 \mathrm{E}+00$ & & & & & & 3 \\
\hline 10/1/1997 & $0.0 \mathrm{E}+00$ & & & & & & 3 \\
\hline $4 / 1 / 1998$ & $0.0 \mathrm{E}+00$ & & & & & & 3 \\
\hline 10/1/1998 & $0.0 \mathrm{E}+00$ & & & & & & 3 \\
\hline 1/1/1999 & $0.0 \mathrm{E}+00$ & & & & & & 1 \\
\hline 4/1/1999 & $0.0 \mathrm{E}+00$ & & & & & & 3 \\
\hline 7/1/1999 & $0.0 \mathrm{E}+00$ & & & & & & 1 \\
\hline 10/1/1999 & $0.0 \mathrm{E}+00$ & & & & & & 3 \\
\hline $4 / 1 / 2000$ & $0.0 \mathrm{E}+00$ & & & & & & 2 \\
\hline $7 / 1 / 2000$ & $0.0 \mathrm{E}+00$ & & & & & & 1 \\
\hline $10 / 1 / 2000$ & $0.0 \mathrm{E}+00$ & & & & & & 1 \\
\hline $1 / 1 / 2001$ & $0.0 \mathrm{E}+00$ & & & & & & 1 \\
\hline $4 / 1 / 2001$ & $0.0 \mathrm{E}+00$ & & & & & & 1 \\
\hline $7 / 1 / 2001$ & $0.0 \mathrm{E}+00$ & & & & & & 1 \\
\hline $10 / 1 / 2001$ & $0.0 \mathrm{E}+00$ & & & & & & 1 \\
\hline $1 / 1 / 2002$ & $0.0 \mathrm{E}+00$ & & & & & & 1 \\
\hline $4 / 1 / 2002$ & $0.0 \mathrm{E}+00$ & & & & & & 4 \\
\hline $7 / 1 / 2002$ & $0.0 \mathrm{E}+00$ & & & & & & 1 \\
\hline 10/1/2002 & $0.0 \mathrm{E}+00$ & & & & & & 4 \\
\hline $1 / 1 / 2003$ & $0.0 \mathrm{E}+00$ & & & & & & 1 \\
\hline $4 / 1 / 2003$ & $0.0 \mathrm{E}+00$ & & & & & & 1 \\
\hline $7 / 1 / 2003$ & $0.0 \mathrm{E}+00$ & & & & & & 1 \\
\hline $10 / 1 / 2003$ & $0.0 \mathrm{E}+00$ & & & & & & 1 \\
\hline $1 / 1 / 2004$ & $0.0 \mathrm{E}+00$ & & & & & & 1 \\
\hline $4 / 1 / 2004$ & $0.0 \mathrm{E}+00$ & & & & & & 2 \\
\hline $7 / 1 / 2004$ & $0.0 \mathrm{E}+00$ & & & & & & 1 \\
\hline $10 / 1 / 2004$ & $0.0 \mathrm{E}+00$ & & & & & & 1 \\
\hline
\end{tabular}

LEAD

$\begin{array}{rrrrrrrr}1 / 1 / 1996 & 6.2 \mathrm{E}-02 & 62,558 & 29,496 & 624 & 105,764 & 26,911 & 8 \\ 4 / 1 / 1996 & 3.9 \mathrm{E}-02 & 62,368 & 29,374 & 502 & 64,581 & 726 & 7 \\ 7 / 1 / 1996 & 0.0 \mathrm{E}+00 & & & & & 763 & 2 \\ 10 / 1 / 1996 & 5.3 \mathrm{E}-02 & 62,346 & 29,374 & 484 & 55,592 & 7 \\ 4 / 1 / 1997 & 0.0 \mathrm{E}+00 & & & & & 3 \\ 10 / 1 / 1997 & 0.0 \mathrm{E}+00 & & & & 3 \\ 4 / 1 / 1998 & 0.0 \mathrm{E}+00 & & & & 3 \\ 10 / 1 / 1998 & 0.0 \mathrm{E}+00 & & & & \\ 1 / 1 / 1999 & 0.0 \mathrm{E}+00 & & & & 3 \\ 4 / 1 / 1999 & 0.0 \mathrm{E}+00 & & & & 3 \\ 7 / 1 / 1999 & 0.0 \mathrm{E}+00 & & & & 3 \\ 10 / 1 / 1999 & 0.0 \mathrm{E}+00 & & & & & 3 \\ 4 / 1 / 2000 & 0.0 \mathrm{E}+00 & & & & & \\ \end{array}$


Project: Y-12 National Security Complex

Location: Fuel Station

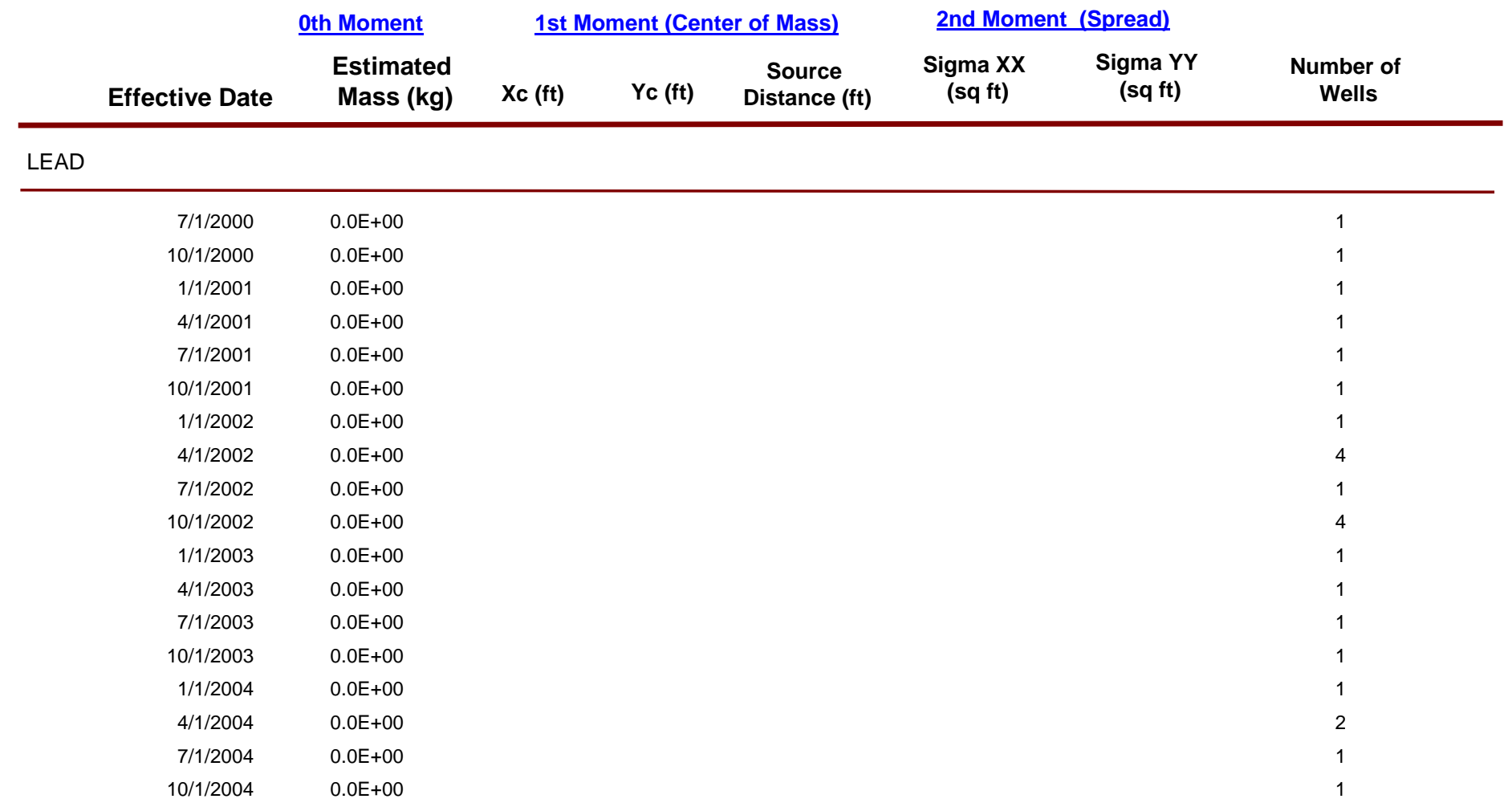

TETRACHLOROETHYLENE(PCE)

\begin{tabular}{|c|c|c|c|c|c|c|c|}
\hline 1/1/1996 & 2.0E-01 & 62,425 & 29,414 & 530 & 75,471 & 13,116 & 8 \\
\hline 4/1/1996 & 1.8E-01 & 62,358 & 29,371 & 495 & 49,795 & 303 & 7 \\
\hline $7 / 1 / 1996$ & $0.0 \mathrm{E}+00$ & & & & & & 2 \\
\hline 10/1/1996 & 4.4E-02 & 62,368 & 29,374 & 502 & 64,581 & 726 & 7 \\
\hline 4/1/1997 & 2.4E-01 & 62,596 & 29,423 & 683 & 18,476 & 21,442 & 6 \\
\hline $7 / 1 / 1997$ & $0.0 \mathrm{E}+00$ & & & & & & 3 \\
\hline 10/1/1997 & $0.0 \mathrm{E}+00$ & & & & & & 5 \\
\hline 1/1/1998 & $0.0 \mathrm{E}+00$ & & & & & & 3 \\
\hline 4/1/1998 & 3.2E-02 & 62,234 & 29,350 & 415 & 16,089 & 1,910 & 6 \\
\hline 7/1/1998 & 2.2E-02 & 62,865 & 29,816 & 923 & 15,885 & 2,745 & 6 \\
\hline 10/1/1998 & $0.0 \mathrm{E}+00$ & & & & & & 3 \\
\hline 1/1/1999 & $0.0 \mathrm{E}+00$ & & & & & & 1 \\
\hline 4/1/1999 & $0.0 \mathrm{E}+00$ & & & & & & 3 \\
\hline 7/1/1999 & $0.0 \mathrm{E}+00$ & & & & & & 1 \\
\hline 10/1/1999 & $0.0 \mathrm{E}+00$ & & & & & & 3 \\
\hline $4 / 1 / 2000$ & $0.0 \mathrm{E}+00$ & & & & & & 2 \\
\hline $7 / 1 / 2000$ & $0.0 \mathrm{E}+00$ & & & & & & 1 \\
\hline $10 / 1 / 2000$ & $0.0 \mathrm{E}+00$ & & & & & & 1 \\
\hline $1 / 1 / 2001$ & $0.0 \mathrm{E}+00$ & & & & & & 1 \\
\hline $4 / 1 / 2001$ & $0.0 \mathrm{E}+00$ & & & & & & 1 \\
\hline $7 / 1 / 2001$ & $0.0 \mathrm{E}+00$ & & & & & & 1 \\
\hline 10/1/2001 & $0.0 \mathrm{E}+00$ & & & & & & 1 \\
\hline $1 / 1 / 2002$ & $0.0 \mathrm{E}+00$ & & & & & & 1 \\
\hline
\end{tabular}


Project: Y-12 National Security Complex

Location: Fuel Station
User Name: $\quad$ MV

State: Tennessee

\begin{tabular}{|c|c|c|c|c|c|}
\hline \multirow[b]{2}{*}{ Effective Date } & \multirow{2}{*}{$\begin{array}{l}\text { Oth Moment } \\
\text { Estimated } \\
\text { Mass (kg) }\end{array}$} & \multicolumn{2}{|c|}{ 1st Moment (Center of Mass) } & $\underline{\text { 2nd Moment (Spread) }}$ & \multirow[b]{2}{*}{$\begin{array}{l}\text { Number of } \\
\text { Wells }\end{array}$} \\
\hline & & Yc (ft) & $\begin{array}{c}\text { Source } \\
\text { Distance (ft) }\end{array}$ & $\begin{array}{cc}\underset{(s q \mathrm{ft})}{\operatorname{Sigma} X X} & \text { Sigma YY } \\
(\mathrm{sq} f t)\end{array}$ & \\
\hline \multicolumn{6}{|c|}{ TETRACHLOROETHYLENE(PCE) } \\
\hline $4 / 1 / 2002$ & $0.0 \mathrm{E}+00$ & & & & 4 \\
\hline $7 / 1 / 2002$ & $0.0 \mathrm{E}+00$ & & & & 1 \\
\hline $10 / 1 / 2002$ & $0.0 \mathrm{E}+00$ & & & & 4 \\
\hline $1 / 1 / 2003$ & $0.0 \mathrm{E}+00$ & & & & 1 \\
\hline $4 / 1 / 2003$ & $0.0 \mathrm{E}+00$ & & & & 4 \\
\hline $7 / 1 / 2003$ & $0.0 \mathrm{E}+00$ & & & & 1 \\
\hline $10 / 1 / 2003$ & $0.0 \mathrm{E}+00$ & & & & 1 \\
\hline $1 / 1 / 2004$ & $0.0 \mathrm{E}+00$ & & & & 1 \\
\hline $4 / 1 / 2004$ & $0.0 \mathrm{E}+00$ & & & & 4 \\
\hline $7 / 1 / 2004$ & $0.0 \mathrm{E}+00$ & & & & 1 \\
\hline $10 / 1 / 2004$ & $0.0 \mathrm{E}+00$ & & & & 1 \\
\hline
\end{tabular}

TRICHLOROETHYLENE (TCE)

\begin{tabular}{|c|c|c|c|c|c|c|c|}
\hline 1/1/1996 & 1.3E-01 & 62,449 & 29,439 & 541 & 88,632 & 18,685 & 8 \\
\hline 4/1/1996 & 1.1E-01 & 62,341 & 29,372 & 481 & 50,059 & 512 & 7 \\
\hline 7/1/1996 & $0.0 \mathrm{E}+00$ & & & & & & 2 \\
\hline 10/1/1996 & 1.9E-01 & 62,339 & 29,371 & 480 & 44,627 & 389 & 7 \\
\hline 4/1/1997 & 1.9E-01 & 62,585 & 29,453 & 662 & 21,063 & 26,619 & 6 \\
\hline $7 / 1 / 1997$ & $0.0 \mathrm{E}+00$ & & & & & & 3 \\
\hline 10/1/1997 & $0.0 \mathrm{E}+00$ & & & & & & 5 \\
\hline 1/1/1998 & $0.0 \mathrm{E}+00$ & & & & & & 3 \\
\hline 4/1/1998 & 4.0E-02 & 62,213 & 29,348 & 404 & 22,180 & 1,773 & 6 \\
\hline $7 / 1 / 1998$ & 2.2E-02 & 62,865 & 29,816 & 923 & 15,885 & 2,745 & 6 \\
\hline 10/1/1998 & $0.0 \mathrm{E}+00$ & & & & & & 3 \\
\hline 1/1/1999 & $0.0 \mathrm{E}+00$ & & & & & & 1 \\
\hline 4/1/1999 & $0.0 \mathrm{E}+00$ & & & & & & 3 \\
\hline 7/1/1999 & $0.0 \mathrm{E}+00$ & & & & & & 1 \\
\hline 10/1/1999 & $0.0 \mathrm{E}+00$ & & & & & & 3 \\
\hline $4 / 1 / 2000$ & $0.0 \mathrm{E}+00$ & & & & & & 2 \\
\hline $7 / 1 / 2000$ & $0.0 \mathrm{E}+00$ & & & & & & 1 \\
\hline $10 / 1 / 2000$ & $0.0 \mathrm{E}+00$ & & & & & & 1 \\
\hline $1 / 1 / 2001$ & $0.0 \mathrm{E}+00$ & & & & & & 1 \\
\hline $4 / 1 / 2001$ & $0.0 \mathrm{E}+00$ & & & & & & 1 \\
\hline $7 / 1 / 2001$ & $0.0 \mathrm{E}+00$ & & & & & & 1 \\
\hline $10 / 1 / 2001$ & $0.0 \mathrm{E}+00$ & & & & & & 1 \\
\hline $1 / 1 / 2002$ & $0.0 \mathrm{E}+00$ & & & & & & 1 \\
\hline $4 / 1 / 2002$ & $0.0 \mathrm{E}+00$ & & & & & & 4 \\
\hline $7 / 1 / 2002$ & $0.0 \mathrm{E}+00$ & & & & & & 1 \\
\hline 10/1/2002 & $0.0 \mathrm{E}+00$ & & & & & & 4 \\
\hline $1 / 1 / 2003$ & $0.0 \mathrm{E}+00$ & & & & & & 1 \\
\hline $4 / 1 / 2003$ & $0.0 \mathrm{E}+00$ & & & & & & 4 \\
\hline $7 / 1 / 2003$ & $0.0 \mathrm{E}+00$ & & & & & & 1 \\
\hline $10 / 1 / 2003$ & $0.0 \mathrm{E}+00$ & & & & & & 1 \\
\hline
\end{tabular}


Project: Y-12 National Security Complex

Location: Fuel Station

\begin{tabular}{|c|c|c|c|c|c|c|c|}
\hline \multirow[b]{2}{*}{ Effective Date } & \multirow{2}{*}{$\begin{array}{r}\text { 0th Moment } \\
\text { Estimated } \\
\text { Mass (kg) }\end{array}$} & \multicolumn{3}{|c|}{ 1st Moment (Center of Mass) } & \multicolumn{2}{|c|}{ 2nd Moment (Spread) } & \multirow[b]{2}{*}{$\begin{array}{c}\text { Number of } \\
\text { Wells }\end{array}$} \\
\hline & & $\mathrm{Xc}(\mathrm{ft})$ & Yc (ft) & $\begin{array}{c}\text { Source } \\
\text { Distance (ft) }\end{array}$ & $\underset{(s q \mathrm{ft})}{\operatorname{Sigma} X X}$ & $\begin{array}{c}\text { Sigma YY } \\
\text { (sq ft) }\end{array}$ & \\
\hline
\end{tabular}

User Name: $\quad$ MV

State: Tennessee

2nd Moment (Spread) 
Project: Y-12 National Security Complex

Location: Fuel Station
User Name: $\mathrm{MV}$

State: Tennessee

\begin{tabular}{|c|c|c|c|c|c|}
\hline Moment Type & Constituent & $\begin{array}{l}\text { Coefficient } \\
\text { of Variation }\end{array}$ & $\begin{array}{l}\text { Mann-Kendall } \\
\text { S Statistic }\end{array}$ & $\begin{array}{l}\text { Confidence } \\
\text { in Trend }\end{array}$ & $\begin{array}{c}\text { Moment } \\
\text { Trend }\end{array}$ \\
\hline \multicolumn{6}{|c|}{ Zeroth Moment: Mass } \\
\hline & BENZENE & 2.31 & -149 & $98.6 \%$ & $\mathrm{D}$ \\
\hline & CHROMIUM III & 3.12 & -85 & $92.3 \%$ & PD \\
\hline & LEAD & 3.16 & -83 & $91.8 \%$ & PD \\
\hline & TETRACHLOROETHYLENE(PCE) & 2.84 & -157 & $99.0 \%$ & $\mathrm{D}$ \\
\hline & TRICHLOROETHYLENE (TCE) & 2.60 & -153 & $98.8 \%$ & $\mathrm{D}$ \\
\hline \multicolumn{6}{|c|}{ 1st Moment: Distance to Source } \\
\hline & BENZENE & 0.22 & 3 & $64.0 \%$ & NT \\
\hline & CHROMIUM III & 0.00 & 0 & $0.0 \%$ & $\mathrm{~N} / \mathrm{A}$ \\
\hline & LEAD & 0.00 & 0 & $0.0 \%$ & $\mathrm{~N} / \mathrm{A}$ \\
\hline & TETRACHLOROETHYLENE(PCE) & 0.31 & 3 & $64.0 \%$ & NT \\
\hline & TRICHLOROETHYLENE (TCE) & 0.32 & 1 & $50.0 \%$ & NT \\
\hline \multicolumn{6}{|c|}{ 2nd Moment: Sigma XX } \\
\hline & BENZENE & 0.66 & -11 & $97.2 \%$ & $\mathrm{D}$ \\
\hline & CHROMIUM III & 0.00 & 0 & $0.0 \%$ & $\mathrm{~N} / \mathrm{A}$ \\
\hline & LEAD & 0.00 & 0 & $0.0 \%$ & $\mathrm{~N} / \mathrm{A}$ \\
\hline & TETRACHLOROETHYLENE(PCE) & 0.67 & -13 & $99.2 \%$ & $\mathrm{D}$ \\
\hline & TRICHLOROETHYLENE (TCE) & 0.68 & -13 & $99.2 \%$ & $\mathrm{D}$ \\
\hline \multicolumn{6}{|c|}{ 2nd Moment: Sigma YY } \\
\hline & BENZENE & 1.36 & 3 & $64.0 \%$ & NT \\
\hline & CHROMIUM III & 0.00 & 0 & $0.0 \%$ & $\mathrm{~N} / \mathrm{A}$ \\
\hline & LEAD & 0.00 & 0 & $0.0 \%$ & $\mathrm{~N} / \mathrm{A}$ \\
\hline & TETRACHLOROETHYLENE(PCE) & 1.29 & 3 & $64.0 \%$ & NT \\
\hline & TRICHLOROETHYLENE (TCE) & 1.34 & 1 & $50.0 \%$ & NT \\
\hline
\end{tabular}

Note: The following assumptions were applied for the calculation of the Zeroth Moment:

Porosity: $0.10 \quad$ Saturated Thickness: Uniform: $50 \mathrm{ft}$

Mann-Kendall Trend test performed on all sample events for each constituent. Increasing (I); Probably Increasing (PI); Stable (S); Probably Decreasing (PD); Decreasing (D); No Trend (NT); Not Applicable (N/A)-Due to insufficient Data (< 4 sampling events).

Note: The Sigma XX and Sigma YY components are estimated using the given field coordinate system and then rotated to align with the estimated groundwater flow direction. Moments are not calculated for sample events with less than 6 wells. 


\section{ASSESSMENT OF THE GROUNDWATER PROTECTION PROGRAM \\ Y-12 NATIONAL SECURITY COMPLEX, OAK RIDGE, TENNESSEE}

Y-12 National Security Complex

Oak Ridge, Tennessee

Appendix D.7 East Fork Regime East Y-12 Area

Table D.7.1 Qualitative Analysis East Y-12 Area

Table D.7.2 Aquifer Input Parameters

MAROS Report COC Assessment

MAROS Report Plume Summary

MAROS Report Spatial Moment Analysis Summary

MAROS Chart New Location Analysis CTET East Y-12 
TABLE D.7.1

QUALITATIVE ANALYSIS EAST FORK POPLAR CREEK EAST Y-12 AREA

East Fork Poplar Creek Hydrogeologic Regime

12 National Security Complex

\begin{tabular}{|c|c|c|c|c|c|c|c|c|c|c|c|c|c|}
\hline $\begin{array}{c}\text { Location } \\
\text { Name }\end{array}$ & Location Type & \begin{tabular}{|c|} 
Average Concentration \\
Exceeds Screening Level
\end{tabular} & $\begin{array}{l}\text { Formation } \\
\text { Type }\end{array}$ & $\begin{array}{c}\text { Horizontal } \\
\text { Delineation } \\
\end{array}$ & $\begin{array}{c}\text { Vertical } \\
\text { Delineation }\end{array}$ & Exit Location & RCRA & CERCLA & Unique & Off-Site & $\begin{array}{c}\text { Monitors } \\
\text { Background } \\
\text { Water Quality }\end{array}$ & Early Detection & $\begin{array}{l}\text { Monitor } \\
\text { Source }\end{array}$ \\
\hline GW-148 & $\mathrm{WL}$ & & Aquifer & & & & & & & & $\mathrm{x}$ & & \\
\hline GW-149 & WL & & Aquifer & & & & & & $x$ & & & & \\
\hline GW-150 & WL & $\mathrm{x}$ & Aquifer & & & & & & $\mathrm{x}$ & & & & \\
\hline GW-151 & WL & $x$ & Aquifer & $x$ & $x$ & & & $x$ & & & & $x$ & \\
\hline GW-152 & WL & $\mathrm{x}$ & Aquifer & $\mathrm{x}$ & & & & & & & & & \\
\hline GW-153 & $\overline{W L}$ & $x$ & Aquifer & $x$ & & & & $x$ & & & & & \\
\hline GW-154 & WL & $\mathrm{x}$ & Aquifer & & & & & $x$ & & & & & \\
\hline GW-167 & WL & $x$ & Aquifer & $x$ & & & & & & & & $\mathrm{x}$ & \\
\hline GW-169 & WL & $\mathrm{x}$ & Aquifer & & & $\mathrm{x}$ & & $x$ & & $\mathrm{x}$ & & & \\
\hline GW-170 & $\mathrm{WL}$ & $x$ & Aquifer & $\mathrm{x}$ & & $x$ & & $x$ & & $x$ & & & \\
\hline GW-171 & WL & & Aquifer & & & $\mathrm{x}$ & & $x$ & & & & & \\
\hline GW-172 & WL & & Aquifer & & & $x$ & & $x$ & & & & & \\
\hline GW-207 & WL & & Aquitard & & & $x$ & & & & & & & \\
\hline GW-208 & WL & & Aquitard & & & $x$ & & & & & & & \\
\hline GW-220 & WL & $\mathrm{x}$ & Aquifer & $\mathrm{x}$ & & & & $\mathrm{x}$ & & & & $\mathrm{x}$ & \\
\hline GW-222 & $\mathrm{WL}$ & $\mathrm{X}$ & Aquifer & & & & & & & & & & \\
\hline GW-223 & WL & $x$ & Aquifer & $x$ & & & & $\mathrm{x}$ & & & & & \\
\hline GW-230 & WL & $x$ & Aquifer & & & $\mathrm{x}$ & & $\mathrm{x}$ & & & & & \\
\hline GW-232 & WL & & Aquifer & & & $x$ & & $x$ & & $x$ & & & \\
\hline GW-239 & WL & & Aquifer & & & & & & $\mathrm{x}$ & & $\mathrm{x}$ & & \\
\hline GW-240 & $\mathrm{WL}$ & $x$ & Aquifer & & & & & & & & & & \\
\hline GW-380 & WL & $x$ & Aquifer & & & & & $\mathrm{x}$ & & & & & $x$ \\
\hline GW-381 & WL & $x$ & Aquifer & & & & & $x$ & & & & & $x$ \\
\hline GW-382 & WL & $\mathrm{x}$ & Aquifer & $\mathrm{x}$ & & & & $x$ & & & & & $x$ \\
\hline GW-383 & WL & $x$ & Aquitard & & & & & $x$ & & & & & \\
\hline GW-384 & $\mathrm{WL}$ & & Aquitard & & & & & & $\mathrm{x}$ & & & & \\
\hline GW-385 & WL & & Aquitard & & & & & & $x$ & & & & \\
\hline GW-603 & WL & & Aquifer & & & & & & & & & & \\
\hline GW-733 & WL & $\mathrm{x}$ & Aquifer & $x$ & & $x$ & $x$ & & & & & & \\
\hline GW-735 & WL & & Aquifer & $x$ & & $\mathrm{x}$ & & & & & & & \\
\hline
\end{tabular}

Notes:

1. $W L=$ Monitoring Well; $S P=$ Spring

2. Well data taken from BWXT Y-12 Analytical Database. Sample locations shown on Figures A.1 and A.3.

RCRA indicates wells monitored as part of compliance with RCRA Post-Closure Corrective Action Monitoring or designated Alternate location;

4. Average Concentration Exceeds Screening = The average concentration over the entire sampling record for the priority constituent is above the MCL or other designated screening level

as defined in Table B.1.

5. Aquifer and aquitard formations identified in Fig. A.2 from BWXT Y12, 2003 Groundwater Monitoring Report, (12/01/2003).

6. Details of the decision criteria for each category are presented in the text. 
TABLE D.7.1

QUALITATIVE ANALYSIS EAST FORK POPLAR CREEK EAST Y-12 AREA

East Fork Poplar Creek Hydrogeologic Regime

-12 National Security Complex
Oak Ridge, Tennessee

\begin{tabular}{|c|c|c|c|c|c|c|c|c|c|c|c|c|c|}
\hline $\begin{array}{l}\text { Location } \\
\text { Name }\end{array}$ & Location Type & \begin{tabular}{|c|} 
Average Concentration \\
Exceeds Screening Level
\end{tabular} & $\begin{array}{c}\text { Formation } \\
\text { Type }\end{array}$ & $\begin{array}{l}\text { Horizontal } \\
\text { Delineation } \\
\end{array}$ & $\begin{array}{c}\text { Vertical } \\
\text { Delineation }\end{array}$ & Exit Location & RCRA & CERCLA & Unique & Off-Site & $\begin{array}{c}\text { Monitors } \\
\text { Background } \\
\text { Water Quality }\end{array}$ & Early Detection & $\begin{array}{l}\text { Monitor } \\
\text { Source }\end{array}$ \\
\hline GW-744 & $\mathrm{WL}$ & & Aquitard & $\mathrm{x}$ & & & & $\mathrm{x}$ & & & & & \\
\hline GW-745 & WL & & Aquitard & $x$ & & & & & $x$ & & & & \\
\hline GW-746 & WL & & Aquitard & $\mathrm{x}$ & & & & & & & & & \\
\hline GW-747 & WL & & Aquitard & $\mathrm{x}$ & $\mathrm{x}$ & & & $x$ & & & $\mathrm{x}$ & & \\
\hline GW-748 & WL & & Aquitard & $\mathrm{x}$ & $x$ & & & & $\mathrm{x}$ & & & & \\
\hline GW-749 & $\mathrm{WL}$ & & Aquitard & $\mathrm{x}$ & $\mathrm{x}$ & & & & & & $\mathrm{x}$ & & \\
\hline GW-750 & WL & & Aquifer & $x$ & & $\mathrm{x}$ & & & & & $x$ & $x$ & \\
\hline GW-816 & WL & & Aquitard & & & $x$ & & $x$ & & & & & \\
\hline GW-817 & WL & & Aquitard & $\mathrm{x}$ & & & & & & & & & \\
\hline GW-832 & WL & $\mathrm{x}$ & Aquifer & $\mathrm{x}$ & & & & $\mathrm{x}$ & & & & & \\
\hline GW-845 & $\overline{W L}$ & $\mathrm{x}$ & Extraction & & & & & & & & & & \\
\hline LRSPW & SP & $x$ & Not Used & & & $x$ & & & & & & & \\
\hline RGQWWSP & SP & & Spring & $x$ & & $x$ & & & & & $\mathrm{x}$ & & \\
\hline SCR7.10SP & $\mathrm{SP}$ & & Spring & $x$ & & $x$ & & & & & $\mathrm{x}$ & & \\
\hline SCR7.14SP & SP & & Spring & $x$ & & $\mathrm{x}$ & & & & & & & \\
\hline SCR7.16SP & $\mathrm{SP}$ & & Spring & $x$ & & $x$ & & & & & & & \\
\hline SCR7.18SP & $\mathrm{SP}$ & & Spring & $x$ & & $x$ & & & & & & & \\
\hline SCR7.1SP & SP & & Spring & $x$ & & $x$ & & & & & & & \\
\hline SCR7.4SP & $\mathrm{SP}$ & & Spring & $x$ & & $\mathrm{x}$ & & & & & $\mathrm{x}$ & & \\
\hline SCR7.6SP & $\mathrm{SP}$ & & Spring & $x$ & & $x$ & & & & & $x$ & & \\
\hline SCR7.7SP & $\mathrm{SP}$ & & Spring & $x$ & & $x$ & & & & & $x$ & & \\
\hline SCR7.8SP & SP & & Spring & $x$ & & $x$ & & & & & & & \\
\hline SCR7.8SSP & $\mathrm{SP}$ & & Spring & $\mathrm{x}$ & & $\mathrm{x}$ & & & & & & & \\
\hline UV8.5SP & $\mathrm{SP}$ & & Spring & $x$ & & $x$ & & & & & $\mathrm{x}$ & & \\
\hline UV8.6SP & $\mathrm{SP}$ & & Spring & $x$ & & $x$ & & & & & $\mathrm{x}$ & & \\
\hline
\end{tabular}

Notes:

1. $W L=$ Monitoring Well; $S P=S$ Spring

2. Well data taken from BWXT Y-12 Analytical Database. Sample locations shown on Figures A.1 and A.3.

3. RCRA indicates wells monitored as part of compliance with RCRA Post-Closure Corrective Action Monitoring or designated Alternate location:

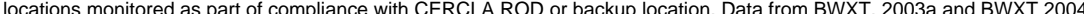

Average Concentration Exceeds Screening $=$ The average concentration over the entire sampling record for the priority constituent is above the MCL or other designated screening level

. Aquifer an in Table B.1.

ard formations identified in Fig. A.2 from BWXT Y12, 2003 Groundwater Monitoring Report, (12/01/2003).

of the decision criteria for each category are presented in the text. 
GSI Job No. G-3038

Issued $12 / 12 / 2005$

Page 1 of 1

GROUNDWATER SERVICES, INC.

\section{TABLE D.7.2 \\ AQUIFER INPUT PARAMETERS}

East Y-12

East Fork Poplar Creek Regime

Y-12 National Security Complex

\begin{tabular}{|l|c|c|}
\hline \multicolumn{1}{|c|}{ Parameter } & Value & Units \\
\hline Current Plume Length & 8500 & $\mathrm{ft}$ \\
Maximum Plume Length & 8500 & $\mathrm{ft}$ \\
PlumeWidth & 1500 & $\mathrm{ft}$ \\
SeepageVelocity (ft/yr) & 200 & $\mathrm{ft} / \mathrm{yr}$ \\
Distance to Receptors & 5000 & $\mathrm{ft}$ \\
GWFluctuations & Yes & -- \\
SourceTreatment & None & -- \\
PlumeType & Chlorinated Solvent & -- \\
Free NAPL Present & Yes & -- \\
\hline \multicolumn{1}{|c|}{ Parameter } & Value & 0 \\
\hline Groundwater flow direction & $\mathrm{E}$ & -- \\
Effective Porosity & 0.1 & -- \\
Source Location near Well & GW-381 & $\mathrm{ft}$ \\
Source X-Coordinate & 62947.7 & $\mathrm{ft}^{\star}$ \\
Source Y-Coordinate & 28715.04 & $\mathrm{ft}$ \\
Saturated Thickness & 50 & \\
\hline \multicolumn{2}{|c|}{ Source Wells } & \multicolumn{2}{|c|}{ GW-381,GW-382, GW 151, GW-223, GW-383, GW-154 } \\
\hline \multicolumn{2}{|c|}{} \\
\hline
\end{tabular}

\section{Notes:}

1. Aquifer data are general values for the hydrologic regime.

2. Priority COCs defined by prevalence, toxicty and mobility.

3. $\mathrm{ft}^{*}=$ Coordinates in $\mathrm{Y}-12$ Plant coordinates, feet.

4. Screening Levels are USEPA MCLs, except in the case of compounds without MCLs where the level is the Region 9 PRG for tap water.

5. Effective Porosity estimated based on average high and low values for aquifer and aquitard suburfaces. 


\section{MAROS Plume Analysis Summary}

Project: $\quad$ Y-12 National Security Complex

Location: East Y-12

Time Period: 1/1/1996

to $1 / 1 / 2005$

Consolidation Period: No Time Consolidation

Consolidation Type: Median

Duplicate Consolidation: Average

ND Values: Specified Detection Limit

J Flag Values : Actual Value

\begin{tabular}{|c|c|c|c|c|c|c|c|c|c|c|c|}
\hline Constituent & Well & $\begin{array}{l}\text { Sourcel } \\
\text { Tail }\end{array}$ & $\begin{array}{l}\text { Number } \\
\text { of } \\
\text { Samples }\end{array}$ & $\begin{array}{c}\text { Number } \\
\text { of } \\
\text { Detects }\end{array}$ & $\begin{array}{c}\text { Average } \\
\text { (mg/L) }\end{array}$ & $\begin{array}{l}\text { Median } \\
(\mathrm{mg} / \mathrm{L})\end{array}$ & $\begin{array}{c}\text { All } \\
\text { Samples } \\
\text { "ND" ? }\end{array}$ & $\begin{array}{l}\text { Mann- } \\
\text { Kendall }\end{array}$ & $\begin{array}{c}\text { Linear } \\
\text { Regression }\end{array}$ & Modeling & Empirical \\
\hline \multicolumn{12}{|c|}{ CARBON TETRACHLORIDE } \\
\hline & GW-151 & $\mathrm{s}$ & 19 & 18 & $1.1 \mathrm{E}+00$ & $1.1 \mathrm{E}+00$ & No & 1 & 1 & $\mathrm{~N} / \mathrm{A}$ & $\mathrm{N} / \mathrm{A}$ \\
\hline & GW-381 & s & 10 & 10 & $2.9 \mathrm{E}-01$ & 2.1E-01 & No & NT & NT & $\mathrm{N} / \mathrm{A}$ & N/A \\
\hline & GW-382 & $\mathrm{s}$ & 11 & 11 & $9.3 \mathrm{E}-01$ & 9.3E-01 & No & $\mathrm{D}$ & PD & N/A & $\mathrm{N} / \mathrm{A}$ \\
\hline & GW-383 & s & 21 & 2 & $1.4 \mathrm{E}-04$ & $5.0 \mathrm{E}-05$ & No & NT & $\mathrm{D}$ & N/A & N/A \\
\hline & GW-154 & s & 20 & 0 & $5.0 \mathrm{E}-05$ & 5.0E-05 & Yes & $\mathrm{s}$ & $\mathrm{D}$ & $\mathrm{N} / \mathrm{A}$ & N/A \\
\hline & GW-223 & $\mathrm{s}$ & 15 & 5 & 4.6E-03 & 5.0E-05 & No & D & D & N/A & $\mathrm{N} / \mathrm{A}$ \\
\hline & GW-222 & $\mathrm{T}$ & 8 & 5 & $5.8 \mathrm{E}-03$ & $1.0 \mathrm{E}-03$ & No & NT & D & N/A & $\mathrm{N} / \mathrm{A}$ \\
\hline & GW-832 & $T$ & 17 & 17 & $1.8 \mathrm{E}-02$ & 1.7E-02 & No & $\mathrm{D}$ & D & $\mathrm{N} / \mathrm{A}$ & N/A \\
\hline & GW-148 & $\mathrm{T}$ & 10 & 0 & $5.0 \mathrm{E}-05$ & 5.0E-05 & Yes & s & $D$ & N/A & $\mathrm{N} / \mathrm{A}$ \\
\hline & GW-845 & $\mathrm{T}$ & 1 & 1 & $1.6 \mathrm{E}+00$ & $1.6 \mathrm{E}+00$ & No & $\mathrm{N} / \mathrm{A}$ & $\mathrm{N} / \mathrm{A}$ & $\mathrm{N} / \mathrm{A}$ & $\mathrm{N} / \mathrm{A}$ \\
\hline & GW-750 & $\mathrm{T}$ & 20 & 0 & 5.0E-05 & 5.0E-05 & Yes & $\mathrm{s}$ & $\mathrm{D}$ & $N / A$ & $\mathrm{~N} / \mathrm{A}$ \\
\hline & GW-733 & $\mathrm{T}$ & 21 & 21 & $1.5 \mathrm{E}-02$ & 1.0E-02 & No & D & D & $\mathrm{N} / \mathrm{A}$ & $\mathrm{N} / \mathrm{A}$ \\
\hline & GW-817 & $\mathrm{T}$ & 6 & 0 & $5.0 \mathrm{E}-05$ & 5.0E-05 & Yes & $\mathrm{s}$ & $\mathrm{s}$ & N/A & $\mathrm{N} / \mathrm{A}$ \\
\hline & LRSPW & $\mathrm{T}$ & 14 & 11 & $1.4 \mathrm{E}-02$ & 1.2E-02 & No & NT & NT & N/A & N/A \\
\hline & GW-230 & $\mathrm{T}$ & 17 & 0 & $5.0 \mathrm{E}-05$ & 5.0E-05 & Yes & S & D & $\mathrm{N} / \mathrm{A}$ & N/A \\
\hline & GW-816 & $\mathrm{T}$ & 20 & 0 & $5.0 \mathrm{E}-05$ & 5.0E-05 & Yes & $\mathrm{s}$ & D & N/A & $\mathrm{N} / \mathrm{A}$ \\
\hline & GW-232 & $T$ & 26 & 0 & $5.0 \mathrm{E}-05$ & 5.0E-05 & Yes & s & D & N/A & $\mathrm{N} / \mathrm{A}$ \\
\hline & GW-240 & $\mathrm{T}$ & 8 & 8 & $6.7 \mathrm{E}-03$ & 5.5E-03 & No & NT & S & $\mathrm{N} / \mathrm{A}$ & $\mathrm{N} / \mathrm{A}$ \\
\hline & GW-208 & $\mathrm{T}$ & 20 & 0 & $5.0 \mathrm{E}-05$ & 5.0E-05 & Yes & $\mathrm{s}$ & D & N/A & $\mathrm{N} / \mathrm{A}$ \\
\hline & GW-380 & $\mathrm{T}$ & 16 & 1 & 1.4E-04 & 5.0E-05 & No & NT & NT & $N / A$ & $\mathrm{~N} / \mathrm{A}$ \\
\hline & GW-220 & $\mathrm{T}$ & 22 & 22 & 7.4E-01 & 6.6E-01 & No & I & 1 & $N / A$ & $\mathrm{~N} / \mathrm{A}$ \\
\hline & GW-748 & $\mathrm{T}$ & 6 & 0 & $5.0 \mathrm{E}-05$ & 5.0E-05 & Yes & s & s & N/A & $\mathrm{N} / \mathrm{A}$ \\
\hline
\end{tabular}

User Name: MV

State: Tennessee 
Project: Y-12 National Security Complex

Location: East $\mathrm{Y}-12$
User Name: MV

State: Tennessee

\begin{tabular}{|c|c|c|c|c|c|c|c|c|c|c|c|}
\hline Constituent & Well & $\begin{array}{l}\text { Sourcel } \\
\text { Tail }\end{array}$ & $\begin{array}{l}\text { Number } \\
\text { of } \\
\text { Samples }\end{array}$ & $\begin{array}{c}\text { Number } \\
\text { of } \\
\text { Detects }\end{array}$ & $\begin{array}{c}\text { Average } \\
\text { (mg/L) }\end{array}$ & $\begin{array}{r}\text { Median } \\
(\mathrm{mg} / \mathrm{L})\end{array}$ & $\begin{array}{c}\text { All } \\
\text { Samples } \\
\text { "ND" ? }\end{array}$ & $\begin{array}{l}\text { Mann- } \\
\text { Kendall }\end{array}$ & $\begin{array}{c}\text { Linear } \\
\text { Regression }\end{array}$ & Modeling & Empirical \\
\hline \multicolumn{12}{|c|}{ CARBON TETRACHLORIDE } \\
\hline & GW-747 & $T$ & 20 & 0 & $5.0 \mathrm{E}-05$ & $5.0 \mathrm{E}-05$ & Yes & $\mathrm{S}$ & $\mathrm{D}$ & N/A & N/A \\
\hline & GW-746 & $\mathrm{T}$ & 6 & 0 & $5.0 \mathrm{E}-05$ & $5.0 \mathrm{E}-05$ & Yes & s & $\mathrm{s}$ & N/A & N/A \\
\hline & GW-745 & $\mathrm{T}$ & 6 & 0 & $5.0 \mathrm{E}-05$ & 5.0E-05 & Yes & s & $\mathrm{s}$ & N/A & N/A \\
\hline & GW-384 & $\mathrm{T}$ & 4 & 0 & $5.0 \mathrm{E}-05$ & 5.0E-05 & Yes & s & S & N/A & N/A \\
\hline & GW-744 & $\mathrm{T}$ & 20 & 0 & $5.0 \mathrm{E}-05$ & 5.0E-05 & Yes & S & $\mathrm{D}$ & N/A & N/A \\
\hline & GW-385 & $\mathrm{T}$ & 4 & 0 & $5.0 \mathrm{E}-05$ & $5.0 \mathrm{E}-05$ & Yes & S & s & N/A & $N / A$ \\
\hline & GW-735 & $\mathrm{T}$ & 21 & 0 & $5.0 \mathrm{E}-05$ & $5.0 \mathrm{E}-05$ & Yes & s & $\mathrm{s}$ & N/A & $N / A$ \\
\hline & GW-603 & $\mathrm{T}$ & 4 & 0 & $5.0 \mathrm{E}-05$ & $5.0 \mathrm{E}-05$ & Yes & s & s & N/A & N/A \\
\hline & GW-749 & $\mathrm{T}$ & 6 & 0 & $5.0 \mathrm{E}-05$ & 5.0E-05 & Yes & S & s & N/A & $N / A$ \\
\hline & SCR7_16SP & $\mathrm{T}$ & 1 & 0 & $5.0 \mathrm{E}-05$ & $5.0 \mathrm{E}-05$ & Yes & N/A & N/A & N/A & $N / A$ \\
\hline & GW-153 & $\mathrm{T}$ & 20 & 19 & $1.6 \mathrm{E}-01$ & 1.4E-01 & No & PD & NT & N/A & N/A \\
\hline & RGQWWSP & $\mathrm{T}$ & 2 & 0 & $5.0 \mathrm{E}-05$ & 5.0E-05 & Yes & $N / A$ & N/A & N/A & $N / A$ \\
\hline & SCR7_1SP & $\mathrm{T}$ & 19 & 9 & 7.1E-04 & 5.0E-05 & No & $\mathrm{D}$ & $\mathrm{D}$ & N/A & $N / A$ \\
\hline & GW-169 & $\mathrm{T}$ & 22 & 0 & $5.0 \mathrm{E}-05$ & $5.0 \mathrm{E}-05$ & Yes & s & I & N/A & N/A \\
\hline & SCR7_7SP & $\mathrm{T}$ & 4 & 0 & $5.0 \mathrm{E}-05$ & 5.0E-05 & Yes & s & s & $N / A$ & N/A \\
\hline & SCR7_18SP & $\mathrm{T}$ & 10 & 1 & $2.5 \mathrm{E}-04$ & 5.0E-05 & No & NT & PD & N/A & $N / A$ \\
\hline & GW-170 & $\mathrm{T}$ & 27 & 27 & 1.7E-02 & $2.0 \mathrm{E}-03$ & No & $D$ & $D$ & N/A & N/A \\
\hline & SCR7_6SP & $\mathrm{T}$ & 4 & 0 & $5.0 \mathrm{E}-05$ & $5.0 \mathrm{E}-05$ & Yes & s & S & N/A & N/A \\
\hline & SCR7_8SP & $\mathrm{T}$ & 17 & 0 & $5.0 \mathrm{E}-05$ & 5.0E-05 & Yes & s & I & N/A & $N / A$ \\
\hline & SCR7_4SP & $\mathrm{T}$ & 4 & 0 & $5.0 \mathrm{E}-05$ & $5.0 \mathrm{E}-05$ & Yes & s & S & N/A & $N / A$ \\
\hline & SCR7_8SSP & $\mathrm{T}$ & 2 & 0 & $5.0 \mathrm{E}-05$ & 5.0E-05 & Yes & $\mathrm{N} / \mathrm{A}$ & N/A & N/A & N/A \\
\hline & GW-171 & $\mathrm{T}$ & 18 & 0 & $5.0 \mathrm{E}-05$ & 5.0E-05 & Yes & $\mathrm{s}$ & $\mathrm{s}$ & N/A & $N / A$ \\
\hline & SCR7_14SP & $\mathrm{T}$ & 1 & 0 & $5.0 \mathrm{E}-05$ & 5.0E-05 & Yes & $N / A$ & N/A & N/A & $N / A$ \\
\hline & GW-172 & $\mathrm{T}$ & 17 & 0 & $5.0 \mathrm{E}-05$ & 5.0E-05 & Yes & S & $\mathrm{D}$ & N/A & N/A \\
\hline & SCR7_10SP & $\mathrm{T}$ & 4 & 0 & $5.0 \mathrm{E}-05$ & $5.0 \mathrm{E}-05$ & Yes & s & $\mathrm{s}$ & N/A & N/A \\
\hline & GW-149 & $\mathrm{T}$ & 4 & 0 & $5.0 \mathrm{E}-05$ & 5.0E-05 & Yes & s & $\mathrm{s}$ & $N / A$ & $N / A$ \\
\hline & UV8_5SP & $\mathrm{T}$ & 2 & 0 & $5.0 \mathrm{E}-05$ & $5.0 \mathrm{E}-05$ & Yes & N/A & N/A & N/A & N/A \\
\hline & GW-207 & $T$ & 20 & 0 & $5.0 \mathrm{E}-05$ & $5.0 \mathrm{E}-05$ & Yes & $\mathrm{s}$ & $\mathrm{D}$ & N/A & N/A \\
\hline & UV8_6SP & $\mathrm{T}$ & 1 & 0 & $5.0 \mathrm{E}-05$ & $5.0 \mathrm{E}-05$ & Yes & $\mathrm{N} / \mathrm{A}$ & N/A & $\mathrm{N} / \mathrm{A}$ & N/A \\
\hline \multicolumn{12}{|l|}{ LEAD } \\
\hline & GW-383 & $\mathrm{s}$ & 21 & 4 & $8.0 \mathrm{E}-04$ & $5.2 \mathrm{E}-05$ & No & NT & NT & $\mathrm{N} / \mathrm{A}$ & $\mathrm{N} / \mathrm{A}$ \\
\hline & GW-151 & s & 18 & 1 & $5.9 \mathrm{E}-05$ & $5.2 \mathrm{E}-05$ & No & s & $\mathrm{s}$ & N/A & N/A \\
\hline & GW-381 & s & 10 & 3 & $6.0 \mathrm{E}-04$ & $5.2 \mathrm{E}-05$ & No & NT & NT & N/A & $N / A$ \\
\hline
\end{tabular}


Project: Y-12 National Security Complex

Location: East $\mathrm{Y}-12$
User Name: MV

State: Tennessee

\begin{tabular}{|c|c|c|c|c|c|c|c|c|c|c|c|}
\hline Constituent & Well & $\begin{array}{c}\text { Sourcel } \\
\text { Tail }\end{array}$ & $\begin{array}{c}\text { Number } \\
\text { of } \\
\text { Samples }\end{array}$ & $\begin{array}{c}\text { Number } \\
\text { of } \\
\text { Detects }\end{array}$ & $\begin{array}{c}\text { Average } \\
\text { (mg/L) }\end{array}$ & $\begin{array}{c}\text { Median } \\
(\mathrm{mg} / \mathrm{L})\end{array}$ & $\begin{array}{c}\text { All } \\
\text { Samples } \\
\text { "ND" ? }\end{array}$ & $\begin{array}{l}\text { Mann- } \\
\text { Kendall }\end{array}$ & $\begin{array}{l}\text { Linear } \\
\text { Regression }\end{array}$ & Modeling & Empirical \\
\hline \multicolumn{12}{|l|}{ LEAD } \\
\hline & GW-382 & $\mathrm{s}$ & 11 & 0 & $5.2 \mathrm{E}-05$ & $5.2 \mathrm{E}-05$ & Yes & $\mathrm{S}$ & $\mathrm{s}$ & $\mathrm{N} / \mathrm{A}$ & $\mathrm{N} / \mathrm{A}$ \\
\hline & GW-154 & s & 17 & 6 & $1.4 \mathrm{E}-03$ & $5.2 \mathrm{E}-05$ & No & NT & NT & $N / A$ & $N / A$ \\
\hline & GW-223 & s & 15 & 0 & $5.2 \mathrm{E}-05$ & $5.2 \mathrm{E}-05$ & Yes & $\mathrm{s}$ & $\mathrm{s}$ & $N / A$ & $N / A$ \\
\hline & GW-222 & $\mathrm{T}$ & 8 & 1 & $6.8 \mathrm{E}-05$ & $5.2 \mathrm{E}-05$ & No & S & s & $N / A$ & $N / A$ \\
\hline & GW-603 & $\mathrm{T}$ & 4 & 2 & 1.7E-04 & $1.3 \mathrm{E}-04$ & No & NT & NT & $N / A$ & $N / A$ \\
\hline & GW-207 & $\mathrm{T}$ & 20 & 11 & $1.2 \mathrm{E}-03$ & $4.9 \mathrm{E}-04$ & No & NT & NT & $N / A$ & $N / A$ \\
\hline & GW-385 & $\mathrm{T}$ & 4 & 2 & 3.6E-04 & $2.5 \mathrm{E}-04$ & No & NT & $\mathrm{PI}$ & N/A & $N / A$ \\
\hline & GW-148 & $\mathrm{T}$ & 10 & 2 & $1.6 \mathrm{E}-04$ & $5.2 \mathrm{E}-05$ & No & NT & NT & N/A & $N / A$ \\
\hline & GW-172 & $\mathrm{T}$ & 1 & 0 & $5.2 \mathrm{E}-05$ & $5.2 \mathrm{E}-05$ & Yes & $N / A$ & $N / A$ & $N / A$ & $N / A$ \\
\hline & GW-384 & $\mathrm{T}$ & 4 & 2 & $2.3 \mathrm{E}-04$ & $1.3 \mathrm{E}-04$ & No & NT & NT & $N / A$ & $N / A$ \\
\hline & GW-208 & $\mathrm{T}$ & 20 & 16 & $2.2 \mathrm{E}-03$ & $1.1 \mathrm{E}-03$ & No & NT & NT & $N / A$ & $N / A$ \\
\hline & GW-240 & $\mathrm{T}$ & 8 & 3 & $2.8 \mathrm{E}-04$ & $5.2 \mathrm{E}-05$ & No & NT & NT & $N / A$ & $N / A$ \\
\hline & GW-171 & $\mathrm{T}$ & 1 & 1 & $1.4 \mathrm{E}-03$ & $1.4 \mathrm{E}-03$ & No & $N / A$ & $N / A$ & $N / A$ & $N / A$ \\
\hline & GW-153 & $\mathrm{T}$ & 20 & 10 & 4.0E-04 & $1.9 \mathrm{E}-04$ & No & NT & NT & $N / A$ & $N / A$ \\
\hline & GW-170 & $\mathrm{T}$ & 15 & 4 & $2.4 \mathrm{E}-03$ & $5.2 \mathrm{E}-05$ & No & NT & NT & N/A & $N / A$ \\
\hline & GW-230 & $\mathrm{T}$ & 2 & 0 & $5.2 \mathrm{E}-05$ & $5.2 \mathrm{E}-05$ & Yes & $\mathrm{N} / \mathrm{A}$ & $\mathrm{N} / \mathrm{A}$ & $N / A$ & $N / A$ \\
\hline & GW-169 & $\mathrm{T}$ & 13 & 1 & 4.4E-04 & $5.2 \mathrm{E}-05$ & No & NT & NT & N/A & $N / A$ \\
\hline & GW-232 & $\mathrm{T}$ & 14 & 1 & $2.8 \mathrm{E}-04$ & $5.2 \mathrm{E}-05$ & No & NT & NT & $N / A$ & $N / A$ \\
\hline & GW-220 & $\mathrm{T}$ & 22 & 6 & $2.3 \mathrm{E}-04$ & 5.2E-05 & No & NT & NT & $N / A$ & $N / A$ \\
\hline & GW-380 & $\mathrm{T}$ & 17 & 3 & $1.4 \mathrm{E}-04$ & $5.2 \mathrm{E}-05$ & No & NT & $\mathrm{D}$ & $\mathrm{N} / \mathrm{A}$ & $N / A$ \\
\hline & GW-149 & $\mathrm{T}$ & 4 & 1 & $2.1 \mathrm{E}-04$ & $5.2 \mathrm{E}-05$ & No & NT & NT & $N / A$ & $N / A$ \\
\hline & GW-749 & $\mathrm{T}$ & 6 & 1 & $1.0 \mathrm{E}-04$ & 5.2E-05 & No & NT & NT & $N / A$ & $N / A$ \\
\hline & LRSPW & $\mathrm{T}$ & 14 & 6 & 7.0E-04 & $5.2 \mathrm{E}-05$ & No & $\mathrm{PI}$ & $\mathrm{PI}$ & N/A & $N / A$ \\
\hline & SCR7_14SP & $\mathrm{T}$ & 1 & 0 & $5.2 \mathrm{E}-05$ & $5.2 \mathrm{E}-05$ & Yes & $N / A$ & $N / A$ & $N / A$ & N/A \\
\hline & GW-845 & $\mathrm{T}$ & 1 & 0 & $5.2 \mathrm{E}-05$ & $5.2 \mathrm{E}-05$ & Yes & $N / A$ & $N / A$ & $N / A$ & $N / A$ \\
\hline & GW-733 & $\mathrm{T}$ & 20 & 4 & $5.2 \mathrm{E}-04$ & $5.2 \mathrm{E}-05$ & No & PD & $\mathrm{D}$ & N/A & $N / A$ \\
\hline & SCR7_16SP & $\mathrm{T}$ & 1 & 1 & $2.5 \mathrm{E}-03$ & $2.5 \mathrm{E}-03$ & No & $\mathrm{N} / \mathrm{A}$ & $\mathrm{N} / \mathrm{A}$ & $\mathrm{N} / \mathrm{A}$ & N/A \\
\hline & GW-817 & $\mathrm{T}$ & 6 & 4 & $2.5 \mathrm{E}-04$ & $2.2 \mathrm{E}-04$ & No & 1 & I & $N / A$ & $N / A$ \\
\hline & GW-816 & $\mathrm{T}$ & 20 & 5 & $6.7 \mathrm{E}-04$ & $5.2 \mathrm{E}-05$ & No & NT & NT & N/A & $N / A$ \\
\hline & SCR7_18SP & $\mathrm{T}$ & 2 & 0 & $5.2 \mathrm{E}-05$ & $5.2 \mathrm{E}-05$ & Yes & $N / A$ & $N / A$ & $N / A$ & $N / A$ \\
\hline & GW-750 & $\mathrm{T}$ & 20 & 4 & $1.5 \mathrm{E}-04$ & $5.2 \mathrm{E}-05$ & No & NT & PD & $N / A$ & $N / A$ \\
\hline & RGQWWSP & $\mathrm{T}$ & 1 & 0 & $5.2 \mathrm{E}-05$ & $5.2 \mathrm{E}-05$ & Yes & N/A & $\mathrm{N} / \mathrm{A}$ & N/A & $N / A$ \\
\hline & SCR7_1SP & $\mathrm{T}$ & 2 & 0 & $5.2 \mathrm{E}-05$ & $5.2 \mathrm{E}-05$ & Yes & $\mathrm{N} / \mathrm{A}$ & N/A & N/A & N/A \\
\hline & GW-832 & $\mathrm{T}$ & 13 & 0 & $5.2 \mathrm{E}-05$ & $5.2 \mathrm{E}-05$ & Yes & $\mathrm{s}$ & $\mathrm{S}$ & $N / A$ & $N / A$ \\
\hline
\end{tabular}


Project: Y-12 National Security Complex

Location: East $\mathrm{Y}-12$
User Name: MV

State: Tennessee

\begin{tabular}{|c|c|c|c|c|c|c|c|c|c|c|c|}
\hline Constituent & Well & $\begin{array}{c}\text { Sourcel } \\
\text { Tail }\end{array}$ & $\begin{array}{l}\text { Number } \\
\text { of } \\
\text { Samples }\end{array}$ & $\begin{array}{c}\text { Number } \\
\text { of } \\
\text { Detects }\end{array}$ & $\begin{array}{c}\text { Average } \\
\text { (mg/L) }\end{array}$ & $\begin{array}{r}\text { Median } \\
(\mathrm{mg} / \mathrm{L})\end{array}$ & $\begin{array}{c}\text { All } \\
\text { Samples } \\
\text { "ND" ? }\end{array}$ & $\begin{array}{l}\text { Mann- } \\
\text { Kendall }\end{array}$ & $\begin{array}{c}\text { Linear } \\
\text { Regression }\end{array}$ & Modeling & Empirical \\
\hline \multicolumn{12}{|l|}{ LEAD } \\
\hline & GW-748 & $T$ & 6 & 2 & $2.0 \mathrm{E}-04$ & $5.2 \mathrm{E}-05$ & No & NT & NT & $\mathrm{N} / \mathrm{A}$ & $\mathrm{N} / \mathrm{A}$ \\
\hline & GW-747 & $\mathrm{T}$ & 20 & 3 & $1.2 \mathrm{E}-04$ & $5.2 \mathrm{E}-05$ & No & NT & $\mathrm{PD}$ & N/A & $N / A$ \\
\hline & GW-744 & $\mathrm{T}$ & 20 & 5 & $5.9 \mathrm{E}-04$ & 5.2E-05 & No & NT & NT & N/A & $N / A$ \\
\hline & GW-735 & T & 21 & 5 & 3.0E-04 & 5.2E-05 & No & NT & PD & N/A & N/A \\
\hline & SCR7_8SSP & $\mathrm{T}$ & 2 & 0 & $5.2 \mathrm{E}-05$ & 5.2E-05 & Yes & N/A & $\mathrm{N} / \mathrm{A}$ & N/A & $N / A$ \\
\hline & GW-746 & $\mathrm{T}$ & 6 & 2 & $1.9 \mathrm{E}-04$ & 5.2E-05 & No & NT & NT & $\mathrm{N} / \mathrm{A}$ & $N / A$ \\
\hline & GW-745 & $\mathrm{T}$ & 6 & 2 & 2.3E-04 & $5.2 \mathrm{E}-05$ & No & NT & NT & N/A & N/A \\
\hline \multicolumn{12}{|c|}{ TETRACHLOROETHYLENE(PCE) } \\
\hline & GW-223 & $\mathrm{S}$ & 15 & 15 & $1.1 \mathrm{E}-01$ & 4.5E-02 & No & $\mathrm{D}$ & $\mathrm{D}$ & $\mathrm{N} / \mathrm{A}$ & N/A \\
\hline & GW-151 & s & 19 & 19 & 2.4E-01 & $1.2 \mathrm{E}-01$ & No & 1 & I & N/A & $N / A$ \\
\hline & GW-383 & s & 21 & 21 & 4.4E-01 & 4.4E-01 & No & NT & NT & N/A & $N / A$ \\
\hline & GW-154 & s & 20 & 1 & 5.3E-04 & $5.0 \mathrm{E}-04$ & No & s & PD & N/A & $N / A$ \\
\hline & GW-381 & s & 10 & 9 & 3.3E-03 & 2.3E-03 & No & S & NT & N/A & $N / A$ \\
\hline & GW-382 & s & 11 & 11 & 2.4E-02 & $1.2 \mathrm{E}-02$ & No & NT & NT & N/A & $N / A$ \\
\hline & RGQWWSP & $T$ & 2 & 0 & $5.0 \mathrm{E}-04$ & 5.0E-04 & Yes & N/A & $\mathrm{N} / \mathrm{A}$ & $\mathrm{N} / \mathrm{A}$ & $N / A$ \\
\hline & SCR7_10SP & $\mathrm{T}$ & 4 & 0 & $5.0 \mathrm{E}-04$ & 5.0E-04 & Yes & S & s & N/A & $N / A$ \\
\hline & UV8_5SP & $T$ & 2 & 0 & $5.0 \mathrm{E}-04$ & 5.0E-04 & Yes & N/A & N/A & N/A & $N / A$ \\
\hline & GW-171 & $T$ & 18 & 0 & $5.0 \mathrm{E}-04$ & 5.0E-04 & Yes & $\mathrm{s}$ & $\mathrm{s}$ & N/A & $N / A$ \\
\hline & GW-148 & $T$ & 10 & 0 & $5.0 \mathrm{E}-04$ & 5.0E-04 & Yes & $\mathrm{s}$ & $\mathrm{D}$ & N/A & $N / A$ \\
\hline & SCR7_8SSP & $\mathrm{T}$ & 2 & 1 & 7.5E-04 & 7.5E-04 & No & N/A & N/A & N/A & $N / A$ \\
\hline & SCR7_14SP & $T$ & 1 & 0 & $5.0 \mathrm{E}-04$ & 5.0E-04 & Yes & $\mathrm{N} / \mathrm{A}$ & N/A & $\mathrm{N} / \mathrm{A}$ & $N / A$ \\
\hline & GW-169 & $\mathrm{T}$ & 22 & 20 & 8.0E-04 & $1.0 \mathrm{E}-03$ & No & NT & NT & $\mathrm{N} / \mathrm{A}$ & $N / A$ \\
\hline & SCR7_6SP & $\mathrm{T}$ & 4 & 0 & $5.0 \mathrm{E}-04$ & $5.0 \mathrm{E}-04$ & Yes & S & s & N/A & $N / A$ \\
\hline & SCR7_16SP & $T$ & 1 & 0 & $5.0 \mathrm{E}-04$ & 5.0E-04 & Yes & N/A & N/A & $\mathrm{N} / \mathrm{A}$ & $N / A$ \\
\hline & GW-149 & $\mathrm{T}$ & 4 & 0 & $5.0 \mathrm{E}-04$ & 5.0E-04 & Yes & $\mathrm{s}$ & $\mathrm{s}$ & $\mathrm{N} / \mathrm{A}$ & $N / A$ \\
\hline & SCR7_1SP & $\mathrm{T}$ & 19 & 2 & $5.0 \mathrm{E}-04$ & $5.0 \mathrm{E}-04$ & No & S & s & N/A & $N / A$ \\
\hline & SCR7_4SP & $T$ & 4 & 0 & $5.0 \mathrm{E}-04$ & 5.0E-04 & Yes & $\mathrm{s}$ & s & N/A & $N / A$ \\
\hline & GW-153 & $\mathrm{T}$ & 20 & 19 & $3.2 \mathrm{E}-03$ & 2.3E-03 & No & D & PD & N/A & $N / A$ \\
\hline & SCR7_7SP & $\mathrm{T}$ & 4 & 0 & $5.0 \mathrm{E}-04$ & 5.0E-04 & Yes & s & s & $\mathrm{N} / \mathrm{A}$ & $N / A$ \\
\hline & SCR7_18SP & $T$ & 10 & 0 & $5.0 \mathrm{E}-04$ & 5.0E-04 & Yes & $\mathrm{s}$ & $\mathrm{s}$ & N/A & $N / A$ \\
\hline & SCR7_8SP & $T$ & 17 & 4 & $5.9 \mathrm{E}-04$ & 5.0E-04 & No & PD & D & $\mathrm{N} / \mathrm{A}$ & $N / A$ \\
\hline & GW-170 & $\mathrm{T}$ & 27 & 26 & $1.5 \mathrm{E}-03$ & $1.0 \mathrm{E}-03$ & No & D & D & N/A & $N / A$ \\
\hline & GW-749 & $T$ & 6 & 0 & $5.0 \mathrm{E}-04$ & 5.0E-04 & Yes & $\mathrm{s}$ & $\mathrm{s}$ & N/A & $N / A$ \\
\hline
\end{tabular}


Project: Y-12 National Security Complex

Location: East $\mathrm{Y}-12$
User Name: MV

State: Tennessee

\begin{tabular}{|c|c|c|c|c|c|c|c|c|c|c|c|}
\hline Constituent & Well & $\begin{array}{c}\text { Sourcel } \\
\text { Tail }\end{array}$ & $\begin{array}{c}\text { Number } \\
\text { of } \\
\text { Samples }\end{array}$ & $\begin{array}{c}\text { Number } \\
\text { of } \\
\text { Detects }\end{array}$ & $\begin{array}{c}\text { Average } \\
\text { (mg/L) }\end{array}$ & $\begin{array}{r}\text { Median } \\
(\mathrm{mg} / \mathrm{L})\end{array}$ & $\begin{array}{c}\text { All } \\
\text { Samples } \\
\text { "ND" ? }\end{array}$ & $\begin{array}{l}\text { Mann- } \\
\text { Kendall }\end{array}$ & $\begin{array}{c}\text { Linear } \\
\text { Regression }\end{array}$ & Modeling & Empirical \\
\hline \multicolumn{12}{|c|}{ TETRACHLOROETHYLENE(PCE) } \\
\hline & UV8_6SP & $T$ & 1 & 0 & $5.0 \mathrm{E}-04$ & $5.0 \mathrm{E}-04$ & Yes & $\mathrm{N} / \mathrm{A}$ & N/A & N/A & $\mathrm{N} / \mathrm{A}$ \\
\hline & GW-385 & $\mathrm{T}$ & 4 & 0 & $5.0 \mathrm{E}-04$ & 5.0E-04 & Yes & s & s & $\mathrm{N} / \mathrm{A}$ & $N / A$ \\
\hline & GW-733 & T & 21 & 5 & $5.2 \mathrm{E}-04$ & $5.0 \mathrm{E}-04$ & No & $\mathrm{s}$ & PD & N/A & N/A \\
\hline & GW-384 & $\mathrm{T}$ & 4 & 0 & $5.0 \mathrm{E}-04$ & 5.0E-04 & Yes & s & $\mathrm{s}$ & N/A & $N / A$ \\
\hline & GW-735 & $\mathrm{T}$ & 21 & 0 & $5.0 \mathrm{E}-04$ & $5.0 \mathrm{E}-04$ & Yes & s & s & N/A & N/A \\
\hline & GW-744 & $\mathrm{T}$ & 20 & 0 & $5.0 \mathrm{E}-04$ & $5.0 \mathrm{E}-04$ & Yes & $\mathrm{s}$ & D & $\mathrm{N} / \mathrm{A}$ & N/A \\
\hline & GW-745 & $\mathrm{T}$ & 6 & 0 & $5.0 \mathrm{E}-04$ & 5.0E-04 & Yes & s & s & $\mathrm{N} / \mathrm{A}$ & N/A \\
\hline & GW-746 & $\mathrm{T}$ & 6 & 0 & $5.0 \mathrm{E}-04$ & $5.0 \mathrm{E}-04$ & Yes & s & s & $\mathrm{N} / \mathrm{A}$ & $N / A$ \\
\hline & GW-380 & $\mathrm{T}$ & 16 & 1 & $5.0 \mathrm{E}-04$ & $5.0 \mathrm{E}-04$ & No & $\mathrm{s}$ & $\mathrm{s}$ & $\mathrm{N} / \mathrm{A}$ & $N / A$ \\
\hline & GW-747 & $\mathrm{T}$ & 20 & 0 & $5.0 \mathrm{E}-04$ & $5.0 \mathrm{E}-04$ & Yes & s & D & $\mathrm{N} / \mathrm{A}$ & $N / A$ \\
\hline & GW-240 & $\mathrm{T}$ & 8 & 0 & $5.0 \mathrm{E}-04$ & 5.0E-04 & Yes & $\mathrm{s}$ & $\mathrm{s}$ & N/A & N/A \\
\hline & GW-832 & $\mathrm{T}$ & 17 & 17 & 4.1E-03 & $5.0 \mathrm{E}-03$ & No & $\mathrm{s}$ & $\mathrm{s}$ & N/A & $N / A$ \\
\hline & GW-232 & $T$ & 26 & 0 & $5.0 \mathrm{E}-04$ & $5.0 \mathrm{E}-04$ & Yes & s & s & $\mathrm{N} / \mathrm{A}$ & $N / A$ \\
\hline & GW-172 & $\mathrm{T}$ & 17 & 0 & $5.0 \mathrm{E}-04$ & 5.0E-04 & Yes & $\mathrm{s}$ & $\mathrm{s}$ & N/A & N/A \\
\hline & GW-230 & $\mathrm{T}$ & 17 & 0 & $5.0 \mathrm{E}-04$ & $5.0 \mathrm{E}-04$ & Yes & $\mathrm{s}$ & $\mathrm{s}$ & N/A & N/A \\
\hline & GW-750 & $T$ & 20 & 0 & $5.0 \mathrm{E}-04$ & $5.0 \mathrm{E}-04$ & Yes & s & D & $\mathrm{N} / \mathrm{A}$ & $N / A$ \\
\hline & GW-816 & $T$ & 20 & 0 & $5.0 \mathrm{E}-04$ & 5.0E-04 & Yes & s & D & $\mathrm{N} / \mathrm{A}$ & $N / A$ \\
\hline & GW-222 & $\mathrm{T}$ & 8 & 8 & 3.6E-02 & 1.1E-02 & No & NT & $\mathrm{PD}$ & N/A & $N / A$ \\
\hline & GW-817 & $\mathrm{T}$ & 6 & 1 & $1.4 \mathrm{E}-03$ & $5.0 \mathrm{E}-04$ & No & NT & 1 & $\mathrm{~N} / \mathrm{A}$ & $N / A$ \\
\hline & GW-220 & $\mathrm{T}$ & 22 & 22 & $1.5 \mathrm{E}-01$ & 7.0E-02 & No & I & I & $\mathrm{N} / \mathrm{A}$ & $N / A$ \\
\hline & GW-603 & $\mathrm{T}$ & 4 & 0 & $5.0 \mathrm{E}-04$ & $5.0 \mathrm{E}-04$ & Yes & s & s & N/A & N/A \\
\hline & GW-208 & $T$ & 20 & 0 & $5.0 \mathrm{E}-04$ & $5.0 \mathrm{E}-04$ & Yes & $\mathrm{s}$ & D & $\mathrm{N} / \mathrm{A}$ & $N / A$ \\
\hline & GW-845 & $\mathrm{T}$ & 1 & 1 & 8.5E-02 & 8.5E-02 & No & $\mathrm{N} / \mathrm{A}$ & $N / A$ & $\mathrm{~N} / \mathrm{A}$ & N/A \\
\hline & GW-207 & $T$ & 20 & 0 & $5.0 \mathrm{E}-04$ & $5.0 \mathrm{E}-04$ & Yes & $\mathrm{s}$ & $\mathrm{D}$ & $\mathrm{N} / \mathrm{A}$ & $N / A$ \\
\hline & LRSPW & $T$ & 14 & 12 & 2.3E-03 & $1.8 \mathrm{E}-03$ & No & NT & NT & N/A & $\mathrm{N} / \mathrm{A}$ \\
\hline & GW-748 & $\mathrm{T}$ & 6 & 0 & $5.0 \mathrm{E}-04$ & $5.0 \mathrm{E}-04$ & Yes & $\mathrm{s}$ & $\mathrm{s}$ & $\mathrm{N} / \mathrm{A}$ & $N / A$ \\
\hline
\end{tabular}

Note: Increasing (I); Probably Increasing (PI); Stable (S); Probably Decreasing (PD); Decreasing (D); No Trend (NT); Not Applicable (N/A) - Due to insufficient Data (< 4 sampling events); Source/Tail (S/T)

The Number of Samples and Number of Detects shown above are post-consolidation values. 


\section{MAROS Spatial Moment Analysis Summary}

Project: Y-12 National Security Complex

Location: East Y-12
User Name: $\mathrm{MV}$

State: Tennessee 2nd Moment (Spread)

$\underline{1 \text { st Moment (Center of Mass) }}$

Source

Estimated

Effective Date
Sigma XX Sigma YY

(sq ft)

(sq ft)
Number of

Wells

\section{CARBON TETRACHLORIDE}

\begin{tabular}{|c|c|c|c|c|c|c|c|}
\hline 1/1/1996 & $1.8 \mathrm{E}+00$ & 65,010 & 28,843 & 2,066 & $1,394,861$ & 188,908 & 39 \\
\hline 4/1/1996 & $2.2 \mathrm{E}+00$ & 65,962 & 28,977 & 3,025 & $2,943,728$ & 285,725 & 36 \\
\hline 7/1/1996 & $1.2 \mathrm{E}+00$ & 65,830 & 29,053 & 2,902 & $8,551,303$ & 192,789 & 30 \\
\hline 10/1/1996 & 8.0E-01 & 64,000 & 28,863 & 1,062 & 99,894 & 52,374 & 27 \\
\hline $1 / 1 / 1997$ & $0.0 \mathrm{E}+00$ & & & & & & 2 \\
\hline 4/1/1997 & $1.4 \mathrm{E}+00$ & 65,299 & 28,547 & 2,357 & $4,461,565$ & 489,833 & 32 \\
\hline $7 / 1 / 1997$ & $4.5 \mathrm{E}+00$ & 65,754 & 28,442 & 2,819 & 560,201 & 204,560 & 14 \\
\hline 10/1/1997 & 1.3E-01 & 64,175 & 29,182 & 1,313 & 74,321 & 131,957 & 18 \\
\hline 1/1/1998 & $1.2 \mathrm{E}+00$ & 65,162 & 28,554 & 2,220 & $1,279,377$ & 306,647 & 16 \\
\hline 4/1/1998 & $8.1 \mathrm{E}+00$ & 64,192 & 28,785 & 1,246 & 62,800 & 60,173 & 16 \\
\hline 7/1/1998 & $3.8 \mathrm{E}+00$ & 65,612 & 28,492 & 2,673 & 761,754 & 197,217 & 16 \\
\hline 10/1/1998 & 8.9E-01 & 64,094 & 28,933 & 1,167 & 56,540 & 43,818 & 13 \\
\hline 1/1/1999 & $2.3 \mathrm{E}+00$ & 65,317 & 28,721 & 2,369 & 644,397 & 20,021 & 13 \\
\hline 4/1/1999 & $1.5 \mathrm{E}+00$ & 64,115 & 29,148 & 1,245 & 42,523 & 131,060 & 13 \\
\hline 7/1/1999 & $1.6 \mathrm{E}+01$ & 64,314 & 28,710 & 1,366 & 363,886 & 6,025 & 16 \\
\hline 10/1/1999 & $1.3 \mathrm{E}+00$ & 64,112 & 29,132 & 1,236 & 44,828 & 130,670 & 13 \\
\hline $1 / 1 / 2000$ & 4.9E-02 & 66,915 & 28,301 & 3,989 & 268,031 & 716 & 10 \\
\hline $4 / 1 / 2000$ & $2.9 \mathrm{E}+00$ & 64,160 & 28,997 & 1,244 & 456,962 & 112,080 & 28 \\
\hline $7 / 1 / 2000$ & $1.6 \mathrm{E}+01$ & 64,109 & 28,711 & 1,161 & 125,311 & 5,018 & 16 \\
\hline $10 / 1 / 2000$ & $1.5 \mathrm{E}+00$ & 64,025 & 29,131 & 1,154 & 157,622 & 122,906 & 17 \\
\hline $1 / 1 / 2001$ & $2.0 \mathrm{E}+01$ & 64,158 & 28,711 & 1,210 & 152,290 & 3,968 & 13 \\
\hline $4 / 1 / 2001$ & $1.9 \mathrm{E}+00$ & 63,872 & 29,083 & 995 & 181,869 & 115,116 & 16 \\
\hline $7 / 1 / 2001$ & $1.5 \mathrm{E}+01$ & 64,154 & 28,715 & 1,206 & 146,163 & 4,290 & 14 \\
\hline 10/1/2001 & $1.4 \mathrm{E}+00$ & 63,982 & 29,044 & 1,085 & 174,617 & 96,295 & 15 \\
\hline $1 / 1 / 2002$ & $1.2 \mathrm{E}+01$ & 64,151 & 28,716 & 1,203 & 150,707 & 3,425 & 15 \\
\hline $4 / 1 / 2002$ & $1.9 \mathrm{E}+00$ & 63,980 & 29,047 & 1,084 & 230,309 & 114,442 & 15 \\
\hline $7 / 1 / 2002$ & $8.3 \mathrm{E}+00$ & 64,144 & 28,719 & 1,196 & 157,818 & 4,057 & 15 \\
\hline $10 / 1 / 2002$ & $2.2 \mathrm{E}+00$ & 64,074 & 29,060 & 1,178 & 250,454 & 116,137 & 15 \\
\hline $1 / 1 / 2003$ & $1.6 \mathrm{E}+01$ & 64,071 & 28,730 & 1,124 & 126,249 & 4,751 & 12 \\
\hline $4 / 1 / 2003$ & $1.4 \mathrm{E}+00$ & 63,932 & 28,964 & 1,015 & 283,953 & 92,077 & 14 \\
\hline $7 / 1 / 2003$ & $1.4 \mathrm{E}+01$ & 64,114 & 28,718 & 1,166 & 108,685 & 3,073 & 15 \\
\hline $10 / 1 / 2003$ & $1.1 \mathrm{E}+00$ & 64,074 & 28,958 & 1,152 & 250,589 & 76,881 & 14 \\
\hline $1 / 1 / 2004$ & $1.6 \mathrm{E}+01$ & 64,134 & 28,716 & 1,186 & 127,815 & 3,115 & 15 \\
\hline $4 / 1 / 2004$ & $1.2 \mathrm{E}+00$ & 64,074 & 28,956 & 1,151 & 306,954 & 97,107 & 15 \\
\hline $7 / 1 / 2004$ & $1.2 \mathrm{E}+01$ & 64,139 & 28,716 & 1,191 & 141,238 & 3,393 & 15 \\
\hline $10 / 1 / 2004$ & $1.0 \mathrm{E}+00$ & 64,150 & 28,961 & 1,227 & 291,673 & 90,121 & 15 \\
\hline
\end{tabular}

LEAD

2.2E-01
29,322

3,312

$1,252,059$

343,419

39 
Project: Y-12 National Security Complex

Location: East Y-12
User Name: MV

State: Tennessee

\begin{tabular}{|c|c|c|c|c|c|c|c|}
\hline \multirow{2}{*}{ Effective Date } & \multirow{2}{*}{$\begin{array}{l}\text { 0th Moment } \\
\text { Estimated } \\
\text { Mass (kg) }\end{array}$} & \multicolumn{3}{|c|}{ 1st Moment (Center of Mass) } & \multicolumn{2}{|c|}{ 2nd Moment (Spread) } & \multirow[b]{2}{*}{$\begin{array}{c}\text { Number of } \\
\text { Wells }\end{array}$} \\
\hline & & $X c(f t)$ & Yc (ft) & $\begin{array}{c}\text { Source } \\
\text { Distance (ft) }\end{array}$ & $\begin{array}{l}\text { Sigma } X X \\
(\mathrm{sq} \mathrm{ft})\end{array}$ & $\begin{array}{l}\text { Sigma } Y Y \\
\text { (sq ft) }\end{array}$ & \\
\hline \multicolumn{8}{|l|}{ LEAD } \\
\hline 4/1/1996 & $1.8 \mathrm{E}-01$ & 68,088 & 29,391 & 5,184 & $6,020,503$ & 227,962 & 34 \\
\hline 7/1/1996 & 2.1E-01 & 63,807 & 29,679 & 1,291 & 160,514 & 289,965 & 28 \\
\hline 10/1/1996 & 1.1E-01 & 64,038 & 29,341 & 1,257 & 195,355 & 357,257 & 27 \\
\hline 1/1/1997 & $0.0 \mathrm{E}+00$ & & & & & & 2 \\
\hline 4/1/1997 & 3.2E-01 & 63,898 & 29,606 & 1,302 & 109,580 & 331,746 & 18 \\
\hline 7/1/1997 & $0.0 \mathrm{E}+00$ & & & & & & 1 \\
\hline 10/1/1997 & 3.8E-02 & 64,109 & 29,772 & 1,570 & 117,243 & 283,616 & 18 \\
\hline 1/1/1998 & $0.0 \mathrm{E}+00$ & & & & & & 2 \\
\hline 4/1/1998 & 7.2E-02 & 63,909 & 29,713 & 1,386 & 141,560 & 236,284 & 14 \\
\hline 7/1/1998 & $0.0 \mathrm{E}+00$ & & & & & & 2 \\
\hline 10/1/1998 & $3.7 \mathrm{E}-02$ & 63,822 & 29,709 & 1,324 & 118,231 & 294,710 & 13 \\
\hline 1/1/1999 & $0.0 \mathrm{E}+00$ & & & & & & 4 \\
\hline 4/1/1999 & $5.1 \mathrm{E}-02$ & 63,897 & 29,844 & 1,475 & 100,109 & 265,475 & 13 \\
\hline 7/1/1999 & $5.0 \mathrm{E}-03$ & 63,988 & 28,788 & 1,043 & 70,276 & 13,452 & 7 \\
\hline 10/1/1999 & 3.3E-02 & 63,831 & 29,795 & 1,395 & 118,294 & 246,399 & 13 \\
\hline $1 / 1 / 2000$ & $0.0 \mathrm{E}+00$ & & & & & & 1 \\
\hline $4 / 1 / 2000$ & $2.5 \mathrm{E}-01$ & 63,623 & 29,834 & 1,307 & 51,719 & 127,548 & 19 \\
\hline $7 / 1 / 2000$ & 1.4E-02 & 63,789 & 28,894 & 860 & 129,927 & 16,881 & 8 \\
\hline $10 / 1 / 2000$ & $6.8 \mathrm{E}-02$ & 63,890 & 29,851 & 1,475 & 100,938 & 249,604 & 15 \\
\hline $1 / 1 / 2001$ & $5.0 \mathrm{E}-03$ & 63,988 & 28,788 & 1,043 & 67,683 & 13,184 & 6 \\
\hline $4 / 1 / 2001$ & $1.9 \mathrm{E}-01$ & 64,009 & 30,197 & 1,822 & 58,465 & 244,847 & 13 \\
\hline $7 / 1 / 2001$ & $5.0 \mathrm{E}-03$ & 63,988 & 28,788 & 1,043 & 67,683 & 13,184 & 6 \\
\hline 10/1/2001 & $1.8 \mathrm{E}-01$ & 63,875 & 29,628 & 1,301 & 339,688 & 243,683 & 15 \\
\hline $1 / 1 / 2002$ & $1.0 \mathrm{E}-02$ & 64,659 & 28,750 & 1,711 & 508,266 & 8,175 & 10 \\
\hline $4 / 1 / 2002$ & $1.1 \mathrm{E}-01$ & 64,144 & 29,875 & 1,667 & 365,763 & 246,665 & 15 \\
\hline 7/1/2002 & $1.3 \mathrm{E}-02$ & 64,474 & 28,807 & 1,529 & 526,298 & 18,079 & 10 \\
\hline 10/1/2002 & $6.7 \mathrm{E}-01$ & 64,533 & 30,190 & 2,165 & 299,447 & 136,862 & 15 \\
\hline $1 / 1 / 2003$ & $1.0 \mathrm{E}-02$ & 64,659 & 28,750 & 1,711 & 508,266 & 8,175 & 10 \\
\hline $4 / 1 / 2003$ & $6.4 \mathrm{E}-02$ & 64,376 & 29,535 & 1,646 & 572,821 & 337,417 & 14 \\
\hline $7 / 1 / 2003$ & 4.1E-02 & 65,186 & 28,721 & 2,238 & 215,903 & 2,291 & 10 \\
\hline $10 / 1 / 2003$ & $5.6 \mathrm{E}-02$ & 64,472 & 29,548 & 1,737 & 471,136 & 298,348 & 14 \\
\hline $1 / 1 / 2004$ & $1.0 \mathrm{E}-02$ & 64,679 & 28,750 & 1,732 & 525,855 & 8,048 & 10 \\
\hline $4 / 1 / 2004$ & $1.0 \mathrm{E}-01$ & 64,862 & 29,341 & 2,014 & 502,822 & 275,863 & 16 \\
\hline $7 / 1 / 2004$ & $2.8 \mathrm{E}-02$ & 64,921 & 28,761 & 1,973 & 458,427 & 11,747 & 10 \\
\hline $10 / 1 / 2004$ & $5.2 \mathrm{E}-02$ & 64,572 & 29,462 & 1,787 & 493,136 & 364,659 & 15 \\
\hline
\end{tabular}

TETRACHLOROETHYLENE(PCE)

\begin{tabular}{rrrrrrrr}
\hline $1 / 1 / 1996$ & $1.5 \mathrm{E}+00$ & 65,088 & 29,267 & 2,210 & $2,119,129$ & 338,311 \\
$4 / 1 / 1996$ & $2.8 \mathrm{E}+00$ & 67,937 & 29,281 & 5,021 & $10,733,516$ & 258,498 & 39 \\
$7 / 1 / 1996$ & $2.3 \mathrm{E}+00$ & 67,711 & 29,419 & 4,815 & $10,063,449$ & 297,733 & 30 \\
$10 / 1 / 1996$ & $8.2 \mathrm{E}-01$ & 63,885 & 29,090 & 1,010 & 172,162 & 216,474 \\
$1 / 1 / 1997$ & $0.0 \mathrm{E}+00$ & & & & 27 \\
$4 / 1 / 1997$ & $4.5 \mathrm{E}+00$ & 66,909 & 28,762 & 3,962 & $8,959,309$ & 970,931 & 32 \\
\end{tabular}


Project: Y-12 National Security Complex

Location: East Y-12
User Name: $\quad$ MV

State: Tennessee

\begin{tabular}{|c|c|c|c|c|c|c|c|}
\hline \multirow[b]{2}{*}{ Effective Date } & \multirow{2}{*}{$\begin{array}{l}\text { Oth Moment } \\
\text { Estimated } \\
\text { Mass (kg) }\end{array}$} & \multicolumn{3}{|c|}{ 1st Moment (Center of Mass) } & \multicolumn{2}{|c|}{ 2nd Moment (Spread) } & \multirow[b]{2}{*}{$\begin{array}{c}\text { Number of } \\
\text { Wells }\end{array}$} \\
\hline & & $\mathrm{Xc}(\mathrm{ft})$ & Yc (ft) & $\begin{array}{c}\text { Source } \\
\text { Distance (ft) }\end{array}$ & $\begin{array}{l}\text { Sigma } X X \\
(s q \mathrm{ft})\end{array}$ & $\begin{array}{c}\text { Sigma YY } \\
(\mathrm{sq} \mathrm{ft})\end{array}$ & \\
\hline \multicolumn{8}{|c|}{ TETRACHLOROETHYLENE(PCE) } \\
\hline 7/1/1997 & $8.9 \mathrm{E}-01$ & 66,817 & 27,938 & 3,946 & $1,686,917$ & 426,160 & 14 \\
\hline 10/1/1997 & $1.2 \mathrm{E}+00$ & 63,815 & 29,577 & 1,223 & 131,534 & 251,109 & 18 \\
\hline $1 / 1 / 1998$ & $9.7 \mathrm{E}-01$ & 66,615 & 28,019 & 3,733 & $2,040,731$ & 461,891 & 16 \\
\hline 4/1/1998 & $2.3 \mathrm{E}+00$ & 64,098 & 29,429 & 1,354 & 370,614 & 346,411 & 16 \\
\hline 7/1/1998 & $1.2 \mathrm{E}+00$ & 66,602 & 27,973 & 3,729 & $1,778,636$ & 393,303 & 16 \\
\hline 10/1/1998 & $1.7 \mathrm{E}+00$ & 63,748 & 29,424 & 1,069 & 116,512 & 287,734 & 13 \\
\hline 1/1/1999 & $6.1 \mathrm{E}-01$ & 65,693 & 28,677 & 2,745 & $1,886,604$ & 49,651 & 13 \\
\hline 4/1/1999 & $2.3 \mathrm{E}+00$ & 63,808 & 29,559 & 1,205 & 100,523 & 298,421 & 13 \\
\hline 7/1/1999 & $3.0 \mathrm{E}+00$ & 64,108 & 28,777 & 1,162 & $1,061,893$ & 20,436 & 16 \\
\hline 10/1/1999 & $2.0 \mathrm{E}+00$ & 63,790 & 29,575 & 1,204 & 105,059 & 282,117 & 13 \\
\hline $1 / 1 / 2000$ & 1.1E-01 & 67,508 & 28,295 & 4,579 & 702,353 & 3,051 & 10 \\
\hline $4 / 1 / 2000$ & $4.0 \mathrm{E}+00$ & 64,232 & 29,442 & 1,476 & $1,147,100$ & 285,165 & 28 \\
\hline $7 / 1 / 2000$ & $1.9 \mathrm{E}+00$ & 64,310 & 28,862 & 1,370 & $1,193,398$ & 52,007 & 16 \\
\hline $10 / 1 / 2000$ & $5.9 \mathrm{E}+00$ & 63,850 & 29,332 & 1,093 & 134,657 & 199,565 & 17 \\
\hline $1 / 1 / 2001$ & $1.7 \mathrm{E}+00$ & 64,263 & 28,759 & 1,316 & $1,328,929$ & 26,200 & 13 \\
\hline $4 / 1 / 2001$ & $4.5 \mathrm{E}+00$ & 63,893 & 29,325 & 1,124 & 163,169 & 197,298 & 16 \\
\hline $7 / 1 / 2001$ & $1.8 \mathrm{E}+00$ & 64,349 & 28,764 & 1,402 & $1,471,563$ & 26,254 & 14 \\
\hline $10 / 1 / 2001$ & $5.6 \mathrm{E}+00$ & 63,897 & 29,271 & 1,100 & 131,686 & 171,155 & 15 \\
\hline $1 / 1 / 2002$ & $1.7 \mathrm{E}+00$ & 64,291 & 28,763 & 1,344 & $1,394,879$ & 25,914 & 15 \\
\hline 4/1/2002 & $5.1 \mathrm{E}+00$ & 63,913 & 29,322 & 1,140 & 159,202 & 179,029 & 15 \\
\hline $7 / 1 / 2002$ & $1.8 \mathrm{E}+00$ & 64,276 & 28,767 & 1,329 & $1,335,946$ & 25,873 & 15 \\
\hline $10 / 1 / 2002$ & $5.5 \mathrm{E}+00$ & 63,957 & 29,274 & 1,153 & 157,580 & 166,752 & 15 \\
\hline $1 / 1 / 2003$ & $3.3 \mathrm{E}+00$ & 63,949 & 28,787 & 1,004 & 429,495 & 14,657 & 12 \\
\hline $4 / 1 / 2003$ & $6.3 \mathrm{E}+00$ & 63,936 & 29,341 & 1,170 & 151,662 & 216,111 & 14 \\
\hline $7 / 1 / 2003$ & $2.6 \mathrm{E}+00$ & 64,228 & 28,763 & 1,281 & $1,031,938$ & 19,874 & 15 \\
\hline $10 / 1 / 2003$ & $6.2 \mathrm{E}+00$ & 63,985 & 29,332 & 1,207 & 133,075 & 202,715 & 14 \\
\hline $1 / 1 / 2004$ & $2.8 \mathrm{E}+00$ & 64,210 & 28,766 & 1,263 & $1,002,690$ & 19,359 & 15 \\
\hline $4 / 1 / 2004$ & $5.5 \mathrm{E}+00$ & 63,975 & 29,372 & 1,219 & 148,020 & 214,172 & 15 \\
\hline $7 / 1 / 2004$ & $2.3 \mathrm{E}+00$ & 64,221 & 28,765 & 1,274 & $1,104,198$ & 21,911 & 15 \\
\hline $10 / 1 / 2004$ & $5.8 \mathrm{E}+00$ & 63,996 & 29,342 & 1,221 & 134,243 & 201,889 & 15 \\
\hline
\end{tabular}


Project: Y-12 National Security Complex

Location: East $\mathrm{Y}-12$
User Name: $\mathrm{MV}$

State: Tennessee

\begin{tabular}{|c|c|c|c|c|c|}
\hline Moment Type & Constituent & $\begin{array}{l}\text { Coefficient } \\
\text { of Variation }\end{array}$ & $\begin{array}{l}\text { Mann-Kendall } \\
\text { S Statistic }\end{array}$ & $\begin{array}{l}\text { Confidence } \\
\text { in Trend }\end{array}$ & $\begin{array}{c}\text { Moment } \\
\text { Trend }\end{array}$ \\
\hline \multicolumn{6}{|c|}{ Zeroth Moment: Mass } \\
\hline & CARBON TETRACHLORIDE & 1.14 & 120 & $94.7 \%$ & $\mathrm{PI}$ \\
\hline & LEAD & 1.49 & 1 & $50.0 \%$ & NT \\
\hline & TETRACHLOROETHYLENE(PCE) & 0.66 & 242 & $100.0 \%$ & 1 \\
\hline \multicolumn{6}{|c|}{ 1st Moment: Distance to Source } \\
\hline & CARBON TETRACHLORIDE & 0.47 & -279 & $100.0 \%$ & $\mathrm{D}$ \\
\hline & LEAD & 0.47 & 137 & $99.3 \%$ & 1 \\
\hline & TETRACHLOROETHYLENE(PCE) & 0.66 & -185 & $99.6 \%$ & $\mathrm{D}$ \\
\hline \multicolumn{6}{|c|}{ 2nd Moment: Sigma XX } \\
\hline & CARBON TETRACHLORIDE & 2.23 & -105 & $93.0 \%$ & PD \\
\hline & LEAD & 2.20 & 89 & $94.1 \%$ & $\mathrm{PI}$ \\
\hline & TETRACHLOROETHYLENE(PCE) & 1.72 & -175 & $99.4 \%$ & $\mathrm{D}$ \\
\hline \multicolumn{6}{|c|}{ 2nd Moment: Sigma YY } \\
\hline & CARBON TETRACHLORIDE & 1.06 & -261 & $100.0 \%$ & $\mathrm{D}$ \\
\hline & LEAD & 0.73 & -121 & $98.4 \%$ & $\mathrm{D}$ \\
\hline & TETRACHLOROETHYLENE(PCE) & 0.92 & -267 & $100.0 \%$ & $\mathrm{D}$ \\
\hline
\end{tabular}

Note: The following assumptions were applied for the calculation of the Zeroth Moment:

Porosity: $0.10 \quad$ Saturated Thickness: Uniform: $50 \mathrm{ft}$

Mann-Kendall Trend test performed on all sample events for each constituent. Increasing (I); Probably Increasing (PI); Stable (S);

Probably Decreasing (PD); Decreasing (D); No Trend (NT); Not Applicable (N/A)-Due to insufficient Data (< 4 sampling events).

Note: The Sigma $X X$ and Sigma $Y Y$ components are estimated using the given field coordinate system and then rotated to align with the estimated groundwater flow direction. Moments are not calculated for sample events with less than 6 wells. 


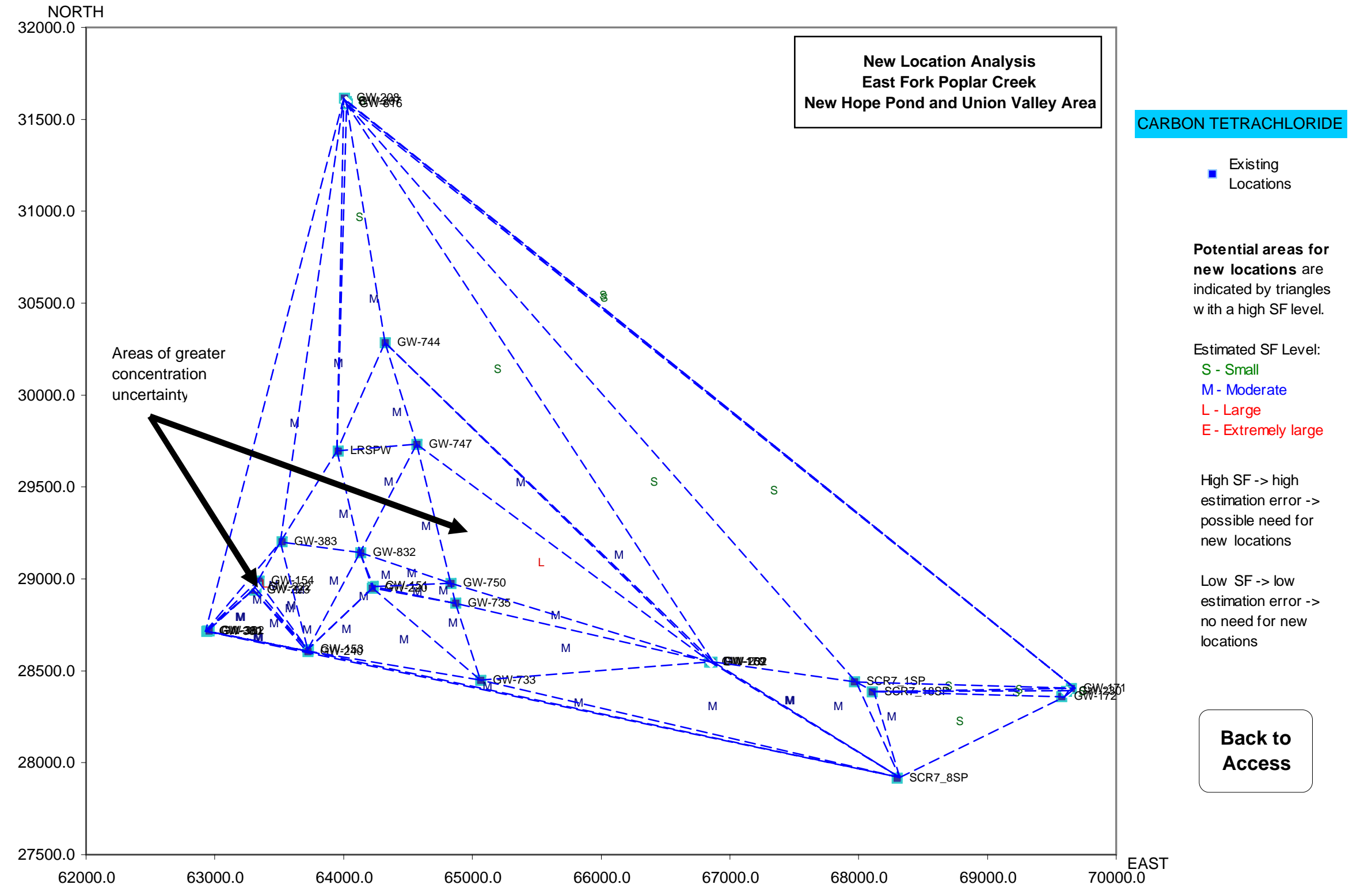




\section{ASSESSMENT OF THE GROUNDWATER PROTECTION PROGRAM \\ Y-12 NATIONAL SECURITY COMPLEX, OAK RIDGE, TENNESSEE}

Y-12 National Security Complex

Oak Ridge, Tennessee

\section{Appendix D.8 Chestnut Ridge Regime West Chestnut Ridge}

Table D.8.1 Qualitative Analysis West Chestnut Ridge Area

Table D.8.2 Aquifer Input Parameters

MAROS Report $\quad$ COC Assessment

MAROS Report Plume Summary

MAROS Report Spatial Moment Analysis Summary

(No Well Sufficiency areas of interest identified) 
TABLE D.8.1

QUALITATIVE ANALYSIS CHESTNUT RIDGE REGIME WEST CHESTNUT RIDGE

Chestnut Ridge Hydrogeologic Regime

Y-12 National Security Complex

Oak Ridge, Tennessee

\begin{tabular}{|c|c|c|c|c|c|c|c|c|c|c|c|c|c|}
\hline $\begin{array}{l}\text { Location } \\
\text { Name }\end{array}$ & $\begin{array}{c}\text { Location } \\
\text { Type } \\
\end{array}$ & $\begin{array}{c}\text { Average } \\
\text { Concentration } \\
\text { Exceeds } \\
\text { Screening } \\
\end{array}$ & $\begin{array}{c}\text { Formation } \\
\text { Type }\end{array}$ & $\begin{array}{l}\text { Horizontal } \\
\text { Delineation }\end{array}$ & $\begin{array}{c}\text { Vertical } \\
\text { Delineation }\end{array}$ & Exit Location & RCRA & CERCLA & Unique & $\begin{array}{c}\text { Monitors } \\
\text { Background } \\
\text { Water Quality }\end{array}$ & $\begin{array}{c}\text { Early } \\
\text { Detection }\end{array}$ & $\begin{array}{c}\text { Monitor } \\
\text { Source }\end{array}$ & SCORE \\
\hline $\begin{array}{c}1090 \\
\text { GW-141 } \\
\text { GW-203 } \\
\text { GW-205 } \\
\text { GW-217 }\end{array}$ & $\begin{array}{l}\text { WL } \\
W L \\
W L \\
W L \\
W L\end{array}$ & & $\begin{array}{l}\text { Aquifer } \\
\text { Aquifer } \\
\text { Aquifer } \\
\text { Aquifer } \\
\text { Aquifer }\end{array}$ & & $\begin{array}{l}X \\
X \\
X \\
X\end{array}$ & & & $\begin{array}{l}X \\
x \\
X\end{array}$ & & & & & \\
\hline $\begin{array}{l}\text { GW-221 } \\
\text { GW-302 } \\
\text { GW-305 } \\
\text { GW-339 } \\
\text { GW-521 }\end{array}$ & $\begin{array}{l}\text { WL } \\
W L \\
W L \\
W L \\
W L\end{array}$ & $x$ & $\begin{array}{l}\text { Aquifer } \\
\text { Aquifer } \\
\text { Aquifer } \\
\text { Aquifer } \\
\text { Aquifer }\end{array}$ & & $\begin{array}{l}X \\
X \\
X \\
X \\
X\end{array}$ & & $\mathrm{x}$ & $\begin{array}{l}X \\
X \\
X \\
X\end{array}$ & & & & & \\
\hline $\begin{array}{l}\text { GW-522 } \\
\text { GW-539 } \\
\text { GW-540 } \\
\text { GW-541 } \\
\text { GW-542 }\end{array}$ & $\begin{array}{l}\text { WL } \\
W L \\
W L \\
W L \\
W L\end{array}$ & $x$ & $\begin{array}{l}\text { Aquifer } \\
\text { Aquifer } \\
\text { Aquifer } \\
\text { Aquifer } \\
\text { Aquifer }\end{array}$ & & $\begin{array}{l}X \\
X \\
X \\
X\end{array}$ & & & & & & & & \\
\hline $\begin{array}{l}\text { GW-543 } \\
\text { GW-544 } \\
\text { GW-546 } \\
\text { GW-709 } \\
\text { GW-757 }\end{array}$ & $\begin{array}{l}\text { WL } \\
W L \\
W L \\
W L \\
\text { WL }\end{array}$ & & $\begin{array}{l}\text { Aquifer } \\
\text { Aquifer } \\
\text { Aquifer } \\
\text { Aquifer } \\
\text { Aquifer }\end{array}$ & & $\mathrm{x}$ & & & & & & & & \\
\hline $\begin{array}{c}\text { GW-827 } \\
\text { SCR1.25SP } \\
\text { SCR2.1SP } \\
\text { SCR2.2SP }\end{array}$ & $\begin{array}{l}\text { WL } \\
\text { SP } \\
\text { SP } \\
\text { SP }\end{array}$ & & $\begin{array}{l}\text { Aquifer } \\
\text { Spring } \\
\text { Spring } \\
\text { Spring }\end{array}$ & & $\mathrm{X}$ & $\begin{array}{l}X \\
X \\
X\end{array}$ & & & & & & & \\
\hline
\end{tabular}

Notes:

1. $\mathrm{WL}=$ Monitoring Well; $\mathrm{SP}=$ Spring

2. Well data taken from BWXT Y-12 Analytical Database. Sample locations shown on Figures A.1 and A.4.

3. RCRA indicates wells monitored as part of compliance with RCRA Post-Closure Corrective Action Monitoring or designated Alternate location;

CERCLA indicates locations monitored as part of compliance with CERCLA ROD or backup location. Data from BWXT, 2003a and BWXT 2004a.

4. Average Concentration Exceeds Screening = The average concentration over the entire sampling record for the priority constituent is above the MCL or other designated screening leve

as defined in Table B.1.

5. Aquifer and aquitard formations identified in Fig. A.2 from BWXT Y12, 2003 Groundwater Monitoring Report , (12/01/2003). 


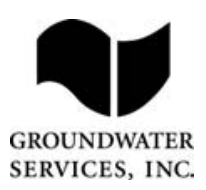

TABLE D.8.2

\title{
AQUIFER INPUT PARAMETERS
}

\author{
West Chestnut Ridge \\ Chestnut Ridge Regime \\ Y-12 National Security Complex
}

\begin{tabular}{|c|c|c|}
\hline Parameter & Value & Units \\
\hline Current Plume Length & 5500 & $\mathrm{ft}$ \\
\hline Maximum Plume Length & 5500 & $\mathrm{ft}$ \\
\hline PlumeWidth & 2000 & $\mathrm{ft}$ \\
\hline SeepageVelocity (ft/yr) & 200 & $\mathrm{ft} / \mathrm{yr}$ \\
\hline Distance to Receptors & 6000 & $\mathrm{ft}$ \\
\hline GWFluctuations & Yes & -- \\
\hline SourceTreatment & None & -- \\
\hline PlumeType & VOC/Metals & -- \\
\hline Free NAPL Present & Yes & -- \\
\hline Parameter & Value & \\
\hline Groundwater flow direction & $\overline{\mathrm{S}}$ & 270 \\
\hline Effective Porosity & 0.1 & -- \\
\hline Source Location near Well & GW-205 & -- \\
\hline Source X-Coordinate & 54008.3 & $\mathrm{ft}^{*}$ \\
\hline Source Y-Coordinate & 28362.98 & $\mathrm{ft}^{\star}$ \\
\hline Saturated Thickness & 50 & $\mathrm{ft}$ \\
\hline \multicolumn{3}{|l|}{ Source Wells } \\
\hline 11 & $090, \mathrm{C}$ & 41 \\
\hline
\end{tabular}

\section{Notes:}

1. Aquifer data are general values for the hydrologic regime.

2. Priority COCs defined by prevalence, toxicty and mobility.

3. $\mathrm{ft}^{\star}=$ Coordinates in Y-12 Plant coordinates, feet.

4. Screening Levels are USEPA MCLs, except in the case of compounds without MCLs where the level is the Region 9 PRG for tap water.

5. Effective Porosity estimated based on average high and low values for aquifer and aquitard suburfaces. 


\section{MAROS Plume Analysis Summary}

Project: $\quad$ Y-12 National Security Complex

Location: West Chestnut Ridge

Time Period: 1/1/1996

to $1 / 1 / 2005$

Consolidation Period: No Time Consolidation

Consolidation Type: Median

Duplicate Consolidation: Average

ND Values: Specified Detection Limit

J Flag Values : Actual Value

\begin{tabular}{|c|c|c|c|c|c|c|c|c|c|c|c|}
\hline Constituent & Well & $\begin{array}{c}\text { Sourcel } \\
\text { Tail }\end{array}$ & $\begin{array}{l}\text { Number } \\
\text { of } \\
\text { Samples }\end{array}$ & $\begin{array}{c}\text { Number } \\
\text { of } \\
\text { Detects }\end{array}$ & $\begin{array}{c}\text { Average } \\
\text { (mg/L) }\end{array}$ & $\begin{array}{r}\text { Median } \\
\text { (mg/L) }\end{array}$ & $\begin{array}{c}\text { All } \\
\text { Samples } \\
\text { "ND" ? }\end{array}$ & $\begin{array}{l}\text { Mann- } \\
\text { Kendall }\end{array}$ & $\begin{array}{c}\text { Linear } \\
\text { Regression }\end{array}$ & Modeling & Empirical \\
\hline \multicolumn{12}{|c|}{ 1,1,1-TRICHLOROETHANE } \\
\hline & GW-205 & $\mathrm{S}$ & 3 & 0 & $6.0 \mathrm{E}-06$ & $6.0 \mathrm{E}-06$ & Yes & N/A & N/A & N/A & N/A \\
\hline & 1090 & $\mathrm{~s}$ & 3 & 0 & $6.0 \mathrm{E}-06$ & 6.0E-06 & Yes & N/A & N/A & N/A & N/A \\
\hline & GW-203 & $\mathrm{s}$ & 3 & 0 & $6.0 \mathrm{E}-06$ & $6.0 \mathrm{E}-06$ & Yes & N/A & N/A & N/A & N/A \\
\hline & GW-141 & $\mathrm{s}$ & 18 & 0 & $6.0 \mathrm{E}-06$ & $6.0 \mathrm{E}-06$ & Yes & $\mathrm{s}$ & $\mathrm{s}$ & $N / A$ & N/A \\
\hline & GW-217 & $\mathrm{s}$ & 18 & 0 & $6.0 \mathrm{E}-06$ & $6.0 \mathrm{E}-06$ & Yes & s & $\mathrm{s}$ & N/A & N/A \\
\hline & GW-543 & $\mathrm{T}$ & 18 & 1 & $6.1 \mathrm{E}-05$ & $6.0 \mathrm{E}-06$ & No & NT & NT & $\mathrm{N} / \mathrm{A}$ & N/A \\
\hline & GW-541 & $\mathrm{T}$ & 1 & 0 & $6.0 \mathrm{E}-06$ & $6.0 \mathrm{E}-06$ & Yes & N/A & N/A & $N / A$ & N/A \\
\hline & GW-544 & $\mathrm{T}$ & 18 & 0 & $6.0 \mathrm{E}-06$ & $6.0 \mathrm{E}-06$ & Yes & $S$ & $S$ & $\mathrm{~N} / \mathrm{A}$ & $\mathrm{N} / \mathrm{A}$ \\
\hline & GW-540 & $\mathrm{T}$ & 16 & 0 & $6.0 \mathrm{E}-06$ & $6.0 \mathrm{E}-06$ & Yes & $\mathrm{s}$ & s & $N / A$ & N/A \\
\hline & GW-305 & $\mathrm{T}$ & 27 & 27 & 1.7E-02 & 1.8E-02 & No & I & I & N/A & N/A \\
\hline & GW-542 & $\mathrm{T}$ & 17 & 0 & $6.0 \mathrm{E}-06$ & 6.0E-06 & Yes & S & s & $N / A$ & N/A \\
\hline & GW-539 & $\mathrm{T}$ & 10 & 0 & $6.0 \mathrm{E}-06$ & $6.0 \mathrm{E}-06$ & Yes & $\mathrm{s}$ & $\mathrm{s}$ & $N / A$ & N/A \\
\hline & GW-546 & $\mathrm{T}$ & 1 & 0 & $6.0 \mathrm{E}-06$ & $6.0 \mathrm{E}-06$ & Yes & N/A & $N / A$ & $\mathrm{~N} / \mathrm{A}$ & N/A \\
\hline & GW-522 & $\mathrm{T}$ & 18 & 0 & $6.0 \mathrm{E}-06$ & $6.0 \mathrm{E}-06$ & Yes & $\mathrm{s}$ & $\mathrm{s}$ & $N / A$ & N/A \\
\hline & GW-709 & $\mathrm{T}$ & 18 & 0 & $6.0 \mathrm{E}-06$ & 6.0E-06 & Yes & $\mathrm{s}$ & s & N/A & N/A \\
\hline & GW-521 & $\mathrm{T}$ & 20 & 0 & $6.0 \mathrm{E}-06$ & $6.0 \mathrm{E}-06$ & Yes & $\mathrm{S}$ & $\mathrm{S}$ & N/A & N/A \\
\hline & GW-339 & $\mathrm{T}$ & 3 & 0 & $6.0 \mathrm{E}-06$ & $6.0 \mathrm{E}-06$ & Yes & N/A & N/A & $N / A$ & $N / A$ \\
\hline & GW-757 & $\mathrm{T}$ & 18 & 1 & 2.3E-04 & 6.0E-06 & No & NT & NT & $N / A$ & N/A \\
\hline & GW-827 & $\mathrm{T}$ & 18 & 0 & $6.0 \mathrm{E}-06$ & 6.0E-06 & Yes & $\mathrm{s}$ & $\mathrm{S}$ & N/A & N/A \\
\hline & GW-302 & $\mathrm{T}$ & 3 & 0 & $6.0 \mathrm{E}-06$ & $6.0 \mathrm{E}-06$ & Yes & N/A & $N / A$ & $N / A$ & $N / A$ \\
\hline & SCR2_2SP & $\mathrm{T}$ & 14 & 0 & $6.0 \mathrm{E}-06$ & 6.0E-06 & Yes & $\mathrm{S}$ & $\mathrm{S}$ & $N / A$ & $\mathrm{~N} / \mathrm{A}$ \\
\hline & GW-221 & $\mathrm{T}$ & 3 & 0 & $6.0 \mathrm{E}-06$ & $6.0 \mathrm{E}-06$ & Yes & N/A & $N / A$ & $N / A$ & N/A \\
\hline
\end{tabular}

MAROS Version 2.1, 2004, AFCEE
User Name: MV

State: Tennessee 
Project: Y-12 National Security Complex

Location: West Chestnut Ridge
User Name: MV

State: Tennessee

\begin{tabular}{|c|c|c|c|c|c|c|c|c|c|c|c|}
\hline Constituent & Well & $\begin{array}{c}\text { Sourcel } \\
\text { Tail }\end{array}$ & $\begin{array}{c}\text { Number } \\
\text { of } \\
\text { Samples }\end{array}$ & $\begin{array}{c}\text { Number } \\
\text { of } \\
\text { Detects }\end{array}$ & $\begin{array}{c}\text { Average } \\
\text { (mg/L) }\end{array}$ & $\begin{array}{r}\text { Median } \\
(\mathrm{mg} / \mathrm{L})\end{array}$ & $\begin{array}{c}\text { All } \\
\text { Samples } \\
\text { "ND" ? }\end{array}$ & $\begin{array}{l}\text { Mann- } \\
\text { Kendall }\end{array}$ & $\begin{array}{l}\text { Linear } \\
\text { Regression }\end{array}$ & Modeling & Empirical \\
\hline \multicolumn{12}{|c|}{ 1,1,1-TRICHLOROETHANE } \\
\hline & SCR1_25SP & $T$ & 16 & 0 & $6.0 \mathrm{E}-06$ & $6.0 \mathrm{E}-06$ & Yes & $\mathrm{S}$ & S & N/A & N/A \\
\hline & SCR2_1SP & $\mathrm{T}$ & 14 & 0 & $6.0 \mathrm{E}-06$ & $6.0 \mathrm{E}-06$ & Yes & $\mathrm{s}$ & s & N/A & N/A \\
\hline \multicolumn{12}{|c|}{ GROSS ALPHA ACTIVITY } \\
\hline & GW-217 & $\mathrm{S}$ & 15 & 5 & $4.0 \mathrm{E}-01$ & $1.0 \mathrm{E}-02$ & No & $\mathrm{D}$ & $\mathrm{D}$ & $\mathrm{N} / \mathrm{A}$ & $\mathrm{N} / \mathrm{A}$ \\
\hline & 1090 & s & 16 & 11 & 9.3E-01 & 7.0E-01 & No & NT & NT & $N / A$ & $N / A$ \\
\hline & GW-141 & s & 17 & 7 & $1.8 \mathrm{E}+00$ & $1.0 \mathrm{E}-02$ & No & $\mathrm{D}$ & $\mathrm{D}$ & N/A & N/A \\
\hline & GW-203 & s & 15 & 11 & $2.2 \mathrm{E}+00$ & 5.4E-01 & No & 1 & NT & $N / A$ & $N / A$ \\
\hline & GW-205 & s & 17 & 12 & $1.3 \mathrm{E}+00$ & $6.1 \mathrm{E}-01$ & No & NT & NT & $N / A$ & $N / A$ \\
\hline & GW-305 & $\mathrm{T}$ & 26 & 8 & $7.1 \mathrm{E}-01$ & $1.0 \mathrm{E}-02$ & No & $\mathrm{D}$ & $\mathrm{D}$ & N/A & N/A \\
\hline & GW-539 & $\mathrm{T}$ & 9 & 4 & $1.6 \mathrm{E}+00$ & $1.0 \mathrm{E}-02$ & No & $D$ & D & N/A & $N / A$ \\
\hline & GW-302 & $\mathrm{T}$ & 15 & 11 & $1.9 \mathrm{E}+00$ & 8.4E-01 & No & NT & NT & $N / A$ & $N / A$ \\
\hline & GW-521 & $\mathrm{T}$ & 15 & 5 & 3.7E-01 & $1.0 \mathrm{E}-02$ & No & $\mathrm{D}$ & $\mathrm{D}$ & N/A & N/A \\
\hline & GW-540 & $\mathrm{T}$ & 15 & 5 & 6.7E-01 & $1.0 \mathrm{E}-02$ & No & $D$ & $\mathrm{D}$ & $\mathrm{N} / \mathrm{A}$ & $N / A$ \\
\hline & GW-221 & $\mathrm{T}$ & 16 & 7 & $1.2 \mathrm{E}+00$ & 1.0E-02 & No & NT & NT & $N / A$ & $N / A$ \\
\hline & GW-541 & $\mathrm{T}$ & 1 & 1 & $2.6 \mathrm{E}+00$ & $2.6 \mathrm{E}+00$ & No & $\mathrm{N} / \mathrm{A}$ & $\mathrm{N} / \mathrm{A}$ & N/A & N/A \\
\hline & GW-339 & $\mathrm{T}$ & 15 & 8 & $9.0 \mathrm{E}-01$ & $4.2 \mathrm{E}-01$ & No & NT & NT & $N / A$ & $N / A$ \\
\hline & GW-542 & $\mathrm{T}$ & 15 & 5 & $9.5 \mathrm{E}-01$ & $1.0 \mathrm{E}-02$ & No & $\mathrm{D}$ & $\mathrm{D}$ & N/A & N/A \\
\hline & GW-522 & $\mathrm{T}$ & 15 & 5 & 8.7E-01 & $1.0 \mathrm{E}-02$ & No & $\mathrm{D}$ & D & $N / A$ & $N / A$ \\
\hline & GW-757 & $\mathrm{T}$ & 18 & 15 & $2.4 \mathrm{E}+00$ & $2.0 \mathrm{E}+00$ & No & $\mathrm{D}$ & D & $N / A$ & $N / A$ \\
\hline & GW-546 & $\mathrm{T}$ & 1 & 1 & $3.3 \mathrm{E}+00$ & $3.3 \mathrm{E}+00$ & No & $N / A$ & $\mathrm{~N} / \mathrm{A}$ & $N / A$ & N/A \\
\hline & GW-543 & $\mathrm{T}$ & 16 & 6 & 3.3E-01 & $1.0 \mathrm{E}-02$ & No & $D$ & $\mathrm{D}$ & $N / A$ & $N / A$ \\
\hline & GW-827 & $\mathrm{T}$ & 15 & 6 & $3.2 \mathrm{E}-01$ & $1.0 \mathrm{E}-02$ & No & $\mathrm{D}$ & D & $N / A$ & $N / A$ \\
\hline & SCR2_1SP & $\mathrm{T}$ & 14 & 14 & $3.3 E+00$ & $2.8 \mathrm{E}+00$ & No & $\mathrm{s}$ & $\mathrm{S}$ & $N / A$ & $N / A$ \\
\hline & SCR1_25SP & $\mathrm{T}$ & 12 & 11 & $3.6 \mathrm{E}+00$ & $2.1 \mathrm{E}+00$ & No & NT & NT & $N / A$ & $N / A$ \\
\hline & SCR2_2SP & $\mathrm{T}$ & 11 & 11 & $1.6 \mathrm{E}+00$ & $1.2 \mathrm{E}+00$ & No & $\mathrm{s}$ & NT & N/A & N/A \\
\hline & GW-544 & $\mathrm{T}$ & 18 & 11 & $1.5 \mathrm{E}+00$ & $1.0 \mathrm{E}+00$ & No & $\mathrm{D}$ & $\mathrm{D}$ & $N / A$ & N/A \\
\hline & GW-709 & $\mathrm{T}$ & 15 & 7 & $1.1 \mathrm{E}+00$ & $1.0 \mathrm{E}-02$ & No & $D$ & $D$ & N/A & N/A \\
\hline \multicolumn{12}{|c|}{ GROSS BETA ACTIVITY } \\
\hline & GW-205 & $\mathrm{s}$ & 16 & 15 & $5.0 \mathrm{E}+01$ & $6.2 \mathrm{E}+01$ & No & 1 & $\mathrm{PI}$ & $N / A$ & $N / A$ \\
\hline & GW-141 & s & 16 & 9 & $2.4 \mathrm{E}+00$ & $1.0 \mathrm{E}+00$ & No & $\mathrm{D}$ & $\mathrm{D}$ & $N / A$ & $N / A$ \\
\hline & GW-203 & s & 14 & 12 & $6.4 \mathrm{E}+00$ & $3.0 \mathrm{E}+00$ & No & NT & NT & $N / A$ & $N / A$ \\
\hline & GW-217 & s & 17 & 11 & $1.2 \mathrm{E}+00$ & $9.0 \mathrm{E}-01$ & No & $D$ & $\mathrm{D}$ & $N / A$ & $N / A$ \\
\hline
\end{tabular}


Project: Y-12 National Security Complex

Location: West Chestnut Ridge
User Name: MV

State: Tennessee

\begin{tabular}{|c|c|c|c|c|c|c|c|c|c|c|c|}
\hline Constituent & Well & $\begin{array}{c}\text { Sourcel } \\
\text { Tail }\end{array}$ & $\begin{array}{c}\text { Number } \\
\text { of } \\
\text { Samples }\end{array}$ & $\begin{array}{c}\text { Number } \\
\text { of } \\
\text { Detects }\end{array}$ & $\begin{array}{c}\text { Average } \\
\text { (mg/L) }\end{array}$ & $\begin{array}{c}\text { Median } \\
(\mathrm{mg} / \mathrm{L})\end{array}$ & $\begin{array}{c}\text { All } \\
\text { Samples } \\
\text { "ND" ? }\end{array}$ & $\begin{array}{l}\text { Mann- } \\
\text { Kendall }\end{array}$ & $\begin{array}{c}\text { Linear } \\
\text { Regression }\end{array}$ & Modeling & Empirical \\
\hline \multicolumn{12}{|c|}{ GROSS BETA ACTIVITY } \\
\hline & 1090 & $\mathrm{~s}$ & 16 & 11 & $2.7 \mathrm{E}+00$ & $1.3 \mathrm{E}+00$ & No & NT & NT & N/A & $\mathrm{N} / \mathrm{A}$ \\
\hline & GW-305 & $\mathrm{T}$ & 24 & 6 & $9.8 \mathrm{E}-01$ & $1.0 \mathrm{E}-03$ & No & $\mathrm{D}$ & D & N/A & N/A \\
\hline & SCR1_25SP & $\mathrm{T}$ & 12 & 10 & $1.3 E+00$ & $1.2 \mathrm{E}+00$ & No & 1 & 1 & N/A & N/A \\
\hline & GW-302 & $\mathrm{T}$ & 14 & 8 & $2.2 \mathrm{E}+00$ & $1.2 \mathrm{E}+00$ & No & NT & NT & N/A & N/A \\
\hline & GW-709 & $\mathrm{T}$ & 14 & 9 & $2.0 \mathrm{E}+00$ & $1.0 \mathrm{E}+00$ & No & NT & NT & N/A & N/A \\
\hline & GW-339 & $\mathrm{T}$ & 13 & 10 & $5.8 \mathrm{E}+00$ & $1.5 \mathrm{E}+00$ & No & NT & NT & N/A & N/A \\
\hline & GW-827 & $\mathrm{T}$ & 16 & 9 & $1.8 \mathrm{E}+00$ & $1.0 \mathrm{E}+00$ & No & D & D & N/A & $N / A$ \\
\hline & GW-542 & $\mathrm{T}$ & 16 & 13 & $1.9 \mathrm{E}+00$ & $1.3 \mathrm{E}+00$ & No & D & $P D$ & N/A & $\mathrm{N} / \mathrm{A}$ \\
\hline & GW-544 & $T$ & 16 & 12 & $2.8 \mathrm{E}+00$ & $2.2 \mathrm{E}+00$ & No & $\mathrm{PD}$ & $\mathrm{s}$ & N/A & N/A \\
\hline & SCR2_2SP & $\mathrm{T}$ & 9 & 9 & $2.9 \mathrm{E}+00$ & $2.5 \mathrm{E}+00$ & No & NT & s & N/A & $N / A$ \\
\hline & GW-543 & $T$ & 17 & 10 & $1.3 \mathrm{E}+00$ & $1.0 \mathrm{E}+00$ & No & D & $\mathrm{D}$ & N/A & N/A \\
\hline & GW-540 & $\mathrm{T}$ & 14 & 8 & $1.4 \mathrm{E}+00$ & $1.5 \mathrm{E}+00$ & No & $\mathrm{PD}$ & PD & N/A & $\mathrm{N} / \mathrm{A}$ \\
\hline & GW-539 & $\mathrm{T}$ & 9 & 3 & 7.7E-01 & $1.0 \mathrm{E}-03$ & No & PD & $\mathrm{D}$ & N/A & $N / A$ \\
\hline & GW-221 & $\mathrm{T}$ & 16 & 9 & $1.9 \mathrm{E}+00$ & 3.9E-01 & No & NT & NT & N/A & N/A \\
\hline & SCR2_1SP & $\mathrm{T}$ & 11 & 10 & $2.0 \mathrm{E}+00$ & $1.6 \mathrm{E}+00$ & No & $\mathrm{PI}$ & NT & $\mathrm{N} / \mathrm{A}$ & N/A \\
\hline & GW-522 & $\mathrm{T}$ & 14 & 5 & 8.7E-01 & $1.0 \mathrm{E}-03$ & No & D & $\mathrm{D}$ & N/A & $N / A$ \\
\hline & GW-546 & $\mathrm{T}$ & 1 & 1 & 5.2E-01 & 5.2E-01 & No & N/A & N/A & N/A & $\mathrm{N} / \mathrm{A}$ \\
\hline & GW-757 & $\mathrm{T}$ & 18 & 18 & $1.3 \mathrm{E}+01$ & $1.5 \mathrm{E}+01$ & No & 1 & 1 & N/A & $\mathrm{N} / \mathrm{A}$ \\
\hline & GW-521 & $T$ & 15 & 5 & $1.0 \mathrm{E}+00$ & 1.0E-03 & No & D & D & N/A & N/A \\
\hline \multicolumn{12}{|l|}{ LEAD } \\
\hline & GW-205 & $\mathrm{s}$ & 17 & 2 & $2.4 \mathrm{E}-04$ & $5.2 \mathrm{E}-05$ & No & NT & $\bar{D}$ & $\mathrm{~N} / \mathrm{A}$ & N/A \\
\hline & GW-203 & s & 16 & 2 & $1.3 \mathrm{E}-04$ & 5.2E-05 & No & NT & D & N/A & $\mathrm{N} / \mathrm{A}$ \\
\hline & GW-217 & s & 18 & 5 & $1.6 \mathrm{E}-04$ & $5.2 \mathrm{E}-05$ & No & $\mathrm{D}$ & D & N/A & N/A \\
\hline & 1090 & s & 17 & 2 & $1.2 \mathrm{E}-04$ & $5.2 \mathrm{E}-05$ & No & NT & D & N/A & $N / A$ \\
\hline & GW-141 & s & 18 & 4 & $1.6 \mathrm{E}-03$ & $5.2 \mathrm{E}-05$ & No & $\mathrm{D}$ & D & N/A & N/A \\
\hline & SCR2_1SP & $\mathrm{T}$ & 14 & 4 & $2.0 \mathrm{E}-04$ & $5.2 \mathrm{E}-05$ & No & NT & NT & $\mathrm{N} / \mathrm{A}$ & N/A \\
\hline & GW-827 & $\mathrm{T}$ & 18 & 0 & $5.2 \mathrm{E}-05$ & $5.2 \mathrm{E}-05$ & Yes & $\mathrm{s}$ & s & N/A & $N / A$ \\
\hline & SCR1_25SP & $\mathrm{T}$ & 12 & 0 & $5.2 \mathrm{E}-05$ & $5.2 \mathrm{E}-05$ & Yes & $\mathrm{s}$ & s & N/A & N/A \\
\hline & GW-546 & $T$ & 1 & 0 & $5.2 \mathrm{E}-05$ & 5.2E-05 & Yes & N/A & $\mathrm{N} / \mathrm{A}$ & $\mathrm{N} / \mathrm{A}$ & $\mathrm{N} / \mathrm{A}$ \\
\hline & GW-542 & $\mathrm{T}$ & 17 & 6 & 2.4E-04 & 5.2E-05 & No & $\mathrm{D}$ & $\mathrm{D}$ & N/A & $N / A$ \\
\hline & GW-541 & $\mathrm{T}$ & 1 & 0 & $5.2 \mathrm{E}-05$ & $5.2 \mathrm{E}-05$ & Yes & N/A & $\mathrm{N} / \mathrm{A}$ & $\mathrm{N} / \mathrm{A}$ & $\mathrm{N} / \mathrm{A}$ \\
\hline & GW-540 & $\mathrm{T}$ & 16 & 2 & $1.8 \mathrm{E}-04$ & 5.2E-05 & No & NT & NT & N/A & $N / A$ \\
\hline & GW-543 & $\mathrm{T}$ & 18 & 0 & $5.2 \mathrm{E}-05$ & $5.2 \mathrm{E}-05$ & Yes & $\mathrm{s}$ & $\mathrm{s}$ & N/A & $N / A$ \\
\hline
\end{tabular}


Project: Y-12 National Security Complex

Location: West Chestnut Ridge
User Name: $\quad$ MV

State: Tennessee

\begin{tabular}{|c|c|c|c|c|c|c|c|c|c|c|c|}
\hline Constituent & Well & $\begin{array}{c}\text { Sourcel } \\
\text { Tail }\end{array}$ & $\begin{array}{c}\text { Number } \\
\text { of } \\
\text { Samples }\end{array}$ & $\begin{array}{c}\text { Number } \\
\text { of } \\
\text { Detects }\end{array}$ & $\begin{array}{c}\text { Average } \\
\text { (mg/L) }\end{array}$ & $\begin{array}{r}\text { Median } \\
(\mathrm{mg} / \mathrm{L})\end{array}$ & $\begin{array}{c}\text { All } \\
\text { Samples } \\
\text { "ND" ? }\end{array}$ & $\begin{array}{l}\text { Mann- } \\
\text { Kendall }\end{array}$ & $\begin{array}{c}\text { Linear } \\
\text { Regression }\end{array}$ & Modeling & Empirical \\
\hline \multicolumn{12}{|l|}{ LEAD } \\
\hline & GW-539 & $T$ & 10 & 3 & $2.1 \mathrm{E}-03$ & $5.2 \mathrm{E}-05$ & No & PD & $\mathrm{D}$ & N/A & N/A \\
\hline & SCR2_2SP & $\mathrm{T}$ & 14 & 2 & 4.7E-03 & 5.2E-05 & No & NT & $\mathrm{D}$ & N/A & $\mathrm{N} / \mathrm{A}$ \\
\hline & GW-544 & $\mathrm{T}$ & 18 & 1 & $6.5 \mathrm{E}-05$ & $5.2 \mathrm{E}-05$ & No & s & s & N/A & $\mathrm{N} / \mathrm{A}$ \\
\hline & GW-521 & $\mathrm{T}$ & 20 & 4 & 2.1E-04 & 5.2E-05 & No & PD & D & N/A & N/A \\
\hline & GW-221 & $\mathrm{T}$ & 17 & 1 & $1.2 \mathrm{E}-04$ & 5.2E-05 & No & NT & NT & N/A & N/A \\
\hline & GW-305 & $\mathrm{T}$ & 28 & 1 & 9.7E-05 & $5.2 \mathrm{E}-05$ & No & NT & $\mathrm{PD}$ & N/A & $\mathrm{N} / \mathrm{A}$ \\
\hline & GW-339 & $\mathrm{T}$ & 15 & 1 & $9.7 \mathrm{E}-05$ & 5.2E-05 & No & NT & NT & N/A & N/A \\
\hline & GW-709 & $\mathrm{T}$ & 18 & 1 & $9.4 \mathrm{E}-05$ & $5.2 \mathrm{E}-05$ & No & NT & PD & N/A & $\mathrm{N} / \mathrm{A}$ \\
\hline & GW-757 & $\mathrm{T}$ & 18 & 1 & $9.2 \mathrm{E}-05$ & $5.2 \mathrm{E}-05$ & No & NT & $\mathrm{PD}$ & N/A & $\mathrm{N} / \mathrm{A}$ \\
\hline & GW-522 & $\mathrm{T}$ & 18 & 5 & $4.8 \mathrm{E}-04$ & 5.2E-05 & No & D & D & N/A & $\mathrm{N} / \mathrm{A}$ \\
\hline & GW-302 & $\mathrm{T}$ & 15 & 2 & $1.8 \mathrm{E}-04$ & $5.2 \mathrm{E}-05$ & No & NT & D & N/A & $\mathrm{N} / \mathrm{A}$ \\
\hline \multicolumn{12}{|l|}{ NICKEL } \\
\hline & GW-205 & $\mathrm{s}$ & 1 & 0 & $1.0 \mathrm{E}-04$ & 1.0E-04 & Yes & $\mathrm{N} / \mathrm{A}$ & N/A & N/A & $\mathrm{N} / \mathrm{A}$ \\
\hline & GW-141 & s & 3 & 3 & 1.1E-01 & 5.3E-02 & No & $N / A$ & $N / A$ & N/A & $\mathrm{N} / \mathrm{A}$ \\
\hline & 1090 & s & 1 & 0 & $1.0 \mathrm{E}-04$ & 1.0E-04 & Yes & $N / A$ & N/A & N/A & N/A \\
\hline & GW-221 & $\mathrm{T}$ & 1 & 0 & $1.0 \mathrm{E}-04$ & 1.0E-04 & Yes & $\mathrm{N} / \mathrm{A}$ & N/A & N/A & $\mathrm{N} / \mathrm{A}$ \\
\hline & GW-522 & $\mathrm{T}$ & 1 & 1 & 1.2E-02 & $1.2 \mathrm{E}-02$ & No & $N / A$ & $\mathrm{~N} / \mathrm{A}$ & N/A & $\mathrm{N} / \mathrm{A}$ \\
\hline & GW-305 & $\mathrm{T}$ & 26 & 26 & $2.8 \mathrm{E}-01$ & 2.3E-01 & No & 1 & 1 & N/A & N/A \\
\hline & GW-339 & $\mathrm{T}$ & 15 & 15 & 2.3E-01 & 2.2E-01 & No & S & $\mathrm{PI}$ & N/A & N/A \\
\hline & GW-302 & $T$ & 14 & 14 & 2.0E-01 & 2.0E-01 & No & D & D & N/A & $\mathrm{N} / \mathrm{A}$ \\
\hline & SCR1_25SP & $\mathrm{T}$ & 1 & 0 & $1.0 \mathrm{E}-04$ & 1.0E-04 & Yes & $\mathrm{N} / \mathrm{A}$ & N/A & N/A & $\mathrm{N} / \mathrm{A}$ \\
\hline & GW-539 & $T$ & 10 & 10 & 1.6E-01 & 5.0E-02 & No & $\mathrm{D}$ & $\mathrm{D}$ & N/A & $\mathrm{N} / \mathrm{A}$ \\
\hline
\end{tabular}

Note: Increasing (I); Probably Increasing (PI); Stable (S); Probably Decreasing (PD); Decreasing (D); No Trend (NT); Not Applicable (N/A) - Due to insufficient Data (< 4 sampling events); Source/Tail (S/T)

The Number of Samples and Number of Detects shown above are post-consolidation values. 


\section{MAROS Spatial Moment Analysis Summary}

Project: Y-12 National Security Complex

Location: West Chestnut Ridge
User Name: MV

State: Tennessee

\begin{tabular}{|c|c|c|c|c|c|c|c|}
\hline \multirow[b]{2}{*}{ Effective Date } & \multirow{2}{*}{$\frac{\text { 0th Moment }}{\text { Estimated }}$} & \multicolumn{3}{|c|}{ 1st Moment (Center of Mass) } & \multicolumn{2}{|c|}{ 2nd Moment (Spread) } & \multirow[b]{2}{*}{$\begin{array}{c}\text { Number o } \\
\text { Wells }\end{array}$} \\
\hline & & $\mathrm{Xc}(\mathrm{ft})$ & Yc (ft) & $\begin{array}{c}\text { Source } \\
\text { Distance (ft) }\end{array}$ & $\underset{\text { (sq ft) }}{\text { Sigma } X X}$ & $\underset{\text { (sq ft) }}{\text { Sigma YY }}$ & \\
\hline
\end{tabular}

\section{1,1,1-TRICHLOROETHANE}

\begin{tabular}{|c|c|c|c|c|c|c|c|}
\hline 1/1/1996 & $0.0 \mathrm{E}+00$ & & & & & & 5 \\
\hline 4/1/1996 & 5.7E-03 & 52,889 & 27,208 & 1,608 & 348,635 & 549,281 & 17 \\
\hline $7 / 1 / 1996$ & $0.0 \mathrm{E}+00$ & & & & & & 5 \\
\hline 10/1/1996 & 5.7E-03 & 52,889 & 27,208 & 1,608 & 336,296 & 546,956 & 14 \\
\hline 1/1/1997 & $1.6 \mathrm{E}-02$ & 53,106 & 27,000 & 1,634 & 27,495 & 288,984 & 6 \\
\hline 4/1/1997 & $6.1 \mathrm{E}-03$ & 53,075 & 26,897 & 1,738 & 393,420 & 520,085 & 15 \\
\hline $7 / 1 / 1997$ & $1.5 \mathrm{E}-02$ & 53,098 & 27,243 & 1,443 & 54,148 & 723,946 & 8 \\
\hline 10/1/1997 & $1.6 \mathrm{E}-03$ & 52,296 & 26,384 & 2,617 & 122,010 & 210,178 & 6 \\
\hline 1/1/1998 & $2.6 \mathrm{E}-02$ & 53,040 & 27,150 & 1,552 & 37,063 & 499,099 & 9 \\
\hline 4/1/1998 & $1.9 \mathrm{E}-03$ & 52,324 & 26,468 & 2,535 & 79,961 & 244,970 & 7 \\
\hline 7/1/1998 & $1.0 \mathrm{E}-02$ & 53,087 & 27,095 & 1,568 & 66,904 & 916,671 & 8 \\
\hline 10/1/1998 & $3.2 \mathrm{E}-03$ & 52,119 & 26,534 & 2,630 & 111,622 & 187,398 & 7 \\
\hline 1/1/1999 & $1.6 \mathrm{E}-02$ & 53,099 & 27,257 & 1,431 & 52,894 & 702,864 & 8 \\
\hline 4/1/1999 & $1.2 \mathrm{E}-02$ & 52,471 & 26,303 & 2,570 & 16,070 & 150,437 & 7 \\
\hline 7/1/1999 & $1.6 \mathrm{E}-02$ & 53,099 & 27,261 & 1,428 & 52,530 & 696,675 & 8 \\
\hline 10/1/1999 & $1.9 \mathrm{E}-03$ & 52,324 & 26,468 & 2,535 & 79,961 & 244,970 & 7 \\
\hline $1 / 1 / 2000$ & 3.3E-02 & 53,022 & 26,940 & 1,731 & 117,334 & 581,551 & 16 \\
\hline $7 / 1 / 2000$ & 3.7E-02 & 53,027 & 26,950 & 1,720 & 112,733 & 562,251 & 16 \\
\hline $10 / 1 / 2000$ & $0.0 \mathrm{E}+00$ & & & & & & 1 \\
\hline $1 / 1 / 2001$ & $3.5 \mathrm{E}-02$ & 53,024 & 26,943 & 1,728 & 115,975 & 575,849 & 16 \\
\hline $4 / 1 / 2001$ & $0.0 \mathrm{E}+00$ & & & & & & 1 \\
\hline $7 / 1 / 2001$ & $3.4 \mathrm{E}-02$ & 53,023 & 26,941 & 1,730 & 116,632 & 578,608 & 16 \\
\hline $10 / 1 / 2001$ & $0.0 \mathrm{E}+00$ & & & & & & 1 \\
\hline $1 / 1 / 2002$ & $2.2 \mathrm{E}-02$ & 52,800 & 27,338 & 1,584 & 52,225 & 324,522 & 14 \\
\hline $4 / 1 / 2002$ & $0.0 \mathrm{E}+00$ & & & & & & 1 \\
\hline $7 / 1 / 2002$ & $2.1 \mathrm{E}-02$ & 52,796 & 27,333 & 1,590 & 54,494 & 329,839 & 14 \\
\hline $10 / 1 / 2002$ & $0.0 \mathrm{E}+00$ & & & & & & 1 \\
\hline $1 / 1 / 2003$ & 3.5E-02 & 53,024 & 26,943 & 1,728 & 113,889 & 576,206 & 15 \\
\hline $4 / 1 / 2003$ & $0.0 \mathrm{E}+00$ & & & & & & 1 \\
\hline $7 / 1 / 2003$ & $3.5 \mathrm{E}-02$ & 53,024 & 26,943 & 1,728 & 113,889 & 576,206 & 15 \\
\hline $10 / 1 / 2003$ & $0.0 \mathrm{E}+00$ & & & & & & 1 \\
\hline $1 / 1 / 2004$ & $3.2 \mathrm{E}-02$ & 53,020 & 26,936 & 1,735 & 116,665 & 588,473 & 15 \\
\hline $4 / 1 / 2004$ & $0.0 \mathrm{E}+00$ & & & & & & 1 \\
\hline $7 / 1 / 2004$ & $3.2 \mathrm{E}-02$ & 53,020 & 26,934 & 1,737 & 117,495 & 592,139 & 15 \\
\hline $10 / 1 / 2004$ & $0.0 \mathrm{E}+00$ & & & & & & 1 \\
\hline
\end{tabular}

GROSS ALPHA ACTIVITY

1/1/1996

4/1/1996

\section{$0.0 \mathrm{E}+00$}

$2.1 \mathrm{E}+03$
52,746

26,782
$2,023 \quad 262,900$ 
Project: Y-12 National Security Complex

Location: West Chestnut Ridge

\begin{tabular}{|c|c|c|c|c|c|c|c|}
\hline \multirow[b]{2}{*}{ Effective Date } & 0th Moment & \multicolumn{3}{|c|}{ 1st Moment (Center of Mass) } & \multicolumn{2}{|c|}{ 2nd Moment (Spread) } & \multirow[b]{2}{*}{$\begin{array}{l}\text { Number of } \\
\text { Wells }\end{array}$} \\
\hline & $\begin{array}{l}\text { Estimated } \\
\text { Mass (kg) }\end{array}$ & $X c(f t)$ & Yc (ft) & $\begin{array}{c}\text { Source } \\
\text { Distance (ft) }\end{array}$ & $\begin{array}{c}\text { Sigma } X X \\
(\mathrm{sq} \mathrm{ft})\end{array}$ & $\begin{array}{l}\text { Sigma } Y Y \\
\text { (sq ft) }\end{array}$ & \\
\hline
\end{tabular}

GROSS ALPHA ACTIVITY

\begin{tabular}{|c|c|c|c|c|c|c|c|}
\hline 10/1/1996 & $3.1 \mathrm{E}+03$ & 52,914 & 27,062 & 1,700 & 199,783 & 473,412 & 13 \\
\hline 1/1/1997 & $0.0 \mathrm{E}+00$ & & & & & & 4 \\
\hline 4/1/1997 & $1.1 \mathrm{E}+03$ & 52,907 & 26,657 & 2,030 & 275,104 & 588,312 & 11 \\
\hline 7/1/1997 & $7.6 \mathrm{E}+02$ & 53,130 & 26,634 & 1,939 & 39,803 & 841,888 & 7 \\
\hline 10/1/1997 & $1.2 \mathrm{E}+03$ & 52,795 & 26,793 & 1,985 & 286,249 & 353,513 & 12 \\
\hline 1/1/1998 & $7.8 \mathrm{E}+02$ & 53,082 & 26,718 & 1,888 & 87,030 & $1,006,974$ & 7 \\
\hline 4/1/1998 & $0.0 \mathrm{E}+00$ & & & & & & 5 \\
\hline 7/1/1998 & $1.1 \mathrm{E}+03$ & 53,528 & 26,883 & 1,556 & 48,143 & 785,185 & 12 \\
\hline 10/1/1998 & $0.0 \mathrm{E}+00$ & & & & & & 4 \\
\hline 1/1/1999 & $1.3 \mathrm{E}+03$ & 53,165 & 26,779 & 1,795 & 151,515 & $1,254,414$ & 14 \\
\hline 4/1/1999 & $0.0 \mathrm{E}+00$ & & & & & & 5 \\
\hline 7/1/1999 & $3.6 \mathrm{E}+02$ & 53,244 & 26,943 & 1,613 & 128,777 & 419,895 & 11 \\
\hline 10/1/1999 & $0.0 \mathrm{E}+00$ & & & & & & 5 \\
\hline $1 / 1 / 2000$ & $3.1 \mathrm{E}+02$ & 53,733 & 26,298 & 2,083 & 148,154 & 582,776 & 22 \\
\hline $7 / 1 / 2000$ & $5.0 \mathrm{E}+02$ & 53,142 & 25,468 & 3,021 & 395,168 & $1,027,840$ & 22 \\
\hline $10 / 1 / 2000$ & $0.0 \mathrm{E}+00$ & & & & & & 1 \\
\hline $1 / 1 / 2001$ & 4. $4 \mathrm{E}+02$ & 53,650 & 26,863 & 1,542 & 160,130 & 837,462 & 21 \\
\hline $4 / 1 / 2001$ & $0.0 \mathrm{E}+00$ & & & & & & 1 \\
\hline 7/1/2001 & 2.7E+02 & 53,508 & 24,871 & 3,527 & 63,037 & 462,299 & 22 \\
\hline $10 / 1 / 2001$ & $0.0 \mathrm{E}+00$ & & & & & & 1 \\
\hline $1 / 1 / 2002$ & $2.6 \mathrm{E}+02$ & 53,666 & 27,352 & 1,067 & 191,840 & 277,866 & 20 \\
\hline $4 / 1 / 2002$ & $0.0 \mathrm{E}+00$ & & & & & & 1 \\
\hline $7 / 1 / 2002$ & $4.2 \mathrm{E}+02$ & 53,580 & 27,263 & 1,180 & 171,107 & 246,993 & 20 \\
\hline $10 / 1 / 2002$ & $0.0 \mathrm{E}+00$ & & & & & & 1 \\
\hline $1 / 1 / 2003$ & $1.8 \mathrm{E}+03$ & 53,646 & 26,225 & 2,168 & 79,277 & $1,219,495$ & 21 \\
\hline 4/1/2003 & $0.0 \mathrm{E}+00$ & & & & & & 1 \\
\hline $7 / 1 / 2003$ & $5.3 \mathrm{E}+02$ & 53,493 & 25,026 & 3,377 & 71,305 & 592,234 & 21 \\
\hline $10 / 1 / 2003$ & $0.0 \mathrm{E}+00$ & & & & & & 1 \\
\hline $1 / 1 / 2004$ & $8.1 \mathrm{E}+02$ & 53,641 & 26,183 & 2,210 & 85,318 & 973,294 & 19 \\
\hline $4 / 1 / 2004$ & $0.0 \mathrm{E}+00$ & & & & & & 1 \\
\hline $7 / 1 / 2004$ & $2.9 \mathrm{E}+02$ & 53,597 & 26,935 & 1,485 & 536,079 & 633,939 & 18 \\
\hline $10 / 1 / 2004$ & $0.0 \mathrm{E}+00$ & & & & & & 1 \\
\hline
\end{tabular}

GROSS BETA ACTIVITY

$\begin{array}{rrrrrrrr}1 / 1 / 1996 & 0.0 \mathrm{E}+00 & & & & & 3 \\ 4 / 1 / 1996 & 1.0 \mathrm{E}+03 & 53,049 & 27,269 & 1,454 & 271,678 & 511,865 & 9 \\ 7 / 1 / 1996 & 0.0 \mathrm{E}+00 & & & & & & 2 \\ 10 / 1 / 1996 & 2.4 \mathrm{E}+03 & 52,938 & 27,078 & 1,672 & 223,999 & 189,183 & 9 \\ 1 / 1 / 1997 & 0.0 \mathrm{E}+00 & & & & & 4 \\ 4 / 1 / 1997 & 3.8 \mathrm{E}+03 & 52,897 & 26,947 & 1,800 & 135,014 & 358,195 & 8 \\ 7 / 1 / 1997 & 0.0 \mathrm{E}+00 & & & & & & 4 \\ 10 / 1 / 1997 & 4.9 \mathrm{E}+03 & 52,982 & 27,079 & 1,643 & 244,873 & 382,858 & 12 \\ 1 / 1 / 1998 & 0.0 \mathrm{E}+00 & & & & & & 5\end{array}$


Project: Y-12 National Security Complex

Location: West Chestnut Ridge
User Name: MV

State: Tennessee

\begin{tabular}{|c|c|c|c|c|c|c|c|}
\hline \multirow[b]{2}{*}{ Effective Date } & \multirow{2}{*}{$\begin{array}{l}\text { Esth Moment } \\
\text { Mass (kg) }\end{array}$} & \multicolumn{3}{|c|}{ 1st Moment (Center of Mass) } & \multicolumn{2}{|c|}{ 2nd Moment (Spread) } & \multirow[b]{2}{*}{$\begin{array}{c}\text { Number of } \\
\text { Wells }\end{array}$} \\
\hline & & $\mathrm{Xc}(\mathrm{ft})$ & $Y c(f t)$ & $\begin{array}{c}\text { Source } \\
\text { Distance (ft) }\end{array}$ & $\begin{array}{l}\text { Sigma } X X \\
(s q \mathrm{ft})\end{array}$ & $\begin{array}{l}\text { Sigma YY } \\
\text { (sq ft) }\end{array}$ & \\
\hline \multicolumn{8}{|l|}{ GROSS BETA ACTIVITY } \\
\hline $4 / 1 / 1998$ & $0.0 \mathrm{E}+00$ & & & & & & 4 \\
\hline 7/1/1998 & $4.6 \mathrm{E}+03$ & 53,395 & 27,063 & 1,437 & 104,294 & 574,170 & 13 \\
\hline 10/1/1998 & $8.0 \mathrm{E}+02$ & 52,362 & 26,677 & 2,356 & 50,778 & 60,475 & 6 \\
\hline $1 / 1 / 1999$ & $1.1 \mathrm{E}+02$ & 53,011 & 28,235 & 1,005 & 128,584 & 722,619 & 12 \\
\hline 4/1/1999 & $9.0 \mathrm{E}+02$ & 52,328 & 26,722 & 2,348 & 64,308 & 84,715 & 6 \\
\hline 7/1/1999 & $4.9 \mathrm{E}+03$ & 53,299 & 26,977 & 1,557 & 120,493 & 716,776 & 13 \\
\hline 10/1/1999 & $1.9 \mathrm{E}+03$ & 52,397 & 26,373 & 2,560 & 53,075 & 220,139 & 7 \\
\hline $1 / 1 / 2000$ & $4.6 \mathrm{E}+02$ & 53,310 & 26,777 & 1,733 & 65,993 & 425,128 & 21 \\
\hline $7 / 1 / 2000$ & 4.7E+02 & 53,362 & 27,277 & 1,263 & 103,626 & 388,248 & 20 \\
\hline $10 / 1 / 2000$ & $0.0 \mathrm{E}+00$ & & & & & & 1 \\
\hline $1 / 1 / 2001$ & $2.2 \mathrm{E}+03$ & 53,723 & 27,084 & 1,310 & 131,824 & 522,816 & 21 \\
\hline 4/1/2001 & $0.0 \mathrm{E}+00$ & & & & & & 1 \\
\hline $7 / 1 / 2001$ & $1.2 \mathrm{E}+03$ & 53,495 & 26,513 & 1,920 & 99,897 & $1,309,001$ & 21 \\
\hline $10 / 1 / 2001$ & $0.0 \mathrm{E}+00$ & & & & & & 1 \\
\hline $1 / 1 / 2002$ & $1.5 \mathrm{E}+03$ & 53,510 & 27,362 & 1,119 & 373,526 & 340,271 & 20 \\
\hline $4 / 1 / 2002$ & $0.0 \mathrm{E}+00$ & & & & & & 1 \\
\hline $7 / 1 / 2002$ & 1.7E+03 & 53,730 & 27,389 & 1,013 & 106,354 & 160,483 & 20 \\
\hline $10 / 1 / 2002$ & $0.0 \mathrm{E}+00$ & & & & & & 1 \\
\hline $1 / 1 / 2003$ & $2.6 \mathrm{E}+03$ & 53,646 & 26,578 & 1,821 & 120,440 & $1,010,469$ & 21 \\
\hline $4 / 1 / 2003$ & $0.0 \mathrm{E}+00$ & & & & & & 1 \\
\hline $7 / 1 / 2003$ & $2.1 \mathrm{E}+03$ & 53,380 & 26,529 & 1,938 & 314,091 & 789,566 & 21 \\
\hline $10 / 1 / 2003$ & $0.0 \mathrm{E}+00$ & & & & & & 1 \\
\hline $1 / 1 / 2004$ & $6.9 \mathrm{E}+03$ & 53,361 & 27,042 & 1,471 & 88,515 & 252,314 & 18 \\
\hline $4 / 1 / 2004$ & $0.0 \mathrm{E}+00$ & & & & & & 1 \\
\hline $7 / 1 / 2004$ & $2.7 \mathrm{E}+03$ & 53,378 & 26,154 & 2,297 & 339,335 & 935,191 & 19 \\
\hline $10 / 1 / 2004$ & $0.0 \mathrm{E}+00$ & & & & & & 1 \\
\hline
\end{tabular}

LEAD

\begin{tabular}{|c|c|c|c|c|c|c|c|}
\hline 1/1/1996 & $0.0 \mathrm{E}+00$ & & & & & & 5 \\
\hline 4/1/1996 & 4.9E-02 & 52,889 & 27,208 & 1,608 & 348,635 & 549,281 & 17 \\
\hline 7/1/1996 & $0.0 \mathrm{E}+00$ & & & & & & 5 \\
\hline 10/1/1996 & $1.5 \mathrm{E}+00$ & 52,872 & 27,139 & 1,670 & 147,730 & 565,884 & 14 \\
\hline 1/1/1997 & 4.5E-01 & 52,934 & 27,210 & 1,576 & 59,586 & 554,428 & 6 \\
\hline $4 / 1 / 1997$ & 3.5E-01 & 53,255 & 27,321 & 1,286 & 323,369 & 456,289 & 14 \\
\hline $7 / 1 / 1997$ & 1.6E-01 & 53,190 & 26,107 & 2,399 & 33,592 & 639,990 & 7 \\
\hline 10/1/1997 & 6.9E-02 & 52,923 & 27,042 & 1,710 & 276,021 & 437,941 & 12 \\
\hline 1/1/1998 & 1.4E-01 & 53,043 & 26,765 & 1,867 & 130,923 & $1,182,476$ & 8 \\
\hline 4/1/1998 & 2.0E-02 & 52,247 & 26,619 & 2,479 & 97,728 & 314,435 & 7 \\
\hline 7/1/1998 & 4.8E-02 & 53,417 & 27,007 & 1,479 & 160,442 & 904,206 & 13 \\
\hline 10/1/1998 & 1.9E-02 & 52,265 & 26,582 & 2,491 & 94,549 & 301,850 & 7 \\
\hline 1/1/1999 & 1.4E-01 & 53,204 & 26,914 & 1,657 & 217,072 & 951,448 & 14 \\
\hline 4/1/1999 & 4.1E-02 & 52,282 & 26,757 & 2,357 & 81,383 & 241,202 & 7 \\
\hline 7/1/1999 & 5.7E-02 & 53,330 & 26,749 & 1,751 & 221,809 & $1,184,371$ & 14 \\
\hline
\end{tabular}


Project: Y-12 National Security Complex

Location: West Chestnut Ridge

\begin{tabular}{|c|c|c|c|c|c|c|c|}
\hline \multirow[b]{2}{*}{ Effective Date } & \multirow{2}{*}{$\begin{array}{l}\text { 0th Moment } \\
\begin{array}{r}\text { Estimated } \\
\text { Mass (kg) }\end{array}\end{array}$} & \multicolumn{3}{|c|}{ 1st Moment (Center of Mass) } & \multicolumn{2}{|c|}{$\underline{\text { 2nd Moment (Spread) }}$} & \multirow[b]{2}{*}{$\begin{array}{c}\text { Number o } \\
\text { Wells }\end{array}$} \\
\hline & & $\mathrm{Xc}(\mathrm{ft})$ & $Y c(f t)$ & $\begin{array}{c}\text { Source } \\
\text { Distance (ft) }\end{array}$ & $\begin{array}{c}\text { Sigma } X X \\
(s q \mathrm{ft})\end{array}$ & $\underset{(s q \mathrm{ft})}{\operatorname{Sigma} Y Y}$ & \\
\hline \multicolumn{8}{|l|}{ LEAD } \\
\hline 10/1/1999 & $2.9 \mathrm{E}-02$ & 52,453 & 26,851 & 2,168 & 129,804 & 364,966 & 8 \\
\hline $1 / 1 / 2000$ & $8.8 \mathrm{E}-02$ & 53,226 & 26,515 & 2,007 & 401,198 & $1,355,159$ & 22 \\
\hline $7 / 1 / 2000$ & $9.1 \mathrm{E}-02$ & 53,235 & 26,449 & 2,064 & 388,759 & $1,380,651$ & 21 \\
\hline $10 / 1 / 2000$ & $0.0 \mathrm{E}+00$ & & & & & & 1 \\
\hline $1 / 1 / 2001$ & 7.1E-02 & 53,104 & 26,714 & 1,881 & 403,426 & $1,270,495$ & 22 \\
\hline $4 / 1 / 2001$ & $0.0 \mathrm{E}+00$ & & & & & & 1 \\
\hline $7 / 1 / 2001$ & 7.1E-02 & 53,104 & 26,714 & 1,881 & 403,426 & $1,270,495$ & 22 \\
\hline $10 / 1 / 2001$ & $0.0 \mathrm{E}+00$ & & & & & & 1 \\
\hline $1 / 1 / 2002$ & 5.1E-02 & 52,886 & 27,219 & 1,602 & 354,277 & 635,920 & 20 \\
\hline $4 / 1 / 2002$ & $0.0 \mathrm{E}+00$ & & & & & & 1 \\
\hline $7 / 1 / 2002$ & 5.1E-02 & 52,886 & 27,219 & 1,602 & 354,277 & 635,920 & 20 \\
\hline $10 / 1 / 2002$ & $0.0 \mathrm{E}+00$ & & & & & & 1 \\
\hline $1 / 1 / 2003$ & 7.1E-02 & 53,104 & 26,714 & 1,881 & 400,075 & $1,266,147$ & 21 \\
\hline 4/1/2003 & $0.0 \mathrm{E}+00$ & & & & & & 1 \\
\hline $7 / 1 / 2003$ & 7.1E-02 & 53,104 & 26,714 & 1,881 & 400,075 & $1,266,147$ & 21 \\
\hline $10 / 1 / 2003$ & $0.0 \mathrm{E}+00$ & & & & & & 1 \\
\hline $1 / 1 / 2004$ & 7.1E-02 & 53,095 & 26,697 & 1,900 & 392,921 & $1,246,287$ & 19 \\
\hline $4 / 1 / 2004$ & $0.0 \mathrm{E}+00$ & & & & & & 1 \\
\hline $7 / 1 / 2004$ & 7.1E-02 & 53,095 & 26,697 & 1,900 & 392,921 & $1,246,287$ & 19 \\
\hline $10 / 1 / 2004$ & $0.0 \mathrm{E}+00$ & & & & & & 1 \\
\hline
\end{tabular}

NICKEL
User Name: MV

State: Tennessee

\begin{tabular}{|c|c|c|c|c|c|c|c|}
\hline 1/1/1996 & $0.0 \mathrm{E}+00$ & & & & & & 2 \\
\hline 4/1/1996 & $0.0 \mathrm{E}+00$ & & & & & & 3 \\
\hline 7/1/1996 & $0.0 \mathrm{E}+00$ & & & & & & 1 \\
\hline 10/1/1996 & $0.0 \mathrm{E}+00$ & & & & & & 3 \\
\hline 1/1/1997 & $0.0 \mathrm{E}+00$ & & & & & & 2 \\
\hline 4/1/1997 & $0.0 \mathrm{E}+00$ & & & & & & 3 \\
\hline $7 / 1 / 1997$ & $0.0 \mathrm{E}+00$ & & & & & & 1 \\
\hline $10 / 1 / 1997$ & $0.0 \mathrm{E}+00$ & & & & & & 3 \\
\hline 1/1/1998 & $0.0 \mathrm{E}+00$ & & & & & & 1 \\
\hline 7/1/1998 & $0.0 \mathrm{E}+00$ & & & & & & 3 \\
\hline 1/1/1999 & $0.0 \mathrm{E}+00$ & & & & & & 3 \\
\hline 7/1/1999 & $0.0 \mathrm{E}+00$ & & & & & & 3 \\
\hline 10/1/1999 & $0.0 \mathrm{E}+00$ & & & & & & 1 \\
\hline $1 / 1 / 2000$ & $0.0 \mathrm{E}+00$ & & & & & & 3 \\
\hline $7 / 1 / 2000$ & $1.3 \mathrm{E}+00$ & 53,190 & 27,900 & 940 & 119,940 & 233,991 & 8 \\
\hline $10 / 1 / 2000$ & $0.0 \mathrm{E}+00$ & & & & & & 1 \\
\hline $1 / 1 / 2001$ & $0.0 \mathrm{E}+00$ & & & & & & 4 \\
\hline $4 / 1 / 2001$ & $0.0 \mathrm{E}+00$ & & & & & & 1 \\
\hline $7 / 1 / 2001$ & $0.0 \mathrm{E}+00$ & & & & & & 4 \\
\hline 10/1/2001 & $0.0 \mathrm{E}+00$ & & & & & & 1 \\
\hline $1 / 1 / 2002$ & $0.0 \mathrm{E}+00$ & & & & & & 4 \\
\hline
\end{tabular}


Project: Y-12 National Security Complex

Location: West Chestnut Ridge

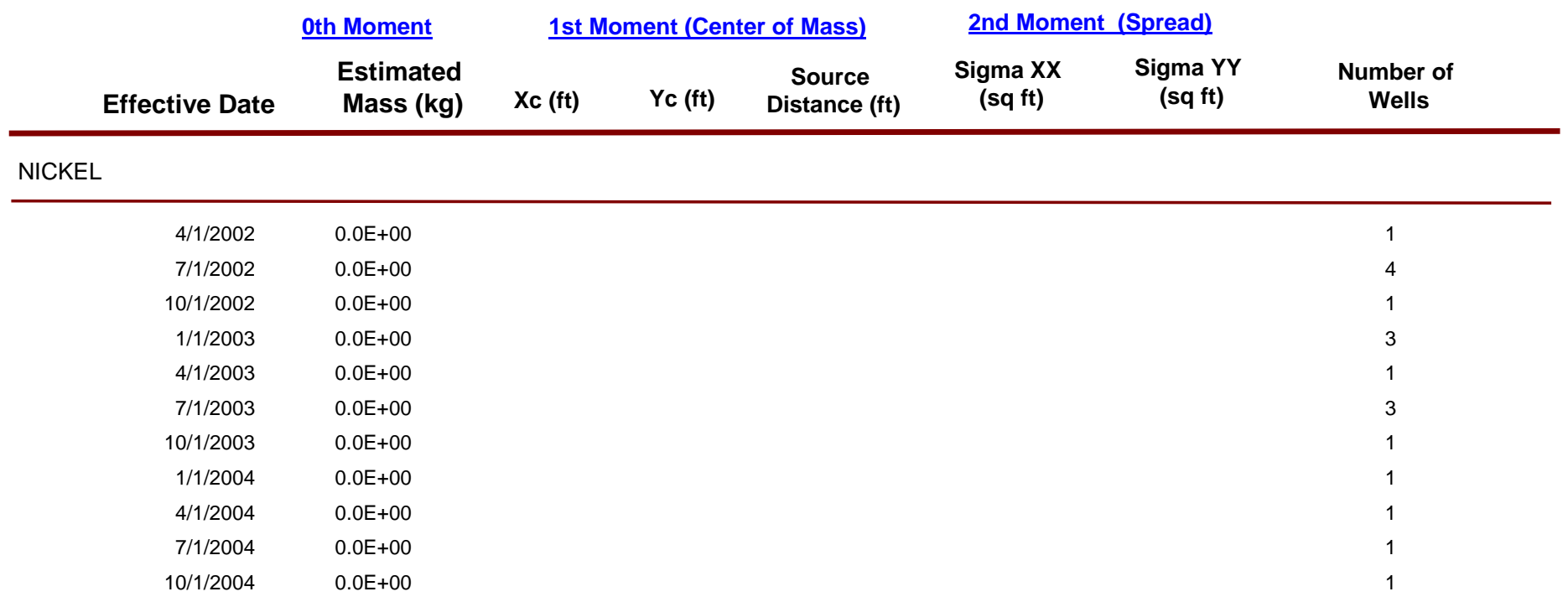


Project: Y-12 National Security Complex

Location: West Chestnut Ridge
User Name: $\mathrm{MV}$

State: Tennessee

\begin{tabular}{|c|c|c|c|c|c|}
\hline Moment Type & Constituent & $\begin{array}{l}\text { Coefficient } \\
\text { of Variation }\end{array}$ & $\begin{array}{l}\text { Mann-Kendall } \\
\text { S Statistic }\end{array}$ & $\begin{array}{l}\text { Confidence } \\
\text { in Trend }\end{array}$ & $\begin{array}{c}\text { Moment } \\
\text { Trend }\end{array}$ \\
\hline \multicolumn{6}{|c|}{ Zeroth Moment: Mass } \\
\hline & 1,1,1-TRICHLOROETHANE & 1.06 & 34 & $67.9 \%$ & NT \\
\hline & GROSS ALPHA ACTIVITY & 1.41 & -116 & $95.6 \%$ & $\mathrm{D}$ \\
\hline & GROSS BETA ACTIVITY & 1.33 & 10 & $55.1 \%$ & NT \\
\hline & LEAD & 2.38 & -154 & $98.6 \%$ & $\mathrm{D}$ \\
\hline & NICKEL & 5.66 & -3 & $51.3 \%$ & NT \\
\hline \multicolumn{6}{|c|}{ 1st Moment: Distance to Source } \\
\hline & 1,1,1-TRICHLOROETHANE & 0.22 & 22 & $69.7 \%$ & NT \\
\hline & GROSS ALPHA ACTIVITY & 0.33 & -1 & $50.0 \%$ & $\mathrm{~S}$ \\
\hline & GROSS BETA ACTIVITY & 0.27 & 4 & $53.8 \%$ & NT \\
\hline & LEAD & 0.17 & 42 & $84.4 \%$ & NT \\
\hline & NICKEL & 0.00 & 0 & $0.0 \%$ & $\mathrm{~N} / \mathrm{A}$ \\
\hline \multicolumn{6}{|c|}{ 2nd Moment: Sigma XX } \\
\hline & 1,1,1-TRICHLOROETHANE & 0.85 & 18 & $66.2 \%$ & NT \\
\hline & GROSS ALPHA ACTIVITY & 0.72 & -9 & $60.9 \%$ & $\mathrm{~S}$ \\
\hline & GROSS BETA ACTIVITY & 0.64 & 0 & $48.7 \%$ & $\mathrm{~S}$ \\
\hline & LEAD & 0.52 & 118 & $99.9 \%$ & 1 \\
\hline & NICKEL & 0.00 & 0 & $0.0 \%$ & $\mathrm{~N} / \mathrm{A}$ \\
\hline \multicolumn{6}{|c|}{ 2nd Moment: Sigma YY } \\
\hline & 1,1,1-TRICHLOROETHANE & 0.40 & 42 & $84.4 \%$ & NT \\
\hline & GROSS ALPHA ACTIVITY & 0.45 & 15 & $68.6 \%$ & NT \\
\hline & GROSS BETA ACTIVITY & 0.67 & 42 & $90.7 \%$ & $\mathrm{PI}$ \\
\hline & LEAD & 0.48 & 90 & $98.7 \%$ & 1 \\
\hline & NICKEL & 0.00 & 0 & $0.0 \%$ & $\mathrm{~N} / \mathrm{A}$ \\
\hline
\end{tabular}

Note: The following assumptions were applied for the calculation of the Zeroth Moment:

Porosity: $0.10 \quad$ Saturated Thickness: Uniform: $50 \mathrm{ft}$

Mann-Kendall Trend test performed on all sample events for each constituent. Increasing (I); Probably Increasing (PI); Stable (S); Probably Decreasing (PD); Decreasing (D); No Trend (NT); Not Applicable (N/A)-Due to insufficient Data (< 4 sampling events).

Note: The Sigma $X X$ and Sigma $Y Y$ components are estimated using the given field coordinate system and then rotated to align with the estimated groundwater flow direction. Moments are not calculated for sample events with less than 6 wells. 


\section{ASSESSMENT OF THE \\ GROUNDWATER PROTECTION PROGRAM \\ Y-12 NATIONAL SECURITY COMPLEX, OAK RIDGE, TENNESSEE}

Y-12 National Security Complex

Oak Ridge, Tennessee

\section{Appendix D.9 Chestnut Ridge Regime Security Pits}

Table D.9.1 Qualitative Analysis Chestnut Ridge Security Pits

Table D.9.2 $\quad$ Aquifer Input Parameters

MAROS Report $\quad$ COC Assessment

MAROS Report Plume Summary

MAROS Report Spatial Moment Analysis Summary

MAROS Chart New Location Analysis 11DCE Chestnut Ridge Security Pits

(No Well Sufficiency areas of interest identified) 
TABLE D.9.1

QUALITATIVE ANALYSIS CHESTNUT RIDGE REGIME SECURITY PITS

Chestnut Ridge Hydrogeologic Regime

Y-12 National Security Complex

Oak Ridge, Tennessee

\begin{tabular}{|c|c|c|c|c|c|c|c|c|c|c|c|c|}
\hline $\begin{array}{c}\text { Location } \\
\text { Name }\end{array}$ & $\begin{array}{c}\text { Location } \\
\text { Type } \\
\end{array}$ & $\begin{array}{c}\text { Average Concentration } \\
\text { Exceeds Screening } \\
\text { Level }\end{array}$ & $\begin{array}{c}\text { Formation } \\
\text { Type }\end{array}$ & $\begin{array}{c}\text { Horizontal } \\
\text { Delineation }\end{array}$ & $\begin{array}{c}\text { Vertical } \\
\text { Delineation }\end{array}$ & $\begin{array}{c}\text { Exit } \\
\text { Location }\end{array}$ & RCRA & CERCLA & Unique & $\begin{array}{c}\text { Monitors } \\
\text { Background } \\
\text { Water Quality }\end{array}$ & $\begin{array}{c}\text { Early } \\
\text { Detection }\end{array}$ & $\begin{array}{c}\text { Monitor } \\
\text { Source }\end{array}$ \\
\hline GW-173 & WL & $x$ & Aquifer & & $x$ & & & & & & & \\
\hline GW-174 & WL & $x$ & Aquifer & & $x$ & & & & & & & \\
\hline GW-175 & WL & $x$ & Aquifer & $x$ & $x$ & & $x$ & & & & & \\
\hline GW-176 & WL & & Aquifer & $x$ & $x$ & & & & & & & \\
\hline GW-177 & WL & & Aquifer & $x$ & & & $x$ & & & & & \\
\hline GW-178 & WL & & Aquifer & & & & & & & & & \\
\hline GW-179 & WL & & Aquifer & $x$ & & & & & & & & \\
\hline GW-180 & WL & $x$ & Aquifer & $x$ & & & & & & & & \\
\hline GW-181 & WL & & Aquifer & & & & & & $x$ & & & \\
\hline GW-184 & WL & & Aquitard & & & & & & & & & \\
\hline \begin{tabular}{|l|} 
GW-186 \\
\end{tabular} & WL & & Aquitard & & & & & & & & & \\
\hline GW-188 & WL & & Aquitard & & & & & & & & & \\
\hline GW-322 & WL & $x$ & Aquifer & & & & & & & & & $x$ \\
\hline GW-511 & WL & & Aquifer & & & & & & & & & \\
\hline GW-512 & WL & & Aquifer & & & & & & & & & \\
\hline GW-513 & WL & & Aquifer & & & & & & $x$ & & & \\
\hline GW-514 & WL & & Aquifer & & & & $x$ & & & & & \\
\hline GW-608 & WL & & Aquifer & $x$ & & & $\hat{x}$ & & & & & \\
\hline GW-609 & WL & $x$ & Aquifer & $x$ & $x$ & & $x$ & & & & & \\
\hline GW-610 & WL & & Aquifer & $x$ & $x$ & & & & & & & \\
\hline \begin{tabular}{|l|} 
GW-611 \\
\end{tabular} & WL & & Aquifer & & & & & & & & & \\
\hline GW-612 & WL & & Aquifer & & $x$ & & & & & & & \\
\hline GW-679 & WL & & Aquifer & & & & & & & & & \\
\hline GW-680 & WL & & Aquifer & & & & & & & & & \\
\hline GW-742 & WL & & Aquifer & & $x$ & & & & $x$ & & & \\
\hline \begin{tabular}{|l} 
GW-743 \\
\end{tabular} & WL & & Aquifer & & $x$ & & & & & & & \\
\hline GW-831 & WL & & Aquifer & & & & $x$ & & & & & \\
\hline SCR3.4SP & $\mathrm{SP}$ & & Spring & & & $x$ & & & & & & \\
\hline SCR3.5SP & $\mathrm{SP}$ & & Spring & & & $x$ & & & & & & \\
\hline
\end{tabular}

\section{Notes:}

1. $W L=$ Monitoring Well; $S P=$ Spring

2. Well data taken from BWXT Y-12 Analytical Database. Sample locations shown on Figures A.1 and A.4.

3. RCRA indicates wells monitored as part of compliance with RCRA Post-Closure Corrective Action Monitoring or designated Alternate location;

4. Average Concentration Exceeds Screening $=$ The average concentration over the entire sampling record for the priority constituent is above the MCL or other designated screening level

as defined in Table B.1.

tions identified in Fig. A.2 from BWXT Y12, 2003 Groundwater Monitoring Report, (12/01/2003).

6. Details of the decision criteria for each category are presented in the text. 
GSI Job No. G-3038

Issued $12 / 12 / 2005$

Page 1 of 1

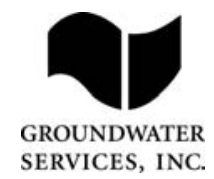

TABLE D.9.2

AQUIFER INPUT PARAMETERS

\author{
Security Pits \\ Chestnut Ridge Regime \\ Y-12 National Security Complex
}

\begin{tabular}{|c|c|c|}
\hline Parameter & Value & Units \\
\hline Current Plume Length & 5500 & $\mathrm{ft}$ \\
\hline Maximum Plume Length & 5500 & $\mathrm{ft}$ \\
\hline PlumeWidth & 2000 & $\mathrm{ft}$ \\
\hline SeepageVelocity (ft/yr) & 200 & $\mathrm{ft} / \mathrm{yr}$ \\
\hline Distance to Receptors & 6000 & $\mathrm{ft}$ \\
\hline GWFluctuations & Yes & -- \\
\hline SourceTreatment & None & -- \\
\hline PlumeType & voc/Metals & -- \\
\hline Free NAPL Present & Yes & -- \\
\hline Parameter & Value & \\
\hline Groundwater flow directio & $\mathrm{S}$ & 270 \\
\hline Effective Porosity & 0.1 & -- \\
\hline Source Location near We & GW-322 & -- \\
\hline Source X-Coordinate & 58912.05 & $\mathrm{ft}^{\star}$ \\
\hline Source Y-Coordinate & 28240.69 & $\mathrm{ft}^{\star}$ \\
\hline Saturated Thickness & 50 & $\mathrm{ft}$ \\
\hline \multicolumn{3}{|l|}{ Source Wells } \\
\hline GW-322, & SW-173, & 80 \\
\hline
\end{tabular}

\title{
Notes:
}

1. Aquifer data are general values for the hydrologic regime.

2. Priority COCs defined by prevalence, toxicty and mobility.

3. $\mathrm{ft}^{\star}=$ Coordinates in $\mathrm{Y}-12$ Plant coordinates, feet.

4. Screening Levels are USEPA MCLs, except in the case of compounds without MCLs where the level is the Region 9 PRG for tap water.

5. Effective Porosity estimated based on average high and low values for aquifer and aquitard suburfaces. 


\section{MAROS COC Assessment}

Project: $\quad$ Y-12 National Security Complex

Location: CRSP

Toxicity:

\begin{tabular}{lccc}
\hline Toxicity: & $\begin{array}{c}\text { Representative } \\
\text { Concentration } \\
\text { (mg/L) }\end{array}$ & $\begin{array}{c}\text { PRG } \\
\text { (mg/L) }\end{array}$ & $\begin{array}{c}\text { Percent } \\
\text { Above } \\
\text { PRG }\end{array}$ \\
\hline TETRACHLOROETHYLENE(PCE) & $8.9 \mathrm{E}-03$ & $5.0 \mathrm{E}-03$ & $78.6 \%$ \\
1,1-DICHLOROETHENE & $9.7 \mathrm{E}-03$ & $7.0 \mathrm{E}-03$ & $38.2 \%$
\end{tabular}

Note: Top COCs by toxicity were determined by examining a representative concentration for each compound over the entire site. The compound representative concentrations are then compared with the chosen PRG for that compound, with the percentage excedence from the PRG determining the compound's toxicity. All compounds above exceed the PRG.

\section{Prevalence:}

Contaminant of Concern

Total

Class

Wells

ORG

ORG

29

29

Exce

Total xcedences

\section{Percent}

Total

TETRACHLOROETHYLENE(PCE)

1,1-DICHLOROETHENE

Note: Top COCs by prevalence were determined by examining a representative concentration for each well location at the site. The total excedences (values above the chosen PRGs) are compared to the total number of wells to determine the prevalence of the compound.

\section{Mobility:}

Contaminant of Concern

Kd

\begin{tabular}{lr}
\hline 1,1-DICHLOROETHENE & 0.13 \\
TETRACHLOROETHYLENE(PCE) & 0.923
\end{tabular}

Note: Top COCs by mobility were determined by examining each detected compound in the dataset and comparing their mobilities (Koc's for organics, assume foc $=0.001$, and Kd's for metals).

Contaminants of Concern (COC's)

LEAD

TETRACHLOROETHYLENE(PCE)

1,1-DICHLOROETHENE

GROSS ALPHA ACTIVITY

TRICHLOROETHYLENE (TCE) 


\section{MAROS Plume Analysis Summary}

Project: $\quad \mathrm{Y}-12$ National Security Complex

Location: CRSP

Time Period: 1/1/1996

to $1 / 1 / 2005$

Consolidation Period: No Time Consolidation

Consolidation Type: Median

Duplicate Consolidation: Average

ND Values: Specified Detection Limit

J Flag Values : Actual Value

\begin{tabular}{|c|c|c|c|c|c|c|c|c|c|c|c|}
\hline Constituent & Well & $\begin{array}{c}\text { Sourcel } \\
\text { Tail }\end{array}$ & $\begin{array}{c}\text { Number } \\
\text { of } \\
\text { Samples }\end{array}$ & $\begin{array}{c}\text { Number } \\
\text { of } \\
\text { Detects }\end{array}$ & $\begin{array}{c}\text { Average } \\
\text { (mg/L) }\end{array}$ & $\begin{array}{r}\text { Median } \\
(\mathrm{mg} / \mathrm{L})\end{array}$ & $\begin{array}{c}\text { All } \\
\text { Samples } \\
\text { "ND" ? }\end{array}$ & $\begin{array}{l}\text { Mann- } \\
\text { Kendall }\end{array}$ & $\begin{array}{c}\text { Linear } \\
\text { Regression }\end{array}$ & Modeling & Empirical \\
\hline \multicolumn{12}{|c|}{ 1,1-DICHLOROETHENE } \\
\hline & GW-173 & $\mathrm{S}$ & 2 & 0 & $5.0 \mathrm{E}-04$ & $5.0 \mathrm{E}-04$ & Yes & N/A & $\mathrm{N} / \mathrm{A}$ & N/A & $\mathrm{N} / \mathrm{A}$ \\
\hline & GW-322 & S & 3 & 3 & $1.1 \mathrm{E}-01$ & 9.3E-02 & No & N/A & $\mathrm{N} / \mathrm{A}$ & N/A & N/A \\
\hline & GW-174 & s & 3 & 0 & $5.0 \mathrm{E}-04$ & 5.0E-04 & Yes & N/A & N/A & N/A & N/A \\
\hline & GW-180 & S & 2 & 0 & $5.0 \mathrm{E}-04$ & 5.0E-04 & Yes & N/A & N/A & N/A & N/A \\
\hline & GW-609 & S & 14 & 1 & $5.4 \mathrm{E}-04$ & 5.0E-04 & No & S & S & N/A & N/A \\
\hline & GW-188 & $\mathrm{T}$ & 1 & 0 & $5.0 \mathrm{E}-04$ & 5.0E-04 & Yes & N/A & N/A & N/A & N/A \\
\hline & GW-743 & $\mathrm{T}$ & 3 & 0 & $5.0 \mathrm{E}-04$ & 5.0E-04 & Yes & N/A & N/A & N/A & N/A \\
\hline & GW-612 & $T$ & 5 & 5 & $3.5 \mathrm{E}-02$ & 3.4E-02 & No & $\mathrm{s}$ & s & N/A & N/A \\
\hline & GW-610 & $\mathrm{T}$ & 3 & 0 & $5.0 \mathrm{E}-04$ & 5.0E-04 & Yes & N/A & N/A & N/A & N/A \\
\hline & GW-742 & $\mathrm{T}$ & 3 & 0 & $5.0 \mathrm{E}-04$ & 5.0E-04 & Yes & N/A & N/A & N/A & N/A \\
\hline & GW-178 & $T$ & 2 & 2 & $1.3 \mathrm{E}-03$ & 1.3E-03 & No & N/A & $\mathrm{N} / \mathrm{A}$ & N/A & N/A \\
\hline & GW-679 & $\mathrm{T}$ & 2 & 0 & $5.0 \mathrm{E}-04$ & 5.0E-04 & Yes & N/A & N/A & N/A & N/A \\
\hline & GW-179 & $\mathrm{T}$ & 2 & 2 & $1.8 \mathrm{E}-02$ & $1.8 \mathrm{E}-02$ & No & N/A & N/A & N/A & N/A \\
\hline & GW-611 & $T$ & 3 & 1 & $5.0 \mathrm{E}-04$ & 5.0E-04 & No & N/A & $\mathrm{N} / \mathrm{A}$ & $\mathrm{N} / \mathrm{A}$ & N/A \\
\hline & GW-184 & $\mathrm{T}$ & 1 & 0 & $5.0 \mathrm{E}-04$ & 5.0E-04 & Yes & N/A & N/A & N/A & N/A \\
\hline & GW-186 & $\mathrm{T}$ & 1 & 0 & $5.0 \mathrm{E}-04$ & $5.0 \mathrm{E}-04$ & Yes & N/A & N/A & N/A & N/A \\
\hline & GW-680 & $T$ & 2 & 0 & $5.0 \mathrm{E}-04$ & 5.0E-04 & Yes & N/A & $\mathrm{N} / \mathrm{A}$ & N/A & N/A \\
\hline & GW-514 & $\mathrm{T}$ & 3 & 0 & $5.0 \mathrm{E}-04$ & $5.0 \mathrm{E}-04$ & Yes & N/A & N/A & N/A & N/A \\
\hline & GW-513 & $\mathrm{T}$ & 3 & 0 & $5.0 \mathrm{E}-04$ & $5.0 \mathrm{E}-04$ & Yes & N/A & N/A & N/A & N/A \\
\hline & GW-177 & $T$ & 9 & 9 & 3.6E-03 & $2.5 \mathrm{E}-03$ & No & S & NT & N/A & N/A \\
\hline & SCR3_5SP & $T$ & 16 & 0 & $5.0 \mathrm{E}-04$ & $5.0 \mathrm{E}-04$ & Yes & s & $\mathrm{s}$ & N/A & N/A \\
\hline & GW-175 & $\mathrm{T}$ & 5 & 0 & $5.0 \mathrm{E}-04$ & 5.0E-04 & Yes & S & 1 & $\mathrm{~N} / \mathrm{A}$ & N/A \\
\hline
\end{tabular}

MAROS Version 2.1, 2004, AFCEE
User Name: MV

State: Tennessee 
Project: Y-12 National Security Complex

Location: CRSP
User Name: MV

State: Tennessee

\begin{tabular}{|c|c|c|c|c|c|c|c|c|c|c|c|}
\hline Constituent & Well & $\begin{array}{c}\text { Sourcel } \\
\text { Tail }\end{array}$ & $\begin{array}{c}\text { Number } \\
\text { of } \\
\text { Samples }\end{array}$ & $\begin{array}{c}\text { Number } \\
\text { of } \\
\text { Detects }\end{array}$ & $\begin{array}{c}\text { Average } \\
\text { (mg/L) }\end{array}$ & $\begin{array}{c}\text { Median } \\
(\mathrm{mg} / \mathrm{L})\end{array}$ & $\begin{array}{c}\text { All } \\
\text { Samples } \\
\text { "ND" ? }\end{array}$ & $\begin{array}{l}\text { Mann- } \\
\text { Kendall }\end{array}$ & $\begin{array}{l}\text { Linear } \\
\text { Regression }\end{array}$ & Modeling & Empirical \\
\hline \multicolumn{12}{|c|}{ 1,1-DICHLOROETHENE } \\
\hline & SCR3_4SP & $T$ & 9 & 0 & $5.0 \mathrm{E}-04$ & $5.0 \mathrm{E}-04$ & Yes & $\mathrm{S}$ & S & N/A & $\mathrm{N} / \mathrm{A}$ \\
\hline & GW-608 & $\mathrm{T}$ & 7 & 1 & $6.4 \mathrm{E}-04$ & $5.0 \mathrm{E}-04$ & No & s & PD & $N / A$ & $N / A$ \\
\hline & GW-831 & $\mathrm{T}$ & 18 & 0 & $5.0 \mathrm{E}-04$ & $5.0 \mathrm{E}-04$ & Yes & $\mathrm{s}$ & $\mathrm{s}$ & $N / A$ & $N / A$ \\
\hline & GW-176 & $\mathrm{T}$ & 2 & 2 & $2.1 \mathrm{E}-02$ & $2.1 \mathrm{E}-02$ & No & N/A & N/A & N/A & N/A \\
\hline & GW-512 & $\mathrm{T}$ & 1 & 0 & $5.0 \mathrm{E}-04$ & $5.0 \mathrm{E}-04$ & Yes & $\mathrm{N} / \mathrm{A}$ & $\mathrm{N} / \mathrm{A}$ & N/A & $N / A$ \\
\hline \multicolumn{12}{|c|}{ GROSS ALPHA ACTIVITY } \\
\hline & GW-173 & $\mathrm{s}$ & 1 & 1 & $3.7 \mathrm{E}+00$ & $3.7 \mathrm{E}+00$ & No & $\mathrm{N} / \mathrm{A}$ & $\mathrm{N} / \mathrm{A}$ & $\mathrm{N} / \mathrm{A}$ & $\mathrm{N} / \mathrm{A}$ \\
\hline & GW-609 & s & 11 & 7 & $1.1 \mathrm{E}+00$ & $8.2 \mathrm{E}-01$ & No & $\mathrm{D}$ & $\mathrm{D}$ & N/A & $N / A$ \\
\hline & GW-174 & s & 3 & 3 & 8.5E-01 & 6.3E-01 & No & $N / A$ & $N / A$ & $N / A$ & $N / A$ \\
\hline & GW-322 & s & 2 & 2 & $2.7 \mathrm{E}+00$ & $2.7 \mathrm{E}+00$ & No & $N / A$ & $N / A$ & $N / A$ & $N / A$ \\
\hline & GW-186 & $\mathrm{T}$ & 1 & 1 & $2.4 \mathrm{E}+00$ & $2.4 \mathrm{E}+00$ & No & $N / A$ & $N / A$ & $N / A$ & $N / A$ \\
\hline & GW-611 & $\mathrm{T}$ & 1 & 1 & $2.1 \mathrm{E}+00$ & $2.1 \mathrm{E}+00$ & No & $\mathrm{N} / \mathrm{A}$ & N/A & N/A & N/A \\
\hline & GW-514 & $\mathrm{T}$ & 2 & 2 & $6.4 \mathrm{E}-01$ & $6.4 \mathrm{E}-01$ & No & $N / A$ & $N / A$ & $N / A$ & $N / A$ \\
\hline & GW-610 & $\mathrm{T}$ & 1 & 1 & $1.4 \mathrm{E}+00$ & $1.4 \mathrm{E}+00$ & No & $\mathrm{N} / \mathrm{A}$ & $N / A$ & $N / A$ & $N / A$ \\
\hline & GW-512 & $\mathrm{T}$ & 1 & 1 & $1.6 \mathrm{E}+00$ & $1.6 \mathrm{E}+00$ & No & $\mathrm{N} / \mathrm{A}$ & N/A & N/A & N/A \\
\hline & GW-608 & $\mathrm{T}$ & 4 & 4 & $2.6 \mathrm{E}+00$ & $2.6 \mathrm{E}+00$ & No & $\mathrm{s}$ & $\mathrm{S}$ & $N / A$ & $N / A$ \\
\hline & GW-188 & $\mathrm{T}$ & 1 & 1 & $1.7 \mathrm{E}+00$ & $1.7 \mathrm{E}+00$ & No & $N / A$ & $N / A$ & $N / A$ & $N / A$ \\
\hline & GW-177 & $\mathrm{T}$ & 9 & 8 & $2.7 \mathrm{E}+00$ & $3.0 \mathrm{E}+00$ & No & NT & NT & $N / A$ & $N / A$ \\
\hline & SCR3_5SP & $\mathrm{T}$ & 16 & 8 & $1.2 \mathrm{E}+00$ & $2.9 \mathrm{E}-01$ & No & NT & NT & $N / A$ & $N / A$ \\
\hline & SCR3_4SP & $\mathrm{T}$ & 6 & 5 & $5.9 \mathrm{E}-01$ & $6.2 \mathrm{E}-01$ & No & $\mathrm{PI}$ & $\mathrm{PI}$ & $N / A$ & N/A \\
\hline & GW-175 & $\mathrm{T}$ & 1 & 1 & $3.2 \mathrm{E}-01$ & $3.2 \mathrm{E}-01$ & No & $\mathrm{N} / \mathrm{A}$ & $\mathrm{N} / \mathrm{A}$ & $N / A$ & $N / A$ \\
\hline & GW-831 & $\mathrm{T}$ & 17 & 13 & $1.1 \mathrm{E}+00$ & 8.3E-01 & No & NT & NT & $N / A$ & $N / A$ \\
\hline & GW-176 & $\mathrm{T}$ & 1 & 1 & $1.2 \mathrm{E}+00$ & $1.2 \mathrm{E}+00$ & No & $N / A$ & $\mathrm{~N} / \mathrm{A}$ & $N / A$ & N/A \\
\hline & GW-743 & $\mathrm{T}$ & 2 & 2 & $2.2 \mathrm{E}+00$ & $2.2 \mathrm{E}+00$ & No & $N / A$ & $N / A$ & $N / A$ & $N / A$ \\
\hline & GW-612 & $\mathrm{T}$ & 4 & 4 & $2.3 \mathrm{E}+00$ & $1.5 \mathrm{E}+00$ & No & NT & PI & $N / A$ & $N / A$ \\
\hline & GW-742 & $\mathrm{T}$ & 3 & 3 & $3.4 \mathrm{E}+00$ & $1.6 \mathrm{E}+00$ & No & $N / A$ & $N / A$ & $N / A$ & N/A \\
\hline & GW-513 & $\mathrm{T}$ & 2 & 2 & $2.8 \mathrm{E}+00$ & $2.8 \mathrm{E}+00$ & No & $\mathrm{N} / \mathrm{A}$ & $\mathrm{N} / \mathrm{A}$ & $N / A$ & $N / A$ \\
\hline & GW-184 & $\mathrm{T}$ & 1 & 1 & $1.4 \mathrm{E}+00$ & $1.4 \mathrm{E}+00$ & No & $N / A$ & $N / A$ & $N / A$ & $N / A$ \\
\hline & GW-679 & $\mathrm{T}$ & 2 & 2 & $6.7 \mathrm{E}-01$ & 6.7E-01 & No & $N / A$ & $N / A$ & $N / A$ & N/A \\
\hline & GW-178 & $\mathrm{T}$ & 1 & 1 & $1.6 \mathrm{E}+00$ & $1.6 \mathrm{E}+00$ & No & N/A & N/A & N/A & N/A \\
\hline \multicolumn{12}{|l|}{ LEAD } \\
\hline & GW-180 & $\mathrm{s}$ & 2 & 0 & $4.5 \mathrm{E}-04$ & $4.5 \mathrm{E}-04$ & Yes & $\mathrm{N} / \mathrm{A}$ & $\mathrm{N} / \mathrm{A}$ & $\mathrm{N} / \mathrm{A}$ & $\mathrm{N} / \mathrm{A}$ \\
\hline
\end{tabular}


Project: Y-12 National Security Complex

Location: CRSP
User Name: MV

State: Tennessee

\begin{tabular}{|c|c|c|c|c|c|c|c|c|c|c|c|}
\hline Constituent & Well & $\begin{array}{c}\text { Sourcel } \\
\text { Tail }\end{array}$ & $\begin{array}{l}\text { Number } \\
\text { of } \\
\text { Samples }\end{array}$ & $\begin{array}{c}\text { Number } \\
\text { of } \\
\text { Detects }\end{array}$ & $\begin{array}{c}\text { Average } \\
\text { (mg/L) }\end{array}$ & $\begin{array}{r}\text { Median } \\
(\mathrm{mg} / \mathrm{L})\end{array}$ & $\begin{array}{c}\text { All } \\
\text { Samples } \\
\text { "ND" ? }\end{array}$ & $\begin{array}{l}\text { Mann- } \\
\text { Kendall }\end{array}$ & $\begin{array}{c}\text { Linear } \\
\text { Regression }\end{array}$ & Modeling & Empirical \\
\hline \multicolumn{12}{|l|}{ LEAD } \\
\hline & GW-609 & $\mathrm{S}$ & 12 & 4 & $5.8 \mathrm{E}-04$ & $4.5 \mathrm{E}-04$ & No & $\mathrm{S}$ & $\mathrm{S}$ & $\mathrm{N} / \mathrm{A}$ & $\mathrm{N} / \mathrm{A}$ \\
\hline & GW-174 & s & 3 & 2 & $1.8 \mathrm{E}-03$ & $1.9 \mathrm{E}-03$ & No & N/A & N/A & N/A & $N / A$ \\
\hline & GW-173 & s & 2 & 0 & 4.5E-04 & 4.5E-04 & Yes & N/A & N/A & N/A & $N / A$ \\
\hline & GW-322 & $\mathrm{s}$ & 3 & 0 & 4.5E-04 & 4.5E-04 & Yes & N/A & N/A & N/A & N/A \\
\hline & GW-179 & $\mathrm{T}$ & 2 & 1 & $6.0 \mathrm{E}-04$ & 6.0E-04 & No & N/A & $\mathrm{N} / \mathrm{A}$ & N/A & $N / A$ \\
\hline & GW-186 & $\mathrm{T}$ & 1 & 0 & 4.5E-04 & 4.5E-04 & Yes & N/A & $\mathrm{N} / \mathrm{A}$ & $\mathrm{N} / \mathrm{A}$ & $N / A$ \\
\hline & GW-176 & $\mathrm{T}$ & 2 & 0 & 4.5E-04 & 4.5E-04 & Yes & N/A & N/A & N/A & $N / A$ \\
\hline & GW-177 & $\mathrm{T}$ & 9 & 0 & 4.5E-04 & 4.5E-04 & Yes & $\mathrm{s}$ & $\mathrm{D}$ & N/A & $N / A$ \\
\hline & GW-188 & $\mathrm{T}$ & 1 & 0 & 4.5E-04 & $4.5 \mathrm{E}-04$ & Yes & N/A & N/A & N/A & N/A \\
\hline & GW-175 & $\mathrm{T}$ & 5 & 1 & $4.8 \mathrm{E}-04$ & 4.5E-04 & No & NT & NT & $\mathrm{N} / \mathrm{A}$ & $N / A$ \\
\hline & GW-513 & $\mathrm{T}$ & 3 & 1 & $6.3 \mathrm{E}-04$ & 4.5E-04 & No & N/A & $\mathrm{N} / \mathrm{A}$ & $\mathrm{N} / \mathrm{A}$ & $N / A$ \\
\hline & GW-512 & $\mathrm{T}$ & 1 & 0 & 4.5E-04 & 4.5E-04 & Yes & N/A & N/A & N/A & $N / A$ \\
\hline & GW-178 & $\mathrm{T}$ & 2 & 1 & $5.0 \mathrm{E}-04$ & 5.0E-04 & No & N/A & $\mathrm{N} / \mathrm{A}$ & N/A & $\mathrm{N} / \mathrm{A}$ \\
\hline & GW-184 & $\mathrm{T}$ & 1 & 0 & 4.5E-04 & 4.5E-04 & Yes & N/A & $\mathrm{N} / \mathrm{A}$ & $\mathrm{N} / \mathrm{A}$ & $N / A$ \\
\hline & GW-743 & $T$ & 3 & 0 & 4.5E-04 & 4.5E-04 & Yes & N/A & $\mathrm{N} / \mathrm{A}$ & $\mathrm{N} / \mathrm{A}$ & $N / A$ \\
\hline & GW-679 & $T$ & 2 & 2 & $5.8 \mathrm{E}-04$ & $5.8 \mathrm{E}-04$ & No & N/A & N/A & N/A & $N / A$ \\
\hline & GW-612 & $T$ & 5 & 5 & $5.4 \mathrm{E}-03$ & 9.7E-04 & No & NT & NT & $\mathrm{N} / \mathrm{A}$ & $N / A$ \\
\hline & GW-680 & $T$ & 2 & 2 & $6.3 \mathrm{E}-04$ & 6.3E-04 & No & N/A & N/A & N/A & $N / A$ \\
\hline & GW-611 & $T$ & 3 & 0 & 4.5E-04 & 4.5E-04 & Yes & N/A & N/A & N/A & $\mathrm{N} / \mathrm{A}$ \\
\hline & GW-742 & $T$ & 3 & 0 & 4.5E-04 & 4.5E-04 & Yes & $\mathrm{N} / \mathrm{A}$ & $\mathrm{N} / \mathrm{A}$ & $\mathrm{N} / \mathrm{A}$ & $N / A$ \\
\hline & GW-610 & $\mathrm{T}$ & 3 & 0 & 4.5E-04 & 4.5E-04 & Yes & N/A & N/A & N/A & $N / A$ \\
\hline & GW-831 & $T$ & 18 & 6 & 2.3E-03 & 4.5E-04 & No & $\mathrm{D}$ & $\mathrm{PD}$ & N/A & $\mathrm{N} / \mathrm{A}$ \\
\hline & GW-608 & $T$ & 5 & 0 & 4.5E-04 & 4.5E-04 & Yes & $\mathrm{s}$ & $\mathrm{s}$ & N/A & $\mathrm{N} / \mathrm{A}$ \\
\hline & GW-514 & $\mathrm{T}$ & 3 & 1 & $5.0 \mathrm{E}-04$ & 4.5E-04 & No & N/A & N/A & N/A & $N / A$ \\
\hline & SCR3_4SP & $\mathrm{T}$ & 9 & 3 & $6.6 \mathrm{E}-04$ & 4.5E-04 & No & NT & s & $\mathrm{N} / \mathrm{A}$ & $N / A$ \\
\hline & SCR3_5SP & $\mathrm{T}$ & 16 & 0 & 4.5E-04 & 4.5E-04 & Yes & NT & s & N/A & $N / A$ \\
\hline \multicolumn{12}{|c|}{ TETRACHLOROETHYLENE(PCE) } \\
\hline & GW-322 & $\mathrm{S}$ & 3 & 3 & $7.3 \mathrm{E}-03$ & $6.0 \mathrm{E}-03$ & No & $\mathrm{N} / \mathrm{A}$ & $\mathrm{N} / \mathrm{A}$ & $\mathrm{N} / \mathrm{A}$ & $\mathrm{N} / \mathrm{A}$ \\
\hline & GW-609 & s & 14 & 13 & $5.1 \mathrm{E}-03$ & $3.2 \mathrm{E}-03$ & No & $\mathrm{D}$ & D & N/A & $N / A$ \\
\hline & GW-180 & $\mathrm{s}$ & 2 & 2 & 2.0E-02 & 2.0E-02 & No & N/A & $\mathrm{N} / \mathrm{A}$ & N/A & $\mathrm{N} / \mathrm{A}$ \\
\hline & GW-174 & s & 3 & 3 & 8.0E-03 & $6.0 \mathrm{E}-03$ & No & $\mathrm{N} / \mathrm{A}$ & $\mathrm{N} / \mathrm{A}$ & $\mathrm{N} / \mathrm{A}$ & $N / A$ \\
\hline & GW-173 & s & 2 & 2 & $8.5 \mathrm{E}-03$ & 8.5E-03 & No & N/A & N/A & N/A & $N / A$ \\
\hline & GW-742 & $T$ & 3 & 0 & $5.0 \mathrm{E}-04$ & 5.0E-04 & Yes & N/A & N/A & N/A & $\mathrm{N} / \mathrm{A}$ \\
\hline
\end{tabular}


Project: Y-12 National Security Complex

Location: CRSP
User Name: MV

State: Tennessee

\begin{tabular}{|c|c|c|c|c|c|c|c|c|c|c|c|}
\hline Constituent & Well & $\begin{array}{c}\text { Sourcel } \\
\text { Tail }\end{array}$ & $\begin{array}{c}\text { Number } \\
\text { of } \\
\text { Samples }\end{array}$ & $\begin{array}{c}\text { Number } \\
\text { of } \\
\text { Detects }\end{array}$ & $\begin{array}{c}\text { Average } \\
\text { (mg/L) }\end{array}$ & $\begin{array}{r}\text { Median } \\
(\mathrm{mg} / \mathrm{L})\end{array}$ & $\begin{array}{c}\text { All } \\
\text { Samples } \\
\text { "ND" ? }\end{array}$ & $\begin{array}{l}\text { Mann- } \\
\text { Kendall }\end{array}$ & $\begin{array}{l}\text { Linear } \\
\text { Regression }\end{array}$ & Modeling & Empirical \\
\hline \multicolumn{12}{|c|}{ TETRACHLOROETHYLENE(PCE) } \\
\hline & SCR3_5SP & $T$ & 16 & 0 & $5.0 \mathrm{E}-04$ & $5.0 \mathrm{E}-04$ & Yes & $\mathrm{S}$ & S & N/A & N/A \\
\hline & GW-679 & $\mathrm{T}$ & 2 & 0 & $5.0 \mathrm{E}-04$ & $5.0 \mathrm{E}-04$ & Yes & N/A & N/A & N/A & N/A \\
\hline & GW-513 & $\mathrm{T}$ & 3 & 0 & $5.0 \mathrm{E}-04$ & 5.0E-04 & Yes & $\mathrm{N} / \mathrm{A}$ & $\mathrm{N} / \mathrm{A}$ & $N / A$ & $N / A$ \\
\hline & GW-178 & $\mathrm{T}$ & 2 & 0 & $5.0 \mathrm{E}-04$ & $5.0 \mathrm{E}-04$ & Yes & N/A & N/A & N/A & N/A \\
\hline & GW-680 & $\mathrm{T}$ & 2 & 0 & $5.0 \mathrm{E}-04$ & $5.0 \mathrm{E}-04$ & Yes & N/A & N/A & N/A & N/A \\
\hline & GW-743 & $\mathrm{T}$ & 3 & 0 & $5.0 \mathrm{E}-04$ & $5.0 \mathrm{E}-04$ & Yes & $N / A$ & $N / A$ & $N / A$ & $N / A$ \\
\hline & SCR3_4SP & $\mathrm{T}$ & 9 & 0 & $5.0 \mathrm{E}-04$ & 5.0E-04 & Yes & S & S & $N / A$ & $N / A$ \\
\hline & GW-176 & $\mathrm{T}$ & 2 & 2 & $1.0 \mathrm{E}-03$ & $1.0 \mathrm{E}-03$ & No & N/A & N/A & N/A & N/A \\
\hline & GW-179 & $\mathrm{T}$ & 2 & 1 & $5.0 \mathrm{E}-04$ & $5.0 \mathrm{E}-04$ & No & $N / A$ & $N / A$ & $N / A$ & $N / A$ \\
\hline & GW-175 & $\mathrm{T}$ & 5 & 5 & $1.0 \mathrm{E}-02$ & $1.0 \mathrm{E}-02$ & No & $\mathrm{s}$ & NT & $N / A$ & $N / A$ \\
\hline & GW-831 & $\mathrm{T}$ & 18 & 0 & $5.0 \mathrm{E}-04$ & $5.0 \mathrm{E}-04$ & Yes & s & $S$ & N/A & N/A \\
\hline & GW-177 & $\mathrm{T}$ & 9 & 0 & $5.0 \mathrm{E}-04$ & $5.0 \mathrm{E}-04$ & Yes & s & s & $N / A$ & $N / A$ \\
\hline & GW-610 & $\mathrm{T}$ & 3 & 0 & $5.0 \mathrm{E}-04$ & $5.0 \mathrm{E}-04$ & Yes & $N / A$ & $N / A$ & $N / A$ & $N / A$ \\
\hline & GW-512 & $\mathrm{T}$ & 1 & 0 & $5.0 \mathrm{E}-04$ & $5.0 \mathrm{E}-04$ & Yes & $\mathrm{N} / \mathrm{A}$ & N/A & N/A & N/A \\
\hline & GW-514 & $\mathrm{T}$ & 3 & 0 & $5.0 \mathrm{E}-04$ & 5.0E-04 & Yes & $N / A$ & $N / A$ & $N / A$ & N/A \\
\hline & GW-608 & $\mathrm{T}$ & 7 & 1 & $6.4 \mathrm{E}-04$ & $5.0 \mathrm{E}-04$ & No & $\mathrm{s}$ & PD & $N / A$ & $N / A$ \\
\hline & GW-188 & $\mathrm{T}$ & 1 & 0 & $5.0 \mathrm{E}-04$ & $5.0 \mathrm{E}-04$ & Yes & $\mathrm{N} / \mathrm{A}$ & N/A & N/A & $N / A$ \\
\hline & GW-186 & $\mathrm{T}$ & 1 & 0 & $5.0 \mathrm{E}-04$ & $5.0 \mathrm{E}-04$ & Yes & $\mathrm{N} / \mathrm{A}$ & N/A & N/A & $N / A$ \\
\hline & GW-611 & $\mathrm{T}$ & 3 & 0 & $5.0 \mathrm{E}-04$ & 5.0E-04 & Yes & $N / A$ & $N / A$ & $N / A$ & $N / A$ \\
\hline & GW-612 & $\mathrm{T}$ & 5 & 3 & 2.4E-03 & $1.8 \mathrm{E}-03$ & No & NT & NT & $\mathrm{N} / \mathrm{A}$ & $N / A$ \\
\hline & GW-184 & $\mathrm{T}$ & 1 & 0 & $5.0 \mathrm{E}-04$ & $5.0 \mathrm{E}-04$ & Yes & $N / A$ & $N / A$ & $N / A$ & $N / A$ \\
\hline \multicolumn{12}{|c|}{ TRICHLOROETHYLENE (TCE) } \\
\hline & GW-180 & $\mathrm{s}$ & 2 & 0 & $5.0 \mathrm{E}-04$ & $5.0 \mathrm{E}-04$ & Yes & $\mathrm{N} / \mathrm{A}$ & $\mathrm{N} / \mathrm{A}$ & $\mathrm{N} / \mathrm{A}$ & $\mathrm{N} / \mathrm{A}$ \\
\hline & GW-173 & s & 2 & 0 & $5.0 \mathrm{E}-04$ & $5.0 \mathrm{E}-04$ & Yes & $N / A$ & $N / A$ & $N / A$ & $N / A$ \\
\hline & GW-322 & s & 3 & 0 & $5.0 \mathrm{E}-04$ & 5.0E-04 & Yes & $N / A$ & $N / A$ & $N / A$ & $N / A$ \\
\hline & GW-174 & s & 3 & 0 & $5.0 \mathrm{E}-04$ & 5.0E-04 & Yes & $N / A$ & $N / A$ & $N / A$ & N/A \\
\hline & GW-609 & s & 14 & 4 & $1.3 \mathrm{E}-02$ & $5.0 \mathrm{E}-04$ & No & NT & NT & $N / A$ & $N / A$ \\
\hline & GW-743 & $\mathrm{T}$ & 3 & 0 & $5.0 \mathrm{E}-04$ & $5.0 \mathrm{E}-04$ & Yes & $N / A$ & $N / A$ & $N / A$ & $N / A$ \\
\hline & GW-513 & $\mathrm{T}$ & 3 & 0 & $5.0 \mathrm{E}-04$ & $5.0 \mathrm{E}-04$ & Yes & $N / A$ & $N / A$ & $N / A$ & N/A \\
\hline & GW-512 & $\mathrm{T}$ & 1 & 0 & $5.0 \mathrm{E}-04$ & $5.0 \mathrm{E}-04$ & Yes & $N / A$ & $\mathrm{~N} / \mathrm{A}$ & $N / A$ & $N / A$ \\
\hline & SCR3_4SP & $\mathrm{T}$ & 9 & 0 & $5.0 \mathrm{E}-04$ & $5.0 \mathrm{E}-04$ & Yes & $\mathrm{s}$ & $\mathrm{s}$ & $\mathrm{N} / \mathrm{A}$ & $N / A$ \\
\hline & SCR3_5SP & $\mathrm{T}$ & 16 & 0 & $5.0 \mathrm{E}-04$ & $5.0 \mathrm{E}-04$ & Yes & $\mathrm{s}$ & $\mathrm{s}$ & $N / A$ & $N / A$ \\
\hline & GW-514 & $\mathrm{T}$ & 3 & 0 & $5.0 \mathrm{E}-04$ & 5.0E-04 & Yes & $N / A$ & $N / A$ & $N / A$ & $N / A$ \\
\hline
\end{tabular}


Project: Y-12 National Security Complex

Location: CRSP
User Name: $\quad$ MV

State: Tennessee

\begin{tabular}{|c|c|c|c|c|c|c|c|c|c|c|c|}
\hline Constituent & Well & $\begin{array}{c}\text { Sourcel } \\
\text { Tail }\end{array}$ & $\begin{array}{c}\text { Number } \\
\text { of } \\
\text { Samples }\end{array}$ & $\begin{array}{c}\text { Number } \\
\text { of } \\
\text { Detects }\end{array}$ & $\begin{array}{c}\text { Average } \\
\text { (mg/L) }\end{array}$ & $\begin{array}{r}\text { Median } \\
(\mathrm{mg} / \mathrm{L})\end{array}$ & $\begin{array}{c}\text { All } \\
\text { Samples } \\
\text { "ND" ? }\end{array}$ & $\begin{array}{l}\text { Mann- } \\
\text { Kendall }\end{array}$ & $\begin{array}{l}\text { Linear } \\
\text { Regression }\end{array}$ & Modeling & Empirical \\
\hline \multicolumn{12}{|c|}{ TRICHLOROETHYLENE (TCE) } \\
\hline & GW-831 & $T$ & 18 & 0 & $5.0 \mathrm{E}-04$ & $5.0 \mathrm{E}-04$ & Yes & $\mathrm{S}$ & $\mathrm{S}$ & N/A & N/A \\
\hline & GW-608 & $\mathrm{T}$ & 7 & 0 & $5.0 \mathrm{E}-04$ & $5.0 \mathrm{E}-04$ & Yes & s & I & N/A & N/A \\
\hline & GW-175 & $\mathrm{T}$ & 5 & 0 & $5.0 \mathrm{E}-04$ & 5.0E-04 & Yes & s & I & N/A & $N / A$ \\
\hline & GW-179 & $\mathrm{T}$ & 2 & 0 & $5.0 \mathrm{E}-04$ & $5.0 \mathrm{E}-04$ & Yes & N/A & N/A & N/A & $N / A$ \\
\hline & GW-611 & $\mathrm{T}$ & 3 & 0 & $5.0 \mathrm{E}-04$ & $5.0 \mathrm{E}-04$ & Yes & N/A & N/A & N/A & N/A \\
\hline & GW-612 & $\mathrm{T}$ & 5 & 0 & $5.0 \mathrm{E}-04$ & $5.0 \mathrm{E}-04$ & Yes & $\mathrm{s}$ & $\mathrm{D}$ & $\mathrm{N} / \mathrm{A}$ & $N / A$ \\
\hline & GW-176 & $\mathrm{T}$ & 2 & 0 & 5.0E-04 & 5.0E-04 & Yes & N/A & N/A & N/A & $N / A$ \\
\hline & GW-742 & $\mathrm{T}$ & 3 & 0 & $5.0 \mathrm{E}-04$ & $5.0 \mathrm{E}-04$ & Yes & N/A & N/A & N/A & N/A \\
\hline & GW-186 & $\mathrm{T}$ & 1 & 0 & $5.0 \mathrm{E}-04$ & $5.0 \mathrm{E}-04$ & Yes & $\mathrm{N} / \mathrm{A}$ & N/A & $\mathrm{N} / \mathrm{A}$ & $N / A$ \\
\hline & GW-679 & $\mathrm{T}$ & 2 & 0 & $5.0 \mathrm{E}-04$ & $5.0 \mathrm{E}-04$ & Yes & N/A & N/A & N/A & $N / A$ \\
\hline & GW-610 & $\mathrm{T}$ & 3 & 0 & $5.0 \mathrm{E}-04$ & $5.0 \mathrm{E}-04$ & Yes & $\mathrm{N} / \mathrm{A}$ & N/A & N/A & N/A \\
\hline & GW-177 & $\mathrm{T}$ & 9 & 0 & $5.0 \mathrm{E}-04$ & $5.0 \mathrm{E}-04$ & Yes & $\mathrm{s}$ & $\mathrm{s}$ & $\mathrm{N} / \mathrm{A}$ & $N / A$ \\
\hline & GW-680 & $\mathrm{T}$ & 2 & 0 & $5.0 \mathrm{E}-04$ & 5.0E-04 & Yes & N/A & N/A & N/A & $N / A$ \\
\hline & GW-178 & $\mathrm{T}$ & 2 & 0 & $5.0 \mathrm{E}-04$ & $5.0 \mathrm{E}-04$ & Yes & N/A & N/A & N/A & N/A \\
\hline & GW-184 & $\mathrm{T}$ & 1 & 0 & $5.0 \mathrm{E}-04$ & $5.0 \mathrm{E}-04$ & Yes & $\mathrm{N} / \mathrm{A}$ & N/A & N/A & $\mathrm{N} / \mathrm{A}$ \\
\hline & GW-188 & $\mathrm{T}$ & 1 & 0 & 5.0E-04 & 5.0E-04 & Yes & $N / A$ & N/A & N/A & $N / A$ \\
\hline
\end{tabular}

Note: Increasing (I); Probably Increasing (PI); Stable (S); Probably Decreasing (PD); Decreasing (D); No Trend (NT); Not Applicable (N/A) - Due to insufficient Data (< 4 sampling events); Source/Tail (S/T)

The Number of Samples and Number of Detects shown above are post-consolidation values. 


\section{MAROS Spatial Moment Analysis Summary}

Project: Y-12 National Security Complex

Location: CRSP
User Name: MV

State: Tennessee

2nd Moment (Spread)

Oth Moment

$\underline{1 \text { st Moment (Center of Mass) }}$

Estimated

Effective Date

Mass (Kg)
Source Xc (t)

Yc (ft) $\quad \begin{gathered}\text { Source } \\ \text { Distance }(\mathrm{ft})\end{gathered}$

Sigma $X X$

(sq ft)
Number of

Wells

1,1-DICHLOROETHENE

\begin{tabular}{|c|c|c|c|c|c|c|c|}
\hline 1/1/1996 & 1.1E-01 & 58,851 & 28,357 & 131 & 340,757 & 24,259 & 8 \\
\hline 4/1/1996 & 5.5E-01 & 57,829 & 25,979 & 2,507 & 387,913 & 670,167 & 7 \\
\hline $7 / 1 / 1996$ & $0.0 \mathrm{E}+00$ & & & & & & 3 \\
\hline 10/1/1996 & $0.0 \mathrm{E}+00$ & & & & & & 2 \\
\hline 1/1/1997 & $0.0 \mathrm{E}+00$ & & & & & & 2 \\
\hline 4/1/1997 & $0.0 \mathrm{E}+00$ & & & & & & 4 \\
\hline $7 / 1 / 1997$ & $0.0 \mathrm{E}+00$ & & & & & & 5 \\
\hline 10/1/1997 & $0.0 \mathrm{E}+00$ & & & & & & 1 \\
\hline 1/1/1998 & 8.6E-01 & 58,273 & 27,240 & 1,187 & 312,901 & 455,781 & 6 \\
\hline 7/1/1998 & $0.0 \mathrm{E}+00$ & & & & & & 5 \\
\hline 1/1/1999 & $0.0 \mathrm{E}+00$ & & & & & & 4 \\
\hline $7 / 1 / 1999$ & $0.0 \mathrm{E}+00$ & & & & & & 4 \\
\hline $1 / 1 / 2000$ & $0.0 \mathrm{E}+00$ & & & & & & 4 \\
\hline $7 / 1 / 2000$ & $0.0 \mathrm{E}+00$ & & & & & & 4 \\
\hline $1 / 1 / 2001$ & 8.2E-01 & 57,975 & 27,495 & 1,198 & 307,145 & 774,310 & 11 \\
\hline $7 / 1 / 2001$ & 8.8E-01 & 57,922 & 27,565 & 1,199 & 186,271 & 721,598 & 9 \\
\hline $1 / 1 / 2002$ & $0.0 \mathrm{E}+00$ & & & & & & 3 \\
\hline $7 / 1 / 2002$ & $0.0 \mathrm{E}+00$ & & & & & & 3 \\
\hline $1 / 1 / 2003$ & $0.0 \mathrm{E}+00$ & & & & & & 3 \\
\hline $7 / 1 / 2003$ & $0.0 \mathrm{E}+00$ & & & & & & 3 \\
\hline $1 / 1 / 2004$ & $0.0 \mathrm{E}+00$ & & & & & & 3 \\
\hline $4 / 1 / 2004$ & 9.4E-01 & 58,426 & 28,217 & 487 & 195,385 & 87,130 & 14 \\
\hline $7 / 1 / 2004$ & $0.0 \mathrm{E}+00$ & & & & & & 3 \\
\hline $10 / 1 / 2004$ & $1.1 \mathrm{E}+00$ & 58,439 & 28,240 & 473 & 183,460 & 80,668 & 14 \\
\hline
\end{tabular}

GROSS ALPHA ACTIVITY

\begin{tabular}{|c|c|c|c|c|c|c|}
\hline 1/1/1996 & $0.0 \mathrm{E}+00$ & & & & & \\
\hline 4/1/1996 & $1.8 \mathrm{E}+03$ & 57,842 & 25,993 & 2,489 & 383,906 & 667,855 \\
\hline 7/1/1996 & $0.0 \mathrm{E}+00$ & & & & & \\
\hline 10/1/1996 & $0.0 \mathrm{E}+00$ & & & & & \\
\hline 1/1/1997 & $0.0 \mathrm{E}+00$ & & & & & \\
\hline 4/1/1997 & $0.0 \mathrm{E}+00$ & & & & & \\
\hline 7/1/1997 & $0.0 E+00$ & & & & & \\
\hline 1/1/1998 & $0.0 \mathrm{E}+00$ & & & & & \\
\hline 7/1/1998 & $0.0 E+00$ & & & & & \\
\hline 1/1/1999 & $0.0 \mathrm{E}+00$ & & & & & \\
\hline 7/1/1999 & $0.0 \mathrm{E}+00$ & & & & & \\
\hline $1 / 1 / 2000$ & $0.0 \mathrm{E}+00$ & & & & & \\
\hline $7 / 1 / 2000$ & $0.0 \mathrm{E}+00$ & & & & & \\
\hline
\end{tabular}


Project: Y-12 National Security Complex

Location: CRSP
User Name: $\quad$ MV

State: Tennessee

\begin{tabular}{|c|c|c|c|c|c|c|c|}
\hline \multirow[b]{2}{*}{ Effective Date } & \multirow{2}{*}{$\begin{array}{l}\text { Oth Moment } \\
\text { Estimated } \\
\text { Mass (kg) }\end{array}$} & \multicolumn{3}{|c|}{ 1st Moment (Center of Mass) } & \multicolumn{2}{|c|}{$\underline{\text { 2nd Moment (Spread) }}$} & \multirow[b]{2}{*}{$\begin{array}{c}\text { Number of } \\
\text { Wells }\end{array}$} \\
\hline & & $\mathrm{Xc}(\mathrm{ft})$ & Yc (ft) & $\begin{array}{c}\text { Source } \\
\text { Distance (ft) }\end{array}$ & $\begin{array}{l}\text { Sigma } X X \\
(s q \mathrm{ft})\end{array}$ & $\begin{array}{l}\text { Sigma } Y Y \\
\quad(s q \mathrm{ft})\end{array}$ & \\
\hline \multicolumn{8}{|l|}{ GROSS ALPHA ACTIVITY } \\
\hline $1 / 1 / 2001$ & $6.7 \mathrm{E}+02$ & 57,594 & 27,142 & 1,716 & 345,756 & 678,540 & 9 \\
\hline $7 / 1 / 2001$ & $4.2 \mathrm{E}+02$ & 58,040 & 27,523 & 1,130 & 197,701 & 581,902 & 7 \\
\hline $1 / 1 / 2002$ & $0.0 \mathrm{E}+00$ & & & & & & 3 \\
\hline $7 / 1 / 2002$ & $0.0 \mathrm{E}+00$ & & & & & & 3 \\
\hline $1 / 1 / 2003$ & $0.0 \mathrm{E}+00$ & & & & & & 3 \\
\hline $7 / 1 / 2003$ & $0.0 \mathrm{E}+00$ & & & & & & 3 \\
\hline $1 / 1 / 2004$ & $0.0 \mathrm{E}+00$ & & & & & & 3 \\
\hline $4 / 1 / 2004$ & $3.6 \mathrm{E}+02$ & 58,161 & 28,116 & 761 & 189,345 & 66,416 & 9 \\
\hline $7 / 1 / 2004$ & $0.0 \mathrm{E}+00$ & & & & & & 3 \\
\hline $10 / 1 / 2004$ & $1.1 \mathrm{E}+02$ & 58,388 & 27,979 & 586 & 185,221 & 41,374 & 6 \\
\hline \multicolumn{8}{|l|}{ LEAD } \\
\hline 1/1/1996 & 7.8E-02 & 58,818 & 28,375 & 164 & 313,641 & 23,227 & 8 \\
\hline $4 / 1 / 1996$ & 4.9E-01 & 57,829 & 25,979 & 2,507 & 387,913 & 670,167 & 7 \\
\hline $7 / 1 / 1996$ & $0.0 \mathrm{E}+00$ & & & & & & 3 \\
\hline 10/1/1996 & $0.0 \mathrm{E}+00$ & & & & & & 2 \\
\hline 1/1/1997 & $0.0 \mathrm{E}+00$ & & & & & & 2 \\
\hline 4/1/1997 & $0.0 \mathrm{E}+00$ & & & & & & 2 \\
\hline $7 / 1 / 1997$ & $0.0 \mathrm{E}+00$ & & & & & & 4 \\
\hline 1/1/1998 & 5.4E-01 & 57,783 & 26,564 & 2,022 & 243,747 & 402,897 & 6 \\
\hline 7/1/1998 & $0.0 \mathrm{E}+00$ & & & & & & 5 \\
\hline 1/1/1999 & $0.0 \mathrm{E}+00$ & & & & & & 4 \\
\hline 7/1/1999 & $0.0 \mathrm{E}+00$ & & & & & & 4 \\
\hline $1 / 1 / 2000$ & $0.0 \mathrm{E}+00$ & & & & & & 4 \\
\hline $7 / 1 / 2000$ & $0.0 \mathrm{E}+00$ & & & & & & 4 \\
\hline $1 / 1 / 2001$ & $7.9 \mathrm{E}-01$ & 58,075 & 27,489 & 1,125 & 347,944 & 726,122 & 11 \\
\hline $7 / 1 / 2001$ & 4.8E-01 & 57,886 & 27,163 & 1,488 & 204,103 & 744,043 & 9 \\
\hline $1 / 1 / 2002$ & $0.0 \mathrm{E}+00$ & & & & & & 3 \\
\hline $7 / 1 / 2002$ & $0.0 \mathrm{E}+00$ & & & & & & 3 \\
\hline $1 / 1 / 2003$ & $0.0 \mathrm{E}+00$ & & & & & & 3 \\
\hline $7 / 1 / 2003$ & $0.0 \mathrm{E}+00$ & & & & & & 3 \\
\hline $1 / 1 / 2004$ & $0.0 \mathrm{E}+00$ & & & & & & 3 \\
\hline $4 / 1 / 2004$ & 1.6E-01 & 58,145 & 28,014 & 800 & 309,772 & 140,619 & 14 \\
\hline $7 / 1 / 2004$ & $0.0 \mathrm{E}+00$ & & & & & & 3 \\
\hline $10 / 1 / 2004$ & 1.9E-01 & 58,097 & 27,975 & 857 & 303,965 & 138,038 & 14 \\
\hline
\end{tabular}

TETRACHLOROETHYLENE(PCE)

\begin{tabular}{rrrrrrrr}
\hline $1 / 1 / 1996$ & $1.6 \mathrm{E}-01$ & 58,912 & 28,389 & 148 & 254,511 & 23,487 & 352,982 \\
$4 / 1 / 1996$ & $1.4 \mathrm{E}+00$ & 58,102 & 26,318 & 2,086 & 205,430 & 7 \\
$7 / 1 / 1996$ & $0.0 \mathrm{E}+00$ & & & & & \\
10 & & & 2 \\
$1 / 1 / 1996$ & $0.0 \mathrm{E}+00$ & & & & 4 \\
$4 / 1 / 1997$ & $0.0 \mathrm{E}+00$ & & & & \\
\hline
\end{tabular}


Project: Y-12 National Security Complex

Location: CRSP
User Name: $\quad$ MV

State: Tennessee

\begin{tabular}{|c|c|c|c|c|c|c|c|}
\hline \multirow[b]{2}{*}{ Effective Date } & \multirow{2}{*}{$\begin{array}{l}\text { Oth Moment } \\
\text { Estimated } \\
\text { Mass (kg) }\end{array}$} & \multicolumn{3}{|c|}{ 1st Moment (Center of Mass) } & \multicolumn{2}{|c|}{ 2nd Moment (Spread) } & \multirow[b]{2}{*}{$\begin{array}{c}\text { Number of } \\
\text { Wells }\end{array}$} \\
\hline & & $\mathrm{Xc}(\mathrm{ft})$ & Yc (ft) & $\begin{array}{c}\text { Source } \\
\text { Distance (ft) }\end{array}$ & $\begin{array}{c}\text { Sigma } X X \\
(\mathrm{sq} f t)\end{array}$ & $\begin{array}{c}\text { Sigma } Y Y \\
(\mathrm{sq} f t)\end{array}$ & \\
\hline \multicolumn{8}{|c|}{ TETRACHLOROETHYLENE(PCE) } \\
\hline 7/1/1997 & $0.0 \mathrm{E}+00$ & & & & & & 5 \\
\hline 10/1/1997 & $0.0 \mathrm{E}+00$ & & & & & & 1 \\
\hline $1 / 1 / 1998$ & $5.4 \mathrm{E}-01$ & 58,179 & 27,001 & 1,440 & 444,525 & 578,852 & 6 \\
\hline 7/1/1998 & $0.0 \mathrm{E}+00$ & & & & & & 5 \\
\hline 1/1/1999 & $0.0 \mathrm{E}+00$ & & & & & & 4 \\
\hline 7/1/1999 & $0.0 \mathrm{E}+00$ & & & & & & 4 \\
\hline $1 / 1 / 2000$ & $0.0 \mathrm{E}+00$ & & & & & & 4 \\
\hline $7 / 1 / 2000$ & $0.0 \mathrm{E}+00$ & & & & & & 4 \\
\hline $1 / 1 / 2001$ & 7.6E-01 & 58,264 & 27,458 & 1,016 & 548,833 & 825,130 & 11 \\
\hline $7 / 1 / 2001$ & $6.7 \mathrm{E}-01$ & 58,136 & 27,384 & 1,155 & 371,120 & 804,652 & 9 \\
\hline $1 / 1 / 2002$ & $0.0 \mathrm{E}+00$ & & & & & & 3 \\
\hline $7 / 1 / 2002$ & $0.0 \mathrm{E}+00$ & & & & & & 3 \\
\hline $1 / 1 / 2003$ & $0.0 \mathrm{E}+00$ & & & & & & 3 \\
\hline $7 / 1 / 2003$ & $0.0 \mathrm{E}+00$ & & & & & & 3 \\
\hline $1 / 1 / 2004$ & $0.0 \mathrm{E}+00$ & & & & & & 3 \\
\hline $4 / 1 / 2004$ & 3.9E-01 & 58,553 & 28,197 & 362 & 321,832 & 116,390 & 14 \\
\hline $7 / 1 / 2004$ & $0.0 \mathrm{E}+00$ & & & & & & 3 \\
\hline $10 / 1 / 2004$ & 3.2E-01 & 58,466 & 28,182 & 450 & 330,131 & 128,156 & 14 \\
\hline
\end{tabular}

TRICHLOROETHYLENE (TCE)

\begin{tabular}{|c|c|c|c|c|c|c|c|}
\hline 1/1/1996 & 8.6E-02 & 58,818 & 28,375 & 164 & 313,641 & 23,227 & 8 \\
\hline 4/1/1996 & 6.4E-01 & 57,895 & 26,061 & 2,405 & 357,610 & 614,646 & 7 \\
\hline 7/1/1996 & $0.0 \mathrm{E}+00$ & & & & & & 3 \\
\hline 10/1/1996 & $0.0 \mathrm{E}+00$ & & & & & & 2 \\
\hline $1 / 1 / 1997$ & $0.0 \mathrm{E}+00$ & & & & & & 2 \\
\hline 4/1/1997 & $0.0 \mathrm{E}+00$ & & & & & & 4 \\
\hline $7 / 1 / 1997$ & $0.0 \mathrm{E}+00$ & & & & & & 5 \\
\hline 10/1/1997 & $0.0 \mathrm{E}+00$ & & & & & & 1 \\
\hline 1/1/1998 & 3.5E-01 & 57,873 & 26,642 & 1,906 & 292,246 & 474,812 & 6 \\
\hline $7 / 1 / 1998$ & $0.0 \mathrm{E}+00$ & & & & & & 5 \\
\hline 1/1/1999 & $0.0 \mathrm{E}+00$ & & & & & & 4 \\
\hline 7/1/1999 & $0.0 \mathrm{E}+00$ & & & & & & 4 \\
\hline $1 / 1 / 2000$ & $0.0 \mathrm{E}+00$ & & & & & & 4 \\
\hline $7 / 1 / 2000$ & $0.0 \mathrm{E}+00$ & & & & & & 4 \\
\hline $1 / 1 / 2001$ & 4.9E-01 & 57,902 & 27,015 & 1,588 & 389,291 & 705,876 & 11 \\
\hline $7 / 1 / 2001$ & 4.6E-01 & 57,835 & 26,996 & 1,646 & 192,779 & 654,374 & 9 \\
\hline $1 / 1 / 2002$ & $0.0 \mathrm{E}+00$ & & & & & & 3 \\
\hline $7 / 1 / 2002$ & $0.0 \mathrm{E}+00$ & & & & & & 3 \\
\hline $1 / 1 / 2003$ & $0.0 \mathrm{E}+00$ & & & & & & 3 \\
\hline $7 / 1 / 2003$ & $0.0 \mathrm{E}+00$ & & & & & & 3 \\
\hline $1 / 1 / 2004$ & $0.0 \mathrm{E}+00$ & & & & & & 3 \\
\hline $4 / 1 / 2004$ & 1.7E-01 & 58,162 & 28,017 & 782 & 311,500 & 136,840 & 14 \\
\hline $7 / 1 / 2004$ & $0.0 \mathrm{E}+00$ & & & & & & 3 \\
\hline
\end{tabular}


Project: Y-12 National Security Complex

Location: CRSP

\begin{tabular}{|c|c|c|c|c|c|c|c|}
\hline \multirow[b]{2}{*}{ Effective Date } & \multirow{2}{*}{$\begin{array}{l}\text { Oth Moment } \\
\text { Estimated } \\
\text { Mass (kg) }\end{array}$} & \multicolumn{3}{|c|}{ 1st Moment (Center of Mass) } & \multicolumn{2}{|c|}{ 2nd Moment (Spread) } & \multirow[b]{2}{*}{$\begin{array}{c}\text { Number of } \\
\text { Wells }\end{array}$} \\
\hline & & $\mathrm{Xc}(\mathrm{ft})$ & Yc (ft) & $\begin{array}{c}\text { Source } \\
\text { Distance (ft) }\end{array}$ & $\underset{(s q \mathrm{ft})}{\operatorname{Sigma} X X}$ & $\begin{array}{l}\text { Sigma } Y Y \\
\text { (sq ft) }\end{array}$ & \\
\hline
\end{tabular}

1.7E-01

58,162

28,017

782

311,500

136,840 
Project: Y-12 National Security Complex

Location: CRSP
User Name: $\mathrm{MV}$

State: Tennessee

\begin{tabular}{|c|c|c|c|c|c|}
\hline Moment Type & Constituent & $\begin{array}{l}\text { Coefficient } \\
\text { of Variation }\end{array}$ & $\begin{array}{c}\text { Mann-Kendall } \\
\text { S Statistic }\end{array}$ & $\begin{array}{c}\text { Confidence } \\
\text { in Trend }\end{array}$ & $\begin{array}{c}\text { Moment } \\
\text { Trend }\end{array}$ \\
\hline
\end{tabular}

Zeroth Moment: Mass

$\begin{array}{llcll}\text { 1,1-DICHLOROETHENE } & 1.77 & 22 & 69.7 \% & \text { NT } \\ \text { GROSS ALPHA ACTIVITY } & 2.74 & 20 & 69.0 \% & \text { NT } \\ \text { LEAD } & 1.91 & -1 & 50.0 \% & \text { NT } \\ \text { TETRACHLOROETHYLENE(PCE) } & 1.96 & -2 & 51.0 \% & \text { NT } \\ \text { TRICHLOROETHYLENE (TCE) } & 1.91 & 0 & 49.0 \% & \text { NT }\end{array}$

1st Moment: Distance to Source

1,1-DICHLOROETHENE
GROSS ALPHA ACTIVITY
LEAD
TETRACHLOROETHYLENE(PCE)
TRICHLOROETHYLENE (TCE)

0.76
0.58
0.62
0.72
0.59

2nd Moment: Sigma XX

1,1-DICHLOROETHENE
GROSS ALPHA ACTIVITY
LEAD
TETRACHLOROETHYLENE(PCE)
TRICHLOROETHYLENE (TCE)

$\begin{array}{lccc}0.31 & -17 & 99.5 \% & \text { D } \\ 0.37 & -10 & 99.2 \% & \text { D } \\ 0.20 & -7 & 80.9 \% & \text { S } \\ 0.33 & 3 & 61.4 \% & \text { NT } \\ 0.20 & -5 & 71.9 \% & \text { S }\end{array}$

2nd Moment: Sigma YY

\begin{tabular}{llclc} 
1,1-DICHLOROETHENE & 0.83 & -1 & $50.0 \%$ & $\mathrm{~S}$ \\
GROSS ALPHA ACTIVITY & 0.80 & -8 & $95.8 \%$ & $\mathrm{D}$ \\
LEAD & 0.76 & 1 & $50.0 \%$ & NT \\
TETRACHLOROETHYLENE(PCE) & 0.83 & 3 & $61.4 \%$ & NT \\
TRICHLOROETHYLENE (TCE) & 0.73 & 1 & $50.0 \%$ & NT \\
\hline
\end{tabular}

Note: The following assumptions were applied for the calculation of the Zeroth Moment:

Porosity: $0.10 \quad$ Saturated Thickness: Uniform: $50 \mathrm{ft}$

Mann-Kendall Trend test performed on all sample events for each constituent. Increasing (I); Probably Increasing (PI); Stable (S); Probably Decreasing (PD); Decreasing (D); No Trend (NT); Not Applicable (N/A)-Due to insufficient Data (< 4 sampling events).

Note: The Sigma $X X$ and Sigma $Y Y$ components are estimated using the given field coordinate system and then rotated to align with the estimated groundwater flow direction. Moments are not calculated for sample events with less than 6 wells. 


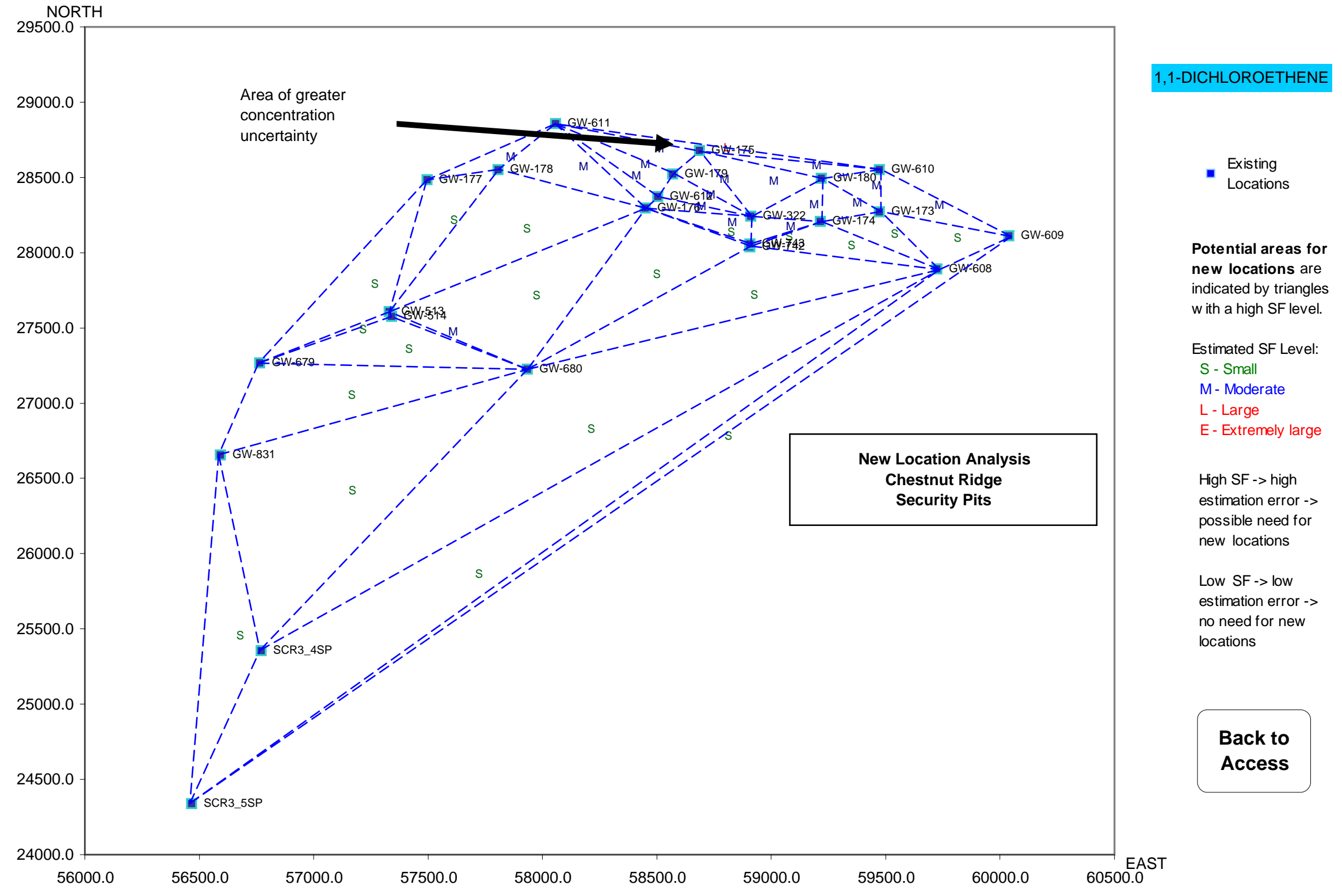




\title{
ASSESSMENT OF THE GROUNDWATER PROTECTION PROGRAM \\ Y-12 NATIONAL SECURITY COMPLEX, OAK RIDGE, TENNESSEE
}

Y-12 National Security Complex

Oak Ridge, Tennessee

\author{
Appendix D.10 Chestnut Ridge Regime Landfills V and VII \\ Table D.10.1 Qualitative Analysis Chestnut Ridge Landfills V and VII \\ Table D.10.2 Aquifer Input Parameters \\ MAROS Report COC Assessment \\ MAROS Report Plume Summary \\ MAROS Report Spatial Moment Analysis Summary \\ (No Well Sufficiency areas of interest identified)
}


TABLE D.10.1

QUALITATIVE ANALYSIS CHESTNUT RIDGE REGIME LANDFILL V AND VII

Chestnut Ridge Hydrogeologic Regime

Y-12 National Security Complex

Oak Ridge, Tennessee

\begin{tabular}{|c|c|c|c|c|c|c|c|c|c|c|c|c|}
\hline $\begin{array}{c}\text { Location } \\
\text { Name }\end{array}$ & $\begin{array}{c}\text { Location } \\
\text { Type }\end{array}$ & \begin{tabular}{|c|} 
Average \\
Concentration \\
Exceeds \\
Screening Level \\
\end{tabular} & $\begin{array}{c}\text { Formation } \\
\text { Type }\end{array}$ & $\begin{array}{c}\text { Horizontal } \\
\text { Delineation }\end{array}$ & $\begin{array}{c}\text { Vertical } \\
\text { Delineation }\end{array}$ & $\begin{array}{c}\text { Exit } \\
\text { Location }\end{array}$ & RCRA & CERCLA & Unique & \begin{tabular}{|c} 
Monitors \\
Background \\
Water Quality \\
\end{tabular} & $\begin{array}{c}\text { Early } \\
\text { Detection }\end{array}$ & $\begin{array}{l}\text { Monitor } \\
\text { source }\end{array}$ \\
\hline $\begin{array}{l}\text { GW-557 } \\
\text { GW-560 } \\
\text { GW-562 } \\
\text { GW-564 } \\
\text { GW-796 }\end{array}$ & $\begin{array}{l}\text { WL } \\
W L \\
W L \\
W L \\
W L\end{array}$ & & $\begin{array}{l}\text { Aquifer } \\
\text { Aquifer } \\
\text { Aquifer } \\
\text { Aquifer } \\
\text { Aquifer }\end{array}$ & $\begin{array}{l}\mathrm{X} \\
\mathrm{x}\end{array}$ & & & $\begin{array}{l}\mathrm{X} \\
\mathrm{x}\end{array}$ & & & $\begin{array}{l}X \\
X\end{array}$ & & $\bar{x}$ \\
\hline \begin{tabular}{|l|} 
GW-797 \\
GW-798 \\
GW-799 \\
GW-801 \\
SCR4.3SP
\end{tabular} & $\begin{array}{l}\text { WL } \\
W L \\
W L \\
W L \\
\text { SP }\end{array}$ & & $\begin{array}{c}\text { Aquifer } \\
\text { Aquifer } \\
\text { Aquifer } \\
\text { Aquifer } \\
\text { Spring }\end{array}$ & $\begin{array}{l}X \\
X\end{array}$ & $x$ & $x$ & $\begin{array}{l}X \\
X \\
X\end{array}$ & & & $\begin{array}{l}x \\
x\end{array}$ & & $x$ \\
\hline
\end{tabular}

\section{Notes:}

1. $\mathrm{WL}=$ Monitoring Well; $\mathrm{SP}=$ Spring

2. Well data taken from BWXT Y-12 Analytical Database. Sample locations shown on Figures A.1 and A.4.

3. RCRA indicates wells monitored as part of compliance with RCRA Post-Closure Corrective Action Monitoring or designated Alternate location;

CERCLA indicates locations monitored as part of compliance with CERCLA ROD or backup location. Data from BWXT, 2003a and BWXT 2004a.

4. Average Concentration Exceeds Screening = The average concentration over the entire sampling record for the priority constituent is above the MCL or other designated screening level as defined in Table B.1.

5. Aquifer and aquitard formations identified in Fig. A.2 from BWXT Y12, 2003 Groundwater Monitoring Report, (12/01/2003).

6. Details of the decision criteria for each category are presented in the text. 
GSI Job No. G-3038

Issued $12 / 12 / 2005$

Page 1 of 1

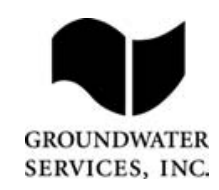

TABLE D.10.2

AQUIFER INPUT PARAMETERS

\author{
Landfills $\mathrm{V}$ and VII \\ Chestnut Ridge Regime \\ Y-12 National Security Complex
}

\begin{tabular}{|l|c|c|}
\hline \multicolumn{1}{|c|}{ Parameter } & Value & Units \\
\hline Current Plume Length & 5500 & $\mathrm{ft}$ \\
Maximum Plume Length & 5500 & $\mathrm{ft}$ \\
PlumeWidth & 2000 & $\mathrm{ft}$ \\
SeepageVelocity (ft/yr) & 200 & $\mathrm{ft} / \mathrm{yr}$ \\
Distance to Receptors & 6000 & $\mathrm{ft}$ \\
GWFluctuations & Yes & -- \\
SourceTreatment & None & -- \\
PlumeType & VOC/Metals & -- \\
Free NAPL Present & Yes & -- \\
\hline \multicolumn{1}{|c|}{ Parameter } & Value & \\
\hline Groundwater flow direction & $\mathrm{S}$ & 270 \\
Effective Porosity & 0.1 & -- \\
Source Location near Well & GW-796 & -- \\
Source X-Coordinate & 58206.4 & $\mathrm{ft}$ \\
Source Y-Coordinate & 27923.9 & $\mathrm{ft}^{\star}$ \\
Saturated Thickness & 50 & $\mathrm{ft}$ \\
\hline \multicolumn{2}{|c|}{ Source Wells $\mathrm{GW-796,} \mathrm{GW-797,} \mathrm{GW-801}$} \\
\hline \multicolumn{2}{|c|}{} \\
\hline
\end{tabular}

Notes:

1. Aquifer data are general values for the hydrologic regime.

2. Priority COCs defined by prevalence, toxicty and mobility.

3. $\mathrm{ft}^{\star}=$ Coordinates in $\mathrm{Y}-12$ Plant coordinates, feet.

4. Screening Levels are USEPA MCLs, except in the case of compounds without MCLs where the level is the Region 9 PRG for tap water.

5. Effective Porosity estimated based on average high and low values for aquifer and aquitard suburfaces. 


\section{MAROS COC Assessment}

Project: $\quad$ Y-12 Security Complex

Location: CR_LandfillsV_VII

Toxicity:

Contaminant of Concern

LEAD
User Name: $\mathrm{MV}$

State: Tennessee

Note: Top COCs by toxicity were determined by examining a representative concentration for each compound over the entire site. The compound representative concentrations are then compared with the chosen PRG for that compound, with the percentage excedence from the PRG determining the compound's toxicity. All compounds above exceed the PRG.

\section{Prevalence:}

\begin{tabular}{|c|c|c|c|c|c|}
\hline Contaminant of Concern & Class & $\begin{array}{l}\text { Total } \\
\text { Wells }\end{array}$ & $\begin{array}{c}\text { Total } \\
\text { Excedences }\end{array}$ & $\begin{array}{c}\text { Percent } \\
\text { Excedences }\end{array}$ & $\begin{array}{l}\text { Total } \\
\text { detects }\end{array}$ \\
\hline LEAD & MET & 10 & 10 & $100.0 \%$ & 10 \\
\hline
\end{tabular}

Note: Top COCs by prevalence were determined by examining a representative concentration for each well location at the site. The total excedences (values above the chosen PRGs) are compared to the total number of wells to determine the prevalence of the compound.

\section{Mobility:}

Contaminant of Concern

Kd

\begin{tabular}{ll}
\hline LEAD & 10
\end{tabular}

Note: Top COCs by mobility were determined by examining each detected compound in the dataset and comparing their mobilities (Koc's for organics, assume foc $=0.001$, and Kd's for metals).

\section{Contaminants of Concern (COC's)}

LEAD

TETRACHLOROETHYLENE(PCE)

BERYLLIUM

CHROMIUM III 


\section{MAROS Plume Analysis Summary}

Project: Y-12 Security Complex

Location: Landfills V and VII

Time Period: 1/1/1996

to $1 / 1 / 2005$

Consolidation Period: No Time Consolidation

Consolidation Type: Median

Duplicate Consolidation: Average

ND Values: Specified Detection Limit

J Flag Values : Actual Value

\begin{tabular}{|c|c|c|c|c|c|c|c|c|c|c|c|}
\hline Constituent & Well & $\begin{array}{l}\text { Sourcel } \\
\text { Tail }\end{array}$ & $\begin{array}{c}\text { Number } \\
\text { of } \\
\text { Samples }\end{array}$ & $\begin{array}{c}\text { Number } \\
\text { of } \\
\text { Detects }\end{array}$ & $\begin{array}{c}\text { Average } \\
\text { (mg/L) }\end{array}$ & $\begin{array}{r}\text { Median } \\
\text { (mg/L) }\end{array}$ & $\begin{array}{c}\text { All } \\
\text { Samples } \\
\text { "ND" ? }\end{array}$ & $\begin{array}{l}\text { Mann- } \\
\text { Kendall }\end{array}$ & $\begin{array}{c}\text { Linear } \\
\text { Regression }\end{array}$ & Modeling & Empirical \\
\hline \multicolumn{12}{|l|}{ BERYLLIUM } \\
\hline & GW-796 & $\mathrm{s}$ & 18 & 0 & $2.0 \mathrm{E}-05$ & 2.0E-05 & Yes & $\mathrm{S}$ & 1 & $\mathrm{~N} / \mathrm{A}$ & $\mathrm{N} / \mathrm{A}$ \\
\hline & GW-801 & s & 18 & 0 & 2.0E-05 & 2.0E-05 & Yes & $\mathrm{s}$ & I & $\mathrm{N} / \mathrm{A}$ & $\mathrm{N} / \mathrm{A}$ \\
\hline & GW-797 & $\mathrm{s}$ & 18 & 0 & $2.0 \mathrm{E}-05$ & 2.0E-05 & Yes & $\mathrm{s}$ & 1 & $\mathrm{~N} / \mathrm{A}$ & $\mathrm{N} / \mathrm{A}$ \\
\hline & GW-799 & $\mathrm{T}$ & 18 & 1 & 7.6E-05 & 2.0E-05 & No & NT & NT & $N / A$ & $N / A$ \\
\hline & SCR4_3SP & $\mathrm{T}$ & 18 & 0 & 2.0E-05 & 2.0E-05 & Yes & $\mathrm{s}$ & I & $\mathrm{N} / \mathrm{A}$ & N/A \\
\hline & GW-560 & $\mathrm{T}$ & 15 & 0 & $2.0 \mathrm{E}-05$ & 2.0E-05 & Yes & $\mathrm{s}$ & $\mathrm{s}$ & $\mathrm{N} / \mathrm{A}$ & N/A \\
\hline & GW-562 & $\mathrm{T}$ & 15 & 0 & 2.0E-05 & 2.0E-05 & Yes & $\mathrm{s}$ & S & $N / A$ & N/A \\
\hline & GW-798 & $\mathrm{T}$ & 21 & 0 & 2.0E-05 & 2.0E-05 & Yes & $\mathrm{s}$ & $\mathrm{s}$ & N/A & $\mathrm{N} / \mathrm{A}$ \\
\hline & GW-557 & $\mathrm{T}$ & 18 & 0 & 2.0E-05 & 2.0E-05 & Yes & $\mathrm{s}$ & I & N/A & N/A \\
\hline & GW-564 & $\mathrm{T}$ & 15 & 0 & $2.0 \mathrm{E}-05$ & 2.0E-05 & Yes & $\mathrm{s}$ & $\mathrm{s}$ & $N / A$ & N/A \\
\hline \multicolumn{12}{|l|}{ CHROMIUM III } \\
\hline & GW-797 & $\mathrm{S}$ & 18 & 1 & $5.4 \mathrm{E}-04$ & 2.6E-04 & No & NT & NT & $\mathrm{N} / \mathrm{A}$ & $\mathrm{N} / \mathrm{A}$ \\
\hline & GW-796 & $\mathrm{s}$ & 18 & 1 & $1.6 \mathrm{E}-03$ & 2.6E-04 & No & NT & NT & $N / A$ & $N / A$ \\
\hline & GW-801 & $\mathrm{s}$ & 18 & 0 & $2.6 \mathrm{E}-04$ & 2.6E-04 & Yes & $\mathrm{S}$ & $\mathrm{S}$ & $N / A$ & $\mathrm{~N} / \mathrm{A}$ \\
\hline & GW-798 & $\mathrm{T}$ & 21 & 0 & $2.6 \mathrm{E}-04$ & 2.6E-04 & Yes & $\mathrm{s}$ & D & $N / A$ & N/A \\
\hline & GW-564 & $\mathrm{T}$ & 15 & 0 & 2.6E-04 & 2.6E-04 & Yes & $\mathrm{S}$ & $\mathrm{s}$ & $N / A$ & $N / A$ \\
\hline & GW-562 & $\mathrm{T}$ & 15 & 0 & $2.6 \mathrm{E}-04$ & 2.6E-04 & Yes & $\mathrm{s}$ & $\mathrm{s}$ & $\mathrm{N} / \mathrm{A}$ & N/A \\
\hline & GW-560 & $\mathrm{T}$ & 15 & 1 & $1.2 \mathrm{E}-03$ & 2.6E-04 & No & NT & NT & $N / A$ & N/A \\
\hline & SCR4_3SP & $\mathrm{T}$ & 18 & 0 & 2.6E-04 & 2.6E-04 & Yes & $S$ & $S$ & $\mathrm{~N} / \mathrm{A}$ & $\mathrm{N} / \mathrm{A}$ \\
\hline & GW-557 & $\mathrm{T}$ & 18 & 2 & 4.4E-03 & 2.6E-04 & No & NT & PD & $N / A$ & $\mathrm{~N} / \mathrm{A}$ \\
\hline & GW-799 & $\mathrm{T}$ & 18 & 14 & 3.4E-02 & 3.9E-02 & No & I & I & $N / A$ & $N / A$ \\
\hline
\end{tabular}

User Name: MV

State: Tennessee 
Project: Y-12 Security Complex

Location: LandfillsV and VII
User Name: $\quad$ MV

State: Tennessee

\begin{tabular}{|c|c|c|c|c|c|c|c|c|c|c|c|}
\hline Constituent & Well & $\begin{array}{c}\text { Sourcel } \\
\text { Tail }\end{array}$ & $\begin{array}{c}\text { Number } \\
\text { of } \\
\text { Samples }\end{array}$ & $\begin{array}{c}\text { Number } \\
\text { of } \\
\text { Detects }\end{array}$ & $\begin{array}{c}\text { Average } \\
\text { (mg/L) }\end{array}$ & $\begin{array}{r}\text { Median } \\
(\mathrm{mg} / \mathrm{L})\end{array}$ & $\begin{array}{c}\text { All } \\
\text { Samples } \\
\text { "ND" ? }\end{array}$ & $\begin{array}{l}\text { Mann- } \\
\text { Kendall }\end{array}$ & $\begin{array}{c}\text { Linear } \\
\text { Regression }\end{array}$ & Modeling & Empirical \\
\hline \multicolumn{12}{|l|}{ LEAD } \\
\hline \multicolumn{12}{|l|}{ LEAD } \\
\hline & GW-797 & $\mathrm{s}$ & 18 & 5 & $9.9 \mathrm{E}-03$ & $5.2 \mathrm{E}-05$ & No & $\mathrm{D}$ & $\mathrm{D}$ & $\mathrm{N} / \mathrm{A}$ & $\mathrm{N} / \mathrm{A}$ \\
\hline & GW-801 & $\mathrm{s}$ & 18 & 1 & $1.3 \mathrm{E}-04$ & 5.2E-05 & No & NT & NT & N/A & N/A \\
\hline & GW-796 & s & 18 & 3 & $2.4 \mathrm{E}-04$ & $5.2 \mathrm{E}-05$ & No & NT & PD & N/A & $\mathrm{N} / \mathrm{A}$ \\
\hline & GW-562 & $\mathrm{T}$ & 15 & 3 & $1.1 \mathrm{E}-04$ & $5.2 \mathrm{E}-05$ & No & PD & D & N/A & $\mathrm{N} / \mathrm{A}$ \\
\hline & SCR4_3SP & $\mathrm{T}$ & 18 & 4 & $1.8 \mathrm{E}-04$ & $5.2 \mathrm{E}-05$ & No & NT & D & $N / A$ & $\mathrm{~N} / \mathrm{A}$ \\
\hline & GW-557 & $\mathrm{T}$ & 18 & 4 & $3.0 \mathrm{E}-04$ & $5.2 \mathrm{E}-05$ & No & NT & D & N/A & N/A \\
\hline & GW-560 & $\mathrm{T}$ & 15 & 3 & $2.1 \mathrm{E}-04$ & 5.2E-05 & No & PD & D & N/A & $\mathrm{N} / \mathrm{A}$ \\
\hline & GW-799 & $\mathrm{T}$ & 18 & 2 & $2.1 \mathrm{E}-04$ & $5.2 \mathrm{E}-05$ & No & NT & PD & N/A & N/A \\
\hline & GW-798 & $\mathrm{T}$ & 21 & 2 & $7.9 \mathrm{E}-05$ & 5.2E-05 & No & NT & D & N/A & $\mathrm{N} / \mathrm{A}$ \\
\hline & GW-564 & $\mathrm{T}$ & 15 & 2 & $2.2 \mathrm{E}-04$ & $5.2 \mathrm{E}-05$ & No & NT & D & N/A & $\mathrm{N} / \mathrm{A}$ \\
\hline \multicolumn{12}{|c|}{ TETRACHLOROETHYLENE(PCE) } \\
\hline & GW-796 & $\mathrm{s}$ & 18 & 0 & $5.0 \mathrm{E}-04$ & $5.0 \mathrm{E}-04$ & Yes & $\mathrm{s}$ & $\mathrm{s}$ & N/A & $\mathrm{N} / \mathrm{A}$ \\
\hline & GW-801 & $\mathrm{s}$ & 18 & 0 & $5.0 \mathrm{E}-04$ & $5.0 \mathrm{E}-04$ & Yes & $\mathrm{s}$ & s & N/A & N/A \\
\hline & GW-797 & s & 18 & 0 & $5.0 \mathrm{E}-04$ & 5.0E-04 & Yes & s & s & N/A & $\mathrm{N} / \mathrm{A}$ \\
\hline & GW-562 & $\mathrm{T}$ & 15 & 0 & $5.0 \mathrm{E}-04$ & 5.0E-04 & Yes & $\mathrm{s}$ & s & N/A & $\mathrm{N} / \mathrm{A}$ \\
\hline & GW-798 & $\mathrm{T}$ & 21 & 13 & $2.8 \mathrm{E}-03$ & $2.0 \mathrm{E}-03$ & No & 1 & I & N/A & N/A \\
\hline & GW-560 & $\mathrm{T}$ & 15 & 0 & $5.0 \mathrm{E}-04$ & 5.0E-04 & Yes & $\mathrm{s}$ & s & N/A & N/A \\
\hline & GW-564 & $\mathrm{T}$ & 15 & 0 & $5.0 \mathrm{E}-04$ & 5.0E-04 & Yes & s & s & N/A & N/A \\
\hline & GW-557 & $\mathrm{T}$ & 18 & 0 & $5.0 \mathrm{E}-04$ & $5.0 \mathrm{E}-04$ & Yes & S & S & N/A & N/A \\
\hline & SCR4_3SP & $\mathrm{T}$ & 18 & 0 & $5.0 \mathrm{E}-04$ & 5.0E-04 & Yes & S & s & N/A & N/A \\
\hline & GW-799 & $\mathrm{T}$ & 18 & 0 & $5.0 \mathrm{E}-04$ & 5.0E-04 & Yes & $\mathrm{s}$ & s & N/A & N/A \\
\hline
\end{tabular}

Note: Increasing (I); Probably Increasing (PI); Stable (S); Probably Decreasing (PD); Decreasing (D); No Trend (NT); Not Applicable (N/A) - Due to insufficient Data (< 4 sampling events); Source/Tail (S/T)

The Number of Samples and Number of Detects shown above are post-consolidation values. 


\section{MAROS Spatial Moment Analysis Summary}

Project: Y-12 Security Complex

Location: Landfills V and VII
User Name: MV

State: Tennessee

\begin{tabular}{|c|c|c|c|c|c|c|c|}
\hline \multirow[b]{2}{*}{ Effective Date } & \multirow{2}{*}{$\begin{array}{l}\text { 0th Moment } \\
\text { Estimated } \\
\text { Mass (Kg) }\end{array}$} & \multicolumn{3}{|c|}{ 1st Moment (Center of Mass) } & \multicolumn{2}{|c|}{ 2nd Moment (Spread) } & \multirow[b]{2}{*}{$\begin{array}{c}\text { Number o } \\
\text { Wells }\end{array}$} \\
\hline & & $X c(f t)$ & Yc (ft) & $\begin{array}{c}\text { Source } \\
\text { Distance (ft) }\end{array}$ & $\underset{(\mathrm{sq} \mathrm{ft})}{\operatorname{Sigma} X X}$ & $\begin{array}{c}\text { Sigma YY } \\
(\mathrm{sq} \mathrm{ft})\end{array}$ & \\
\hline
\end{tabular}

BERYLLIUM

\begin{tabular}{|c|c|c|c|c|c|c|c|}
\hline 4/1/1996 & 1.2E-02 & 60,145 & 26,465 & 2,427 & 599,576 & 394,724 & 10 \\
\hline 10/1/1996 & 1.2E-02 & 60,145 & 26,465 & 2,427 & 599,576 & 394,724 & 10 \\
\hline 1/1/1997 & 1.2E-02 & 60,145 & 26,465 & 2,427 & 599,576 & 394,724 & 10 \\
\hline $7 / 1 / 1997$ & $1.2 \mathrm{E}-02$ & 60,145 & 26,465 & 2,427 & 599,576 & 394,724 & 10 \\
\hline 1/1/1998 & 8.8E-03 & 59,795 & 26,615 & 2,059 & 330,386 & 324,786 & 7 \\
\hline 7/1/1998 & 8.8E-03 & 59,795 & 26,615 & 2,059 & 330,386 & 324,786 & 7 \\
\hline 1/1/1999 & 8.8E-03 & 59,795 & 26,615 & 2,059 & 330,386 & 324,786 & 7 \\
\hline 7/1/1999 & 8.8E-03 & 59,795 & 26,615 & 2,059 & 330,386 & 324,786 & 7 \\
\hline $1 / 1 / 2000$ & 2.7E-02 & 59,896 & 26,552 & 2,176 & 298,732 & 252,946 & 7 \\
\hline $7 / 1 / 2000$ & 1.2E-02 & 60,145 & 26,465 & 2,427 & 599,576 & 394,724 & 10 \\
\hline $10 / 1 / 2000$ & $0.0 \mathrm{E}+00$ & & & & & & 4 \\
\hline $1 / 1 / 2001$ & 8.8E-03 & 59,795 & 26,615 & 2,059 & 330,386 & 324,786 & 7 \\
\hline 4/1/2001 & $0.0 \mathrm{E}+00$ & & & & & & 4 \\
\hline $7 / 1 / 2001$ & 1.2E-02 & 60,145 & 26,465 & 2,427 & 599,576 & 394,724 & 10 \\
\hline $10 / 1 / 2001$ & $0.0 \mathrm{E}+00$ & & & & & & 4 \\
\hline $1 / 1 / 2002$ & 1.2E-02 & 60,145 & 26,465 & 2,427 & 599,576 & 394,724 & 10 \\
\hline $7 / 1 / 2002$ & 1.2E-02 & 60,145 & 26,465 & 2,427 & 599,576 & 394,724 & 10 \\
\hline $1 / 1 / 2003$ & 1.2E-02 & 60,145 & 26,465 & 2,427 & 599,576 & 394,724 & 10 \\
\hline $7 / 1 / 2003$ & 1.2E-02 & 60,145 & 26,465 & 2,427 & 599,576 & 394,724 & 10 \\
\hline $1 / 1 / 2004$ & 1.2E-02 & 60,145 & 26,465 & 2,427 & 599,576 & 394,724 & 10 \\
\hline $7 / 1 / 2004$ & 1.2E-02 & 60,145 & 26,465 & 2,427 & 599,576 & 394,724 & 10 \\
\hline
\end{tabular}

CHROMIUM III

$\begin{array}{rrrrrrrr}4 / 1 / 1996 & 2.6 \mathrm{E}-01 & 59,911 & 26,469 & 2,241 & 469,790 & 251,462 & 10 \\ 10 / 1 / 1996 & 1.6 \mathrm{E}-01 & 60,145 & 26,465 & 2,427 & 599,576 & 394,724 & 10 \\ 1 / 1 / 1997 & 1.6 \mathrm{E}-01 & 60,145 & 26,465 & 2,427 & 599,576 & 394,724 & 10 \\ 7 / 1 / 1997 & 1.6 \mathrm{E}-01 & 60,145 & 26,465 & 2,427 & 599,576 & 394,724 & 10 \\ 1 / 1 / 1998 & 4.8 \mathrm{E}-01 & 59,909 & 26,544 & 2,192 & 293,134 & 243,045 & 7 \\ 7 / 1 / 1998 & 4.2 \mathrm{E}-01 & 59,904 & 26,547 & 2,186 & 295,097 & 246,464 & 7 \\ 1 / 1 / 1999 & 4.4 \mathrm{E}-01 & 59,905 & 26,546 & 2,188 & 294,627 & 245,641 & 7 \\ 7 / 1 / 1999 & 9.6 \mathrm{E}-01 & 59,975 & 26,348 & 2,369 & 212,154 & 203,926 & 7 \\ 1 / 1 / 2000 & 4.5 \mathrm{E}-01 & 59,907 & 26,546 & 2,189 & 294,062 & 244,655 & 7 \\ 7 / 1 / 2000 & 4.9 \mathrm{E}-01 & 59,889 & 26,617 & 2,131 & 359,813 & 240,324 & 10 \\ 10 / 1 / 2000 & 0.0 \mathrm{E}+00 & & & & & & 4 \\ 1 / 1 / 2001 & 6.1 \mathrm{E}-01 & 59,824 & 26,634 & 2,069 & 343,879 & 299,616 & 7 \\ 4 / 1 / 2001 & 0.0 \mathrm{E}+00 & & & & & & 4 \\ 7 / 1 / 2001 & 5.4 \mathrm{E}-01 & 59,878 & 26,623 & 2,118 & 347,006 & 232,910 & 10 \\ 10 / 1 / 2001 & 0.0 \mathrm{E}+00 & & & & & & 4 \\ 1 / 1 / 2002 & 5.4 \mathrm{E}-01 & 59,878 & 26,623 & 2,118 & 347,006 & 232,910 & 10\end{array}$


Project: Y-12 Security Complex

Location: LandfillsV and VII
User Name: MV

State: Tennessee

\begin{tabular}{|c|c|c|c|c|c|c|c|}
\hline \multirow[b]{2}{*}{ Effective Date } & \multirow{2}{*}{$\begin{array}{l}\text { Oth Moment } \\
\text { Estimated } \\
\text { Mass (kg) }\end{array}$} & \multicolumn{3}{|c|}{ 1st Moment (Center of Mass) } & \multicolumn{2}{|c|}{ 2nd Moment (Spread) } & \multirow[b]{2}{*}{$\begin{array}{c}\text { Number of } \\
\text { Wells }\end{array}$} \\
\hline & & $X c(f t)$ & Yc (ft) & $\begin{array}{c}\text { Source } \\
\text { Distance (ft) }\end{array}$ & $\begin{array}{l}\text { Sigma } X X \\
(s q \mathrm{ft})\end{array}$ & $\begin{array}{l}\text { Sigma YY } \\
(\mathrm{sq} f t)\end{array}$ & \\
\hline JM III & & & & & & & \\
\hline $7 / 1 / 2002$ & 8.6E-01 & 59,670 & 26,822 & 1,832 & 309,568 & 210,387 & 10 \\
\hline $1 / 1 / 2003$ & 4.9E-01 & 59,890 & 26,616 & 2,132 & 361,694 & 241,417 & 10 \\
\hline $7 / 1 / 2003$ & 5.0E-01 & 59,887 & 26,618 & 2,129 & 358,030 & 239,288 & 10 \\
\hline $1 / 1 / 2004$ & 4.7E-01 & 59,896 & 26,613 & 2,139 & 368,023 & 245,104 & 10 \\
\hline $7 / 1 / 2004$ & 5.1E-01 & 59,885 & 26,619 & 2,126 & 355,517 & 237,831 & 10 \\
\hline
\end{tabular}

LEAD

\begin{tabular}{|c|c|c|c|c|c|c|c|}
\hline 4/1/1996 & $3.2 \mathrm{E}-02$ & 60,145 & 26,465 & 2,427 & 599,576 & 394,724 & 10 \\
\hline 10/1/1996 & 2.2E-01 & 59,979 & 26,665 & 2,174 & 591,738 & 442,789 & 10 \\
\hline $1 / 1 / 1997$ & 2.3E-01 & 60,535 & 26,207 & 2,894 & 423,145 & 361,862 & 10 \\
\hline 7/1/1997 & 4.6E-01 & 59,696 & 26,695 & 1,931 & 593,115 & 350,095 & 10 \\
\hline 1/1/1998 & 7.0E-02 & 59,892 & 26,441 & 2,246 & 263,924 & 279,755 & 7 \\
\hline 7/1/1998 & 2.3E-01 & 59,234 & 27,226 & 1,242 & 171,842 & 171,206 & 7 \\
\hline 1/1/1999 & 4.8E-02 & 59,747 & 26,679 & 1,981 & 340,459 & 340,932 & 7 \\
\hline 7/1/1999 & 5.7E-02 & 60,017 & 26,293 & 2,437 & 210,579 & 215,996 & 7 \\
\hline $1 / 1 / 2000$ & 2.3E-02 & 59,795 & 26,615 & 2,059 & 330,386 & 324,786 & 7 \\
\hline $7 / 1 / 2000$ & 3.2E-02 & 60,145 & 26,465 & 2,427 & 599,576 & 394,724 & 10 \\
\hline $10 / 1 / 2000$ & $0.0 \mathrm{E}+00$ & & & & & & 4 \\
\hline $1 / 1 / 2001$ & 2.3E-02 & 59,795 & 26,615 & 2,059 & 330,386 & 324,786 & 7 \\
\hline $4 / 1 / 2001$ & $0.0 \mathrm{E}+00$ & & & & & & 4 \\
\hline $7 / 1 / 2001$ & 3.2E-02 & 60,145 & 26,465 & 2,427 & 599,576 & 394,724 & 10 \\
\hline $10 / 1 / 2001$ & $0.0 \mathrm{E}+00$ & & & & & & 4 \\
\hline $1 / 1 / 2002$ & 3.2E-02 & 60,145 & 26,465 & 2,427 & 599,576 & 394,724 & 10 \\
\hline $7 / 1 / 2002$ & 3.2E-02 & 60,145 & 26,465 & 2,427 & 599,576 & 394,724 & 10 \\
\hline $1 / 1 / 2003$ & 3.2E-02 & 60,145 & 26,465 & 2,427 & 599,576 & 394,724 & 10 \\
\hline $7 / 1 / 2003$ & 3.2E-02 & 60,145 & 26,465 & 2,427 & 599,576 & 394,724 & 10 \\
\hline $1 / 1 / 2004$ & 3.2E-02 & 60,145 & 26,465 & 2,427 & 599,576 & 394,724 & 10 \\
\hline $7 / 1 / 2004$ & 3.2E-02 & 60,145 & 26,465 & 2,427 & 599,576 & 394,724 & 10 \\
\hline
\end{tabular}

TETRACHLOROETHYLENE(PCE)

\begin{tabular}{|c|c|c|c|c|c|c|c|}
\hline 4/1/1996 & 3.1E-01 & 60,145 & 26,465 & 2,427 & 599,576 & 394,724 & 10 \\
\hline 10/1/1996 & 3.1E-01 & 60,145 & 26,465 & 2,427 & 599,576 & 394,724 & 10 \\
\hline 1/1/1997 & 3.1E-01 & 60,145 & 26,465 & 2,427 & 599,576 & 394,724 & 10 \\
\hline 7/1/1997 & 3.1E-01 & 60,145 & 26,465 & 2,427 & 599,576 & 394,724 & 10 \\
\hline 1/1/1998 & 2.2E-01 & 59,795 & 26,615 & 2,059 & 330,386 & 324,786 & 7 \\
\hline 7/1/1998 & 2.2E-01 & 59,795 & 26,615 & 2,059 & 330,386 & 324,786 & 7 \\
\hline 1/1/1999 & 2.2E-01 & 59,795 & 26,615 & 2,059 & 330,386 & 324,786 & 7 \\
\hline 7/1/1999 & 2.2E-01 & 59,795 & 26,615 & 2,059 & 330,386 & 324,786 & 7 \\
\hline $1 / 1 / 2000$ & 2.2E-01 & 59,795 & 26,615 & 2,059 & 330,386 & 324,786 & 7 \\
\hline $7 / 1 / 2000$ & 4.0E-01 & 60,145 & 26,545 & 2,379 & 584,633 & 373,618 & 10 \\
\hline $10 / 1 / 2000$ & $0.0 \mathrm{E}+00$ & & & & & & 4 \\
\hline $1 / 1 / 2001$ & $2.5 \mathrm{E}-01$ & 59,802 & 26,643 & 2,047 & 336,585 & 328,165 & 7 \\
\hline $4 / 1 / 2001$ & $0.0 \mathrm{E}+00$ & & & & & & 4 \\
\hline
\end{tabular}


Project: Y-12 Security Complex

Location: LandfillsV and VII

\begin{tabular}{|c|c|c|c|c|c|c|c|}
\hline \multirow[b]{2}{*}{ Effective Date } & \multirow{2}{*}{$\begin{array}{l}\text { 0th Moment } \\
\begin{array}{r}\text { Estimated } \\
\text { Mass (kg) }\end{array}\end{array}$} & \multicolumn{3}{|c|}{ 1st Moment (Center of Mass) } & \multicolumn{2}{|c|}{ 2nd Moment (Spread) } & \multirow[b]{2}{*}{$\begin{array}{c}\text { Number of } \\
\text { Wells }\end{array}$} \\
\hline & & $X c(f t)$ & Yc (ft) & $\begin{array}{c}\text { Source } \\
\text { Distance (ft) }\end{array}$ & $\underset{(s q \mathrm{ft})}{\text { Sigma } X X}$ & $\underset{\text { (sq ft) }}{\text { Sigma YY }}$ & \\
\hline
\end{tabular}

TETRACHLOROETHYLENE(PCE)

\begin{tabular}{rrrrrrrr}
\hline $7 / 1 / 2001$ & $4.2 \mathrm{E}-01$ & 60,145 & 26,563 & 2,369 & 581,457 & 367,473 & 10 \\
$10 / 1 / 2001$ & $0.0 \mathrm{E}+00$ & & & & & 4 \\
$1 / 1 / 2002$ & $4.5 \mathrm{E}-01$ & 60,145 & 26,579 & 2,360 & 578,425 & 361,062 & 10 \\
$7 / 1 / 2002$ & $5.2 \mathrm{E}-01$ & 60,145 & 26,610 & 2,342 & 572,570 & 347,184 & 10 \\
$1 / 1 / 2003$ & $5.8 \mathrm{E}-01$ & 60,145 & 26,633 & 2,330 & 568,246 & 335,666 & 10 \\
$7 / 1 / 2003$ & $5.1 \mathrm{E}-01$ & 60,145 & 26,605 & 2,345 & 573,531 & 349,599 & 10 \\
$1 / 1 / 2004$ & $4.9 \mathrm{E}-01$ & 60,145 & 26,596 & 2,350 & 575,222 & 353,715 & 10 \\
$7 / 1 / 2004$ & $4.4 \mathrm{E}-01$ & 60,145 & 26,573 & 2,364 & 579,519 & 363,438 & 10
\end{tabular}

User Name: MV

State: Tennessee 
Project: Y-12 Security Complex

Location: LandfillsV and VII
User Name: $\mathrm{MV}$

State: Tennessee

\begin{tabular}{|c|c|c|c|c|c|}
\hline Moment Type & Constituent & $\begin{array}{l}\text { Coefficient } \\
\text { of Variation }\end{array}$ & $\begin{array}{c}\text { Mann-Kendall } \\
\text { S Statistic }\end{array}$ & $\begin{array}{l}\text { Confidence } \\
\text { in Trend }\end{array}$ & $\begin{array}{c}\text { Moment } \\
\text { Trend }\end{array}$ \\
\hline \multicolumn{6}{|c|}{ Zeroth Moment: Mass } \\
\hline & BERYLLIUM & 0.55 & -15 & $66.2 \%$ & S \\
\hline & CHROMIUM III & 0.64 & 55 & $94.8 \%$ & $\mathrm{PI}$ \\
\hline & LEAD & 1.44 & -81 & $99.3 \%$ & $\mathrm{D}$ \\
\hline & TETRACHLOROETHYLENE(PCE) & 0.56 & 59 & $96.0 \%$ & 1 \\
\hline \multicolumn{6}{|c|}{ 1st Moment: Distance to Source } \\
\hline & BERYLLIUM & 0.07 & 41 & $93.4 \%$ & $\mathrm{PI}$ \\
\hline & CHROMIUM III & 0.07 & -87 & $100.0 \%$ & $\mathrm{D}$ \\
\hline & LEAD & 0.15 & 29 & $85.3 \%$ & NT \\
\hline & TETRACHLOROETHYLENE(PCE) & 0.07 & -25 & $81.6 \%$ & $\mathrm{~S}$ \\
\hline \multicolumn{6}{|c|}{ 2nd Moment: Sigma XX } \\
\hline & BERYLLIUM & 0.26 & 7 & $58.9 \%$ & NT \\
\hline & CHROMIUM III & 0.30 & -1 & $50.0 \%$ & $\mathrm{~S}$ \\
\hline & LEAD & 0.33 & 43 & $94.4 \%$ & $\mathrm{PI}$ \\
\hline & TETRACHLOROETHYLENE(PCE) & 0.25 & -9 & $61.7 \%$ & $\mathrm{~S}$ \\
\hline \multicolumn{6}{|c|}{ 2nd Moment: Sigma YY } \\
\hline & BERYLLIUM & 0.12 & 5 & $55.9 \%$ & NT \\
\hline & CHROMIUM III & 0.23 & -69 & $99.6 \%$ & $\mathrm{D}$ \\
\hline & LEAD & 0.20 & 29 & $85.3 \%$ & NT \\
\hline & TETRACHLOROETHYLENE(PCE) & 0.08 & -15 & $70.0 \%$ & $\mathrm{~S}$ \\
\hline
\end{tabular}

Note: The following assumptions were applied for the calculation of the Zeroth Moment:

$$
\text { Porosity: } 0.10 \quad \text { Saturated Thickness: Uniform: } 50 \mathrm{ft}
$$

Mann-Kendall Trend test performed on all sample events for each constituent. Increasing (I); Probably Increasing (PI); Stable (S); Probably Decreasing (PD); Decreasing (D); No Trend (NT); Not Applicable (N/A)-Due to insufficient Data (< 4 sampling events).

Note: The Sigma $X X$ and Sigma $Y Y$ components are estimated using the given field coordinate system and then rotated to align with the estimated groundwater flow direction. Moments are not calculated for sample events with less than 6 wells. 


\section{ASSESSMENT OF THE GROUNDWATER PROTECTION PROGRAM \\ Y-12 NATIONAL SECURITY COMPLEX, OAK RIDGE, TENNESSEE}

Y-12 National Security Complex

Oak Ridge, Tennessee

\section{Appendix D.11 Chestnut Ridge Regime East Chestnut Ridge}

Table D.11.1 Qualitative Analysis East Chestnut Ridge

Table D.11.2 Aquifer Input Parameters

MAROS Report $\quad$ COC Assessment

MAROS Report Plume Summary

MAROS Report Spatial Moment Analysis Summary

(No Well Sufficiency areas of interest identified) 
TABLE D 111

QUALITATIVE ANALYSIS CHESTNUT RIDGE REGIME EAST CHESTNUT RIDGE

Chestnut Ridge Hydrogeologic Regime

Y-12 National Security Complex

Oak Ridge, Tennessee

\begin{tabular}{|c|c|c|c|c|c|c|c|c|c|c|c|c|}
\hline $\begin{array}{c}\text { Location } \\
\text { Name }\end{array}$ & $\begin{array}{c}\text { Location } \\
\text { Type }\end{array}$ & \begin{tabular}{|c|} 
Average \\
Concentration \\
Exceeds \\
Screening Level
\end{tabular} & $\begin{array}{c}\text { Formation } \\
\text { Type }\end{array}$ & $\begin{array}{c}\text { Horizontal } \\
\text { Delineation }\end{array}$ & $\begin{array}{c}\text { Vertical } \\
\text { Delineation }\end{array}$ & $\begin{array}{c}\text { Exit } \\
\text { Location }\end{array}$ & RCRA & CERCLA & Unique & $\begin{array}{c}\text { Monitors } \\
\text { Background } \\
\text { Water Quality }\end{array}$ & $\begin{array}{c}\text { Early } \\
\text { Detection }\end{array}$ & $\begin{array}{c}\text { Monitor } \\
\text { source }\end{array}$ \\
\hline \begin{tabular}{|l|}
$G W-142$ \\
$G W-143$ \\
GW-144 \\
GW-145 \\
GW-156
\end{tabular} & $\begin{array}{l}\text { WL } \\
\text { WL } \\
\text { WL } \\
\text { WL } \\
\text { WL }\end{array}$ & & $\begin{array}{l}\text { Aquifer } \\
\text { Aquifer } \\
\text { Aquifer } \\
\text { Aquifer } \\
\text { Aquifer }\end{array}$ & $\begin{array}{l}X \\
X \\
X \\
X \\
X\end{array}$ & $\begin{array}{l}x \\
x \\
x\end{array}$ & & $\begin{array}{l}X \\
X \\
X \\
x \\
X\end{array}$ & $\begin{array}{l}x \\
X \\
X\end{array}$ & & X & & $x$ \\
\hline $\begin{array}{l}\text { GW-159 } \\
\text { GW-160 } \\
\text { GW-231 } \\
\text { GW-241 } \\
\text { GW-292 }\end{array}$ & $\begin{array}{l}\text { WL } \\
W L \\
W L \\
W L \\
W L\end{array}$ & $\begin{array}{l}X \\
x\end{array}$ & $\begin{array}{l}\text { Aquifer } \\
\text { Aquifer } \\
\text { Aquifer } \\
\text { Aquifer } \\
\text { Aquifer }\end{array}$ & $\begin{array}{l}x \\
x \\
x\end{array}$ & $\begin{array}{l}x \\
x\end{array}$ & & $\begin{array}{l}x \\
x\end{array}$ & $x$ & & & & \\
\hline $\begin{array}{l}G W-293 \\
G W-298 \\
G W-299 \\
G W-300 \\
G W-301\end{array}$ & $\begin{array}{l}\text { WL } \\
W L \\
W L \\
W L \\
W L\end{array}$ & & $\begin{array}{l}\text { Aquifer } \\
\text { Aquifer } \\
\text { Aquifer } \\
\text { Aquifer } \\
\text { Aquifer }\end{array}$ & $x$ & $\begin{array}{l}X \\
X \\
X\end{array}$ & & $\mathrm{X}$ & & $\mathrm{X}$ & & & \\
\hline $\begin{array}{l}\text { GW-303 } \\
\text { GW-304 } \\
\text { GW-731 } \\
\text { GW-732 } \\
\text { GW-841 } \\
\end{array}$ & $\begin{array}{l}\text { WL } \\
W L \\
W L \\
W L \\
W L\end{array}$ & $x$ & $\begin{array}{c}\text { Aquifer } \\
\text { Aquifer } \\
\text { Aquifer } \\
\text { Aquifer } \\
\text { Aquitard }\end{array}$ & $\begin{array}{l}x \\
x\end{array}$ & $\begin{array}{l}X \\
X \\
X\end{array}$ & & $\begin{array}{l}x \\
x\end{array}$ & X & & $\begin{array}{l}x \\
x \\
x\end{array}$ & & \\
\hline $\begin{array}{l}\text { GW-842 } \\
\text { GW-843 } \\
\text { GW-844 } \\
\text { SCR5.1SP } \\
\text { SCR5.2SP } \\
\text { SCR5.4SP }\end{array}$ & $\begin{array}{l}\text { WL } \\
W L \\
W L \\
\text { SP } \\
\text { SP } \\
\text { SP }\end{array}$ & & $\begin{array}{c}\text { Aquitard } \\
\text { Aquitard } \\
\text { Aquitard } \\
\text { Spring } \\
\text { Spring } \\
\text { Spring }\end{array}$ & $\begin{array}{l}X \\
X\end{array}$ & $x$ & $\begin{array}{l}X \\
X \\
X\end{array}$ & & $\begin{array}{l}x \\
x \\
x\end{array}$ & & $\begin{array}{l}x \\
x\end{array}$ & & \\
\hline
\end{tabular}

\section{Notes:}

1. $\mathrm{WL}=$ Monitoring Well; $\mathrm{SP}=$ Spring

2. Well data taken from BWXT Y-12 Analytical Database. Sample locations shown on Figures A.1 and A.4.

3. RCRA indicates wells monitored as part of compliance with RCRA Post-Closure Corrective Action Monitoring or designated Alternate location;

CERCLA indicates locations monitored as part of compliance with CERCLA ROD or backup location. Data from BWXT, 2003a and BWXT 2004a.

4. Average Concentration Exceeds Screening = The average concentration over the entire sampling record for the priority constituent is above the MCL or other designated screening leve

as defined in Table B.1.

5. Aquifer and aquitard formations identified in Fig. A.2 from BWXT Y12, 2003 Groundwater Monitoring Report , (12/01/2003).

6. Details of the decision criteria for each category are presented in the text. 
GSI Job No. G-3038

Issued 12/12/2005

Page 1 of 1

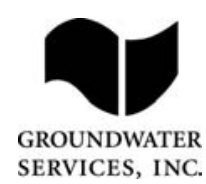

TABLE D.11.2

AQUIFER INPUT PARAMETERS

\author{
East Chestnut Ridge \\ Chestnut Ridge Regime \\ Y-12 National Security Complex
}

\begin{tabular}{|c|c|c|}
\hline Parameter & Value & Units \\
\hline Current Plume Length & 6500 & $\mathrm{ft}$ \\
\hline Maximum Plume Length & 6500 & $\mathrm{ft}$ \\
\hline PlumeWidth & 2000 & $\mathrm{ft}$ \\
\hline SeepageVelocity (ft/yr) & 200 & $\mathrm{ft} / \mathrm{yr}$ \\
\hline Distance to Receptors & 6000 & $\mathrm{ft}$ \\
\hline GWFluctuations & Yes & -- \\
\hline SourceTreatment & None & -- \\
\hline PlumeType & VOC & -- \\
\hline Free NAPL Present & Yes & -- \\
\hline Parameter & Value & \\
\hline Groundwater flow direction & $\bar{S}$ & 270 \\
\hline Effective Porosity & 0.1 & -- \\
\hline Source Location near Well & GW-292 & - \\
\hline Source X-Coordinate & 62146.29 & $\mathrm{ft}^{\star}$ \\
\hline Source Y-Coordinate & 28140.54 & $\mathrm{ft}^{\star}$ \\
\hline Saturated Thickness & 50 & $\mathrm{ft}$ \\
\hline \multicolumn{3}{|l|}{ Source Wells } \\
\hline & $93, \mathrm{C}$ & \\
\hline
\end{tabular}

Notes:

1. Aquifer data are general values for the hydrologic regime.

2. Priority COCs defined by prevalence, toxicty and mobility.

3. $\mathrm{ft}^{*}=$ Coordinates in $\mathrm{Y}-12$ Plant coordinates, feet.

4. Screening Levels are USEPA MCLs, except in the case of compounds without MCLS where the level is the Region 9 PRG for tap water.

5. Effective Porosity estimated based on average high and low values for aquifer and aquitard suburfaces. 


\section{MAROS COC Assessment}

Project: Y-12 Scurity Complex

Location: East Chestnut Ridge

\section{Toxicity:}

Contaminant of Concern

TRICHLOROETHYLENE (TCE)
User Name: $\mathrm{MV}$

State: Tennessee

Note: Top COCs by toxicity were determined by examining a representative concentration for each compound over the entire site. The compound representative concentrations are then compared with the chosen PRG for that compound, with the percentage excedence from the PRG determining the compound's toxicity. All compounds above exceed the PRG.

\section{Prevalence:}

\begin{tabular}{|c|c|c|c|c|c|}
\hline Contaminant of Concern & Class & $\begin{array}{l}\text { Total } \\
\text { Wells }\end{array}$ & $\begin{array}{c}\text { Total } \\
\text { Excedences }\end{array}$ & $\begin{array}{c}\text { Percent } \\
\text { Excedences }\end{array}$ & $\begin{array}{l}\text { Total } \\
\text { detects }\end{array}$ \\
\hline TRICHLOROETHYLENE (TCE) & ORG & 26 & 1 & $3.8 \%$ & 12 \\
\hline
\end{tabular}

Note: Top COCs by prevalence were determined by examining a representative concentration for each well location at the site. The total excedences (values above the chosen PRGs) are compared to the total number of wells to determine the prevalence of the compound.

\section{Mobility:}

Contaminant of Concern

TRICHLOROETHYLENE (TCE)
$\mathrm{Kd}$

0.297

Note: Top COCs by mobility were determined by examining each detected compound in the dataset and comparing their mobilities (Koc's for organics, assume foc $=0.001$, and Kd's for metals).

\section{Contaminants of Concern (COC's)}

TRICHLOROETHYLENE (TCE)

LEAD

CADMIUM

BENZENE

GROSS ALPHA ACTIVITY 


\section{MAROS Plume Analysis Summary}

Project: $\quad \mathrm{Y}-12$ Scurity Complex

Location: East Chestnut Ridge

Time Period: 1/1/1996

to $1 / 1 / 2005$

Consolidation Period: No Time Consolidation

Consolidation Type: Median

Duplicate Consolidation: Average

ND Values: Specified Detection Limit

J Flag Values : Actual Value

\begin{tabular}{|c|c|c|c|c|c|c|c|c|c|c|c|}
\hline Constituent & Well & $\begin{array}{c}\text { Sourcel } \\
\text { Tail }\end{array}$ & $\begin{array}{l}\text { Number } \\
\text { of } \\
\text { Samples }\end{array}$ & $\begin{array}{c}\text { Number } \\
\text { of } \\
\text { Detects }\end{array}$ & $\begin{array}{c}\text { Average } \\
\text { (mg/L) }\end{array}$ & $\begin{array}{r}\text { Median } \\
\text { (mg/L) }\end{array}$ & $\begin{array}{c}\text { All } \\
\text { Samples } \\
\text { "ND" ? }\end{array}$ & $\begin{array}{l}\text { Mann- } \\
\text { Kendall }\end{array}$ & $\begin{array}{c}\text { Linear } \\
\text { Regression }\end{array}$ & Modeling & Empirical \\
\hline \multicolumn{12}{|l|}{ BENZENE } \\
\hline & GW-293 & $\mathrm{S}$ & 1 & 0 & $5.0 \mathrm{E}-04$ & 5.0E-04 & Yes & N/A & N/A & N/A & N/A \\
\hline & GW-292 & s & 1 & 0 & $5.0 \mathrm{E}-04$ & 5.0E-04 & Yes & $\mathrm{N} / \mathrm{A}$ & N/A & $\mathrm{N} / \mathrm{A}$ & $\mathrm{N} / \mathrm{A}$ \\
\hline & GW-160 & $\mathrm{s}$ & 1 & 0 & $5.0 \mathrm{E}-04$ & 5.0E-04 & Yes & N/A & $\mathrm{N} / \mathrm{A}$ & $\mathrm{N} / \mathrm{A}$ & N/A \\
\hline & GW-844 & $\mathrm{T}$ & 1 & 1 & $6.0 \mathrm{E}-03$ & $6.0 \mathrm{E}-03$ & No & $N / A$ & $N / A$ & $N / A$ & N/A \\
\hline & GW-301 & $\mathrm{T}$ & 18 & 0 & $5.0 \mathrm{E}-04$ & 5.0E-04 & Yes & $\mathrm{s}$ & $\mathrm{s}$ & $\mathrm{N} / \mathrm{A}$ & N/A \\
\hline & GW-300 & $\mathrm{T}$ & 3 & 0 & $5.0 \mathrm{E}-04$ & 5.0E-04 & Yes & N/A & $\mathrm{N} / \mathrm{A}$ & $\mathrm{N} / \mathrm{A}$ & N/A \\
\hline & GW-299 & $\mathrm{T}$ & 1 & 0 & $5.0 \mathrm{E}-04$ & 5.0E-04 & Yes & $N / A$ & N/A & $N / A$ & N/A \\
\hline & GW-841 & $\mathrm{T}$ & 8 & 0 & $5.0 \mathrm{E}-04$ & 5.0E-04 & Yes & $\mathrm{s}$ & $\mathrm{s}$ & N/A & $\mathrm{N} / \mathrm{A}$ \\
\hline & GW-142 & $\mathrm{T}$ & 17 & 0 & $5.0 \mathrm{E}-04$ & 5.0E-04 & Yes & $\mathrm{s}$ & s & N/A & N/A \\
\hline & GW-842 & $\mathrm{T}$ & 11 & 0 & $5.0 \mathrm{E}-04$ & $5.0 \mathrm{E}-04$ & Yes & $\mathrm{s}$ & D & N/A & N/A \\
\hline & GW-843 & $\mathrm{T}$ & 11 & 0 & $5.0 \mathrm{E}-04$ & 5.0E-04 & Yes & $\mathrm{s}$ & D & $N / A$ & $N / A$ \\
\hline & GW-241 & $\mathrm{T}$ & 2 & 0 & $5.0 \mathrm{E}-04$ & 5.0E-04 & Yes & $\mathrm{N} / \mathrm{A}$ & $\mathrm{N} / \mathrm{A}$ & $\mathrm{N} / \mathrm{A}$ & N/A \\
\hline & GW-298 & $\mathrm{T}$ & 1 & 0 & $5.0 \mathrm{E}-04$ & 5.0E-04 & Yes & N/A & N/A & $N / A$ & N/A \\
\hline & GW-231 & $\mathrm{T}$ & 19 & 0 & $5.0 \mathrm{E}-04$ & 5.0E-04 & Yes & $\mathrm{s}$ & $\mathrm{s}$ & $N / A$ & $N / A$ \\
\hline & GW-145 & $\mathrm{T}$ & 19 & 0 & $5.0 \mathrm{E}-04$ & 5.0E-04 & Yes & $\mathrm{s}$ & $\mathrm{s}$ & $N / A$ & $\mathrm{~N} / \mathrm{A}$ \\
\hline & GW-143 & $\mathrm{T}$ & 19 & 0 & $5.0 \mathrm{E}-04$ & 5.0E-04 & Yes & $\mathrm{s}$ & $\mathrm{s}$ & N/A & N/A \\
\hline & SCR5_4SP & $\mathrm{T}$ & 10 & 0 & $5.0 \mathrm{E}-04$ & $5.0 \mathrm{E}-04$ & Yes & $\mathrm{s}$ & S & $N / A$ & $N / A$ \\
\hline & GW-144 & $\mathrm{T}$ & 19 & 0 & $5.0 \mathrm{E}-04$ & 5.0E-04 & Yes & $\mathrm{s}$ & $\mathrm{s}$ & $N / A$ & N/A \\
\hline & SCR5_1SP & $\mathrm{T}$ & 10 & 0 & $5.0 \mathrm{E}-04$ & 5.0E-04 & Yes & $\mathrm{s}$ & s & N/A & N/A \\
\hline & SCR5_2SP & $\mathrm{T}$ & 4 & 0 & $5.0 \mathrm{E}-04$ & 5.0E-04 & Yes & $\mathrm{S}$ & S & $N / A$ & $N / A$ \\
\hline CADMIUM & & & & & & & & & & & \\
\hline
\end{tabular}


Project: Y-12 Scurity Complex

Location: East Chestnut Ridge
User Name: MV

State: Tennessee

\begin{tabular}{|c|c|c|c|c|c|c|c|c|c|c|c|}
\hline Constituent & Well & $\begin{array}{c}\text { Sourcel } \\
\text { Tail }\end{array}$ & $\begin{array}{l}\text { Number } \\
\text { of } \\
\text { Samples }\end{array}$ & $\begin{array}{c}\text { Number } \\
\text { of } \\
\text { Detects }\end{array}$ & $\begin{array}{c}\text { Average } \\
\text { (mg/L) }\end{array}$ & $\begin{array}{r}\text { Median } \\
(\mathrm{mg} / \mathrm{L})\end{array}$ & $\begin{array}{c}\text { All } \\
\text { Samples } \\
\text { "ND" ? }\end{array}$ & $\begin{array}{l}\text { Mann- } \\
\text { Kendall }\end{array}$ & $\begin{array}{c}\text { Linear } \\
\text { Regression }\end{array}$ & Modeling & Empirical \\
\hline \multicolumn{12}{|l|}{ CADMIUM } \\
\hline & GW-293 & $\mathrm{s}$ & 1 & 0 & $8.0 \mathrm{E}-05$ & $8.0 \mathrm{E}-05$ & Yes & N/A & $\mathrm{N} / \mathrm{A}$ & N/A & N/A \\
\hline & GW-292 & s & 1 & 0 & $8.0 \mathrm{E}-05$ & 8.0E-05 & Yes & $N / A$ & $\mathrm{~N} / \mathrm{A}$ & $N / A$ & N/A \\
\hline & GW-160 & s & 1 & 0 & $8.0 \mathrm{E}-05$ & 8.0E-05 & Yes & $\mathrm{N} / \mathrm{A}$ & $\mathrm{N} / \mathrm{A}$ & $\mathrm{N} / \mathrm{A}$ & N/A \\
\hline & GW-145 & $\mathrm{T}$ & 20 & 0 & $8.0 \mathrm{E}-05$ & 8.0E-05 & Yes & s & s & N/A & N/A \\
\hline & GW-241 & $\mathrm{T}$ & 2 & 0 & $8.0 \mathrm{E}-05$ & 8.0E-05 & Yes & $N / A$ & N/A & N/A & N/A \\
\hline & GW-301 & $\mathrm{T}$ & 18 & 0 & $8.0 \mathrm{E}-05$ & 8.0E-05 & Yes & $\mathrm{s}$ & $\mathrm{s}$ & N/A & N/A \\
\hline & GW-231 & $\mathrm{T}$ & 20 & 0 & $8.0 \mathrm{E}-05$ & 8.0E-05 & Yes & S & S & $\mathrm{N} / \mathrm{A}$ & N/A \\
\hline & GW-156 & $\mathrm{T}$ & 18 & 0 & $8.0 \mathrm{E}-05$ & 8.0E-05 & Yes & s & s & $\mathrm{N} / \mathrm{A}$ & N/A \\
\hline & GW-298 & $\mathrm{T}$ & 1 & 0 & $8.0 \mathrm{E}-05$ & 8.0E-05 & Yes & $N / A$ & $N / A$ & N/A & N/A \\
\hline & GW-159 & $\mathrm{T}$ & 18 & 0 & $8.0 \mathrm{E}-05$ & 8.0E-05 & Yes & s & s & N/A & N/A \\
\hline & GW-143 & $\mathrm{T}$ & 20 & 0 & $8.0 \mathrm{E}-05$ & 8.0E-05 & Yes & s & s & N/A & N/A \\
\hline & GW-299 & $\mathrm{T}$ & 1 & 0 & $8.0 \mathrm{E}-05$ & 8.0E-05 & Yes & $N / A$ & $N / A$ & N/A & N/A \\
\hline & GW-300 & $\mathrm{T}$ & 3 & 0 & $8.0 \mathrm{E}-05$ & 8.0E-05 & Yes & $N / A$ & $N / A$ & N/A & N/A \\
\hline & GW-142 & $\mathrm{T}$ & 18 & 1 & $9.3 \mathrm{E}-05$ & 8.0E-05 & No & NT & NT & $\mathrm{N} / \mathrm{A}$ & N/A \\
\hline & GW-144 & $\mathrm{T}$ & 20 & 0 & $8.0 \mathrm{E}-05$ & 8.0E-05 & Yes & $\mathrm{s}$ & $\mathrm{s}$ & N/A & N/A \\
\hline & GW-732 & $\mathrm{T}$ & 18 & 0 & $8.0 \mathrm{E}-05$ & 8.0E-05 & Yes & s & S & N/A & N/A \\
\hline & GW-842 & $\mathrm{T}$ & 3 & 0 & 8.0E-05 & 8.0E-05 & Yes & $\mathrm{N} / \mathrm{A}$ & N/A & $\mathrm{N} / \mathrm{A}$ & N/A \\
\hline & GW-841 & $\mathrm{T}$ & 2 & 0 & 8.0E-05 & 8.0E-05 & Yes & $\mathrm{N} / \mathrm{A}$ & N/A & $\mathrm{N} / \mathrm{A}$ & N/A \\
\hline & SCR5_4SP & $\mathrm{T}$ & 8 & 0 & $8.0 \mathrm{E}-05$ & 8.0E-05 & Yes & S & S & N/A & N/A \\
\hline & SCR5_2SP & $\mathrm{T}$ & 4 & 0 & 8.0E-05 & 8.0E-05 & Yes & s & s & N/A & N/A \\
\hline & GW-843 & $\mathrm{T}$ & 3 & 0 & 8.0E-05 & 8.0E-05 & Yes & $N / A$ & $N / A$ & $N / A$ & N/A \\
\hline & GW-731 & $\mathrm{T}$ & 18 & 1 & $1.1 \mathrm{E}-04$ & 8.0E-05 & No & NT & NT & N/A & N/A \\
\hline & SCR5_1SP & $\mathrm{T}$ & 8 & 0 & 8.0E-05 & 8.0E-05 & Yes & $\mathrm{s}$ & $S$ & $\mathrm{~N} / \mathrm{A}$ & N/A \\
\hline \multicolumn{12}{|c|}{ GROSS ALPHA ACTIVITY } \\
\hline & GW-292 & $\mathrm{S}$ & 1 & 1 & $1.5 \mathrm{E}+00$ & $1.5 \mathrm{E}+00$ & No & N/A & $\mathrm{N} / \mathrm{A}$ & $\mathrm{N} / \mathrm{A}$ & $\mathrm{N} / \mathrm{A}$ \\
\hline & GW-160 & s & 1 & 1 & $3.0 \mathrm{E}+00$ & $3.0 \mathrm{E}+00$ & No & N/A & N/A & N/A & N/A \\
\hline & GW-293 & $\mathrm{s}$ & 1 & 1 & $2.1 \mathrm{E}+00$ & $2.1 \mathrm{E}+00$ & No & $\mathrm{N} / \mathrm{A}$ & $N / A$ & $\mathrm{~N} / \mathrm{A}$ & N/A \\
\hline & SCR5_1SP & $\mathrm{T}$ & 4 & 4 & $1.2 \mathrm{E}+00$ & $1.1 \mathrm{E}+00$ & No & NT & NT & N/A & N/A \\
\hline & GW-298 & $\mathrm{T}$ & 1 & 1 & $3.4 \mathrm{E}+00$ & $3.4 \mathrm{E}+00$ & No & $\mathrm{N} / \mathrm{A}$ & $\mathrm{N} / \mathrm{A}$ & N/A & N/A \\
\hline & GW-231 & $\mathrm{T}$ & 19 & 14 & $9.0 \mathrm{E}-01$ & 4.9E-01 & No & $\mathrm{D}$ & $\mathrm{D}$ & N/A & N/A \\
\hline & GW-159 & $\mathrm{T}$ & 2 & 2 & $6.0 \mathrm{E}+01$ & $6.0 \mathrm{E}+01$ & No & $\mathrm{N} / \mathrm{A}$ & $\mathrm{N} / \mathrm{A}$ & N/A & N/A \\
\hline & GW-156 & $\mathrm{T}$ & 2 & 2 & $3.6 \mathrm{E}+00$ & $3.6 \mathrm{E}+00$ & No & $\mathrm{N} / \mathrm{A}$ & $\mathrm{N} / \mathrm{A}$ & N/A & N/A \\
\hline & GW-145 & $\mathrm{T}$ & 19 & 19 & $1.1 \mathrm{E}+01$ & $1.1 \mathrm{E}+01$ & No & $\mathrm{s}$ & $\mathrm{s}$ & N/A & N/A \\
\hline
\end{tabular}


Project: Y-12 Scurity Complex

Location: East Chestnut Ridge
User Name: MV

State: Tennessee

\begin{tabular}{|c|c|c|c|c|c|c|c|c|c|c|c|}
\hline Constituent & Well & $\begin{array}{c}\text { Sourcel } \\
\text { Tail }\end{array}$ & $\begin{array}{c}\text { Number } \\
\text { of } \\
\text { Samples }\end{array}$ & $\begin{array}{c}\text { Number } \\
\text { of } \\
\text { Detects }\end{array}$ & $\begin{array}{c}\text { Average } \\
\text { (mg/L) }\end{array}$ & $\begin{array}{c}\text { Median } \\
(\mathrm{mg} / \mathrm{L})\end{array}$ & $\begin{array}{c}\text { All } \\
\text { Samples } \\
\text { "ND" ? }\end{array}$ & $\begin{array}{l}\text { Mann- } \\
\text { Kendall }\end{array}$ & $\begin{array}{c}\text { Linear } \\
\text { Regression }\end{array}$ & Modeling & Empirical \\
\hline \multicolumn{12}{|c|}{ GROSS ALPHA ACTIVITY } \\
\hline & SCR5_2SP & $T$ & 1 & 1 & $1.4 \mathrm{E}+00$ & $1.4 \mathrm{E}+00$ & No & N/A & N/A & N/A & $\mathrm{N} / \mathrm{A}$ \\
\hline & GW-241 & $\mathrm{T}$ & 1 & 1 & 3.4E-01 & 3.4E-01 & No & N/A & N/A & N/A & N/A \\
\hline & GW-143 & $\mathrm{T}$ & 19 & 17 & $3.1 \mathrm{E}+00$ & $2.8 \mathrm{E}+00$ & No & $\mathrm{D}$ & $\mathrm{D}$ & N/A & N/A \\
\hline & GW-144 & $\mathrm{T}$ & 19 & 19 & $2.1 \mathrm{E}+00$ & $2.0 \mathrm{E}+00$ & No & NT & NT & N/A & N/A \\
\hline & GW-301 & $\mathrm{T}$ & 15 & 9 & 7.7E-01 & 4.9E-01 & No & NT & NT & N/A & N/A \\
\hline & GW-731 & T & 2 & 2 & $1.7 \mathrm{E}+00$ & $1.7 \mathrm{E}+00$ & No & N/A & $\mathrm{N} / \mathrm{A}$ & N/A & N/A \\
\hline & GW-142 & $\mathrm{T}$ & 17 & 16 & $3.3 \mathrm{E}+00$ & 1.7E+00 & No & D & D & N/A & $N / A$ \\
\hline & GW-732 & $\mathrm{T}$ & 2 & 2 & $1.1 \mathrm{E}+00$ & $1.1 \mathrm{E}+00$ & No & N/A & N/A & N/A & $\mathrm{N} / \mathrm{A}$ \\
\hline & GW-299 & $T$ & 1 & 1 & $2.0 \mathrm{E}+00$ & $2.0 \mathrm{E}+00$ & No & N/A & $\mathrm{N} / \mathrm{A}$ & N/A & N/A \\
\hline & SCR5_4SP & $\mathrm{T}$ & 8 & 8 & 8.9E-01 & 6.3E-01 & No & NT & NT & N/A & $N / A$ \\
\hline & GW-300 & $T$ & 3 & 3 & $5.5 \mathrm{E}+00$ & $3.2 E+00$ & No & N/A & N/A & N/A & N/A \\
\hline \multicolumn{12}{|l|}{ LEAD } \\
\hline & GW-160 & $\mathrm{S}$ & 1 & 1 & $3.1 \mathrm{E}-02$ & 3.1E-02 & No & $\mathrm{N} / \mathrm{A}$ & $\mathrm{N} / \mathrm{A}$ & $\mathrm{N} / \mathrm{A}$ & $\mathrm{N} / \mathrm{A}$ \\
\hline & GW-292 & s & 1 & 0 & $5.2 \mathrm{E}-05$ & $5.2 \mathrm{E}-05$ & Yes & N/A & N/A & N/A & $\mathrm{N} / \mathrm{A}$ \\
\hline & GW-293 & s & 1 & 0 & $5.2 \mathrm{E}-05$ & 5.2E-05 & Yes & N/A & N/A & N/A & $N / A$ \\
\hline & SCR5_4SP & $\mathrm{T}$ & 8 & 1 & $1.5 \mathrm{E}-04$ & 5.2E-05 & No & NT & $P D$ & N/A & $\mathrm{N} / \mathrm{A}$ \\
\hline & GW-142 & $\mathrm{T}$ & 18 & 5 & 4.7E-04 & $5.2 \mathrm{E}-05$ & No & $\mathrm{PD}$ & NT & $\mathrm{N} / \mathrm{A}$ & $\mathrm{N} / \mathrm{A}$ \\
\hline & GW-159 & $\mathrm{T}$ & 18 & 3 & $3.8 \mathrm{E}-04$ & 5.2E-05 & No & $\mathrm{D}$ & $\mathrm{D}$ & N/A & $N / A$ \\
\hline & GW-156 & $\mathrm{T}$ & 18 & 5 & $5.6 \mathrm{E}-04$ & 5.2E-05 & No & D & D & N/A & $\mathrm{N} / \mathrm{A}$ \\
\hline & GW-843 & $T$ & 3 & 0 & $5.2 \mathrm{E}-05$ & $5.2 \mathrm{E}-05$ & Yes & N/A & $\mathrm{N} / \mathrm{A}$ & N/A & N/A \\
\hline & GW-145 & $\mathrm{T}$ & 20 & 4 & 1.3E-04 & 5.2E-05 & No & $\mathrm{D}$ & $\mathrm{D}$ & N/A & $N / A$ \\
\hline & GW-143 & $\mathrm{T}$ & 20 & 4 & 7.6E-05 & 5.2E-05 & No & D & D & N/A & $\mathrm{N} / \mathrm{A}$ \\
\hline & SCR5_2SP & $\mathrm{T}$ & 4 & 1 & $5.0 \mathrm{E}-04$ & $5.2 \mathrm{E}-05$ & No & NT & NT & N/A & N/A \\
\hline & GW-144 & $\mathrm{T}$ & 20 & 4 & 8.3E-05 & $5.2 \mathrm{E}-05$ & No & $\mathrm{s}$ & D & N/A & $N / A$ \\
\hline & SCR5_1SP & $\mathrm{T}$ & 8 & 1 & $9.2 \mathrm{E}-05$ & $5.2 \mathrm{E}-05$ & No & NT & NT & N/A & N/A \\
\hline & GW-842 & $\mathrm{T}$ & 3 & 0 & $5.2 \mathrm{E}-05$ & $5.2 \mathrm{E}-05$ & Yes & N/A & N/A & N/A & $\mathrm{N} / \mathrm{A}$ \\
\hline & GW-732 & $\mathrm{T}$ & 18 & 5 & 3.4E-04 & $5.2 \mathrm{E}-05$ & No & D & $\mathrm{D}$ & N/A & $N / A$ \\
\hline & GW-298 & $\mathrm{T}$ & 1 & 0 & $5.2 \mathrm{E}-05$ & $5.2 \mathrm{E}-05$ & Yes & N/A & N/A & N/A & N/A \\
\hline & GW-299 & $T$ & 1 & 0 & $5.2 \mathrm{E}-05$ & 5.2E-05 & Yes & N/A & $\mathrm{N} / \mathrm{A}$ & $\mathrm{N} / \mathrm{A}$ & $\mathrm{N} / \mathrm{A}$ \\
\hline & GW-841 & $\mathrm{T}$ & 2 & 0 & $5.2 \mathrm{E}-05$ & 5.2E-05 & Yes & N/A & N/A & N/A & $N / A$ \\
\hline & GW-731 & $\mathrm{T}$ & 18 & 4 & $2.5 \mathrm{E}-04$ & $5.2 \mathrm{E}-05$ & No & $\mathrm{D}$ & $\mathrm{D}$ & $\mathrm{N} / \mathrm{A}$ & $\mathrm{N} / \mathrm{A}$ \\
\hline & GW-241 & $T$ & 2 & 1 & $5.8 \mathrm{E}-04$ & $5.8 \mathrm{E}-04$ & No & N/A & $\mathrm{N} / \mathrm{A}$ & $\mathrm{N} / \mathrm{A}$ & $N / A$ \\
\hline & GW-300 & $\mathrm{T}$ & 3 & 2 & 4.4E-04 & 2.8E-04 & No & N/A & N/A & N/A & N/A \\
\hline
\end{tabular}


Project: Y-12 Scurity Complex

Location: East Chestnut Ridge
User Name: $\quad$ MV

State: Tennessee

\begin{tabular}{|c|c|c|c|c|c|c|c|c|c|c|c|}
\hline Constituent & Well & $\begin{array}{c}\text { Sourcel } \\
\text { Tail }\end{array}$ & $\begin{array}{l}\text { Number } \\
\text { of } \\
\text { Samples }\end{array}$ & $\begin{array}{c}\text { Number } \\
\text { of } \\
\text { Detects }\end{array}$ & $\begin{array}{c}\text { Average } \\
\text { (mg/L) }\end{array}$ & $\begin{array}{r}\text { Median } \\
(\mathrm{mg} / \mathrm{L})\end{array}$ & $\begin{array}{c}\text { All } \\
\text { Samples } \\
\text { "ND" ? }\end{array}$ & $\begin{array}{l}\text { Mann- } \\
\text { Kendall }\end{array}$ & $\begin{array}{c}\text { Linear } \\
\text { Regression }\end{array}$ & Modeling & Empirical \\
\hline \multicolumn{12}{|l|}{ LEAD } \\
\hline & GW-231 & $T$ & 20 & 4 & $1.8 \mathrm{E}-04$ & $5.2 \mathrm{E}-05$ & No & $\mathrm{D}$ & D & $\mathrm{N} / \mathrm{A}$ & $\mathrm{N} / \mathrm{A}$ \\
\hline & GW-301 & $\mathrm{T}$ & 18 & 4 & $4.9 \mathrm{E}-04$ & $5.2 \mathrm{E}-05$ & No & $\mathrm{D}$ & $\mathrm{D}$ & $\mathrm{N} / \mathrm{A}$ & N/A \\
\hline \multicolumn{12}{|c|}{ TRICHLOROETHYLENE (TCE) } \\
\hline & GW-292 & $\mathrm{S}$ & 1 & 0 & $5.0 \mathrm{E}-04$ & $5.0 \mathrm{E}-04$ & Yes & N/A & N/A & $\mathrm{N} / \mathrm{A}$ & N/A \\
\hline & GW-293 & $\mathrm{s}$ & 1 & 0 & $5.0 \mathrm{E}-04$ & 5.0E-04 & Yes & $\mathrm{N} / \mathrm{A}$ & N/A & N/A & N/A \\
\hline & GW-160 & $\mathrm{s}$ & 1 & 0 & $5.0 \mathrm{E}-04$ & 5.0E-04 & Yes & N/A & N/A & N/A & N/A \\
\hline & GW-142 & $\mathrm{T}$ & 17 & 0 & $5.0 \mathrm{E}-04$ & $5.0 \mathrm{E}-04$ & Yes & $\mathrm{s}$ & $\mathrm{s}$ & $N / A$ & $N / A$ \\
\hline & GW-300 & $\mathrm{T}$ & 3 & 0 & $5.0 \mathrm{E}-04$ & $5.0 \mathrm{E}-04$ & Yes & N/A & N/A & N/A & $N / A$ \\
\hline & GW-143 & $\mathrm{T}$ & 19 & 0 & $5.0 \mathrm{E}-04$ & $5.0 \mathrm{E}-04$ & Yes & $\mathrm{s}$ & s & N/A & N/A \\
\hline & SCR5_2SP & $\mathrm{T}$ & 4 & 0 & $5.0 \mathrm{E}-04$ & $5.0 \mathrm{E}-04$ & Yes & $\mathrm{s}$ & $\mathrm{s}$ & N/A & $N / A$ \\
\hline & GW-299 & $\mathrm{T}$ & 1 & 0 & $5.0 \mathrm{E}-04$ & 5.0E-04 & Yes & $N / A$ & $\mathrm{~N} / \mathrm{A}$ & $N / A$ & $\mathrm{~N} / \mathrm{A}$ \\
\hline & GW-298 & $\mathrm{T}$ & 1 & 0 & $5.0 \mathrm{E}-04$ & 5.0E-04 & Yes & N/A & N/A & $\mathrm{N} / \mathrm{A}$ & N/A \\
\hline & GW-231 & $\mathrm{T}$ & 19 & 1 & $5.1 \mathrm{E}-04$ & 5.0E-04 & No & $\mathrm{s}$ & $\mathrm{s}$ & $N / A$ & $\mathrm{~N} / \mathrm{A}$ \\
\hline & GW-144 & $\mathrm{T}$ & 19 & 0 & $5.0 \mathrm{E}-04$ & 5.0E-04 & Yes & $S$ & $S$ & $\mathrm{~N} / \mathrm{A}$ & $\mathrm{N} / \mathrm{A}$ \\
\hline & GW-841 & $\mathrm{T}$ & 8 & 8 & $1.1 \mathrm{E}-01$ & 3.0E-02 & No & D & D & N/A & $\mathrm{N} / \mathrm{A}$ \\
\hline & GW-145 & $\mathrm{T}$ & 19 & 2 & $5.3 \mathrm{E}-04$ & 5.0E-04 & No & $\mathrm{s}$ & $\mathrm{s}$ & $N / A$ & N/A \\
\hline & SCR5_1SP & $\mathrm{T}$ & 10 & 0 & $5.0 \mathrm{E}-04$ & $5.0 \mathrm{E}-04$ & Yes & $\mathrm{s}$ & $\mathrm{s}$ & $N / A$ & $N / A$ \\
\hline & GW-842 & $\mathrm{T}$ & 11 & 11 & $3.2 \mathrm{E}-03$ & 1.5E-03 & No & NT & NT & $\mathrm{N} / \mathrm{A}$ & N/A \\
\hline & GW-241 & $\mathrm{T}$ & 2 & 0 & $5.0 \mathrm{E}-04$ & 5.0E-04 & Yes & N/A & N/A & $\mathrm{N} / \mathrm{A}$ & $\mathrm{N} / \mathrm{A}$ \\
\hline & GW-301 & $\mathrm{T}$ & 18 & 0 & $5.0 \mathrm{E}-04$ & $5.0 \mathrm{E}-04$ & Yes & $\mathrm{s}$ & $\mathrm{s}$ & $N / A$ & $N / A$ \\
\hline & GW-843 & $\mathrm{T}$ & 11 & 0 & $5.0 \mathrm{E}-04$ & 5.0E-04 & Yes & $\mathrm{s}$ & D & $N / A$ & N/A \\
\hline & GW-844 & $\mathrm{T}$ & 1 & 0 & $5.0 \mathrm{E}-04$ & 5.0E-04 & Yes & N/A & $\mathrm{N} / \mathrm{A}$ & $\mathrm{N} / \mathrm{A}$ & $\mathrm{N} / \mathrm{A}$ \\
\hline & SCR5_4SP & T & 10 & 0 & $5.0 \mathrm{E}-04$ & $5.0 \mathrm{E}-04$ & Yes & $\mathrm{s}$ & $\mathrm{s}$ & $N / A$ & $N / A$ \\
\hline
\end{tabular}

Note: Increasing (I); Probably Increasing (PI); Stable (S); Probably Decreasing (PD); Decreasing (D); No Trend (NT); Not Applicable (N/A) - Due to insufficient Data (< 4 sampling events); Source/Tail (S/T)

The Number of Samples and Number of Detects shown above are post-consolidation values. 


\section{MAROS Spatial Moment Analysis Summary}

Project: Y-12 Scurity Complex

Location: East Chestnut Ridge
User Name: MV

State: Tennessee

\begin{tabular}{|c|c|c|c|c|c|c|c|}
\hline \multirow[b]{2}{*}{ Effective Date } & \multirow{2}{*}{$\begin{array}{l}\text { 0th Moment } \\
\text { Estimated } \\
\text { Mass (Kg) }\end{array}$} & \multicolumn{3}{|c|}{$\underline{1 s t}$ Moment (Center of Mass) } & \multicolumn{2}{|c|}{ 2nd Moment (Spread) } & \multirow[b]{2}{*}{$\begin{array}{c}\text { Number of } \\
\text { Wells }\end{array}$} \\
\hline & & Xc (ft) & Yc (ft) & $\begin{array}{c}\text { Source } \\
\text { Distance (ft) }\end{array}$ & $\underset{(s q \mathrm{ft})}{\operatorname{Sigma} x X}$ & $\begin{array}{c}\text { Sigma YY } \\
(\mathrm{sq} f \mathrm{ft})\end{array}$ & \\
\hline
\end{tabular}

BENZENE

\begin{tabular}{|c|c|c|c|c|c|c|}
\hline 1/1/1996 & $0.0 \mathrm{E}+00$ & & & & & \\
\hline $4 / 1 / 1996$ & 1.7E-01 & 62,954 & 26,063 & 2,230 & 145,839 & 683,097 \\
\hline 10/1/1996 & 9.7E-02 & 63,118 & 25,473 & 2,839 & 48,570 & 151,188 \\
\hline 1/1/1997 & $0.0 \mathrm{E}+00$ & & & & & \\
\hline 4/1/1997 & 8.0E-02 & 63,545 & 24,753 & 3,665 & 15,165 & 263,660 \\
\hline $7 / 1 / 1997$ & $0.0 \mathrm{E}+00$ & & & & & \\
\hline 10/1/1997 & $0.0 \mathrm{E}+00$ & & & & & \\
\hline 1/1/1998 & $0.0 \mathrm{E}+00$ & & & & & \\
\hline $4 / 1 / 1998$ & $0.0 \mathrm{E}+00$ & & & & & \\
\hline $7 / 1 / 1998$ & $0.0 \mathrm{E}+00$ & & & & & \\
\hline 10/1/1998 & $0.0 \mathrm{E}+00$ & & & & & \\
\hline 1/1/1999 & 5.1E-01 & 63,837 & 24,754 & 3,785 & 503,531 & 580,491 \\
\hline 4/1/1999 & $0.0 \mathrm{E}+00$ & & & & & \\
\hline 7/1/1999 & 5.1E-01 & 63,837 & 24,754 & 3,785 & 503,531 & 580,491 \\
\hline 10/1/1999 & $0.0 \mathrm{E}+00$ & & & & & \\
\hline $1 / 1 / 2000$ & $0.0 \mathrm{E}+00$ & & & & & \\
\hline $4 / 1 / 2000$ & $0.0 \mathrm{E}+00$ & & & & & \\
\hline $7 / 1 / 2000$ & $0.0 \mathrm{E}+00$ & & & & & \\
\hline $10 / 1 / 2000$ & $0.0 \mathrm{E}+00$ & & & & & \\
\hline $1 / 1 / 2001$ & $6.5 \mathrm{E}-01$ & 63,865 & 25,034 & 3,550 & 496,180 & $1,012,586$ \\
\hline $4 / 1 / 2001$ & $0.0 \mathrm{E}+00$ & & & & & \\
\hline $7 / 1 / 2001$ & $6.5 \mathrm{E}-01$ & 63,865 & 25,034 & 3,550 & 496,180 & $1,012,586$ \\
\hline $10 / 1 / 2001$ & $0.0 \mathrm{E}+00$ & & & & & \\
\hline $1 / 1 / 2002$ & $0.0 \mathrm{E}+00$ & & & & & \\
\hline $4 / 1 / 2002$ & $0.0 \mathrm{E}+00$ & & & & & \\
\hline $7 / 1 / 2002$ & $0.0 \mathrm{E}+00$ & & & & & \\
\hline $10 / 1 / 2002$ & $0.0 \mathrm{E}+00$ & & & & & \\
\hline $1 / 1 / 2003$ & $0.0 \mathrm{E}+00$ & & & & & \\
\hline 4/1/2003 & $0.0 \mathrm{E}+00$ & & & & & \\
\hline 7/1/2003 & $0.0 \mathrm{E}+00$ & & & & & \\
\hline $10 / 1 / 2003$ & $0.0 \mathrm{E}+00$ & & & & & \\
\hline $1 / 1 / 2004$ & $0.0 \mathrm{E}+00$ & & & & & \\
\hline $4 / 1 / 2004$ & $0.0 \mathrm{E}+00$ & & & & & \\
\hline $7 / 1 / 2004$ & $0.0 \mathrm{E}+00$ & & & & & \\
\hline $10 / 1 / 2004$ & $0.0 \mathrm{E}+00$ & & & & & \\
\hline
\end{tabular}

CADMIUM

\begin{tabular}{|c|c|c|c|c|c|c|c|}
\hline 1/1/1996 & $0.0 \mathrm{E}+00$ & & & & & & 5 \\
\hline 4/1/1996 & $6.3 \mathrm{E}-02$ & 63,285 & 26,470 & 2,022 & 223,472 & 618,607 & 16 \\
\hline
\end{tabular}


Project: Y-12 Scurity Complex

Location: East Chestnut Ridge
State: Tennessee
User Name: MV

\begin{tabular}{|c|c|c|c|c|c|c|c|}
\hline \multirow[b]{2}{*}{ Effective Date } & \multirow{2}{*}{$\begin{array}{r}\text { 0th Moment } \\
\begin{array}{r}\text { Estimated } \\
\text { Mass (kg) }\end{array}\end{array}$} & \multicolumn{3}{|c|}{ 1st Moment (Center of Mass) } & \multicolumn{2}{|c|}{$\underline{\text { 2nd Moment (Spread) }}$} & \multirow[b]{2}{*}{$\begin{array}{c}\text { Number of } \\
\text { Wells }\end{array}$} \\
\hline & & $\mathrm{Xc}(\mathrm{ft})$ & Yc (ft) & $\begin{array}{c}\text { Source } \\
\text { Distance (ft) }\end{array}$ & $\begin{array}{c}\text { Sigma XX } \\
\text { (sq ft) }\end{array}$ & $\begin{array}{l}\text { Sigma YY } \\
(\mathrm{sq} \mathrm{ft})\end{array}$ & \\
\hline \multicolumn{8}{|l|}{ CADMIUM } \\
\hline 10/1/1996 & $5.8 \mathrm{E}-02$ & 63,337 & 26,340 & 2,159 & 157,231 & 433,565 & 10 \\
\hline $1 / 1 / 1997$ & $0.0 \mathrm{E}+00$ & & & & & & 1 \\
\hline $4 / 1 / 1997$ & $2.9 \mathrm{E}-02$ & 63,758 & 26,062 & 2,630 & 41,990 & 517,221 & 9 \\
\hline 7/1/1997 & $1.5 \mathrm{E}-02$ & 63,118 & 25,473 & 2,839 & 48,570 & 151,188 & 6 \\
\hline 10/1/1997 & $2.9 \mathrm{E}-02$ & 63,758 & 26,062 & 2,630 & 41,990 & 517,221 & 9 \\
\hline $1 / 1 / 1998$ & $0.0 \mathrm{E}+00$ & & & & & & 3 \\
\hline $4 / 1 / 1998$ & $2.9 \mathrm{E}-02$ & 63,758 & 26,062 & 2,630 & 41,990 & 517,221 & 9 \\
\hline 7/1/1998 & $0.0 \mathrm{E}+00$ & & & & & & 3 \\
\hline 10/1/1998 & $5.4 \mathrm{E}-02$ & 63,779 & 26,129 & 2,591 & 37,497 & 431,763 & 9 \\
\hline 1/1/1999 & $8.1 \mathrm{E}-02$ & 63,837 & 24,754 & 3,785 & 503,531 & 580,491 & 6 \\
\hline $4 / 1 / 1999$ & $2.9 \mathrm{E}-02$ & 63,758 & 26,062 & 2,630 & 41,990 & 517,221 & 9 \\
\hline $7 / 1 / 1999$ & $0.0 \mathrm{E}+00$ & & & & & & 3 \\
\hline 10/1/1999 & $2.9 \mathrm{E}-02$ & 63,758 & 26,062 & 2,630 & 41,990 & 517,221 & 9 \\
\hline $1 / 1 / 2000$ & $0.0 \mathrm{E}+00$ & & & & & & 3 \\
\hline $4 / 1 / 2000$ & $2.9 \mathrm{E}-02$ & 63,758 & 26,062 & 2,630 & 41,990 & 517,221 & 9 \\
\hline $7 / 1 / 2000$ & $0.0 \mathrm{E}+00$ & & & & & & 3 \\
\hline $10 / 1 / 2000$ & 4.0E-02 & 63,786 & 26,008 & 2,691 & 38,964 & 489,746 & 9 \\
\hline $1 / 1 / 2001$ & $0.0 \mathrm{E}+00$ & & & & & & 5 \\
\hline $4 / 1 / 2001$ & $2.9 \mathrm{E}-02$ & 63,758 & 26,062 & 2,630 & 41,990 & 517,221 & 9 \\
\hline $7 / 1 / 2001$ & $0.0 \mathrm{E}+00$ & & & & & & 5 \\
\hline $10 / 1 / 2001$ & $2.9 \mathrm{E}-02$ & 63,758 & 26,062 & 2,630 & 41,990 & 517,221 & 9 \\
\hline $1 / 1 / 2002$ & $0.0 \mathrm{E}+00$ & & & & & & 2 \\
\hline $4 / 1 / 2002$ & $2.9 \mathrm{E}-02$ & 63,758 & 26,062 & 2,630 & 41,990 & 517,221 & 9 \\
\hline $7 / 1 / 2002$ & $0.0 \mathrm{E}+00$ & & & & & & 2 \\
\hline $10 / 1 / 2002$ & $2.9 \mathrm{E}-02$ & 63,758 & 26,062 & 2,630 & 41,990 & 517,221 & 9 \\
\hline $1 / 1 / 2003$ & $0.0 \mathrm{E}+00$ & & & & & & 1 \\
\hline 4/1/2003 & $2.9 \mathrm{E}-02$ & 63,758 & 26,062 & 2,630 & 41,990 & 517,221 & 9 \\
\hline 7/1/2003 & $0.0 \mathrm{E}+00$ & & & & & & 1 \\
\hline $10 / 1 / 2003$ & $2.9 \mathrm{E}-02$ & 63,758 & 26,062 & 2,630 & 41,990 & 517,221 & 9 \\
\hline $1 / 1 / 2004$ & $0.0 \mathrm{E}+00$ & & & & & & 4 \\
\hline $4 / 1 / 2004$ & $4.7 \mathrm{E}-02$ & 63,255 & 26,453 & 2,019 & 97,915 & 321,951 & 9 \\
\hline 7/1/2004 & $0.0 \mathrm{E}+00$ & & & & & & 3 \\
\hline $10 / 1 / 2004$ & 4.7E-02 & 63,255 & 26,453 & 2,019 & 97,915 & 321,951 & 9 \\
\hline \multicolumn{8}{|l|}{ GROSS ALPHA ACTIVITY } \\
\hline 1/1/1996 & $0.0 \mathrm{E}+00$ & & & & & & 5 \\
\hline 4/1/1996 & $2.5 \mathrm{E}+03$ & 63,197 & 26,783 & 1,717 & 212,358 & 862,900 & 15 \\
\hline 10/1/1996 & $9.7 \mathrm{E}+02$ & 63,421 & 26,160 & 2,356 & 176,327 & 635,407 & 10 \\
\hline 1/1/1997 & $0.0 \mathrm{E}+00$ & & & & & & 1 \\
\hline $4 / 1 / 1997$ & $0.0 \mathrm{E}+00$ & & & & & & 5 \\
\hline 7/1/1997 & $0.0 \mathrm{E}+00$ & & & & & & 1 \\
\hline 10/1/1997 & $0.0 \mathrm{E}+00$ & & & & & & 5 \\
\hline $1 / 1 / 1998$ & $0.0 \mathrm{E}+00$ & & & & & & 1 \\
\hline
\end{tabular}


Project: Y-12 Scurity Complex

Location: East Chestnut Ridge

\begin{tabular}{|c|c|c|c|c|c|c|c|}
\hline \multirow[b]{2}{*}{ Effective Date } & \multirow{2}{*}{$\begin{array}{l}\text { 0th Moment } \\
\text { Estimated } \\
\text { Mass (kg) }\end{array}$} & \multicolumn{3}{|c|}{ 1st Moment (Center of Mass) } & \multicolumn{2}{|c|}{ 2nd Moment (Spread) } & \multirow[b]{2}{*}{$\begin{array}{c}\text { Number of } \\
\text { Wells }\end{array}$} \\
\hline & & $\mathrm{Xc}(\mathrm{ft})$ & Yc (ft) & $\begin{array}{c}\text { Source } \\
\text { Distance (ft) }\end{array}$ & $\underset{\text { (sq ft) }}{\text { Sigma } X X}$ & $\begin{array}{c}\text { Sigma YY } \\
\text { (sq ft) }\end{array}$ & \\
\hline \multicolumn{8}{|l|}{ GROSS ALPHA ACTIVITY } \\
\hline 4/1/1998 & $0.0 E+00$ & & & & & & 5 \\
\hline 7/1/1998 & $0.0 \mathrm{E}+00$ & & & & & & 2 \\
\hline 10/1/1998 & $0.0 \mathrm{E}+00$ & & & & & & 5 \\
\hline 1/1/1999 & $0.0 \mathrm{E}+00$ & & & & & & 2 \\
\hline 4/1/1999 & $0.0 \mathrm{E}+00$ & & & & & & 5 \\
\hline 7/1/1999 & $0.0 \mathrm{E}+00$ & & & & & & 2 \\
\hline 10/1/1999 & $0.0 \mathrm{E}+00$ & & & & & & 5 \\
\hline $1 / 1 / 2000$ & $0.0 \mathrm{E}+00$ & & & & & & 3 \\
\hline $4 / 1 / 2000$ & $0.0 \mathrm{E}+00$ & & & & & & 5 \\
\hline 7/1/2000 & $0.0 \mathrm{E}+00$ & & & & & & 3 \\
\hline $10 / 1 / 2000$ & $0.0 \mathrm{E}+00$ & & & & & & 5 \\
\hline $1 / 1 / 2001$ & $0.0 \mathrm{E}+00$ & & & & & & 4 \\
\hline 4/1/2001 & $0.0 \mathrm{E}+00$ & & & & & & 5 \\
\hline 7/1/2001 & $0.0 \mathrm{E}+00$ & & & & & & 2 \\
\hline 10/1/2001 & $0.0 \mathrm{E}+00$ & & & & & & 5 \\
\hline $1 / 1 / 2002$ & $0.0 \mathrm{E}+00$ & & & & & & 1 \\
\hline 4/1/2002 & $0.0 \mathrm{E}+00$ & & & & & & 5 \\
\hline 7/1/2002 & $0.0 \mathrm{E}+00$ & & & & & & 2 \\
\hline 10/1/2002 & $0.0 \mathrm{E}+00$ & & & & & & 5 \\
\hline $1 / 1 / 2003$ & $0.0 \mathrm{E}+00$ & & & & & & 1 \\
\hline 4/1/2003 & $0.0 \mathrm{E}+00$ & & & & & & 5 \\
\hline 7/1/2003 & $0.0 \mathrm{E}+00$ & & & & & & 1 \\
\hline $10 / 1 / 2003$ & $0.0 \mathrm{E}+00$ & & & & & & 5 \\
\hline $1 / 1 / 2004$ & $0.0 \mathrm{E}+00$ & & & & & & 1 \\
\hline 4/1/2004 & $0.0 \mathrm{E}+00$ & & & & & & 5 \\
\hline $7 / 1 / 2004$ & $0.0 E+00$ & & & & & & 1 \\
\hline $10 / 1 / 2004$ & $0.0 E+00$ & & & & & & 5 \\
\hline
\end{tabular}

User Name: MV

State: Tennessee

\begin{abstract}
Location: East Chestnut Ridge
\end{abstract}
Moment

10/1/2004

LEAD

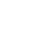

\begin{tabular}{|c|c|c|c|c|c|c|c|}
\hline 1/1/1996 & $0.0 \mathrm{E}+00$ & & & & & & 5 \\
\hline 4/1/1996 & 7.7E-02 & 62,939 & 27,057 & 1,343 & 388,564 & 743,005 & 16 \\
\hline 10/1/1996 & 5.1E-01 & 63,250 & 26,529 & 1,953 & 125,762 & 384,340 & 10 \\
\hline 1/1/1997 & $0.0 \mathrm{E}+00$ & & & & & & 1 \\
\hline 4/1/1997 & $3.9 \mathrm{E}-01$ & 63,780 & 26,286 & 2,472 & 42,291 & 498,833 & 9 \\
\hline 7/1/1997 & $1.1 \mathrm{E}-01$ & 63,107 & 25,560 & 2,754 & 28,288 & 75,226 & 6 \\
\hline 10/1/1997 & 1.3E-01 & 63,798 & 26,170 & 2,571 & 40,663 & 419,897 & 9 \\
\hline 1/1/1998 & $0.0 \mathrm{E}+00$ & & & & & & 3 \\
\hline 4/1/1998 & $2.9 \mathrm{E}-02$ & 63,810 & 26,085 & 2,645 & 43,847 & 639,701 & 9 \\
\hline 7/1/1998 & $0.0 \mathrm{E}+00$ & & & & & & 3 \\
\hline 10/1/1998 & $7.5 \mathrm{E}-02$ & 63,790 & 26,143 & 2,587 & 36,941 & 387,976 & 9 \\
\hline 1/1/1999 & $1.0 \mathrm{E}-01$ & 63,813 & 24,783 & 3,749 & 478,574 & 545,780 & 6 \\
\hline 4/1/1999 & 1.9E-02 & 63,758 & 26,062 & 2,630 & 41,990 & 517,221 & 9 \\
\hline $7 / 1 / 1999$ & $0.0 \mathrm{E}+00$ & & & & & & 3 \\
\hline
\end{tabular}


Project: Y-12 Scurity Complex

Location: East Chestnut Ridge

0th Moment

Estimated

Effective Date 1st Moment (Center of Mass)

Source $\mathrm{Xc}(\mathrm{ft})$
Distance (ft)
User Name: MV

State: Tennessee

2nd Moment (Spread)

$\begin{array}{ccc}\text { Sigma XX } & \begin{array}{c}\text { Sigma YY } \\ (\mathrm{sq} f \mathrm{ft})\end{array} & \begin{array}{c}\text { Number of } \\ \text { Wells }\end{array}\end{array}$

Wells

LEAD

$\begin{array}{rr}10 / 1 / 1999 & 2.0 \mathrm{E}-02 \\ 1 / 1 / 2000 & 0.0 \mathrm{E}+00 \\ 4 / 1 / 2000 & 1.9 \mathrm{E}-02 \\ 7 / 1 / 2000 & 0.0 \mathrm{E}+00 \\ 10 / 1 / 2000 & 1.9 \mathrm{E}-02 \\ 1 / 1 / 2001 & 0.0 \mathrm{E}+00 \\ 4 / 1 / 2001 & 1.9 \mathrm{E}-02 \\ 7 / 1 / 2001 & 0.0 \mathrm{E}+00 \\ 10 / 1 / 2001 & 1.9 \mathrm{E}-02 \\ 1 / 1 / 2002 & 0.0 \mathrm{E}+00 \\ 4 / 1 / 2002 & 1.9 \mathrm{E}-02 \\ 7 / 1 / 2002 & 0.0 \mathrm{E}+00 \\ 10 / 1 / 2002 & 5.3 \mathrm{E}-02 \\ 1 / 1 / 2003 & 0.0 \mathrm{E}+00 \\ 4 / 1 / 2003 & 1.9 \mathrm{E}-02 \\ 7 / 1 / 2003 & 0.0 \mathrm{E}+00 \\ 10 / 1 / 2003 & 1.9 \mathrm{E}-02 \\ 1 / 1 / 2004 & 0.0 \mathrm{E}+00 \\ 4 / 1 / 2004 & 7.0 \mathrm{E}-02 \\ 7 / 1 / 2004 & 0.0 \mathrm{E}+00 \\ 10 / 1 / 2004 & 4.8 \mathrm{E}-02\end{array}$

$\begin{array}{llllll}63,765 & 26,168 & 2,552 & 40,604 & 638,997 & 9 \\ 63,758 & 26,062 & 2,630 & 41,990 & 517,221 & 3 \\ 63,758 & 26,062 & 2,630 & 41,990 & 517,221 & 9 \\ 63,758 & 26,062 & 2,630 & 41,990 & 517,221 & 9 \\ 63,758 & 26,062 & 2,630 & 41,990 & 517,221 & 5 \\ & & & & 5 \\ 63,758 & 26,062 & 2,630 & 41,990 & 517,221 & 9 \\ & & & & 9 \\ 63,827 & 25,931 & 2,777 & 31,886 & 440,880 & 2 \\ 63,758 & 26,062 & 2,630 & 41,990 & 517,221 & 9 \\ & & & & 9 \\ 63,758 & 26,062 & 2,630 & 41,990 & 517,221 & 1 \\ & & & & & 9 \\ 63,158 & 26,490 & 1,936 & 53,037 & 261,660 & 4 \\ & & & & & 9 \\ 63,192 & 26,477 & 1,965 & 70,929 & 283,178 & 9\end{array}$

TRICHLOROETHYLENE (TCE)

\begin{tabular}{|c|c|c|c|c|c|c|c|}
\hline 1/1/1996 & $0.0 \mathrm{E}+00$ & & & & & & 5 \\
\hline 4/1/1996 & 1.7E-01 & 62,954 & 26,063 & 2,230 & 145,839 & 683,097 & 12 \\
\hline 10/1/1996 & $9.7 \mathrm{E}-02$ & 63,118 & 25,473 & 2,839 & 48,570 & 151,188 & 6 \\
\hline $1 / 1 / 1997$ & $0.0 \mathrm{E}+00$ & & & & & & 5 \\
\hline 4/1/1997 & 8.1E-02 & 63,541 & 24,749 & 3,667 & 15,643 & 264,710 & 7 \\
\hline 7/1/1997 & $0.0 \mathrm{E}+00$ & & & & & & 3 \\
\hline 10/1/1997 & $0.0 \mathrm{E}+00$ & & & & & & 5 \\
\hline 1/1/1998 & $0.0 \mathrm{E}+00$ & & & & & & 3 \\
\hline 4/1/1998 & $0.0 \mathrm{E}+00$ & & & & & & 5 \\
\hline 7/1/1998 & $0.0 \mathrm{E}+00$ & & & & & & 3 \\
\hline 10/1/1998 & $0.0 E+00$ & & & & & & 5 \\
\hline 1/1/1999 & $3.2 \mathrm{E}+00$ & 64,244 & 24,321 & 4,357 & 148,168 & 181,055 & 6 \\
\hline 4/1/1999 & $0.0 \mathrm{E}+00$ & & & & & & 5 \\
\hline 7/1/1999 & $3.0 \mathrm{E}+00$ & 64,238 & 24,327 & 4,350 & 155,130 & 188,824 & 6 \\
\hline 10/1/1999 & $0.0 \mathrm{E}+00$ & & & & & & 5 \\
\hline $1 / 1 / 2000$ & $0.0 \mathrm{E}+00$ & & & & & & 3 \\
\hline $4 / 1 / 2000$ & $0.0 \mathrm{E}+00$ & & & & & & 5 \\
\hline $7 / 1 / 2000$ & $0.0 \mathrm{E}+00$ & & & & & & 3 \\
\hline $10 / 1 / 2000$ & $0.0 \mathrm{E}+00$ & & & & & & 5 \\
\hline $1 / 1 / 2001$ & $1.6 \mathrm{E}+00$ & 64,147 & 24,735 & 3,950 & 285,398 & 780,760 & 8 \\
\hline
\end{tabular}


Project: Y-12 Scurity Complex

Location: East Chestnut Ridge

\begin{tabular}{|c|c|c|c|c|c|c|c|}
\hline \multirow[b]{2}{*}{ Effective Date } & th Moment & \multicolumn{3}{|c|}{ 1st Moment (Center of Mass) } & \multicolumn{2}{|c|}{ 2nd Moment (Spread) } & \multirow[b]{2}{*}{$\begin{array}{c}\text { Number of } \\
\text { Wells }\end{array}$} \\
\hline & $\begin{array}{l}\text { Estimated } \\
\text { Mass (kg) }\end{array}$ & $X c(f t)$ & Yc (ft) & $\begin{array}{c}\text { Source } \\
\text { Distance (ft) }\end{array}$ & $\begin{array}{c}\text { Sigma } X X \\
(\mathrm{sq} \mathrm{ft})\end{array}$ & $\begin{array}{l}\text { Sigma } Y Y \\
\text { (sq ft) }\end{array}$ & \\
\hline
\end{tabular}

TRICHLOROETHYLENE (TCE)

\begin{tabular}{|c|c|c|c|c|c|c|c|}
\hline $4 / 1 / 2001$ & $0.0 \mathrm{E}+00$ & & & & & & 5 \\
\hline $7 / 1 / 2001$ & $1.9 \mathrm{E}+00$ & 64,187 & 24,692 & 4,007 & 242,866 & 733,622 & 8 \\
\hline $10 / 1 / 2001$ & $0.0 \mathrm{E}+00$ & & & & & & 5 \\
\hline $1 / 1 / 2002$ & $0.0 \mathrm{E}+00$ & & & & & & 5 \\
\hline 4/1/2002 & $0.0 \mathrm{E}+00$ & & & & & & 5 \\
\hline $7 / 1 / 2002$ & $0.0 \mathrm{E}+00$ & & & & & & 4 \\
\hline 10/1/2002 & $0.0 \mathrm{E}+00$ & & & & & & 5 \\
\hline $1 / 1 / 2003$ & $0.0 \mathrm{E}+00$ & & & & & & 4 \\
\hline $4 / 1 / 2003$ & $0.0 \mathrm{E}+00$ & & & & & & 5 \\
\hline $7 / 1 / 2003$ & $0.0 \mathrm{E}+00$ & & & & & & 3 \\
\hline $10 / 1 / 2003$ & $0.0 \mathrm{E}+00$ & & & & & & 5 \\
\hline $1 / 1 / 2004$ & $0.0 \mathrm{E}+00$ & & & & & & 4 \\
\hline $4 / 1 / 2004$ & $0.0 \mathrm{E}+00$ & & & & & & 5 \\
\hline $7 / 1 / 2004$ & $0.0 \mathrm{E}+00$ & & & & & & 3 \\
\hline $10 / 1 / 2004$ & $0.0 \mathrm{E}+00$ & & & & & & 5 \\
\hline
\end{tabular}


Project: Y-12 Scurity Complex

Location: East Chestnut Ridge
User Name: $\mathrm{MV}$

State: Tennessee

\begin{tabular}{|c|c|c|c|c|c|}
\hline Moment Type & Constituent & $\begin{array}{l}\text { Coefficient } \\
\text { of Variation }\end{array}$ & $\begin{array}{l}\text { Mann-Kendall } \\
\text { S Statistic }\end{array}$ & $\begin{array}{l}\text { Confidence } \\
\text { in Trend }\end{array}$ & $\begin{array}{c}\text { Moment } \\
\text { Trend }\end{array}$ \\
\hline \multicolumn{6}{|c|}{ Zeroth Moment: Mass } \\
\hline & BENZENE & 2.47 & -81 & $87.1 \%$ & NT \\
\hline & CADMIUM & 1.04 & -26 & $63.8 \%$ & NT \\
\hline & GROSS ALPHA ACTIVITY & 4.54 & -63 & $80.9 \%$ & NT \\
\hline & LEAD & 2.12 & -126 & $96.2 \%$ & $\mathrm{D}$ \\
\hline & TRICHLOROETHYLENE (TCE) & 2.84 & -91 & $89.9 \%$ & NT \\
\hline \multicolumn{6}{|c|}{ 1st Moment: Distance to Source } \\
\hline & BENZENE & 0.18 & 9 & $88.1 \%$ & NT \\
\hline & CADMIUM & 0.15 & -22 & $75.0 \%$ & $\mathrm{~S}$ \\
\hline & GROSS ALPHA ACTIVITY & 0.00 & 0 & $0.0 \%$ & N/A \\
\hline & LEAD & 0.18 & 28 & $80.7 \%$ & NT \\
\hline & TRICHLOROETHYLENE (TCE) & 0.22 & 11 & $93.2 \%$ & $\mathrm{PI}$ \\
\hline \multicolumn{6}{|c|}{ 2nd Moment: Sigma XX } \\
\hline & BENZENE & 0.74 & 5 & $71.9 \%$ & NT \\
\hline & CADMIUM & 1.28 & 20 & $72.9 \%$ & NT \\
\hline & GROSS ALPHA ACTIVITY & 0.00 & 0 & $0.0 \%$ & $\mathrm{~N} / \mathrm{A}$ \\
\hline & LEAD & 1.42 & -18 & $70.7 \%$ & NT \\
\hline & TRICHLOROETHYLENE (TCE) & 0.65 & 13 & $96.5 \%$ & 1 \\
\hline \multicolumn{6}{|c|}{ 2nd Moment: Sigma YY } \\
\hline & BENZENE & 0.54 & 11 & $93.2 \%$ & $\mathrm{PI}$ \\
\hline & CADMIUM & 0.22 & -28 & $80.7 \%$ & $\mathrm{~S}$ \\
\hline & GROSS ALPHA ACTIVITY & 0.00 & 0 & $0.0 \%$ & N/A \\
\hline & LEAD & 0.31 & -24 & $77.0 \%$ & $\mathrm{~S}$ \\
\hline & TRICHLOROETHYLENE (TCE) & 0.68 & 7 & $80.9 \%$ & NT \\
\hline
\end{tabular}

Note: The following assumptions were applied for the calculation of the Zeroth Moment:

Porosity: $0.10 \quad$ Saturated Thickness: Uniform: $50 \mathrm{ft}$

Mann-Kendall Trend test performed on all sample events for each constituent. Increasing (I); Probably Increasing (PI); Stable (S); Probably Decreasing (PD); Decreasing (D); No Trend (NT); Not Applicable (N/A)-Due to insufficient Data (< 4 sampling events).

Note: The Sigma $X X$ and Sigma $Y Y$ components are estimated using the given field coordinate system and then rotated to align with the estimated groundwater flow direction. Moments are not calculated for sample events with less than 6 wells. 


\section{DISTRIBUTION}

U.S. DEPARTMENT OF ENERGY

J.D. Darby, DOE-EM

J.P. Donnelly, DOE-NNSA

TENNESSEE DEPARTMENT OF

ENVIRONMENT AND CONSERVATION

DOE-ORR OVERSIGHT DIVISION

D. Gilmore

BWXT Y-12, L.L.C.

ENVIRONMENTAL COMPLIANCE

DEPARTMENT

S.M. Field

C.C. Hill

S.B. Jones

E.R. Schultz

O. D. Stevens

L.O. Vaughan

File - GWPP (2)

EC Document Center

Y-12 Central Files

Y-12 Records Services (Electronic copy- OSTI)

YDCC - RC $\underline{\text { BECHTEL JACOBS COMPANY LLC }}$

R.H. Ketelle

L.M. Sims

ELVADO ENVIRONMENTAL LLC

T.R. Harrison

J.R. Walker

UT-BATTELLE, LLC

D.B. Watson

SCIENCE APPLICATIONS

INTERNATIONAL CORPORATION

W.K. Jago 